\title{
MANUAL
}

INL/EXT-19-53844

Revision 0

Printed May 26, 2021

\section{RAVEN regression tests' description}

Andrea Alfonsi, Paul W. Talbot, Diego Mandelli, Congjian Wang, Joshua Cogliati, Cristian Rabiti

Prepared by

Idaho National Laboratory

Idaho Falls, Idaho 83415

The Idaho National Laboratory is a multiprogram laboratory operated by

Battelle Energy Alliance for the United States Department of Energy

under DOE Idaho Operations Office. Contract DE-AC07-05ID14517.

Approved for unlimited release.

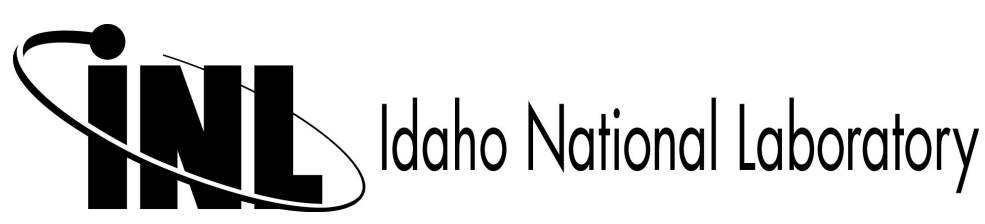


Issued by the Idaho National Laboratory, operated for the United States Department of Energy by Battelle Energy Alliance.

NOTICE: This report was prepared as an account of work sponsored by an agency of the United States Government. Neither the United States Government, nor any agency thereof, nor any of their employees, nor any of their contractors, subcontractors, or their employees, make any warranty, express or implied, or assume any legal liability or responsibility for the accuracy, completeness, or usefulness of any information, apparatus, product, or process disclosed, or represent that its use would not infringe privately owned rights. Reference herein to any specific commercial product, process, or service by trade name, trademark, manufacturer, or otherwise, does not necessarily constitute or imply its endorsement, recommendation, or favoring by the United States Government, any agency thereof, or any of their contractors or subcontractors. The views and opinions expressed herein do not necessarily state or reflect those of the United States Government, any agency thereof, or any of their contractors.

Printed in the United States of America. This report has been reproduced directly from the best available copy.

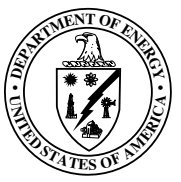


INL/EXT-19-53844

Revision 0

Printed May 26, 2021

\title{
RAVEN regression tests' description
}

\author{
Andrea Alfonsi \\ Paul W. Talbot \\ Diego Mandelli \\ Congjian Wang \\ Joshua Cogliati \\ Cristian Rabiti
}




\section{Introduction}

This document has been automatically generated by the script "raven \developer_tools $\backslash$ createRegressionTestDocumentation.py" Currently there are 887 regression tests in the RAVEN framework. The \% of tests that are commented is currently equal to $97.519729425 \%$. 


\section{Documented Tests}

Regression tests for the Python RAVEN framework are found in raven/tests/framework. There is a hierarchy of folders with tests collected by similar testing. Every test is described in a special XML node $(<$ TestInfo $>$ ) within the $<$ Simulation $>$ block. An example is reported below:

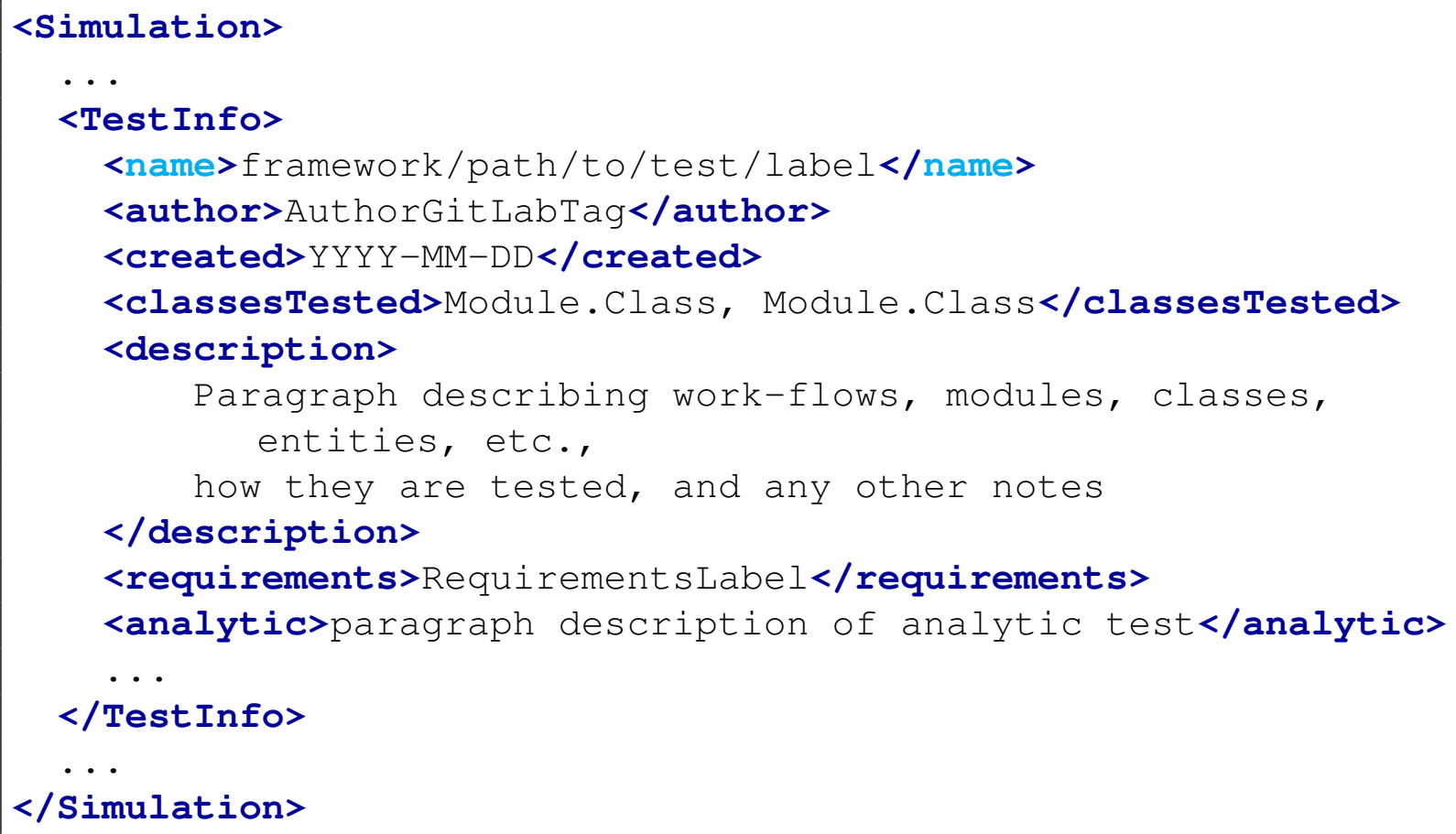

The $<$ requirements $>$ and $<$ analytic $>$ nodes are optional, for those tests who satisfy an NQA design requirement and or have an analytic solution documented in the analytic tests document. Other notes on block contents:

- $<$ name $>$ : this is the test framework path, as well as the name (label) assigned in the tests file block. This is the path and name that show up when running the tests using the testing harness (run_tests)

- $<$ author $>$ : this is the GitLab tag of the author who constructed this test originally, i.e. alfoafor@alfoa

- $<$ created $>$ : this is the date on which the test was originally created, in year-month-day $Y Y Y Y-M M-D D X S D$ date format

- $<$ classesTested $>$ : a list of the classes tested in the python framework, listed as Entity.Class, i.e. Samplers.MonteCarlo 
- $<$ description $>$ : general notes about what work-flows or other methods are tested

- < requirements $>$ (optional): lists the NQA requirement that this test satisfies

- $<$ analytic $>$ (optional): describes the analytic nature of this test and how it is documented in the analytic tests documentation

An additional node is optionally available to demonstrate significant revisions to a test:

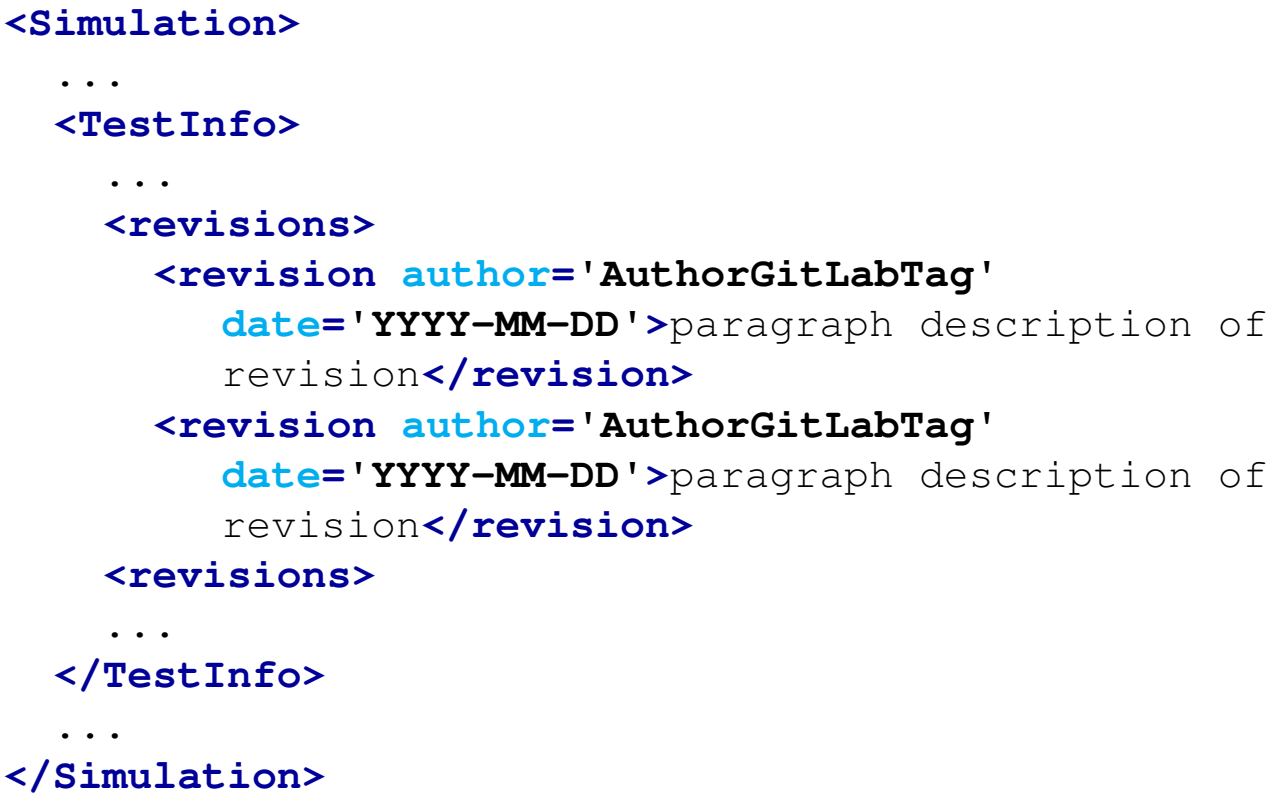

The following sub-sections collect all the documented tests.

\subsection{Requirement tests' description}

This section contains the description of all the requirement tests.

\subsubsection{PLUGINS FARM.RG PARAMETERIZED SIMO}

This test can be found at ". / raven/plugins/FARM/tests/test_RefGov_para_xmlABC. xml". This test can be called executing the following command:

./run_tests --re=plugins/FARM.RG_parameterized_SIMO

or 
./run_framework_tests --re=plugins/FARM.RG_parameterized_SIMO

- Test Description:

- This is a test run of parameterized reference governor. It loads ABC matrices from external xml file and calculate the feedback from RefGov_parameterized_SIMO external model.

- Original Author:

- HaoyuWang

- Creation date:

$-2021-02-01$

- The classes tested in this test are:

- Models.ExternalModel.FARM

- This test fulfills the following requirement:

$-\mathrm{F}-1$

\subsubsection{PLUGINS FARM.RG NOT PARAMETERIZED SIMO}

This test can be found at "./raven/plugins/FARM/tests/test_RefGov_unpara_ xmlABC.xml". This test can be called executing the following command:

./run_tests --re=plugins/FARM.RG_not_parameterized_SIMO

or

./run_framework_tests --re=plugins/FARM.RG_not_parameterized_sIMф

- Test Description:

- This is a test run of single reference governor. It loads ABC matrices from external xml file.

- Original Author:

- HaoyuWang 
- Creation date:

- 2020-07-29

- The classes tested in this test are:

- Models.ExternalModel.FARM

- This test fulfills the following requirement:

$-\mathrm{F}-1$

\subsubsection{FRAMEWORK.TESTGRAPHMODEL}

This test can be found at “. / raven/plugins/SR2ML/tests/test_graphModel.xml”. This test can be called executing the following command:

./run_tests --re=framework. TestGraphModel

or

./run_framework_tests --re=framework. TestGraphModel

- Test Description:

- This test is aimed to check the Graph Model

- Original Author:

- mandd, wangc

- Creation date:

- 2019-05-08

- The classes tested in this test are:

- Models.ExternalModel.GraphModel

- This test fulfills the following requirement:

- SR2ML-PRA-4

- Since the creation of this test, the following main revisions have been performed:

1. revision info: 
- author: wangc

- date : 2019-11-27

- description: Added Plugin name extension

\subsubsection{FRAMEWORK.TESTMARKOVMODEL 2STATES}

This test can be found at ". / raven/plugins/SR2ML/tests/test_markovModel_2states. xml". This test can be called executing the following command:

. Irun_tests --re=framework. TestMarkovModel_2states

or

./run_framework_tests --re=framework. TestMarkovModel_2states

- Test Description:

- This test is aimed to check the Markov Model

- Original Author:

- mandd, wangc

- Creation date:

- 2019-05-08

- The classes tested in this test are:

- Models.ExternalModel.MarkovModel

- This test fulfills the following requirement:

- SR2ML-PRA-3

- Since the creation of this test, the following main revisions have been performed:

1. revision info:

- author: wangc

- date : 2019-11-27

- description: Added Plugin name extension 


\subsubsection{FRAMEWORK.TESTMARKOVMODEL 3STATES}

This test can be found at ". /raven/plugins/SR2ML/tests/test_markovModel_3states. xml". This test can be called executing the following command:

. /run_tests --re=framework. TestMarkovModel_3states

or

./run_framework_tests --re=framework. TestMarkovModel_3states

- Test Description:

- This test is aimed to check Markov model with 3 states

- Original Author:

- mandd, wangc

- Creation date:

- 2019-05-08

- The classes tested in this test are:

- Models.ExternalModel.MarkovModel

- This test fulfills the following requirement:

- SR2ML-F-2

- Since the creation of this test, the following main revisions have been performed:

1. revision info:

- author: wangc

- date : 2019-11-27

- description: Added Plugin name extension

\subsubsection{FRAMEWORK.TESTETMODEL}

This test can be found at ". /raven/plugins/SR2ML/tests/test_ETmodel.xml". This test can be called executing the following command:

. /run_tests --re=framework. TesteTModel 
or

./run_framework_tests --re=framework. TestETModel

- Test Description:

- This test is aimed to check the event tree external model

- Original Author:

- mandd, wangc

- Creation date:

- 2019-05-08

- The classes tested in this test are:

- Models.ExternalModel.ETModel

- This test fulfills the following requirement:

- SR2ML-PRA-1

- Since the creation of this test, the following main revisions have been performed:

1. revision info:

- author: wangc

- date : 2019-11-27

- description: Added Plugin name extension

\subsubsection{FRAMEWORK.TESTFTMODEL}

This test can be found at “. / raven/plugins/SR2ML/tests/test_FTmodel.xml”. This test can be called executing the following command:

./run_tests --re=framework. TestFTModel

or

./run_framework_tests --re=framework. TestFTModel

- Test Description: 
- This test is aimed to check fault tree external model

- Original Author:

- mandd, wangc

- Creation date:

- 2019-05-08

- The classes tested in this test are:

- Models.ExternalModel.FTModel

- This test fulfills the following requirement:

- SR2ML-PRA-2

- Since the creation of this test, the following main revisions have been performed:

1. revision info:

- author: wangc

- date : 2019-11-27

- description: Added Plugin name extension

\subsubsection{FRAMEWORK.TESTGRAPHMODELTD}

This test can be found at "./raven/plugins/SR2ML/tests/test_graphModel_TD. xml". This test can be called executing the following command:

. Irun_tests --re=framework. TestGraphModelTD

or

. Irun_framework_tests --re=framework. TestGraphModelTD

- Test Description:

- This test is aimed to check the time dependent graph model

- Original Author:

- mandd, wangc

- Creation date: 
- 2019-05-08

- The classes tested in this test are:

- Models.ExternalModel.GraphModel

- This test fulfills the following requirement:

- SR2ML-PRA-4

- Since the creation of this test, the following main revisions have been performed:

1. revision info:

- author: wangc

- date : 2019-11-27

- description: Added Plugin name extension

\subsubsection{FRAMEWORK.TESTFTMODELTD}

This test can be found at ". / raven/plugins/SR2ML/tests/test_FTmodel_TD.xml". This test can be called executing the following command:

. Irun_tests --re=framework. TestFTModelTD

or

./run_framework_tests --re=framework. TestFTModelTD

- Test Description:

- This test is aimed to check time dependent fault tree external model

- Original Author:

- mandd, wangc

- Creation date:

- 2019-05-08

- The classes tested in this test are:

- Models.ExternalModel.FTModel

- This test fulfills the following requirement: 
- SR2ML-PRA-2

- Since the creation of this test, the following main revisions have been performed:

1. revision info:

- author: wangc

- date : 2019-11-27

- description: Added Plugin name extension

\subsubsection{FRAMEWORK.TESTETMODELTD}

This test can be found at ". / raven/plugins/SR2ML/tests/test_ETmodel_TD.xml”. This test can be called executing the following command:

. Irun_tests --re=framework. TestETModelTD

or

./run_framework_tests --re=framework.TestETModelTD

- Test Description:

- This test is aimed to check the fault tree external model

- Original Author:

- mandd, wangc

- Creation date:

- 2019-05-08

- The classes tested in this test are:

- Models.ExternalModel

- This test fulfills the following requirement:

- SR2ML-PRA-1

- Since the creation of this test, the following main revisions have been performed:

1. revision info:

- author: wangc

- date : 2019-11-27

- description: Added Plugin name extension 


\subsubsection{FRAMEWORK.TESTENESEMBLEDISCRETE}

This test can be found at “. / raven/plugins/SR2ML/tests/test_ensemblePRAModel_ discrete. xml". This test can be called executing the following command:

. Irun_tests --re=framework. TestEnesembleDiscrete

or

. /run_framework_tests --re=framework. TestEnesembleDiscrete

- Test Description:

- This test is aimed to check ensemble model with fault tree and exvent tree external model

- Original Author:

- mandd, wangc

- Creation date:

- 2019-05-08

- The classes tested in this test are:

- Models.ExternalModel.EnsembleModel

- This test fulfills the following requirement:

- SR2ML-F-1

- Since the creation of this test, the following main revisions have been performed:

1. revision info:

- author: wangc

- date : 2019-11-27

- description: Added Plugin name extension 


\subsubsection{FRAMEWORK.TESTENESEMBLEMIXED}

This test can be found at “. / raven/plugins/SR2ML/tests/test_ensemblePRAModel_ mixed.xml". This test can be called executing the following command:

. Irun_tests --re=framework. TestEnesembleMixed

or

. /run_framework_tests --re=framework. TestEnesembleMixed

- Test Description:

- This test is aimed to check combined PRA external models

- Original Author:

- mandd, wangc

- Creation date:

- 2019-05-08

- The classes tested in this test are:

- Models.ExternalModel

- This test fulfills the following requirement:

- SR2ML-SI-1

- Since the creation of this test, the following main revisions have been performed:

1. revision info:

- author: wangc

- date : 2019-11-27

- description: Added Plugin name extension

\subsubsection{PLUGINS SR2ML.RELIABILITYMODEL.EXPON}

This test can be found at ". / raven/plugins/SR2ML/tests/reliabilityModel/test_ expon.xml". This test can be called executing the following command:

. Irun_tests --re=plugins/SR2ML.reliabilityModel.expon 
or

./run_framework_tests --re=plugins/SR2ML.reliabilityModel.expon

- Test Description:

- This input tests reliability external model (i.e. exponential function) within SR2ML plugin

- Original Author:

- wangc

- Creation date:

- 2020-02-04

- The classes tested in this test are:

- Models.ExternalModel

- This test fulfills the following requirement:

- R-SR2ML-2

- Since the creation of this test, the following main revisions have been performed:

1. revision info:

- author: wangc

- date : 2020-02-04

- description: Add test

\subsubsection{PLUGINS SR2ML.RELIABILITYMODEL.ERLANG}

This test can be found at ". / raven/plugins/SR2ML/tests/reliabilityModel/test_ erlangian. xml". This test can be called executing the following command:

. Irun_tests --re=plugins/SR2ML.reliabilityModel.erlang

or

./run_framework_tests --re=plugins/SR2ML.reliabilityModel.erlang

- Test Description: 
- This input tests reliability external model (i.e. erlangian function) within SR2ML plugin

- Original Author:

- wangc

- Creation date:

- 2020-02-04

- The classes tested in this test are:

- Models.ExternalModel

- This test fulfills the following requirement:

- R-SR2ML-2

- Since the creation of this test, the following main revisions have been performed:

1. revision info:

- author: wangc

- date : 2020-02-04

- description: Add test

\subsubsection{PLUGINS SR2ML.RELIABILITYMODEL.LOGNORM}

This test can be found at ". / raven/plugins/SR2ML/tests/reliabilityModel/test_ lognorm. xml". This test can be called executing the following command:

. /run_tests --re=plugins/SR2ML.reliabilityModel.lognorm

or

./run_framework_tests --re=plugins/SR2ML.reliabilityModel.lognorm

- Test Description:

- This input tests reliability external model (i.e. lognorm function) within SR2ML plugin

- Original Author:

- wangc 
- Creation date:

- 2020-02-04

- The classes tested in this test are:

- Models.ExternalModel

- This test fulfills the following requirement:

- R-SR2ML-2

- Since the creation of this test, the following main revisions have been performed:

1. revision info:

- author: wangc

- date : 2020-02-04

- description: Add test

\subsubsection{PLUGINS SR2ML.RELIABILITYMODEL.GAMMA}

This test can be found at “. /raven/plugins/SR2ML/tests/reliabilityModel/test_ gamma.xml". This test can be called executing the following command:

./run_tests --re=plugins/SR2ML.reliabilityModel.gamma

or

./run_framework_tests --re=plugins/SR2ML.reliabilityModel.gamma

- Test Description:

- This input tests reliability external model (i.e. Gamma function) within SR2ML plugin

- Original Author:

- wangc

- Creation date:

- 2020-02-04

- The classes tested in this test are:

- Models.ExternalModel 
- This test fulfills the following requirement:

- R-SR2ML-2

- Since the creation of this test, the following main revisions have been performed:

1. revision info:

- author: wangc

- date : 2020-02-04

- description: Add test

\subsubsection{PLUGINS SR2ML.RELIABILITYMODEL.WEIBULL}

This test can be found at ". / raven/plugins/SR2ML/tests/reliabilityModel/test_ weibull.xml". This test can be called executing the following command:

./run_tests --re=plugins/SR2ML.reliabilityModel.weibull

or

./run_framework_tests --re=plugins/SR2ML.reliabilityModel.weibull

- Test Description:

- This input tests reliability external model (i.e. Weibull function) within SR2ML plugin

- Original Author:

- wangc

- Creation date:

- 2020-02-04

- The classes tested in this test are:

- Models.ExternalModel

- This test fulfills the following requirement:

- R-SR2ML-2

- Since the creation of this test, the following main revisions have been performed:

1. revision info: 
- author: wangc

- date : 2020-02-04

- description: Add test

\subsubsection{PLUGINS SR2ML.RELIABILITYMODEL.BATHTUB}

This test can be found at “. / raven/plugins/SR2ML/tests/reliabilityModel/test_ bathtub.xml". This test can be called executing the following command:

. /run_tests --re=plugins/SR2ML.reliabilityModel.bathtub

or

./run_framework_tests --re=plugins/SR2ML.reliabilityModel.bathtuld

- Test Description:

- This input tests reliability external model (i.e. bathtub function) within SR2ML plugin

- Original Author:

- wangc

- Creation date:

- 2020-02-04

- The classes tested in this test are:

- Models.ExternalModel

- This test fulfills the following requirement:

- R-SR2ML-2

- Since the creation of this test, the following main revisions have been performed:

1. revision info:

- author: wangc

- date : 2020-02-04

- description: Add test 


\subsubsection{PLUGINS SR2ML.RELIABILITYMODEL.FATIGUELIFE}

This test can be found at ". / raven/plugins/SR2ML/tests/reliabilityModel/test_ fatiguelife.xml". This test can be called executing the following command:

. /run_tests --re=plugins/SR2ML.reliabilityModel.fatiguelife

or

./run_framework_tests --re=plugins/SR2ML.reliabilityModel.fatiguelife

- Test Description:

- This input tests reliability external model (i.e. fatiguelife function) within SR2ML plugin

- Original Author:

- wangc

- Creation date:

- 2020-02-04

- The classes tested in this test are:

- Models.ExternalModel

- This test fulfills the following requirement:

- R-SR2ML-2

- Since the creation of this test, the following main revisions have been performed:

1. revision info:

- author: wangc

- date : 2020-02-04

- description: Add test 


\subsubsection{PLUGINS SR2ML.RELIABILITYMODEL.LOGLINEAR}

This test can be found at ". /raven/plugins/SR2ML/tests/reliabilityModel/test_ loglinear.xml". This test can be called executing the following command:

. /run_tests --re=plugins/SR2ML.reliabilityModel.loglinear

or

./run_framework_tests --re=plugins/SR2ML.reliabilityModel.loglinfar

- Test Description:

- This input tests reliability external model (i.e. loglinear function) within SR2ML plugin

- Original Author:

- wangc

- Creation date:

- 2020-02-11

- The classes tested in this test are:

- Models.ExternalModel

- This test fulfills the following requirement:

- R-SR2ML-2

- Since the creation of this test, the following main revisions have been performed:

1. revision info:

- author: wangc

- date : 2020-02-11

- description: Add test

\subsubsection{PLUGINS SR2ML.RELIABILITYMODEL.NORMAL}

This test can be found at ". / raven/plugins/SR2ML/tests/reliabilityModel/test_ normal.xml". This test can be called executing the following command:

./run_tests --re=plugins/SR2ML.reliabilityModel.normal 
or

./run_framework_tests --re=plugins/SR2ML.reliabilityModel.normal

- Test Description:

- This input tests reliability external model (i.e. norm function) within SR2ML plugin

- Original Author:

- wangc

- Creation date:

- 2020-02-04

- The classes tested in this test are:

- Models.ExternalModel

- This test fulfills the following requirement:

- R-SR2ML-2

- Since the creation of this test, the following main revisions have been performed:

1. revision info:

- author: wangc

- date : 2020-02-04

- description: Add test

\subsubsection{PLUGINS SR2ML.RELIABILITYMODEL.POWERLAW}

This test can be found at ". / raven/plugins/SR2ML/tests/reliabilityModel/test_ powerlaw.xml". This test can be called executing the following command:

./run_tests --re=plugins/SR2ML.reliabilityModel.powerlaw

or

./run_framework_tests --re=plugins/SR2ML.reliabilityModel.powerlaw

- Test Description: 
- This input tests reliability external model (i.e. powerlaw function) within SR2ML plugin

- Original Author:

- wangc

- Creation date:

$-2020-02-11$

- The classes tested in this test are:

- Models.ExternalModel

- This test fulfills the following requirement:

- R-SR2ML-2

- Since the creation of this test, the following main revisions have been performed:

1. revision info:

- author: wangc

- date : 2020-02-11

- description: Add test

\subsubsection{PLUGINS SR2ML.RELIABILITYMODEL.EXPONWEIBULL}

This test can be found at ". / raven/plugins/SR2ML/tests/reliabilityModel/test_ exponweibull.xml". This test can be called executing the following command:

./run_tests --re=plugins/SR2ML.reliabilityModel.exponweibull

or

./run_framework_tests --re=plugins/SR2ML.reliabilityModel.exponweibull

- Test Description:

- This input tests reliability external model (i.e. exponweibull function) within SR2ML plugin

- Original Author:

- wangc 
- Creation date:

- 2020-02-04

- The classes tested in this test are:

- Models.ExternalModel

- This test fulfills the following requirement:

- R-SR2ML-2

- Since the creation of this test, the following main revisions have been performed:

1. revision info:

- author: wangc

- date : 2020-02-04

- description: Add test

\subsubsection{PLUGINS EXAMPLEPLUGIN.TESTPLUGINEXAMPLE}

This test can be found at ". / raven/plugins/ExamplePlugin/tests/test_example_ plugin.xml". This test can be called executing the following command:

./run_tests --re=plugins /ExamplePlugin. TestPluginExample

or

./run_framework_tests --re=plugins/ExamplePlugin. TestPluginExample

- Test Description:

- This input tests an example of external developed plugin. In this case it is just a plugin that computes a sum of exponentials: $X i(t)=\sum \_i=1^{n} \operatorname{coe}_{\_} i * e^{v a r_{-} i * t}$

- Original Author:

- alfoa

- Creation date:

- 2017-11-17

- The classes tested in this test are: 
- Models.ExternalModel

- This test fulfills the following requirement:

- R-SI-2

- Since the creation of this test, the following main revisions have been performed:

1. revision info:

- author : alfoa

- date : 2018-10-01

- description: This is a requirement test now.

\subsubsection{PLUGINS EXAMPLEPLUGIN.TESTPLUGINEXAMPLE}

This test can be found at ". /raven/plugins/ExamplePlugin/tests/test_raven_ running_raven_plugin.xml”. This test can be called executing the following command:

./run_tests --re=plugins/ExamplePlugin. TestPluginExample

or

./run_framework_tests --re=plugins/ExamplePlugin. TestPluginExample

- Test Description:

- This test is aimed to check the functionality of the RAVEN code interface (RAVEN running RAVEN) executing an external plugin (ExternalModel) plugin

- Original Author:

- alfoa

- Creation date:

- 2017-11-17

- The classes tested in this test are:

- Models.ExternalModel

- This test fulfills the following requirement:

- R-SI-2 
- Since the creation of this test, the following main revisions have been performed:

1. revision info:

- author : alfoa

- date : 2018-10-01

- description: This is a requirement test now.

2. revision info:

- author : alfoa

- date : 2019-06-05

- description: Removed HDF5 dumping in order to reduce overhead of IO on HPC slow systems. In addition, it invokes python directly to remove a bit of overhead for establishing conda

\subsubsection{MULTIMARKETFIXEDPRICE}

This test can be found at "./raven/plugins/HERON/tests/integration_tests/ mechanics/multimarket_fix_price/heron_input.xml". This test can be called executing the following command:

. /run_tests --re=MultiMarketFixedPrice

or

. /run_framework_tests --re=MultiMarketFixedPrice

- Test Description:

- Tests HERON creating workflows and running with a fixed deregulated market price.

- Original Author:

- gaira

- Creation date:

- 2019-12-12

- The classes tested in this test are:

- HERON

- This test fulfills the following requirement:

- H-EA-3 


\subsubsection{VARDEMANDVARPRICE}

This test can be found at "./raven/plugins/HERON/tests/integration_tests/ mechanics/var_demand_var_price/heron_input.xml". This test can be called executing the following command:

. /run_tests --re=VarDemandVarPrice

or

. /run_framework_tests --re=VarDemandVarPrice

- Test Description:

- Tests HERON for a variable demand and variable price.

- Original Author:

- gaira

- Creation date:

- 2019-12-12

- The classes tested in this test are:

- HERON

- This test fulfills the following requirement:

- H-EA-2

\subsubsection{VARDEMANDFIXEDPRICE}

This test can be found at "./raven/plugins/HERON/tests/integration_tests/ mechanics/var_demand_fix_price/heron_input.xml". This test can be called executing the following command:

./run_tests --re=VarDemandFixedPrice

or

./run_framework_tests --re=VarDemandFixedPrice 
- Test Description:

- Tests HERON creating workflows and running with a variable demand but fixed market price.

- Original Author:

- gaira

- Creation date:

- 2019-12-12

- The classes tested in this test are:

- HERON

- This test fulfills the following requirement:

- H-F-1

- H-F-2

- H-EA-1

- H-SI-1

\subsubsection{CASHFLOW TEST PI}

This test can be found at ". / raven/plugins/TEAL/tests/CashFlow_test_PI.xml". This test can be called executing the following command:

. Irun_tests --re=CashFlow_test_PI

or

./run_framework_tests --re=CashFlow_test_PI

- Test Description:

- This input tests the RAVEN plugin TEAL. The tested functionality is called PI.

- Original Author:

- A. Epiney

- Creation date: 
$-2017-10-25$

- The classes tested in this test are:

- Models.ExternalModel.TEAL

- This test fulfills the following requirement:

- CF-F-1

- CF-F-2

- CF-EA-3

- Since the creation of this test, the following main revisions have been performed:

1. revision info:

- author : alfoa

- date : 2018-12-13

- description: Added classTested node

2. revision info:

- author : alfoa

- date : 2019-07-30

- description: Added requirements

3. revision info:

- author: wangc

- date : 2019-11-08

- description: update ExternalXML with new structure cash flow input

4. revision info:

- author : alfoa

- date : 2019-11-25

- description: Added Plugin name extension

5. revision info:

- author : alfoa

- date : 2020-07-28

- description: Modified name from CashFlow to TEAL 


\subsubsection{CASHFLOW TEST NPV}

This test can be found at ". /raven/plugins/TEAL/tests/CashFlow_test_NPV.xml". This test can be called executing the following command:

. Irun_tests --re=CashFlow_test_NPV

or

./run_framework_tests --re=CashFlow_test_NPV

- Test Description:

- This input tests the RAVEN plugin TEAL. The tested functionality is called NPV.

- Original Author:

- A. Epiney

- Creation date:

- 2017-10-25

- The classes tested in this test are:

- Models.ExternalModel.TEAL

- This test fulfills the following requirement:

- CF-F-1

- CF-F-2

- CF-EA-1

- Since the creation of this test, the following main revisions have been performed:

1. revision info:

- author: alfoa

- date : 2018-12-13

- description: Added classTested node

2. revision info:

- author: alfoa

- date : 2019-07-30

- description: Added requirements 
3. revision info:

- author: wangc

- date : 2019-11-08

- description: update ExternalXML with new structure cash flow input

4. revision info:

- author : alfoa

- date : 2019-11-25

- description: Added Plugin name extension

5. revision info:

- author: alfoa

- date : 2020-07-28

- description: Modified name from CashFlow to TEAL

\subsubsection{CASHFLOW TEST NPVSEARCH}

This test can be found at "./raven/plugins/TEAL/tests/CashFlow_test_NPVsearch. xml". This test can be called executing the following command:

./run_tests --re=CashFlow_test_NPVsearch

or

./run_framework_tests --re=CashFlow_test_NPVsearch

- Test Description:

- This input tests the RAVEN plugin TEAL. The tested functionality is called NPV search.

- Original Author:

- A. Epiney

- Creation date:

- 2017-10-25

- The classes tested in this test are:

- Models.ExternalModel.TEAL 
- This test fulfills the following requirement:

- CF-EA-4

- Since the creation of this test, the following main revisions have been performed:

1. revision info:

- author : alfoa

- date : 2018-12-13

- description: Added classTested node

2. revision info:

- author : alfoa

- date : 2019-07-30

- description: Added requirements

3. revision info:

- author: wangc

- date : 2019-11-08

- description: update ExternalXML with new structure cash flow input

4. revision info:

- author : alfoa

- date : 2019-11-25

- description: Added Plugin name extension

5. revision info:

- author : alfoa

- date : 2020-07-28

- description: Modified name from CashFlow to TEAL

\subsubsection{CASHFLOW TEST IRR}

This test can be found at ". / raven/plugins/TEAL/tests/CashFlow_test_IRR.xml”. This test can be called executing the following command:

. /run_tests --re=CashFlow_test_IRR

or

./run_framework_tests --re=CashFlow_test_IRR 
- Test Description:

- This input tests the RAVEN plugin TEAL. The tested functionality is called IRR.

- Original Author:

- A. Epiney

- Creation date:

- 2017-10-25

- The classes tested in this test are:

- Models.ExternalModel.TEAL

- This test fulfills the following requirement:

- CF-F-1

- CF-F-2

- CF-EA-2

- Since the creation of this test, the following main revisions have been performed:

1. revision info:

- author: alfoa

- date : 2018-12-13

- description: Added classTested node

2. revision info:

- author : alfoa

- date : 2019-07-30

- description: Added requirements

3. revision info:

- author: wangc

- date : 2019-11-08

- description: update ExternalXML with new structure cash flow input

4. revision info:

- author: alfoa

- date : 2019-11-25

- description: Added Plugin name extension

5. revision info:

- author : alfoa

- date : 2020-07-28

- description: Modified name from CashFlow to TEAL 


\subsubsection{CASHFLOW TEST NPV MULTIPLY}

This test can be found at “. / raven/plugins/TEAL/tests/CashFlow_test_NPV_multiplier. xml". This test can be called executing the following command:

. /run_tests --re=CashFlow_test_NPV_multiply

or

./run_framework_tests --re=CashFlow_test_NPV_multiply

- Test Description:

- This input tests the RAVEN plugin TEAL. The tested functionality is called NPV. This test uses the "Multiplier" functionality of CashFlow.

- Original Author:

- A. Epiney

- Creation date:

- 2018-03-22

- The classes tested in this test are:

- Models.ExternalModel.TEAL

- This test fulfills the following requirement:

- CF-EA-1

- Since the creation of this test, the following main revisions have been performed:

1. revision info:

- author : alfoa

- date : 2018-12-13

- description: Added classTested node

2. revision info:

- author : alfoa

- date : 2019-07-30

- description: Added requirements

3. revision info: 
- author: wangc

- date : 2019-11-08

- description: update ExternalXML with new structure cash flow input

4. revision info:

- author : alfoa

- date : 2019-11-25

- description: Added Plugin name extension

5. revision info:

- author : alfoa

- date : 2020-07-28

- description: Modified name from CashFlow to TEAL

\subsubsection{CASHFLOW TEST NPV}

This test can be found at “./raven/plugins/TEAL/tests/CashFlow_test_NPV_expandedDrive xml". This test can be called executing the following command:

. /run_tests --re=CashFlow_test_NPV

or

./run_framework_tests --re=CashFlow_test_NPV

- Test Description:

- This input tests the RAVEN plugin CashFlow. The tested functionality is called NPV. The BOP revenue is an expanded driver, i.e. values for every year are provided instead of just one value that is repeated for all years.

- Original Author:

- A. Epiney

- Creation date:

- 2018-03-23

- The classes tested in this test are:

- Models.ExternalModel.TEAL

- This test fulfills the following requirement: 


\section{- CF-EA-1}

- Since the creation of this test, the following main revisions have been performed:

1. revision info:

- author : alfoa

- date : 2018-12-13

- description: Added classTested node

2. revision info:

- author : alfoa

- date : 2019-07-30

- description: Added requirements

3. revision info:

- author: wangc

- date : 2019-11-08

- description: update ExternalXML with new structure cash flow input

4. revision info:

- author : alfoa

- date : 2019-11-25

- description: Added Plugin name extension

5. revision info:

- author : alfoa

- date : 2020-07-28

- description: Modified name from CashFlow to TEAL

\subsubsection{CASHFLOW TEST NPV}

This test can be found at ". / raven/plugins/TEAL/tests/CashFlow_test_NPV_componentTax. xml". This test can be called executing the following command:

. Irun_tests --re=CashFlow_test_NPV

or

./run_framework_tests --re=CashFlow_test_NPV

- Test Description: 
- This input tests the RAVEN plugin TEAL. The tested functionality is called NPV. The functionality of different tax and inflation reates for different components is tested here.

- Original Author:

- A. Epiney

- Creation date:

- 2018-03-23

- The classes tested in this test are:

- Models.ExternalModel.TEAL

- This test fulfills the following requirement:

- CF-EA-1

- Since the creation of this test, the following main revisions have been performed:

1. revision info:

- author : alfoa

- date : 2018-12-13

- description: Added classTested node

2. revision info:

- author : alfoa

- date : 2019-07-30

- description: Added requirements

3. revision info:

- author: wangc

- date : 2019-11-08

- description: update ExternalXML with new structure cash flow input

4. revision info:

- author : alfoa

- date : 2019-11-25

- description: Added Plugin name extension

5. revision info:

- author : alfoa

- date : 2020-07-28

- description: Modified name from CashFlow to TEAL 


\subsubsection{CASHFLOW TEST PI}

This test can be found at “. / raven/plugins/TEAL/tests/CashFlow_test_NPV_RunAsCode. py". This test can be called executing the following command:

./run_tests --re=CashFlow_test_PI

or

./run_framework_tests --re=CashFlow_test_PI

- Test Description:

- \# This input tests the RAVEN plugin CashFlow in standalone mode. \#

- Original Author:

- A. Epiney

- Creation date:

- 2017-10-25

- The classes tested in this test are:

- Models.ExternalModel.CashFlow

- This test fulfills the following requirement:

- CF-EA-5

- Since the creation of this test, the following main revisions have been performed:

1. revision info:

- author : alfoa

- date : 2019-11-25

- description: Added classTested node

\subsubsection{CASHFLOW TEST NPV}

This test can be found at “. / raven/plugins/TEAL/tests/CashFlow_test_NPV_customTime. xml". This test can be called executing the following command:

. Irun_tests --re=CashFlow_test_NPV 
or

./run_framework_tests --re=CashFlow_test_NPV

- Test Description:

- This input tests the RAVEN plugin CashFlow. The tested functionality is called NPV. Tests the ProjectTime, StartTime and Repetition functionalities. All sub-projects, i.e. components lifetimes (including repetition) finish before the ProjectTime finishes.

- Original Author:

- A. Epiney

- Creation date:

- 2018-04-06

- The classes tested in this test are:

- Models.ExternalModel.TEAL

- This test fulfills the following requirement:

- CF-EA-1

- CF-SI-1

- Since the creation of this test, the following main revisions have been performed:

1. revision info:

- author : alfoa

- date : 2018-12-13

- description: Added classTested node

2. revision info:

- author : alfoa

- date : 2019-07-30

- description: Added requirements

3. revision info:

- author: wangc

- date : 2019-11-08

- description: update ExternalXML with new structure cash flow input

4. revision info: 
- author : alfoa

- date : 2019-11-25

- description: Added Plugin name extension

5. revision info:

- author : alfoa

- date : 2020-07-28

- description: Modified name from CashFlow to TEAL

\subsubsection{CASHFLOW TEST NPV}

This test can be found at ". / raven/plugins/TEAL/tests/CashFlow_test_NPV_customTime2 . xml". This test can be called executing the following command:

. Irun_tests --re=CashFlow_test_NPV

or

./run_framework_tests --re=CashFlow_test_NPV

- Test Description:

- This input tests the RAVEN plugin TEAL. The tested functionality is called NPV. Tests the ProjectTime, StartTime and Repetition functionalities. No sub-project, i.e. component lifetime (including repetition) finish before the ProjectTime finishes.

- Original Author:

- A. Epiney

- Creation date:

- 2018-04-06

- The classes tested in this test are:

- Models.ExternalModel.CashFlow

- This test fulfills the following requirement:

- CF-EA-1

- CF-IS-1

- Since the creation of this test, the following main revisions have been performed: 
1. revision info:

- author : alfoa

- date : 2018-12-13

- description: Added classTested node

2. revision info:

- author : alfoa

- date : 2019-07-30

- description: Added requirements

3. revision info:

- author: wangc

- date : 2019-11-08

- description: update ExternalXML with new structure cash flow input

4. revision info:

- author : alfoa

- date : 2019-11-25

- description: Added Plugin name extension

5. revision info:

- author : alfoa

- date : 2020-07-28

- description: Modified name from CashFlow to TEAL

\subsubsection{CASHFLOW TEST NPV CUSTOM MACRS}

This test can be found at “. / raven/plugins/TEAL/tests/CashFlow_test_NPV_custom_ MACRS . xml". This test can be called executing the following command:

./run_tests --re=CashFlow_test_NPV_custom_MACRS

or

./run_framework_tests --re=CashFlow_test_NPV_custom_MACRS

- Test Description:

- This input tests the RAVEN plugin CashFlow. The tested functionality is called NPV with custom macrs. 
- Original Author:

- wangc

- Creation date:

- 2019-11-08

- The classes tested in this test are:

- Models.ExternalModel.TEAL

- This test fulfills the following requirement:

- CF-EA-1

- Since the creation of this test, the following main revisions have been performed:

1. revision info:

- author : alfoa

- date : 2019-11-25

- description: Added Plugin name extension

2. revision info:

- author : alfoa

- date : 2019-11-25

- description: Added requirements

3. revision info:

- author : alfoa

- date : 2020-07-28

- description: Modified name from CashFlow to TEAL

\subsubsection{CASHFLOW TEST NPV REPETITIONS}

This test can be found at ". / raven/plugins/TEAL/tests/CashFlow_test_repetitions. xml". This test can be called executing the following command:

. /run_tests --re=CashFlow_test_NPV_repetitions

or

./run_framework_tests --re=CashFlow_test_NPV_repetitions 
- Test Description:

- This input finds the differential NPV between Li-ion batteries and other hydrogen production technologies. This test is showing the usage of periodic (repetition) Operation and Maintainence chasflows for components. This test is aimed to show that the issue \#20 (https://github.com/idaholab/TEAL/issues/20) is resolved.

- Original Author:

- alfoa

- Creation date:

- 2020-10-20

- The classes tested in this test are:

- Models.ExternalModel.TEAL

- This test fulfills the following requirement:

$-\mathrm{CF}-\mathrm{F}-1$

- CF-F-2

- CF-EA-1

- Since the creation of this test, the following main revisions have been performed:

1. revision info:

- author : alfoa

- date : 2021-01-28

- description: Added test for checking issue \#20 is resolved

\subsubsection{FRAMEWORK.TEST OUTPUT}

This test can be found at ". /raven/tests/framework/test_output.xml". This test can be called executing the following command:

. Irun_tests --re=framework.test_output

or

./run_framework_tests --re=framework.test_output

- Test Description: 
- This test is aimed to check the OutStreams system in RAVEN (BOTH TYPE: Plot (all available plot types) and Print(all the available combination of options))

- Original Author:

$-\operatorname{cogljj}$

- Creation date:

- 2013-09-24

- The classes tested in this test are:

- OutStreams.Plot, OutStreams.Print

- This test fulfills the following requirement:

- R-RA-3

- R-F-5

- R-F-6

- Since the creation of this test, the following main revisions have been performed:

1. revision info:

- author : crisr

- date : 2014-04-03

- description: new imput stile for the rom and the dummy. SVN r26236

2. revision info:

- author : alfoa

- date : 2014-04-10

- description: Interactive mode for Steps + moved exec on the fly outside the modules in order to avoid unneeded slowing down. SVN r26340

3. revision info:

- author : senrs

- date : 2015-03-17

- description: Scatter plot corrected

4. revision info:

- author : mandd

- date : 2015-04-17

- description: conversion to Database and DataObjects

5. revision info: 
- author : alfoa

- date : 2015-06-18

- description: Changed Name of all DataObjects

6. revision info:

- author : maljdan

- date : 2015-06-22

- description: Adding the outstream manager to the XSD and adjusting some of the input files to play nicely with it.

7. revision info:

- author : senrs

- date : 2015-07-08

- description: Adding subPlot capability

8. revision info:

- author : cogljj

- date : 2015-10-08

- description: Switching from RAVEN model to external model, so RELAP-7 not needed. This is checking raven's output, so it shouldn't depend on RELAP-7. This removes the raven $\mathrm{c}++$ input files. This updates gold files because of changing the model.

9. revision info:

- author : maljdan

- date : 2016-05-20

- description: Updating the test cases to remove references to Point or History.

10. revision info:

- author : alfoa

- date : 2016-09-01

- description: Close \#650

11. revision info:

- author : cogljj

- date : 2016-10-07

- description: Having both a color and a colormap doesn't make sense.

12. revision info:

- author : alfoa

- date : 2017-01-21

- description: Adding this test description. 


\subsubsection{FRAMEWORK.TEST ROM TRAINER}

This test can be found at ". / raven/tests/framework/test_rom_trainer.xml". This test can be called executing the following command:

. Irun_tests --re=framework.test_rom_trainer

or

./run_framework_tests --re=framework.test_rom_trainer

- Test Description:

- This test is aimed to test the capability of RAVEN to train ROMs based on the data sets constructed by whatever sampling strategy

- Original Author:

- alfoa

- Creation date:

- 2013-09-26

- The classes tested in this test are:

- Steps.RomTrainer, Models.ROM

- This test fulfills the following requirement:

- R-RA-4

- R-F-1

- Since the creation of this test, the following main revisions have been performed:

1. revision info:

- author : alfoa

- date : 2014-03-06

- description: changed name of DataBase handling, modified all tests were using it, improved Step output handling etc r 25347

2. revision info:

- author : alfoa

- date : 2014-07-15 
- description: 2) eliminated redondancy type for distribution specification in samplers... all inputs and tests found in repository have been modified as well r28610

3. revision info:

- author : cogljj

- date : 2014-10-06

- description: Renaming blocks with IODataBase and OutStreamStep to IOStep. The two different steps were combined. r29902

4. revision info:

- author : alfoa

- date : 2014-10-22

- description: added variable samping MC for RAVEN code interface + eliminated need to specify variable type for Functions and ExternalModels r30198

5. revision info:

- author : alfoa

- date : 2015-05-19

- description: Closes \#171

6. revision info:

- author : alfoa

- date : 2015-06-18

- description: Changed Name of all DataObjects

7. revision info:

- author : alfoa

- date : 2015-09-12

- description: removed syntax inconsistency with respect standards for Samplers (underscores instead of camelBack) + created conversion script

8. revision info:

- author : cogljj

- date : 2015-10-12

- description: Moving test_rom_trainer to test_rom_trainer_raven.

9. revision info:

- author : cogljj

- date : 2015-10-12

- description: Made a test rom trainer with an external model.

10. revision info:

- author : cogljj 
- date : 2015-11-17

- description: Updating to work with scikit-learn 0.17. In the new version of sklearn the rom can not use a classifier anymore.

11. revision info:

- author : maljdan

- date : 2016-05-20

- description: Updating the test cases to remove references to Point or History.

12. revision info:

- author : alfoa

- date : 2017-01-21

- description: Adding this test description.

\subsubsection{FRAMEWORK.TESTGRID}

This test can be found at "./raven/tests/framework/test_Grid_Sampler.xml". This test can be called executing the following command:

./run_tests --re=framework.testGrid

or

./run_framework_tests --re=framework.testGrid

- Test Description:

- This test is aimed to check the capability in RAVEN to employ a Grid sampling strategy. Since the goal of the test is to testify that the Grid sampling strategy is functional, a Dummy model is used.

- Original Author:

- crisr

- Creation date:

- 2013-10-15

- The classes tested in this test are:

- Sampler.Grid

- This test fulfills the following requirement: 
- R-RE-3

- Since the creation of this test, the following main revisions have been performed:

1. revision info:

- author : mandd

- date : 2015-04-17

- description: conversion to Database and DataObjects

2. revision info:

- author : talbpaul

- date : 2015-05-06

- description: improved verbosity in tests and manual

3. revision info:

- author : alfoa

- date : 2015-05-18

- description: Closes \#167

4. revision info:

- author : alfoa

- date : 2015-05-18

- description: Closes \#169

5. revision info:

- author : alfoa

- date : 2015-05-26

- description: grid done

6. revision info:

- author : alfoa

- date : 2015-06-18

- description: Changed Name of all DataObjects

7. revision info:

- author : maljdan

- date : 2016-04-06

- description: Updating test cases to reflect the changes to the user input.

8. revision info:

- author : talbpaul

- date : 2016-04-26

- description: added check for pre-existing backup files when validating 
9. revision info:

- author : alfoa

- date : 2017-01-21

- description: Adding this test description.

10. revision info:

- author : alfoa

- date : 2019-06-05

- description: Modified grid to test resolution of issue \#1003

\subsubsection{FRAMEWORK.TESTRANDOM}

This test can be found at "./raven/tests/framework/test_random.xml". This test can be called executing the following command:

. Irun_tests --re=framework.testRandom

or

./run_framework_tests --re=framework.testRandom

- Test Description:

- This test is aimed to check the functionality of RAVEN to perform random sampling (MonteCarlo) on simple 1Dimensional distributions. Since the goal of the test is to check the Sampler only, a Dummy Model is used.

- Original Author:

$-\operatorname{cog} l \mathrm{jj}$

- Creation date:

- 2014-02-18

- The classes tested in this test are:

- Samplers.MonteCarlo

- This test fulfills the following requirement:

- R-F-1

- Since the creation of this test, the following main revisions have been performed: 
1. revision info:

- author : mandd

- date : 2015-04-17

- description: conversion to Database and DataObjects

2. revision info:

- author : alfoa

- date : 2015-05-18

- description: Closes \#169

3. revision info:

- author: alfoa

- date : 2015-06-18

- description: Changed Name of all DataObjects

4. revision info:

- author : maljdan

- date : 2016-04-06

- description: Updating test cases to reflect the changes to the user input.

5. revision info:

- author : alfoa

- date : 2017-01-21

- description: Adding this test description.

\subsubsection{FRAMEWORK.TESTEXTERNALMODEL}

This test can be found at "./raven/tests/framework/test_Lorentz.xml”. This test can be called executing the following command:

. Irun_tests --re=framework.testExternalModel

or

./run_framework_tests --re=framework.testExternalModel

- Test Description:

- This test is aimed to check the functionality of RAVEN to use ExternalModel entities.

- Original Author: 
- mandd

- Creation date:

- 2013-10-24

- The classes tested in this test are:

- Models.ExternalModel

- This test fulfills the following requirement:

- R-IS-4

- Since the creation of this test, the following main revisions have been performed:

1. revision info:

- author : mandd

- date : 2015-04-17

- description: conversion to Database and DataObjects

2. revision info:

- author : alfoa

- date : 2015-04-21

- description: Closes \#122

3. revision info:

- author : cogljj

- date : 2015-09-29

- description: Modifing external modules to use relative to working directory.

4. revision info:

- author : alfoa

- date : 2017-01-21

- description: Adding this test description.

5. revision info:

- author : talbpaul

- date : 2018-02-26

- description: Adding maxQueueSize of 1 for consistency with previous behavior 


\subsubsection{FRAMEWORK.TEST IOSTEP LOAD}

This test can be found at ". / raven/tests/framework/test_iostep_load.xml". This test can be called executing the following command:

. /run_tests --re=framework.test_iostep_load

or

./run_framework_tests --re=framework.test_iostep_load

- Test Description:

- This test is aimed to check the capability of RAVEN to perform IO operation on its own object. This case checks the capability to create DataObjects from a folder containing CSVs and to dump them into HDF5 (and viceversa)

- Original Author:

$-\operatorname{cog} l \mathrm{jj}$

- Creation date:

- 2014-09-29

- The classes tested in this test are:

- Steps.IOStep, DataObjects.PointSet, DataObjects.HistorySet, Databases.HDF5

- This test fulfills the following requirement:

- R-RA-2

- R-F-6

- Since the creation of this test, the following main revisions have been performed:

1. revision info:

- author : cogljj

- date : 2014-10-06

- description: Renaming blocks with IODataBase and OutStreamStep to IOStep. The two different steps were combined. SVN r29902

2. revision info:

- author : cogljj

- date : 2014-10-08 
- description: Modify input to use its own unique database. SVN r29924

3. revision info:

- author : cogljj

- date : 2015-02-27

- description: Adding documentation to the manual about relative working directory. Also changing one of tests to do this.

4. revision info:

- author : alfoa

- date : 2015-03-04

- description: Modified batch sizes because sometimes, if we run the tests in parallel, the order of values in csv can be different

5. revision info:

- author : mandd

- date : 2015-04-17

- description: conversion to Database and DataObjects

6. revision info:

- author : cogljj

- date : 2015-04-23

- description: Switching to relative directories for working directory and database directory. Switching to default to relative to the $\mathrm{xml}$ file, not relative to the run dir. Passing the run info to the DataBases so that the working directory is known.

7. revision info:

- author : maljdan

- date : 2015-06-16

- description: Fixing the input files to pass the validation

8. revision info:

- author : alfoa

- date : 2015-06-18

- description: Changed Name of all DataObjects

9. revision info:

- author : talbpaul

- date : 2015-07-07

- description: updated to restrict partial-node comments

10. revision info:

- author : maljdan 
- date : 2016-04-06

- description: Updating test cases to reflect the changes to the user input.

11. revision info:

- author : maljdan

- date : 2016-05-20

- description: Updating the test cases to remove references to Point or History.

12. revision info:

- author : maljdan

- date : 2016-06-27

- description: Adjusting the inputs and outputs of two test cases to match what the loaded files read.

13. revision info:

- author : alfoa

- date : 2017-01-21

- description: Adding this test description.

\subsubsection{FRAMEWORK.ND EXTERNAL MC}

This test can be found at “./raven/tests/framework/test_simple_ND_external_ MC . xml". This test can be called executing the following command:

./run_tests --re=framework.ND_external_MC

or

./run_framework_tests --re=framework.ND_external_MC

- Test Description:

- This test is aimed to check the capability of RAVEN to handle ND (custom) probability density functions (distributions), when a MonteCarlo sampling strategy is performed.

- Original Author:

- mandd

- Creation date:

- 2015-03-04 
- The classes tested in this test are:

- Distributions.NDInverseWeight, Distributions.NDCartesianSpline, Samplers.MonteCarlo

- This test fulfills the following requirement:

- R-RE-2

- Since the creation of this test, the following main revisions have been performed:

1. revision info:

- author : mandd

- date : 2015-04-17

- description: conversion to Database and DataObjects

2. revision info:

- author : alfoa

- date : 2015-06-18

- description: Changed Name of all DataObjects

3. revision info:

- author : cogljj

- date : 2015-09-29

- description: Modifing external modules to use relative to working directory.

4. revision info:

- author : maljdan

- date : 2016-04-06

- description: Updating test cases to reflect the changes to the user input.

5. revision info:

- author : alfoa

- date : 2017-01-21

- description: Adding this test description.

\subsubsection{FRAMEWORK CALCULATE AND TRANSFER}

This test can be found at ". /raven/tests/framework/test_calc_and_transfer. xml". This test can be called executing the following command:

./run_tests --re=framework/calculate_and_transfer

or 
./run_framework_tests --re=framework/calculate_and_transfer

- Test Description:

- Creates data with an external model, then creates a rom from that, and then compares the data generated by both with the ComparisonStatistics class.

- Original Author:

$-\operatorname{cog} \mathrm{ljj}$

- Creation date:

- 2015-11-24

- The classes tested in this test are:

- ComparisonStatistics,IOStep

- This test fulfills the following requirement:

- R-IS-5

\subsubsection{FRAMEWORK DATABASES HDF5.2STEPS SAME DB}

This test can be found at ". / raven/tests / framework/Databases/HDF5/test_2steps_ same_db.xml". This test can be called executing the following command:

./run_tests --re=framework/Databases/HDF5.2steps_same_db

or

./run_framework_tests --re=framework/Databases/HDF5.2steps_same_t

- Test Description:

- This test is aimed to test the capability of the RAVEN database strucuture to use the same database (HDF5) for subsequential analyses in order to collect all the results in the same HDF5 container

- Original Author:

-@alfoa 
- Creation date:

- 2015-05-01

- The classes tested in this test are:

- Databases.HDF5

- This test fulfills the following requirement:

- R-IS-3

- Since the creation of this test, the following main revisions have been performed:

1. revision info:

- author: @maljdan

- date : 2016-05-20

- description: Updating the test cases to remove references to Point or History

2. revision info:

- author : alfoa

- date : 2019-03-01

- description: Modified test location

\subsubsection{FRAMEWORK DATABASES HDF5.LOAD AND PUSH REUSING SAME HDF5}

This test can be found at ". / raven/tests/framework/Databases/HDF5/test_load_ and_push_reusing_same_hdf5.xml". This test can be called executing the following command:

./run_tests --re=framework/Databases/HDF5.load_and_push_reusing_\$ame_hdf5

or

./run_framework_tests --re=framework/Databases/HDF5.1oad_and_push_reusing_

- Test Description:

- This test is aimed to check the possiblity in RAVEN to load an HDF5 (pre-generated) and use the same database to store new results.

- Original Author:

- alfoa 
- Creation date:

- 2016-12-12

- The classes tested in this test are:

- Databases.HDF5

- This test fulfills the following requirement:

- R-F-6

- Since the creation of this test, the following main revisions have been performed:

1. revision info:

- author : alfoa

- date : 2016-12-12

- description: Added test

2. revision info:

- author : alfoa

- date : 2017-01-21

- description: Adding this test description.

3. revision info:

- author: alfoa

- date : 2017-12-14

- description: Modified input since new structure of DataObject

4. revision info:

- author : alfoa

- date : 2019-03-01

- description: Modified test location

\subsubsection{FRAMEWORK.TEST DISTRIBUTIONS}

This test can be found at ". /raven/tests/framework/unit_tests/Distributions/ TestDistributions.py". This test can be called executing the following command:

./run_tests --re=framework.test_distributions

or

./run_framework_tests --re=framework.test_distributions 
- Test Description:

- This test is a Unit Test for the Distributions classes. It tests all the distributions and all the methods.

- Original Author:

$-\operatorname{cogljj}$

- Creation date:

- 2013-12-10

- The classes tested in this test are:

- This test fulfills the following requirement:

- R-RE-1

- Since the creation of this test, the following main revisions have been performed:

1. revision info:

- author : cogljj

- date : 2013-12-10

- description: Adding test of all the rest of the distributions except for binomial. r23360

2. revision info:

- author : senrs

- date : 2015-01-26

- description: fixed Bug in Distribution.py the attribute mean is obsolete use untrMean instead

3. revision info:

- author : senrs

- date : 2015-01-26

- description: Fixed bugs in the if statements, etc...and included tests for Distributions.py

4. revision info:

- author : talbpaul

- date : 2015-02-05

- description: added pickle methods and tests for distributionsw 
5. revision info:

- author : alfoa

- date : 2015-02-10

- description: finished caching of data

6. revision info:

- author : talbpaul

- date : 2015-03-11

- description: added way to do beta through keywords, still need to test stochcoll, but testdistros is passing

7. revision info:

- author : cogljj

- date : 2015-04-29

- description: Adding test of standard deviation.

8. revision info:

- author : cogljj

- date : 2015-05-05

- description: Adding check of std deviation of a binomial.

9. revision info:

- author : cogljj

- date : 2015-05-06

- description: Adding additional checks of the mean and standard deviation.

10. revision info:

- author: alfoa

- date : 2015-05-18

- description: modified test distribution

11. revision info:

- author : mandd

- date : 2015-06-16

- description: fixed testDistributions

12. revision info:

- author: alfoa

- date : 2016-03-31

- description: Closes \#478

13. revision info:

- author : maljdan 
- date : 2016-04-12

- description: Improving readability of our own code and removing extraneous functions.

14. revision info:

- author : cogljj

- date : 2016-04-12

- description: Converting Distributions to use the new input system. All distributions have been converted.

15. revision info:

- author : alfoa

- date : 2017-01-21

- description: Adding this test description.

16. revision info:

- author : alfoa

- date : 2018-05-10

- description: Added Log Uniform distribution unit test

\subsubsection{FRAMEWORK CODEINTERFACETESTS.GENERICINTERFACE2}

This test can be found at “. / raven/tests/framework/CodeInterfaceTests/test_ generic_interface.xml”. This test can be called executing the following command:

. /run_tests --re=framework/CodeInterfaceTests.genericInterface2

or

./run_framework_tests --re=framework/CodeInterfaceTests.genericInterface2

- Test Description:

- An example of using the the Model Code of type GenericCode. This test is aimed to show the usage of the GenericCode interface present in RAVEN in order to drive an external code using the wild cards approach

- Original Author:

- senrs

- Creation date: 
- 2015-10-06

- The classes tested in this test are:

- Models.Code.GenericCode

- This test fulfills the following requirement:

- R-IS-6

- Since the creation of this test, the following main revisions have been performed:

1. revision info:

- author : talbpaul

- date : 2016-01-04

- description: Re-golded for updated external model variables

2. revision info:

- author : maljdan

- date : 2016-04-06

- description: Updating test cases to reflect the changes to the user input.

3. revision info:

- author : alfoa

- date : 2017-01-21

- description: Adding this test description.

4. revision info:

- author : alfoa

- date : 2018-10-01

- description: This is a requirement test now.

\subsubsection{FRAMEWORK CODEINTERFACETESTS.GENERICINTERFACEPARALLEL}

This test can be found at ". / raven/tests / framework/CodeInterfaceTests/generic_ parallel.xml". This test can be called executing the following command:

./run_tests --re=framework/CodeInterfaceTests.genericInterfaceParallel

or

. Irun_framework_tests --re=framework/CodeInterfaceTests.genericInterfacePa 
- Test Description:

- Tests using "Files" in an internalParallel run (which tests pickling and unpickling them). This test is also a requirement test where we test the capability of RAVEN to dispatch multiple parallel jobs based on user-defined information (batchSize)

- Original Author:

- talbpaul

- Creation date:

- 2017-08-24

- The classes tested in this test are:

- Models.Code.GenericCode, Files

- This test fulfills the following requirement:

- R-F-2

- Since the creation of this test, the following main revisions have been performed:

1. revision info:

- author : talbpaul

- date : 2017-08-24

- description: Created

\subsubsection{FRAMEWORK CODEINTERFACETESTS.GENERICINTERFACEIO}

This test can be found at ". / raven/tests / framework/CodeInterfaceTests/test_ generic_IO.xml”. This test can be called executing the following command:

. /run_tests --re=framework/CodeInterfaceTests.genericInterfaceIo

or

./run_framework_tests --re=framework/CodeInterfaceTests.genericInterfaceIo

- Test Description:

- An example of using the the Model Code of type GenericCode. This test is aimed to test the IO system present in the GenericCode interface. 
- Original Author:

- senrs

- Creation date:

- 2015-10-06

- The classes tested in this test are:

- Models.Code.GenericCode

- This test fulfills the following requirement:

- R-IS-2

- Since the creation of this test, the following main revisions have been performed:

1. revision info:

- author : maljdan

- date : 2016-04-06

- description: Updating test cases to reflect the changes to the user input.

2. revision info:

- author : alfoa

- date : 2017-01-21

- description: Adding this test description.

3. revision info:

- author : alfoa

- date : 2020-10-15

- description: This is now a requirement test (R-IS-2)

\subsubsection{FRAMEWORK CODEINTERFACETESTS.GENERICINTERFACEIOCUSTOMOUTPUT}

This test can be found at ". /raven/tests/framework/CodeInterfaceTests/test_ generic_interface_custom_out_file.xml". This test can be called executing the following command:

./run_tests --re=framework/CodeInterfaceTests.genericInterfaceIoqustomoutp

or

./run_framework_tests --re=framework/CodeInterfaceTests.genericInterfaceIo 
- Test Description:

- An example of using the the Model Code of type GenericCode with a code that produces CSV output file. This test is aimed to test the IO system present in the GenericCode interface. In XML node outputFile the user can specify the specific output file name RAVEN is going to load.

- Original Author:

- alfoa

- Creation date:

$-2017-01-24$

- The classes tested in this test are:

- Models.Code.GenericCode

- This test fulfills the following requirement:

- R-IS-6

- Since the creation of this test, the following main revisions have been performed:

1. revision info:

- author : alfoa

- date : 2018-01-24

- description: Adding this test description.

2. revision info:

- author : alfoa

- date : 2018-10-01

- description: This is a requirement test now.

\subsubsection{FRAMEWORK CODEINTERFACETESTS.RAVEN.ROM}

This test can be found at ". / raven/tests / framework/CodeInterfaceTests/RAVEN/ rom.xml". This test can be called executing the following command:

. /run_tests --re=framework/CodeInterfaceTests.RAVEN.ROM

or

./run_framework_tests --re=framework/CodeInterfaceTests.RAVEN.ROM 
- Test Description:

- This test is aimed to check the functionality of the RAVEN code interface (RAVEN running RAVEN). It tests the MPI implementation of the SLAVE RAVEN runs. In this case, 3 simultaneous SLAVE RAVEN (batchSize=3) runs are going to be spawned, each of them using 2 processors (NumMPI=2). The NumMPI XML node MUST BE INPUTTED if the SLAVE RAVEN runs must run in multiple processors!

- Original Author:

- alfoa

- Creation date:

- 2017-09-16

- The classes tested in this test are:

- Models.Code.RAVEN

- This test fulfills the following requirement:

- R-SI-1

- Since the creation of this test, the following main revisions have been performed:

1. revision info:

- author : talbpaul

- date : 2018-08-31

- description: added VariableGroups to inner/slave input file

2. revision info:

- author : alfoa

- date : 2018-10-01

- description: This is a requirement test now.

\subsubsection{FRAMEWORK CODEINTERFACETESTS RELAP5.RELAP5INTERFACETESTNOEXECUTA}

This test can be found at ". / raven/tests / framework/CodeInterfaceTests/RELAP 5 / test_relap5_code_interface.xml". This test can be called executing the following command:

. Irun_tests --re=framework/CodeInterfaceTests/RELAP 5.RELAP 5 interfaceTestNol 
. Irun_framework_tests --re=framework/CodeInterfaceTests/RELAP 5.RELAP 5inter

- Test Description:

- An example of using the RELAP5 code interface. This test is aimed to test the mechanics of the interface (no executable).

- Original Author:

- alfoa

- Creation date:

- 2015-10-06

- The classes tested in this test are:

- Models.Code.RELAP5

- This test fulfills the following requirement:

- R-SI-1

- Since the creation of this test, the following main revisions have been performed:

1. revision info:

- author : alfoa

- date : 2016-03-24

- description: New relap5 test case

2. revision info:

- author : talbpaul

- date : 2016-04-26

- description: Added check for pre-existing backup files when validating

3. revision info:

- author : alfoa

- date : 2016-08-02

- description: Added new gold, since the case has been changed

4. revision info:

- author : talbpaul

- date : 2016-09-15

- description: Test updates 
5. revision info:

- author : alfoa

- date : 2016-11-17

- description: Closes \#750

6. revision info:

- author : alfoa

- date : 2016-11-17

- description: Added alias for output system

7. revision info:

- author : alfoa

- date : 2017-01-21

- description: Adding this test description.

8. revision info:

- author : alfoa

- date : 2018-10-01

- description: This is a requirement test now. Req. R-SI-1

9. revision info:

- author : alfoa

- date : 2019-12-05

- description: Modified to check we can handle multiple words in the same card.

10. revision info:

- author : alfoa

- date : 2020-10-31

- description: Added csv xml node in the Code block for showing how to use it.

\subsubsection{FRAMEWORK POSTPROCESSORS BASICSTATISTICS GENERAL}

This test can be found at ". / raven/tests/framework/PostProcessors/BasicStatistics / test_Basicstatistics.xml". This test can be called executing the following command:

./run_tests --re=framework/PostProcessors/Basicstatistics/general

or

./run_framework_tests --re=framework/PostProcessors/Basicstatistilcs/genera 


\section{- Test Description:}

- This requirements test checks operation of the basic statistics postprocessor.

- Original Author:

- alfoa

- Creation date:

- 2014-05-21

- The classes tested in this test are:

- PostProcessors.BasicStatistics

- This test fulfills the following requirement:

- R-RA-5

- Since the creation of this test, the following main revisions have been performed:

1. revision info:

- author: wangc

- date : 2017-12-05

- description: convert test to use the new dataobjects

2. revision info:

- author: wangc

- date : 2018-12-13

- description: In PR \#882, the standard errors on statistics moments are implemented, these quantities are stored in the pointwise metadata, and will be printed out in the output csv files by default. In order to avoid regolding this test, the optional node 'what' with text values 'input, output' is added. In this case, only the user requested variables as specified in the DataObjects are printed out.

3. revision info:

- author : alfoa

- date : 2019-03-07

- description: Modified syntax in Functions as for issue \#934

4. revision info:

- author: wangc

- date : 2020-07-16

- description: Disable multipleFeatures option for input output sensitivity calcuation, since the condition number is ver big for this case which will cause unstable test results in Windows system. 


\subsubsection{FRAMEWORK POSTPROCESSORS LIMITSURFACE.TESTLIMITSURFACEPOSTPROCESS}

This test can be found at ". / raven/tests/framework/PostProcessors/LimitSurface/ test_LimitSurface.xml". This test can be called executing the following command:

. Irun_tests --re=framework/PostProcessors/Limitsurface.testLimit\$urfacePos

or

. Irun_framework_tests --re=framework/PostProcessors/Limitsurface.testLimit

- Test Description:

- This test is aimed to check the capability of RAVEN to generate Limit Surfaces from a pre-generated data set (in this case, generated with a MonteCarlo sampling), outputting the generated Limit Surface in DataObject.PointSet(s) and Databases.HDF5 reporting both transition boundaries (-1 1) or just one of them.

- Original Author:

- alfoa

- Creation date:

- 2014-07-10

- The classes tested in this test are:

- Models.PostProcessors.LimitSurface, Functions.External, Databases.HDF5

- This test fulfills the following requirement:

- R-RA-1

- R-RA-7

- Since the creation of this test, the following main revisions have been performed:

1. revision info:

- author : alfoa

- date : 2014-07-11

- description: Limit Surface pp fix. SVN r28533

2. revision info:

- author: alfoa

- date : 2014-11-17 
- description: restructuring Steps (removed Adaptive and PostProcessor), adding Assembler capability, removed datatype input for Functions and External models, modified all the inputs, added SafestPoint postprocessor and relative regression test, fixed Sampling for descrete distributions, added check for the distributions consistency between framework and RELAP7. SVN r30411

3. revision info:

- author : alfoa

- date : 2015-06-18

- description: Changed Name of all DataObjects

4. revision info:

- author : talbpaul

- date : 2015-07-06

- description: standardization of tests

5. revision info:

- author : talbpaul

- date : 2015-07-06

- description: Revert "standardization of tests". This reverts commit 68099325e8daba0f756179a201

6. revision info:

- author : talbpaul

- date : 2015-07-06

- description: updated tests, added script

7. revision info:

- author : talbpaul

- date : 2015-07-07

- description: updated to restrict partial-node comments

8. revision info:

- author : cogljj

- date : 2015-09-29

- description: Modifing external modules to use relative to working directory.

9. revision info:

- author : maljdan

- date : 2016-04-06

- description: Updating test cases to reflect the changes to the user input.

10. revision info:

- author : alfoa 
- date : 2017-01-21

- description: Adding this test description.

11. revision info:

- author : alfoa

- date : 2017-12-14

- description: Moved LimitSurface Postprocessor tests in a specific directory (tests/framework/PostProcessors/LimitSurface) and updated for new DataObject structure

12. revision info:

- author : alfoa

- date : 2018-09-11

- description: Added an additional IOStep to check that the limit surface DataObject can be dumped into a Database of type HDF5

13. revision info:

- author : alfoa

- date : 2019-03-07

- description: Modified syntax in Functions as for issue \#934

\subsubsection{FRAMEWORK POSTPROCESSORS LIMITSURFACE.TESTLIMITSURFACEINTEGRALPP}

This test can be found at ". / raven/tests/framework/PostProcessors/LimitSurface/ test_LimitSurface_and_integral.xml". This test can be called executing the following command:

. Irun_tests --re=framework/PostProcessors/LimitSurface.testLimit\$urfaceInt

or

. Irun_framework_tests --re=framework/PostProcessors/Limitsurface. testLimit

- Test Description:

- This test is aimed to check the capability of RAVEN to generate Limit Surfaces from a pre-generated data set (in this case, generated with a MonteCarlo sampling). In addition, this test is aimed to check the capability of RAVEN to compute the integral of the Limit Surface (e.g. Failure probability) both probability-weighted and not.

- Original Author: 
- alfoa

- Creation date:

$-2015-05-08$

- The classes tested in this test are:

- Models.PostProcessors.LimitSurface, Models.PostProcessors.LimitSurfaceIntegral

- This test fulfills the following requirement:

- R-RA-7

- Since the creation of this test, the following main revisions have been performed:

1. revision info:

- author : alfoa

- date : 2015-05-11

- description: Limit surface integral

2. revision info:

- author : alfoa

- date : 2015-05-12

- description: Closes \#153

3. revision info:

- author : alfoa

- date : 2015-06-17

- description: Closes \#213

4. revision info:

- author : alfoa

- date : 2015-06-18

- description: Changed Name of all DataObjects

5. revision info:

- author : maljdan

- date : 2016-04-06

- description: Updating test cases to reflect the changes to the user input.

6. revision info:

- author : alfoa

- date : 2017-01-21

- description: Adding this test description. 
7. revision info:

- author : alfoa

- date : 2017-12-14

- description: Moved LimitSurface Postprocessor tests in a specific directory (tests/framework/PostProcessors/LimitSurface) and updated for new DataObject structure

8. revision info:

- author : alfoa

- date : 2018-09-20

- description: This test is now a requirement test.

9. revision info:

- author : alfoa

- date : 2019-03-07

- description: Modified syntax in Functions as for issue \#934

\subsubsection{FRAMEWORK POSTPROCESSORS LIMITSURFACE.TESTLIMITSURFACEINTEGRALPPV}

This test can be found at ". /raven/tests/framework/PostProcessors/LimitSurface/ test_LimitSurface_with_err_bounds.xml". This test can be called executing the following command:

./run_tests --re=framework/PostProcessors/LimitSurface.testLimit\$urfaceInt or

./run_framework_tests --re=framework/PostProcessors/LimitSurface.testLimit.

- Test Description:

- This test is aimed to check the capability of RAVEN to compute the integral of the Limit Surface (e.g. Failure probability) reporting the bounding error as well (the maximum error, in probability, in the computation of the limit surface )

- Original Author:

- alfoa

- Creation date:

- 2020-04-15 
- The classes tested in this test are:

- Models.PostProcessors.LimitSurface, Models.PostProcessors.LimitSurfaceIntegral

- This test fulfills the following requirement:

- R-RA-8

- Since the creation of this test, the following main revisions have been performed:

1. revision info:

- author : alfoa

- date : 2020-04-15

- description: Added test for computation of the error bound and linked to new requirement

\subsubsection{FRAMEWORK POSTPROCESSORS DATAMININGPOSTPROCESSOR DIMEN- SIONALITYREDUCTION EXACTPCA}

This test can be found at ". / raven/tests / framework/PostProcessors/DataMiningPostProce DimensionalityReduction/test_dataMiningExactPCA.xml". This test can be called executing the following command:

. Irun_tests --re=framework/PostProcessors/DataMiningPostProcessor/Dimensio

or

. Irun_framework_tests --re=framework/PostProcessors/DataMiningPoßtProcesso

- Test Description:

- Tests decomposition with PCA model.

- Original Author:

- senrs

- Creation date:

- 2015-11-24

- The classes tested in this test are:

- DataMining 
- This test fulfills the following requirement:

- R-RA-6

- Since the creation of this test, the following main revisions have been performed:

1. revision info:

- author: wangc

- date : 2018-09-27

- description: As reported in issue \#805, RAVEN will not allow input DataObject of PostProcessor to be output DataObject, the output data object will be renamed, and the variable name of labels will be explicitly defined.

\subsubsection{FRAMEWORK OPTIMIZERS.MINIMAL}

This test can be found at ". / raven/tests / framework/Optimizers / GradientDescent/ minimal.xml". This test can be called executing the following command:

./run_tests --re=framework/Optimizers.Minimal

or

./run_framework_tests --re=framework/Optimizers.Minimal

- Test Description:

- Provides the minimum possible options for which the Optimizer can run.

- This test is analytic:

- This test uses Beale's function, which is documented in the analytic tests documentation under the Optimizer functions section.

- Original Author:

- talbpaul

- Creation date:

- 2020-03-06

- The classes tested in this test are:

- Optimizer

- This test fulfills the following requirement:

- R-RM-1 


\subsubsection{FRAMEWORK OPTIMIZERS.CONSTRAINBOUNDARY}

This test can be found at ". /raven/tests / framework/Optimizers/GradientDescent/ constrain_boundary.xml". This test can be called executing the following command:

./run_tests --re=framework/Optimizers. ConstrainBoundary

or

./run_framework_tests --re=framework/Optimizers.ConstrainBoundary

- Test Description:

- This test uses a multidimensional linear function with optima outside the domain space to test boundary constraint handling.

- This test is analytic:

- This test uses the NDSlant function, which is documented in the analytic tests documentation under the Optimizer functions section.

- Original Author:

- talbpaul

- Creation date:

$-2020-01-30$

- The classes tested in this test are:

- Optimizer

- This test fulfills the following requirement:

- R-RM-2

\subsubsection{FRAMEWORK OPTIMIZERS.CONSTRAINFUNCTION}

This test can be found at ". /raven/tests / framework / Optimizers/GradientDescent/ constrain_function.xml". This test can be called executing the following command:

./run_tests --re=framework/Optimizers. Constrainfunction

or 
./run_framework_tests --re=framework/Optimizers.ConstrainFunction

- Test Description:

- This test uses a multidimensional linear function such that the trajectory must pass through a functional constraint to reach the optimal point.

- Original Author:

- talbpaul

- Creation date:

- 2020-02-05

- The classes tested in this test are:

- Optimizer

- This test fulfills the following requirement:

- R-RM-2

- Since the creation of this test, the following main revisions have been performed:

1. revision info:

- author: zhouj

- date : 2020-05-15

- description: Add implicit constriant node in optimizer and function node with objective as variable.

2. revision info:

- author: zhouj

- date : 2020-06-04

- description: Add reject opt point reason.

\subsubsection{FRAMEWORK ROM TIMESERIES DMD.TRADITIONALDMD}

This test can be found at ". /raven/tests/framework/ROM/TimeSeries/DMD/test_ traditional_dmd.xml". This test can be called executing the following command:

./run_tests --re=framework/ROM/TimeSeries/DMD.traditionalDMD 
or

./run_framework_tests --re=framework/ROM/TimeSeries/DMD.traditionalDMD

- Test Description:

- This test is aimed to check the mechanics of the DMD ROM using the standard SVDbased algorithm. In addition, it tests the capability for the user to export the ROM info in an XML output file (requirement).

- Original Author:

- alfoa

- Creation date:

- 2018-04-25

- The classes tested in this test are:

- SupervisedLearning.DynamicModeDecomposition

- This test fulfills the following requirement:

- R-F-5

- Since the creation of this test, the following main revisions have been performed:

1. revision info:

- author : alfoa

- date : 2018-04-25

- description: Adding this test description.

2. revision info:

- author : alfoa

- date : 2018-10-01

- description: This test is a requirement test now.

3. revision info:

- author : talbpaul

- date : 2019-01-09

- description: moving from ROM-files to ROM-dataobjects 


\subsubsection{FRAMEWORK SAMPLERS RESTART.MC}

This test can be found at "./raven/tests/framework/Samplers/Restart/test_ restart_MC.xml". This test can be called executing the following command:

. /run_tests --re=framework/Samplers/Restart.MC

or

. Irun_framework_tests --re=framework/Samplers/Restart.MC

- Test Description:

- Tests restarting a Monte Carlo sampling from restart. makeCoarse samples initial data, then makeRestart makes additional samples, restarting from the first set of samples. makeF ine does all the samples without restart for comparison. The model for "coarse" always returns a value of 1 , while the model for "restart" returns a value of 2, so you can tell which samples came from which sampling strategy.

- Original Author:

- talbpaul

- Creation date:

- 2015-07-07

- The classes tested in this test are:

- Samplers.MonteCarlo

- This test fulfills the following requirement:

- R-F-4

- Since the creation of this test, the following main revisions have been performed:

1. revision info:

- author : alfoa

- date : 2018-10-01

- description: This Test is now a requirement test. 


\subsubsection{FRAMEWORK SAMPLERS RESTART.CSV}

This test can be found at "./raven/tests/framework/Samplers/Restart/test_ restart_csv.xml". This test can be called executing the following command:

./run_tests --re=framework/Samplers/Restart.CSV

or

./run_framework_tests --re=framework/Samplers/Restart.CSV

- Test Description:

- This test demonstrates that a restart can be performed from a loaded CSV file, not just an internal data object from an earlier step. As with the other restart tests, in output data objects samples from "course" have an output of 1 , while samples from "fine" have an output of 2 .

- Original Author:

- talbpaul

- Creation date:

- 2015-07-27

- The classes tested in this test are:

- Files

- This test fulfills the following requirement:

- R-F-4

- Since the creation of this test, the following main revisions have been performed:

1. revision info:

- author : alfoa

- date : 2018-10-01

- description: This Test is now a requirement test. 


\subsubsection{FRAMEWORK SAMPLERS RESTART.CONSTANT}

This test can be found at "./raven/tests/framework/Samplers/Restart/test_ restart_constant.xml". This test can be called executing the following command:

. /run_tests --re=framework/Samplers/Restart. Constant

or

. /run_framework_tests --re=framework/Samplers/Restart. Constant

- Test Description:

- The essence of this test is to demonstrate the restart capability with constant variable, i.e. test the scaling factor for constant samples as mentioned in issue \#690

- Original Author:

- wangc

- Creation date:

- 2018-08-04

- The classes tested in this test are:

- Samplers.Grid

- This test fulfills the following requirement:

- R-F-4

- Since the creation of this test, the following main revisions have been performed:

1. revision info:

- author : alfoa

- date : 2018-10-01

- description: This Test is now a requirement test. 


\subsubsection{FRAMEWORK MCMC.METROPOLIS}

This test can be found at ". /raven/tests/framework/MCMC/test_metropolis.xml". This test can be called executing the following command:

. /run_tests --re=framework/MCMC.Metropolis

or

./run_framework_tests --re=framework/MCMC.Metropolis

- Test Description:

- Test the Markov Chain Monte Carlo algorithm: Metropolis Sampling A 2-D multivariate normal distribution is used as the likelihood function. mean: [5, 5], cov $=[[1,0.9]$, $[0.9,1]]$ Both input parameters have the standard normal distribution as their prior distribution. The proposal distributions for the input variables are also standard normal distribution. "TargetEvaluation" is used to collect the inputs and outputs from the likelihood model. "SolutionExport" is used to store the posterior values for the input variables, i.e. "xin" and "yin"

- Original Author:

- wangc

- Creation date:

- 2020-06-26

- The classes tested in this test are:

- MCMC.Metropolis

- This test fulfills the following requirement:

- R-RA-9

\subsubsection{FRAMEWORK INTERNALPARALLELTESTS.ROMSCIKIT}

This test can be found at "./raven/tests/framework/InternalParallelTests/ test_internal_parallel_ROM_scikit.xml”. This test can be called executing the following command:

. /run_tests --re=framework/InternalParallelTests.ROMscikit 
or

./run_framework_tests --re=framework/InternalParalleltests.RoMscilkit

- Test Description:

- This test is aimed to check the functionality of the RAVEN parallelization scheme for Internal Objects. In this case the functionality of the parallelization is tested for the Model ROM of type SKLearn

- Original Author:

- alfoa

- Creation date:

- 2015-10-07

- The classes tested in this test are:

- JobHandler.ParallelPython, Models.ROM.SKLearn

- This test fulfills the following requirement:

- R-IS-8

- Since the creation of this test, the following main revisions have been performed:

1. revision info:

- author : maljdan

- date : 2016-04-06

- description: Updating test cases to reflect the changes to the user input.

2. revision info:

- author : maljdan

- date : 2016-04-25

- description: Fixing the internal parallel tests to use support vector regression and making the step sizes look the same in the cluster and local test cases. This creates a more robust test case since the data points will be floating point values.

3. revision info:

- author : maljdan

- date : 2016-04-25

- description: Switching to a different regressor that yields more consistent results.

4. revision info: 
- author : maljdan

- date : 2016-04-25

- description: Regolding based on 1-nearest neighbor regression. This should effectively report the same information as the training data.

5. revision info:

- author : alfoa

- date : 2017-01-21

- description: Adding this test description.

6. revision info:

- author : maljdan

- date : 2017-09-19

- description: Adding a maxQueueSize for testing a new feature.

7. revision info:

- author : alfoa

- date : 2018-10-01

- description: This is a requirement test now.

\subsubsection{FRAMEWORK INTERNALPARALLELTESTS.EXTERNALMODEL}

This test can be found at "./raven/tests/framework/InternalParallelTests/ test_internal_parallel_extModel.xml”. This test can be called executing the following command:

./run_tests --re=framework/InternalParallelTests.ExternalModel

or

./run_framework_tests --re=framework/InternalParallelTests.ExternalModel

- Test Description:

- This test is aimed to check the functionality of the RAVEN parallelization scheme for Internal Objects. In this case the functionality of the parallelization is tested for the Model External Model

- Original Author:

- alfoa 
- Creation date:

- 2015-10-07

- The classes tested in this test are:

- JobHandler.ParallelPython, Models.ExternalModel

- This test fulfills the following requirement:

- R-IS-8

- Since the creation of this test, the following main revisions have been performed:

1. revision info:

- author : alfoa

- date : 2015-10-19

- description: New syntax form samplerInit: from sampler_init -i samplerInit

2. revision info:

- author : maljdan

- date : 2016-04-06

- description: Updating test cases to reflect the changes to the user input.

3. revision info:

- author : maljdan

- date : 2016-05-20

- description: Updating the test cases to remove references to Point or History.

4. revision info:

- author : alfoa

- date : 2017-01-21

- description: Adding this test description.

5. revision info:

- author : alfoa

- date : 2018-10-01

- description: This is a requirement test now.

\subsubsection{FRAMEWORK INTERNALPARALLELTESTS.POSTPROCESSOR}

This test can be found at "./raven/tests/framework/InternalParallelTests/ test_internal_parallel_PP_LS.xml”. This test can be called executing the following command: 
./run_tests --re=framework/InternalParallelTests.PostProcessor

or

./run_framework_tests --re=framework/InternalParallelTests.PostProcessor

- Test Description:

- This test is aimed to check the functionality of the RAVEN parallelization scheme for Internal Objects. In this case the functionality of the parallelization is tested for the Model PostProcessor

- Original Author:

- alfoa

- Creation date:

- 2015-10-07

- The classes tested in this test are:

- JobHandler.ParallelPython, Models.PostProcessor

- This test fulfills the following requirement:

- R-IS-8

- Since the creation of this test, the following main revisions have been performed:

1. revision info:

- author : alfoa

- date : 2015-10-07

- description: Made the test faster

2. revision info:

- author : alfoa

- date : 2015-10-19

- description: New syntax form samplerInit: from sampler_init - i samplerInit

3. revision info:

- author : maljdan

- date : 2016-04-06

- description: Updating test cases to reflect the changes to the user input. 
4. revision info:

- author : alfoa

- date : 2017-01-21

- description: Adding this test description.

5. revision info:

- author : alfoa

- date : 2018-01-09

- description: Modified for new dataobject structure

6. revision info:

- author : alfoa

- date : 2018-10-01

- description: This is a requirement test now.

7. revision info:

- author : alfoa

- date : 2019-03-07

- description: Modified syntax in Functions as for issue \#934

\subsection{Analytical tests' description}

This section contains the description of all the analytical tests.

\subsubsection{FRAMEWORK CODEINTERFACETESTS RAVEN.BASIC}

This test can be found at ". / raven/tests / framework/CodeInterfaceTests/RAVEN/ basic.xml". This test can be called executing the following command:

./run_tests --re=framework/CodeInterfaceTests/RAVEN.Basic

or

./run_framework_tests --re=framework/CodeInterfaceTests/RAVEN.Baßic

- Test Description:

- Basic test of the raven-runs-raven interface. Outer raven runs a grid sampling by changing the upper and lower bounds of the sampledVars distribution on the inner raven. Inner raven runs $100 \mathrm{MC}$ samples, does basic stats, and returns the mean, sigma to the outer raven. 
- This test is analytic:

- Each sample in this test converges towards the analytic solutions discussed in the "changing lower, upper bounds" section of the "attenuate" analytic model documentation.

- Original Author:

- talbpaul

- Creation date:

- 2018-01-18

- The classes tested in this test are:

- Models.Code.RAVEN

- Since the creation of this test, the following main revisions have been performed:

1. revision info:

- author : talbpaul

- date : 2018-05-02

- description: added "fully-correlated" variable for innerUpperBound as a test of the mechanic

2. revision info:

- author : talbpaul

- date : 2018-11-06

- description: modified inner run to use grid for speed, consistent results

\subsubsection{FRAMEWORK CODEINTERFACETESTS RAVEN.RETURNDATABASE}

This test can be found at ". / raven/tests/framework/CodeInterfaceTests/RAVEN/ return_database.xml”. This test can be called executing the following command:

. /run_tests --re=framework/CodeInterfaceTests/RAVEN. ReturnDataba \$e

or

./run_framework_tests --re=framework/CodeInterfaceTests/RAVEN.ReturnDataba

- Test Description: 
- Tests returning a NetCDF database instead of a set of dataobject CSVs.

- This test is analytic:

- Each sample in this test converges towards the analytic solutions discussed in the "changing lower, upper bounds" section of the "attenuate" analytic model documentation.

- Original Author:

- talbpaul

- Creation date:

- 2020-03-04

- The classes tested in this test are:

- Models.Code.RAVEN

\subsubsection{FRAMEWORK PCA SPARSEGRID ATTENUCORRELATION}

This test can be found at ". / raven/tests/framework/pca_sparseGridCollocation/ test_attenu_correlation.xml". This test can be called executing the following command:

./run_tests --re=framework/pca_sparseGrid/attenuCorrelation

or

./run_framework_tests --re=framework/pca_sparseGrid/attenuCorrelation

- Test Description:

- Tests the SparseGridCollocation sampler combined wit input reductions via PCA method, using correlated multivariate normal distributions.

- This test is analytic:

- This test is analytic in mean and variance using the "attenuation" analytic model documented in the analytic tests.

- Original Author:

- wangc 
- Creation date:

- 2015-11-23

- The classes tested in this test are:

- Samplers.SparseGridCollocation,SupervisedLearning.GaussPolynomialROM

- Since the creation of this test, the following main revisions have been performed:

1. revision info:

- author : talbpaul

- date : 2019-01-09

- description: moving from ROM-files to ROM-dataobject

\subsubsection{FRAMEWORK PCA SPARSEGRID ATTENUCORRELATIONMC}

This test can be found at ". /raven/tests/framework/pca_sparseGridCollocation/ test_attenu_correlation_mc.xml”. This test can be called executing the following command:

./run_tests --re=framework/pca_sparseGrid/attenuCorrelationMC

or

./run_framework_tests --re=framework/pca_sparseGrid/attenuCorrelattionMC

- Test Description:

- Tests the SparseGridCollocation sampler combined wit input reductions via PCA method, using correlated multivariate normal distributions.

- This test is analytic:

- This test is analytic in mean and variance using the "attenuation" analytic model documented in the analytic tests.

- Original Author:

- wangc

- Creation date:

- 2015-11-23

- The classes tested in this test are:

- Samplers.MonteCarlo 


\subsubsection{FRAMEWORK PCA SPARSEGRID POLYCORRELATION}

This test can be found at ". / raven/tests/framework/pca_sparseGridCollocation/ test_poly_correlation.xml". This test can be called executing the following command:

./run_tests --re=framework/pca_sparseGrid/polyCorrelation

or

./run_framework_tests --re=framework/pca_sparseGrid/polyCorrelation

- Test Description:

- Tests the SparseGridCollocation sampler combined wit input reductions via PCA method, using correlated multivariate normal distributions.

- This test is analytic:

- This test is analytic in mean and variance using the "tensor polynomial" analytic model documented in the analytic tests.

- Original Author:

- wangc

- Creation date:

$-2015-11-23$

- The classes tested in this test are:

- Samplers.SparseGridCollocation,SupervisedLearning.GaussPolynomialROM

- Since the creation of this test, the following main revisions have been performed:

1. revision info:

- author : talbpaul

- date : 2019-01-09

- description: moving from ROM-file to ROM-dataobject 


\subsubsection{FRAMEWORK PCA SPARSEGRID POLYCORRELATIONMC}

This test can be found at ". / raven/tests/framework/pca_sparseGridCollocation/ test_poly_correlation_mc.xml”. This test can be called executing the following command:

./run_tests --re=framework/pca_sparseGrid/polyCorrelationMC

or

. /run_framework_tests --re=framework/pca_sparseGrid/polyCorrelationMC

- Test Description:

- Tests the SparseGridCollocation sampler combined with input reductions via PCA method, using correlated multivariate normal distributions.

- This test is analytic:

- This test is analytic in mean and variance using the "tensor polynomial" analytic model documented in the analytic tests.

- Original Author:

- wangc

- Creation date:

- 2015-11-23

- The classes tested in this test are:

- Samplers.SparseGridCollocation,SupervisedLearning.GaussPolynomialROM

\subsubsection{FRAMEWORK PCA SPARSEGRID POLYANALYTICALTEST}

This test can be found at ". / raven/tests/framework/pca_sparseGridCollocation/ test_poly.xml". This test can be called executing the following command:

. /run_tests --re=framework/pca_sparseGrid/polyAnalyticalTest

or

./run_framework_tests --re=framework/pca_sparseGrid/polyAnalyticalitest 
- Test Description:

- Tests the SparseGridCollocation sampler combined wit input reductions via PCA method, using uncorrelated multivariate normal distributions.

- This test is analytic:

- This test is analytic in mean and variance using the "tensor polynomial" analytic model documented in the analytic tests.

- Original Author:

- wangc

- Creation date:

- 2015-11-23

- The classes tested in this test are:

- Samplers.SparseGridCollocation,SupervisedLearning.GaussPolynomialROM

- Since the creation of this test, the following main revisions have been performed:

1. revision info:

- author : talbpaul

- date : 2019-01-09

- description: moving from ROM-file to ROM-dataobject

\subsubsection{FRAMEWORK.MODELS.EXTERNAL.ALL METHODS}

This test can be found at ". / raven/tests/framework/Models/External/all_methods. xml". This test can be called executing the following command:

./run_tests --re=framework.Models.External.all_methods

or

./run_framework_tests --re=framework.Models.External.all_methods

- Test Description:

- This test is used to exercise all of the optional ExternalModel methods and assure they behave as expected. 
- This test is analytic:

- This test uses the analytic model "attenuate", which is documented in the analytical test documentation. Additionally, values of the "from" variables are exactly determined because they are set in the extmod methods:

* fromReadMoreXML: pi (3.14159)

* fromInit : sqrt(pi) (1.77245)

$*$ fromCNISelf : pi/2 (1.57080)

* fromCNIDict : $2 * \operatorname{sqrt}(\mathrm{pi})(3.54491)$

The exit strength "ans" is analytic with results as follows (Note that for testing purposes, the model always adds 0.05 to $\mathrm{y} 2$ before calculating the exit strength):

* y1, y2, ans

* $0.0,0.0,0.97531$

* $0.0,1.0,0.59156$

* 1.0, 0.0, 0.59156

* 1.0, 1.0, 0.35880

- Original Author:

- talbpaul

- Creation date:

- 2018-01-17

- The classes tested in this test are:

- Models.ExternalModel

\subsubsection{FRAMEWORK.MODELS.EXTERNAL.SERIALIZE EXT MODEL}

This test can be found at "./raven/tests/framework/Models/External/serialize_ ext_model_and_use.xml". This test can be called executing the following command:

./run_tests --re=framework.Models.External.serialize_ext_model

or

./run_framework_tests --re=framework.Models.External.serialize_ext_model

- Test Description: 
- This test is used to exercise the ability or RAVEN to serialize (pickle) the external model class and re-use it in the framework.

- This test is analytic:

- This test uses the analytic model "attenuate", which is documented in the analytical test documentation. Additionally, values of the "from" variables are exactly determined because they are set in the extmod methods:

* fromReadMoreXML: pi (3.14159)

* fromInit : sqrt(pi) (1.77245)

* fromCNISelf : pi/2 (1.57080)

* fromCNIDict : $2 * \operatorname{sqrt}($ pi) $(3.54491)$

The exit strength "ans" is analytic with results as follows (Note that for testing purposes, the model always adds 0.05 to $\mathrm{y} 2$ before calculating the exit strength):

* y $1, \mathrm{y}^{2}$, ans

* $0.0,0.0,0.97531$

* $0.0,1.0,0.59156$

* 1.0, $0.0,0.59156$

* 1.0, 1.0, 0.35880

- Original Author:

- alfoa

- Creation date:

- 2020-09-26

- The classes tested in this test are:

- Models.ExternalModel

\subsubsection{FRAMEWORK OPTIMIZERS SIMULATEDANNEALING.EXPONENTIALSA}

This test can be found at ". / raven/tests / framework/Optimizers/SimulatedAnnealing/ ExponentialSA/test_simulatedAnnealing.xml". This test can be called executing the following command:

./run_tests --re=framework/Optimizers/SimulatedAnnealing.ExponentialsA

or

./run_framework_tests --re=framework/Optimizers/SimulatedAnnealing. Exponen 
- Test Description:

- This test assesses the Simulated Annealing algorithm using Boltzmann cooling schedule on the Beale function. The nominal dimensionality of the problem is 2, i.e., variables are $\mathrm{x}, \mathrm{y}$. The objective variable is ans. The global minimum is at: ans $(3,0.5)=$ 0.0 Parameter range is $-4.5 \leq x, y \leq 4.5$.

- This test is analytic:

- This test uses beale's function, which is documented in the analytic tests documentation under the Optimizer functions section.

- Original Author:

- MohammadAbdo

- Creation date:

$-2020-02-11$

- The classes tested in this test are:

- SimulatedAnnealing

\subsubsection{FRAMEWORK OPTIMIZERS SIMULATEDANNEALING.EXPONENTIALEGGHOLDER}

This test can be found at ". / raven/tests/framework/Optimizers/SimulatedAnnealing/ ExponentialEggHolder/test_simulatedAnnealing_eggHold.xml". This test can be called executing the following command:

./run_tests --re=framework/Optimizers/SimulatedAnnealing.ExponentialEggHol

or

./run_framework_tests --re=framework/Optimizers/SimulatedAnnealing.Exponen

- Test Description:

- This test assesses the Simulated Annealing algorithm using Boltzmann cooling schedule on the Eggholder function. The eggholder function is a very challenging objective function, as it has enourmous number of local minima, The nominal dimensionality of the problem is 2, i.e., variables are $\mathrm{x}, \mathrm{y}$. The objective variable is ans. The global minimum is at: ans $(512,404.2319)=-959.6407$ parameter range is $-512 \leq x, y \leq 512$

- This test is analytic: 
- This test uses eggHolder's function, which is documented in the analytic tests documentation under the Optimizer functions section.

- Original Author:

- MohammadAbdo

- Creation date:

- 2020-02-11

- The classes tested in this test are:

- SimulatedAnnealing

\subsubsection{FRAMEWORK OPTIMIZERS SIMULATEDANNEALING.BOLTZMANSA}

This test can be found at ". / raven/tests/framework/Optimizers/SimulatedAnnealing/ BoltzmanSA/test_simulatedAnnealing.xml". This test can be called executing the following command:

./run_tests --re=framework/Optimizers/SimulatedAnnealing.Boltzman.SA

or

./run_framework_tests --re=framework/Optimizers/SimulatedAnnealing. Boltzma

- Test Description:

- This test assesses the Simulated Annealing algorithm using Boltzmann cooling schedule on the Beale function. The nominal dimensionality of the problem is 2 , i.e., variables are $\mathrm{x}, \mathrm{y}$. The objective variable is ans. The global minimum is at: ans $(3,0.5)=$ 0.0 Parameter range is $-4.5 \leq x, y \leq 4.5$.

- This test is analytic:

- This test uses Beale's function, which is documented in the analytic tests documentation under the Optimizer functions section.

- Original Author:

- MohammadAbdo

- Creation date: 
- 2020-02-11

- The classes tested in this test are:

- SimulatedAnnealing

\subsubsection{FRAMEWORK OPTIMIZERS SIMULATEDANNEALING.BOLTZMANEGGHOLDER}

This test can be found at ". / raven/tests/framework/Optimizers/SimulatedAnnealing/ BoltzmanEggHolder/test_simulatedAnnealing_eggHold.xml”. This test can be called executing the following command:

./run_tests --re=framework/Optimizers/SimulatedAnnealing.BoltzmanEggHolder

or

./run_framework_tests --re=framework/Optimizers/SimulatedAnnealing.Boltzma

- Test Description:

- This test assesses the Simulated Annealing algorithm using Boltzmann cooling schedule on the Eggholder function. The eggholder function is a very challenging objective function, as it has enourmous number of local minima, The nominal dimensionality of the problem is 2, i.e., variables are $\mathrm{x}$, y. The objective variable is ans. The global minimum is at: ans $(512,404.2319)=-959.6407$ parameter range is $-512 \leq x, y \leq 512$

- This test is analytic:

- This test uses eggHolder's function, which is documented in the analytic tests documentation under the Optimizer functions section.

- Original Author:

- MohammadAbdo

- Creation date:

- 2020-02-11

- The classes tested in this test are:

- SimulatedAnnealing 


\subsubsection{FRAMEWORK OPTIMIZERS SIMULATEDANNEALING.CAUCHYSA}

This test can be found at ". /raven/tests/framework/Optimizers/SimulatedAnnealing/ CauchySA/test_simulatedAnnealing.xml”. This test can be called executing the following command:

./run_tests --re=framework/Optimizers/SimulatedAnnealing.CauchysA

or

./run_framework_tests --re=framework/Optimizers/SimulatedAnnealing. Cauchys

- Test Description:

- This test assesses the Simulated Annealing algorithm using Boltzmann cooling schedule on the Beale function. The nominal dimensionality of the problem is 2, i.e., variables are $\mathrm{x}, \mathrm{y}$. The objective variable is ans. The global minimum is at: ans $(3,0.5)=$ 0.0 Parameter range is $-4.5 \leq x, y \leq 4.5$.

- This test is analytic:

- This test uses Beale's function, which is documented in the analytic tests documentation under the Optimizer functions section.

- Original Author:

- MohammadAbdo

- Creation date:

$-2020-02-11$

- The classes tested in this test are:

- SimulatedAnnealing

\subsubsection{FRAMEWORK OPTIMIZERS SIMULATEDANNEALING.CAUCHYEGGHOLDER}

This test can be found at ". /raven/tests/framework/Optimizers/SimulatedAnnealing/ CauchyEggHolder/test_simulatedAnnealing_eggHold.xml". This test can be called executing the following command:

./run_tests --re=framework/Optimizers/SimulatedAnnealing.CauchyEqgHolder 
or

. Irun_framework_tests --re=framework/Optimizers/SimulatedAnnealing. CauchyE

- Test Description:

- This test assesses the Simulated Annealing algorithm using Boltzmann cooling schedule on the Eggholder function. The eggholder function is a very challenging objective function, as it has enourmous number of local minima, The nominal dimensionality of the problem is 2, i.e., variables are $\mathrm{x}, \mathrm{y}$. The objective variable is ans. The global minimum is at: ans $(512,404.2319)=-959.6407$ parameter range is $-512 \leq x, y \leq 512$

- This test is analytic:

- This test uses eggHolder's function, which is documented in the analytic tests documentation under the Optimizer functions section.

- Original Author:

- MohammadAbdo

- Creation date:

- 2020-02-11

- The classes tested in this test are:

- SimulatedAnnealing

\subsubsection{FRAMEWORK OPTIMIZERS SIMULATEDANNEALING.VERYFASTSA}

This test can be found at ". / raven/tests/framework/Optimizers/SimulatedAnnealing/ VeryFastSA/test_simulatedAnnealing.xml". This test can be called executing the following command:

./run_tests --re=framework/Optimizers/SimulatedAnnealing.VeryFastsA

or

./run_framework_tests --re=framework/Optimizers/SimulatedAnnealing.VeryFas

- Test Description: 
- This test assesses the Simulated Annealing algorithm using Boltzmann cooling schedule on the Beale function. The nominal dimensionality of the problem is 2, i.e., variables are $x, y$. The objective variable is ans. The global minimum is at: ans $(3,0.5)=$ 0.0 Parameter range is $-4.5 \leq x, y \leq 4.5$.

- This test is analytic:

- This test uses beale's function, which is documented in the analytic tests documentation under the Optimizer functions section.

- Original Author:

- MohammadAbdo

- Creation date:

$-2020-02-11$

- The classes tested in this test are:

- SimulatedAnnealing

\subsubsection{FRAMEWORK OPTIMIZERS.MINIMAL}

This test can be found at ". / raven/tests/framework/Optimizers/GradientDescent/ minimal.xml". This test can be called executing the following command:

. Irun_tests --re=framework/Optimizers.Minimal

Or

. /run_framework_tests --re=framework/Optimizers.Minimal

- Test Description:

- Provides the minimum possible options for which the Optimizer can run.

- This test is analytic:

- This test uses Beale's function, which is documented in the analytic tests documentation under the Optimizer functions section.

- Original Author:

- talbpaul 
- Creation date:

- 2020-03-06

- The classes tested in this test are:

- Optimizer

- This test fulfills the following requirement:

- R-RM-1

\subsubsection{FRAMEWORK OPTIMIZERS.BASIC}

This test can be found at ". /raven/tests / framework/Optimizers/GradientDescent/ basic.xml". This test can be called executing the following command:

./run_tests --re=framework/Optimizers.Basic

or

./run_framework_tests --re=framework/Optimizers.Basic

- Test Description:

- This is the most basic test of the Optimizer. It employs finite difference for the gradient estimation, gradient history for the step determination, and strict improvement acceptance. It converges only on the number of samples taken with a single trajectory on the Beale function. It also demonstrates typical outstream options for optimization. It also tests passing through constants and functions.

- This test is analytic:

- This test uses Beale's function, which is documented in the analytic tests documentation under the Optimizer functions section.

- Original Author:

- talbpaul

- Creation date:

- 2020-01-29

- The classes tested in this test are:

- Optimizer 


\subsubsection{FRAMEWORK OPTIMIZERS.CONVERGEGRADIENT}

This test can be found at ". / raven/tests/framework/Optimizers/GradientDescent/ converge_gradient.xml". This test can be called executing the following command:

./run_tests --re=framework/Optimizers. ConvergeGradient

or

./run_framework_tests --re=framework/Optimizers.ConvergeGradient

- Test Description:

- Extending on the Basic test, this test converges on gradient magnitude instead of number of samples. Also converges with persistence.

- This test is analytic:

- This test uses Beale's function, which is documented in the analytic tests documentation under the Optimizer functions section.

- Original Author:

- talbpaul

- Creation date:

- 2020-01-29

- The classes tested in this test are:

- Optimizer

\subsubsection{FRAMEWORK OPTIMIZERS.CONVERGESTEPSIZE}

This test can be found at ". / raven/tests/framework/Optimizers/GradientDescent/ converge_stepsize.xml". This test can be called executing the following command:

./run_tests --re=framework/Optimizers.ConvergeStepsize

or

./run_framework_tests --re=framework/Optimizers.ConvergeStepsize 
- Test Description:

- Extending on the Basic test, this test converges on step size instead of number of samples.

- This test is analytic:

- This test uses Beale's function, which is documented in the analytic tests documentation under the Optimizer functions section.

- Original Author:

- talbpaul

- Creation date:

- 2020-01-29

- The classes tested in this test are:

- Optimizer

\subsubsection{FRAMEWORK OPTIMIZERS.CONVERGEOBJECTIVE}

This test can be found at ". / raven/tests / framework/Optimizers/GradientDescent / converge_objective.xml”. This test can be called executing the following command:

. Irun_tests --re=framework/Optimizers.Convergeobjective

or

./run_framework_tests --re=framework/Optimizers.Convergeobjective

- Test Description:

- Extending on the Basic test, this test converges on change in objective function instead of number of samples.

- This test is analytic:

- This test uses Beale's function, which is documented in the analytic tests documentation under the Optimizer functions section.

- Original Author:

- talbpaul 
- Creation date:

- 2020-01-29

- The classes tested in this test are:

- Optimizer

\subsubsection{FRAMEWORK OPTIMIZERS.CONSTRAINBOUNDARY}

This test can be found at ". /raven/tests / framework/Optimizers/GradientDescent/ constrain_boundary.xml". This test can be called executing the following command:

./run_tests --re=framework/Optimizers. ConstrainBoundary

or

./run_framework_tests --re=framework/Optimizers.ConstrainBoundary

- Test Description:

- This test uses a multidimensional linear function with optima outside the domain space to test boundary constraint handling.

- This test is analytic:

- This test uses the NDSlant function, which is documented in the analytic tests documentation under the Optimizer functions section.

- Original Author:

- talbpaul

- Creation date:

- 2020-01-30

- The classes tested in this test are:

- Optimizer

- This test fulfills the following requirement:

- R-RM-2 


\subsubsection{FRAMEWORK OPTIMIZERS CONVERGEGRADIENT.INITSAMPLER}

This test can be found at ". /raven/tests / framework/Optimizers/GradientDescent/ init_sampler.xml". This test can be called executing the following command:

./run_tests --re=framework/Optimizers/ConvergeGradient.InitSampler

or

./run_framework_tests --re=framework/Optimizers/ConvergeGradient. Initsampl

- Test Description:

- Extending on the Basic test, this test initializes values using a Sampler.

- This test is analytic:

- This test uses Beale's function, which is documented in the analytic tests documentation under the Optimizer functions section.

- Original Author:

- talbpaul

- Creation date:

- 2020-02-10

- The classes tested in this test are:

- Optimizer

\subsubsection{FRAMEWORK OPTIMIZERS.CENTRALDIFF}

This test can be found at ". / raven/tests/framework/Optimizers/GradientDescent/ central_diff.xml". This test can be called executing the following command:

./run_tests --re=framework/Optimizers.CentralDiff

or

./run_framework_tests --re=framework/Optimizers.CentralDiff

- Test Description: 
- Tests Central Difference gradient approximation, otherwise as the Basic case.

- This test is analytic:

- This test uses Beale's function, which is documented in the analytic tests documentation under the Optimizer functions section.

- Original Author:

- talbpaul

- Creation date:

- 2020-03-03

- The classes tested in this test are:

- Optimizer

\subsubsection{FRAMEWORK OPTIMIZERS.FDCONJGRAD}

This test can be found at ". / raven/tests / framework/Optimizers/GradientDescent/ fd_conjgrad.xml". This test can be called executing the following command:

. Irun_tests --re=framework/Optimizers.FDConjgrad

Or

./run_framework_tests --re=framework/Optimizers.FDConjgrad

- Test Description:

- Tests the Conjugate Gradient StepManipulator with the FiniteDifference GradientApproximator for the GradientDescent Optimizer.

- This test is analytic:

- This test uses Beale's function, which is documented in the analytic tests documentation under the Optimizer functions section.

- Original Author:

- talbpaul

- Creation date: 
- 2020-02-26

- The classes tested in this test are:

- Optimizer

\subsubsection{FRAMEWORK OPTIMIZERS.MAX}

This test can be found at ". / raven/tests / framework/Optimizers/GradientDescent/ max.xml". This test can be called executing the following command:

. /run_tests --re=framework/Optimizers.Max

or

./run_framework_tests --re=framework/Optimizers.Max

- Test Description:

- This tests the capability of the optimizer to perform a maximization instead of minimization.

- This test is analytic:

- This test uses an inverted Beale's function, which is documented in the analytic tests documentation under the Optimizer functions section.

- Original Author:

- talbpaul

- Creation date:

- 2020-03-02

- The classes tested in this test are:

- Optimizer 


\subsubsection{FRAMEWORK OPTIMIZERS.SPSA}

This test can be found at ". /raven/tests / framework/Optimizers/GradientDescent/ spsa.xml". This test can be called executing the following command:

./run_tests --re=framework/Optimizers.SPSA

or

./run_framework_tests --re=framework/Optimizers.SPSA

- Test Description:

- Tests SPSA gradient approximation, otherwise as the Basic case.

- This test is analytic:

- This test uses Beale's function, which is documented in the analytic tests documentation under the Optimizer functions section.

- Original Author:

- talbpaul

- Creation date:

- 2020-03-03

- The classes tested in this test are:

- Optimizer

\subsubsection{FRAMEWORK OPTIMIZERS.MULTITRAJ}

This test can be found at ". / raven/tests/framework/Optimizers/GradientDescent/ multitraj.xml". This test can be called executing the following command:

. /run_tests --re=framework/Optimizers.MultiTraj

or

./run_framework_tests --re=framework/Optimizers.MultiTraj

- Test Description: 
- Same as Basic, but adds several trajectories to check proper handling.

- This test is analytic:

- This test uses Beale's function, which is documented in the analytic tests documentation under the Optimizer functions section.

- Original Author:

- talbpaul

- Creation date:

- 2020-03-01

- The classes tested in this test are:

- Optimizer

\subsubsection{FRAMEWORK OPTIMIZERS.WRITEFINAL}

This test can be found at ". / raven/tests / framework/Optimizers/GradientDescent/ write_final.xml". This test can be called executing the following command:

. Irun_tests --re=framework/Optimizers.WriteFinal

or

./run_framework_tests --re=framework/Optimizers.WriteFinal

- Test Description:

- Tests writing only final solution to the solution export.

- This test is analytic:

- This test uses Beale's function, which is documented in the analytic tests documentation under the Optimizer functions section.

- Original Author:

- talbpaul

- Creation date:

- 2020-03-04 
- The classes tested in this test are:

- Optimizer

\subsubsection{FRAMEWORK OPTIMIZERS.INITIALSTEP}

This test can be found at “. / raven/tests / framework/Optimizers/GradientDescent / initial_step.xml". This test can be called executing the following command:

./run_tests --re=framework/Optimizers.Initialstep

or

./run_framework_tests --re=framework/Optimizers.Initialstep

- Test Description:

- Same as basic test, but increases the initial step size. The first perturbed point in optOut for $x$ should be -1.98 instead of -1.99 , which is the default. Similarly, $y$ should be -2.01 in the second perturbed point instead of the default -2.006 .

- This test is analytic:

- This test uses Beale's function, which is documented in the analytic tests documentation under the Optimizer functions section.

- Original Author:

- talbpaul

- Creation date:

- 2020-01-19

- The classes tested in this test are:

- Optimizer.StepManipulator

\subsubsection{FRAMEWORK OPTIMIZERS.1VARIABLE}

This test can be found at ". / raven/tests / framework/Optimizers / GradientDescent/ 1_variable_gradient.xml". This test can be called executing the following command:

. /run_tests --re=framework/Optimizers.1variable 
or

./run_framework_tests --re=framework/Optimizers.1variable

- Test Description:

- This test is inspired by the the test "framework/Optimizers.Basic". The only two differences are: 1) a single parameter is optimized and, 2) all the gradient options (finite difference, spsa, central diff, etc.) are tested. This test is aimed to show how a single variable optimization can be performed with RAVEN.

- This test is analytic:

- This test uses Beale's function, which is documented in the analytic tests documentation under the Optimizer functions section.

- Original Author:

- alfoa

- Creation date:

$-2021-01-04$

- The classes tested in this test are:

- Optimizer

\subsubsection{FRAMEWORK OPTIMIZERS GA MINWREPLACEMENT}

This test can be found at ". / raven/tests / framework/Optimizers/GeneticAlgorithms / testGAMinwRep.xml". This test can be called executing the following command:

. /run_tests --re=framework/Optimizers/GA/MinwReplacement

or

./run_framework_tests --re=framework/Optimizers/GA/MinwReplacement

- Test Description:

- This test assesses the Genetic algorithm using on the myLocalSum function. The nominal dimensionality of the problem is 5 . The objective variable is ans. 
- This test is analytic:

- This test uses myLocalSum's function, which is documented in the analytic tests documentation under the Optimizer functions section.

- Original Author:

- MohammadAbdo

- Creation date:

- 2020-05-16

- The classes tested in this test are:

- GeneticAlgorithm

\subsubsection{FRAMEWORK OPTIMIZERS GA MINWREPLACEMENTCONVAHDP}

This test can be found at ". / raven/tests / framework/Optimizers/GeneticAlgorithms / testGAMinwRepConvAHDp.xml”. This test can be called executing the following command:

. /run_tests --re=framework/Optimizers/GA/MinwReplacementConvAHDp

or

./run_framework_tests --re=framework/Optimizers/GA/MinwReplacementConvAHDp

- Test Description:

- This test assesses the Genetic algorithm using on the myLocalSum function. The nominal dimensionality of the problem is 5 . The objective variable is ans.

- This test is analytic:

- This test uses myLocalSum's function, which is documented in the analytic tests documentation under the Optimizer functions section.

- Original Author:

- MohammadAbdo

- Creation date:

- 2020-05-16

- The classes tested in this test are:

- GeneticAlgorithm 


\subsubsection{FRAMEWORK OPTIMIZERS GA MINWOREPLACEMENT}

This test can be found at ". / raven/tests/framework/Optimizers/GeneticAlgorithms/ testGAMinwoRep.xml". This test can be called executing the following command:

./run_tests --re=framework/Optimizers/GA/MinwoReplacement

or

./run_framework_tests --re=framework/Optimizers/GA/MinwoReplacement

- Test Description:

- This test assesses the Genetic algorithm using on the myLocalSum function. The nominal dimensionality of the problem is 5 . The objective variable is ans.

- This test is analytic:

- This test uses myLocalSum's function, which is documented in the analytic tests documentation under the Optimizer functions section.

- Original Author:

- MohammadAbdo

- Creation date:

- 2020-05-16

- The classes tested in this test are:

- GeneticAlgorithm

\subsubsection{FRAMEWORK OPTIMIZERS GA MINWOREPLACEMENTCONVAHDP}

This test can be found at ". / raven/tests / framework/Optimizers/GeneticAlgorithms/ testGAMinwoRepConvAHDp.xml". This test can be called executing the following command:

./run_tests --re=framework/Optimizers/GA/MinwoReplacementConvAHD

or

./run_framework_tests --re=framework/Optimizers/GA/MinwoReplacementConvAHD 
- Test Description:

- This test assesses the Genetic algorithm using on the myLocalSum function. The nominal dimensionality of the problem is 5 . The objective variable is ans.

- This test is analytic:

- This test uses myLocalSum's function, which is documented in the analytic tests documentation under the Optimizer functions section.

- Original Author:

- MohammadAbdo

- Creation date:

- 2020-05-16

- The classes tested in this test are:

- GeneticAlgorithm

\subsubsection{FRAMEWORK OPTIMIZERS GA MAXWREPLACEMENT}

This test can be found at ". / raven/tests / framework/Optimizers/GeneticAlgorithms / testGAMaxwRep. xml”. This test can be called executing the following command:

./run_tests --re=framework/Optimizers/GA/MaxwReplacement

or

./run_framework_tests --re=framework/Optimizers/GA/MaxwReplacement

- Test Description:

- This test assesses the Genetic algorithm using on the myLocalSum function. The nominal dimensionality of the problem is 5 . The objective variable is ans.

- This test is analytic:

- This test uses myLocalSum's function, which is documented in the analytic tests documentation under the Optimizer functions section.

- Original Author:

- MohammadAbdo 
- Creation date:

- 2020-05-16

- The classes tested in this test are:

- GeneticAlgorithm

\subsubsection{FRAMEWORK OPTIMIZERS GA MAXWREPLACEMENTCONVAHDP}

This test can be found at ". / raven/tests/framework/Optimizers/GeneticAlgorithms/ testGAMaxwRepConvAHDp.xml". This test can be called executing the following command:

./run_tests --re=framework/Optimizers/GA/MaxwReplacementConvAHDp

or

./run_framework_tests --re=framework/Optimizers/GA/MaxwReplacementConvAHDp

- Test Description:

- This test assesses the Genetic algorithm using on the myLocalSum function. The nominal dimensionality of the problem is 5 . The objective variable is ans.

- This test is analytic:

- This test uses myLocalSum's function, which is documented in the analytic tests documentation under the Optimizer functions section.

- Original Author:

- MohammadAbdo

- Creation date:

- 2020-05-16

- The classes tested in this test are:

- GeneticAlgorithm 


\subsubsection{FRAMEWORK OPTIMIZERS GA MAXWOREPLACEMENT}

This test can be found at ". / raven/tests/framework/Optimizers/GeneticAlgorithms/ test GAMaxwoRep.xml". This test can be called executing the following command:

./run_tests --re=framework/Optimizers/GA/MaxwoReplacement

or

./run_framework_tests --re=framework/Optimizers/GA/MaxwoReplacement

- Test Description:

- This test assesses the Genetic algorithm using on the myLocalSum function. The nominal dimensionality of the problem is 5 . The objective variable is ans.

- This test is analytic:

- This test uses myLocalSum's function, which is documented in the analytic tests documentation under the Optimizer functions section.

- Original Author:

- MohammadAbdo

- Creation date:

- 2020-05-16

- The classes tested in this test are:

- GeneticAlgorithm

\subsubsection{FRAMEWORK OPTIMIZERS GA MAXWOREPLACEMENTCONVAHDP}

This test can be found at ". / raven/tests / framework/Optimizers/GeneticAlgorithms/ testGAMaxwoRepConvAHDp.xml". This test can be called executing the following command:

./run_tests --re=framework/Optimizers/GA/MaxwoReplacementConvAHDR

or

./run_framework_tests --re=framework/Optimizers/GA/MaxwoReplacementConvAHD 
- Test Description:

- This test assesses the Genetic algorithm using on the myLocalSum function. The nominal dimensionality of the problem is 5 . The objective variable is ans.

- This test is analytic:

- This test uses myLocalSum's function, which is documented in the analytic tests documentation under the Optimizer functions section.

- Original Author:

- MohammadAbdo

- Creation date:

- 2020-05-16

- The classes tested in this test are:

- GeneticAlgorithm

\subsubsection{FRAMEWORK SAMPLERS ROM VERIFYGAUSSPOLYROM}

This test can be found at "./raven/tests/framework/ROM/verify_time_scgpc. xml”. This test can be called executing the following command:

. /run_tests --re=framework/Samplers/ROM/verifyGaussPolyRom

or

./run_framework_tests --re=framework/Samplers/ROM/verifyGaussPolyRom

- Test Description:

- This tests validates the time-dependent GaussPolynomialROM by sampling it and comparing to the original model.

- This test is analytic:

- This test uses the "projectile.py" ballistic model and tracks position in time. The evaluations of this model as well as the ROMs should match the results documented there.

- Original Author:

- talbpaul 
- Creation date:

- 2016-03-09

- The classes tested in this test are:

- SupervisedLearning.GaussPolynomialROM

- Since the creation of this test, the following main revisions have been performed:

1. revision info:

- author: wangc

- date : 2018-08-09

- description: Add pivotParameter for time dependent ROM

\subsubsection{FRAMEWORK SAMPLERS ROM SOBOL SPARSEGRID SCGPCSUDRETANA- LYTIC}

This test can be found at ". /raven/tests/framework/ROM/SparseGrid/test_scgpc_ sudret. xml". This test can be called executing the following command:

./run_tests --re=framework/Samplers/ROM/Sobol/SparseGrid/scgpcSudretAnalyt

or

./run_framework_tests --re=framework/Samplers/ROM/Sobol/SparseGrid/scgpcSu

- Test Description:

- This analytic test checks the performance of HDMRRom against the simple Sudret polynomial

- This test is analytic:

- dumprom.xml has analytic mean and variance, documented in the analytic tests documentation under "Global Sobol Sensitivity: Sudret".

- Original Author:

- talbpaul

- Creation date:

- 2016-03-18 
- The classes tested in this test are:

- SupervisedLearning.GaussPolynomialROM

- Since the creation of this test, the following main revisions have been performed:

1. revision info:

- author : talbpaul

- date : 2018-12-20

- description: moved ROM metadata writing from direct-to-file to direct-to-dataobject

\subsubsection{FRAMEWORK SAMPLERS ROM SOBOL SOBOLSUDRETANALYTIC}

This test can be found at". / raven/tests/framework/ROM/Sobol/test_sobol_sudret. xml". This test can be called executing the following command:

./run_tests --re=framework/Samplers/ROM/Sobol/sobolsudretAnalytic

or

. Irun_framework_tests --re=framework/Samplers/ROM/Sobol/sobolsudretAnalyti

- Test Description:

- This analytic test checks the performance of HDMRRom against the simple Sudret polynomial.

- This test is analytic:

- dumprom.xml has analytic mean and variance, and is documented in the analytic test documentation under "Global Sobol Sensitivity: Sudret"

- Original Author:

- talbpaul

- Creation date:

- 2016-03-09

- The classes tested in this test are:

- SupervisedLearning.HDMRRom

- Since the creation of this test, the following main revisions have been performed: 
1. revision info:

- author : talbpaul

- date : 2018-12-20

- description: moved ROM metadata writing from direct-to-file to direct-to-dataobject

\subsubsection{FRAMEWORK SAMPLERS ROM SOBOL SOBOLISHIGAMIANALYTIC}

This test can be found at ". /raven/tests/framework/ROM/Sobol/test_sobol_ishigami. xml". This test can be called executing the following command:

./run_tests --re=framework/Samplers/ROM/Sobol/sobolIshigamiAnalytic

or

./run_framework_tests --re=framework/Samplers/ROM/Sobol/sobolIshilgamiAnaly

- Test Description:

- This analytic test checks the performance of HDMRRom against the sinusoidal Ishigami function.

- This test is analytic:

- dumprom.xml has analytic mean and variance, and is documented in the analytic test document under "Global Sobol Sensitivity: Ishigami".

- Original Author:

- talbpaul

- Creation date:

- 2016-03-08

- The classes tested in this test are:

- SupervisedLearning.HDMRRom

- Since the creation of this test, the following main revisions have been performed:

1. revision info:

- author : talbpaul

- date : 2018-12-20

- description: moved ROM metadata writing from direct-to-file to direct-to-dataobject 


\subsubsection{FRAMEWORK SAMPLERS ROM SOBOL SOBOLGFUNCTION}

This test can be found at "./raven/tests/framework/ROM/Sobol/test_sobol_gfunc. xml". This test can be called executing the following command:

. /run_tests --re=framework/Samplers/ROM/Sobol/sobolGFunction

or

./run_framework_tests --re=framework/Samplers/ROM/Sobol/sobolGFunction

- Test Description:

- This analytic test checks the performance of HDMRRom against the discontinuous Sobol G-Function.

- This test is analytic:

- dumprom.xml has analytic mean and variance, but is poorly converged for this model. It is documented in the analytic test documentation under "Sobol G-Function"

- Original Author:

- talbpaul

- Creation date:

- 2016-03-08

- The classes tested in this test are:

- SupervisedLearning.HDMRRom

- Since the creation of this test, the following main revisions have been performed:

1. revision info:

- author : talbpaul

- date : 2018-12-20

- description: moved ROM metadata writing from direct-to-file to direct-to-dataobject 


\subsubsection{FRAMEWORK SAMPLERS ROM SOBOL ANOVAONCUTHDMR}

This test can be found at ". / raven/tests/framework/ROM/Sobol/test_anova_cut. xml". This test can be called executing the following command:

. /run_tests --re=framework/Samplers/ROM/Sobol/AnovaOnCutHDMR

or

./run_framework_tests --re=framework/Samplers/ROM/Sobol/AnovaOnCytHDMR

- Test Description:

- This analytically tests calculating variance using ANOVA on cut-HDMR.

- This test is analytic:

- This test is analytic in the variance, Sobol sensitivities, and mean of the response. These parameters are documented in the analytic tests documentation under "Second-Order ANOVA of Second-Order Cut-HDMR Expansion of Sudret".

- Original Author:

- talbpaul

- Creation date:

- 2016-03-11

- The classes tested in this test are:

- SupervisedLearning.HDMRRom

- Since the creation of this test, the following main revisions have been performed:

1. revision info:

- author : talbpaul

- date : 2018-12-20

- description: moved ROM metadata writing from direct-to-file to direct-to-dataobject 


\subsubsection{FRAMEWORK SAMPLERS ROM SOBOL ADAPTIVESOBOL}

This test can be found at “. / raven/tests / framework/ROM/Sobol/test_adapt_sobol. xml". This test can be called executing the following command:

./run_tests --re=framework/Samplers/ROM/Sobol/AdaptiveSobol

or

./run_framework_tests --re=framework/Samplers/ROM/Sobol/Adaptive\$obol

- Test Description:

- This tests using the AdaptiveSobol sampler to construct HDMRRom ROMs.

- This test is analytic:

- dumprom.xml has analytic results for mean and variance in that are documented in the Attenuation section of the analytic tests manual.

- Original Author:

- talbpaul

- Creation date:

- 2016-02-08

- The classes tested in this test are:

- Samplers.AdaptiveSobol,SupervisedLearning.HDMRRom

- Since the creation of this test, the following main revisions have been performed:

1. revision info:

- author : talbpaul

- date : 2018-12-20

- description: moved ROM metadata writing from direct-to-file to direct-to-dataobject 


\subsubsection{FRAMEWORK SAMPLERS ROM SOBOL VERIFYHDMRROM}

This test can be found at "./raven/tests/framework/ROM/Sobol/verify_time_ dep_sobol.xml". This test can be called executing the following command:

. /run_tests --re=framework/Samplers/ROM/Sobol/verifyHDMRRom

or

. /run_framework_tests --re=framework/Samplers/ROM/Sobol/verifyHDMRRom

- Test Description:

- This analytic test checks that the time-dependent HDMRRom performs the same as the model it's representing.

- This test is analytic:

- This test uses "projectile.py" as documented in the analytic tests document, and makes several evaluations of position based on time.

- Original Author:

- talbpaul

- Creation date:

- 2016-11-08

- The classes tested in this test are:

- SupervisedLearning.HDMRRom

- Since the creation of this test, the following main revisions have been performed:

1. revision info:

- author: wangc

- date : 2018-08-09

- description: Add pivotParameter for time dependent ROM 


\subsubsection{FRAMEWORK SAMPLERS SPARSEGRID.GAMMA LARGE SCALE}

This test can be found at ". / raven/tests/framework/Samplers/SparseGrid/test_ scgpc_gamma_large_scale.xml". This test can be called executing the following command:

./run_tests --re=framework/Samplers/SparseGrid.gamma_large_scale

or

./run_framework_tests --re=framework/Samplers/SparseGrid.gamma_large_scale

- Test Description:

- This tests using SparseGridCollocation with gamma-distributed inputs with large scale parameters to test the fix for issue \#693

- This test is analytic:

- This test is analytic in mean and variance documented in the analytic tests. Two models are used: ans $=\mathrm{x} 1+\mathrm{x} 2$ with analytic mean $=4.7$, variance $=3.565$ ans $=x 1 * * 2+\mathrm{x} 2 * * 2$ with analytic mean $=14.655$, variance $=215.638125$

- Original Author:

- wangc

- Creation date:

- 2018-07-26

- The classes tested in this test are:

- Samplers.SparseGridCollocation

\subsubsection{FRAMEWORK PCA ADAPTIVE SGC TEST ADAPTIVE SGC ATTENUATION}

This test can be found at "./raven/tests/framework/pca_adaptive_sgc/test_ adaptive_sgc_attenuation.xml". This test can be called executing the following command:

./run_tests --re=framework/pca_adaptive_sgc/test_adaptive_sgc_attenuation

or 
./run_framework_tests --re=framework/pca_adaptive_sgc/test_adaptilve_sgc_at

- Test Description:

- Tests use of the PCA method for adaptive sparse grids on exponential decay models with uncorrelated inputs.

- This test is analytic:

- Attenuation with Multivariate Normal Distribution

- Original Author:

- wangc

- Creation date:

- 2015-11-18

- The classes tested in this test are:

- Distributions.MultivariateNormal,Samplers.AdaptiveSparseGrid,SupervisedLearning.GaussPolynomi

- Since the creation of this test, the following main revisions have been performed:

1. revision info:

- author : talbpaul

- date : 2019-01-09

- description: moving rom ROM-file to ROM-dataobject

\subsubsection{FRAMEWORK PCA ADAPTIVE SGC.TEST ADAPTIVE SGC POLY CORRE- LATION}

This test can be found at "./raven/tests/framework/pca_adaptive_sgc/test_ adaptive_sgc_poly_correlation.xml". This test can be called executing the following command:

./run_tests --re=framework/pca_adaptive_sgc.test_adaptive_sgc_poly_correla

or

./run_framework_tests --re=framework/pca_adaptive_sgc.test_adaptilve_sgc_po 
- Test Description:

- Tests use of the PCA method for adaptive sparse grids on polynomial models.

- This test is analytic:

_ "Tensor Polynomial (First-Order) with Multivariate Normal Distribution"

- Original Author:

- wangc

- Creation date:

- 2015-11-20

- The classes tested in this test are:

- Distributions.MultivariateNormal,Samplers.AdaptiveSparseGrid,SupervisedLearning.GaussPolynomi

- Since the creation of this test, the following main revisions have been performed:

1. revision info:

- author : talbpaul

- date : 2019-01-09

- description: moving from ROM-file to ROM-dataobject

\subsubsection{FRAMEWORK PCA ADAPTIVE SGC.TEST ADAPTIVE SGC POLY PCS ANA- LYTIC}

This test can be found at "./raven/tests/framework/pca_adaptive_sgc/test_ adaptive_sgc_poly_pca_analytic.xml". This test can be called executing the following command:

./run_tests --re=framework/pca_adaptive_sgc.test_adaptive_sgc_pol_y_pcs_ana

or

.Irun_framework_tests --re=framework/pca_adaptive_sgc.test_adaptivve_sgc_po

- Test Description:

- Tests the SparseGridCollocation sampler combined with input reductions via PCA method, using uncorrelated multivariate normal distributions. 
- This test is analytic:

- This test is analytic in mean and variance using the "tensor polynomial" analytic model documented in the analytic tests.

- Original Author:

- wangc

- Creation date:

$-2015-12-01$

- The classes tested in this test are:

- Samplers.AdaptiveSparseGrid,SupervisedLearning.GaussPolynomialROM

- Since the creation of this test, the following main revisions have been performed:

1. revision info:

- author : talbpaul

- date : 2019-01-09

- description: moving from ROM-file to ROM-dataobject

\subsubsection{FRAMEWORK PCA SOBOL TEST ADAPTIVE SOBOL POLY}

This test can be found at “. / raven/tests/framework/pca_sobol/test_adapt_sobol_ poly.xml". This test can be called executing the following command:

./run_tests --re=framework/pca_sobol/test_adaptive_sobol_poly

or

./run_framework_tests --re=framework/pca_sobol/test_adaptive_sobol_poly

- Test Description:

- Tests the Adaptive Sobol sampler combined wit input reductions via PCA method, using uncorrelated multivariate normal distributions.

- This test is analytic:

- This test is analytic in mean and variance using the "tensor polynomial" analytic model documented in the analytic tests. 
- Original Author:

- wangc

- Creation date:

- 2016-01-06

- The classes tested in this test are:

- Samplers.AdaptiveSobol,SupervisedLearning.HDMRRom

- Since the creation of this test, the following main revisions have been performed:

1. revision info:

- author : talbpaul

- date : 2019-01-09

- description: moving from ROM-file to ROM-dataobject

\subsubsection{FRAMEWORK PCA SOBOL TEST ADAPTIVE SOBOL ATTENUATION}

This test can be found at ". / raven/tests/framework/pca_sobol/test_adapt_sobol_ attenu.xml". This test can be called executing the following command:

./run_tests --re=framework/pca_sobol/test_adaptive_sobol_attenuation

or

./run_framework_tests --re=framework/pca_sobol/test_adaptive_sobql_attenua

- Test Description:

- Tests the Adaptive Sobol sampler combined wit input reductions via PCA method, using uncorrelated multivariate normal distribution.

- This test is analytic:

- This test is analytic in mean and variance using the "attenuation" analytic model documented in the analytic tests.

- Original Author:

- wangc

- Creation date: 
- 2016-01-06

- The classes tested in this test are:

- Samplers.AdaptiveSobol,SupervisedLearning.HDMRRom

- Since the creation of this test, the following main revisions have been performed:

1. revision info:

- author : talbpaul

- date : 2019-01-09

- description: moving from ROM-file to ROM-dataobject

\subsubsection{FRAMEWORK PCA SOBOL TEST ADAPTIVE SOBOL ATTENU CORRELA- TION}

This test can be found at ". / raven/tests/framework/pca_sobol/test_adapt_sobol_ correlation.xml". This test can be called executing the following command:

.Irun_tests --re=framework/pca_sobol/test_adaptive_sobol_attenu_gorrelatio

or

./run_framework_tests --re=framework/pca_sobol/test_adaptive_sobql_attenu_

- Test Description:

- Tests the Adaptive Sobol sampler combined wit input reductions via PCA method, using correlated multivariate normal distributions.

- This test is analytic:

- This test is analytic in mean and variance using the "attenuation" and "tensor polynomial" analytic models documented in the analytic tests.

- Original Author:

- wangc

- Creation date:

- 2016-02-17

- The classes tested in this test are: 
- Samplers.AdaptiveSobol,SupervisedLearning.HDMRRom

- Since the creation of this test, the following main revisions have been performed:

1. revision info:

- author : talbpaul

- date : 2019-01-09

- description: moving from ROM-file to ROM-dataobject

\subsubsection{FRAMEWORK PCA SOBOL TEST SOBOL POLY CORRELATION}

This test can be found at". / raven/tests/framework/pca_sobol/test_sobol_correlation. xml". This test can be called executing the following command:

./run_tests --re=framework/pca_sobol/test_sobol_poly_correlation

or

.Irun_framework_tests --re=framework/pca_sobol/test_sobol_poly_correlation

- Test Description:

- Tests the Sobol sampler combined wit input reductions via PCA method, using correlated multivariate normal distributions.

- This test is analytic:

- This test is analytic in mean and variance using the "attenuation" and "tensor polynomial" analytic models documented in the analytic tests.

- Original Author:

- wangc

- Creation date:

- 2016-01-06

- The classes tested in this test are:

- Samplers.Sobol,SupervisedLearning.HDMRRom

- Since the creation of this test, the following main revisions have been performed:

1. revision info: 
- author : talbpaul

- date : 2019-01-09

- description: moving from ROM-file to ROM-dataobject

\subsubsection{FRAMEWORK PCA SOBOL TEST SOBOL POLY CORRELATION}

This test can be found at ". / raven/tests/cluster_tests/test_pbs.xmlcluster_ runinfo_legacy.xml". This test can be called executing the following command:

./run_tests --re=framework/pca_sobol/test_sobol_poly_correlation

or

. Irun_framework_tests --re=framework/pca_sobol/test_sobol_poly_correlation

- Test Description:

- Tests the Sobol sampler combined wit input reductions via PCA method, using correlated multivariate normal distributions.

- This test is analytic:

- This test is analytic in mean and variance using the "attenuation" and "tensor polynomial" analytic models documented in the analytic tests.

- Original Author:

- wangc

- Creation date:

- 2016-01-06

- The classes tested in this test are:

- Samplers.Sobol,SupervisedLearning.HDMRRom

- Since the creation of this test, the following main revisions have been performed:

1. revision info:

- author : talbpaul

- date : 2019-01-09

- description: moving from ROM-file to ROM-dataobject 


\subsubsection{FRAMEWORK PCA SOBOL TEST SOBOL POLY CORRELATION}

This test can be found at "./raven/tests/cluster_tests/test_mpiqsub_local. xmlpbspro_mpi.xmlcluster_runinfo_legacy.xml”. This test can be called executing the following command:

./run_tests --re=framework/pca_sobol/test_sobol_poly_correlation

or

./run_framework_tests --re=framework/pca_sobol/test_sobol_poly_correlation

- Test Description:

- Tests the Sobol sampler combined wit input reductions via PCA method, using correlated multivariate normal distributions.

- This test is analytic:

- This test is analytic in mean and variance using the "attenuation" and "tensor polynomial" analytic models documented in the analytic tests.

- Original Author:

- wangc

- Creation date:

- 2016-01-06

- The classes tested in this test are:

- Samplers.Sobol,SupervisedLearning.HDMRRom

- Since the creation of this test, the following main revisions have been performed:

1. revision info:

- author : talbpaul

- date : 2019-01-09

- description: moving from ROM-file to ROM-dataobject 


\subsubsection{FRAMEWORK PCA SOBOL TEST SOBOL POLY CORRELATION}

This test can be found at "./raven/tests/cluster_tests/test_mpiqsub_flex. xmlcluster_runinfo_legacy.xml". This test can be called executing the following command:

./run_tests --re=framework/pca_sobol/test_sobol_poly_correlation

or

./run_framework_tests --re=framework/pca_sobol/test_sobol_poly_correlation

- Test Description:

- Tests the Sobol sampler combined wit input reductions via PCA method, using correlated multivariate normal distributions.

- This test is analytic:

- This test is analytic in mean and variance using the "attenuation" and "tensor polynomial" analytic models documented in the analytic tests.

- Original Author:

- wangc

- Creation date:

- 2016-01-06

- The classes tested in this test are:

- Samplers.Sobol,SupervisedLearning.HDMRRom

- Since the creation of this test, the following main revisions have been performed:

1. revision info:

- author : talbpaul

- date : 2019-01-09

- description: moving from ROM-file to ROM-dataobject 


\subsubsection{FRAMEWORK PCA SOBOL TEST SOBOL POLY CORRELATION}

This test can be found at "./raven/tests/cluster_tests/test_mpiqsub_limitnode. xmlcluster_runinfo_legacy.xml”. This test can be called executing the following command:

./run_tests --re=framework/pca_sobol/test_sobol_poly_correlation

or

./run_framework_tests --re=framework/pca_sobol/test_sobol_poly_correlation

- Test Description:

- Tests the Sobol sampler combined wit input reductions via PCA method, using correlated multivariate normal distributions.

- This test is analytic:

- This test is analytic in mean and variance using the "attenuation" and "tensor polynomial" analytic models documented in the analytic tests.

- Original Author:

- wangc

- Creation date:

- 2016-01-06

- The classes tested in this test are:

- Samplers.Sobol,SupervisedLearning.HDMRRom

- Since the creation of this test, the following main revisions have been performed:

1. revision info:

- author : talbpaul

- date : 2019-01-09

- description: moving from ROM-file to ROM-dataobject 


\subsubsection{FRAMEWORK PCA SOBOL TEST SOBOL POLY CORRELATION}

This test can be found at “. /raven/tests/cluster_tests/test_mpiqsub_nosplit. xmlcluster_runinfo_legacy.xml". This test can be called executing the following command:

./run_tests --re=framework/pca_sobol/test_sobol_poly_correlation

or

./run_framework_tests --re=framework/pca_sobol/test_sobol_poly_correlation

- Test Description:

- Tests the Sobol sampler combined wit input reductions via PCA method, using correlated multivariate normal distributions.

- This test is analytic:

- This test is analytic in mean and variance using the "attenuation" and "tensor polynomial" analytic models documented in the analytic tests.

- Original Author:

- wangc

- Creation date:

- 2016-01-06

- The classes tested in this test are:

- Samplers.Sobol,SupervisedLearning.HDMRRom

- Since the creation of this test, the following main revisions have been performed:

1. revision info:

- author : talbpaul

- date : 2019-01-09

- description: moving from ROM-file to ROM-dataobject 


\subsubsection{FRAMEWORK PCA SOBOL TEST SOBOL POLY CORRELATION}

This test can be found at "./raven/tests/cluster_tests/test_mpiqsub_forced. xmlcluster_runinfo_legacy.xml”. This test can be called executing the following command:

./run_tests --re=framework/pca_sobol/test_sobol_poly_correlation

or

./run_framework_tests --re=framework/pca_sobol/test_sobol_poly_correlation

- Test Description:

- Tests the Sobol sampler combined wit input reductions via PCA method, using correlated multivariate normal distributions.

- This test is analytic:

- This test is analytic in mean and variance using the "attenuation" and "tensor polynomial" analytic models documented in the analytic tests.

- Original Author:

- wangc

- Creation date:

- 2016-01-06

- The classes tested in this test are:

- Samplers.Sobol,SupervisedLearning.HDMRRom

- Since the creation of this test, the following main revisions have been performed:

1. revision info:

- author : talbpaul

- date : 2019-01-09

- description: moving from ROM-file to ROM-dataobject 


\subsubsection{FRAMEWORK PCA SOBOL TEST SOBOL POLY CORRELATION}

This test can be found at "./raven/tests/cluster_tests/test_mpiqsub_1_proc. xmlpbspro_mpi.xmlcluster_runinfo_legacy.xml”. This test can be called executing the following command:

./run_tests --re=framework/pca_sobol/test_sobol_poly_correlation

or

./run_framework_tests --re=framework/pca_sobol/test_sobol_poly_correlation

- Test Description:

- Tests the Sobol sampler combined wit input reductions via PCA method, using correlated multivariate normal distributions.

- This test is analytic:

- This test is analytic in mean and variance using the "attenuation" and "tensor polynomial" analytic models documented in the analytic tests.

- Original Author:

- wangc

- Creation date:

- 2016-01-06

- The classes tested in this test are:

- Samplers.Sobol,SupervisedLearning.HDMRRom

- Since the creation of this test, the following main revisions have been performed:

1. revision info:

- author : talbpaul

- date : 2019-01-09

- description: moving from ROM-file to ROM-dataobject 


\subsubsection{FRAMEWORK PCA SOBOL TEST SOBOL POLY CORRELATION}

This test can be found at "./raven/tests/cluster_tests/AdaptiveSobol/test_ adapt_sobol_parallel.xml../pbspro_mpi.xml../cluster_runinfo_legacy. xml". This test can be called executing the following command:

./run_tests --re=framework/pca_sobol/test_sobol_poly_correlation

or

./run_framework_tests --re=framework/pca_sobol/test_sobol_poly_correlation

- Test Description:

- Tests the Sobol sampler combined wit input reductions via PCA method, using correlated multivariate normal distributions.

- This test is analytic:

- This test is analytic in mean and variance using the "attenuation" and "tensor polynomial" analytic models documented in the analytic tests.

- Original Author:

- wangc

- Creation date:

- 2016-01-06

- The classes tested in this test are:

- Samplers.Sobol,SupervisedLearning.HDMRRom

- Since the creation of this test, the following main revisions have been performed:

1. revision info:

- author : talbpaul

- date : 2019-01-09

- description: moving from ROM-file to ROM-dataobject 


\subsubsection{FRAMEWORK PCA SOBOL TEST SOBOL POLY CORRELATION}

This test can be found at "./raven/tests/cluster_tests/InternalParallel/test_ internal_parallel_extModel.xml../pbspro_mpi.xml../cluster_runinfo_ legacy.xml”. This test can be called executing the following command:

./run_tests --re=framework/pca_sobol/test_sobol_poly_correlation

or

./run_framework_tests --re=framework/pca_sobol/test_sobol_poly_correlation

- Test Description:

- Tests the Sobol sampler combined wit input reductions via PCA method, using correlated multivariate normal distributions.

- This test is analytic:

- This test is analytic in mean and variance using the "attenuation" and "tensor polynomial" analytic models documented in the analytic tests.

- Original Author:

- wangc

- Creation date:

- 2016-01-06

- The classes tested in this test are:

- Samplers.Sobol,SupervisedLearning.HDMRRom

- Since the creation of this test, the following main revisions have been performed:

1. revision info:

- author : talbpaul

- date : 2019-01-09

- description: moving from ROM-file to ROM-dataobject 


\subsubsection{FRAMEWORK PCA SOBOL TEST SOBOL POLY CORRELATION}

This test can be found at “. / raven/tests/cluster_tests/InternalParallel/test_ internal_parallel_ROM_scikit.xml../pbspro_mpi.xml../cluster_runinfo_ legacy.xml". This test can be called executing the following command:

./run_tests --re=framework/pca_sobol/test_sobol_poly_correlation

or

./run_framework_tests --re=framework/pca_sobol/test_sobol_poly_correlation

- Test Description:

- Tests the Sobol sampler combined wit input reductions via PCA method, using correlated multivariate normal distributions.

- This test is analytic:

- This test is analytic in mean and variance using the "attenuation" and "tensor polynomial" analytic models documented in the analytic tests.

- Original Author:

- wangc

- Creation date:

- 2016-01-06

- The classes tested in this test are:

- Samplers.Sobol,SupervisedLearning.HDMRRom

- Since the creation of this test, the following main revisions have been performed:

1. revision info:

- author : talbpaul

- date : 2019-01-09

- description: moving from ROM-file to ROM-dataobject 


\subsubsection{FRAMEWORK PCA SOBOL TEST SOBOL POLY CORRELATION}

This test can be found at "./raven/tests/cluster_tests/InternalParallel/test_ internal_parallel_PP_LS.xml../pbspro_mpi.xml../cluster_runinfo_legacy. xml". This test can be called executing the following command:

./run_tests --re=framework/pca_sobol/test_sobol_poly_correlation

or

./run_framework_tests --re=framework/pca_sobol/test_sobol_poly_correlation

- Test Description:

- Tests the Sobol sampler combined wit input reductions via PCA method, using correlated multivariate normal distributions.

- This test is analytic:

- This test is analytic in mean and variance using the "attenuation" and "tensor polynomial" analytic models documented in the analytic tests.

- Original Author:

- wangc

- Creation date:

- 2016-01-06

- The classes tested in this test are:

- Samplers.Sobol,SupervisedLearning.HDMRRom

- Since the creation of this test, the following main revisions have been performed:

1. revision info:

- author : talbpaul

- date : 2019-01-09

- description: moving from ROM-file to ROM-dataobject 


\subsubsection{FRAMEWORK PCA SOBOL TEST SOBOL POLY CORRELATION}

This test can be found at ". /raven/tests/cluster_tests/InternalParallel/test_ internal_MSR.xml../pbspro_mpi.xml../cluster_runinfo_legacy.xml”. This test can be called executing the following command:

./run_tests --re=framework/pca_sobol/test_sobol_poly_correlation

or

./run_framework_tests --re=framework/pca_sobol/test_sobol_poly_correlation

- Test Description:

- Tests the Sobol sampler combined wit input reductions via PCA method, using correlated multivariate normal distributions.

- This test is analytic:

- This test is analytic in mean and variance using the "attenuation" and "tensor polynomial" analytic models documented in the analytic tests.

- Original Author:

- wangc

- Creation date:

- 2016-01-06

- The classes tested in this test are:

- Samplers.Sobol,SupervisedLearning.HDMRRom

- Since the creation of this test, the following main revisions have been performed:

1. revision info:

- author : talbpaul

- date : 2019-01-09

- description: moving from ROM-file to ROM-dataobject 


\subsubsection{FRAMEWORK PCA SOBOL TEST SOBOL POLY CORRELATION}

This test can be found at “. / raven/tests/cluster_tests/InternalParallel/test_ ensemble_model_picard_parallel.xml../pbspro_mpi.xml../cluster_runinfo_ legacy.xml". This test can be called executing the following command:

./run_tests --re=framework/pca_sobol/test_sobol_poly_correlation

or

./run_framework_tests --re=framework/pca_sobol/test_sobol_poly_correlation

- Test Description:

- Tests the Sobol sampler combined wit input reductions via PCA method, using correlated multivariate normal distributions.

- This test is analytic:

- This test is analytic in mean and variance using the "attenuation" and "tensor polynomial" analytic models documented in the analytic tests.

- Original Author:

- wangc

- Creation date:

- 2016-01-06

- The classes tested in this test are:

- Samplers.Sobol,SupervisedLearning.HDMRRom

- Since the creation of this test, the following main revisions have been performed:

1. revision info:

- author : talbpaul

- date : 2019-01-09

- description: moving from ROM-file to ROM-dataobject 


\subsubsection{FRAMEWORK PCA SOBOL TEST SOBOL POLY CORRELATION}

This test can be found at “. / raven/tests/cluster_tests/InternalParallel/test_ ensemble_model_linear_internal_parallel.xml../pbspro_mpi.xml../cluster_ runinfo_legacy.xml". This test can be called executing the following command:

./run_tests --re=framework/pca_sobol/test_sobol_poly_correlation

or

./run_framework_tests --re=framework/pca_sobol/test_sobol_poly_correlation

- Test Description:

- Tests the Sobol sampler combined wit input reductions via PCA method, using correlated multivariate normal distributions.

- This test is analytic:

- This test is analytic in mean and variance using the "attenuation" and "tensor polynomial" analytic models documented in the analytic tests.

- Original Author:

- wangc

- Creation date:

- 2016-01-06

- The classes tested in this test are:

- Samplers.Sobol,SupervisedLearning.HDMRRom

- Since the creation of this test, the following main revisions have been performed:

1. revision info:

- author : talbpaul

- date : 2019-01-09

- description: moving from ROM-file to ROM-dataobject 


\subsubsection{FRAMEWORK PCA SOBOL TEST SOBOL POLY CORRELATION}

This test can be found at ". / raven/tests/cluster_tests/InternalParallel/test_ hybrid_model_code.xml../pbspro_mpi.xml../cluster_runinfo_legacy.xml”. This test can be called executing the following command:

./run_tests --re=framework/pca_sobol/test_sobol_poly_correlation

or

./run_framework_tests --re=framework/pca_sobol/test_sobol_poly_correlation

- Test Description:

- Tests the Sobol sampler combined wit input reductions via PCA method, using correlated multivariate normal distributions.

- This test is analytic:

- This test is analytic in mean and variance using the "attenuation" and "tensor polynomial" analytic models documented in the analytic tests.

- Original Author:

- wangc

- Creation date:

- 2016-01-06

- The classes tested in this test are:

- Samplers.Sobol,SupervisedLearning.HDMRRom

- Since the creation of this test, the following main revisions have been performed:

1. revision info:

- author : talbpaul

- date : 2019-01-09

- description: moving from ROM-file to ROM-dataobject 


\subsubsection{FRAMEWORK PCA SOBOL TEST SOBOL POLY CORRELATION}

This test can be found at ". /raven/tests/cluster_tests/InternalParallel/test_ hybrid_model_external.xml../pbspro_mpi.xml../cluster_runinfo_legacy. xml". This test can be called executing the following command:

./run_tests --re=framework/pca_sobol/test_sobol_poly_correlation

or

./run_framework_tests --re=framework/pca_sobol/test_sobol_poly_correlation

- Test Description:

- Tests the Sobol sampler combined wit input reductions via PCA method, using correlated multivariate normal distributions.

- This test is analytic:

- This test is analytic in mean and variance using the "attenuation" and "tensor polynomial" analytic models documented in the analytic tests.

- Original Author:

- wangc

- Creation date:

- 2016-01-06

- The classes tested in this test are:

- Samplers.Sobol,SupervisedLearning.HDMRRom

- Since the creation of this test, the following main revisions have been performed:

1. revision info:

- author : talbpaul

- date : 2019-01-09

- description: moving from ROM-file to ROM-dataobject 


\subsubsection{FRAMEWORK PCA SOBOL TEST SOBOL POLY CORRELATION}

This test can be found at "./raven/tests/cluster_tests/RavenRunsRaven/rom. xml...pbspro_mpi.xml../cluster_runinfo.xml”. This test can be called executing the following command:

./run_tests --re=framework/pca_sobol/test_sobol_poly_correlation

or

./run_framework_tests --re=framework/pca_sobol/test_sobol_poly_correlation

- Test Description:

- Tests the Sobol sampler combined wit input reductions via PCA method, using correlated multivariate normal distributions.

- This test is analytic:

- This test is analytic in mean and variance using the "attenuation" and "tensor polynomial" analytic models documented in the analytic tests.

- Original Author:

- wangc

- Creation date:

- 2016-01-06

- The classes tested in this test are:

- Samplers.Sobol,SupervisedLearning.HDMRRom

- Since the creation of this test, the following main revisions have been performed:

1. revision info:

- author : talbpaul

- date : 2019-01-09

- description: moving from ROM-file to ROM-dataobject 


\subsubsection{FRAMEWORK PCA SOBOL TEST SOBOL POLY CORRELATION}

This test can be found at "./raven/tests/cluster_tests/RavenRunsRaven/code. xml../pbspro_mpi.xml../cluster_runinfo.xml”. This test can be called executing the following command:

./run_tests --re=framework/pca_sobol/test_sobol_poly_correlation

or

./run_framework_tests --re=framework/pca_sobol/test_sobol_poly_correlation

- Test Description:

- Tests the Sobol sampler combined wit input reductions via PCA method, using correlated multivariate normal distributions.

- This test is analytic:

- This test is analytic in mean and variance using the "attenuation" and "tensor polynomial" analytic models documented in the analytic tests.

- Original Author:

- wangc

- Creation date:

- 2016-01-06

- The classes tested in this test are:

- Samplers.Sobol,SupervisedLearning.HDMRRom

- Since the creation of this test, the following main revisions have been performed:

1. revision info:

- author : talbpaul

- date : 2019-01-09

- description: moving from ROM-file to ROM-dataobject

\subsection{Verification tests' description}

This section contains the description of all the verification tests. 


\subsubsection{FRAMEWORK CODEINTERFACETESTS ACCELERATEDCFD.PERTURBACFD1}

This test can be found at "./raven/ForACFDBranch/AcceleratedCFD/test_perturb_ acdf.xml". This test can be called executing the following command:

./run_tests --re=framework/CodeInterfaceTests/AcceleratedCFD.perturbACFD1

or

./run_framework_tests --re=framework/CodeInterfaceTests/AcceleratedCFD.per

- Test Description:

- An example of using the AcceleratedCFD code interface. This test is aimed to test the mechanics of the interface (no executable).

- Original Author:

- alfoa

- Creation date:

- 2020-11-03

- The classes tested in this test are:

- Models.Code.AcceleratedCFD

\subsubsection{FRAMEWORK.TESTMARKOVMODEL 3STATES COMPLEXTRANS}

This test can be found at ". /raven/plugins/SR2ML/tests/test_markovModel_3states_ complextrans.xml". This test can be called executing the following command:

./run_tests --re=framework. TestMarkovModel_3states_complexTrans

or

./run_framework_tests --re=framework. TestMarkovModel_3states_complexTrans

- Test Description:

- This test is aimed to check Markov model with 3 states and complex transition 
- Original Author:

- mandd, wangc

- Creation date:

- 2019-05-08

- The classes tested in this test are:

- Models.ExternalModel.MarkovModel

- Since the creation of this test, the following main revisions have been performed:

1. revision info:

- author: wangc

- date : 2019-11-27

- description: Added Plugin name extension

\subsubsection{FRAMEWORK.TESTMARKOVMODEL 3STATES INSTANTTRANS}

This test can be found at “. / raven/plugins/SR2ML/tests/test_markovModel_3states_ instantTrans.xml". This test can be called executing the following command:

./run_tests --re=framework. TestMarkovModel_3states_instantTrans

or

./run_framework_tests --re=framework. TestMarkovModel_3states_instantTrans

- Test Description:

- This test is aimed to check Markov model with 3 states with instant transition

- Original Author:

- mandd, wangc

- Creation date:

- 2019-05-08

- The classes tested in this test are:

- Models.ExternalModel.MarkovModel 
- Since the creation of this test, the following main revisions have been performed:

1. revision info:

- author: wangc

- date : 2019-11-27

- description: Added Plugin name extension

\subsubsection{FRAMEWORK.TESTMARKOVMODEL 2STATES TAU}

This test can be found at ". / raven/plugins/SR2ML/tests/test_markovModel_2states_ tau. xml". This test can be called executing the following command:

. Irun_tests --re=framework. TestMarkovModel_2states_tau

Or

./run_framework_tests --re=framework. TestMarkovModel_2states_tau

- Test Description:

- This test is aimed to check Markov model with 2 states

- Original Author:

- mandd, wangc

- Creation date:

- 2019-05-08

- The classes tested in this test are:

- Models.ExternalModel.MarkovModel

- Since the creation of this test, the following main revisions have been performed:

1. revision info:

- author: wangc

- date : 2019-11-27

- description: Added Plugin name extension 


\subsubsection{FRAMEWORK.MC FW COST}

This test can be found at “./raven/plugins/SR2ML/tests/CRAFT/test_mc_fw_cost. xml". This test can be called executing the following command:

. /run_tests --re=framework.mc_fw_cost

or

./run_framework_tests --re=framework.mc_fw_cost

- Test Description:

- This test is aimed to check the craft workflow

- Original Author:

- mandd, wangc

- Creation date:

- 2019-05-14

- The classes tested in this test are:

- Models.EnsembleModel

\subsubsection{FRAMEWORK.TIME DEP FW COST}

This test can be found at ". / raven/plugins/SR2ML/tests/CRAFT/test_time_dep_ $\mathrm{fw}$ _cost. xml". This test can be called executing the following command:

. Irun_tests --re=framework.time_dep_fw_cost

or

.Irun_framework_tests --re=framework.time_dep_fw_cost

- Test Description:

- This test is aimed to check craft workflow with time dependent data

- Original Author: 
- mandd, wangc

- Creation date:

$-2019-05-14$

- The classes tested in this test are:

- Models.EnsembleModel

\subsubsection{FRAMEWORK.SEPT MILESTONE}

This test can be found at “. / raven/plugins/SR2ML/tests/CRAFT/test_sept_milestone. xml". This test can be called executing the following command:

./run_tests --re=framework.sept_milestone

or

./run_framework_tests --re=framework.sept_milestone

- Test Description:

- This test is aimed to check the craft workflow for Sept. 2018 milestone

- Original Author:

- mandd, wangc

- Creation date:

- 2019-05-14

- The classes tested in this test are:

- Models.EnsembleModel

\subsubsection{FRAMEWORK.RELIABILITYMODEL.TIMEDEPRELIABILITY}

This test can be found at ". / raven/plugins/SR2ML/tests/reliabilityModel/test_ time_dep_ensemble_reliability.xml". This test can be called executing the following command:

./run_tests --re=framework.reliabilityModel.timedepReliability 
or

./run_framework_tests --re=framework.reliabilityModel.timeDepReliability

- Test Description:

- This test is aimed to check the reliablity model with time series input. The reliability model will return time-dependent reliablity data, such as failure rate, cumulative failure value, probability failure value, and reliablity value.

- Original Author:

- wangc

- Creation date:

- 2020-02-12

- The classes tested in this test are:

- Models.ExternalModel

\subsubsection{FRAMEWORK.SKP OPTIMIZATION}

This test can be found at ". / raven/plugins/LOGOS/tests/test_skp.xml". This test can be called executing the following command:

./run_tests --re=framework.skp_optimization

or

./run_framework_tests --re=framework.skp_optimization

- Test Description:

- This test is aimed to check the capital investment model for single knapsack problem

- Original Author:

- mandd, wangc

- Creation date:

- 2019-05-16 
- The classes tested in this test are:

- Models.ExternalModel.CapitalInvestmentModel

- Since the creation of this test, the following main revisions have been performed:

1. revision info:

- author: wangc

- date : 2019-05-17

- description: rename models to PyomoModels and utils to investment_utils

2. revision info:

- author: wangc

- date : 2019-05-17

- description: add raven CapitalInvestment external model test

3. revision info:

- author: wangc

- date : 2019-05-17

- description: fix import issue for raven plugins

4. revision info:

- author: wangc

- date : 2019-05-20

- description: Dump PyomoModel's outputs into raven data objects

\subsubsection{FRAMEWORK.BKP OPTIMIZATION}

This test can be found at ". /raven/plugins/LOGOS/tests/test_bkp.xml". This test can be called executing the following command:

. /run_tests --re=framework.bkp_optimization

or

./run_framework_tests --re=framework.bkp_optimization

- Test Description:

- This test is aimed to check the capital investment model for bounded knapsack problem

- Original Author: 
- mandd, wangc

- Creation date:

$-2019-05-20$

- The classes tested in this test are:

- Models.ExternalModel.CapitalInvestmentModel

\subsubsection{FRAMEWORK.MCKP OPTIMIZATION}

This test can be found at ". / raven/plugins/LOGOS/tests/test_mckp.xml". This test can be called executing the following command:

./run_tests --re=framework.mckp_optimization

Or

./run_framework_tests --re=framework.mckp_optimization

- Test Description:

- This test is aimed to check the capital investment model for multi-choice knapsack problem

- Original Author:

- mandd, wangc

- Creation date:

- 2019-05-20

- The classes tested in this test are:

- Models.ExternalModel.CapitalInvestmentModel

- Since the creation of this test, the following main revisions have been performed:

1. revision info:

- author: wangc

- date : 2019-05-20

- description: update test_mckp 
2. revision info:

- author: wangc

- date : 2019-05-20

- description: change variable names, this is because RAVEN External model currently can only store variables that follow the python variable rules, I think this should be changed

\subsubsection{FRAMEWORK.MKP OPTIMIZATION}

This test can be found at "./raven/plugins/LOGOS/tests/test_mkp.xml". This test can be called executing the following command:

. /run_tests --re=framework.mkp_optimization

or

./run_framework_tests --re=framework.mkp_optimization

- Test Description:

- This test is aimed to check the capital investment model for multiple knapsacks problem

- Original Author:

- mandd, wangc

- Creation date:

- 2019-05-20

- The classes tested in this test are:

- Models.ExternalModel.CapitalInvestmentModel

- Since the creation of this test, the following main revisions have been performed:

1. revision info:

- author: wangc

- date : 2019-05-20

- description: debug

2. revision info:

- author: wangc

- date : 2019-05-20

- description: fix 


\subsubsection{PLUGINS NUSAR.TESTBATTERYREPLACEMENT}

This test can be found at ". /raven/plugins/LOGOS/tests/test_battery_replacement_ cashflow.xml". This test can be called executing the following command:

. Irun_tests --re=plugins/NuSAR. TestBatteryReplacement

or

. /run_framework_tests --re=plugins/NuSAR. TestBatteryReplacement

- Test Description:

- This input tests an example of external model: battery replacement cashflow calculations.

- Original Author:

- wangc

- Creation date:

- 2019-07-12

- The classes tested in this test are:

- Models.ExternalModel

\subsubsection{PLUGINS NUSAR.TESTINCREMENTALNPV}

This test can be found at ". /raven/plugins/LOGOS/tests/test_incremental_npv . xml". This test can be called executing the following command:

./run_tests --re=plugins/NuSAR. Test IncrementalNPV

or

./run_framework_tests --re=plugins/NuSAR. Test IncrementalNPV

- Test Description:

- This input tests an example of external model: incremental cashflow calculations. 
- Original Author:

- wangc

- Creation date:

- 2019-07-12

- The classes tested in this test are:

- Models.ExternalModel

\subsubsection{PLUGINS NUSAR.TESTINCREMENTALNPV}

This test can be found at ". / raven/plugins/LOGOS/tests/test_incremental_npv_ options.xml". This test can be called executing the following command:

. /run_tests --re=plugins/NuSAR. Test IncrementalNPV

or

./run_framework_tests --re=plugins/NuSAR. TestIncrementalNPV

- Test Description:

- This input tests an example of external model: incremental cashflow calculations.

- Original Author:

- wangc

- Creation date:

- 2019-07-12

- The classes tested in this test are:

- Models.ExternalModel 


\subsubsection{LOGOS.LOGOS MCKP}

This test can be found at "./raven/plugins/LOGOS/tests/CapitalInvestments/ mckp/test_mckp.xml". This test can be called executing the following command:

./run_tests --re=Logos.logos_mckp

or

./run_framework_tests --re=Logos.logos_mckp

- Test Description:

- This test is aimed to check logos_mckp

- Original Author:

- wangc

- Creation date:

- 2019-11-18

- The classes tested in this test are:

- MCKP

\subsubsection{LOGOS.LOGOS MCKP SCENARIO}

This test can be found at "./raven/plugins/LOGOS/tests/CapitalInvestments/ mckp/test_mckp_scenario.xml". This test can be called executing the following command:

./run_tests --re=Logos.logos_mckp_scenario

or

./run_framework_tests --re=Logos.logos_mckp_scenario

- Test Description:

- This test is aimed to check logos_mckp_scenario 
- Original Author:

- wangc

- Creation date:

- 2019-11-18

- The classes tested in this test are:

- MCKP

\subsubsection{LOGOS.LOGOS SKP}

This test can be found at "./raven/plugins/LOGos/tests/Capitalinvestments/ skp/test_skp.xml". This test can be called executing the following command:

. /run_tests --re=Logos.logos_skp

Or

. Irun_framework_tests --re=Logos.logos_skp

- Test Description:

- This test is aimed to check logos_skp

- Original Author:

- wangc

- Creation date:

- 2019-11-18

- The classes tested in this test are:

- SingleKnapsack 


\subsubsection{LOGOS.LOGOS SKP}

This test can be found at "./raven/plugins/LOGoS/tests/CapitalInvestments/ skp/test_skp_external_constraint.xml". This test can be called executing the following command:

./run_tests --re=Logos.logos_skp

or

./run_framework_tests --re=Logos.logos_skp

- Test Description:

- This test is aimed to check logos_skp

- Original Author:

- wangc

- Creation date:

- 2019-11-18

- The classes tested in this test are:

- SingleKnapsack

\subsubsection{LOGOS.LOGOS BKP}

This test can be found at "./raven/plugins/LOGoS/tests/CapitalInvestments/ skp/test_bkp.xml". This test can be called executing the following command:

./run_tests --re=Logos.logos_bkp

or

./run_framework_tests --re=Logos.logos_bkp

- Test Description:

- This test is aimed to check logos_bkp 
- Original Author:

- wangc

- Creation date:

- 2019-11-18

- The classes tested in this test are:

- SingleKnapsack

\subsubsection{LOGOS.LOGOS BKP SCENARIOS}

This test can be found at "./raven/plugins/LOGos/tests/Capitalinvestments/ skp/test_bkp_scenarios.xml". This test can be called executing the following command:

./run_tests --re=Logos.logos_bkp_scenarios

or

./run_framework_tests --re=Logos.logos_bkp_scenarios

- Test Description:

- This test is aimed to check logos_bkp_scenarios

- Original Author:

- wangc

- Creation date:

- 2019-11-18

- The classes tested in this test are:

- SingleKnapsack 


\subsubsection{LOGOS.LOGOS DKP}

This test can be found at "./raven/plugins/LOGoS/tests/CapitalInvestments/ skp/test_dkp.xml". This test can be called executing the following command:

./run_tests --re=Logos.logos_dkp

or

./run_framework_tests --re=Logos.logos_dkp

- Test Description:

- This test is aimed to check logos_dkp

- Original Author:

- wangc

- Creation date:

- 2019-11-18

- The classes tested in this test are:

- SingleKnapsack

\subsubsection{LOGOS.LOGOS SKP MANDATED}

This test can be found at "./raven/plugins/LOGOS/tests/CapitalInvestments/ skp/test_mandated.xml". This test can be called executing the following command:

./run_tests --re=Logos.logos_skp_mandated

or

./run_framework_tests --re=Logos.logos_skp_mandated

- Test Description:

- This test is aimed to check logos_skp_mandated

- Original Author: 
- wangc

- Creation date:

- 2019-11-18

- The classes tested in this test are:

- SingleKnapsack

\subsubsection{LOGOS.LOGOS SKP MULTI VARIATION}

This test can be found at ". / raven/plugins/LOGOS/tests/Capital Investments / skp/test_multi_variation.xml". This test can be called executing the following command:

./run_tests --re=Logos.logos_skp_multi_variation

or

./run_framework_tests --re=Logos.logos_skp_multi_variation

- Test Description:

- This test is aimed to check logos_skp_multi_variation

- Original Author:

- wangc

- Creation date:

- 2019-11-18

- The classes tested in this test are:

- SingleKnapsack

\subsubsection{LOGOS.LOGOS SKP NO INDEX}

This test can be found at ". / raven/plugins/LOGOS/tests/Capitalinvestments / skp/test_noIndex.xml". This test can be called executing the following command:

./run_tests --re=Logos.logos_skp_no_index 
or

./run_framework_tests --re=Logos.logos_skp_no_index

- Test Description:

- This test is aimed to check logos_skp_no_index

- Original Author:

- wangc

- Creation date:

- 2019-11-18

- The classes tested in this test are:

- SingleKnapsack

\subsubsection{LOGOS.LOGOS SKP NPV}

This test can be found at ". /raven/plugins/LOGOS/tests/Capitalinvestments / skp/test_npv.xml". This test can be called executing the following command:

./run_tests --re=Logos.logos_skp_npv

or

./run_framework_tests --re=Logos.logos_skp_npv

- Test Description:

- This test is aimed to check logos_skp_npv

- Original Author:

- wangc

- Creation date:

- 2019-11-18

- The classes tested in this test are:

- SingleKnapsack 


\subsubsection{LOGOS.LOGOS SKP SCENARIOS}

This test can be found at "./raven/plugins/LOGOS/tests/Capitalinvestments/ skp/test_skp_scenarios.xml". This test can be called executing the following command:

./run_tests --re=Logos.logos_skp_scenarios

or

./run_framework_tests --re=Logos.logos_skp_scenarios

- Test Description:

- This test is aimed to check logos_skp_scenarios

- Original Author:

- wangc

- Creation date:

- 2019-11-18

- The classes tested in this test are:

- SingleKnapsack

\subsubsection{LOGOS.LOGOS SKP VARIATION}

This test can be found at "./raven/plugins/LOGoS/tests/CapitalInvestments/ skp/test_variation.xml". This test can be called executing the following command:

./run_tests --re=Logos.logos_skp_variation

or

./run_framework_tests --re=Logos.logos_skp_variation

- Test Description:

- This test is aimed to check logos_skp_variation 
- Original Author:

- wangc

- Creation date:

- 2019-11-18

- The classes tested in this test are:

- SingleKnapsack

\subsubsection{LOGOS.LOGOS SKP EE 41 PROJECTS}

This test can be found at "./raven/plugins/LOGos/tests/Capitalinvestments/ skp/test_EE_41_projects.xml". This test can be called executing the following command:

./run_tests --re=Logos.logos_skp_EE_41_projects

or

./run_framework_tests --re=Logos.logos_skp_EE_41_projects

- Test Description:

- This test is aimed to check logos_skp_EE_41_projects

- Original Author:

- wangc

- Creation date:

- 2019-11-18

- The classes tested in this test are:

- SingleKnapsack 


\subsubsection{CAPITALINVESTMENTS DRO.TEST DROMCKP SCENARIO}

This test can be found at "./raven/plugins/LOGOS/tests/CapitalInvestments/ dro/test_dromckp_scenario.xml”. This test can be called executing the following command:

./run_tests --re=Capitalinvestments/dro.test_dromckp_scenario

or

./run_framework_tests --re=Capitalinvestments/dro.test_dromckp_scenario

- Test Description:

- This test is aimed to check distributional robust optimization. In this case, radius_ambiguity is setted to zero, the test is equivalent to the sthochastic optimization MCKP test.

- Original Author:

- wangc

- Creation date:

- 2020-04-29

- The classes tested in this test are:

- DROMCKP

\subsubsection{CAPITALINVESTMENTS DRO.TEST DROMCKP SCENARIO I}

This test can be found at "./raven/plugins/LOGoS/tests/CapitalInvestments/ dro/test_dromckp_scenario_I.xml". This test can be called executing the following command:

./run_tests --re=CapitalInvestments/dro.test_dromckp_scenario_I

or

./run_framework_tests --re=Capitalinvestments/dro.test_dromckp_scenario_I

- Test Description: 
- This test is aimed to check distributional robust optimization. In this case, radius_ambiguity is setted to $2 \mathrm{E} 9$.

- Original Author:

- wangc

- Creation date:

- 2020-04-29

- The classes tested in this test are:

- DROMCKP

\subsubsection{CAPITALINVESTMENTS DRO.TEST DROMCKP SCENARIO II}

This test can be found at "./raven/plugins/LOGOS/tests/Capitalinvestments/ dro/test_dromckp_scenario_II.xml". This test can be called executing the following command:

./run_tests --re=Capitalinvestments/dro.test_dromckp_scenario_II

or

./run_framework_tests --re=Capitalinvestments/dro.test_dromckp_scenario_II

- Test Description:

- This test is aimed to check distributional robust optimization. In this case, radius_ambiguity is setted to $10 \mathrm{E} 9$.

- Original Author:

- wangc

- Creation date:

- 2020-04-29

- The classes tested in this test are:

- DROMCKP 


\subsubsection{CAPITALINVESTMENTS DRO.TEST DRO ADJUSTED USE CASE 2A}

This test can be found at "./raven/plugins/LOGoS/tests/CapitalInvestments/ dro/test_dro_adjusted_use_case_2a.xml". This test can be called executing the following command:

./run_tests --re=Capitalinvestments/dro.test_dro_adjusted_use_całe_2a

or

./run_framework_tests --re=CapitalInvestments/dro.test_dro_adjusted_use_ca

- Test Description:

- This test is aimed to check the distributionally robust optimization for use case 2 for FY19 In this case, we only consider capital budget (OM is not included). In this test, radius_ambiguity $=0.0$, and this test is equivalent to the stochastic optimization problem.

- Original Author:

- wangc

- Creation date:

- 2020-04-29

- The classes tested in this test are:

- DROMCKP

\subsubsection{CAPITALINVESTMENTS DRO.TEST DRO ADJUSTED USE CASE 2A}

This test can be found at "./raven/plugins/LOGOS/tests/Capitalinvestments/ dro/test_dro_adjusted_use_case_2a_I.xml”. This test can be called executing the following command:

./run_tests --re=Capitalinvestments/dro.test_dro_adjusted_use_ca\$e_2a

or

./run_framework_tests --re=CapitalInvestments/dro.test_dro_adjusted_use_ca 
- Test Description:

- This test is aimed to check the distributionally robust optimization for use case 2 for FY19 In this case, we only consider capital budget (OM is not included). In this test, radius_ambiguity $=0.01$

- Original Author:

- wangc

- Creation date:

- 2020-04-29

- The classes tested in this test are:

- DROMCKP

\subsubsection{CAPITALINVESTMENTS DRO.TEST DRO ADJUSTED USE CASE 2A}

This test can be found at ". /raven/plugins/LOGOS/tests/Capitalinvestments/ dro/test_dro_adjusted_use_case_2a_II.xml". This test can be called executing the following command:

./run_tests --re=CapitalInvestments/dro.test_dro_adjusted_use_całe_2a

or

./run_framework_tests --re=CapitalInvestments/dro.test_dro_adjusted_use_ca

- Test Description:

- This test is aimed to check the distributionally robust optimization for use case 2 for FY19 In this case, we only consider capital budget (OM is not included). In this test, radius_ambiguity $=0.1$

- Original Author:

- wangc

- Creation date:

- 2020-04-29

- The classes tested in this test are:

- DROMCKP 


\subsubsection{CAPITALINVESTMENTS DRO.TEST DRO ADJUSTED USE CASE 2A}

This test can be found at "./raven/plugins/LOGOS/tests/CapitalInvestments/ dro/test_dro_adjusted_use_case_2a_III.xml”. This test can be called executing the following command:

./run_tests --re=CapitalInvestments/dro.test_dro_adjusted_use_caße_2a

or

./run_framework_tests --re=CapitalInvestments/dro.test_dro_adjusted_use_ca

- Test Description:

- This test is aimed to check the distributionally robust optimization for use case 2 for FY19 In this case, we only consider capital budget (OM is not included). In this test, radius_ambiguity $=1.0$

- Original Author:

- wangc

- Creation date:

- 2020-04-29

- The classes tested in this test are:

- DROMCKP

\subsubsection{CAPITALINVESTMENTS DRO.TEST DRO ADJUSTED USE CASE 2A}

This test can be found at "./raven/plugins/LOGOS/tests/Capitalinvestments/ dro/test_dro_adjusted_use_case_2a_IV.xml”. This test can be called executing the following command:

./run_tests --re=Capitalinvestments/dro.test_dro_adjusted_use_caße_2a

or

./run_framework_tests --re=CapitalInvestments/dro.test_dro_adjusted_use_ca

- Test Description: 
- This test is aimed to check the distributionally robust optimization for use case 2 for FY19 In this case, we only consider capital budget (OM is not included). In this test, radius_ambiguity $=10.0$

- Original Author:

- wangc

- Creation date:

- 2020-04-29

- The classes tested in this test are:

- DROMCKP

\subsubsection{CAPITALINVESTMENTS DRO.TEST DROSKP SCENARIO}

This test can be found at "./raven/plugins/LOGOS/tests/Capital Investments/ dro/test_droskp_scenario.xml”. This test can be called executing the following command:

./run_tests --re=CapitalInvestments/dro.test_droskp_scenario

or

./run_framework_tests --re=Capitalinvestments/dro.test_droskp_scenario

- Test Description:

- This test is aimed to check the distributionally robust optimization for Single Knapsack Problem, we only consider capital budget. In this test, radius_ambiguity $=0.0$, and this test is equivalent to the stochastic optimization problem.

- Original Author:

- wangc

- Creation date:

- 2020-04-30

- The classes tested in this test are:

- DROSKP 


\subsubsection{CAPITALINVESTMENTS DRO.TEST DROSKP SCENARIO I}

This test can be found at "./raven/plugins/LOGOS/tests/CapitalInvestments/ dro/test_droskp_scenario_I.xml”. This test can be called executing the following command:

./run_tests --re=Capitalinvestments/dro.test_droskp_scenario_I

or

./run_framework_tests --re=CapitalInvestments/dro.test_droskp_scenario_I

- Test Description:

- This test is aimed to check the distributionally robust optimization for Single Knapsack Problem, we only consider capital budget. In this test, radius_ambiguity $=0.01$.

- Original Author:

- wangc

- Creation date:

- 2020-04-30

- The classes tested in this test are:

- DROSKP

\subsubsection{CAPITALINVESTMENTS DRO.TEST DROSKP SCENARIO II}

This test can be found at "./raven/plugins/LOGoS/tests/CapitalInvestments/ dro/test_droskp_scenario_II.xml". This test can be called executing the following command:

./run_tests --re=CapitalInvestments/dro.test_droskp_scenario_II

or

./run_framework_tests --re=Capitalinvestments/dro.test_droskp_scenario_II

- Test Description: 
- This test is aimed to check the distributionally robust optimization for Single Knapsack Problem, we only consider capital budget. In this test, radius_ambiguity $=0.05$.

- Original Author:

- wangc

- Creation date:

- 2020-04-30

- The classes tested in this test are:

- DROSKP

\subsubsection{CAPITALINVESTMENTS DRO.TEST DROMKP SCENARIO}

This test can be found at "./raven/plugins/LOGOS/tests/Capital Investments/ dro/test_dromkp_scenarios.xml". This test can be called executing the following command:

./run_tests --re=Capitalinvestments/dro.test_dromkp_scenario

or

./run_framework_tests --re=Capitalinvestments/dro.test_dromkp_scenario

- Test Description:

- This test is aimed to check the distributionally robust optimization for Multiple Knapsack Problem, we only consider capital budget. In this test, radius_ambiguity $=0.0$, which is equivalent to the stochastic programming

- Original Author:

- wangc

- Creation date:

- 2020-04-30

- The classes tested in this test are:

- DROMKP 


\subsubsection{CAPITALINVESTMENTS DRO.TEST DROMKP SCENARIO}

This test can be found at "./raven/plugins/LOGOS/tests/CapitalInvestments/ dro/test_dromkp_scenarios_I.xml". This test can be called executing the following command:

./run_tests --re=CapitalInvestments/dro.test_dromkp_scenario

or

./run_framework_tests --re=Capitalinvestments/dro.test_dromkp_scenario

- Test Description:

- This test is aimed to check the distributionally robust optimization for Multiple Knapsack Problem, we only consider capital budget. In this test, radius_ambiguity $=1.0$.

- Original Author:

- wangc

- Creation date:

$-2020-04-30$

- The classes tested in this test are:

- DROMKP

\subsubsection{CAPITALINVESTMENTS DRO.TEST DROSKP MULTIPLE VARIATIONS}

This test can be found at "./raven/plugins/LOGoS/tests/Capitalinvestments/ dro/test_droskp_multiple_variations.xml". This test can be called executing the following command:

./run_tests --re=CapitalInvestments/dro.test_droskp_multiple_variations

or

./run_framework_tests --re=CapitalInvestments/dro.test_droskp_multiple_var

- Test Description: 
- This test is aimed to test scenarios of multiple parameters using distributionally robust optimization. In this case, radius_ambiguity $=0.0$. In other words, this problem is equivalent to the stochastic single knapsack problem.

- Original Author:

- wangc

- Creation date:

- 2020-06-01

- The classes tested in this test are:

- DROSKP

\subsubsection{CAPITALINVESTMENTS DRO.TEST DROSKP MULTIPLE VARIATIONS I}

This test can be found at ". /raven/plugins/LOGOS/tests/CapitalInvestments/ dro/test_droskp_multiple_variations_I.xml". This test can be called executing the following command:

. Irun_tests --re=CapitalInvestments/dro.test_droskp_multiple_varilations_I

or

.Irun_framework_tests --re=CapitalInvestments/dro.test_droskp_muftiple_var

- Test Description:

- This test is aimed to test scenarios of multiple parameters using distributionally robust optimization. In this case, radius_ambiguity $=0.01$. However, we are using the same values for different scenarios of net_present_values. Thus, this problem is equivalent to droskp problem with single parameter variations.

- Original Author:

- wangc

- Creation date:

- 2020-06-01

- The classes tested in this test are:

- DROSKP 


\subsubsection{CAPITALINVESTMENTS DRO.TEST DROSKP MULTIPLE VARIATIONS II}

This test can be found at "./raven/plugins/LOGoS/tests/CapitalInvestments/ dro/test_droskp_multiple_variations_II.xml". This test can be called executing the following command:

./run_tests --re=CapitalInvestments/dro.test_droskp_multiple_variations_II

or

./run_framework_tests --re=CapitalInvestments/dro.test_droskp_multiple_var

- Test Description:

- This test is aimed to test scenarios of multiple parameters using distributionally robust optimization. In this case, radius_ambiguity $=0.01$. In this case, the net_present_values at the second scenario is reduced by 10the optimized max npv will be also reduced by 10

- Original Author:

- wangc

- Creation date:

- 2020-06-01

- The classes tested in this test are:

- DROSKP

\subsubsection{LOGOS.LOGOS MKP}

This test can be found at "./raven/plugins/LOGoS/tests/CapitalInvestments/ mkp/test_mkp.xml". This test can be called executing the following command:

./run_tests --re=Logos.logos_mkp

or

./run_framework_tests --re=Logos.logos_mkp

- Test Description: 
- This test is aimed to check logos_mkp

- Original Author:

- wangc

- Creation date:

- 2019-11-18

- The classes tested in this test are:

- MultipleKnapsack

\subsubsection{LOGOS.LOGOS MKP GLPK}

This test can be found at ". / raven/plugins/LOGOS/tests/Capital Investments/ mkp/test_mkp_1.xml". This test can be called executing the following command:

. /run_tests --re=Logos.logos_mkp_glpk

or

./run_framework_tests --re=Logos.logos_mkp_glpk

- Test Description:

- This test is aimed to check logos_mkp_glpk

- Original Author:

- wangc

- Creation date:

- 2019-11-18

- The classes tested in this test are:

- MultipleKnapsack 


\subsubsection{LOGOS.LOGOS MKP NO INDEX}

This test can be found at "./raven/plugins/LOGOS/tests/CapitalInvestments/ mkp/test_mkp_noindex.xml”. This test can be called executing the following command:

./run_tests --re=Logos.logos_mkp_no_index

or

./run_framework_tests --re=Logos.logos_mkp_no_index

- Test Description:

- This test is aimed to check logos_mkp_no_index

- Original Author:

- wangc

- Creation date:

- 2019-11-18

- The classes tested in this test are:

- MultipleKnapsack

\subsubsection{LOGOS.LOGOS MKP SCENARIOS}

This test can be found at "./raven/plugins/LOGOS/tests/Capital Investments/ mkp/test_mkp_scenarios.xml". This test can be called executing the following command:

./run_tests --re=Logos.logos_mkp_scenarios

or

./run_framework_tests --re=Logos.logos_mkp_scenarios

- Test Description:

- This test is aimed to check logos_mkp_scenarios 
- Original Author:

- wangc

- Creation date:

- 2019-11-18

- The classes tested in this test are:

- MultipleKnapsack

\subsubsection{LOGOS.LOGOS MKP T}

This test can be found at "./raven/plugins/LOGos/tests/CapitalInvestments/ mkp/test_mkp_t.xml". This test can be called executing the following command:

. Irun_tests --re=Logos.logos_mkp_t

Or

./run_framework_tests --re=Logos.logos_mkp_t

- Test Description:

- This test is aimed to check logos_mkp_t

- Original Author:

- wangc

- Creation date:

- 2019-11-18

- The classes tested in this test are:

- MultipleKnapsack 


\subsubsection{LOGOS.LOGOS MKP WEING}

This test can be found at "./raven/plugins/LOGOS/tests/CapitalInvestments/ mkp/test_mkp_weing.xml". This test can be called executing the following command:

./run_tests --re=Logos.logos_mkp_weing

or

./run_framework_tests --re=Logos.logos_mkp_weing

- Test Description:

- This test is aimed to check logos_mkp_weing

- Original Author:

- wangc

- Creation date:

- 2019-11-18

- The classes tested in this test are:

- MultipleKnapsack

\subsubsection{LOGOS.LOGOS EXAMPLES P16}

This test can be found at "./raven/plugins/LOGOS/tests/CapitalInvestments/ examples/test_p16.xml". This test can be called executing the following command:

./run_tests --re=Logos.logos_examples_p16

or

./run_framework_tests --re=Logos.logos_examples_p16

- Test Description:

- This test is aimed to check logos_examples_p16

- Original Author: 
- wangc

- Creation date:

- 2019-11-18

- The classes tested in this test are:

- MCKP

\subsubsection{LOGOS.LOGOS EXAMPLES P16 EXT}

This test can be found at ". / raven/plugins/LOGOS/tests/Capital Investments / examples/test_p16_ext.xml”. This test can be called executing the following command:

. Irun_tests --re=Logos.logos_examples_p16_ext

Or

./run_framework_tests --re=Logos.logos_examples_p16_ext

- Test Description:

- This test is aimed to check logos_examples_p16_ext

- Original Author:

- wangc

- Creation date:

- 2019-11-18

- The classes tested in this test are:

- MCKP

\subsubsection{LOGOS.LOGOS EXAMPLES USE CASE 1A FY19}

This test can be found at "./raven/plugins/LOGOS/tests/Capitalinvestments/ examples/use_case_la_fy19.xml". This test can be called executing the following command:

./run_tests --re=Logos.logos_examples_use_case_la_fyl9 
or

./run_framework_tests --re=Logos.logos_examples_use_case_1a_fy19

- Test Description:

- This test is aimed to check logos_examples_use_case_1a_fy 19

- Original Author:

- wangc

- Creation date:

- 2019-11-18

- The classes tested in this test are:

- MCKP

\subsubsection{LOGOS.LOGOS EXAMPLES USE CASE 1B FY19}

This test can be found at ". /raven/plugins/LOGOS/tests/Capital Investments / examples/use_case_1b_fy19.xml". This test can be called executing the following command:

./run_tests --re=Logos.logos_examples_use_case_1b_fy19

or

./run_framework_tests --re=Logos.logos_examples_use_case_1b_fy19

- Test Description:

- This test is aimed to check logos_examples_use_case_1b_fy19

- Original Author:

- wangc

- Creation date:

- 2019-11-18

- The classes tested in this test are:

- MCKP 


\subsubsection{LOGOS.LOGOS EXAMPLES USE CASE 1C FY19}

This test can be found at "./raven/plugins/LOGOS/tests/CapitalInvestments/ examples/use_case_1c_fy19.xml". This test can be called executing the following command:

./run_tests --re=Logos.logos_examples_use_case_1c_fy19

or

./run_framework_tests --re=Logos.logos_examples_use_case_1c_fy19

- Test Description:

- This test is aimed to check logos_examples_use_case_1c_fy 19

- Original Author:

- wangc

- Creation date:

- 2019-11-18

- The classes tested in this test are:

- MCKP

\subsubsection{LOGOS.LOGOS EXAMPLES USE CASE 2A FY19}

This test can be found at "./raven/plugins/LOGOS/tests/Capitalinvestments/ examples/use_case_2a_fy19.xml". This test can be called executing the following command:

./run_tests --re=Logos.logos_examples_use_case_2a_fy19

or

./run_framework_tests --re=Logos.logos_examples_use_case_2a_fy19

- Test Description:

- This test is aimed to check logos_examples_use_case_2a_fy 19 
- Original Author:

- wangc

- Creation date:

- 2019-11-18

- The classes tested in this test are:

- MCKP

\subsubsection{LOGOS.LOGOS EXAMPLES USE CASE 2A FY19 CONSISTENT CONSTRAINT I}

This test can be found at ". /raven/plugins/LOGOS/tests/Capitalinvestments/ examples/use_case_2a_fy19_consistent_constraint_I.xml". This test can be called executing the following command:

. Irun_tests --re=Logos.logos_examples_use_case_2a_fy19_consistent_constrai

or

./run_framework_tests --re=Logos.logos_examples_use_case_2a_fy19_consisten

- Test Description:

- This test is aimed to check logos_examples_use_case_2a_fy19_consistent_constraint_I

- Original Author:

- wangc

- Creation date:

- 2019-11-18

- The classes tested in this test are:

- MCKP 


\subsubsection{LOGOS.LOGOS EXAMPLES USE CASE 2A FY19 CONSISTENT CONSTRAINT I II}

This test can be found at "./raven/plugins/LOGOS/tests/Capitalinvestments/ examples/use_case_2a_fy19_consistent_constraint_I_II.xml". This test can be called executing the following command:

.Irun_tests --re=Logos.logos_examples_use_case_2a_fy19_consistent_constrai

or

.Irun_framework_tests --re=Logos.logos_examples_use_case_2a_fy19_consisten

- Test Description:

- This test is aimed to check logos_examples_use_case_2a_fy19_consistent_constraint_I_II

- Original Author:

- wangc

- Creation date:

- 2019-11-18

- The classes tested in this test are:

- MCKP

\subsubsection{LOGOS.LOGOS EXAMPLES USE CASE 2A FY19 NO CONSISTENT CONSTRAINT}

This test can be found at ". /raven/plugins/LOGOS/tests/Capitalinvestments/ examples/use_case_2a_fy19_no_consistent_constraint.xml”. This test can be called executing the following command:

.Irun_tests --re=Logos.logos_examples_use_case_2a_fy19_no_consistent_const.

or

./run_framework_tests --re=Logos.logos_examples_use_case_2a_fy19_no_consis

- Test Description: 
- This test is aimed to check logos_examples_use_case_2a_fy19_no_consistent_constraint

- Original Author:

- wangc

- Creation date:

- 2019-11-18

- The classes tested in this test are:

- MCKP

\subsubsection{LOGOS.LOGOS EXAMPLES USE CASE 2B FY19 CONSISTENT CONSTRAINT I}

This test can be found at ". /raven/plugins/LOGOS/tests/Capitalinvestments/ examples/use_case_2b_fy19_consistent_constraint_I.xml". This test can be called executing the following command:

.Irun_tests --re=Logos.logos_examples_use_case_2b_fy19_consistent_constrai

or

.Irun_framework_tests --re=Logos.logos_examples_use_case_2b_fy19_consisten

- Test Description:

- This test is aimed to check logos_examples_use_case_2b_fy19_consistent_constraint_I

- Original Author:

- wangc

- Creation date:

- 2019-11-18

- The classes tested in this test are:

- MCKP 


\subsubsection{LOGOS.LOGOS EXAMPLES USE CASE 2B FY19 CONSISTENT CONSTRAINT I II}

This test can be found at "./raven/plugins/LOGOS/tests/Capitalinvestments/ examples/use_case_2b_fy19_consistent_constraint_I_II.xml”. This test can be called executing the following command:

.Irun_tests --re=Logos.logos_examples_use_case_2b_fy19_consistent_constrai

or

.Irun_framework_tests --re=Logos.logos_examples_use_case_2b_fy19_consisten

- Test Description:

- This test is aimed to check logos_examples_use_case_2b_fy19_consistent_constraint_I_II

- Original Author:

- wangc

- Creation date:

- 2019-11-18

- The classes tested in this test are:

- MCKP

\subsubsection{LOGOS.LOGOS EXAMPLES USE CASE 2B FY19 NO CONSISTENT CONSTRAINT}

This test can be found at "./raven/plugins/LOGOS/tests/Capitalinvestments/ examples/use_case_2b_fy19_no_consistent_constraint.xml”. This test can be called executing the following command:

.Irun_tests --re=Logos.logos_examples_use_case_2b_fy19_no_consistent_const

or

. Irun_framework_tests --re=Logos.logos_examples_use_case_2b_fy19_no_consis

- Test Description: 
- This test is aimed to check logos_examples_use_case_2b_fy19_no_consistent_constraint

- Original Author:

- wangc

- Creation date:

- 2019-11-18

- The classes tested in this test are:

- MCKP

\subsubsection{LOGOS.LOGOS EXAMPLES USE CASE 2C FY19 CONSISTENT CONSTRAINT I II}

This test can be found at ". /raven/plugins/LOGOS/tests/Capitalinvestments/ examples/use_case_2c_fy19_consistent_constraint_I_II.xml". This test can be called executing the following command:

.Irun_tests --re=Logos.logos_examples_use_case_2c_fy19_consistent_constrai

or

.Irun_framework_tests --re=Logos.logos_examples_use_case_2c_fy19_consisten

- Test Description:

- This test is aimed to check logos_examples_use_case_2c_fy19_consistent_constraint_I_II

- Original Author:

- wangc

- Creation date:

- 2019-11-18

- The classes tested in this test are:

- MCKP 


\subsubsection{LOGOS.LOGOS EXAMPLES USE CASE 2D FY19 CONSISTENT CONSTRAINT I II}

This test can be found at "./raven/plugins/LOGOS/tests/Capitalinvestments/ examples/use_case_2d_fy19_consistent_constraint_I_II.xml". This test can be called executing the following command:

.Irun_tests --re=Logos.logos_examples_use_case_2d_fy19_consistent_constrai

or

.Irun_framework_tests --re=Logos.logos_examples_use_case_2d_fy19_consisten

- Test Description:

- This test is aimed to check logos_examples_use_case_2d_fy19_consistent_constraint_I_II

- Original Author:

- wangc

- Creation date:

- 2019-11-18

- The classes tested in this test are:

- MCKP

\subsubsection{LOGOS.LOGOS EXAMPLES USE CASE 3A FY19 CONSISTENT CONSTRAINT I II}

This test can be found at ". / raven/plugins/LOGOS/tests/Capitalinvestments/ examples/use_case_3a_fy19_consistent_constraint_I_II.xml”. This test can be called executing the following command:

.Irun_tests --re=Logos.logos_examples_use_case_3a_fy19_consistent_constrai

or

.Irun_framework_tests --re=Logos.logos_examples_use_case_3a_fy19_consisten

- Test Description: 
- This test is aimed to check logos_examples_use_case_3a_fy19_consistent_constraint_I_II

- Original Author:

- wangc

- Creation date:

- 2019-11-18

- The classes tested in this test are:

- MCKP

\subsubsection{LOGOS.LOGOS EXAMPLES USE CASE 3B FY19 CONSISTENT CONSTRAINT I II}

This test can be found at "./raven/plugins/LOGOS/tests/Capitalinvestments/ examples/use_case_3b_fy19_consistent_constraint_I_II.xml". This test can be called executing the following command:

.Irun_tests --re=Logos.logos_examples_use_case_3b_fy19_consistent_constrai

or

.Irun_framework_tests --re=Logos.logos_examples_use_case_3b_fy19_consisten

- Test Description:

- This test is aimed to check logos_examples_use_case_3b_fy19_consistent_constraint_I_II

- Original Author:

- wangc

- Creation date:

- 2019-11-18

- The classes tested in this test are:

- MCKP 


\subsubsection{PRODUCTIONFLEX}

This test can be found at "./raven/plugins/HERON/tests/integration_tests/ workflows/production_flex/heron_input.xml". This test can be called executing the following command:

./run_tests --re=Productionflex

or

./run_framework_tests --re=ProductionFlex

- Test Description:

- Tests including a consume-and-produce unit, and the pyomo dispatcher. Uses an ARMA for "electr_flex" pricing. May be able to serve as a timing test in the future. "steamer" produces steam which is consumed by "generator" to produce electricity. This electricity can then be sold at the "electr_market" at a profit or "electr_flex" at a variable profit/cost.

- Original Author:

- talbpaul

- Creation date:

$-2020-06-15$

- The classes tested in this test are:

- HERON

\subsubsection{PRODUCTION}

This test can be found at "./raven/plugins/HERON/tests/integration_tests/ workflows/production_flex_opt/heron_input.xml". This test can be called executing the following command:

./run_tests $--r e=$ production

or

./run_framework_tests --re=production 


\section{- Test Description:}

- Demonstrates using an optimization strategy on a simple system, instead of a sweep strategy. A steam source feeds a generator, which then sells electricity to a low-capacity high-value market and a high-capacity negative-value market. The size of the steam source and high-capacity market are optimized. The market size is irrelevant; the optimal steam source size is equal to the high-value market size.

- Original Author:

- talbpaul

- Creation date:

- 2020-06-15

- The classes tested in this test are:

- HERON

\subsubsection{VALIDATOR}

This test can be found at "./raven/plugins/HERON/tests/integration_tests/ mechanics/validator/heron_input.xml”. This test can be called executing the following command:

. Irun_tests --re=validator

or

. Irun_framework_tests --re=validator

- Test Description:

- Tests using a "validator" to modify behavior of a dispatch optimizer. Uses the Example validator.

- Original Author:

- talbpaul

- Creation date:

- 2020-09-14

- The classes tested in this test are:

- HERON 


\subsubsection{MINDEMAND}

This test can be found at "./raven/plugins/HERON/tests/integration_tests/ mechanics/min_demand/heron_input.xml". This test can be called executing the following command:

./run_tests --re=MinDemand

or

./run_framework_tests --re=MinDemand

- Test Description:

- Tests having a minimum level for a demand component.

- Original Author:

- E. Worsham

- Creation date:

- 2020-10-15

- The classes tested in this test are:

- HERON

\subsubsection{LABELS}

This test can be found at "./raven/plugins/HERON/tests/integration_tests/ mechanics/labels/heron_input.xml". This test can be called executing the following command:

. Irun_tests --re=Labels

or

. Irun_framework_tests --re=Labels

- Test Description:

- Tests including case-info user defined labels. Extends from production_flex. 
- Original Author:

- dylanjm

- Creation date:

$-2020-10-12$

- The classes tested in this test are:

- HERON

\subsubsection{CASHFLOWS}

This test can be found at "./raven/plugins/HERON/tests/integration_tests/ mechanics/cashflows/heron_input.xml". This test can be called executing the following command:

. /run_tests --re=Cashflows

or

. Irun_framework_tests --re=Cashflows

- Test Description:

- Tests various kinds of cashflows

- Original Author:

- talbpaul

- Creation date:

- 2020-10-16

- The classes tested in this test are:

- HERON 


\subsubsection{ARMA SINE}

This test can be found at "./raven/plugins/HERON/tests/integration_tests/ ARMA/train_sine.xml". This test can be called executing the following command:

. Irun_tests --re=ARMA_sine

or

./run_framework_tests --re=ARMA_sine

- Test Description:

- This trains a non-stochastic sine function for use in HERON tests.

- Original Author:

- gaira

- Creation date:

- 2020-05-12

- The classes tested in this test are:

- SupervisedLearning.ARMA

\subsubsection{ARMA SINE}

This test can be found at “. / raven/plugins / SRAW/tests/sraw_capital_budgeting . xml". This test can be called executing the following command:

. Irun_tests --re=ARMA_sine

or

./run_framework_tests --re=ARMA_sine

- Test Description:

- This trains a non-stochastic sine function for use in HERON tests.

- Original Author: 
- gaira

- Creation date:

- 2020-05-12

- The classes tested in this test are:

- SupervisedLearning.ARMA

\subsubsection{FRAMEWORK PCARESPONSESURFACE}

This test can be found at “. / raven/tests/framework/test_pca_responseSurface. xml". This test can be called executing the following command:

./run_tests --re=framework/pcaResponseSurface

or

./run_framework_tests --re=framework/pcaResponseSurface

- Test Description:

- Tests responseSurface sampler in connection with PCA.

- Original Author:

- wangc

- Creation date:

- 2015-12-16

- The classes tested in this test are:

- Samplers.responseSurface

\subsubsection{FRAMEWORK.EXTERNAL XML}

This test can be found at "./raven/tests/framework/test_External_XML.xml". This test can be called executing the following command:

.Irun_tests --re=framework.external_xml 
or

./run_framework_tests --re=framework.external_xml

- Test Description:

- This test is aimed to check the capability in RAVEN to load external XML files as part of the input file. Indeed, allows the user to inject in the input files XML blocks defined in external files

- Original Author:

- wangc

- Creation date:

$-2015-07-22$

- The classes tested in this test are:

- Simulation.ExternalXML

- Since the creation of this test, the following main revisions have been performed:

1. revision info:

- author : maljdan

- date : 2016-04-06

- description: Updating test cases to reflect the changes to the user input.

2. revision info:

- author : talbpaul

- date : 2016-04-26

- description: added check for pre-existing backup files when validating

3. revision info:

- author : alfoa

- date : 2017-01-21

- description: Adding this test description.

4. revision info:

- author : talbpaul

- date : 2018-03-26

- description: Nested ExternalXML nodes in OutStream 


\subsubsection{FRAMEWORK.TEST MULTI TARGET ROM}

This test can be found at “./raven/tests/framework/test_multi_target_rom. xml". This test can be called executing the following command:

. Irun_tests --re=framework.test_multi_target_rom

or

./run_framework_tests --re=framework.test_multi_target_rom

- Test Description:

- This test is aimed to test the capability of RAVEN to train ROMs that can predict multiple targets at the same time (multiple FOMs)

- Original Author:

- alfoa

- Creation date:

- 2014-11-30

- The classes tested in this test are:

- Steps.RomTrainer, Models.ROM

- Since the creation of this test, the following main revisions have been performed:

1. revision info:

- author : mandd

- date : 2015-04-17

- description: conversion to Database and DataObjects

2. revision info:

- author : mandd

- date : 2015-04-20

- description: Merge remote-tracking branch 'origin/devel' into mandd/DataRename

3. revision info:

- author : alfoa

- date : 2015-06-18

- description: Changed Name of all DataObjects 
4. revision info:

- author : alfoa

- date : 2015-06-21

- description: modified Names + added new capabilities and flexibility in DataObjects + added options block. Modified XSD schema and manual to reflect the modifications

5. revision info:

- author : alfoa

- date : 2015-09-12

- description: removed syntax inconsistency with respect standards for Samplers (underscores instead of camelBack) + created conversion script

6. revision info:

- author : cogljj

- date : 2015-10-13

- description: Updating test_multi_target_rom to use external models.

7. revision info:

- author : cogljj

- date : 2015-11-17

- description: Updating to work with scikit-learn 0.17. In the new version of sklearn the rom can not use a classifier anymore.

8. revision info:

- author : maljdan

- date : 2016-05-20

- description: Updating the test cases to remove references to Point or History.

9. revision info:

- author : alfoa

- date : 2017-01-21

- description: Adding this test description.

10. revision info:

- author : alfoa

- date : 2017-12-17

- description: Modified for adapting to new DataObject structure

\subsubsection{FRAMEWORK.TEST ROM TRAIN FROM ALREADY DUMPED HDF5}

This test can be found at ". /raven/tests/framework/test_rom_train_from_already_ dumped_HDF 5.xml". This test can be called executing the following command: 
./run_tests --re=framework.test_rom_train_from_already_dumped_HDF5

or

. Irun_framework_tests --re=framework.test_rom_train_from_already_dumped_HD.

- Test Description:

- This test is aimed to test the capability of RAVEN to train ROMs based on the pregerenated data sets (HDF5)

- Original Author:

- alfoa

- Creation date:

- 2014-02-26

- The classes tested in this test are:

- Steps.RomTrainer, Models.ROM, Databases.HDF5

- Since the creation of this test, the following main revisions have been performed:

1. revision info:

- author : alfoa

- date : 2014-03-06

- description: changed name of DataBase handling, modified all tests were using it, improved Step output handling etc r 25347

2. revision info:

- author : maljdan

- date : 2015-06-16

- description: Adding steps to the XSD, and fixing the input files to pass the validation. Currently, using sequences.

3. revision info:

- author : alfoa

- date : 2015-06-18

- description: Changed Name of all DataObjects

4. revision info: 
- author : alfoa

- date : 2015-06-21

- description: modified Names + added new capabilities and flexibility in DataObjects + added options block. Modified XSD schema and manual to reflect the modifications

5. revision info:

- author : cogljj

- date : 2015-10-13

- description: Converting test_rom_train_from_already_dumped_HDF5 to use external model.

6. revision info:

- author : cogljj

- date : 2015-11-17

- description: Updating to work with scikit-learn 0.17. In the new version of sklearn the rom can not use a classifier anymore.

7. revision info:

- author : maljdan

- date : 2016-04-06

- description: Updating test cases to reflect the changes to the user input.

8. revision info:

- author : alfoa

- date : 2017-01-21

- description: Adding this test description.

9. revision info:

- author : talbpaul

- date : 2017-07-10

- description: Fixed location of database to be specified relative to working directory

10. revision info:

- author : alfoa

- date : 2017-12-18

- description: Adapted for new DataObject structure

\subsubsection{FRAMEWORK.TESTRESPONSESURFACEDESIGNS}

This test can be found at “. / raven/tests/framework/test_ResponseSurfaceDesign_ sampler.xml". This test can be called executing the following command: 
. Irun_tests --re=framework.testResponseSurfacedesigns

or

./run_framework_tests --re=framework.testResponseSurfacedesigns

- Test Description:

- This test is aimed to check the capability in RAVEN to employ a ResponseSurfaceDesign sampling strategy. Since the goal of the test is to testify that the Grid sampling strategy is functional, a Dummy model is used. Both available Response Surface design strategies are tested (BoxBehnken, CentralComposite)

- Original Author:

- alfoa

- Creation date:

- 2014-12-06

- The classes tested in this test are:

- Sampler.ResponseSurfaceDesign

- Since the creation of this test, the following main revisions have been performed:

1. revision info:

- author : alfoa

- date : 2015-02-04

- description: added algorithm_type in tests and fixed a bug

2. revision info:

- author : mandd

- date : 2015-04-17

- description: conversion to Database and DataObjects

3. revision info:

- author : alfoa

- date : 2015-05-18

- description: Closes \#167

4. revision info: 
- author : alfoa

- date : 2015-05-18

- description: Closes \#169

5. revision info:

- author : alfoa

- date : 2015-05-29

- description: finalized all the samplers

6. revision info:

- author : alfoa

- date : 2015-06-18

- description: Changed Name of all DataObjects

7. revision info:

- author : maljdan

- date : 2016-04-06

- description: Updating test cases to reflect the changes to the user input.

8. revision info:

- author : talbpaul

- date : 2016-04-26

- description: added check for pre-existing backup files when validating

9. revision info:

- author : alfoa

- date : 2017-01-21

- description: Adding this test description.

\subsubsection{FRAMEWORK.TESTGRIDWITHCONSTANTS}

This test can be found at "./raven/tests/framework/test_Grid_Sampler_with_ constants.xml". This test can be called executing the following command:

. Irun_tests --re=framework.testGridWithConstants

or

./run_framework_tests --re=framework.testGridWithConstants

- Test Description: 
- This test is aimed to check the capability of RAVEN to input constant variables values. This test is valid to demonstrate this capability for any Sampler in RAVEN. In addition, it shows how the constant variables can be used in conjunction with a function.

- Original Author:

- alfoa

- Creation date:

- 2017-04-05

- The classes tested in this test are:

- Sampler.Grid

- Since the creation of this test, the following main revisions have been performed:

1. revision info:

- author : alfoa

- date : 2017-04-24

- description: added a function to show how the constant variables can be used in conjunction with a function

2. revision info:

- author : alfoa

- date : 2019-03-07

- description: Modified syntax in Functions as for issue \#934

\subsubsection{FRAMEWORK.TESTLHS}

This test can be found at “. / raven/tests / framework/test_LHS_Sampler.xml”. This test can be called executing the following command:

. Irun_tests --re=framework.testLHS

or

./run_framework_tests --re=framework.testLHS

- Test Description:

- This test is aimed to check the functionality of the Stratified sampling. In order to check this sampling strategy a Model of type Dummy. 
- Original Author:

- crisr

- Creation date:

- 2013-10-15

- The classes tested in this test are:

- Samplers.Stratified, Models.Dummy, Steps.MultiRun

- Since the creation of this test, the following main revisions have been performed:

1. revision info:

- author : alfoa

- date : 2014-01-21

- description: fixed tests, added new capability in OutStreamPrint + modified check_output test, bug fixes in Datas.py and Steps... Deletede Plotting Step... Not needed SVN r24086

2. revision info:

- author : alfoa

- date : 2015-03-04

- description: modified batch sizes because sometimes, if we run the tests in parallel, the order of values in csv can be different

3. revision info:

- author : mandd

- date : 2015-04-17

- description: conversion to Database and DataObjects

4. revision info:

- author : alfoa

- date : 2015-04-24

- description: From LHS to Stratified. Closes \#130

5. revision info:

- author : alfoa

- date : 2015-05-18

- description: Closes \#167

6. revision info:

- author : alfoa

- date : 2015-05-18 
- description: Closes \#169

7. revision info:

- author : alfoa

- date : 2015-06-18

- description: Changed Name of all DataObjects

8. revision info:

- author : maljdan

- date : 2015-08-20

- description: Fixing a bug associated with the grid construction used by the LHS sampler. (See issue \#266)

9. revision info:

- author: wangc

- date : 2015-09-10

- description: update validate_xml.sh, convert some test inputs into something mostly RAVEN-preferred

10. revision info:

- author : alfoa

- date : 2015-09-12

- description: removed syntax inconsistency with respect standards for Samplers (underscores instead of camelBack) + created conversion script

11. revision info:

- author : maljdan

- date : 2016-04-06

- description: Updating test cases to reflect the changes to the user input.

12. revision info:

- author : alfoa

- date : 2017-01-21

- description: Adding this test description.

\subsubsection{FRAMEWORK.TESTEXTERNALMODELRESEED}

This test can be found at ". / raven/tests / framework/test_external_reseed.xml". This test can be called executing the following command:

. /run_tests --re=framework.testExternalModelReseed 
or

./run_framework_tests --re=framework.testExternalModelReseed

- Test Description:

- This test is aimed to test the capability of the random-based sampling strategies (e.g.MonteCarlo, Stratified, etc.) to change the seeding of the random number generator every time a new realization is requested.

- Original Author:

- alfoa

- Creation date:

- 2015-01-26

- The classes tested in this test are:

- Sampler.MonteCarlo

- Since the creation of this test, the following main revisions have been performed:

1. revision info:

- author : mandd

- date : 2015-04-17

- description: conversion to Database and DataObjects

2. revision info:

- author : alfoa

- date : 2015-04-21

- description: Closes \#122

3. revision info:

- author : alfoa

- date : 2015-06-18

- description: Changed Name of all DataObjects

4. revision info:

- author : alfoa

- date : 2015-09-12

- description: removed syntax inconsistency with respect standards for Samplers (underscores instead of camelBack) + created conversion script 
5. revision info:

- author : maljdan

- date : 2016-04-06

- description: Updating test cases to reflect the changes to the user input.

6. revision info:

- author : talbpaul

- date : 2016-04-26

- description: added check for pre-existing backup files when validating

7. revision info:

- author : maljdan

- date : 2016-05-20

- description: Updating the test cases to remove references to Point or History.

8. revision info:

- author : alfoa

- date : 2017-01-21

- description: Adding this test description.

\subsubsection{FRAMEWORK.TEST CUSTOM MODE}

This test can be found at ". / raven/tests/framework/test_custom_mode.xml". This test can be called executing the following command:

./run_tests --re=framework.test_custom_mode

or

.Irun_framework_tests --re=framework.test_custom_mode

- Test Description:

- This test is aimed to check the capability of RAVEN to load external Custom dispatching recepies. RunInfo.CustomMode.

- Original Author:

$-\operatorname{cog} l \mathrm{jj}$

- Creation date: 
- 2014-04-02

- The classes tested in this test are:

- JobHandler.ExternalRunner

- Since the creation of this test, the following main revisions have been performed:

1. revision info:

- author : mandd

- date : 2015-04-17

- description: conversion to Database and DataObjects

2. revision info:

- author : alfoa

- date : 2015-05-18

- description: Closes \#167

3. revision info:

- author : alfoa

- date : 2015-06-18

- description: Changed Name of all DataObjects

4. revision info:

- author : alfoa

- date : 2016-04-27

- description: New subdirectories structure

5. revision info:

- author : alfoa

- date : 2017-01-21

- description: Adding this test description.

\subsubsection{FRAMEWORK.TEST XML DIFFER}

This test can be found at ". / raven/tests/framework/TestDiffer/TestXMLDiffer. py". This test can be called executing the following command:

./run_tests --re=framework.test_xml_differ

or

./run_framework_tests --re=framework.test_xml_differ 
- Test Description:

- This test is aimed to check the xml differ program

- Original Author:

- cogljj

- Creation date:

$-2016-10-21$

- The classes tested in this test are:

- None

- Since the creation of this test, the following main revisions have been performed:

1. revision info:

- author : alfoa

- date : 2017-01-21

- description: Adding this test description.

\subsubsection{FRAMEWORK STOCHPOLYINTERPTEST}

This test can be found at "./raven/tests/framework/test_stochpoly_interp. xml". This test can be called executing the following command:

. /run_tests --re=framework/stochPolyInterpTest

or

. Irun_framework_tests --re=framework/stochPolyInterpTest

- Test Description:

- This test checks the use of the "interpolation" blocks for the SCgPC methodology.

- Original Author:

- talbpaul

- Creation date:

- 2015-07-07

- The classes tested in this test are:

- Samplers.SparseGridCollocation,SupervisedLearning.GaussPolynomialROM 


\subsubsection{FRAMEWORK.ND EXTERNAL LHS}

This test can be found at ". / raven/tests/framework/test_simple_ND_external_ LHS . xml". This test can be called executing the following command:

. /run_tests --re=framework.ND_external_LHS

or

. /run_framework_tests --re=framework.ND_external_LHS

- Test Description:

- This test is aimed to check the capability of RAVEN to handle ND probability density functions (distributions), when a Stratified sampling strategy is performed. In this case, the capability to use a globalGrid specification, when the discretization is performed in CDF.

- Original Author:

- mandd

- Creation date:

- 2015-03-04

- The classes tested in this test are:

- Distributions.NDInverseWeight, Distrubtions.NDCartesianSpline, Samplers.Stratified

- Since the creation of this test, the following main revisions have been performed:

1. revision info:

- author : mandd

- date : 2015-04-17

- description: conversion to Database and DataObjects

2. revision info:

- author : alfoa

- date : 2015-04-24

- description: From LHS to Stratified. Closes \#130

3. revision info:

- author : alfoa 
- date : 2015-05-28

- description: fixed Stratified Sampler bug $(=i$ regolding $)+$

4. revision info:

- author: alfoa

- date : 2015-06-18

- description: Changed Name of all DataObjects

5. revision info:

- author : alfoa

- date : 2015-09-12

- description: removed syntax inconsistency with respect standards for Samplers (underscores instead of camelBack) + created conversion script

6. revision info:

- author : cogljj

- date : 2015-09-29

- description: Modifing external modules to use relative to working directory.

7. revision info:

- author: wangc

- date : 2015-10-22

- description: fix several bugs inside stratified sampler, a test is added to prevent the bugs, and xml output file is added for the testing to prevent the bugs

8. revision info:

- author : maljdan

- date : 2016-04-06

- description: Updating test cases to reflect the changes to the user input.

9. revision info:

- author : alfoa

- date : 2017-01-21

- description: Adding this test description.

\subsubsection{FRAMEWORK.ND EXTERNAL GRID VALUE}

This test can be found at “./raven/tests/framework/test_simple_ND_external_ grid_value.xml". This test can be called executing the following command:

./run_tests --re=framework.ND_external_grid_value 
or

./run_framework_tests --re=framework.ND_external_grid_value

- Test Description:

- This test is aimed to check the capability of RAVEN to handle ND probability density functions (distributions), when a Grid sampling strategy is performed. In this case, the capability to construct marginal distributions (for each uncertanties of the ND distribution domain), when the discretization is performed in VALUE.

- Original Author:

- mandd

- Creation date:

- 2015-03-04

- The classes tested in this test are:

- Distributions.NDInverseWeight, Distrubtions.NDCartesianSpline, Samplers.Grid

- Since the creation of this test, the following main revisions have been performed:

1. revision info:

- author : mandd

- date : 2015-04-17

- description: conversion to Database and DataObjects

2. revision info:

- author : alfoa

- date : 2015-06-18

- description: Changed Name of all DataObjects

3. revision info:

- author : cogljj

- date : 2015-09-29

- description: Modifing external modules to use relative to working directory.

4. revision info:

- author: wangc

- date : 2015-09-30

- description: improve efficiency when calculate the SampledVarsPb with N-Dimensional PDF, fix the issue \#298 
5. revision info:

- author : maljdan

- date : 2016-04-06

- description: Updating test cases to reflect the changes to the user input.

6. revision info:

- author : alfoa

- date : 2017-01-21

- description: Adding this test description.

\subsubsection{FRAMEWORK.ND EXTERNAL GRID CDF}

This test can be found at ". / raven/tests/framework/test_simple_ND_external_ grid_cdf.xml". This test can be called executing the following command:

./run_tests --re=framework.ND_external_grid_cdf

or

./run_framework_tests --re=framework.ND_external_grid_cdf

- Test Description:

- This test is aimed to check the capability of RAVEN to handle ND probability density functions (distributions), when a Grid sampling strategy is performed. In this case, the capability to construct marginal distributions (for each uncertanties of the ND distribution domain), when the discretization is performed in CDF.

- Original Author:

- mandd

- Creation date:

- 2015-03-04

- The classes tested in this test are:

- Distributions.NDInverseWeight, Distrubtions.NDCartesianSpline, Samplers.Grid

- Since the creation of this test, the following main revisions have been performed:

1. revision info: 
- author : mandd

- date : 2015-03-09

- description: test fixes

2. revision info:

- author : mandd

- date : 2015-03-09

- description: test fixes

3. revision info:

- author : mandd

- date : 2015-03-09

- description: fix tests

4. revision info:

- author : mandd

- date : 2015-04-17

- description: conversion to Database and DataObjects

5. revision info:

- author : senrs

- date : 2015-05-18

- description: update the test cases

6. revision info:

- author : alfoa

- date : 2015-06-18

- description: Changed Name of all DataObjects

7. revision info:

- author: talbpaul

- date : 2015-07-06

- description: standardization of tests

8. revision info:

- author : talbpaul

- date : 2015-07-06

- description: Revert"standardization of tests" This reverts commit 68099325e8daba0f756179a20b

9. revision info:

- author : talbpaul

- date : 2015-07-06 
- description: updated tests, added script

10. revision info:

- author : talbpaul

- date : 2015-07-07

- description: updated to restrict partial-node comments

11. revision info:

- author : talbpaul

- date : 2015-07-07

- description: standardized tests

12. revision info:

- author : talbpaul

- date : 2015-07-09

- description: tests pass, except LSIntegral needs regolding

13. revision info:

- author : cogljj

- date : 2015-09-29

- description: Modifing external modules to use relative to working directory.

14. revision info:

- author: wangc

- date : 2015-09-30

- description: improve efficiency when calculate the SampledVarsPb with N-Dimensional PDF, fix the issue \#298

15. revision info:

- author: wangc

- date : 2015-09-30

- description: add new tests for the issue \#298

16. revision info:

- author : alfoa

- date : 2015-10-06

- description: modified all the other files

17. revision info:

- author : alfoa

- date : 2015-10-06

- description: final modifications

18. revision info: 
- author : alfoa

- date : 2015-10-06

- description: Merge branch 'devel' of hpcgitlab.inl.gov:idaholab/raven into wangc/fix_bug

19. revision info:

- author : alfoa

- date : 2015-10-15

- description: merged devel and resolved conflicts

20. revision info:

- author : mandd

- date : 2015-11-04

- description: edits

21. revision info:

- author : mandd

- date : 2015-11-06

- description: edits

22. revision info:

- author : mandd

- date : 2015-11-17

- description: final merging with devel

23. revision info:

- author: talbpaul

- date : 2016-02-08

- description: first update, looking for more failing tests to add

24. revision info:

- author : mandd

- date : 2016-02-11

- description: merged with devel

25. revision info:

- author : maljdan

- date : 2016-04-06

- description: Updating test cases to reflect the changes to the user input.

26. revision info:

- author : alfoa

- date : 2017-01-21

- description: Adding this test description. 


\subsubsection{FRAMEWORK.ND TEST MC MVN}

This test can be found at “. / raven/tests/framework/test_simple_ND_external_ MC_MVN.xml". This test can be called executing the following command:

. /run_tests --re=framework.ND_test_MC_MVN

or

./run_framework_tests --re=framework.ND_test_MC_MVN

- Test Description:

- This test is aimed to check the capability of RAVEN to handle MultivariateNormal probability density functions (distributions), when a MonteCarlo sampling strategy is performed.

- Original Author:

- mandd

- Creation date:

$-2015-04-15$

- The classes tested in this test are:

- Distributions.MultivariateNormal, Samplers.MonteCarlo

- Since the creation of this test, the following main revisions have been performed:

1. revision info:

- author : alfoa

- date : 2015-06-18

- description: Changed Name of all DataObjects

2. revision info:

- author: wangc

- date : 2015-07-02

- description: nd multivariate distribution improvement

3. revision info:

- author : maljdan

- date : 2016-04-06 
- description: Updating test cases to reflect the changes to the user input.

4. revision info:

- author : alfoa

- date : 2017-01-21

- description: Adding this test description.

5. revision info:

- author: wangc

- date : 2017-12-20

- description: convert test to use the new DataObjects with the new structure of basic statistic

\subsubsection{FRAMEWORK.DISTS VARS}

This test can be found at ". / raven/tests/framework/test_distribution_combination. xml". This test can be called executing the following command:

. Irun_tests --re=framework.dists_vars

or

./run_framework_tests --re=framework.dists_vars

- Test Description:

- This test is aimed to check the capability in RAVEN to combine 1Dimensional and NDimensional probability density functions in the same sampling strategy. In this test a NDimensional distribution (Custom) is used in conjunction with 3 1Dimensional Exponential distributions, in a MonteCarlo sampling strategy.

- Original Author:

- mandd

- Creation date:

- 2015-05-13

- The classes tested in this test are:

- Distributions.Exponential, Distributions.NDCartesianSpline, Sampler.MonteCarlo

- Since the creation of this test, the following main revisions have been performed: 
1. revision info:

- author : alfoa

- date : 2015-06-18

- description: Changed Name of all DataObjects

2. revision info:

- author : maljdan

- date : 2015-06-24

- description: Adding DataObjects and fixing Distributions in the XSD.

3. revision info:

- author : alfoa

- date : 2015-09-12

- description: removed syntax inconsistency with respect standards for Samplers (underscores instead of camelBack) + created conversion script

4. revision info:

- author : cogljj

- date : 2015-09-29

- description: Modifing external modules to use relative to working directory.

5. revision info:

- author : maljdan

- date : 2016-04-06

- description: Updating test cases to reflect the changes to the user input.

6. revision info:

- author : maljdan

- date : 2016-05-20

- description: Updating the test cases to remove references to Point or History.

7. revision info:

- author: alfoa

- date : 2017-01-21

- description: Adding this test description.

8. revision info:

- author: alfoa

- date : 2017-12-20

- description: Added seed and added additional ND distribution. 


\subsubsection{FRAMEWORK.REDUNDANTINPUTS}

This test can be found at "./raven/tests/framework/test_redundant_inputs. xml". This test can be called executing the following command:

. Irun_tests --re=framework.redundant Inputs

or

./run_framework_tests --re=framework.redundant Inputs

- Test Description:

- This test is aimed to check the capability of RAVEN to treat redundant input variables. This test shows how some of the variables defined in the Samplers, can be associated to Functions (External) instead of Distributions. In this way, the user can "sample" the variable as combination of the other variables.

- Original Author:

- talbpaul

- Creation date:

- 2015-06-25

- The classes tested in this test are:

- Samplers.Sampler, Functions.External

- Since the creation of this test, the following main revisions have been performed:

1. revision info:

- author: wangc

- date : 2015-09-22

- description: fix the bug \#296

2. revision info:

- author : maljdan

- date : 2016-04-06

- description: Updating test cases to reflect the changes to the user input.

3. revision info:

- author : alfoa 
- date : 2017-01-21

- description: Adding this test description.

4. revision info:

- author : alfoa

- date : 2019-03-07

- description: Modified syntax in Functions as for issue \#934

\subsubsection{FRAMEWORK.REDUNDANTINPUTSSCGPC}

This test can be found at ". / raven/tests / framework/test_redundant_scgpc.xml". This test can be called executing the following command:

$$
\text { . /run_tests --re=framework. redundant InputsSCgPC }
$$

or

./run_framework_tests --re=framework.redundant InputsSCgPC

- Test Description:

- This test is aimed to check the capability of RAVEN to treat redundant input variables, when the Sampler of type "SparseGridCollocation" is used. This test shows how some of the variables defined in the Sampler, can be associated to Functions (External) instead of Distributions. In this way, the user can "sample" the variable as combination of the other variables.

- Original Author:

- talbpaul

- Creation date:

- 2016-01-07

- The classes tested in this test are:

- Samplers.SparseGridCollocation, Functions.External

- Since the creation of this test, the following main revisions have been performed:

1. revision info:

- author : talbpaul 
- date : 2016-01-07

- description: Tests updated

2. revision info:

- author : maljdan

- date : 2016-04-06

- description: Updating test cases to reflect the changes to the user input.

3. revision info:

- author: alfoa

- date : 2017-01-21

- description: Adding this test description.

4. revision info:

- author : alfoa

- date : 2019-03-07

- description: Modified syntax in Functions as for issue \#934

\subsubsection{FRAMEWORK.REDUNDANTINPUTSSOBOL}

This test can be found at ". / raven/tests/framework/test_redundant_sobol.xml”. This test can be called executing the following command:

./run_tests --re=framework. redundant InputsSobol

or

./run_framework_tests --re=framework.redundantInputsSobol

- Test Description:

- This test is aimed to check the capability of RAVEN to treat redundant input variables, when the Sampler of type "Sobol" is used. This test shows how some of the variables defined in the Sampler, can be associated to Functions (External) instead of Distributions. In this way, the user can "sample" the variable as combination of the other variables.

- Original Author:

- talbpaul

- Creation date: 
- 2016-01-07

- The classes tested in this test are:

- Samplers.Sobol, Functions.External

- Since the creation of this test, the following main revisions have been performed:

1. revision info:

- author : cogljj

- date : 2016-01-15

- description: Rename step in sobol test to avoid directry name collision. Closes \#400. Both test_redundant_scgpc and test_redundant_sobol were using the directory RedundantInputs/sim_py_SC which could cause test failures. This renames the one that redundant_sobol uses.

2. revision info:

- author : maljdan

- date : 2016-04-06

- description: Updating test cases to reflect the changes to the user input.

3. revision info:

- author : alfoa

- date : 2017-01-21

- description: Adding this test description.

4. revision info:

- author : alfoa

- date : 2019-03-07

- description: Modified syntax in Functions as for issue \#934

\subsubsection{FRAMEWORK.FAILRUNSMC}

This test can be found at “. / raven/tests/framework/test_failruns_MC.xml”. This test can be called executing the following command:

. Irun_tests --re=framework. failrunsMC

or

./run_framework_tests --re=framework.failrunsMC 


\section{- Test Description:}

- This test is aimed to check the capability of RAVEN to identify failures (Model crashes in some realizations) when a sampling strategy is employed.

- Original Author:

- talbpaul

- Creation date:

$-2015-08-25$

- The classes tested in this test are:

- Steps.MultiRun

- Since the creation of this test, the following main revisions have been performed:

1. revision info:

- author : alfoa

- date : 2015-09-12

- description: removed syntax inconsistency with respect standards for Samplers (underscores instead of camelBack) + created conversion script

2. revision info:

- author : cogljj

- date : 2015-09-29

- description: Modifing external modules to use relative to working directory.

3. revision info:

- author : maljdan

- date : 2016-04-06

- description: Updating test cases to reflect the changes to the user input.

4. revision info:

- author : alfoa

- date : 2017-01-21

- description: Adding this test description. 


\subsubsection{FRAMEWORK.NDGRIDPROBABILITYWEIGHTCDF}

This test can be found at"./raven/tests/framework/test_simple_ND_grid_probabilityWei cdf. xml". This test can be called executing the following command:

. Irun_tests --re=framework.NDGridProbabilityWeightCDF

or

. /run_framework_tests --re=framework.NDGridProbabilityWeightCDF

- Test Description:

- This test is aimed to check the capability of RAVEN to handle ND probability density functions (distributions), when a Grid sampling strategy is performed. In this case, the capability to construct marginal distributions (for each uncertanties of the ND distribution domain), when the discretization is performed in CDF.

- Original Author:

- wangc

- Creation date:

- 2015-09-30

- The classes tested in this test are:

- Distributions.NDInverseWeight, Distrubtions.NDCartesianSpline, Samplers.Grid

- Since the creation of this test, the following main revisions have been performed:

1. revision info:

- author : alfoa

- date : 2015-10-15

- description: merged devel and resolved conflicts

2. revision info:

- author : mandd

- date : 2015-11-06

- description: edits

3. revision info:

- author : mandd 
- date : 2015-11-17

- description: final merging with devel

4. revision info:

- author : talbpaul

- date : 2016-02-08

- description: first update, looking for more failing tests to add

5. revision info:

- author : mandd

- date : 2016-02-11

- description: merged with devel

6. revision info:

- author : maljdan

- date : 2016-04-06

- description: Updating test cases to reflect the changes to the user input.

7. revision info:

- author : alfoa

- date : 2017-01-21

- description: Adding this test description.

\subsubsection{FRAMEWORK.NDGRIDPROBABILITYWEIGHTVALUE}

This test can be found at “. / raven/tests/framework/test_simple_ND_grid_probabilityWeic value.xml". This test can be called executing the following command:

. /run_tests --re=framework. NDGridProbabilityWeightValue

Or

./run_framework_tests --re=framework.NDGridProbabilityWeightValue

- Test Description:

- This test is aimed to check the capability of RAVEN to handle ND probability density functions (distributions), when a Grid sampling strategy is performed. In this case, the capability to construct marginal distributions (for each uncertanties of the ND distribution domain), when the discretization is performed in VALUE.

- Original Author: 
- wangc

- Creation date:

- 2015-09-30

- The classes tested in this test are:

- Distributions.NDInverseWeight, Distrubtions.NDCartesianSpline, Samplers.Grid

- Since the creation of this test, the following main revisions have been performed:

1. revision info:

- author : talbpaul

- date : 2016-02-08

- description: first update, looking for more failing tests to add

2. revision info:

- author : maljdan

- date : 2016-04-06

- description: Updating test cases to reflect the changes to the user input.

3. revision info:

- author : alfoa

- date : 2017-01-21

- description: Adding this test description.

\subsubsection{FRAMEWORK.LHSVARIABLES}

This test can be found at ". / raven/tests/framework/test_LHS_variables.xml". This test can be called executing the following command:

. Irun_tests --re=framework. LHSVariables

or

. Irun_framework_tests --re=framework. LHSVariables

- Test Description:

- This test is aimed to check the capability in RAVEN to employ a Stratified sampling strategy (LHS- equally spaced in CDF). Since the goal of the test is to testify that the Stratified sampling strategy is functional, a Dummy model is used. 
- Original Author:

- wangc

- Creation date:

- 2015-10-22

- The classes tested in this test are:

- Sampler.Stratified

- Since the creation of this test, the following main revisions have been performed:

1. revision info:

- author : cogljj

- date : 2015-10-29

- description: Moving comment to third line and removing \#

2. revision info:

- author : talbpaul

- date : 2016-02-08

- description: first update, looking for more failing tests to add

3. revision info:

- author : maljdan

- date : 2016-04-06

- description: Updating test cases to reflect the changes to the user input.

4. revision info:

- author : alfoa

- date : 2017-01-21

- description: Adding this test description.

\subsubsection{FRAMEWORK FAIL CSV}

This test can be found at ". / raven/tests/framework/test_simple_fail_csv.xml”. This test can be called executing the following command:

. /run_tests --re=framework/fail_csv

or

./run_framework_tests --re=framework/fail_csv 
- Test Description:

- Tests the test harness by checking that differences in the csv file cause the test to fail (but it passes because expected_fail is used in the tests file). If the CSV check passes, then this will fail the test.

- Original Author:

$-\operatorname{cog} \mathrm{ljj}$

- Creation date:

- 2016-04-14

- The classes tested in this test are:

- TestHarness.testers.RavenFramework

\subsubsection{FRAMEWORK FAIL XML}

This test can be found at ". / raven/tests/framework/test_simple_fail_xml.xml”. This test can be called executing the following command:

./run_tests --re=framework/fail_xml

or

. /run_framework_tests --re=framework/fail_xml

- Test Description:

- Tests the test harness by checking that differences in the xml file cause the test to fail (but it passes because expected_fail is used in the tests file). If the XML check passes, then this will fail the test.

- Original Author:

$-\operatorname{cog} \mathrm{ljj}$

- Creation date:

- 2016-04-14

- The classes tested in this test are:

- TestHarness.testers.XMLDiff,TestHarness.testers.RavenFramework 


\subsubsection{FRAMEWORK.SIMULATIONTAGS}

This test can be found at "./raven/tests/framework/test_sim_tags.xml". This test can be called executing the following command:

. /run_tests --re=framework. SimulationTags

or

./run_framework_tests --re=framework.SimulationTags

- Test Description:

- This test is aimed to check the functionality of the options that can be defined in the Simulation block (attributes), such as "color", "printTimeStamps" and "verbosity".

- Original Author:

- talbpaul

- Creation date:

- 2016-02-18

- The classes tested in this test are:

- Simulation

- Since the creation of this test, the following main revisions have been performed:

1. revision info:

- author : maljdan

- date : 2016-04-06

- description: Updating test cases to reflect the changes to the user input.

2. revision info:

- author : alfoa

- date : 2017-01-21

- description: Adding this test description. 


\subsubsection{FRAMEWORK.TESTSTRATIFIEDLARGESIZE}

This test can be found at "./raven/tests/framework/test_stratified_dummy_ model_large_grid_size.xml". This test can be called executing the following command:

./run_tests --re=framework.testStratifiedLargesize

or

./run_framework_tests --re=framework.testStratifiedLargeSize

- Test Description:

- This test is aimed to check the capability of RAVEN to perform a Stratified Sampling strategy of large size [(16 uncertanties and 2 nodes each) and (16 uncertanties and 161 nodes each)]. An external model is used, the results are stored the results into a Database of type HDF5 and Dataobjects of type PointSet. In addition, it prints the results (contained in the DataObjects) in a CSV file (OutStreams of type Print)

- Original Author:

- alfoa

- Creation date:

- 2016-04-11

- The classes tested in this test are:

- OutStreams.Print, DataObjects.PointSet, Databases.HDF5, Steps.MultiRun, Samplers.Stratified

- Since the creation of this test, the following main revisions have been performed:

1. revision info:

- author : alfoa

- date : 2016-04-13

- description: new syntax for OutStreamManger - $i$ OutStreams

2. revision info:

- author : talbpaul

- date : 2016-04-26

- description: added check for pre-existing backup files when validating

3. revision info:

- author : alfoa

- date : 2017-01-21

- description: Adding this test description. 


\subsubsection{FRAMEWORK.CUSTOM1D}

This test can be found at “./raven/tests/framework/test_distributionCustom1D . xml". This test can be called executing the following command:

. /run_tests --re=framework. custom1D

or

. Irun_framework_tests --re=framework.custom1D

- Test Description:

- This test is aimed to check the capability of RAVEN to handle custom probability density functions (distributions).

- Original Author:

- mandd

- Creation date:

- 2016-08-08

- The classes tested in this test are:

- Distributions.Custom1D

- Since the creation of this test, the following main revisions have been performed:

1. revision info:

- author : alfoa

- date : 2017-01-21

- description: Adding this test description.

\subsubsection{FRAMEWORK ERRORCHECKS.MISSINGSTEPS}

This test can be found at ". /raven/tests/framework/ErrorChecks/test_noSteps . xml". This test can be called executing the following command:

./run_tests --re=framework/ErrorChecks.missingsteps

or 
./run_framework_tests --re=framework/ErrorChecks.missingsteps

- Test Description:

- This test is aimed to check the functionality of RAVEN to error out in case some input inconsistencies are found. In this case, the error in case of no Step is tested.

- Original Author:

- talbpaul

- Creation date:

- 2016-02-09

- The classes tested in this test are:

- Steps.MultiRun

- Since the creation of this test, the following main revisions have been performed:

1. revision info:

- author : talbpaul

- date : 2016-04-26

- description: Updated test to accept new "overwrite" attribute in HDF5

2. revision info:

- author : alfoa

- date : 2016-04-27

- description: New subdirectories structure

3. revision info:

- author : alfoa

- date : 2017-01-21

- description: Adding this test description.

\subsubsection{FRAMEWORK ERRORCHECKS.MVNNOTSQUARE}

This test can be found at “. / raven/tests / framework/ErrorChecks/test_MVN_not_ square.xml". This test can be called executing the following command:

./run_tests --re=framework/ErrorChecks.MVNnotsquare 
or

./run_framework_tests --re=framework/ErrorChecks.MVNnot Square

- Test Description:

- This test is aimed to check the functionality of RAVEN to error out in case some input inconsistencies are found. In this case, the fact that the MultiVariate distribution errors out when it is not square

- Original Author:

- talbpaul

- Creation date:

- 2016-07-14

- The classes tested in this test are:

- Distributions.MultivariateNormal

- Since the creation of this test, the following main revisions have been performed:

1. revision info:

- author : alfoa

- date : 2017-01-21

- description: Adding this test description.

\subsubsection{FRAMEWORK ERRORCHECKS.MVNBADDIMS}

This test can be found at “. / raven/tests / framework/ErrorChecks/test_MVN_bad_ dims.xml". This test can be called executing the following command:

. /run_tests --re=framework/ErrorChecks.MVNbadDims

or

. Irun_framework_tests --re=framework/ErrorChecks.MVNbadDims

- Test Description: 
- This test is aimed to check the functionality of RAVEN to error out in case some input inconsistencies are found. In this case, the fact that the MultiVariate distribution errors out when not all the dimensions of the MultiVariate are used in the sampling strategy.

- Original Author:

- talbpaul

- Creation date:

- 2016-07-14

- The classes tested in this test are:

- Distributions.MultivariateNormal, Samplers.MonteCarlo

- Since the creation of this test, the following main revisions have been performed:

1. revision info:

- author : alfoa

- date : 2017-01-21

- description: Adding this test description.

\subsubsection{FRAMEWORK ERRORCHECKS.BADHISTORY}

This test can be found at ". / raven/tests / framework/ErrorChecks / loadBadHistory . xml". This test can be called executing the following command:

. /run_tests --re=framework/ErrorChecks.badHistory

or

./run_framework_tests --re=framework/ErrorChecks.badHistory

- Test Description:

- This test is aimed to check the functionality of RAVEN to error out in case some input inconsistencies are found. In this case, the data contained in "badHistorySet.csv" is foulty and the code (HistorySet) needs to error out.

- Original Author:

- maljovec 
- Creation date:

- 2017-07-21

- The classes tested in this test are:

- Steps.IOStep, DataObjects.HistorySet

- Since the creation of this test, the following main revisions have been performed:

1. revision info:

- author : alfoa

- date : 2018-05-15

- description: Adding this test description.

\subsubsection{FRAMEWORK ERRORCHECKS.BADHISTORY}

This test can be found at ". / raven/tests / framework/ErrorChecks / loadBadPoint Set. xml". This test can be called executing the following command:

./run_tests --re=framework/ErrorChecks.badHistory

or

./run_framework_tests --re=framework/ErrorChecks.badHistory

- Test Description:

- This test is aimed to check the functionality of RAVEN to error out in case some input inconsistencies are found. In this case, the data contained in "badHistory.csv" is foulty and the code (PointSet) needs to error out.

- Original Author:

- maljovec

- Creation date:

- 2017-07-21

- The classes tested in this test are:

- Steps.IOStep, DataObjects.PointSet

- Since the creation of this test, the following main revisions have been performed: 
1. revision info:

- author : alfoa

- date : 2018-05-15

- description: Adding this test description.

\subsubsection{FRAMEWORK ERRORCHECKS.BADINSIMULATION}

This test can be found at ". / raven/tests/framework/ErrorChecks/badInSimulation. xml". This test can be called executing the following command:

./run_tests --re=framework/ErrorChecks.badInSimulation

or

./run_framework_tests --re=framework/ErrorChecks.badInSimulation

- Test Description:

- This test is aimed to check the functionality of RAVEN to error out in case some input inconsistencies are found. In this case, the input is malformed.

- Original Author:

- maljovec

- Creation date:

- 2017-07-21

- The classes tested in this test are:

- Simulation

- Since the creation of this test, the following main revisions have been performed:

1. revision info:

- author: alfoa

- date : 2018-05-15

- description: Adding this test description. 


\subsubsection{FRAMEWORK ERRORCHECKS.UNSYNCEDTYPICALHISTORY}

This test can be found at ". /raven/tests/framework/ErrorChecks/unsyncedTypicalHistory xml". This test can be called executing the following command:

./run_tests --re=framework/ErrorChecks.unsyncedTypicalHistory

or

. Irun_framework_tests --re=framework/ErrorChecks.unsyncedTypicalyistory

- Test Description:

- This test is aimed to check the functionality of RAVEN to error out in case some input inconsistencies are found. In this case, the HistorySet passed into the PostProcessor TypicalHistoryFromHistorySet is not syncronized and the code must error out.

- Original Author:

- maljovec

- Creation date:

- 2017-07-21

- The classes tested in this test are:

- Models.PostProcessors.InterfacePostProcessor.TypicalHistoryFromHistorySet

- Since the creation of this test, the following main revisions have been performed:

1. revision info:

- author : alfoa

- date : 2018-05-15

- description: Adding this test description.

\subsubsection{FRAMEWORK DATABASES HDF5.TEST PUSH INTO HDF5}

This test can be found at ". / raven/tests / framework / Databases / HDF 5/test_push_

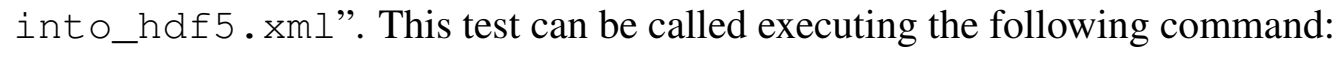

./run_tests --re=framework/Databases/HDF5.test_push_into_hdf5 
or

./run_framework_tests --re=framework/Databases/HDF5.test_push_into_hdf5

- Test Description:

- The idea of this input case is to test che capability of RAVEN create a Database HDF5 from DataObjects.PointSet and DataObjects.HistorySet and to construct from a Database the DataObjects.

- Original Author:

- alfoa

- Creation date:

- 2013-11-11

- The classes tested in this test are:

- DataObjects.PointSet, DataObjects.HistorySet, Databases.HDF5

- Since the creation of this test, the following main revisions have been performed:

1. revision info:

- author : alfoa

- date : 2015-05-19

- description: Closes \#171

2. revision info:

- author : alfoa

- date : 2015-06-18

- description: Changed Name of all DataObjects

3. revision info:

- author : alfoa

- date : 2015-06-21

- description: modified Names + added new capabilities and flexibility in DataObjects + added options block. Modified XSD schema and manual to reflect the modifications

4. revision info:

- author : maljdan

- date : 2016-04-06 
- description: Updating test cases to reflect the changes to the user input.

5. revision info:

- author : talbpaul

- date : 2016-04-26

- description: added check for pre-existing backup files when validating

6. revision info:

- author : maljdan

- date : 2016-05-20

- description: Updating the test cases to remove references to Point or History.

7. revision info:

- author : alfoa

- date : 2017-01-21

- description: Adding this test description.

8. revision info:

- author : alfoa

- date : 2019-03-01

- description: Modified test location

\subsubsection{FRAMEWORK DATABASES HDF5.TEST MERGE 2 DATABASES}

This test can be found at ". / raven/tests / framework/Databases / HDF 5/test_merge_ 2_databases.xml". This test can be called executing the following command:

./run_tests --re=framework/Databases/HDF5.test_merge_2_databases

or

. Irun_framework_tests --re=framework/Databases/HDF5.test_merge_2_databases

- Test Description:

- This test is aimed to check the possiblity in RAVEN to merge 2 different Databases of type HDF5.

- Original Author:

- alfoa

- Creation date: 
- 2015-03-03

- The classes tested in this test are:

- Databases.HDF5

- Since the creation of this test, the following main revisions have been performed:

1. revision info:

- author : maljdan

- date : 2016-04-06

- description: Updating test cases to reflect the changes to the user input.

2. revision info:

- author : talbpaul

- date : 2016-04-26

- description: some tests slipped through

3. revision info:

- author : alfoa

- date : 2016-04-27

- description: New subdirectories structure

4. revision info:

- author : maljdan

- date : 2016-05-20

- description: Updating the test cases to remove references to Point or History.

5. revision info:

- author : maljdan

- date : 2016-05-23

- description: Changing and regolding a test case that uses an external model when unnecessary.

6. revision info:

- author : alfoa

- date : 2017-01-21

- description: Adding this test description.

7. revision info:

- author : alfoa

- date : 2019-03-01

- description: Modified test location 


\subsubsection{FRAMEWORK DATABASES HDF5.NEWERDATABASEWITHFILENAME}

This test can be found at "./raven/tests/framework/Databases/HDF5/test_new_ database_with_filename.xml". This test can be called executing the following command:

./run_tests --re=framework/Databases/HDF5.newerDatabaseWithFilename

or

./run_framework_tests --re=framework/Databases/HDF 5. newerDatabas \&WithFilen

- Test Description:

- This test is aimed to check the possiblity in RAVEN to create a newer Database (HDF5) with a specific name

- Original Author:

- alfoa

- Creation date:

- 2016-12-12

- The classes tested in this test are:

- Databases.HDF5

- Since the creation of this test, the following main revisions have been performed:

1. revision info:

- author : alfoa

- date : 2017-05-19

- description: Added test

2. revision info:

- author: alfoa

- date : 2019-03-01

- description: Modified test location 


\subsubsection{FRAMEWORK DATABASES HDF5.HDF5SELECTIVE}

This test can be found at ". /raven/tests/framework/Databases/HDF5/hdf5_selective. xml". This test can be called executing the following command:

. /run_tests --re=framework/Databases/HDF5.HDF5selective

or

./run_framework_tests --re=framework/Databases/HDF5.HDF5selective

- Test Description:

- Tests the ability for HDF5 databases to selectively store data by defining variable blocks. Without the selectivity, the resulting HDF5 is $220 \mathrm{~KB}$ (all.hdf5). With selectivity, the resulting HDF5 is $187 \mathrm{~KB}$ (sine.hdf5).

- Original Author:

- talbpaul

- Creation date:

- 2017-07-06

- The classes tested in this test are:

- Databases.HDF5

- Since the creation of this test, the following main revisions have been performed:

1. revision info:

- author : alfoa

- date : 2019-03-01

- description: Modified test location

\subsubsection{FRAMEWORK DATABASES HDF5.HDF5LOCATION}

This test can be found at ". / raven/tests/framework/Databases/HDF5/hdf5_location. xml". This test can be called executing the following command:

./run_tests --re=framework/Databases/HDF5.HDF5location 
or

./run_framework_tests --re=framework/Databases/HDF5.HDF5location

- Test Description:

- Tests that the location specified for the HDF5 is relative to the working dir, not to the run dir.

- Original Author:

- talbpaul

- Creation date:

- 2017-07-10

- The classes tested in this test are:

- Databases.HDF5

- Since the creation of this test, the following main revisions have been performed:

1. revision info:

- author : alfoa

- date : 2019-03-01

- description: Modified test location

\subsubsection{FRAMEWORK DATABASES HDF5.LARGE HDF5}

This test can be found at ". / raven/tests/framework/Databases/HDF 5/test_large_ database.xml". This test can be called executing the following command:

./run_tests --re=framework/Databases/HDF 5. large_HDF5

or

./run_framework_tests --re=framework/Databases/HDF5.large_HDF5

- Test Description:

- This test is aimed to test the capability of the RAVEN database strucuture to handle large databases (\#926) in terms of group names. 
- Original Author:

- @alfoa

- Creation date:

- 2019-03-01

- The classes tested in this test are:

- Databases.HDF5

- Since the creation of this test, the following main revisions have been performed:

1. revision info:

- author : alfoa

- date : 2019-03-01

- description: Added test for testing new way to handle group names

\subsubsection{FRAMEWORK DATABASES NETCDF.WRITE}

This test can be found at ". / raven/tests/framework/Databases/NetCDF/write. xml". This test can be called executing the following command:

. /run_tests --re=framework/Databases/NetCDF.Write

or

./run_framework_tests --re=framework/Databases/NetCDF.Write

- Test Description:

- Tests using NetCDF as a writable database format.

- Original Author:

- talbpaul

- Creation date:

$-2021-03-02$

- The classes tested in this test are:

- Databases.NetCDF 


\subsubsection{FRAMEWORK DATABASES NETCDF.READ}

This test can be found at "./raven/tests/framework/Databases/NetCDF/read. xml". This test can be called executing the following command:

. Irun_tests --re=framework/Databases/NetCDF.Read

or

./run_framework_tests --re=framework/Databases/NetCDF.Read

- Test Description:

- Tests using NetCDF as a readable database format.

- Original Author:

- talbpaul

- Creation date:

- 2021-03-03

- The classes tested in this test are:

- Databases.NetCDF

\subsubsection{FRAMEWORK DATABASES NETCDF.SAMPLE}

This test can be found at". / raven/tests/framework/Databases/NetCDF/sample. xml". This test can be called executing the following command:

. /run_tests --re=framework/Databases/NetCDF. Sample

or

./run_framework_tests --re=framework/Databases/NetCDF.Sample

- Test Description:

- Tests using NetCDF as a writable database format while sampling.

- Original Author: 
- talbpaul

- Creation date:

- 2021-03-03

- The classes tested in this test are:

- Databases.NetCDF

\subsubsection{FRAMEWORK DATABASES NETCDF.TWICEWRITE}

This test can be found at ". / raven/tests/framework/Databases/NetCDF/twice_ write.xml". This test can be called executing the following command:

./run_tests --re=framework/Databases/NetCDF. TwiceWrite

Or

./run_framework_tests --re=framework/Databases/NetCDF. TwiceWrite

- Test Description:

- Test using NetCDF as a writable database format, and then adding to it. Given two methods of writing to the database, "sampling" (one realization at a time) and "io" (via IOStep), check that all possible combinations come out the same: sample-sample, sample-io, io-sample, and io-io.

- Original Author:

- talbpaul

- Creation date:

- 2021-03-09

- The classes tested in this test are:

- Databases.NetCDF 


\subsubsection{FRAMEWORK.UTILS}

This test can be found at ". /raven/tests/framework/unit_tests/utils/testutils. py". This test can be called executing the following command:

. /run_tests --re=framework.utils

or

./run_framework_tests --re=framework.utils

- Test Description:

- This test performs Unit Tests for the utils class. It cannot be considered part of the active code but of the regression test system

- Original Author:

- talbpaul

- Creation date:

- 2017-11-01

- The classes tested in this test are:

- utils.utils

- Since the creation of this test, the following main revisions have been performed:

1. revision info:

- author : alfoa

- date : 2018-05-15

- description: Adding this test description.

2. revision info:

- author : alfoa

- date : 2019-03-04

- description: Moved methods isAString, isAFloat, isAInteger, isABoolean from mathUtils to utils 


\subsubsection{FRAMEWORK.MATHUTILS}

This test can be found at ". /raven/tests/framework/unit_tests/utils/testMathutils . py". This test can be called executing the following command:

. Irun_tests --re=framework.mathutils

or

./run_framework_tests --re=framework.mathutils

- Test Description:

- This test performs Unit Tests for the mathUtils methods It cannot be considered part of the active code but of the regression test system

- Original Author:

- talbpaul

- Creation date:

- 2016-11-01

- The classes tested in this test are:

- utils.mathUtils

- Since the creation of this test, the following main revisions have been performed:

1. revision info:

- author : talbpaul

- date : 2016-11-08

- description: Relocated utils tests

2. revision info:

- author : alfoa

- date : 2017-01-21

- description: Adding this test description.

3. revision info:

- author: alfoa

- date : 2019-03-04

- description: Moved methods isAString, isAFloat, isAInteger, isABoolean from mathUtils to utils 


\subsubsection{FRAMEWORK.RANDOMUTILS}

This test can be found at "./raven/tests/framework/unit_tests/utils/testRandomutils. py". This test can be called executing the following command:

. /run_tests --re=framework. randomUtils

or

./run_framework_tests --re=framework.randomUtils

- Test Description:

- This test performs Unit Tests for the randomUtils methods

- Original Author:

- talbpaul

- Creation date:

- 2017-06-16

- The classes tested in this test are:

- utils.randomUtils

\subsubsection{FRAMEWORK.XMLUTILS}

This test can be found at "./raven/tests/framework/unit_tests/utils/testXmlUtils . py". This test can be called executing the following command:

./run_tests --re=framework.xmlutils

or

./run_framework_tests --re=framework.xmlutils

- Test Description:

- This test performs Unit Tests for the xmlUtils class It cannot be considered part of the active code but of the regression test system 
- Original Author:

- talbpaul

- Creation date:

- 2016-11-01

- The classes tested in this test are:

- utils.xmlUtils

- Since the creation of this test, the following main revisions have been performed:

1. revision info:

- author : talbpaul

- date : 2016-11-08

- description: Relocated utils tests

2. revision info:

- author : alfoa

- date : 2017-01-21

- description: Adding this test description.

\subsubsection{FRAMEWORK.CACHEDNDARRAY}

This test can be found at ". /raven/tests/framework/unit_tests/utils/testCachedNDArray py". This test can be called executing the following command:

./run_tests --re=framework. cachedNDArray

or

./run_framework_tests --re=framework.cachedNDArray

- Test Description:

- This test performs Unit Tests for the cached_ndarray module It cannot be considered part of the active code but of the regression test system

- Original Author:

- talbpaul 
- Creation date:

- 2016-11-01

- The classes tested in this test are:

- utils.cachedNDArray

- Since the creation of this test, the following main revisions have been performed:

1. revision info:

- author : talbpaul

- date : 2016-11-08

- description: Relocated utils tests

2. revision info:

- author: alfoa

- date : 2017-01-21

- description: Adding this test description.

\subsubsection{FRAMEWORK.TREESTRUCTURE}

This test can be found at "./raven/tests/framework/unit_tests/utils/testTreeStructure py". This test can be called executing the following command:

. Irun_tests $--r e=f r a m e w o r k . t r e e S t r u c t u r e$

or

./run_framework_tests --re=framework.treeStructure

- Test Description:

- This test performs Unit Tests for the TreeStructure classes It cannot be considered part of the active code but of the regression test system

- Original Author:

- talbpaul

- Creation date:

- 2016-11-01 
- The classes tested in this test are:

- utils.TreeStructure

- Since the creation of this test, the following main revisions have been performed:

1. revision info:

- author : talbpaul

- date : 2016-11-08

- description: Relocated utils tests

2. revision info:

- author : alfoa

- date : 2017-01-21

- description: Adding this test description.

\subsubsection{FRAMEWORK.INPUTPARSING}

This test can be found at ". / raven/tests/framework/unit_tests/utils/testParse. py". This test can be called executing the following command:

. Irun_tests --re=framework. inputParsing

or

./run_framework_tests --re=framework.inputParsing

- Test Description:

- This test performs Unit Tests for the utils class (TreeStructure) It cannot be considered part of the active code but of the regression test system

- Original Author:

- talbpaul

- Creation date:

- 2017-11-01

- The classes tested in this test are:

- utils.utils.TreeStructure 
- Since the creation of this test, the following main revisions have been performed:

1. revision info:

- author : alfoa

- date : 2018-05-15

- description: Adding this test description.

\subsubsection{FRAMEWORK.INPUTDATA}

This test can be found at ". / raven/tests/framework/unit_tests/utils/test InputData. py". This test can be called executing the following command:

. /run_tests --re=framework.inputData

or

./run_framework_tests --re=framework.inputData

- Test Description:

- This test performs Unit Tests for the InputData methods

- Original Author:

- talbpaul

- Creation date:

- 2020-01-08

- The classes tested in this test are:

- utils.InputData

\subsubsection{FRAMEWORK.DEBUGGING}

This test can be found at ". / raven/tests/framework/unit_tests/utils/testDebugging . py". This test can be called executing the following command:

. Irun_tests --re=framework.Debugging

or 
./run_framework_tests --re=framework. Debugging

- Test Description:

- This test performs Unit Tests for the Debugging methods It cannot be considered part of the active code but of the regression test system

- Original Author:

- talbpaul

- Creation date:

$-2020-01-18$

- The classes tested in this test are:

- utils.Debugging

\subsubsection{FRAMEWORK.TEST DATASETS}

This test can be found at “. / raven/tests / framework/unit_tests/SupervisedLearning/ test ARMA. py". This test can be called executing the following command:

.Irun_tests --re=framework.test_datasets

or

./run_framework_tests --re=framework.test_datasets

- Test Description:

- This test is a Unit Test for the DataSet classes.

- Original Author:

- talbpaul

- Creation date:

- 2017-10-20

- The classes tested in this test are:

- DataSet 


\subsubsection{FRAMEWORK.UNIT TESTS.TSA.FOURIER}

This test can be found at "./raven/tests/framework/unit_tests/TSA/testFourier. py". This test can be called executing the following command:

. /run_tests --re=framework.unit_tests.TSA.Fourier

or

./run_framework_tests --re=framework.unit_tests.TSA.Fourier

- Test Description:

- This test is a Unit Test for the Fourier TimeSeriesAnalyzer classes.

- Original Author:

- talbpaul

- Creation date:

$-2021-01-05$

- The classes tested in this test are:

- TSA.Fourier

\subsubsection{FRAMEWORK.UNIT TESTS.TSA.POLYNOMIALREGRESSION}

This test can be found at ". / raven/tests/framework/unit_tests/TSA/testPolynomialRegre. py". This test can be called executing the following command:

./run_tests --re=framework.unit_tests.TSA.PolynomialRegression

or

./run_framework_tests --re=framework.unit_tests.TSA.PolynomialReqression

- Test Description:

- This test is a Unit Test for the PolynomialRegression TimeSeriesAnalyzer classes.

- Original Author: 
- dylanjm

- Creation date:

- 2021-02-16

- The classes tested in this test are:

- TSA.PolynomialRegression

\subsubsection{FRAMEWORK.UNIT TESTS.TSA.WAVELET}

This test can be found at ". / raven/tests/framework/unit_tests/TSA/testWavelet. py". This test can be called executing the following command:

. Irun_tests --re=framework.unit_tests.TSA.Wavelet

or

./run_framework_tests --re=framework.unit_tests.TSA.Wavelet

- Test Description:

- This test is a Unit Test for the Wavelet TimeSeriesAnalyzer classes.

- Original Author:

- dylanjm

- Creation date:

$-2021-02-24$

- The classes tested in this test are:

- TSA.Wavelet

\subsubsection{FRAMEWORK.TEST DATASETS}

This test can be found at "./raven/tests/framework/unit_tests/Dataobjects/ TestDataSet.py". This test can be called executing the following command:

./run_tests --re=framework.test_datasets 
or

./run_framework_tests --re=framework.test_datasets

- Test Description:

- This test is a Unit Test for the DataSet classes.

- Original Author:

- talbpaul

- Creation date:

- 2017-10-20

- The classes tested in this test are:

- DataSet

\subsubsection{FRAMEWORK.TEST DATASETS}

This test can be found at "./raven/tests/framework/unit_tests/Dataobjects/ TestHistoryset.py". This test can be called executing the following command:

.Irun_tests --re=framework.test_datasets

or

./run_framework_tests --re=framework.test_datasets

- Test Description:

- This test is a Unit Test for the HistorySet classes.

- Original Author:

- talbpaul

- Creation date:

- 2017-10-20

- The classes tested in this test are:

- HistorySet 


\subsubsection{FRAMEWORK.TEST DATASETS}

This test can be found at "./raven/tests/framework/unit_tests/Dataobjects/ TestPoint Set.py". This test can be called executing the following command:

. Irun_tests --re=framework.test_datasets

or

./run_framework_tests --re=framework.test_datasets

- Test Description:

- This test is a Unit Test for the DataSet classes.

- Original Author:

- talbpaul

- Creation date:

- 2017-10-20

- The classes tested in this test are:

- DataSet

\subsubsection{FRAMEWORK.TEST DATASETS}

This test can be found at "./raven/tests/framework/unit_tests/DataObjects/ TestHierarchal.py". This test can be called executing the following command:

./run_tests $--r e=f r a m e w o r k . t e s t \_d a t a s e t s$

or

./run_framework_tests --re=framework.test_datasets

- Test Description:

- This test is a Unit Test for the DataSet classes.

- Original Author: 
- talbpaul

- Creation date:

- 2017-10-20

- The classes tested in this test are:

- DataSet

\subsubsection{FRAMEWORK ENSEMBLEMODELTESTS.TESTENSEMBLEMODELLINEARPARALLEL}

This test can be found at ". / raven/tests/framework/ensembleModelTests/test_ ensemble_model_linear_internal_parallel.xml”. This test can be called executing the following command:

. Irun_tests --re=framework/ensembleModelTests.testEnsembleModelLinearParal

or

. Irun_framework_tests --re=framework/ensembleModelTests.testEnsembleModelL

- Test Description:

- Example of usage of the Ensemble Model capability in RAVEN, connecting multiple External Models. This test is aimed to check the functionality of assembling multiple Models, when they result in a single chain of Models (linear system). This test checks the functionality of the model using a multi processor (ParallelPython) parallelization scheme.

- Original Author:

- alfoa

- Creation date:

- 2016-04-14

- The classes tested in this test are:

- Models.EnsembleModel, Models.ExternalModel, JobHandler.ParallelPython

- Since the creation of this test, the following main revisions have been performed:

1. revision info: 
- author : alfoa

- date : 2016-04-21

- description: Modified number of processors

2. revision info:

- author : maljdan

- date : 2016-05-20

- description: Updating the test cases to remove references to Point or History.

3. revision info:

- author : alfoa

- date : 2016-09-01

- description: Close \#650

4. revision info:

- author : alfoa

- date : 2016-11-14

- description: New syntax ensemble

5. revision info:

- author : alfoa

- date : 2017-01-21

- description: Adding this test description.

\subsubsection{FRAMEWORK ENSEMBLEMODELTESTS.TESTENSEMBLEMODELLINEARTHREAD}

This test can be found at ". / raven/tests/framework/ensembleModelTests/test_ ensemble_model_linear_threading.xml”. This test can be called executing the following command:

. Irun_tests --re=framework/ensembleModelTests.testEnsembleModel LinearThrea

or

./run_framework_tests --re=framework/ensembleModel Tests.testEnsembleModel L

- Test Description:

- Example of usage of the Ensemble Model capability in RAVEN, connecting multiple External Models. This test is aimed to check the functionality of assembling multiple Models, when they result in a single chain of Models (linear system). This test checks the functionality of the model using a multi threading parallelization scheme. 
- Original Author:

- alfoa

- Creation date:

- 2016-04-14

- The classes tested in this test are:

- Models.EnsembleModel, Models.ExternalModel, JobHandler.Thread

- Since the creation of this test, the following main revisions have been performed:

1. revision info:

- author : maljdan

- date : 2016-05-20

- description: Updating the test cases to remove references to Point or History.

2. revision info:

- author : alfoa

- date : 2016-09-01

- description: Close \#650

3. revision info:

- author : alfoa

- date : 2016-11-14

- description: New syntax ensemble

4. revision info:

- author : alfoa

- date : 2017-01-21

- description: Adding this test description.

\subsubsection{FRAMEWORK ENSEMBLEMODELTESTS.TESTENSEMBLEMODELLINEARTHREADWI}

This test can be found at ". / raven/tests/framework/ensembleModelTests/test_ ensemble_model_linear_threading_with_time_series.xml”. This test can be called executing the following command:

. Irun_tests --re=framework/ensembleModelTests.testEnsembleModel LinearThrea

or 
./run_framework_tests --re=framework/ensembleModelTests.testEnsembleModelL

- Test Description:

- Example of usage of the Ensemble Model capability in RAVEN, connecting multiple External Models. This test is aimed to check the functionality of assembling multiple Models, when they result in a single chain of Models (linear system) and they need to transfer "time series" type of data (vectors) using the DataSet DataObject. This test checks the functionality of the model using a multi threading parallelization scheme.

- Original Author:

- alfoa

- Creation date:

- 2016-08-02

- The classes tested in this test are:

- Models.EnsembleModel, Models.ExternalModel, JobHandler.Thread

- Since the creation of this test, the following main revisions have been performed:

1. revision info:

- author : alfoa

- date : 2016-11-29

- description: Added test for time series

2. revision info:

- author : alfoa

- date : 2016-12-01

- description: Fixed order of objects in test inputs in order to respect the XSD schema

3. revision info:

- author: alfoa

- date : 2017-01-21

- description: Adding this test description.

4. revision info:

- author : alfoa

- date : 2018-02-03

- description: Modified test for the new DataObject system. Usage of DataSet. 


\subsubsection{FRAMEWORK ENSEMBLEMODELTESTS.TESTENSEMBLEMODELNONLINEARPARAL}

This test can be found at ". /raven/tests/framework/ensembleModelTests/test_ ensemble_model_picard_parallel.xml". This test can be called executing the following command:

./run_tests --re=framework/ensembleModelTests.testEnsembleModelNonLinearPa

or

./run_framework_tests --re=framework/ensembleModelTests.testEnsembleModelN

- Test Description:

- Example of usage of the Ensemble Model capability in RAVEN, connecting multiple External Models. This test is aimed to check the functionality of assembling multiple Models, testing the iterative scheme present in RAVEN when the chain of Models turn to be a Non Linear System. This test checks the functionality of the Model with a multi processor (Parallel Python) parallelization scheme.

- Original Author:

- alfoa

- Creation date:

- 2016-04-14

- The classes tested in this test are:

- Models.EnsembleModel, Models.ExternalModel, JobHandler.ParallelPython

- Since the creation of this test, the following main revisions have been performed:

1. revision info:

- author : alfoa

- date : 2016-04-21

- description: Modified number of processors

2. revision info:

- author : maljdan

- date : 2016-05-20

- description: Updating the test cases to remove references to Point or History.

3. revision info: 
- author : alfoa

- date : 2016-09-01

- description: Close \#650

4. revision info:

- author : alfoa

- date : 2016-11-14

- description: New syntax ensemble

5. revision info:

- author : alfoa

- date : 2017-01-21

- description: Adding this test description.

\subsubsection{FRAMEWORK ENSEMBLEMODELTESTS.TESTENSEMBLEMODELNONLINEARTHREA}

This test can be found at ". / raven/tests/framework/ensembleModelTests/test_ ensemble_model_picard_thread.xml". This test can be called executing the following command:

. Irun_tests --re=framework/ensembleModelTests.testEnsembleModelNonLinearTh

or

. Irun_framework_tests --re=framework/ensembleModel Tests.testEnsembleModelN

- Test Description:

- Example of usage of the Ensemble Model capability in RAVEN, connecting multiple External Models. This test is aimed to check the functionality of assembling multiple Models, testing the iterative scheme present in RAVEN when the chain of Models turn to be a Non Linear System. This test checks the functionality of the Model with a multi threading parallelization scheme.

- Original Author:

- alfoa

- Creation date:

- 2016-04-14

- The classes tested in this test are: 
- Models.EnsembleModel, Models.ExternalModel, JobHandler.Thread

- Since the creation of this test, the following main revisions have been performed:

1. revision info:

- author : alfoa

- date : 2016-04-21

- description: Modified number of processors

2. revision info:

- author : maljdan

- date : 2016-05-20

- description: Updating the test cases to remove references to Point or History.

3. revision info:

- author : alfoa

- date : 2016-09-01

- description: Close \#650

4. revision info:

- author : alfoa

- date : 2016-11-14

- description: New syntax ensemble

5. revision info:

- author : alfoa

- date : 2017-01-21

- description: Adding this test description.

6. revision info:

- author: wangc

- date : 2017-09-11

- description: add new sub-node 'initialStartModels'

\subsubsection{FRAMEWORK ENSEMBLEMODELTESTS.TESTENSEMBLEMODELWITHCODE}

This test can be found at “. / raven/tests/framework/ensembleModelTests/test_ ensemble_model_linear_threading_with_code.xml". This test can be called executing the following command:

. /run_tests --re=framework/ensembleModelTests.testEnsembleModelWithCode 
or

. Irun_framework_tests --re=framework/ensembleModel Tests.testEnsenhleModelW.

- Test Description:

- Example of usage of the Ensemble Model capability in RAVEN, connecting multiple External Models and Codes. This test is aimed to check the functionality of assembling multiple Models, when they result in a single chain of Models (linear system). This test checks the functionality of the model using a multi threading parallelization scheme for the External Models and multi processor scheme for the external Code.

- Original Author:

- alfoa

- Creation date:

- 2016-04-14

- The classes tested in this test are:

- Models.EnsembleModel, Models.ExternalModel, Models.Code, JobHandler.Thread

- Since the creation of this test, the following main revisions have been performed:

1. revision info:

- author : alfoa

- date : 2016-04-21

- description: Modified number of processors

2. revision info:

- author : alfoa

- date : 2016-11-14

- description: New syntax ensemble

3. revision info:

- author : alfoa

- date : 2017-01-21

- description: Adding this test description. 


\subsubsection{FRAMEWORK ENSEMBLEMODELTESTS.TESTENSEMBLEMODELLINEARPARALLEL}

This test can be found at ". /raven/tests/framework/ensembleModelTests/test_ ensemble_model_linear_internal_parallel_with_optimizer.xml”. This test can be called executing the following command:

./run_tests --re=framework/ensembleModelTests.testEnsembleModelLi nearParal

or

./run_framework_tests --re=framework/ensembleModelTests.testEnsembleModelL

- Test Description:

- Example of usage of the Ensemble Model capability in RAVEN, connecting multiple External Models. This test is aimed to check the functionality of assembling multiple Models, when they result in a single chain of Models (linear system) and they are sampled with an Optimization scheme. This test checks the functionality of the model using a multi processor (ParallelPython) parallelization scheme.

- Original Author:

- alfoa

- Creation date:

- 2016-09-01

- The classes tested in this test are:

- Models.EnsembleModel, Models.ExternalModel, JobHandler.ParallelPython

- Since the creation of this test, the following main revisions have been performed:

1. revision info:

- author : alfoa

- date : 2016-10-20

- description: Modified XSD and removed dim attribute from all the tests

2. revision info:

- author : chenj

- date : 2016-10-31

- description: Regold parallel EnsembleModel test since change in the optimization algorithm 
3. revision info:

- author : alfoa

- date : 2016-11-14

- description: New syntax ensemble

4. revision info:

- author : alfoa

- date : 2017-01-21

- description: Adding this test description.

\subsubsection{FRAMEWORK ENSEMBLEMODELTESTS.TESTENSEMBLEMODELLATERALINSERTIO}

This test can be found at ". /raven/tests/framework/ensembleModelTests/test_ ensemble_model_linear_with_multiple_lateral_insertions.xml". This test can be called executing the following command:

./run_tests --re=framework/ensembleModelTests.testEnsembleModelLateral Inse

or

. Irun_framework_tests --re=framework/ensembleModelTests.testEnsenbleModelL

- Test Description:

- Example of usage of the Ensemble Model capability in RAVEN, connecting multiple External Models. This test is aimed to check the functionality of assembling multiple Models, when multiple chains of Models are connected "laterly".

- Original Author:

- alfoa

- Creation date:

- 2016-12-13

- The classes tested in this test are:

- Models.EnsembleModel, Models.ExternalModel, JobHandler.ParallelPython

- Since the creation of this test, the following main revisions have been performed:

1. revision info: 
- author : alfoa

- date : 2017-01-21

- description: Adding this test description.

\subsubsection{FRAMEWORK ENSEMBLEMODELTESTS.TESTENSEMBLEMODELWITH2CODESANDA}

This test can be found at ". /raven/tests/framework/ensembleModelTests/test_ ensemble_model_with_2_codes.xml". This test can be called executing the following command:

./run_tests --re=framework/ensembleModelTests.testEnsembleModelWilth2CodesA

or

./run_framework_tests --re=framework/ensembleModelTests.testEnsenbleModelW

- Test Description:

- Example of usage of the Ensemble Model capability in RAVEN, connecting multiple multiple Codes together, with same output variable names. This means that the alias system has been used and this test is aimed to show how to use it in these kinds of situations.

- Original Author:

- alfoa

- Creation date:

- 2017-02-16

- The classes tested in this test are:

- Models.Code, JobHandler.Thread

\subsubsection{FRAMEWORK ENSEMBLEMODELTESTS.TESTENSEMBLEMODELWITH2CODESANDA}

This test can be found at ". /raven/tests/framework/ensembleModel Tests/test_ ensemble_model_2_codes_optional_output.xml". This test can be called executing the following command:

./run_tests --re=framework/ensembleModelTests.testEnsembleModelWi th2CodesA 
or

. Irun_framework_tests --re=framework/ensembleModelTests.testEnsenbleModelW.

- Test Description:

- The main goal of this test is to show how to specify optional output in the Ensemble Model object. All the RAVEN available data storage types are tested (DataObjects and Databases).

- Original Author:

- alfoa

- Creation date:

- 2017-05-17

- The classes tested in this test are:

- Models.Code, JobHandler.Thread

- Since the creation of this test, the following main revisions have been performed:

1. revision info:

- author : alfoa

- date : 2017-07-10

- description: Modified test in order to show how to use a HDF5 as optional output (Issue \#237)

\subsubsection{FRAMEWORK ENSEMBLEMODELTESTS.TESTENSEMBLEMODELLINEAREXPECTED]}

This test can be found at ". / raven/tests/framework/ensembleModelTests/test_ ensemble_model_linear_expected_failures.xml”. This test can be called executing the following command:

. Irun_tests --re=framework/ensembleModelTests.testEnsembleModel Li nearExpec

or

./run_framework_tests --re=framework/ensembleModel Tests.testEnsembleModel L

- Test Description: 
- Mechanic TEST: This test is aimed to check that the failures from the EnsembleModel are correctly collected without abnormal termination of RAVEN.

- Original Author:

- alfoa

- Creation date:

- 2017-09-22

- The classes tested in this test are:

- Models.EnsembleModel, Models.ExternalModel, JobHandler.Thread

\subsubsection{FRAMEWORK ENSEMBLEMODELTESTS.TESTENSEMBLEMODELFULLYCORRVARS}

This test can be found at “. / raven/tests/framework/ensembleModelTests/test_ ensemble_model_fully_correlated_vars.xml". This test can be called executing the following command:

. Irun_tests --re=framework/ensembleModelTests.testEnsembleModelFyllyCorrVa

or

./run_framework_tests --re=framework/ensembleModel Tests.testEnsembleModelF

- Test Description:

- This test is a mechanical test for the EnsembleModel to test that it can correctly handle fully correlated variables

- Original Author:

- alfoa

- Creation date:

- 2017-12-07

- The classes tested in this test are:

- Models.EnsembleModel, Models.ExternalModel, Models.Code, JobHandler.Thread

- Since the creation of this test, the following main revisions have been performed: 
1. revision info:

- author : alfoa

- date : 2017-12-07

- description: Added the test

\subsubsection{FRAMEWORK ENSEMBLEMODELTESTS.INDEXINPUTOUTPUT}

This test can be found at ". / raven/tests/framework/ensembleModel Tests/index_ input_output.xml". This test can be called executing the following command:

./run_tests --re=framework/ensembleModelTests.indexInputoutput

or

./run_framework_tests --re=framework/ensembleModelTests.indexInpytoutput

- Test Description:

- Tests that an index can be depended on by variables in both in input and output space of an ensembled model.

- Original Author:

- talbpaul

- Creation date:

- 2018-02-06

- The classes tested in this test are:

- Models.EnsembleModel, Models.ExternalModel, DataObject.DataSet

\subsubsection{FRAMEWORK ENSEMBLEMODELTESTS.TESTEMCONNECTION}

This test can be found at ". /raven/tests/framework/ensembleModelTests/test_ em_connection.xml". This test can be called executing the following command:

./run_tests --re=framework/ensembleModelTests.testEMConnection

or 
./run_framework_tests --re=framework/ensembleModelTests.testEMConnection

- Test Description:

- Example of usage of the Ensemble Model capability in RAVEN, connecting multiple External Models. This test is aimed to check the connection between multiple models, as reported in issue \#696.

- Original Author:

- alfoa

- Creation date:

- 2018-08-04

- The classes tested in this test are:

- Models.EnsembleModel, Models.ExternalModel, JobHandler.ParallelPython

\subsubsection{FRAMEWORK ENSEMBLEMODELTESTS.TESTEMREUSABILITY}

This test can be found at ". / raven/tests/framework/ensembleModelTests/test_ em_reuse.xml". This test can be called executing the following command:

./run_tests --re=framework/ensembleModelTests.testEMReusability

or

./run_framework_tests --re=framework/ensembleModelTests.testEMReysability

- Test Description:

- Example of re-using the ensemble model in different steps. This test is aimed to fix the bug reported in issue \#859.

- Original Author:

- Jimmy

- Creation date:

- 2019-10-30

- The classes tested in this test are:

- Models.EnsembleModel, Models.ExternalModel, JobHandler.ParallelPython 


\subsubsection{FRAMEWORK ENSEMBLEMODELTESTS.NDENSEMBLE2OUTS}

This test can be found at "./raven/tests/framework/ensembleModelTests/nd_ ensemble_2outs.xml". This test can be called executing the following command:

. /run_tests --re=framework/ensembleModelTests.NDEnsemble2outs

or

./run_framework_tests --re=framework/ensembleModelTests.NDEnsemble2Outs

- Test Description:

- Tests having 2 outputs in an ensemble model with an ND model. See \#1119.

- Original Author:

- talbpaul

- Creation date:

$-2021-02-25$

- The classes tested in this test are:

- Models.EnsembleModel

\subsubsection{FRAMEWORK CODEINTERFACETESTS.TESTMCMAMMOTHNOEXECUTABLE BISONRELAP7}

This test can be found at ". / raven/tests/framework/CodeInterfaceTests/test_ perturb_mammoth_bison_relap7.xml”. This test can be called executing the following command:

. Irun_tests --re=framework/CodeInterfaceTests.testMCMammothNoExequtable_Bi

or

./run_framework_tests --re=framework/CodeInterfaceTests.testMCMammothNoExe

- Test Description: 
- An example of using the MAMMOTH code interface (no executable). It checks Bison and RELAP7. This test is designed to ensure the MAMMOTH interface mechanics is correctly functional. In addition it used the RAVEN alias system.

- Original Author:

- tompjame

- Creation date:

- 2016-08-29

- The classes tested in this test are:

- Models.Code.MAMMOTH, Models.AliasSystem

- Since the creation of this test, the following main revisions have been performed:

1. revision info:

- author : talbpaul

- date : 2016-09-15

- description: Test updates

2. revision info:

- author : alfoa

- date : 2016-11-30

- description: Added alias for MAMMOTH just to test the new system

3. revision info:

- author : alfoa

- date : 2016-12-12

- description: Added type for alias system in mammoth test

4. revision info:

- author : alfoa

- date : 2017-01-21

- description: Adding this test description.

\subsubsection{FRAMEWORK CODEINTERFACETESTS.TESTMCMAMMOTHNOEXECUTABLEBISONR}

This test can be found at ". / raven/tests/framework/CodeInterfaceTests/test_ mammoth_r7_bison_no_exe_hdf5_restart.xml”. This test can be called executing the following command: 
./run_tests --re=framework/CodeInterfaceTests.testMCMammothNoExequtableBis

or

./run_framework_tests --re=framework/CodeInterfaceTests.testMCManmothNoExe

- Test Description:

- An example of using the MAMMOTH code interface (no executable). This test is designed to ensure the MAMMOTH interface mechanics is correctly functional. In addition, the alias system is used

- Original Author:

- alfoa

- Creation date:

- 2016-12-15

- The classes tested in this test are:

- Models.Code.MAMMOTH, Model.AliasSystem

- Since the creation of this test, the following main revisions have been performed:

1. revision info:

- author : alfoa

- date : 2016-12-15

- description: modified test in order to be validable by XSD schema

2. revision info:

- author : alfoa

- date : 2017-01-21

- description: Adding this test description.

\subsubsection{FRAMEWORK CODEINTERFACETESTS.TESTMCMAMMOTHNOEXECUTABLE RATTLESNAKEBISON}

This test can be found at "./raven/tests/framework/CodeInterfaceTests/test_ perturb_all_rattlesnake_bison.xml". This test can be called executing the following command: 
./run_tests --re=framework/CodeInterfaceTests.testMCMAMMOTHNoExequtable_Ra

or

./run_framework_tests --re=framework/CodeInterfaceTests.testMCMAMMOTHNoExe

- Test Description:

- An example of using the MAMMOTH code interface (no executable). It checks Bison and RattleSnake. This test is designed to ensure the MAMMOTH interface mechanics is correctly functional, overal for Neutron Cross section perturbation.

- Original Author:

- tompjame

- Creation date:

- 2016-08-29

- The classes tested in this test are:

- Models.Code.MAMMOTH

- Since the creation of this test, the following main revisions have been performed:

1. revision info:

- author : talbpaul

- date : 2016-09-15

- description: other test updates

2. revision info:

- author : alfoa

- date : 2017-01-21

- description: Adding this test description.

\subsubsection{FRAMEWORK CODEINTERFACETESTS.TESTPERTURBMAMMOTHNOEXECUTABLE RATTLESNAKEBISON}

This test can be found at "./raven/tests/framework/CodeInterfaceTests/test_ perturb_mammoth_rattlesnake_bison.xml". This test can be called executing the following command: 
. Irun_tests --re=framework/CodeInterfaceTests.testPerturbMAMMOTHNoExecutab

or

. Irun_framework_tests --re=framework/CodeInterfaceTests.testPerturbMAMMOTH

- Test Description:

- An example of using the MAMMOTH code interface. This test is designed to ensure the MAMMOTH interface runs correctly. It runs BISON and RattleSnake.

- Original Author:

- tompjame

- Creation date:

- 2016-08-29

- The classes tested in this test are:

- Models.Code.MAMMOTH

- Since the creation of this test, the following main revisions have been performed:

1. revision info:

- author : talbpaul

- date : 2016-09-15

- description: Test updates

2. revision info:

- author : alfoa

- date : 2017-01-21

- description: Adding this test description.

\subsubsection{FRAMEWORK CODEINTERFACETESTS.TESTMC}

This test can be found at ". / raven/tests/framework/CodeInterfaceTests/test_ mc_rattlesnake.xml". This test can be called executing the following command:

./run_tests --re=framework/CodeInterfaceTests.testMC 
or

./run_framework_tests --re=framework/CodeInterfaceTests.testmC

- Test Description:

- An example of using the Rattlesnake code interface. This test is aimed to test the mechanics of the interface (no executable)..

- Original Author:

- wangc

- Creation date:

- 2016-05-09

- The classes tested in this test are:

- Models.Code.Rattlesnake

- Since the creation of this test, the following main revisions have been performed:

1. revision info:

- author : talbpaul

- date : 2016-09-15

- description: Test updates

2. revision info:

- author : alfoa

- date : 2017-01-21

- description: Adding this test description.

\subsubsection{FRAMEWORK CODEINTERFACETESTS OPENMODELICAINTERFACETEST- NOEXECUTABLE}

This test can be found at ". / raven/tests/framework/CodeInterfaceTests/test_ openModelica_code_interface.xml". This test can be called executing the following command:

. Irun_tests --re=framework/CodeInterfaceTests/OpenModelicaInterfadceTestNoE

Or 
./run_framework_tests --re=framework/CodeInterfaceTests/OpenModelicaInterf

- Test Description:

- The purpose of this test is to exercise the OpenModelica external code interface without needing to have a built executable. It is directly modeled on the test_relap5_code_interface.xml test and works by making sure that 1) The input files are perturbed correctly 2) That the raw output CSV files are properly processed for reading by RAVEN

- Original Author:

- @bobk

- Creation date:

- 2015-06-11

- The classes tested in this test are:

- CodeInterfaces.OpenModelica

- Since the creation of this test, the following main revisions have been performed:

1. revision info:

- author : @ talbpaul

- date : 2016-04-26

- description: Added check for pre-existing HDF5 backup files when validating

\subsubsection{FRAMEWORK CODEINTERFACETESTS DYMOLAINTERFACETESTNOEX- ECUTABLE}

This test can be found at ". /raven/tests/framework/CodeInterfaceTests/test_ Dymola_code_interface.xml". This test can be called executing the following command:

./run_tests --re=framework/CodeInterfaceTests /DymolaInterfaceTes tNoExecutal

or

./run_framework_tests --re=framework/CodeInterfaceTests/DymolaInterfaceTes

- Test Description: 
- The purpose of this test is to exercise the Dymola external code interface without needing to have a built executable. It is directly modeled on the test_relap5_code_interface.xml test and works by making sure that 1) The input files are perturbed correctly 2) The raw output MAT files are properly processed for reading by RAVEN

- Original Author:

- @kimj

- Creation date:

- 2015-11-11

- The classes tested in this test are:

- CodeInterfaces.Dymola

- Since the creation of this test, the following main revisions have been performed:

1. revision info:

- author : @ talbpaul

- date : 2016-04-26

- description: Added check for pre-existing HDF5 backup files when validating

\subsubsection{FRAMEWORK CODEINTERFACETESTS.DYMOLATESTLOADSOMEVARSNOEXECUTAF}

This test can be found at ". / raven/tests/framework/CodeInterfaceTests/test_ Dymola_code_interface_load_some_vars.xml". This test can be called executing the following command:

. Irun_tests --re=framework/CodeInterfaceTests. DymolaTestLoadSomeyarsNoExec

or

./run_framework_tests --re=framework/CodeInterfaceTests. DymolaTe\$tLoadSome

- Test Description:

- The purpose of this test is to exercise the Dymola external code interface without needing to have a built executable. This special case shows how to load just few output varibles from the Dymola outputs (e.g. .mat files). In this case only the variables e,g and $\mathrm{h}$ will be loaded

- Original Author: 
-@alfoa

- Creation date:

- 2017-09-16

- The classes tested in this test are:

- CodeInterfaces.Dymola

\subsubsection{FRAMEWORK CODEINTERFACETESTS.DYMOLATESTTIMEDEPNOEXECUTABLEENS}

This test can be found at ". / raven/tests/framework/CodeInterfaceTests/test_ Dymola_code_interface_timedep.xml". This test can be called executing the following command:

. Irun_tests --re=framework/CodeInterfaceTests. DymolaTestTimeDepNoExecutabl

or

./run_framework_tests --re=framework/CodeInterfaceTests.DymolaTe $\$$ timeDepN

- Test Description:

- The purpose of this test is to ensure that the Dyola external code interface functions properly with the new ensemble model functionality. Since the output files have been pre-generated, a Dymola executable is not necessary to run this test.

- Original Author:

- alfoa

- Creation date:

- 2016-11-11

- The classes tested in this test are:

- Models.EnsembleModel,CodeInterfaces.OpenModelica

- Since the creation of this test, the following main revisions have been performed:

1. revision info:

- author : alfoa

- date : 2016-12-01 
- description: Fixed order of test inputs to respect XSD schema

2. revision info:

- author : alfoa

- date : 2018-02-04

- description: Modified test in order to work with the new DataObject structure (Usage of DataSet)

\subsubsection{FRAMEWORK CODEINTERFACETESTS.MELCORINTERFACEFORWARDSAMPLINGNO}

This test can be found at ". / raven/tests/framework/CodeInterfaceTests/test_ melcor_interface. xml”. This test can be called executing the following command:

. Irun_tests --re=framework/CodeInterfaceTests.melcorInterfaceForwardSampli

or

- Irun_framework_tests --re=framework/CodeInterfaceTests.melcorInterfacefor

- Test Description:

- An example of using the Melcor 2.1/2.2 code interface. This test is aimed to test the mechanics of the interface (no executable).

- Original Author:

- alfoa

- Creation date:

- 2017-04-27

- The classes tested in this test are:

- Models.Code.Melcor

- Since the creation of this test, the following main revisions have been performed:

1. revision info:

- author : alfoa

- date : 2017-04-27

- description: Adding this test description.

2. revision info: 
- author : alfoa

- date : 2020-10-31

- description: Added csv xml node in the Code block for showing how to use it.

\subsubsection{FRAMEWORK CODEINTERFACETESTS PRESCIENTINTERFACE}

This test can be found at ". /raven/tests/framework/CodeInterfaceTests/test_ Prescient_code_interface.xml". This test can be called executing the following command:

./run_tests --re=framework/CodeInterfaceTests/PrescientInterface

or

./run_framework_tests --re=framework/CodeInterfaceTests/PrescientInterface

- Test Description:

- Test the Prescient Code Interface. This perturbs the demand on the Abel bus. This should result in different outputs depending on the value.

- Original Author:

- @ cogljj

- Creation date:

- 2020-09-02

- The classes tested in this test are:

- CodeInterfaces.PrescientCodeInterface

\subsubsection{FRAMEWORK CODEINTERFACETESTS PRESCIENTINTERFACE}

This test can be found at “. / raven/tests/framework/CodeInterfaceTests/test_ Prescient_code_interface_fake.xml". This test can be called executing the following command:

. /run_tests --re=framework/CodeInterfaceTests/PrescientInterface

or 
./run_framework_tests --re=framework/CodeInterfaceTests/PrescientInterface

- Test Description:

- Test the Prescient Code Interface. This perturbs the demand on the Abel bus. This should result in different outputs depending on the value. This version is an interface check version.

- Original Author:

- @ cogljj

- Creation date:

- 2020-09-02

- The classes tested in this test are:

- CodeInterfaces.PrescientCodeInterface

\subsubsection{SAPHIRE}

This test can be found at ". / raven/tests/framework/CodeInterfaceTests/SaphireInterfac test_saphire.xml". This test can be called executing the following command:

. Irun_tests --re=saphire

or

. Irun_framework_tests --re=saphire

- Test Description:

- This test is aimed to show the extension of the GenericCode interface for SAPHIRE

- Original Author:

- wangc

- Creation date:

- 2018-07-03

- The classes tested in this test are:

- CodeInterface 


\subsubsection{FRAMEWORK CODEINTERFACETESTS SCALE.SCALEORIGEN}

This test can be found at ". / raven/tests/framework/CodeInterfaceTests/Scale/ test_scale_origen_forward.xml". This test can be called executing the following command:

./run_tests --re=framework/CodeInterfaceTests/Scale.Scaleorigen

or

./run_framework_tests --re=framework/CodeInterfaceTests/Scale.Scaleorigen

- Test Description:

- An example of using the the Model Code of type Scale, with Forward Sampling and driving the ORIGEN sequence

- Original Author:

- alfoa

- Creation date:

- 2018-04-04

- The classes tested in this test are:

- Models.Code.Scale

- Since the creation of this test, the following main revisions have been performed:

1. revision info:

- author : alfoa

- date : 2018-04-04

- description: Test created

\subsubsection{FRAMEWORK CODEINTERFACETESTS SCALE.SCALEORIGEN}

This test can be found at ". / raven/tests/framework/CodeInterfaceTests/Scale/ test_scale_triton_forward.xml". This test can be called executing the following command:

. /run_tests --re=framework/CodeInterfaceTests/Scale.ScaleOrigen 
or

./run_framework_tests --re=framework/CodeInterfaceTests/Scale.Scaleorigen

- Test Description:

- An example of using the the Model Code of type Scale, with Forward Sampling and driving the TRITON (t- dpl) sequence

- Original Author:

- alfoa

- Creation date:

- 2018-04-04

- The classes tested in this test are:

- Models.Code.Scale

- Since the creation of this test, the following main revisions have been performed:

1. revision info:

- author : alfoa

- date : 2018-04-04

- description: Test created

\subsubsection{FRAMEWORK CODEINTERFACETESTS SCALE.SCALETRITONORIGENCOMBINED}

This test can be found at “. / raven/tests/framework/CodeInterfaceTests/Scale/ test_scale_combined_forward.xml". This test can be called executing the following command:

. Irun_tests --re=framework/CodeInterfaceTests/Scale.ScaleTritonorigenCombi

or

./run_framework_tests --re=framework/CodeInterfaceTests/Scale.ScaleTritono

- Test Description: 
- An example of using the the Model Code of type Scale, with Forward Sampling and combined calcolation (Triton to Origen)

- Original Author:

- alfoa

- Creation date:

- 2018-04-04

- The classes tested in this test are:

- Models.Code.Scale

- Since the creation of this test, the following main revisions have been performed:

1. revision info:

- author : alfoa

- date : 2018-04-04

- description: Test created

2. revision info:

- author : alfoa

- date : 2019-03-07

- description: Modified syntax in Functions as for issue \#934

\subsubsection{FRAMEWORK CODEINTERFACETESTS MOOSEBASEAPPS.INPUTPARSER}

This test can be found at ". / raven/tests / framework/CodeInterfaceTests/MoosEBaseApps / input_parser.xml". This test can be called executing the following command:

. /run_tests --re=framework/CodeInterfaceTests/MoosEBaseApps. Inputparser

or

./run_framework_tests --re=framework/CodeInterfaceTests/MoosEBaseApps. Inpu

- Test Description:

- Various challenging moose-based input parsing tests. As far as I know would not actually run in MOOSE.

- Original Author: 
- talbpaul

- Creation date:

- 2020-04-06

- The classes tested in this test are:

- Models.Code.MooseBasedApp

\subsubsection{FRAMEWORK CODEINTERFACETESTS MOOSEBASEAPPS.MOOSEEX18WITHGENERI}

This test can be found at “. / raven/tests/framework/CodeInterfaceTests/MoosEBaseApps / test_MC_MooseEx18_with_generic_file.xml". This test can be called executing the following command:

. Irun_tests --re=framework/CodeInterfaceTests/MOOSEBaseApps.MooseEx18withG

or

. Irun_framework_tests --re=framework/CodeInterfaceTests/MoosEBaseApps.Moos

- Test Description:

- An example of using the MooseBasedApp code interface, perturbing the MOOSE main input file and a generic external input file (e.g. CSV file)

- Original Author:

- alfoa

- Creation date:

$-2020-01-31$

- The classes tested in this test are:

- Models.Code.MooseBasedApp

- Since the creation of this test, the following main revisions have been performed:

1. revision info:

- author : alfoa

- date : 2020-01-31

- description: Created test 


\subsubsection{FRAMEWORK CODEINTERFACETESTS MOOSEBASEAPPS.MOOSEEX18ONLYGENERI}

This test can be found at ". / raven/tests/framework/CodeInterfaceTests/MOOSEBaseApps / test_MC_MooseEx18_only_generic_file.xml". This test can be called executing the following command:

./run_tests --re=framework/CodeInterfaceTests/MoOSEBaseApps.Moos \&x18onlyG

or

./run_framework_tests --re=framework/CodeInterfaceTests/MOOSEBas@Apps.Moos

- Test Description:

- An example of using the MooseBasedApp code interface, perturbing a generic external input file ONLY (e.g. CSV file)

- Original Author:

- alfoa

- Creation date:

- 2020-01-31

- The classes tested in this test are:

- Models.Code.MooseBasedApp

- Since the creation of this test, the following main revisions have been performed:

1. revision info:

- author : alfoa

- date : 2020-01-31

- description: Created test

\subsubsection{FRAMEWORK CODEINTERFACETESTS INSTANT.TESTINSTANT NOEXEC}

This test can be found at ". / raven/tests/framework/CodeInterfaceTests/Instant/ test_instant_noexec.xml". This test can be called executing the following command:

./run_tests --re=framework/CodeInterfaceTests/Instant.testInstant_noexec 
or

. Irun_framework_tests --re=framework/CodeInterfaceTests/Instant. test Instan

- Test Description:

- An example of using the code interface RattleSnake when the INSTANT solver is employed (noexecutable). This test is aimed to check the mechanics of the code interface when a XS library needs to be perturbed (IAEA2D benchmark).

- Original Author:

- wangc

- Creation date:

- 2016-07-14

- The classes tested in this test are:

- Models.Code.Rattlesnake

- Since the creation of this test, the following main revisions have been performed:

1. revision info:

- author : talbpaul

- date : 2016-09-15

- description: other test updates

2. revision info:

- author : alfoa

- date : 2017-01-21

- description: Adding this test description.

\subsubsection{FRAMEWORK CODEINTERFACETESTS INTERFACESWORKSHOPEXAM- PLE PROJECTILEEXAMPLE.PROJWITHOUTCSV}

This test can be found at ". / raven/tests/framework/CodeInterfaceTests/InterfacesWorks ProjectileExample/example_using_interface_without_CSV.xml”. This test can be called executing the following command:

. Irun_tests --re=framework/CodeInterfaceTests/InterfacesWorkshop所ample/Pr

or 
./run_framework_tests --re=framework/CodeInterfaceTests/InterfacesWorkshop

- Test Description:

- Testing the functionality of the Projectile Example interface without the creation of the CSV out of the code output. The output is directly returned as dictionary to RAVEN (no double IO).

- Original Author:

- alfoa

- Creation date:

- 2020-10-31

- The classes tested in this test are:

- Models.Code

\subsubsection{FRAMEWORK CODEINTERFACETESTS INTERFACESWORKSHOPEXAM- PLE PROJECTILEEXAMPLE.PROJWITHCSV}

This test can be found at ". /raven/tests/framework/CodeInterfaceTests/InterfacesWorks ProjectileExample/example_using_interface_with_CSV_creation.xml". This test can be called executing the following command:

./run_tests --re=framework/CodeInterfaceTests/InterfacesWorkshop \&xample/Pr

or

./run_framework_tests --re=framework/CodeInterfaceTests/Interfacesworkshop

- Test Description:

- Testing the functionality of the Projectile Example interface with the creation of the CSV out of the code output. The CSV is then parsed by RAVEN.

- Original Author:

- alfoa

- Creation date: 
- 2020-10-31

- The classes tested in this test are:

- Models.Code

\subsubsection{FRAMEWORK CODEINTERFACETESTS INTERFACESWORKSHOPEXAM- PLE BATEMANEXAMPLE.BATEMAN}

This test can be found at ". / raven/tests/framework/CodeInterfaceTests/InterfacesWorksl BatemanExample/example_using_interface_bateman.xml". This test can be called executing the following command:

. Irun_tests --re=framework/CodeInterfaceTests/InterfacesWorkshop贴ample/Ba

or

. Irun_framework_tests --re=framework/CodeInterfaceTests/InterfacesWorkshop

- Test Description:

- Testing the functionality of the Bateman Example interface. It demonstrates typical usage of the MultiRun with Monte Carlo sampler.

- Original Author:

- alfoa

- Creation date:

- 2020-10-31

- The classes tested in this test are:

- Models.Code

\subsubsection{FRAMEWORK CODEINTERFACETESTS NEUTRINO.BASE}

This test can be found at ". / raven/tests/framework/CodeInterfaceTests/Neutrino/ test_neutrino_base.xml". This test can be called executing the following command:

./run_tests --re=framework/CodeInterfaceTests/Neutrino.Base 
or

. /run_framework_tests --re=framework/CodeInterfaceTests/Neutrino. Base

- Test Description:

- Basic test of the Neutrino base interface. Sample three values for the particle size from a uniform distribution and uses a Monte Carlo sampler. Outputs the results of each and particle size run. This test is used to test the Neutrino intereface with no executable.

- Original Author:

- EmeraldRyan

- Creation date:

- 2019-07-16

- The classes tested in this test are:

- Models.Code.Neutrino

\subsubsection{FRAMEWORK CODEINTERFACETESTS.MAAP5INTERFACEFORWARDSAMPLING}

This test can be found at ". / raven/tests / framework/CodeInterfaceTests/MAAP 5 / test_maap5_code_interface_forward.xml". This test can be called executing the following command:

. Irun_tests --re=framework/CodeInterfaceTests.MAAP5interfaceForwa rdSamplin

or

./run_framework_tests --re=framework/CodeInterfaceTests.MAAP 5interfaceForw

- Test Description:

- An example of using the the Model Code of type MAAP5, with Forward Sampling (e.g. MonteCarlo)

- Original Author:

- alfoa

- Creation date: 
- 2016-06-27

- The classes tested in this test are:

- Models.Code.MAAP5

- Since the creation of this test, the following main revisions have been performed:

1. revision info:

- author : alfoa

- date : 2016-07-01

- description: Addressed Dan's comments on interface (By Claudia Picoco)

2. revision info:

- author : alfoa

- date : 2016-09-01

- description: Close \#650

3. revision info:

- author : talbpaul

- date : 2016-09-15

- description: Test updates

4. revision info:

- author : talbpaul

- date : 2016-10-25

- description: removed dim in OutStreams Plot for validation purposes

5. revision info:

- author : alfoa

- date : 2016-11-15

- description: Updated input files

6. revision info:

- author : alfoa

- date : 2017-01-21

- description: Adding this test description.

\subsubsection{FRAMEWORK CODEINTERFACETESTS.MAAP5INTERFACEDETSAMPLING}

This test can be found at ". / raven/tests / framework/CodeInterfaceTests / MAAP 5 / test_maap5_code_interface_det.xml". This test can be called executing the following command: 
./run_tests --re=framework/CodeInterfaceTests.MAAP 5interfaceDETSampling

or

./run_framework_tests --re=framework/CodeInterfaceTests.MAAP5interfaceDETS

- Test Description:

- An example of using the the Model Code of type MAAP5. This test is aimed to test the mechanics of the MAAP5 interface when used with the Dynamic Event Tree methodology.

- Original Author:

- alfoa

- Creation date:

- 2016-06-27

- The classes tested in this test are:

- Models.Code.MAAP5, Samplers.DynamicEventTree

- Since the creation of this test, the following main revisions have been performed:

1. revision info:

- author : alfoa

- date : 2016-07-01

- description: Addressed Dan's comments on interface (By Claudia Picoco)

2. revision info:

- author : bobk

- date : 2016-07-07

- description: Changes file mode on two XML test file from 775 to 664 so that they will not be flagged as modified after validate_xml is run.

3. revision info:

- author: alfoa

- date : 2016-09-01

- description: Close \#650

4. revision info: 
- author : talbpaul

- date : 2016-09-15

- description: Test updates

5. revision info:

- author : talbpaul

- date : 2016-10-25

- description: removed dim in OutStream Plot for validation purposes

6. revision info:

- author : alfoa

- date : 2016-11-15

- description: Updated input files

7. revision info:

- author : alfoa

- date : 2017-01-21

- description: Adding this test description.

\subsubsection{FRAMEWORK CODEINTERFACETESTS.MAAP5INTERFACEHYBRIDDETSAMPLING}

This test can be found at ". / raven/tests/framework/CodeInterfaceTests/MAAP 5 / test_maap5_code_interface_hybrid_det.xml". This test can be called executing the following command:

. Irun_tests --re=framework/CodeInterfaceTests.MAAP5interfaceHybri dDETSampl

or

. Irun_framework_tests --re=framework/CodeInterfaceTests.MAAP 5 interfaceHybr

- Test Description:

- An example of using the the Model Code of type MAAP5. This test is aimed to test the mechanics of the MAAP5 interface when used with the Dynamic Event Tree (Hybrid Dynamic Event Tree) methodology.

- Original Author:

- alfoa

- Creation date: 
- 2016-07-01

- The classes tested in this test are:

- Models.Code.MAAP5, Samplers.DynamicEventTree

- Since the creation of this test, the following main revisions have been performed:

1. revision info:

- author : maljdan

- date : 2016-07-05

- description: Fixing XSD and user manual to match XSD and code.

2. revision info:

- author : alfoa

- date : 2016-09-01

- description: Close \#650

3. revision info:

- author : talbpaul

- date : 2016-09-15

- description: Test updates

4. revision info:

- author : talbpaul

- date : 2016-10-25

- description: removed dim in OutStream Plot for validation purposes

5. revision info:

- author : alfoa

- date : 2016-11-15

- description: Updated input files

6. revision info:

- author : alfoa

- date : 2017-01-21

- description: Adding this test description.

7. revision info:

- author : alfoa

- date : 2020-10-16

- description: Removed plots since are not checked in this test. 


\subsubsection{FRAMEWORK CODEINTERFACETESTS.MAAP5INTERFACEADETSAMPLING}

This test can be found at ". / raven/tests/framework/CodeInterfaceTests/MAAP5/ test_maap5_code_interface_adaptive_det.xml”. This test can be called executing the following command:

./run_tests --re=framework/CodeInterfaceTests.MAAP 5 interfaceADET \$ampling

or

./run_framework_tests --re=framework/CodeInterfaceTests.MAAP5interfaceADET

- Test Description:

- An example of using the the Model Code of type MAAP5. This test is aimed to test the mechanics of the MAAP5 interface when used with the Adaptive Dynamic Event Tree methodology.

- Original Author:

- alfoa

- Creation date:

- 2016-07-11

- The classes tested in this test are:

- Models.Code.MAAP5, Samplers.AdaptiveDynamicEventTree

- Since the creation of this test, the following main revisions have been performed:

1. revision info:

- author : alfoa

- date : 2016-07-21

- description: Fixed XSD

2. revision info:

- author : alfoa

- date : 2016-07-27

- description: modified xml input tests for MAAP5

3. revision info:

- author : alfoa 
- date : 2017-01-21

- description: Adding this test description.

4. revision info:

- author : maljdan

- date : 2017-05-03

- description: Reducing batch size to ensure consistent execution path

5. revision info:

- author : alfoa

- date : 2019-03-07

- description: Modified syntax in Functions as for issue \#934

\subsubsection{FRAMEWORK CODEINTERFACETESTS.MAAP5INTERFACEAHDETSAMPLING}

This test can be found at ". / raven/tests/framework/CodeInterfaceTests/MAAP 5 / test_maap5_code_interface_adaptive_hybrid_det.xml”. This test can be called executing the following command:

. Irun_tests --re=framework/CodeInterfaceTests.MAAP 5 interfaceAHDETSampling

or

./run_framework_tests --re=framework/CodeInterfaceTests.MAAP 5 interfaceAHDE

- Test Description:

- An example of using the the Model Code of type MAAP5. This test is aimed to test the mechanics of the MAAP5 interface when used with the Adaptive Dynamic Event Tree methodology (Adaptive Hybrid Dynamic Event Tree).

- Original Author:

- alfoa

- Creation date:

- 2016-07-11

- The classes tested in this test are:

- Models.Code.MAAP5, Samplers.AdaptiveDynamicEventTree

- Since the creation of this test, the following main revisions have been performed: 
1. revision info:

- author : alfoa

- date : 2016-07-21

- description: Fixed XSD

2. revision info:

- author : alfoa

- date : 2016-07-27

- description: Modified xml input tests for MAAP5

3. revision info:

- author : alfoa

- date : 2017-01-21

- description: Adding this test description.

4. revision info:

- author : maljdan

- date : 2017-05-03

- description: Reducing batch size to ensure consistent execution path

5. revision info:

- author : alfoa

- date : 2019-03-07

- description: Modified syntax in Functions as for issue \#934

\subsubsection{FRAMEWORK CODEINTERFACETESTS.MAAP5INTERFACEDETSAMPLINGMULTIBRA}

This test can be found at ". / raven/tests/framework/CodeInterfaceTests/MAAP5/ test_maap5_code_interface_det_multibranch.xml". This test can be called executing the following command:

. /run_tests --re=framework/CodeInterfaceTests.MAAP 5 interfaceDETSamplingMul

or

./run_framework_tests --re=framework/CodeInterfaceTests.MAAP 5interfaceDETS

- Test Description:

- An example of using the the Model Code of type MAAP5. This test is aimed to test the mechanics of the MAAP5 interface when used with the Dynamic Event Tree methodology, characterized my a Multi-Branch sampling approach. 
- Original Author:

- alfoa

- Creation date:

- 2016-12-16

- The classes tested in this test are:

- Models.Code.MAAP5, Samplers.DynamicEventTree

- Since the creation of this test, the following main revisions have been performed:

1. revision info:

- author : alfoa

- date : 2017-01-21

- description: Adding this test description.

\subsubsection{FRAMEWORK CODEINTERFACETESTS RAVEN.CODE}

This test can be found at ". / raven/tests / framework/CodeInterfaceTests/RAVEN/ code.xml". This test can be called executing the following command:

./run_tests --re=framework/CodeInterfaceTests/RAVEN. Code

or

./run_framework_tests --re=framework/CodeInterfaceTests/RAVEN. Code

- Test Description:

- Basic test of the raven-runs-raven interface. Outer raven runs a grid sampling by changing the upper and lower bounds of the sampledVars distribution on the inner raven. Inner raven runs Grid samples, does basic stats, and returns the mean, sigma to the outer raven. Specifically uses both a File and Function in the inner, to cover testing

- Original Author:

- talbpaul

- Creation date:

- 2019-10-16 
- The classes tested in this test are:

- Models.Code.RAVEN

- Since the creation of this test, the following main revisions have been performed:

1. revision info:

- author : ZHOUJ2

- date : 2019-10-17

- description: This test will run twice to make sure if a working directory is already exist, warning message will be shown up. Assures the results and the subdirectory generated are identical.

\subsubsection{FRAMEWORK CODEINTERFACETESTS RATTLESNAKEINTERFACE.TESTGRIDRATTL}

This test can be found at ". / raven/tests / framework/CodeInterfaceTests/RattlesnakeInte: test_grid_rattlesnake.xml". This test can be called executing the following command:

. Irun_tests --re=framework/CodeInterfaceTests/RattlesnakeInterface.testGri

or

. Irun_framework_tests --re=framework/CodeInterfaceTests/RattlesnakeInterfa

- Test Description:

- An example of using the code interface RattleSnake when XS need to be perturbed. This test is aimed to check the functionality (running the code) of the Rattlesnake interface when a Grid sampling approach is used.

- Original Author:

- wangc

- Creation date:

- 2016-04-21

- The classes tested in this test are:

- Models.Code.Rattlesnake, Sampler.Grid

- Since the creation of this test, the following main revisions have been performed: 
1. revision info:

- author : talbpaul

- date : 2016-09-15

- description: Test updates

2. revision info:

- author : alfoa

- date : 2017-01-21

- description: Adding this test description.

\subsubsection{FRAMEWORK CODEINTERFACETESTS RATTLESNAKEINTERFACE.TESTMC}

This test can be found at ". / raven/tests/framework/CodeInterfaceTests/RattlesnakeInte test_mc_rattlesnake.xml". This test can be called executing the following command:

./run_tests --re=framework/CodeInterfaceTests/RattlesnakeInterfade.testMC

or

./run_framework_tests --re=framework/CodeInterfaceTests/RattlesnakeInterfa

- Test Description:

- An example of using the code interface RattleSnake when XS need to be perturbed. This test is aimed to check the functionality (running the code) of the Rattlesnake interface when a MonteCarlo sampling approach is used.

- Original Author:

- wangc

- Creation date:

- 2016-04-21

- The classes tested in this test are:

- Models.Code.Rattlesnake, Sampler.MonteCarlo

- Since the creation of this test, the following main revisions have been performed:

1. revision info:

- author: wangc 
- date : 2016-08-04

- description: Modify test to perturb transport XS and regold the tests

2. revision info:

- author : talbpaul

- date : 2016-09-15

- description: Test updates

3. revision info:

- author : alfoa

- date : 2017-01-21

- description: Adding this test description.

\subsubsection{7ＦRAMEWORK CODEINTERFACETESTS RATTLESNAKEINTERFACE.TESTMCRATTLES}

This test can be found at ". / raven/tests/framework/CodeInterfaceTests/RattlesnakeInte test_perturb_rattlesnake_only.xml". This test can be called executing the following command:

./run_tests --re=framework/CodeInterfaceTests/RattlesnakeInterface.testMCR

or

. Irun_framework_tests --re=framework/CodeInterfaceTests/RattlesnakeInterfa

- Test Description:

- An example of using the code interface RattleSnake. This test is aimed to check the functionality (running the code) of the Rattlesnake interface (perturbing only RattleSnake and not the YAK library) when a MonteCarlo sampling approach is used.

- Original Author:

- wangc

- Creation date:

$-2016-04-21$

- The classes tested in this test are:

- Models.Code.Rattlesnake, Sampler.MonteCarlo

- Since the creation of this test, the following main revisions have been performed: 
1. revision info:

- author : talbpaul

- date : 2016-09-15

- description: other test updates

2. revision info:

- author : alfoa

- date : 2017-01-21

- description: Adding this test description.

\subsubsection{FRAMEWORK CODEINTERFACETESTS RATTLESNAKEINTERFACE.TESTMCYAK}

This test can be found at ". / raven/tests/framework/CodeInterfaceTests/RattlesnakeInte test_perturb_yak_only.xml". This test can be called executing the following command:

./run_tests --re=framework/CodeInterfaceTests/RattlesnakeInterface.testMCY

or

./run_framework_tests --re=framework/CodeInterfaceTests/RattlesnakeInterfa

- Test Description:

- An example of using the code interface RattleSnake when XS need to be perturbed. This test is aimed to check the functionality (running the code) of the Rattlesnake interface (perturbing only YAK library) when a MonteCarlo sampling approach is used.

- Original Author:

- wangc

- Creation date:

- 2016-04-21

- The classes tested in this test are:

- Models.Code.Rattlesnake, Sampler.MonteCarlo

- Since the creation of this test, the following main revisions have been performed:

1. revision info:

- author: wangc 
- date : 2016-08-04

- description: Modify test to perturb transport XS and regold the tests

2. revision info:

- author : talbpaul

- date : 2016-09-15

- description: Test updates

3. revision info:

- author : alfoa

- date : 2017-01-21

- description: Adding this test description.

\subsubsection{FRAMEWORK CODEINTERFACETESTS COBRATF.TEST1}

This test can be found at ". / raven/tests / framework/CodeInterfaceTests/CobraTF/ test1_cobratf_code_interface.xml”. This test can be called executing the following command:

./run_tests --re=framework/CodeInterfaceTests/CobraTF.test1

or

./run_framework_tests --re=framework/CodeInterfaceTests/CobraTF.test 1

- Test Description:

- An example of using the CobraTF code interface. This test is aimed to test the mechanics of the interface using a MonteCarlo sampling strategy.

- Original Author:

- jyoo,alptezbasaran

- Creation date:

- 2018-04-03

- The classes tested in this test are:

- Models.Code.CobraTF

- Since the creation of this test, the following main revisions have been performed: 
1. revision info:

- author: jyoo

- date : 2018-04-03

- description: New CobraTF test case

2. revision info:

- author : alptezbasaran

- date : 2018-08-15

- description: Modification for new sampling method (generic)

3. revision info:

- author : alfoa

- date : 2020-10-31

- description: Added csv xml node in the Code block for showing how to use it.

\subsubsection{FRAMEWORK CODEINTERFACETESTS COBRATF.TEST2}

This test can be found at ". / raven/tests/framework/CodeInterfaceTests/CobraTF/ test2_cobratf_code_interface.xml". This test can be called executing the following command:

./run_tests --re=framework/CodeInterfaceTests/CobratF.test2

or

./run_framework_tests --re=framework/CodeInterfaceTests/CobratF. Hest2

- Test Description:

- An example of using the CobraTF code interface. This test is aimed to test the mechanics of the interface using two sampling strategies in cascade (Monte Carlo and Grid sampling).

- Original Author:

- jyoo,alptezbasaran

- Creation date:

- 2018-04-03

- The classes tested in this test are: 
- Models.Code.CobraTF

- Since the creation of this test, the following main revisions have been performed:

1. revision info:

- author : jyoo

- date : 2018-04-03

- description: New CobraTF test case

2. revision info:

- author : alptezbasaran

- date : 2018-08-15

- description: Modification for new sampling method (generic)

3. revision info:

- author : alfoa

- date : 2020-10-31

- description: Added csv xml node in the Code block for showing how to use it.

\subsubsection{FRAMEWORK CODEINTERFACETESTS COBRATF.TEST3}

This test can be found at “. / raven/tests / framework/CodeInterfaceTests/CobraTF/ test3_cobratf_code_interface.xml". This test can be called executing the following command:

./run_tests --re=framework/CodeInterfaceTests/CobraTF.test 3

or

. /run_framework_tests --re=framework/CodeInterfaceTests/CobraTF. test3

- Test Description:

- An example of using the CobraTF code interface. This test is aimed to test the mechanics of the interface using two supplementary input files (vuq_params.txt and vuq_mult.txt). All input files are to be perturbed

- Original Author:

- alptezbasaran

- Creation date: 
$-2018-07-22$

- The classes tested in this test are:

- Models.Code.CobraTF

- Since the creation of this test, the following main revisions have been performed:

1. revision info:

- author : alptezbasaran

- date : 2018-07-22

- description: New CobraTF test case

\subsubsection{FRAMEWORK CODEINTERFACETESTS COBRATF.TEST4}

This test can be found at ". / raven/tests / framework/CodeInterfaceTests/CobraTF / test4_cobratf_code_interface.xml". This test can be called executing the following command:

. /run_tests --re=framework/CodeInterfaceTests/CobraTF.test 4

or

./run_framework_tests --re=framework/CodeInterfaceTests/CobraTF. test 4

- Test Description:

- An example of using the CobraTF code interface. This test is aimed to test the mechanics of the interface using a supplementary input file (vuq_params.txt). All input files are to be perturbed. Newly added heat slab output parser capabilities are tested.

- Original Author:

- alptezbasaran

- Creation date:

- 2018-07-24

- The classes tested in this test are:

- Models.Code.CobraTF

- Since the creation of this test, the following main revisions have been performed: 
1. revision info:

- author : alptezbasaran

- date : 2018-07-24

- description: New CobraTF test case

\subsubsection{FRAMEWORK CODEINTERFACETESTS COBRATF.TEST5}

This test can be found at ". / raven/tests/framework/CodeInterfaceTests/CobraTF/ test5_cobratf_code_interface.xml”. This test can be called executing the following command:

./run_tests --re=framework/CodeInterfaceTests/CobratF.test5

or

./run_framework_tests --re=framework/CodeInterfaceTests/CobraTF. test5

- Test Description:

- An example of using the CobraTF code interface. This test is aimed to test the output conversion if input file name is 'deck.inp'

- Original Author:

- alptezbasaran

- Creation date:

- 2018-07-26

- The classes tested in this test are:

- Models.Code.CobraTF

- Since the creation of this test, the following main revisions have been performed:

1. revision info:

- author : alptezbasaran

- date : 2018-07-26

- description: New CobraTF test case 


\subsubsection{FRAMEWORK CODEINTERFACETESTS RELAP5.RELAP5INTERFACETESTSINGLERUN}

This test can be found at ". / raven/tests/framework/CodeInterfaceTests/RELAP5/ test_relap5_code_interface_single_run.xml”. This test can be called executing the following command:

./run_tests --re=framework/CodeInterfaceTests/RELAP 5.RELAP 5interfaceTestSi

or

./run_framework_tests --re=framework/CodeInterfaceTests/RELAP 5.RELAP 5inter

- Test Description:

- An example of using the RELAP5 code interface with a SingleRun step. This test is aimed to test the mechanics of the interface (no executable).

- Original Author:

- alfoa

- Creation date:

- 2017-04-01

- The classes tested in this test are:

- Models.Code.RELAP5

\subsubsection{FRAMEWORK CODEINTERFACETESTS RELAP5.RELAP5INTERFACETESTALIASALIAS}

This test can be found at ". / raven/tests/framework/CodeInterfaceTests/RELAP 5/ test_relap5_code_interface_alias.xml". This test can be called executing the following command:

./run_tests --re=framework/CodeInterfaceTests/RELAP 5.RELAP5interfaceTestAl.

or

./run_framework_tests --re=framework/CodeInterfaceTests/RELAP 5.RELAP 5 inter

- Test Description: 
- An example of using the RELAP5 code interface. This test is aimed to test the mechanics of the interface (no executable), when the RAVEN alias system is used.

- Original Author:

- alfoa

- Creation date:

- 2016-11-28

- The classes tested in this test are:

- Models.Code.RELAP5

- Since the creation of this test, the following main revisions have been performed:

1. revision info:

- author : alfoa

- date : 2017-01-21

- description: Adding this test description.

2. revision info:

- author : alfoa

- date : 2020-10-31

- description: Added csv xml node in the Code block for showing how to use it.

\subsubsection{FRAMEWORK CODEINTERFACETESTS RELAP5.RELAP5INTERFACEMULTDECKTEST}

This test can be found at ". / raven/tests / framework/CodeInterfaceTests/RELAP 5/ test_relap5_code_interface_multideck.xml". This test can be called executing the following command:

. Irun_tests --re=framework/CodeInterfaceTests/RELAP 5.RELAP5interfaceMultDe

or

./run_framework_tests --re=framework/CodeInterfaceTests/RELAP5.RELAP 5 inter

- Test Description:

- An example of using the Relap5 code interface. This test is aimed to test the mechanics of the interface (no executable) when a multi-deck type of input needs to be perturbed. 
- Original Author:

- alfoa

- Creation date:

- 2016-05-03

- The classes tested in this test are:

- Models.Code.Relap5

- Since the creation of this test, the following main revisions have been performed:

1. revision info:

- author : talbpaul

- date : 2016-09-15

- description: Test updates

2. revision info:

- author : alfoa

- date : 2017-01-21

- description: Adding this test description.

\subsubsection{FRAMEWORK CODEINTERFACETESTS RELAP5.RELAP5INTERFACEMULTDECKTEST}

This test can be found at ". / raven/tests/framework/CodeInterfaceTests/RELAP5/ test_relap5_code_interface_multideck_choosing_deck_output.xml”. This test can be called executing the following command:

. Irun_tests --re=framework/CodeInterfaceTests/RELAP5.RELAP 5 interfaceMultDe

or

. /run_framework_tests --re=framework/CodeInterfaceTests/RELAP 5.RELAP 5 inter

- Test Description:

- An example of using the RELAP5 code interface. This test is aimed to test the mechanics of the interface (no executable), when a multi deck input is used (and needs to be perturbed).

- Original Author: 
- alfoa

- Creation date:

- 2016-07-07

- The classes tested in this test are:

- Models.Code.Relap5

- Since the creation of this test, the following main revisions have been performed:

1. revision info:

- author : talbpaul

- date : 2016-09-15

- description: Test updates

2. revision info:

- author : alfoa

- date : 2017-01-21

- description: Adding this test description.

\subsubsection{8ＦRAMEWORK CODEINTERFACETESTS RELAP5.RELAP5INTERFACETESTALIASANDN}

This test can be found at" . / raven/tests/framework/CodeInterfaceTests/RELAP 5 / test_relap5_code_ensemble_alias_metadata.xml". This test can be called executing the following command:

. Irun_tests --re=framework/CodeInterfaceTests/RELAP5.RELAP 5interfaceTestAl or

./run_framework_tests --re=framework/CodeInterfaceTests/RELAP 5.RELAP 5 inter

- Test Description:

- An example of using the RELAP5 code interface. This test is aimed to test the mechanics of the interface (no executable), when the RAVEN alias system is used and some metadata (i.e. restart prefix) needs to be passed in. This test executes 2 relap5 runs for each realization: the first one establishes the steady state, the second one restarts the calculation and runs a transient.

- Original Author: 
- alfoa

- Creation date:

- 2017-02-19

- The classes tested in this test are:

- Models.Code.RELAP5

- Since the creation of this test, the following main revisions have been performed:

1. revision info:

- author : alfoa

- date : 2017-01-21

- description: Adding this test description.

2. revision info:

- author : alfoa

- date : 2019-02-13

- description: Modified description

\subsubsection{FRAMEWORK CODEINTERFACETESTS RELAP5.RELAP5INTERFACEOPERATORXMLN}

This test can be found at ". / raven/tests/framework/CodeInterfaceTests/RELAP 5/ test_relap5_code_interface_operator.xml”. This test can be called executing the following command:

. Irun_tests --re=framework/CodeInterfaceTests/RELAP 5.RELAP 5interfaceOperat

or

./run_framework_tests --re=framework/CodeInterfaceTests/RELAP5.RELLAP 5 inter

- Test Description:

- An example of using the RELAP5 code interface. This test is aimed to test the mechanics of the interface (no executable).

- Original Author:

- alfoa

- Creation date: 
- 2019-02-04

- The classes tested in this test are:

- Models.Code.RELAP5

- Since the creation of this test, the following main revisions have been performed:

1. revision info:

- author : alfoa

- date : 2019-02-04

- description: Adding this test description.

\subsubsection{FRAMEWORK CODEINTERFACETESTS RELAP5.INSSRELAP5JAPANINTERFACESAMP}

This test can be found at ". / raven/tests / framework/CodeInterfaceTests/RELAP 5/ test_relap5_code_inss.xml". This test can be called executing the following command:

. Irun_tests --re=framework/CodeInterfaceTests/RELAP 5. INSSrelap5JapanInterf

or

. Irun_framework_tests --re=framework/CodeInterfaceTests/RELAP 5. In SSrelap5 J

- Test Description:

- An example of using the RELAP5 code interface (modified version of INSS (Japan)). This test is aimed to test the mechanics of the interface (no executable), that perturbs the RELAP5 input deck and the auxiliary file that the RELAP5 version by INSS reads.

- Original Author:

- alfoa

- Creation date:

$-2016-07-21$

- The classes tested in this test are:

- Models.Code.Relap5inssJp

- Since the creation of this test, the following main revisions have been performed:

1. revision info: 
- author : alfoa

- date : 2016-10-24

- description: finalized branch

2. revision info:

- author : alfoa

- date : 2016-10-25

- description: added xsd

3. revision info:

- author : alfoa

- date : 2016-10-25

- description: ok

4. revision info:

- author : alfoa

- date : 2017-01-21

- description: Adding this test description.

\subsubsection{FRAMEWORK CODEINTERFACETESTS RELAP5.RELAP5INTERFACEDYNAMICET}

This test can be found at ". / raven/tests/framework/CodeInterfaceTests/RELAP5/ test_relap5_code_interface_dynamic_event_tree.xml”. This test can be called executing the following command:

. Irun_tests --re=framework/CodeInterfaceTests/RELAP 5.RELAP 5 interfaceDynami

or

. /run_framework_tests --re=framework/CodeInterfaceTests/RELAP 5 .RELAP 5 inter

- Test Description:

- An example of using the RELAP5 code interface with the Dynamic Event Tree approach (only aleatory uncertanties). This test shows how to set up the Dynamic Event Tree for RELAP5 (i.e. usage of trips) and aliases.

- Original Author:

- alfoa

- Creation date: 
- 2020-11-30

- The classes tested in this test are:

- Models.Code.RELAP5

- Since the creation of this test, the following main revisions have been performed:

1. revision info:

- author : alfoa

- date : 2020-12-01

- description: Added hierarchical dataset

\subsubsection{FRAMEWORK CODEINTERFACETESTS RELAP5.RELAP5INTERFACEHYBRIDDYNAMI}

This test can be found at ". / raven/tests / framework/CodeInterfaceTests/RELAP 5/ test_relap5_code_interface_hybrid_dynamic_event_tree.xml". This testcan be called executing the following command:

. Irun_tests --re=framework/CodeInterfaceTests/RELAP 5.RELAP 5 interfaceHybrid

or

. Irun_framework_tests --re=framework/CodeInterfaceTests/RELAP 5.RELLAP 5 inter

- Test Description:

- An example of using the RELAP5 code interface with the Hybrid Dynamic Event Tree approach (aleatory uncertanties and epistemic uncertanties). This test shows how to set up the Dynamic Event Tree for RELAP5 (i.e. usage of trips) and aliases.

- Original Author:

- alfoa

- Creation date:

- 2020-11-30

- The classes tested in this test are:

- Models.Code.RELAP5

- Since the creation of this test, the following main revisions have been performed: 
1. revision info:

- author : alfoa

- date : 2020-12-01

- description: Added hierarchical dataset

\subsubsection{FRAMEWORK CODEINTERFACETESTS ACCELERATEDCFD.PERTURBACFD1}

This test can be found at ". / raven/tests/framework/CodeInterfaceTests/AcceleratedCFD/ test_perturb_acdf.xml". This test can be called executing the following command:

./run_tests --re=framework/CodeInterfaceTests/AcceleratedCFD.perturbACFD1

or

./run_framework_tests --re=framework/CodeInterfaceTests/AcceleratedCFD.per

- Test Description:

- An example of using the AcceleratedCFD code interface. This test is aimed to test the mechanics of the interface (no executable).

- Original Author:

- alfoa

- Creation date:

- 2020-11-03

- The classes tested in this test are:

- Models.Code.AcceleratedCFD

\subsubsection{FRAMEWORK CODEINTERFACETESTS PHISICS.MRTAU}

This test can be found at ". / raven/tests / framework/CodeInterfaceTests / PHISICS / test_mrtau_standalone.xml". This test can be called executing the following command:

./run_tests --re=framework/CodeInterfaceTests/PHISICS.mrtau

or 
./run_framework_tests --re=framework/CodeInterfaceTests/PHISICS.mitau

- Test Description:

- This test is aimed to test the mechanics of the interface between RAVEN and PHISICS, using the burnup code MRTAU. The decay constant of the PU241 is perturbed and the mass of the NP237. NOTE: The input files and data have been epurated from nuclear data; consequentially the case is not runable for real with PHISICS (MRTAU). The code will error out.

- Original Author:

- rouxpn

- Creation date:

- 2018-04-23

- The classes tested in this test are:

- Models.Code.Phisics

- Since the creation of this test, the following main revisions have been performed:

1. revision info:

- author: alfoa

- date : 2018-07-08

- description: modified to make it work and to remove nuclear data

\subsubsection{FRAMEWORK CODEINTERFACETESTS PHISICS.PHISICS}

This test can be found at ". / raven/tests/framework/CodeInterfaceTests/PHISICS/ test_phisics_interface.xml". This test can be called executing the following command:

./run_tests --re=framework/CodeInterfaceTests/PHISICS.phisics

or

./run_framework_tests --re=framework/CodeInterfaceTests/PHISICS.中hisics

- Test Description: 
- This test is aimed to test the mechanics of the interface between RAVEN and PHISICS, using the full-blonde PHISICS executable. Qvalues, decay constants, initial densities and cross sections get perturbed. NOTE: The input files and data have been epurated from nuclear data; consequentially the case is not runable for real with PHISICS. The code will error out.

- Original Author:

- rouxpn

- Creation date:

- 2018-04-23

- The classes tested in this test are:

- Models.Code.Phisics

- Since the creation of this test, the following main revisions have been performed:

1. revision info:

- author : alfoa

- date : 2018-07-08

- description: modified to make it work and to remove nuclear data

\subsubsection{FRAMEWORK CODEINTERFACETESTS PHISICS.PHISICSRELAP5}

This test can be found at ". / raven/tests/framework/CodeInterfaceTests/PHISICS / test_relapphisics_interface.xml". This test can be called executing the following command:

./run_tests --re=framework/CodeInterfaceTests/PHISICS.phisicsRelap5

or

./run_framework_tests --re=framework/CodeInterfaceTests/PHISICS.phisicsRel

- Test Description:

- This test is aimed to test the mechanics of the combined interface PHISICS/RELAP5 using the RELAP5 (coupled with PHISICS) executable. The density of the I135 (PHISICS side) and the initial temperature for time-dep volume 201 are perturbed. NOTE: The input files and data have been epurated from nuclear data; consequentially the case is not runable for real with PHISICS and RELAP5-3D coupled. The coupled suite will error out. 
- Original Author:

- rouxpn

- Creation date:

$-2018-04-25$

- The classes tested in this test are:

- Models.Code.PhisicsRelap5

- Since the creation of this test, the following main revisions have been performed:

1. revision info:

- author : rouxpn

- date : 2018-08-08

- description: PHISICS RELAP suite test case added

\subsubsection{FRAMEWORK CODEINTERFACETESTS.MOOSEPARSER}

This test can be found at ". / raven/tests / framework/CodeInterfaceTests/MoosEparser/ test_MOOSE_parser.xml". This test can be called executing the following command:

./run_tests --re=framework/CodeInterfaceTests.MoosEparser

or

./run_framework_tests --re=framework/CodeInterfaceTests.MoosEparger

- Test Description:

- A check to make sure the Raven produced input file is the same as the original parsed MOOSE input file.

- Original Author:

- zabrax

- Creation date:

- 2018-09-21

- The classes tested in this test are:

- Models.Code.MooseBasedApp 


\subsubsection{FRAMEWORK PCA SPARSEGRID NORMAL}

This test can be found at ". / raven/tests/framework/pca_sparseGridCollocation/ test_scgpc_normal.xml". This test can be called executing the following command:

. Irun_tests --re=framework/pca_sparseGrid/normal

or

./run_framework_tests --re=framework/pca_sparseGrid/normal

- Test Description:

- Tests the SparseGridCollocation sampler combined with input reductions via PCA method, using correlated multivariate normal distributions. Uses distributions with nonzero means.

- Original Author:

- wangc

- Creation date:

$-2015-11-23$

- The classes tested in this test are:

- Samplers.SparseGridCollocation,SupervisedLearning.GaussPolynomialROM

\subsubsection{FRAMEWORK PCA SPARSEGRID MVNUNCORRELATED}

This test can be found at ". / raven/tests/framework/pca_sparseGridCollocation/ test_scgpc_uncorrelatedMVN.xml". This test can be called executing the following command:

./run_tests --re=framework/pca_sparseGrid/MVNUncorrelated

or

./run_framework_tests --re=framework/pca_sparseGrid/MVNUncorrelated

- Test Description: 
- Tests the SparseGridCollocation sampler combined with input reductions via PCA method, using uncorrelated multivariate normal distributions.

- Original Author:

- wangc

- Creation date:

$-2015-11-23$

- The classes tested in this test are:

- Samplers.SparseGridCollocation,SupervisedLearning.GaussPolynomialROM

\subsubsection{FRAMEWORK PCA SPARSEGRID MVNCORRELATED}

This test can be found at ". / raven/tests/framework/pca_sparseGridCollocation/ test_scgpc_correlatedMVN.xml". This test can be called executing the following command:

./run_tests --re=framework/pca_sparseGrid/MVNCorrelated

or

./run_framework_tests --re=framework/pca_sparseGrid/MVNCorrelated

- Test Description:

- Tests the SparseGridCollocation sampler combined with input reductions via PCA method, using correlated multivariate normal distributions.

- Original Author:

- wangc

- Creation date:

- 2015-11-23

- The classes tested in this test are:

- Samplers.SparseGridCollocation,SupervisedLearning.GaussPolynomialROM 


\subsubsection{FRAMEWORK PCA SPARSEGRID ATTENUATIONTEST}

This test can be found at ". / raven/tests/framework/pca_sparseGridCollocation/ test_attenuation.xml". This test can be called executing the following command:

. /run_tests --re=framework/pca_sparseGrid/attenuationTest

or

./run_framework_tests --re=framework/pca_sparseGrid/attenuationTest

- Test Description:

- Tests the SparseGridCollocation sampler combined wit input reductions via PCA method, using uncorrelated multivariate normal distributions.

- Original Author:

- wangc

- Creation date:

$-2015-11-23$

- The classes tested in this test are:

- Samplers.SparseGridCollocation,SupervisedLearning.GaussPolynomialROM

- Since the creation of this test, the following main revisions have been performed:

1. revision info:

- author : talbpaul

- date : 2019-01-09

- description: moving from ROM-file to ROM-dataobject

\subsubsection{FRAMEWORK POSTPROCESSORS METRIC TEST METRIC}

This test can be found at ". / raven/tests/framework/PostProcessors/Metrics/ test_metric.xml”. This test can be called executing the following command:

./run_tests --re=framework/PostProcessors/Metric/test_metric

or 
./run_framework_tests --re=framework/PostProcessors/Metric/test_metric

- Test Description:

- This test checks the Metric PostProcessor

- Original Author:

- wangc

- Creation date:

- 2017-08-29

- The classes tested in this test are:

- PostProcessors.Metric

- Since the creation of this test, the following main revisions have been performed:

1. revision info:

- author: wangc

- date : 2017-12-06

- description: convert to using new data objects

2. revision info:

- author: wangc

- date : 2017-11-20

- description: restructure metric system, add time-dependent capability

\subsubsection{FRAMEWORK POSTPROCESSORS METRIC TEST MULTIPLE INPUT OB- JECTS}

This test can be found at "./raven/tests/framework/PostProcessors/Metrics/ test_metric_multiple_input_objects.xml". This test can be called executing the following command:

./run_tests --re=framework/PostProcessors/Metric/test_multiple_input_objec

or

./run_framework_tests --re=framework/PostProcessors/Metric/test_nultiple_i 
- Test Description:

- This test checks the Metric PostProcessor to accept multiple input data objects

- Original Author:

- wangc

- Creation date:

- 2017-08-30

- The classes tested in this test are:

- PostProcessors.Metric

- Since the creation of this test, the following main revisions have been performed:

1. revision info:

- author: wangc

- date : 2017-12-06

- description: convert to using new data objects

2. revision info:

- author: wangc

- date : 2017-11-20

- description: restructure metric system, add time-dependent capability

\subsubsection{FRAMEWORK POSTPROCESSORS METRIC TEST METRIC SKL}

This test can be found at "./raven/tests/framework/PostProcessors/Metrics/ test_metric_skl.xml". This test can be called executing the following command:

./run_tests --re=framework/PostProcessors/Metric/test_metric_skl

or

.Irun_framework_tests --re=framework/PostProcessors/Metric/test_metric_skl

- Test Description:

- This test checks metrics from ScikitLearn library

- Original Author: 
- wangc

- Creation date:

- 2017-08-30

- The classes tested in this test are:

- PostProcessors.Metric

- Since the creation of this test, the following main revisions have been performed:

1. revision info:

- author : talbpaul

- date : 2019-01-09

- description: no printing directly from postprocessor to file

2. revision info:

- author: wangc

- date : 2017-12-06

- description: convert to using new data objects

3. revision info:

- author: wangc

- date : 2017-11-20

- description: restructure metric system, add time-dependent capability

\subsubsection{FRAMEWORK POSTPROCESSORS METRIC TEST COMPARISON STATS MET- RICS}

This test can be found at ". /raven/tests/framework/PostProcessors/Metrics/ test_comparison_stats_metrics.xml”. This test can be called executing the following command:

.Irun_tests --re=framework/PostProcessors/Metric/test_comparison_stats_met

or

./run_framework_tests --re=framework/PostProcessors/Metric/test_Gomparison

- Test Description:

- This test checks the Comparison Statistics Metric 
- Original Author:

- joshua-cogliati-inl

- Creation date:

- 2017-09-12

- The classes tested in this test are:

- PostProcessors.Metric

- Since the creation of this test, the following main revisions have been performed:

1. revision info:

- author: wangc

- date : 2017-12-06

- description: convert to using new data objects

\subsubsection{FRAMEWORK POSTPROCESSORS METRIC TEST COMPARISON STATS MET- RICS GRID}

This test can be found at "./raven/tests/framework/PostProcessors/Metrics/ test_comparison_stats_metrics_grid.xml”. This test can be called executing the following command:

./run_tests --re=framework/PostProcessors/Metric/test_comparison_stats_met

or

./run_framework_tests --re=framework/PostProcessors/Metric/test_Gomparison

- Test Description:

- This test checks the Comparison Statistics Metrics with a Grid.

- Original Author:

- joshua-cogliati-inl

- Creation date:

- 2017-09-13

- The classes tested in this test are: 
- PostProcessors.Metric

- Since the creation of this test, the following main revisions have been performed:

1. revision info:

- author: wangc

- date : 2017-12-06

- description: convert to using new data objects

\subsubsection{FRAMEWORK POSTPROCESSORS METRIC TEST COMPARISON STATS MET- RICS}

This test can be found at "./raven/tests/framework/PostProcessors/Metrics/ test_comparison_stats_metrics_dists.xml". This test can be called executing the following command:

./run_tests --re=framework/PostProcessors/Metric/test_comparison_stats_met

or

. Irun_framework_tests --re=framework/PostProcessors/Metric/test__omparison

- Test Description:

- This test checks the Comparison Statistics Metric

- Original Author:

- joshua-cogliati-inl

- Creation date:

- 2017-09-12

- The classes tested in this test are:

- PostProcessors.Metric

- Since the creation of this test, the following main revisions have been performed:

1. revision info:

- author: wangc

- date : 2017-12-06

- description: convert to using new data objects 


\subsubsection{FRAMEWORK.TEST DISTRIBUTIONS}

This test can be found at "./raven/tests/framework/PostProcessors/Metrics/ unit_test_comparison_statistics.py". This test can be called executing the following command:

./run_tests --re=framework.test_distributions

or

./run_framework_tests --re=framework.test_distributions

- Test Description:

- This test is a Unit Test for the comparison statistics metric. It checks all the functions that are available to RAVEN internally.

- Original Author:

$-\operatorname{cog} \mathrm{jj}$

- Creation date:

- 2017-09-08

- The classes tested in this test are:

- Since the creation of this test, the following main revisions have been performed:

1. revision info:

- author: alfoa

- date : 2018-05-10

- description: Added Log Uniform distribution unit test

\subsubsection{FRAMEWORK POSTPROCESSORS METRIC TESTMETRICTD}

This test can be found at "./raven/tests/framework/PostProcessors/Metrics/ test_metric_td.xml". This test can be called executing the following command:

./run_tests --re=framework/PostProcessors/Metric/testMetricTD 
Or

./run_framework_tests --re=framework/PostProcessors/Metric/testMetrictD

- Test Description:

- This test checks the Metric PostProcessor to handle time-dependent data

- Original Author:

- wangc

- Creation date:

- 2017-11-20

- The classes tested in this test are:

- PostProcessors.Metric

- Since the creation of this test, the following main revisions have been performed:

1. revision info:

- author : talbpaul

- date : 2019-01-09

- description: no writing directly to files from postprocessors

\subsubsection{FRAMEWORK POSTPROCESSORS METRIC TEST METRIC SCIPY}

This test can be found at "./raven/tests/framework/PostProcessors/Metrics/ test_metric_scipy.xml”. This test can be called executing the following command:

./run_tests --re=framework/PostProcessors/Metric/test_metric_scipy

or

./run_framework_tests --re=framework/PostProcessors/Metric/test_nhetric_sci]

- Test Description:

- This test checks metrics from Scipy library

- Original Author: 
- wangc

- Creation date:

- 2018-02-16

- The classes tested in this test are:

- PostProcessors.Metric

\subsubsection{FRAMEWORK POSTPROCESSORS METRIC TEST METRIC SCIPY BOOLEAN}

This test can be found at ". /raven/tests/framework/PostProcessors/Metrics/ test_metric_scipy_boolean.xml". This test can be called executing the following command:

./run_tests --re=framework/PostProcessors/Metric/test_metric_scipy_boolean

or

./run_framework_tests --re=framework/PostProcessors/Metric/test_metric_sci

- Test Description:

- This test checks metrics from Scipy library

- Original Author:

- wangc

- Creation date:

- 2018-02-16

- The classes tested in this test are:

- PostProcessors.Metric

\subsubsection{FRAMEWORK POSTPROCESSORS METRIC TEST DTW}

This test can be found at ". /raven/tests/framework/PostProcessors/Metrics/ test_dtw.xml". This test can be called executing the following command:

./run_tests --re=framework/PostProcessors/Metric/test_dtw 
or

./run_framework_tests --re=framework/PostProcessors/Metric/test_dtw

- Test Description:

- This test checks the Metric PostProcessor with DTW metric

- Original Author:

- wangc

- Creation date:

- 2018-02-20

- The classes tested in this test are:

- PostProcessors.Metric

- Since the creation of this test, the following main revisions have been performed:

1. revision info:

- author : talbpaul

- date : 2019-01-09

- description: no writing directly to files from postprocessors

\subsubsection{FRAMEWORK POSTPROCESSORS VALUEDURATION.BASIC}

This test can be found at ". / raven/tests/framework/PostProcessors/ValueDuration/ basic.xml". This test can be called executing the following command:

./run_tests --re=framework/PostProcessors/ValueDuration.Basic

or

./run_framework_tests --re=framework/PostProcessors/ValueDuration. Basic

- Test Description:

- Tests the basic functionality of the ValueDuration postprocessor to create their unique brand of cumulative histograms. 
- Original Author:

- talbpaul

- Creation date:

- 2018-08-28

- The classes tested in this test are:

- PostProcessors.ValueDuration

\subsubsection{FRAMEWORK POSTPROCESSORS ETIMPORTERPOSTPROCESSOR.ET IM- PORTER OPENPSA}

This test can be found at ". / raven/tests / framework/PostProcessors / ETimporterPostProce. test_ETimporter.xml". This test can be called executing the following command:

. Irun_tests --re=framework/PostProcessors/ETimporterPostProcessor.ET_impor

or

. Irun_framework_tests --re=framework/PostProcessors/ETimporterPoßtProcesso

- Test Description:

- Tests of the ETImporter post-processor: it read an event-tree (ET) from an .xml file (eventTree.xml) and it imports the ET structure into a PointSet. Note that the ET needs to be in an OpenPSA format.

- Original Author:

- mandd

- Creation date:

- 2017-11-07

- The classes tested in this test are:

- ETimporter 


\subsubsection{FRAMEWORK POSTPROCESSORS ETIMPORTERPOSTPROCESSOR.ET IM- PORTER OPENPSA SYMBOLIC}

This test can be found at ". / raven/tests / framework/PostProcessors/ETimporterPostProce test_ETimporterSymbolic.xml". This test can be called executing the following command:

.Irun_tests --re=framework/PostProcessors/ETimporterPostProcessor.ET_impor

or

. Irun_framework_tests --re=framework/PostProcessors/ETimporterPoßtProcesso

- Test Description:

- Tests of the ETImporter post-processor: it read an event-tree (ET) from an .xml file (eventTree.xml) and it imports the ET structure into a PointSet. Note that the ET needs to be in an OpenPSA format. In particular, this tests checks that capability of the PP to manage symbolic ID for the ET sequences. Note that in this configuration, a mapping file is generated

- Original Author:

- mandd

- Creation date:

- 2017-11-07

- The classes tested in this test are:

- ETimporter

\subsubsection{FRAMEWORK POSTPROCESSORS ETIMPORTERPOSTPROCESSOR.ET IM- PORTER OPENPSA DEFINE BRANCH}

This test can be found at ". / raven/tests / framework/PostProcessors/ETimporterPostProce. test_ETimporter_DefineBranch.xml". This test can be called executing the following command:

. Irun_tests --re=framework/PostProcessors/ETimporterPostProcessor.ET_impor

or 
./run_framework_tests --re=framework/PostProcessors/ETimporterPołtProcesso

- Test Description:

- Tests of the ETImporter post-processor: it read an event-tree (ET) from an .xml file (eventTree.xml) and it imports the ET structure into a PointSet. Note that the ET needs to be in an OpenPSA format. In particular, this tests checks that capability of the PP to manage ETs that contains by-pass branches.

- Original Author:

- mandd

- Creation date:

- 2017-11-07

- The classes tested in this test are:

- ETimporter

\subsubsection{FRAMEWORK POSTPROCESSORS.ETIMPORTERPOSTPROCESSORMULTIPLEET.ET IMPORTER OPENPSA COUPLEDET}

This test can be found at ". /raven/tests/framework/PostProcessors/ETimporterPostProce test_ETimporterMultipleET.xml”. This test can be called executing the following command:

./run_tests --re=framework/PostProcessors.ETimporterPostProcessohMultipleE

or

./run_framework_tests --re=framework/PostProcessors.ETimporterPoßtProcesso.

- Test Description:

- Tests of the ETImporter post-processor: it read an event-tree (ET) from multiple .xml files (eventTree1.xml and eventTree2.xml). Note that one single ET is provided but its structure is splitted into two files, i.e., the sub ETs are linked. and it imports the fullET structure into a PointSet. Note that the ETs need to be in an OpenPSA format.

- Original Author: 
- mandd

- Creation date:

- 2017-11-07

- The classes tested in this test are:

- ETimporter

\subsubsection{FRAMEWORK POSTPROCESSORS ETIMPORTERPOSTPROCESSOR.ET IM- PORTER OPENPSA EXPAND}

This test can be found at ". / raven/tests / framework/PostProcessors/ETimporterPostProce. test_ETimporter_expand.xml". This test can be called executing the following command:

./run_tests --re=framework/PostProcessors/ETimporterPostProcessor.ET_impor

or

. Irun_framework_tests --re=framework/PostProcessors/ETimporterPo\$tProcesso

- Test Description:

- Tests of the ETImporter post-processor: it read an event-tree (ET) from an .xml file (eventTree.xml) and it imports the ET structure into a PointSet. Note that the ET needs to be in an OpenPSA format.

- Original Author:

- mandd

- Creation date:

- 2017-11-07

- The classes tested in this test are:

- ETimporter 


\subsubsection{FRAMEWORK POSTPROCESSORS ETIMPORTERPOSTPROCESSOR.ET IM- PORTER 3BRANCHES}

This test can be found at ". /raven/tests/framework/PostProcessors/ETimporterPostProce test_ETimporter_3branches.xml". This test can be called executing the following command:

./run_tests --re=framework/PostProcessors/ETimporterPostProcesson.ET_impor

or

./run_framework_tests --re=framework/PostProcessors/ETimporterPoßtProcesso

- Test Description:

- Tests of the ETImporter post-processor: it read an event-tree (ET) from an .xml file (eventTree.xml) and it imports the ET structure into a PointSet. Note that the ET needs to be in an OpenPSA format.

- Original Author:

- mandd

- Creation date:

- 2017-11-07

- The classes tested in this test are:

- ETimporter

\subsubsection{FRAMEWORK POSTPROCESSORS ETIMPORTERPOSTPROCESSOR.ETIMPORTER 3BRANCHES NEWNUMBERING}

This test can be found at ". / raven/tests/framework/PostProcessors/ETimporterPostProce test_ETimporter_3branches_NewNumbering.xml”. This test can be called executing the following command:

./run_tests --re=framework/PostProcessors/ETimporterPostProcesson. ETimport

or

./run_framework_tests --re=framework/PostProcessors/ETimporterPoßtProcesso 
- Test Description:

- Tests of the ETImporter post-processor: it read an event-tree (ET) from an .xml file (eventTree.xml) and it imports the ET structure into a PointSet. Note that the ET needs to be in an OpenPSA format.

- Original Author:

- mandd

- Creation date:

- 2017-11-07

- The classes tested in this test are:

- ETimporter

\subsubsection{FRAMEWORK POSTPROCESSORS ETIMPORTERPOSTPROCESSOR.ETIMPORTER 3BRANCHES NEWNUMBERING EXPANDED}

This test can be found at ". / raven/tests / framework/PostProcessors/ETimporterPostProce test_ETimporter_3branches_NewNumbering_expanded.xml”. This test can be called executing the following command:

. Irun_tests --re=framework/PostProcessors/ETimporterPostProcessor. ETimport

or

./run_framework_tests --re=framework/PostProcessors/ETimporterPo\$tProcesso

- Test Description:

- Tests of the ETImporter post-processor: it read an event-tree (ET) from an .xml file (eventTree.xml) and it imports the ET structure into a PointSet. Note that the ET needs to be in an OpenPSA format.

- Original Author:

- mandd

- Creation date:

- 2017-11-07

- The classes tested in this test are:

- ETimporter 


\subsubsection{FRAMEWORK POSTPROCESSORS BASICSTATISTICS GRID ANALYTIC}

This test can be found at ". /raven/tests/framework/PostProcessors/BasicStatistics/ grid_analytic.xml". This test can be called executing the following command:

./run_tests --re=framework/PostProcessors/BasicStatistics/grid_analytic

or

./run_framework_tests --re=framework/PostProcessors/BasicStatistifcs/grid_a

- Test Description:

- This test checks all the basic statistics on analytic values using a grid sampling; however, the analytic models are not yet documented.

- Original Author:

- alfoa

- Creation date:

$-2015-11-21$

- The classes tested in this test are:

- PostProcessors.BasicStatistics

- Since the creation of this test, the following main revisions have been performed:

1. revision info:

- author: wangc

- date : 2017-12-05

- description: convert test to use the new dataobjects

2. revision info:

- author: wangc

- date : 2018-12-13

- description: In PR \#882, the standard errors on statistics moments are implemented, these quantities are stored in the pointwise metadata, and will be printed out in the output csv files by default. In order to avoid regolding this test, the optional node 'what' with text values 'input, output' is added. In this case, only the user requested variables as specified in the DataObjects are printed out. 


\subsubsection{FRAMEWORK POSTPROCESSORS BASICSTATISTICS GRID INVALUE}

This test can be found at ". / raven/tests/framework/PostProcessors/BasicStatistics/ grid_invalue.xml". This test can be called executing the following command:

./run_tests --re=framework/PostProcessors/BasicStatistics/grid_inValue

or

./run_framework_tests --re=framework/PostProcessors/BasicStatisti_cs/grid_i

- Test Description:

- This test checks many basic statistics using a grid sampling.

- Original Author:

- alfoa

- Creation date:

- 2015-12-16

- The classes tested in this test are:

- PostProcessors.BasicStatistics

- Since the creation of this test, the following main revisions have been performed:

1. revision info:

- author: wangc

- date : 2017-12-05

- description: convert test to use the new dataobjects

2. revision info:

- author: wangc

- date : 2018-12-13

- description: In PR \#882, the standard errors on statistics moments are implemented, these quantities are stored in the pointwise metadata, and will be printed out in the output csv files by default. In order to avoid regolding this test, the optional node 'what' with text values 'input, output' is added. In this case, only the user requested variables as specified in the DataObjects are printed out. 


\subsubsection{FRAMEWORK POSTPROCESSORS BASICSTATISTICS MC}

This test can be found at ". / raven/tests/framework/PostProcessors/BasicStatistics/ mc. xml". This test can be called executing the following command:

- /run_tests --re=framework/PostProcessors/Basicstatistics/mc

or

./run_framework_tests --re=framework/PostProcessors/BasicStatistifcs/mc

- Test Description:

- This test checks basic statistics on Monte Carlo samples.

- Original Author:

- alfoa

- Creation date:

- 2015-11-18

- The classes tested in this test are:

- PostProcessors.BasicStatistics

- Since the creation of this test, the following main revisions have been performed:

1. revision info:

- author: wangc

- date : 2017-12-05

- description: convert test to use the new dataobjects

2. revision info:

- author: wangc

- date : 2018-12-13

- description: In PR \#882, the standard errors on statistics moments are implemented, these quantities are stored in the pointwise metadata, and will be printed out in the output csv files by default. In order to avoid regolding this test, the optional node 'what' with text values 'input, output' is added. In this case, only the user requested variables as specified in the DataObjects are printed out. 


\subsubsection{FRAMEWORK POSTPROCESSORS BASICSTATISTICS.MCFLOATPERCENTILE}

This test can be found at ". /raven/tests/framework/PostProcessors/BasicStatistics/ mc_float_percentile.xml". This test can be called executing the following command:

./run_tests --re=framework/PostProcessors/BasicStatistics.mcFloatPercentil

or

./run_framework_tests --re=framework/PostProcessors/BasicStatistifcs.mcFloa

- Test Description:

- This test checks basic statistics on Monte Carlo samples, requesting percentiles less than 1 percent

- Original Author:

- alfoa

- Creation date:

- 2017-03-26

- The classes tested in this test are:

- PostProcessors.BasicStatistics

- Since the creation of this test, the following main revisions have been performed:

1. revision info:

- author: wangc

- date : 2017-12-05

- description: convert test to use the new dataobjects

\subsubsection{FRAMEWORK POSTPROCESSORS BASICSTATISTICS GRID ANALYTIC}

This test can be found at ". /raven/tests/framework/PostProcessors/BasicStatistics/ stratified_analytic.xml". This test can be called executing the following command:

./run_tests --re=framework/PostProcessors/BasicStatistics/grid_analytic

or 
./run_framework_tests --re=framework/PostProcessors/BasicStatistilcs/grid_a

- Test Description:

- This test checks basic statistics on Stratified samples

- Original Author:

- alfoa

- Creation date:

$-2015-11-22$

- The classes tested in this test are:

- PostProcessors.BasicStatistics

- Since the creation of this test, the following main revisions have been performed:

1. revision info:

- author: wangc

- date : 2017-12-05

- description: convert test to use the new dataobjects

2. revision info:

- author: wangc

- date : 2018-12-13

- description: In PR \#882, the standard errors on statistics moments are implemented, these quantities are stored in the pointwise metadata, and will be printed out in the output csv files by default. In order to avoid regolding this test, the optional node 'what' with text values 'input, output' is added. In this case, only the user requested variables as specified in the DataObjects are printed out.

\subsubsection{FRAMEWORK POSTPROCESSORS BASICSTATISTICS FACTORIAL ANALYTIC}

This test can be found at ". / raven/tests/framework/PostProcessors/BasicStatistics/ factorial_analytic.xml". This test can be called executing the following command:

./run_tests --re=framework/PostProcessors/BasicStatistics/factorilal_analyt

or 
./run_framework_tests --re=framework/PostProcessors/BasicStatistilcs/factor

- Test Description:

- This test checks basic statistics on Factorial samples.

- Original Author:

- alfoa

- Creation date:

- 2015-11-22

- The classes tested in this test are:

- PostProcessors.BasicStatistics

- Since the creation of this test, the following main revisions have been performed:

1. revision info:

- author: wangc

- date : 2017-12-05

- description: convert test to use the new dataobjects

2. revision info:

- author: wangc

- date : 2018-12-13

- description: In PR \#882, the standard errors on statistics moments are implemented, these quantities are stored in the pointwise metadata, and will be printed out in the output csv files by default. In order to avoid regolding this test, the optional node 'what' with text values 'input, output' is added. In this case, only the user requested variables as specified in the DataObjects are printed out.

\subsubsection{FRAMEWORK POSTPROCESSORS BASICSTATISTICS RESPONSESURFACE- DOE ANALYTIC}

This test can be found at ". /raven/tests/framework/PostProcessors/BasicStatistics/ responseSurfaceDoE_analytic.xml". This test can be called executing the following command:

./run_tests --re=framework/PostProcessors/BasicStatistics/responłeSurfaced 
or

./run_framework_tests --re=framework/PostProcessors/BasicStatistilcs/respon

- Test Description:

- This test checks the basic statistics on Response Surface Design of Experiment samples

- Original Author:

- alfoa

- Creation date:

$-2015-11-22$

- The classes tested in this test are:

- PostProcessors.BasicStatistics

- Since the creation of this test, the following main revisions have been performed:

1. revision info:

- author: wangc

- date : 2017-12-05

- description: convert test to use the new dataobjects

2. revision info:

- author: wangc

- date : 2018-12-13

- description: In PR \#882, the standard errors on statistics moments are implemented, these quantities are stored in the pointwise metadata, and will be printed out in the output csv files by default. In order to avoid regolding this test, the optional node 'what' with text values 'input, output' is added. In this case, only the user requested variables as specified in the DataObjects are printed out.

\subsubsection{FRAMEWORK POSTPROCESSORS BASICSTATISTICS MEDIANANDPERCENTILE}

This test can be found at ". /raven/tests/framework/PostProcessors/BasicStatistics/ test_median_and_percentile.xml”. This test can be called executing the following command:

./run_tests --re=framework/PostProcessors/BasicStatistics/medianAndPercent 
or

./run_framework_tests --re=framework/PostProcessors/BasicStatisti_cs/median

- Test Description:

- This requirements test checks median calcuation and the percentile calcuation. This test use CustomSampler to read a pre-genrated data and use BasicStatistic PostProcessor to output the median and the percentile for some variables. In median calculations, RAVEN will gives a 50 percentile. The interpolation method to use when the desired percentile lies between two data points $i, j$ : RAVEN will return the midpoint: $(i+j) / 2$.

- Original Author:

- ZHOUJ2

- Creation date:

- 2020-10-08

- The classes tested in this test are:

- PostProcessors.BasicStatistics

\subsubsection{FRAMEWORK POSTPROCESSORS BASICSTATISTICS PRINTXML}

This test can be found at ". /raven/tests/framework/PostProcessors/BasicStatistics/ print_xml.xml". This test can be called executing the following command:

./run_tests --re=framework/PostProcessors/BasicStatistics/printXml

or

./run_framework_tests --re=framework/PostProcessors/BasicStatisti_cs/printXl

- Test Description:

- This test checks the ability of the basic statistics PP to print RAVEN XML outputs.

- Original Author:

- talbpaul

- Creation date: 
- 2016-05-10

- The classes tested in this test are:

- PostProcessors.BasicStatistics

- Since the creation of this test, the following main revisions have been performed:

1. revision info:

- author : wangc

- date : 2017-12-05

- description: convert test to use the new dataobjects

2. revision info:

- author: wangc

- date : 2018-12-13

- description: In PR \#882, the standard errors on statistics moments are implemented, these quantities are stored in the pointwise metadata, and will be printed out in the output csv files by default. In order to avoid regolding this test, the optional node 'what' with text values 'input, output' is added. In this case, only the user requested variables as specified in the DataObjects are printed out.

3. revision info:

- author : talbpaul

- date : 2020-06-26

- description: greatly increased numerical tolerance due to collinearity

\subsubsection{FRAMEWORK POSTPROCESSORS BASICSTATISTICS SENSITIVITY}

This test can be found at ". / raven/tests/framework/PostProcessors/BasicStatistics / sensitivity.xml". This test can be called executing the following command:

./run_tests --re=framework/PostProcessors/BasicStatistics/sensitivity

or

./run_framework_tests --re=framework/PostProcessors/Basicstatistilcs/sensit

- Test Description:

- This test checks the sensitivities (and other metrics) calculated by basic statistics PP

- Original Author: 
- wangc

- Creation date:

- 2015-12-10

- The classes tested in this test are:

- PostProcessors.BasicStatistics

- Since the creation of this test, the following main revisions have been performed:

1. revision info:

- author: wangc

- date : 2017-12-05

- description: convert test to use the new dataobjects

2. revision info:

- author: wangc

- date : 2018-12-13

- description: In PR \#882, the standard errors on statistics moments are implemented, these quantities are stored in the pointwise metadata, and will be printed out in the output csv files by default. In order to avoid regolding this test, the optional node 'what' with text values 'input, output' is added. In this case, only the user requested variables as specified in the DataObjects are printed out.

\subsubsection{FRAMEWORK POSTPROCESSORS BASICSTATISTICS.SENSITIVITYONLY}

This test can be found at ". / raven/tests/framework/PostProcessors/BasicStatistics / sensitivity_only.xml". This test can be called executing the following command:

./run_tests --re=framework/PostProcessors/Basicstatistics.sensitivityonly

or

./run_framework_tests --re=framework/PostProcessors/Basicstatistilcs.sensit.

- Test Description:

- This test checks the sensitivities only calculated by basic statistics PP

- Original Author: 
- alfoa

- Creation date:

- 2017-07-18

- The classes tested in this test are:

- PostProcessors.BasicStatistics

- Since the creation of this test, the following main revisions have been performed:

1. revision info:

- author: wangc

- date : 2017-12-05

- description: convert test to use the new dataobjects

\subsubsection{FRAMEWORK POSTPROCESSORS BASICSTATISTICS TIMEDEPENDENT}

This test can be found at ". / raven/tests/framework/PostProcessors/BasicStatistics/ time_dep.xml". This test can be called executing the following command:

. /run_tests --re=framework/PostProcessors/Basicstatistics/timeDependent

or

./run_framework_tests --re=framework/PostProcessors/BasicStatistilcs/timeDe

- Test Description:

- This tests time-dependent basic statistics.

- Original Author:

- alfoa

- Creation date:

- 2016-06-20

- The classes tested in this test are:

- PostProcessors.BasicStatistics

- Since the creation of this test, the following main revisions have been performed: 
1. revision info:

- author: wangc

- date : 2017-12-05

- description: convert test to use the new dataobjects

2. revision info:

- author: wangc

- date : 2018-12-13

- description: In PR \#882, the standard errors on statistics moments are implemented, these quantities are stored in the pointwise metadata, and will be printed out in the output csv files by default. In order to avoid regolding this test, the optional node 'what' with text values 'input, output' is added. In this case, only the user requested variables as specified in the DataObjects are printed out.

\subsubsection{FRAMEWORK POSTPROCESSORS BASICSTATISTICS TIMEDEPENDENTA- SYNCHISTORIES}

This test can be found at ". /raven/tests/framework/PostProcessors/BasicStatistics/ time_dep_asyncHists.xml". This test can be called executing the following command:

./run_tests --re=framework/PostProcessors/BasicStatistics/timeDependentAsy

or

./run_framework_tests --re=framework/PostProcessors/BasicStatistilcs/timeDe]

- Test Description:

- This test checks time-dependent basic statistics with histories that are not synchronized a priori.

- Original Author:

- alfoa

- Creation date:

- 2016-06-21

- The classes tested in this test are:

- PostProcessors.BasicStatistics 
- Since the creation of this test, the following main revisions have been performed:

1. revision info:

- author: wangc

- date : 2017-12-05

- description: convert test to use the new dataobjects

2. revision info:

- author: wangc

- date : 2017-12-20

- description: convert test to use the new DataObjects with the new structure of basic statistic

3. revision info:

- author: wangc

- date : 2018-12-13

- description: In PR \#882, the standard errors on statistics moments are implemented, these quantities are stored in the pointwise metadata, and will be printed out in the output csv files by default. In order to avoid regolding this test, the optional node 'what' with text values 'input, output' is added. In this case, only the user requested variables as specified in the DataObjects are printed out.

\subsubsection{FRAMEWORK POSTPROCESSORS BASICSTATISTICS VARIATIONCOEFFI- CIENT}

This test can be found at ". / raven/tests/framework/PostProcessors/BasicStatistics / variationcoefficient. xml”. This test can be called executing the following command:

. Irun_tests --re=framework/PostProcessors/BasicStatistics/variatifoncoeffic

or

./run_framework_tests --re=framework/PostProcessors/Basicstatistilcs/variat

- Test Description:

- This test is used to test the defect mentioned in issue \#666 This test will make sure the expectedValue and the variationCoefficient are correctly computed. If the expectedValues are zero, the variationCoefficient will be INF.

- Original Author: 
- wangc

- Creation date:

$-2015-11-21$

- The classes tested in this test are:

- PostProcessors.BasicStatistics

- Since the creation of this test, the following main revisions have been performed:

1. revision info:

- author: wangc

- date : 2017-12-05

- description: convert test to use the new dataobjects

\subsubsection{FRAMEWORK POSTPROCESSORS BASICSTATISTICS MC UNIFORM}

This test can be found at ". / raven/tests/framework/PostProcessors/BasicStatistics/ mcUnif.xml". This test can be called executing the following command:

./run_tests --re=framework/PostProcessors/BasicStatistics/mc_uniform

or

./run_framework_tests --re=framework/PostProcessors/Basicstatisti_cs/mc_uni

- Test Description:

- Tests basic statistics on uniformly-sampled normally-distributed Monte Carlo sampling.

- Original Author:

- mandd

- Creation date:

- 2016-11-28

- The classes tested in this test are:

- PostProcessors.BasicStatistics 
- Since the creation of this test, the following main revisions have been performed:

1. revision info:

- author: wangc

- date : 2017-12-05

- description: convert test to use the new dataobjects

2. revision info:

- author: wangc

- date : 2018-12-13

- description: In PR \#882, the standard errors on statistics moments are implemented, these quantities are stored in the pointwise metadata, and will be printed out in the output csv files by default. In order to avoid regolding this test, the optional node 'what' with text values 'input, output' is added. In this case, only the user requested variables as specified in the DataObjects are printed out.

\subsubsection{FRAMEWORK POSTPROCESSORS BASICSTATISTICS WRITEHEAVYXML}

This test can be found at ". / raven/tests/framework/PostProcessors/BasicStatistics/ heavy_xml_write.xml". This test can be called executing the following command:

. /run_tests --re=framework/PostProcessors/BasicStatistics/writeHeavyXml

or

./run_framework_tests --re=framework/PostProcessors/BasicStatistilcs/writeH

- Test Description:

- This tests time-dependent basic statistics writing a large number of entries to ensure good speed.

- Original Author:

- talbpaul

- Creation date:

- 2017-03-16

- The classes tested in this test are:

- PostProcessors.BasicStatistics,Files.DynamicXMLOutput 
- Since the creation of this test, the following main revisions have been performed:

1. revision info:

- author: wangc

- date : 2017-12-05

- description: convert test to use the new dataobjects

2. revision info:

- author: wangc

- date : 2018-12-13

- description: In PR \#882, the standard errors on statistics moments are implemented, these quantities are stored in the pointwise metadata, and will be printed out in the output csv files by default. In order to avoid regolding this test, the optional node 'what' with text values 'input, output' is added. In this case, only the user requested variables as specified in the DataObjects are printed out.

\subsubsection{FRAMEWORK POSTPROCESSORS BASICSTATISTICS TIMEDEPOUTCSV}

This test can be found at ". / raven/tests/framework/PostProcessors/BasicStatistics/ test_timedep_out_cSV.xml”. This test can be called executing the following command:

. /run_tests --re=framework/PostProcessors/BasicStatistics/timeDepoutCsv

or

./run_framework_tests --re=framework/PostProcessors/BasicStatistifcs/timeDe

- Test Description:

- This test checks basic statistics for csv ouptut

- Original Author:

- alfoa

- Creation date:

- 2017-03-29

- The classes tested in this test are:

- PostProcessors.BasicStatistics 
- Since the creation of this test, the following main revisions have been performed:

1. revision info:

- author: wangc

- date : 2017-12-20

- description: convert test to use the new DataObjects with the new structure of basic statistic

2. revision info:

- author: wangc

- date : 2018-05-15

- description: remove unecessary plots

3. revision info:

- author: wangc

- date : 2018-12-13

- description: In PR \#882, the standard errors on statistics moments are implemented, these quantities are stored in the pointwise metadata, and will be printed out in the output csv files by default. In order to avoid regolding this test, the optional node 'what' with text values 'input, output' is added. In this case, only the user requested variables as specified in the DataObjects are printed out.

\subsubsection{FRAMEWORK POSTPROCESSORS BASICSTATISTICS TIMEDEPDATASET}

This test can be found at ". / raven/tests/framework/PostProcessors/BasicStatistics / time_dep_dataset.xml". This test can be called executing the following command:

./run_tests --re=framework/PostProcessors/BasicStatistics/timeDepDataset

or

./run_framework_tests --re=framework/PostProcessors/BasicStatistilcs/timeDe

- Test Description:

- This tests dumping time-dependent basic statistics into dataset

- Original Author:

- wangc

- Creation date: 
$-2018-08-25$

- The classes tested in this test are:

- PostProcessors.BasicStatistics

- Since the creation of this test, the following main revisions have been performed:

1. revision info:

- author: wangc

- date : 2018-12-13

- description: avoid metadata output since metadata is added in basicStatistics

2. revision info:

- author: wangc

- date : 2018-12-13

- description: In PR \#882, the standard errors on statistics moments are implemented, these quantities are stored in the pointwise metadata, and will be printed out in the output csv files by default. In this case, the time dependent standard errors will be printed out.

\subsubsection{FRAMEWORK POSTPROCESSORS BASICSTATISTICS NML}

This test can be found at ". / raven/tests/framework/PostProcessors/BasicStatistics/ test_not_multiple_linear.xml". This test can be called executing the following command:

./run_tests --re=framework/PostProcessors/Basicstatistics/nml

or

./run_framework_tests --re=framework/PostProcessors/BasicStatistilcs/nml

- Test Description:

- This test checks sensitivity, variance dependent sensitivity calculations without using the multiple linear regression method. This means the inputs are treated uncorrelated with respective to the output space

- Original Author:

- wangc 
- Creation date:

- 2018-09-08

- The classes tested in this test are:

- PostProcessors.BasicStatistics

\subsubsection{FRAMEWORK POSTPROCESSORS BASICSTATISTICS.STE}

This test can be found at ". /raven/tests/framework/PostProcessors/BasicStatistics/ ste_mc.xml". This test can be called executing the following command:

./run_tests --re=framework/PostProcessors/BasicStatistics.ste

or

./run_framework_tests --re=framework/PostProcessors/BasicStatistilcs.ste

- Test Description:

- This test checks standard error calculations of statistics moments in basic statistics using Monte Carlo samples.

- Original Author:

- wangc

- Creation date:

- 2018-12-13

- The classes tested in this test are:

- PostProcessors.BasicStatistics

- Since the creation of this test, the following main revisions have been performed:

1. revision info:

- author: wangc

- date : 2018-12-13

- description: In PR \#882, the standard errors on statistics moments are implemented, these quantities are stored in the pointwise metadata, and will be printed out in the output csv files by default. This test will test the Monte Carlo-based weighted standard errors calculations. The equivalent sample size will be equal the number of samples in this case, and then standard errors will be computed with unbiased standard deviations and/or the equivalent sample size. 


\subsubsection{FRAMEWORK POSTPROCESSORS BASICSTATISTICS.STEGRID}

This test can be found at ". / raven/tests/framework/PostProcessors/BasicStatistics/ ste_grid.xml". This test can be called executing the following command:

./run_tests --re=framework/PostProcessors/BasicStatistics.steGric

or

./run_framework_tests --re=framework/PostProcessors/BasicStatistifcs.steGri

- Test Description:

- This test checks standard error calculations of statistics moments in basic statistics using Grid samples.

- Original Author:

- wangc

- Creation date:

$-2018-12-13$

- The classes tested in this test are:

- PostProcessors.BasicStatistics

- Since the creation of this test, the following main revisions have been performed:

1. revision info:

- author: wangc

- date : 2018-12-13

- description: In PR \#882, the standard errors on statistics moments are implemented, these quantities are stored in the pointwise metadata, and will be printed out in the output csv files by default. This test will test the grid-based weighted standard errors calculations. The equivalent sample size will be computed first using the probability weights, and then standard errors will be computed with unbiased standard deviations and/or the equivalent sample size. 


\subsubsection{FRAMEWORK POSTPROCESSORS BASICSTATISTICS.TIMEDEPMETA}

This test can be found at ". /raven/tests/framework/PostProcessors/BasicStatistics/ test_time_dep_meta.xml”. This test can be called executing the following command:

./run_tests --re=framework/PostProcessors/BasicStatistics.timeDepMeta

or

./run_framework_tests --re=framework/PostProcessors/BasicStatistifcs.timede

- Test Description:

- This test checks the metadata printing of history set, the scalar meta data should be printed with the input of HistorySet, while vector meta data should be printed with output of HistorySet. See issue \#986. With the fix of \#986, The HistorySet will handle scalar and vector meta data separately.

- Original Author:

- wangc

- Creation date:

- 2019-05-10

- The classes tested in this test are:

- PostProcessors.BasicStatistics, DataObjects.HistorySet

\subsubsection{FRAMEWORK.SAFEST POINT PP}

This test can be found at ". / raven/tests / framework/PostProcessors/SafestPoint/ test_safest_point.xml". This test can be called executing the following command:

./run_tests --re=framework. safest_point_pp

or

./run_framework_tests --re=framework.safest_point_pp

- Test Description: 
- This test is about the identification of the Safest Point in a set of controllable and uncontrollable parameter. In order to do that, a LimitSurfaceSearch sampling is employed and sub-sequentially the PostProcessor of type SafestPoint is used.

- Original Author:

- alfoa

- Creation date:

- 2014-11-17

- The classes tested in this test are:

- Samplers.LimitSurfaceSearch, Models.PostProcessor.SafestPoint, Steps.MultiRun

- Since the creation of this test, the following main revisions have been performed:

1. revision info:

- author : mandd

- date : 2015-04-17

- description: conversion to Database and DataObjects

2. revision info:

- author : alfoa

- date : 2015-05-19

- description: Closes \#176

3. revision info:

- author : alfoa

- date : 2015-06-18

- description: Changed Name of all DataObjects

4. revision info:

- author : talbpaul

- date : 2015-07-07

- description: standardized tests

5. revision info:

- author : cogljj

- date : 2015-09-29

- description: Modifying external modules to use relative to working directory.

6. revision info:

- author : maljdan 
- date : 2016-04-06

- description: Updating test cases to reflect the changes to the user input.

7. revision info:

- author : alfoa

- date : 2017-01-21

- description: Adding this test description.

8. revision info:

- author : alfoa

- date : 2019-03-07

- description: Modified syntax in Functions as for issue \#934

\subsubsection{FRAMEWORK.SAFEST POINT CDF PP}

This test can be found at ". / raven/tests/framework/PostProcessors/SafestPoint/ test_safest_point_cdf.xml". This test can be called executing the following command:

./run_tests --re=framework.safest_point_cdf_pp

or

./run_framework_tests --re=framework.safest_point_cdf_pp

- Test Description:

- This test is about the identification of the Safest Point in a set of controllable and uncontrollable parameter. The un-controllable parameter space is probability weighted in this case. In order to do that, a LimitSurfaceSearch sampling is employed and subsequentially the PostProcessor of type SafestPoint is used.

- Original Author:

- alfoa

- Creation date:

$-2015-01-22$

- The classes tested in this test are:

- Samplers.LimitSurfaceSearch, Models.PostProcessor.SafestPoint, Steps.MultiRun

- Since the creation of this test, the following main revisions have been performed: 
1. revision info:

- author : mandd

- date : 2015-04-17

- description: conversion to Database and DataObjects

2. revision info:

- author : alfoa

- date : 2015-05-19

- description: Closes \#176

3. revision info:

- author : talbpaul

- date : 2015-06-04

- description: regolding after SG feature ordering, also changed Adaptive sampler to LimitSurfaceSearch sampler

4. revision info:

- author : cogljj

- date : 2015-09-29

- description: Modifying external modules to use relative to working directory.

5. revision info:

- author : maljdan

- date : 2016-04-06

- description: Updating test cases to reflect the changes to the user input.

6. revision info:

- author : alfoa

- date : 2017-01-21

- description: Adding this test description.

7. revision info:

- author : alfoa

- date : 2019-03-07

- description: Modified syntax in Functions as for issue \#934

\subsubsection{FRAMEWORK POSTPROCESSORS PARETOFRONTIERPOSTPROCESSOR.PARETOFRO]}

This test can be found at ". / raven/tests/framework/PostProcessors/ParetoFrontierPostP test_paretoFrontier.xml". This test can be called executing the following command:

./run_tests --re=framework/PostProcessors/ParetoFrontierPostProcessor.Pare 
or

. Irun_framework_tests --re=framework/PostProcessors/ParetofrontierPostProc

- Test Description:

- This post-processor identifies the points lying on the Pareto Frontier in a cost-value space

- Original Author:

- mandd

- Creation date:

$-2020-03-25$

- The classes tested in this test are:

- Models.PostProcessors.ParetoFrontier

\subsubsection{FRAMEWORK POSTPROCESSORS PARETOFRONTIERPOSTPROCESSOR.PARETOFRO}

This test can be found at ". / raven/tests / framework/PostProcessors/ParetoFrontierPostP test_paretoFrontierWithCostLimit.xml". This test can be called executing the following command:

. Irun_tests --re=framework/PostProcessors/ParetoFrontierPostProcessor.Pare

or

./run_framework_tests --re=framework/PostProcessors/ParetofrontierPostProc

- Test Description:

- This post-processor identifies the points lying on the Pareto Frontier in a cost-value space when cost limit is specified

- Original Author:

- mandd

- Creation date: 
- 2020-07-05

- The classes tested in this test are:

- Models.PostProcessors.ParetoFrontier

\subsubsection{FRAMEWORK POSTPROCESSORS PARETOFRONTIERPOSTPROCESSOR.PARETOFRO}

This test can be found at ". / raven/tests / framework/PostProcessors/ParetoFrontierPostP test_paretoFrontierWithValueLimit.xml”. This test can be called executing the following command:

. Irun_tests --re=framework/PostProcessors/ParetoFrontierPostProcessor.Pare

or

. Irun_framework_tests --re=framework/PostProcessors/ParetofrontierPostProc

- Test Description:

- This post-processor identifies the points lying on the Pareto Frontier in a cost-value space when value limit is specified.

- Original Author:

- mandd

- Creation date:

- 2020-07-05

- The classes tested in this test are:

- Models.PostProcessors.ParetoFrontier

\subsubsection{FRAMEWORK POSTPROCESSORS PARETOFRONTIERPOSTPROCESSOR.PARETOFRO}

This test can be found at ". / raven/tests/framework/PostProcessors/ParetoFrontierPostP. test_paretoFrontierWithBothLimits.xml”. This test can be called executing the following command:

. Irun_tests --re=framework/PostProcessors/ParetoFrontierPostProcessor.Pare 
or

. Irun_framework_tests --re=framework/PostProcessors/ParetofrontierPostProc

- Test Description:

- This post-processor identifies the points lying on the Pareto Frontier in a cost-value space when cost and value limits are specified.

- Original Author:

- mandd

- Creation date:

- 2020-07-05

- The classes tested in this test are:

- Models.PostProcessors.ParetoFrontier

\subsubsection{FRAMEWORK POSTPROCESSORS PARETOFRONTIERPOSTPROCESSOR.PARETOFRO}

This test can be found at ". / raven/tests / framework/PostProcessors/ParetoFrontierPostP test_paretoFrontierInverted.xml". This test can be called executing the following command:

. Irun_tests --re=framework/PostProcessors/ParetoFrontierPostProcessor.Pare

or

./run_framework_tests --re=framework/PostProcessors/ParetofrontierPostProc

- Test Description:

- This post-processor identifies the points lying on the Pareto Frontier in a cost-value space with data types inverted

- Original Author:

- mandd

- Creation date: 
$-2020-03-25$

- The classes tested in this test are:

- Models.PostProcessors.ParetoFrontier

\subsubsection{FRAMEWORK TEST COMPARISON STATISTICS}

This test can be found at ". / raven/tests / framework/PostProcessors/OldComparisonStatist test_cc_stats.xml". This test can be called executing the following command:

./run_tests --re=framework/test_comparison_statistics

or

./run_framework_tests --re=framework/test_comparison_statistics

- Test Description:

- Tests the comparison statistics class including both two PointSets and PointSet to distribution.

- Original Author:

- cogljj

- Creation date:

- 2014-10-16

- The classes tested in this test are:

- ComparisonStatistics

- Since the creation of this test, the following main revisions have been performed:

1. revision info:

- author : alfoa

- date : 2017-10-17

- description: Removed support for PointSet output for ComparisonStatisticsModule 


\subsubsection{FRAMEWORK POSTPROCESSORS MCSIMPORTERPOSTPROCESSOR.MCSIMPORTER}

This test can be found at ". /raven/tests/framework/PostProcessors/MCSimporterPostProce test_MCSimporter.xml". This test can be called executing the following command:

./run_tests --re=framework/PostProcessors/MCSimporterPostProcessor. MCSimpo

or

./run_framework_tests --re=framework/PostProcessors/MCSimporterP stProcess

- Test Description:

- Tests of the MCSImporter post-processor: it reads a list of MCSs generated from Saphire and it imports it as a PointSet. We specifically test the capability to expand the list of Basic Events from an external file.

- Original Author:

- mandd

- Creation date:

- 2019-11-01

- The classes tested in this test are:

- MCSImporter

\subsubsection{FRAMEWORK POSTPROCESSORS MCSIMPORTERPOSTPROCESSOR.MCSIMPORTER NOEXPAND}

This test can be found at ". / raven/tests / framework/PostProcessors/MCSimporterPostProce test_MCSimporter_noExpand.xml". This test can be called executing the following command:

./run_tests --re=framework/PostProcessors/MCSimporterPostProcess gr. MCSimpo

or

./run_framework_tests --re=framework/PostProcessors/MCSimporterP pstProcess

- Test Description: 
- Tests of the MCSImporter post-processor: it reads a list of MCSs generated from Saphire and it imports it as a PointSet.

- Original Author:

- mandd

- Creation date:

- 2019-11-01

- The classes tested in this test are:

- MCSImporter

\subsubsection{FRAMEWORK POSTPROCESSORS LIMITSURFACE.TESTLIMITSURFACEPOSTPROCES}

This test can be found at ". / raven/tests/framework/PostProcessors/LimitSurface/ test_LimitSurface_regressor_inverseWeight.xml”. This test can be called executing the following command:

. Irun_tests --re=framework/PostProcessors/LimitSurface.testLimit\$urfacePos

or

.Irun_framework_tests --re=framework/PostProcessors/Limitsurface.testLimit

- Test Description:

- This test is aimed to check the capability of RAVEN to generate Limit Surfaces, using a regressor surrogate model instead of a classifier, from a pre-generated data set (in this case, generated with a MonteCarlo sampling), outputting the generated Limit Surface in DataObject.PointSet(s) reporting both transition boundaries (-1 1) or just one of them.

- Original Author:

- alfoa

- Creation date:

- 2014-07-10

- The classes tested in this test are: 
- Models.PostProcessors.LimitSurface, Functions.External, Models.ROM

- Since the creation of this test, the following main revisions have been performed:

1. revision info:

- author : alfoa

- date : 2017-06-02

- description: Adding this test description.

2. revision info:

- author : alfoa

- date : 2017-12-14

- description: Moved LimitSurface Postprocessor tests in a specific directory (tests/framework/PostProcessors/LimitSurface) and updated for new DataObject structure

3. revision info:

- author : alfoa

- date : 2019-03-07

- description: Modified syntax in Functions as for issue \#934

\subsubsection{FRAMEWORK POSTPROCESSORS DATACLASSIFIER CLUSTERING}

This test can be found at ". / raven/tests/framework/PostProcessors/DataClassifier/ test_dataclassifier_postprocessor.xml”. This test can be called executing the following command:

./run_tests --re=framework/PostProcessors/DataClassifier/Clustering/

or

. Irun_framework_tests --re=framework/PostProcessors/Dataclassifier/Cluster

- Test Description:

- Test DataClassifier PostProcessor with PointSet input

- Original Author:

- wangc

- Creation date: 
$-2018-10-18$

- The classes tested in this test are:

- DataClassifier

- Since the creation of this test, the following main revisions have been performed:

1. revision info:

- author: wangc

- date : 2019-04-26

- description: Move the test from PRAPlugin to RAVEN tests/framework/PostProcessors/DataClassifier, this is because the DataClassifier PostProcessor is currently in RAVEN main repository.

2. revision info:

- author: wangc

- date : 2019-05-02

- description: As reported in issue \#805, RAVEN will not allow input DataObject of PostProcessor to be output DataObject, the output data object will be renamed, and the variable name of labels will be explicitly defined.

\subsubsection{FRAMEWORK POSTPROCESSORS DATACLASSIFIER CLUSTERING}

This test can be found at ". / raven/tests/framework/PostProcessors/DataClassifier/ test_dataClassifier_postprocessor_HS.xml”. This test can be called executing the following command:

. /run_tests --re=framework/PostProcessors/Dataclassifier/Clustering/

or

. Irun_framework_tests --re=framework/PostProcessors/DataClassifier/Cluster

- Test Description:

- Test DataClassifier PostProcessor with HistorySet input

- Original Author:

- wangc

- Creation date: 
- 2018-10-18

- The classes tested in this test are:

- DataClassifier

- Since the creation of this test, the following main revisions have been performed:

1. revision info:

- author: wangc

- date : 2019-04-26

- description: Move the test from PRAPlugin to RAVEN tests/framework/PostProcessors/DataClassifier, this is because the DataClassifier PostProcessor is currently in RAVEN main repository.

2. revision info:

- author: wangc

- date : 2019-05-02

- description: As reported in issue \#805, RAVEN will not allow input DataObject of PostProcessor to be output DataObject, the output data object will be renamed, and the variable name of labels will be explicitly defined.

\subsubsection{FRAMEWORK POSTPROCESSORS METRIC TEST CROSS VALIDATION}

This test can be found at ". / raven/tests/framework/PostProcessors/CrossValidations/ test_cross_validation.xml”. This test can be called executing the following command:

./run_tests --re=framework/PostProcessors/Metric/test_cross_validation

or

. Irun_framework_tests --re=framework/PostProcessors/Metric/test_qross_vali

- Test Description:

- This test checks the Cross Validation PostProcessor with KFold method

- Original Author:

- wangc

- Creation date: 
- 2017-09-06

- The classes tested in this test are:

- PostProcessors.Cross Validation

- Since the creation of this test, the following main revisions have been performed:

1. revision info:

- author: wangc

- date : 2017-12-07

- description: convert test to use the new dataobjects

\subsubsection{FRAMEWORK POSTPROCESSORS METRIC TEST LABELKFOLD}

This test can be found at ". / raven/tests / framework/PostProcessors/CrossValidations/ test_LabelKFold.xml". This test can be called executing the following command:

. Irun_tests --re=framework/PostProcessors/Metric/test_LabelKFold

or

. Irun_framework_tests --re=framework/PostProcessors/Metric/test_tabelKFold

- Test Description:

- This test checks the Cross Validation PostProcessor with LabelKFold method

- Original Author:

- wangc

- Creation date:

- 2017-09-07

- The classes tested in this test are:

- PostProcessors.Cross Validation

- Since the creation of this test, the following main revisions have been performed:

1. revision info:

- author: wangc

- date : 2017-12-07

- description: convert test to use the new dataobjects 


\subsubsection{FRAMEWORK POSTPROCESSORS METRIC TEST LEAVEONEOUT}

This test can be found at ". /raven/tests/framework/PostProcessors/CrossValidations/ test_labelShuffleSplit.xml". This test can be called executing the following command:

./run_tests --re=framework/PostProcessors/Metric/test_leaveoneout

or

./run_framework_tests --re=framework/PostProcessors/Metric/test_leaveoneou

- Test Description:

- This test checks the CrossValidation PostProcessor with LabelShuffleSplit method

- Original Author:

- wangc

- Creation date:

- 2017-09-07

- The classes tested in this test are:

- PostProcessors.Cross Validation

- Since the creation of this test, the following main revisions have been performed:

1. revision info:

- author: wangc

- date : 2017-12-07

- description: convert test to use the new dataobjects

\subsubsection{FRAMEWORK POSTPROCESSORS METRIC TEST LEAVEONEOUT}

This test can be found at ". /raven/tests/framework/PostProcessors/CrossValidations/ test_leaveoneOut.xml". This test can be called executing the following command:

./run_tests --re=framework/PostProcessors/Metric/test_leaveoneout

or 
./run_framework_tests --re=framework/PostProcessors/Metric/test_lleaveoneou

- Test Description:

- This test checks the CrossValidation PostProcessor with leaveOneOut method

- Original Author:

- wangc

- Creation date:

- 2017-09-07

- The classes tested in this test are:

- PostProcessors.Cross Validation

- Since the creation of this test, the following main revisions have been performed:

1. revision info:

- author: wangc

- date : 2017-12-07

- description: convert test to use the new dataobjects

\subsubsection{FRAMEWORK POSTPROCESSORS METRIC TEST LEAVEPLABELOUT}

This test can be found at ". / raven/tests/framework/PostProcessors/CrossValidations/ test_leavePLabelout.xml". This test can be called executing the following command:

./run_tests --re=framework/PostProcessors/Metric/test_leavePLabe] Out

or

./run_framework_tests --re=framework/PostProcessors/Metric/test_leavePLabe

- Test Description:

- This test checks the CrossValidation PostProcessor with LeavePLabelOut method

- Original Author: 
- wangc

- Creation date:

- 2017-09-07

- The classes tested in this test are:

- PostProcessors.Cross Validation

- Since the creation of this test, the following main revisions have been performed:

1. revision info:

- author : wangc

- date : 2017-12-07

- description: convert test to use the new dataobjects

\subsubsection{FRAMEWORK POSTPROCESSORS METRIC TEST LEAVEPOUT}

This test can be found at ". / raven/tests/framework/PostProcessors/CrossValidations/ test_leavePout.xml". This test can be called executing the following command:

./run_tests --re=framework/PostProcessors/Metric/test_leavePout

or

./run_framework_tests --re=framework/PostProcessors/Metric/test_leavePout

- Test Description:

- This test checks the Cross Validation PostProcessor with leavePOut method

- Original Author:

- wangc

- Creation date:

- 2017-09-07

- The classes tested in this test are:

- PostProcessors.Cross Validation

- Since the creation of this test, the following main revisions have been performed: 
1. revision info:

- author: wangc

- date : 2017-12-07

- description: convert test to use the new dataobjects

\subsubsection{FRAMEWORK POSTPROCESSORS METRIC TEST SHUFFLESPLIT}

This test can be found at ". /raven/tests/framework/PostProcessors/CrossValidations/ test_shuffleSplit.xml”. This test can be called executing the following command:

./run_tests --re=framework/PostProcessors/Metric/test_shufflesplitt

or

./run_framework_tests --re=framework/PostProcessors/Metric/test_\$hufflespl.

- Test Description:

- This test checks the CrossValidation PostProcessor with shuffle and split method

- Original Author:

- wangc

- Creation date:

- 2017-09-07

- The classes tested in this test are:

- PostProcessors.Cross Validation

- Since the creation of this test, the following main revisions have been performed:

1. revision info:

- author: wangc

- date : 2017-12-07

- description: convert test to use the new dataobjects 


\subsubsection{FRAMEWORK POSTPROCESSORS METRIC TEST STRATIFIEDKFOLD}

This test can be found at ". /raven/tests/framework/PostProcessors/CrossValidations/ test_stratifiedKFold.xml". This test can be called executing the following command:

./run_tests --re=framework/PostProcessors/Metric/test_stratifiedkFold

or

./run_framework_tests --re=framework/PostProcessors/Metric/test_łtratified.

- Test Description:

- This test checks the CrossValidation PostProcessor with Stratified K-Folds method

- Original Author:

- wangc

- Creation date:

- 2017-09-07

- The classes tested in this test are:

- PostProcessors.Cross Validation

- Since the creation of this test, the following main revisions have been performed:

1. revision info:

- author: wangc

- date : 2017-12-07

- description: convert test to use the new dataobjects

\subsubsection{FRAMEWORK POSTPROCESSORS METRIC TEST STRATIFIEDSHUFFLE- SPLIT}

This test can be found at ". / raven/tests / framework/PostProcessors/CrossValidations/ test_stratifiedShufflesplit.xml". This test can be called executing the following command:

./run_tests --re=framework/PostProcessors/Metric/test_stratified\$hufflespl 
or

. Irun_framework_tests --re=framework/PostProcessors/Metric/test_\$tratified

- Test Description:

- This test checks the Cross Validation PostProcessor with StratifiedShuffleSplit method

- Original Author:

- wangc

- Creation date:

- 2017-09-07

- The classes tested in this test are:

- PostProcessors.Cross Validation

- Since the creation of this test, the following main revisions have been performed:

1. revision info:

- author: wangc

- date : 2017-12-07

- description: convert test to use the new dataobjects

\subsubsection{FRAMEWORK POSTPROCESSORS METRIC TEST LEAVEONELABELOUT}

This test can be found at". / raven/tests/framework/PostProcessors/CrossValidations/ test_leaveOneLabelout.xml". This test can be called executing the following command:

./run_tests --re=framework/PostProcessors/Metric/test_leaveOneLabelout

or

./run_framework_tests --re=framework/PostProcessors/Metric/test_leaveoneLa

- Test Description:

- This test checks the CrossValidation PostProcessor with LeaveOneLabelOut method

- Original Author: 
- wangc

- Creation date:

- 2017-09-07

- The classes tested in this test are:

- PostProcessors.Cross Validation

- Since the creation of this test, the following main revisions have been performed:

1. revision info:

- author: wangc

- date : 2017-12-07

- description: convert test to use the new dataobjects

\subsubsection{FRAMEWORK POSTPROCESSORS FASTFOURIERTRANSFORM.BASIC}

This test can be found at ". / raven/tests / framework/PostProcessors/FastFourierTransforr basic.xml". This test can be called executing the following command:

. Irun_tests --re=framework/PostProcessors/FastFourierTransform. Basic

or

./run_framework_tests --re=framework/PostProcessors/FastFourierTransform.B

- Test Description:

- Tests the basic functionality of the FastFourierTransform postprocessor to create a frequency-amplitude Fourier analysis of a signal.

The signals are created using Basic/dataGenerator.py, and use analytic Fourier-based signals. The first realization uses periods of 4, 8 and 20, and in the second realization the periods are $3.333,13$, and 50. At the corresponding periods (in the "fft_*csv" files, under "signal_fft_period"), there should be a peak in the "signal_fft_amplitude" values.

- Original Author:

- talbpaul

- Creation date:

- 2018-09-11 
- The classes tested in this test are:

- PostProcessors.FastFourierTransform

\subsubsection{FRAMEWORK POSTPROCESSORS SAMPLESELECTOR.BASIC}

This test can be found at ". / raven/tests / framework/PostProcessors/SampleSelector/ basic.xml". This test can be called executing the following command:

./run_tests --re=framework/PostProcessors/Sampleselector.Basic

or

./run_framework_tests --re=framework/PostProcessors/SampleSelect

- Test Description:

- This test checks basic functionalities of the Sample Selector. The model multiplies the input $\mathrm{x}$ by 2.5 to obtain $\mathrm{x} 1$, and $\mathrm{y}$ is multiplied by 3.5 to obtain $\mathrm{y} 1 . \mathrm{x}$ and $\mathrm{y}$ are sampled through a Monte Carlo sampler, with 10 realizations. The SampleSelector postprocessor is called several times, testing each of the selection methods.

- Original Author:

$-\mathrm{gm}$

- Creation date:

- 2018-09-12

- The classes tested in this test are:

- PostProcessors.SampleSelector

- Since the creation of this test, the following main revisions have been performed:

1. revision info:

- author : talbpaul

- date : 2018-11-06

- description: added index selection method to test 


\subsubsection{FRAMEWORK POSTPROCESSORS FTIMPORTERPOSTPROCESSOR.FTIMPORTER AND}

This test can be found at ". / raven/tests/framework/PostProcessors /FTimporterPostProce test_FTimporter_and.xml”. This test can be called executing the following command:

./run_tests --re=framework/PostProcessors/FTimporterPostProcessor. FTimport

or

./run_framework_tests --re=framework/PostProcessors/FTimporterPoßtProcesso

- Test Description:

- Tests of the FTImporter post-processor: it read a fault-tree (ET) from an .xml file (eventTree.xml) and it imports the FT structure into a PointSet. Note that the FT needs to be in an OpenPSA format.

- Original Author:

- mandd

- Creation date:

- 2017-11-07

- The classes tested in this test are:

- ETimporter

\subsubsection{FRAMEWORK POSTPROCESSORS FTIMPORTERPOSTPROCESSOR.FTIMPORTER AND WITHNOT}

This test can be found at ". /raven/tests/framework/PostProcessors/FTimporterPostProce test_FTimporter_and_withNOT.xml". This test can be called executing the following command:

./run_tests --re=framework/PostProcessors/FTimporterPostProcessor. FTimport

or

./run_framework_tests --re=framework/PostProcessors/FTimporterPoßtProcesso 
- Test Description:

- Tests of the FTImporter post-processor: it read a fault-tree (ET) from an .xml file (eventTree.xml) and it imports the FT structure into a PointSet. Note that the FT needs to be in an OpenPSA format.

- Original Author:

- mandd

- Creation date:

- 2017-11-07

- The classes tested in this test are:

- ETimporter

\subsubsection{FRAMEWORK POSTPROCESSORS FTIMPORTERPOSTPROCESSOR.FTIMPORTER AND WITHNOT EMBEDDED}

This test can be found at ". / raven/tests / framework/PostProcessors/FTimporterPostProce test_FTimporter_and_withNOT_embedded.xml". This test can be called executing the following command:

. Irun_tests --re=framework/PostProcessors/FTimporterPostProcessor. FTimport

or

./run_framework_tests --re=framework/PostProcessors/FTimporterPo\$tProcesso

- Test Description:

- Tests of the FTImporter post-processor: it read a fault-tree (ET) from an .xml file (eventTree.xml) and it imports the FT structure into a PointSet. Note that the FT needs to be in an OpenPSA format.

- Original Author:

- mandd

- Creation date:

- 2017-11-07

- The classes tested in this test are:

- ETimporter 


\subsubsection{FRAMEWORK POSTPROCESSORS FTIMPORTERPOSTPROCESSOR}

This test can be found at ". /raven/tests/framework/PostProcessors/FTimporterPostProce test_FTimporter_and_withNOT_withNOT_embedded.xml”. This test can be called executing the following command:

./run_tests --re=framework/PostProcessors/FTimporterPostProcessor

or

./run_framework_tests --re=framework/PostProcessors/FTimporterPoßtProcesso

- Test Description:

- Tests of the FTImporter post-processor: it read a fault-tree (ET) from an .xml file (eventTree.xml) and it imports the FT structure into a PointSet. Note that the FT needs to be in an OpenPSA format.

- Original Author:

- mandd

- Creation date:

- 2017-11-07

- The classes tested in this test are:

- ETimporter

\subsubsection{FRAMEWORK POSTPROCESSORS FTIMPORTERPOSTPROCESSOR.FTIMPORTER ATLEAST}

This test can be found at ". / raven/tests/framework/PostProcessors/FTimporterPostProce test_FTimporter_atleast.xml". This test can be called executing the following command:

./run_tests --re=framework/PostProcessors/FTimporterPostProcessor. FTimport

or

./run_framework_tests --re=framework/PostProcessors/FTimporterPoßtProcesso 
- Test Description:

- Tests of the FTImporter post-processor: it read a fault-tree (ET) from an .xml file (eventTree.xml) and it imports the FT structure into a PointSet. Note that the FT needs to be in an OpenPSA format.

- Original Author:

- mandd

- Creation date:

- 2017-11-07

- The classes tested in this test are:

- ETimporter

\subsubsection{FRAMEWORK POSTPROCESSORS FTIMPORTERPOSTPROCESSOR.FTIMPORTER CARDINALITY}

This test can be found at ". / raven/tests/framework/PostProcessors/FTimporterPostProce test_FTimporter_cardinality.xml". This test can be called executing the following command:

. Irun_tests --re=framework/PostProcessors/FTimporterPostProcessor.FTimport

or

./run_framework_tests --re=framework/PostProcessors/FTimporterPo\$tProcesso

- Test Description:

- Tests of the FTImporter post-processor: it read a fault-tree (ET) from an .xml file (eventTree.xml) and it imports the FT structure into a PointSet. Note that the FT needs to be in an OpenPSA format.

- Original Author:

- mandd

- Creation date:

- 2017-11-07

- The classes tested in this test are:

- ETimporter 


\subsubsection{FRAMEWORK POSTPROCESSORS FTIMPORTERPOSTPROCESSOR.FTIMPORTER COMPONENT}

This test can be found at ". / raven/tests / framework/PostProcessors/FTimporterPostProce test_FTimporter_component.xml". This test can be called executing the following command:

. Irun_tests --re=framework/PostProcessors/FTimporterPostProcessor. FTimport

or

. Irun_framework_tests --re=framework/PostProcessors/FTimporterPoßtProcesso

- Test Description:

- Tests of the FTImporter post-processor: it read a fault-tree (ET) from an .xml file (eventTree.xml) and it imports the FT structure into a PointSet. Note that the FT needs to be in an OpenPSA format.

- Original Author:

- mandd

- Creation date:

- 2017-11-07

- The classes tested in this test are:

- ETimporter

\subsubsection{FRAMEWORK POSTPROCESSORS FTIMPORTERPOSTPROCESSOR.FTIMPORTER IFF}

This test can be found at ". / raven/tests / framework/PostProcessors/FTimporterPostProce test_FTimporter_iff.xml". This test can be called executing the following command:

. Irun_tests --re=framework/PostProcessors/FTimporterPostProcessor. FTimport

or

. Irun_framework_tests --re=framework/PostProcessors/FTimporterPo\$tProcesso 
- Test Description:

- Tests of the FTImporter post-processor: it read a fault-tree (ET) from an .xml file (eventTree.xml) and it imports the FT structure into a PointSet. Note that the FT needs to be in an OpenPSA format.

- Original Author:

- mandd

- Creation date:

- 2017-11-07

- The classes tested in this test are:

- ETimporter

\subsubsection{FRAMEWORK POSTPROCESSORS FTIMPORTERPOSTPROCESSOR.FTIMPORTER IMPY}

This test can be found at ". / raven/tests / framework/PostProcessors /FTimporterPostProces test_FTimporter_imply.xml". This test can be called executing the following command:

. Irun_tests --re=framework/PostProcessors/FTimporterPostProcessor. FTimport

or

./run_framework_tests --re=framework/PostProcessors/FTimporterPo\$tProcesso

- Test Description:

- Tests of the FTImporter post-processor: it read a fault-tree (ET) from an .xml file (eventTree.xml) and it imports the FT structure into a PointSet. Note that the FT needs to be in an OpenPSA format.

- Original Author:

- mandd

- Creation date:

- 2017-11-07

- The classes tested in this test are:

- ETimporter 


\subsubsection{FRAMEWORK POSTPROCESSORS FTIMPORTERPOSTPROCESSOR.FTIMPORTER MULTIPLEFTS}

This test can be found at ". / raven/tests / framework/PostProcessors/FTimporterPostProce test_FTimporter_multipleFTs.xml". This test can be called executing the following command:

./run_tests --re=framework/PostProcessors/FTimporterPostProcessor. FTimport

or

. Irun_framework_tests --re=framework/PostProcessors/FTimporterPo\$tProcesso

- Test Description:

- Tests of the FTImporter post-processor: it read a fault-tree (ET) from an .xml file (eventTree.xml) and it imports the FT structure into a PointSet. Note that the FT needs to be in an OpenPSA format.

- Original Author:

- mandd

- Creation date:

- 2017-11-07

- The classes tested in this test are:

- ETimporter

\subsubsection{FRAMEWORK POSTPROCESSORS FTIMPORTERPOSTPROCESSOR.FTIMPORTER NAND}

This test can be found at ". / raven/tests / framework/PostProcessors/FTimporterPostProce test_FTimporter_nand.xml". This test can be called executing the following command:

. Irun_tests --re=framework/PostProcessors/FTimporterPostProcessor. FTimport

or

. Irun_framework_tests --re=framework/PostProcessors/FTimporterPo\$tProcesso 
- Test Description:

- Tests of the FTImporter post-processor: it read a fault-tree (ET) from an .xml file (eventTree.xml) and it imports the FT structure into a PointSet. Note that the FT needs to be in an OpenPSA format.

- Original Author:

- mandd

- Creation date:

- 2017-11-07

- The classes tested in this test are:

- ETimporter

\subsubsection{FRAMEWORK POSTPROCESSORS FTIMPORTERPOSTPROCESSOR.FTIMPORTER NOR}

This test can be found at ". / raven/tests / framework/PostProcessors /FTimporterPostProces test_FTimporter_nor.xml". This test can be called executing the following command:

. Irun_tests --re=framework/PostProcessors/FTimporterPostProcesson.FTimport

or

./run_framework_tests --re=framework/PostProcessors/FTimporterPo\$tProcesso

- Test Description:

- Tests of the FTImporter post-processor: it read a fault-tree (ET) from an .xml file (eventTree.xml) and it imports the FT structure into a PointSet. Note that the FT needs to be in an OpenPSA format.

- Original Author:

- mandd

- Creation date:

- 2017-11-07

- The classes tested in this test are:

- ETimporter 


\subsubsection{FRAMEWORK POSTPROCESSORS FTIMPORTERPOSTPROCESSOR.FTIMPORTER OR}

This test can be found at ". / raven/tests/framework/PostProcessors /FTimporterPostProce test_FTimporter_or.xml”. This test can be called executing the following command:

./run_tests --re=framework/PostProcessors/FTimporterPostProcessor. FTimport

or

./run_framework_tests --re=framework/PostProcessors/FTimporterPoßtProcesso

- Test Description:

- Tests of the FTImporter post-processor: it read a fault-tree (ET) from an .xml file (eventTree.xml) and it imports the FT structure into a PointSet. Note that the FT needs to be in an OpenPSA format.

- Original Author:

- mandd

- Creation date:

- 2017-11-07

- The classes tested in this test are:

- ETimporter

\subsubsection{FRAMEWORK POSTPROCESSORS FTIMPORTERPOSTPROCESSOR.FTIMPORTER OR HOUSEEVENT}

This test can be found at ". /raven/tests/framework/PostProcessors/FTimporterPostProce test_FTimporter_or_houseEvent.xml". This test can be called executing the following command:

./run_tests --re=framework/PostProcessors/FTimporterPostProcessor. FTimport

or

./run_framework_tests --re=framework/PostProcessors/FTimporterPoßtProcesso 
- Test Description:

- Tests of the FTImporter post-processor: it read a fault-tree (ET) from an .xml file (eventTree.xml) and it imports the FT structure into a PointSet. Note that the FT needs to be in an OpenPSA format.

- Original Author:

- mandd

- Creation date:

- 2017-11-07

- The classes tested in this test are:

- ETimporter

\subsubsection{FRAMEWORK POSTPROCESSORS FTIMPORTERPOSTPROCESSOR.FTIMPORTER XOR}

This test can be found at ". / raven/tests / framework/PostProcessors /FTimporterPostProces test_FTimporter_xor.xml". This test can be called executing the following command:

. Irun_tests --re=framework/PostProcessors/FTimporterPostProcesson.FTimport

or

. Irun_framework_tests --re=framework/PostProcessors/FTimporterPo\$tProcesso

- Test Description:

- Tests of the FTImporter post-processor: it read a fault-tree (ET) from an .xml file (eventTree.xml) and it imports the FT structure into a PointSet. Note that the FT needs to be in an OpenPSA format.

- Original Author:

- mandd

- Creation date:

- 2017-11-07

- The classes tested in this test are:

- ETimporter 


\subsubsection{FRAMEWORK POSTPROCESSORS FTIMPORTERPOSTPROCESSOR.FTIMPORTER NOT}

This test can be found at ". / raven/tests / framework/PostProcessors/FTimporterPostProce test_FTimporter_not.xml". This test can be called executing the following command:

. Irun_tests --re=framework/PostProcessors/FTimporterPostProcessor. FTimport

or

./run_framework_tests --re=framework/PostProcessors/FTimporterPo\$tProcesso

- Test Description:

- Tests of the FTImporter post-processor: it read a fault-tree (ET) from an .xml file (eventTree.xml) and it imports the FT structure into a PointSet. Note that the FT needs to be in an OpenPSA format.

- Original Author:

- mandd

- Creation date:

- 2017-11-07

- The classes tested in this test are:

- ETimporter

\subsubsection{FRAMEWORK POSTPROCESSORS FTIMPORTERPOSTPROCESSOR.FTIMPORTER DOUBLENOT}

This test can be found at ". / raven/tests/framework/PostProcessors/FTimporterPostProce test_FTimporter_doubleNot.xml". This test can be called executing the following command:

. Irun_tests --re=framework/PostProcessors/FTimporterPostProcessor. FTimport

or

./run_framework_tests --re=framework/PostProcessors/FTimporterPo\$tProcesso 
- Test Description:

- Tests of the FTImporter post-processor: it read a fault-tree (ET) from an .xml file (eventTree.xml) and it imports the FT structure into a PointSet. Note that the FT needs to be in an OpenPSA format.

- Original Author:

- mandd

- Creation date:

- 2017-11-07

- The classes tested in this test are:

- ETimporter

\subsubsection{FRAMEWORK POSTPROCESSORS TOPOLOGICALPOSTPROCESSOR.TOPOLOGY SIMPLE}

This test can be found at ". / raven/tests / framework/PostProcessors/TopologicalPostProc test_topology_simple.xml”. This test can be called executing the following command:

. Irun_tests --re=framework/PostProcessors/TopologicalpostProcess qr.topolog

or

./run_framework_tests --re=framework/PostProcessors/Topologicalp ostProcess

- Test Description:

- A simple example of the approximate Morse-Smale complex (AMSC) interface using a test function consisting of a 2D Gaussian with a single maximum and 4 local minimum occurring at the corners of the domain space. The hill in the middle is purposefully off-centered so as to create non-uniform cases at each corner allowing us to simplify each local minimum in turn to create a 4 partition case, a 3 partition case, a 2 partition case, and a single partition case.

- Original Author:

- maljdan

- Creation date: 
- 2015-09-02

- The classes tested in this test are:

- PostProcessors.TopologicalDecomposition

- Since the creation of this test, the following main revisions have been performed:

1. revision info:

- author : cogljj

- date : 2015-09-29

- description: Modifying external modules to use relative to working directory.

2. revision info:

- author : maljdan

- date : 2015-10-06

- description: Correcting the user manual and reducing the sleepTime on these tests to reduce their total run time to $15 \mathrm{~s}$ on my local machine.

3. revision info:

- author : talbpaul

- date : 2016-02-08

- description: Converted failing tests.

4. revision info:

- author : maljdan

- date : 2016-04-06

- description: Updating test cases to reflect the changes to the user input.

5. revision info:

- author : maljdan

- date : 2017-01-17

- description: Adding test description.

\subsubsection{FRAMEWORK POSTPROCESSORS TOPOLOGICALPOSTPROCESSOR.PERSISTENCE COUNT}

This test can be found at ". / raven/tests / framework/PostProcessors/TopologicalPostProce test_persistence_count.xml". This test can be called executing the following command:

. Irun_tests --re=framework/PostProcessors/TopologicalpostProcessqr.persist 
or

./run_framework_tests --re=framework/PostProcessors/TopologicalpфstProcess

- Test Description:

- A simple example of the approximate Morse-Smale complex (AMSC) interface where select a persistence level using the count of points in each partition, that is if a partition has fewer than $\mathrm{x}$ points, it will be merged into a neighboring partition. Note, that all of the "persistence" tests will use the Schwefel function for testing.

- Original Author:

- maljdan

- Creation date:

- 2015-09-21

- The classes tested in this test are:

- PostProcessors.TopologicalDecomposition

- Since the creation of this test, the following main revisions have been performed:

1. revision info:

- author : cogljj

- date : 2015-09-29

- description: Modifying external modules to use relative to working directory.

2. revision info:

- author : maljdan

- date : 2015-10-05

- description: Removing the local fits from the topology PP and reducing the problem sizes for the persistence tests.

3. revision info:

- author : maljdan

- date : 2015-10-05

- description: Adjusting the Schwefel function's domain to fit better with fewer data points.

4. revision info:

- author : maljdan 
- date : 2015-10-06

- description: Correcting the user manual and reducing the sleepTime on these tests to reduce their total run time to $15 \mathrm{~s}$ on my local machine.

5. revision info:

- author : talbpaul

- date : 2016-02-08

- description: Converted failing tests.

6. revision info:

- author : maljdan

- date : 2016-04-06

- description: Updating test cases to reflect the changes to the user input.

7. revision info:

- author : maljdan

- date : 2017-01-17

- description: Adding test description.

\subsubsection{FRAMEWORK POSTPROCESSORS TOPOLOGICALPOSTPROCESSOR.PERSISTENCE PROB}

This test can be found at ". /raven/tests/framework/PostProcessors/TopologicalPostProce test_persistence_prob.xml". This test can be called executing the following command:

./run_tests --re=framework/PostProcessors/TopologicalPostProcess $\phi$ r.persist

or

./run_framework_tests --re=framework/PostProcessors/Topologicalp stProcess

- Test Description:

- A simple example of the approximate Morse-Smale complex (AMSC) interface where we select a persistence level based on a minimum amount of probability. That is, if the total probability weight of a partition is too small, then that partition will be merged into a neighboring partition given the normal rules of persistence simplification. Note, that all of the "persistence" tests will use the Schwefel function for testing.

- Original Author:

- maljdan 
- Creation date:

- 2015-09-21

- The classes tested in this test are:

- PostProcessors.TopologicalDecomposition

- Since the creation of this test, the following main revisions have been performed:

1. revision info:

- author : cogljj

- date : 2015-09-29

- description: Modifying external modules to use relative to working directory.

2. revision info:

- author : maljdan

- date : 2015-10-05

- description: Removing the local fits from the topology PP and reducing the problem sizes for the persistence tests.

3. revision info:

- author : maljdan

- date : 2015-10-05

- description: Adjusting the Schwefel function's domain to fit better with fewer data points.

4. revision info:

- author : maljdan

- date : 2015-10-06

- description: Correcting the user manual and reducing the sleepTime on these tests to reduce their total run time to $15 \mathrm{~s}$ on my local machine.

5. revision info:

- author : talbpaul

- date : 2016-02-08

- description: Converted failing tests.

6. revision info:

- author : maljdan

- date : 2016-04-06

- description: Updating test cases to reflect the changes to the user input.

7. revision info: 
- author : maljdan

- date : 2017-01-17

- description: Adding test description.

\subsubsection{FRAMEWORK POSTPROCESSORS TOPOLOGICALPOSTPROCESSOR.PERSISTENCE VALUE}

This test can be found at ". / raven/tests/framework/PostProcessors/TopologicalPostProc test_persistence_value.xml". This test can be called executing the following command:

. Irun_tests --re=framework/PostProcessors/TopologicalpostProcessor.persist

or

. Irun_framework_tests --re=framework/PostProcessors/TopologicalpostProcess

- Test Description:

- A simple example of the approximate Morse-Smale complex (AMSC) interface where we select a persistence level based on the standard notion of the function value difference between the extrema and its nearest valued neighboring critical point. That is, larger peaks and valleys will persist longer than smaller "noisy" features. This is the standard definition of persistence used in Morse topology. Note, that all of the "persistence" tests will use the Schwefel function for testing.

- Original Author:

- maljdan

- Creation date:

- 2015-09-21

- The classes tested in this test are:

- PostProcessors.TopologicalDecomposition

- Since the creation of this test, the following main revisions have been performed:

1. revision info:

- author : cogljj

- date : 2015-09-29 
- description: Modifying external modules to use relative to working directory.

2. revision info:

- author : maljdan

- date : 2015-10-05

- description: Removing the local fits from the topology PP and reducing the problem sizes for the persistence tests.

3. revision info:

- author : maljdan

- date : 2015-10-05

- description: Adjusting the Schwefel function's domain to fit better with fewer data points.

4. revision info:

- author : maljdan

- date : 2015-10-06

- description: Correcting the user manual and reducing the sleepTime on these tests to reduce their total run time to $15 \mathrm{~s}$ on my local machine.

5. revision info:

- author : talbpaul

- date : 2016-02-08

- description: Converted failing tests.

6. revision info:

- author : maljdan

- date : 2016-04-06

- description: Updating test cases to reflect the changes to the user input.

7. revision info:

- author : maljdan

- date : 2017-01-17

- description: Adding test description.

\subsubsection{FRAMEWORK POSTPROCESSORS TOPOLOGICALPOSTPROCESSOR.PERSISTENCE FULL}

This test can be found at ". / raven/tests / framework/PostProcessors/TopologicalPostProce test_persistence_full.xml". This test can be called executing the following command:

. Irun_tests --re=framework/PostProcessors/TopologicalpostProcessor.persist 
or

./run_framework_tests --re=framework/PostProcessors/TopologicalpфstProcess

- Test Description:

- A simple example of the approximate Morse-Smale complex (AMSC) interface where no persistence simplification will be done. Note, that all of the "persistence" tests will use the Schwefel function for testing.

- Original Author:

- maljdan

- Creation date:

- 2015-09-21

- The classes tested in this test are:

- PostProcessors.TopologicalDecomposition

- Since the creation of this test, the following main revisions have been performed:

1. revision info:

- author : cogljj

- date : 2015-09-29

- description: Modifying external modules to use relative to working directory.

2. revision info:

- author : maljdan

- date : 2015-10-05

- description: Removing the local fits from the topology PP and reducing the problem sizes for the persistence tests.

3. revision info:

- author : maljdan

- date : 2015-10-05

- description: Adjusting the Schwefel function's domain to fit better with fewer data points.

4. revision info:

- author : maljdan

- date : 2015-10-06 
- description: Correcting the user manual and reducing the sleepTime on these tests to reduce their total run time to $15 \mathrm{~s}$ on my local machine.

5. revision info:

- author : talbpaul

- date : 2016-02-08

- description: Converted failing tests.

6. revision info:

- author : maljdan

- date : 2016-04-06

- description: Updating test cases to reflect the changes to the user input.

7. revision info:

- author : maljdan

- date : 2017-01-17

- description: Adding test description.

\subsubsection{FRAMEWORK POSTPROCESSORS TOPOLOGICALPOSTPROCESSOR.KNN}

This test can be found at ". / raven/tests / framework/PostProcessors/TopologicalPostProce test_graph_knn.xml”. This test can be called executing the following command:

. Irun_tests --re=framework/PostProcessors/TopologicalPostProcessqr.knn

or

./run_framework_tests --re=framework/PostProcessors/TopologicalPostProcess

- Test Description:

- A simple example of the approximate Morse-Smale complex (AMSC) interface that exercises the k-nearest neighbor graph structure as the underlying connectivity model for the point cloud. Note, each of the "graph" test cases uses the GaussianPeaks test case which consists of 4 distinctly shaped Gaussian peaks in a 2D input domain.

- Original Author:

- maljdan

- Creation date:

- 2015-09-21 
- The classes tested in this test are:

- PostProcessors.TopologicalDecomposition

- Since the creation of this test, the following main revisions have been performed:

1. revision info:

- author : cogljj

- date : 2015-09-29

- description: Modifying external modules to use relative to working directory.

2. revision info:

- author : maljdan

- date : 2015-10-06

- description: Reducing the graph tests for the topology pp, and re-golding them, also adding a plotter to validate the results visually.

3. revision info:

- author : talbpaul

- date : 2016-02-08

- description: Converted failing tests.

4. revision info:

- author : maljdan

- date : 2016-04-06

- description: Updating test cases to reflect the changes to the user input.

5. revision info:

- author : maljdan

- date : 2017-01-17

- description: Adding test description.

\subsubsection{FRAMEWORK POSTPROCESSORS TOPOLOGICALPOSTPROCESSOR.RELAXED BETA SKELETON}

This test can be found at ". / raven/tests/framework/PostProcessors/TopologicalPostProc test_graph_bs.xml". This test can be called executing the following command:

. Irun_tests --re=framework/PostProcessors/TopologicalPostProcess

or

. Irun_framework_tests --re=framework/PostProcessors/TopologicalpostProcess 


\section{- Test Description:}

- A simple example of the approximate Morse-Smale complex (AMSC) interface that exercises the beta skeleton graph structure as the underlying connectivity model for the point cloud. Note, each of the "graph" test cases uses the GaussianPeaks test case which consists of 4 distinctly shaped Gaussian peaks in a 2D input domain.

- Original Author:

- maljdan

- Creation date:

- 2015-09-21

- The classes tested in this test are:

- PostProcessors.TopologicalDecomposition

- Since the creation of this test, the following main revisions have been performed:

1. revision info:

- author : cogljj

- date : 2015-09-29

- description: Modifying external modules to use relative to working directory.

2. revision info:

- author : maljdan

- date : 2015-10-06

- description: Reducing the graph tests for the topology pp, and re-golding them, also adding a plotter to validate the results visually.

3. revision info:

- author : talbpaul

- date : 2016-02-08

- description: Converted failing tests.

4. revision info:

- author : maljdan

- date : 2016-04-06

- description: Updating test cases to reflect the changes to the user input.

5. revision info:

- author : maljdan

- date : 2017-01-17

- description: Adding test description. 


\subsubsection{FRAMEWORK POSTPROCESSORS TOPOLOGICALPOSTPROCESSOR.RELAXED BETA SKELETON}

This test can be found at ". / raven/tests/framework/PostProcessors/TopologicalPostProce test_graph_rbs.xml". This test can be called executing the following command:

./run_tests --re=framework/PostProcessors/TopologicalPostProcess $\phi$ r.relaxed

or

./run_framework_tests --re=framework/PostProcessors/TopologicalpostProcess

- Test Description:

- A simple example of the approximate Morse-Smale complex (AMSC) interface that exercises the relaxed beta skeleton graph structure as the underlying connectivity model for the point cloud. Note, each of the "graph" test cases uses the GaussianPeaks test case which consists of 4 distinctly shaped Gaussian peaks in a 2D input domain.

- Original Author:

- maljdan

- Creation date:

- 2015-09-21

- The classes tested in this test are:

- PostProcessors.TopologicalDecomposition

- Since the creation of this test, the following main revisions have been performed:

1. revision info:

- author : cogljj

- date : 2015-09-29

- description: Modifying external modules to use relative to working directory.

2. revision info:

- author : maljdan

- date : 2015-10-06

- description: Reducing the graph tests for the topology pp, and re-golding them, also adding a plotter to validate the results visually.

3. revision info: 
- author : talbpaul

- date : 2016-02-08

- description: Converted failing tests.

4. revision info:

- author : maljdan

- date : 2016-04-06

- description: Updating test cases to reflect the changes to the user input.

5. revision info:

- author : maljdan

- date : 2017-01-17

- description: Adding test description.

\subsubsection{FRAMEWORK POSTPROCESSORS TOPOLOGICALPOSTPROCESSOR.INTERACTIVE UI}

This test can be found at ". / raven/tests / framework/PostProcessors/TopologicalPostProce test_topology_ui.xml". This test can be called executing the following command:

. Irun_tests --re=framework/PostProcessors/TopologicalpostProcess pr. interac

or

./run_framework_tests --re=framework/PostProcessors/TopologicalpostProcess

- Test Description:

- This test exercises the user interface coupled with the Morse-Smale topology code. This test is basically a copy of test_graph_knn.xml, but it exercises our ability to load, run, and tear down a Pyside UI.

- Original Author:

- maljdan

- Creation date:

- 2016-03-23

- The classes tested in this test are:

- PostProcessors.QTopologicalDecomposition 
- Since the creation of this test, the following main revisions have been performed:

1. revision info:

- author : maljdan

- date : 2016-07-17

- description: Working on updating the syntax of test_topology_ui to match current devel.

2. revision info:

- author : maljdan

- date : 2017-01-17

- description: Adding test description.

3. revision info:

- author : maljdan

- date : 2017-05-09

- description: Adding this test to the automated system.

\subsubsection{FRAMEWORK POSTPROCESSORS REALIZATIONAVERAGER.BASIC}

This test can be found at ". / raven/tests/framework/PostProcessors/RealizationAverager, basic.xml". This test can be called executing the following command:

./run_tests --re=framework/PostProcessors/RealizationAverager.Ba\$ic

or

. Irun_framework_tests --re=framework/PostProcessors/RealizationAyerager.Ba

- Test Description:

- This test checks basic functionalities of the Realization Averager. The data is samples from a multiyear ARMA, with dimensions of 3 years and 10 time steps each. The RealizationAverager simply averages the three samples, maintaining the year and time dimensions. This test was checked analytically at creation to assure the average of the three samples is the resulting averaged values.

- Original Author:

- talbpw

- Creation date: 
- 2019-07-02

- The classes tested in this test are:

- PostProcessors.RealizationAverager

\subsubsection{FRAMEWORK POSTPROCESSORS TEMPORALDATAMININGPOSTPROCES- SOR CLUSTERING KMEANS}

This test can be found at ". / raven/tests / framework/PostProcessors/TemporalDataMiningP clustering/test_tdmSKLearnKMeans.xml". This test can be called executing the following command:

. Irun_tests --re=framework/PostProcessors/TemporalDataMiningPosterocessor/

or

. Irun_framework_tests --re=framework/PostProcessors/TemporalDataMiningPost

- Test Description:

- Tests clustering with KMeans and a pivot parameter.

- Original Author:

- chenj

- Creation date:

- 2016-02-26

- The classes tested in this test are:

- DataMining

- Since the creation of this test, the following main revisions have been performed:

1. revision info:

- author: wangc

- date : 2018-09-27

- description: As reported in issue \#805, RAVEN will not allow input DataObject of PostProcessor to be output DataObject, the output data object will be renamed, and the variable name of labels will be explicitly defined. 


\subsubsection{FRAMEWORK POSTPROCESSORS TEMPORALDATAMININGPOSTPROCES- SOR CLUSTERING MINIBATCHKMEANS}

This test can be found at ". / raven/tests/framework/PostProcessors/TemporalDataMiningP Clustering/test_tdmSKLearnMiniBatchKMeans.xml”. This test can be called executing the following command:

./run_tests --re=framework/PostProcessors/TemporalDataMiningPost Hrocessor/

or

./run_framework_tests --re=framework/PostProcessors / TemporalDataMiningPost

- Test Description:

- Tests clustering with MiniBatchKMeans and a pivot parameter.

- Original Author:

- chenj

- Creation date:

- 2016-02-26

- The classes tested in this test are:

- DataMining

- Since the creation of this test, the following main revisions have been performed:

1. revision info:

- author: wangc

- date : 2018-09-27

- description: As reported in issue \#805, RAVEN will not allow input DataObject of PostProcessor to be output DataObject, the output data object will be renamed, and the variable name of labels will be explicitly defined.

\subsubsection{FRAMEWORK POSTPROCESSORS TEMPORALDATAMININGPOSTPROCES- SOR CLUSTERING MEANSHIFT}

This test can be found at ". / raven/tests/framework/PostProcessors/TemporalDataMiningP clustering/test_tdmSKLearnMeanShift.xml". This test can be called executing the following command: 
./run_tests --re=framework/PostProcessors/TemporalDataMiningPost Hrocessor/

or

./run_framework_tests --re=framework/PostProcessors/TemporalDataMiningPost

- Test Description:

- Tests clustering with MeanShift and a pivot parameter.

- Original Author:

- chenj

- Creation date:

- 2016-02-26

- The classes tested in this test are:

- DataMining

- Since the creation of this test, the following main revisions have been performed:

1. revision info:

- author: wangc

- date : 2018-09-27

- description: As reported in issue \#805, RAVEN will not allow input DataObject of PostProcessor to be output DataObject, the output data object will be renamed, and the variable name of labels will be explicitly defined.

\subsubsection{FRAMEWORK POSTPROCESSORS TEMPORALDATAMININGPOSTPROCES- SOR CLUSTERING DBSCAN}

This test can be found at ". / raven/tests/framework/PostProcessors/TemporalDataMiningP clustering/test_tdmSKLearnDBSCAN.xml”. This test can be called executing the following command:

./run_tests --re=framework/PostProcessors/TemporalDataMiningPostArocessor/

or

./run_framework_tests --re=framework/PostProcessors/TemporalDataMiningPost 
- Test Description:

- Tests clustering with DBSCAN and a pivot parameter.

- Original Author:

- chenj

- Creation date:

$-2016-02-26$

- The classes tested in this test are:

- DataMining

- Since the creation of this test, the following main revisions have been performed:

1. revision info:

- author: wangc

- date : 2018-09-27

- description: As reported in issue \#805, RAVEN will not allow input DataObject of PostProcessor to be output DataObject, the output data object will be renamed, and the variable name of labels will be explicitly defined.

\subsubsection{FRAMEWORK POSTPROCESSORS TEMPORALDATAMININGPOSTPROCES- SOR CLUSTERING AFFINITYPROPOGATION}

This test can be found at ". / raven/tests / framework/PostProcessors/TemporalDataMiningP Clustering/test_tdmSKLearnAffinityPropogation.xml". This test can be called executing the following command:

. Irun_tests --re=framework/PostProcessors/TemporalDataMiningPostprocessor/

or

. Irun_framework_tests --re=framework/PostProcessors/TemporalDataMiningPost

- Test Description:

- Tests clustering with AffinityPropogation and a pivot parameter.

- Original Author: 
- chenj

- Creation date:

- 2016-02-26

- The classes tested in this test are:

- DataMining

- Since the creation of this test, the following main revisions have been performed:

1. revision info:

- author: wangc

- date : 2018-09-27

- description: As reported in issue \#805, RAVEN will not allow input DataObject of PostProcessor to be output DataObject, the output data object will be renamed, and the variable name of labels will be explicitly defined.

\subsubsection{FRAMEWORK POSTPROCESSORS TEMPORALDATAMININGPOSTPROCES- SOR CLUSTERING SPECTRALCLUSTERING}

This test can be found at ". / raven/tests / framework/PostProcessors / TemporalDataMiningP clustering/test_tdmSKLearnSpectralclustering.xml". This test can be called executing the following command:

. Irun_tests --re=framework/PostProcessors/TemporalDataMiningPostprocessor/

or

. Irun_framework_tests --re=framework/PostProcessors/TemporalDataMiningPost

- Test Description:

- Tests clustering with SpectralClustering with pivot parameter.

- Original Author:

- chenj

- Creation date:

- 2016-02-26 
- The classes tested in this test are:

- DataMining

- Since the creation of this test, the following main revisions have been performed:

1. revision info:

- author: wangc

- date : 2018-09-27

- description: As reported in issue \#805, RAVEN will not allow input DataObject of PostProcessor to be output DataObject, the output data object will be renamed, and the variable name of labels will be explicitly defined.

\subsubsection{FRAMEWORK POSTPROCESSORS TEMPORALDATAMININGPOSTPROCES- SOR CLUSTERING GAUSSIANMIXTURE}

This test can be found at ". / raven/tests / framework/PostProcessors / TemporalDataMiningP clustering/test_tdmSKLearnGaussianMixture.xml". This test can be called executing the following command:

. Irun_tests --re=framework/PostProcessors/TemporalDataMiningPostprocessor/

or

. Irun_framework_tests --re=framework/PostProcessors/TemporalDataMiningPost

- Test Description:

- Tests mixtures with Gaussian Mixture Model and a pivot parameter.

- Original Author:

- chenj

- Creation date:

- 2016-02-26

- The classes tested in this test are:

- DataMining

- Since the creation of this test, the following main revisions have been performed: 
1. revision info:

- author: wangc

- date : 2018-09-27

- description: As reported in issue \#805, RAVEN will not allow input DataObject of PostProcessor to be output DataObject, the output data object will be renamed, and the variable name of labels will be explicitly defined.

2. revision info:

- author : alfoa

- date : 2019-06-25

- description: init_param changed from wc to kmeans because the wc option has been removed in scikitlearn v. 0.21

\subsubsection{FRAMEWORK POSTPROCESSORS TEMPORALDATAMININGPOSTPROCES- SOR CLUSTERING VARIATIONALGMM}

This test can be found at ". / raven/tests / framework/PostProcessors/TemporalDataMiningP Clustering/test_tdmSKLearnVariationalGMM.xml”. This test can be called executing the following command:

./run_tests --re=framework/PostProcessors/TemporalDataMiningPostprocessor/

or

./run_framework_tests --re=framework/PostProcessors/TemporalDataMiningPost

- Test Description:

- Testing clustering with VBGMM (Variational Bayesian Gaussian Mixture Modeling) and pivot parameter.

- Original Author:

- chenj

- Creation date:

- 2016-02-26

- The classes tested in this test are:

- DataMining 
- Since the creation of this test, the following main revisions have been performed:

1. revision info:

- author: wangc

- date : 2018-09-27

- description: As reported in issue \#805, RAVEN will not allow input DataObject of PostProcessor to be output DataObject, the output data object will be renamed, and the variable name of labels will be explicitly defined.

\subsubsection{FRAMEWORK POSTPROCESSORS TEMPORALDATAMININGPOSTPROCES- SOR DIMENSIONALITYREDUCTION EXACTPCA}

This test can be found at ". / raven/tests / framework/PostProcessors / TemporalDataMiningP DimensionalityReduction/test_tdmSKLearnExactPCA.xml”. This test can be called executing the following command:

. Irun_tests --re=framework/PostProcessors/TemporalDataMiningPostprocessor/l

or

. Irun_framework_tests --re=framework/PostProcessors/TemporalDataMiningPost

- Test Description:

- Tests decomposition with PCA and a pivot parameter.

- Original Author:

- chenj

- Creation date:

$-2016-02-29$

- The classes tested in this test are:

- DataMining

- Since the creation of this test, the following main revisions have been performed:

1. revision info:

- author: wangc

- date : 2018-09-27 
- description: As reported in issue \#805, RAVEN will not allow input DataObject of PostProcessor to be output DataObject, the output data object will be renamed, and the variable name of labels will be explicitly defined.

\subsubsection{FRAMEWORK POSTPROCESSORS TEMPORALDATAMININGPOSTPROCES- SOR DIMENSIONALITYREDUCTION RANDOMIZEDPCA}

This test can be found at ". / raven/tests / framework/PostProcessors / TemporalDataMiningP DimensionalityReduction/test_tdmSKLearnRandomizedPCA.xml". This test can be called executing the following command:

. Irun_tests --re=framework/PostProcessors/TemporalDataMiningPostprocessor/l

or

. /run_framework_tests --re=framework/PostProcessors/TemporalDataminingPost

- Test Description:

- Tests decomposition with RandomizedPCA and a pivot parameter.

- Original Author:

- chenj

- Creation date:

- 2016-02-29

- The classes tested in this test are:

- DataMining

- Since the creation of this test, the following main revisions have been performed:

1. revision info:

- author: wangc

- date : 2018-09-27

- description: As reported in issue \#805, RAVEN will not allow input DataObject of PostProcessor to be output DataObject, the output data object will be renamed, and the variable name of labels will be explicitly defined. 


\subsubsection{FRAMEWORK POSTPROCESSORS TEMPORALDATAMININGPOSTPROCES- SOR DIMENSIONALITYREDUCTION KERNELPCA}

This test can be found at “. / raven/tests / framework/PostProcessors/TemporalDataMiningP DimensionalityReduction/test_tdmSKLearnKernelPCA.xml". This test can be called executing the following command:

. Irun_tests --re=framework/PostProcessors/TemporalDataMiningPostprocessor/l

or

./run_framework_tests --re=framework/PostProcessors/TemporalDataMiningPost

- Test Description:

- Tests decomposition with KernelPCA and a pivot parameter.

- Original Author:

- chenj

- Creation date:

- 2016-02-29

- The classes tested in this test are:

- DataMining

- Since the creation of this test, the following main revisions have been performed:

1. revision info:

- author : wangc

- date : 2018-09-27

- description: As reported in issue \#805, RAVEN will not allow input DataObject of PostProcessor to be output DataObject, the output data object will be renamed, and the variable name of labels will be explicitly defined.

\subsubsection{FRAMEWORK POSTPROCESSORS TEMPORALDATAMININGPOSTPROCES- SOR DIMENSIONALITYREDUCTION SPARSEPCA}

This test can be found at “. / raven/tests / framework/PostProcessors / TemporalDataMiningP DimensionalityReduction/test_tdmSKLearnSparsePCA.xml". This test can be called executing the following command: 
./run_tests --re=framework/PostProcessors/TemporalDataMiningPost Hrocessor/l

or

./run_framework_tests --re=framework/PostProcessors/TemporalDataMiningPost

- Test Description:

- Tests decomposition with SparsePCA and a pivot parameter.

- Original Author:

- chenj

- Creation date:

- 2016-02-26

- The classes tested in this test are:

- DataMining

- Since the creation of this test, the following main revisions have been performed:

1. revision info:

- author: wangc

- date : 2018-09-27

- description: As reported in issue \#805, RAVEN will not allow input DataObject of PostProcessor to be output DataObject, the output data object will be renamed, and the variable name of labels will be explicitly defined.

\subsubsection{FRAMEWORK POSTPROCESSORS TEMPORALDATAMININGPOSTPROCES- SOR DIMENSIONALITYREDUCTION MINIBATCHSPARSEPCA}

This test can be found at ". /raven/tests/framework/PostProcessors/TemporalDataMiningP DimensionalityReduction/test_tdmSKLearnMiniBatchSparsePCA.xml”. This test can be called executing the following command:

./run_tests --re=framework/PostProcessors/TemporalDataMiningPostArocessor/

or

./run_framework_tests --re=framework/PostProcessors/TemporalDataMiningPost 
- Test Description:

- Tests decomposition with MiniBatchSparsePCA and a pivot parameter.

- Original Author:

- chenj

- Creation date:

$-2016-02-29$

- The classes tested in this test are:

- DataMining

- Since the creation of this test, the following main revisions have been performed:

1. revision info:

- author: wangc

- date : 2018-09-27

- description: As reported in issue \#805, RAVEN will not allow input DataObject of PostProcessor to be output DataObject, the output data object will be renamed, and the variable name of labels will be explicitly defined.

\subsubsection{FRAMEWORK POSTPROCESSORS TEMPORALDATAMININGPOSTPROCES- SOR DIMENSIONALITYREDUCTION TRUNCATEDSVD}

This test can be found at ". / raven/tests / framework/PostProcessors / TemporalDataMiningP DimensionalityReduction/test_tdmSKLearnTruncatedSVD.xml". This test can be called executing the following command:

. Irun_tests --re=framework/PostProcessors/TemporalDataMiningPostprocessor/l

or

. Irun_framework_tests --re=framework/PostProcessors/TemporalDataMiningPost

- Test Description:

- Tests decomposition with TruncatedSVD and a pivot parameter.

- Original Author: 
- chenj

- Creation date:

- 2016-02-26

- The classes tested in this test are:

- DataMining

- Since the creation of this test, the following main revisions have been performed:

1. revision info:

- author: wangc

- date : 2018-09-27

- description: As reported in issue \#805, RAVEN will not allow input DataObject of PostProcessor to be output DataObject, the output data object will be renamed, and the variable name of labels will be explicitly defined.

\subsubsection{FRAMEWORK POSTPROCESSORS TEMPORALDATAMININGPOSTPROCES- SOR DIMENSIONALITYREDUCTION FASTICA}

This test can be found at ". / raven/tests / framework/PostProcessors / TemporalDataMiningP DimensionalityReduction/test_tdmSKLearnFastICA.xml". This test can be called executing the following command:

. Irun_tests --re=framework/PostProcessors/TemporalDataMiningPostprocessor/

or

. Irun_framework_tests --re=framework/PostProcessors/TemporalDataMiningPost

- Test Description:

- Tests decomposition with FastICA and a pivot parameter.

- Original Author:

- chenj

- Creation date:

- 2016-02-29 
- The classes tested in this test are:

- DataMining

- Since the creation of this test, the following main revisions have been performed:

1. revision info:

- author: wangc

- date : 2018-09-27

- description: As reported in issue \#805, RAVEN will not allow input DataObject of PostProcessor to be output DataObject, the output data object will be renamed, and the variable name of labels will be explicitly defined.

\subsubsection{FRAMEWORK POSTPROCESSORS TEMPORALDATAMININGPOSTPROCES- SOR DIMENSIONALITYREDUCTION LOCALLYLINEAREMBEDDING}

This test can be found at ". / raven/tests / framework/PostProcessors / TemporalDataMiningP DimensionalityReduction/test_tdmSKLearnLocallyLinearEmbedding.xml".

This test can be called executing the following command:

. Irun_tests --re=framework/PostProcessors/TemporalDataMiningPostprocessor/

or

. Irun_framework_tests --re=framework/PostProcessors/TemporalDataMiningPost

- Test Description:

- Tests manifold with LocallyLinearEmbedding and a pivot paramater.

- Original Author:

- chenj

- Creation date:

- 2016-02-29

- The classes tested in this test are:

- DataMining

- Since the creation of this test, the following main revisions have been performed: 
1. revision info:

- author: wangc

- date : 2018-09-27

- description: As reported in issue \#805, RAVEN will not allow input DataObject of PostProcessor to be output DataObject, the output data object will be renamed, and the variable name of labels will be explicitly defined.

\subsubsection{FRAMEWORK POSTPROCESSORS TEMPORALDATAMININGPOSTPROCES- SOR DIMENSIONALITYREDUCTION MULTIDIMENSIONALSCALING}

This test can be found at ". /raven/tests/framework/PostProcessors/TemporalDataMiningP DimensionalityReduction/test_tdmSKLearnMultiDimensionalScaling.xml”. This test can be called executing the following command:

./run_tests --re=framework/PostProcessors/TemporalDataMiningPostArocessor/

or

./run_framework_tests --re=framework/PostProcessors/TemporalDataMiningPost

- Test Description:

- Tests manifold with MDS (MultiDimensionalScaling) and a pivot parameter.

- Original Author:

- chenj

- Creation date:

- 2016-02-29

- The classes tested in this test are:

- DataMining

- Since the creation of this test, the following main revisions have been performed:

1. revision info:

- author: wangc

- date : 2018-09-27

- description: As reported in issue \#805, RAVEN will not allow input DataObject of PostProcessor to be output DataObject, the output data object will be renamed, and the variable name of labels will be explicitly defined. 


\subsubsection{FRAMEWORK POSTPROCESSORS INTERFACEDPOSTPROCESSOR INTER- FACEDPOSTPROCESSOR}

This test can be found at ". / raven/tests / framework/PostProcessors/InterfacedPostProce test_interfacedPP.xml". This test can be called executing the following command:

./run_tests --re=framework/PostProcessors/InterfacedPostProcessor/interfac

or

./run_framework_tests --re=framework/PostProcessors/InterfacedPo\$tProcesso

- Test Description:

- Test of the interfaced post-processor interface

- Original Author:

- mandd

- Creation date:

- 2019-01-16

- The classes tested in this test are:

- InterfacedPostProcessor

\subsubsection{FRAMEWORK POSTPROCESSORS INTERFACEDPOSTPROCESSOR INTER- FACEDPOSTPROCESSORPARALLEL}

This test can be found at ". / raven/tests/framework/PostProcessors/InterfacedPostProce. test_interfacedPP_parallel.xml". This test can be called executing the following command:

./run_tests --re=framework/PostProcessors/InterfacedPostProcesson/interfac

or

./run_framework_tests --re=framework/PostProcessors/InterfacedPo\$tProcesso

- Test Description: 
- Test of the interfaced post-processor interface performed in parallel

- Original Author:

- mandd

- Creation date:

- 2019-01-16

- The classes tested in this test are:

- InterfacedPostProcessor

\subsubsection{FRAMEWORK POSTPROCESSORS INTERFACEDPOSTPROCESSOR HISTO- RYSAMPLING}

This test can be found at ". / raven/tests/framework/PostProcessors/InterfacedPostProce test_historySetSamplingIPP.xml". This test can be called executing the following command:

. Irun_tests --re=framework/PostProcessors/InterfacedPostProcessor/historys

or

. /run_framework_tests --re=framework/PostProcessors/InterfacedPo\$tProcesso

- Test Description:

- Tests of the HistorySetSampling interfaced post-processor and all its sampling types

- Original Author:

- mandd

- Creation date:

- 2016-02-09

- The classes tested in this test are:

- InterfacedPostProcessor

- Since the creation of this test, the following main revisions have been performed:

1. revision info: 
- author: wangc

- date : 2018-08-08

- description: Test the defect issue \#739

\subsubsection{FRAMEWORK POSTPROCESSORS INTERFACEDPOSTPROCESSOR HISTO- RYSAMPLINGINTERVALAVE}

This test can be found at ". / raven/tests / framework/PostProcessors/InterfacedPostProce test_historySetSamplingIntervalAve.xml". This test can be called executing the following command:

. Irun_tests --re=framework/PostProcessors/InterfacedPostProcesson/historys

or

./run_framework_tests --re=framework/PostProcessors/InterfacedPo\$tProcesso

- Test Description:

- Tests of the HistorySetSampling post-processor with intervalAverage interpolation technique

- Original Author:

- junc

- Creation date:

- 2016-11-16

- The classes tested in this test are:

- InterfacedPostProcessor

\subsubsection{FRAMEWORK POSTPROCESSORS INTERFACEDPOSTPROCESSOR HISTO- RYSETSNAPSHOT}

This test can be found at ". / raven/tests / framework/PostProcessors / InterfacedPostProce test_historysetsnapshot.xml". This test can be called executing the following command:

./run_tests --re=framework/PostProcessors/InterfacedPostProcessor/Historys 
or

. Irun_framework_tests --re=framework/PostProcessors/InterfacedPo\$tProcesso.

- Test Description:

- Tests of the HistorySetSnapShot interfaced post-processor

- Original Author:

- mandd

- Creation date:

- 2016-02-09

- The classes tested in this test are:

- InterfacedPostProcessor

\subsubsection{FRAMEWORK POSTPROCESSORS INTERFACEDPOSTPROCESSOR HISTO- RYSETSNAPSHOTMIXED}

This test can be found at ". / raven/tests / framework/PostProcessors/InterfacedPostProce: test_historysetSnapshot_mixed.xml”. This test can be called executing the following command:

. Irun_tests --re=framework/PostProcessors/InterfacedPostProcesson/Historys

or

./run_framework_tests --re=framework/PostProcessors/InterfacedPo tProcesso

- Test Description:

- Tests of HistorySetSnapShot and its mixed type of snapshot techniques

- Original Author:

- talbpaul

- Creation date:

- 2016-06-14

- The classes tested in this test are:

- InterfacedPostProcessor 


\subsubsection{FRAMEWORK POSTPROCESSORS INTERFACEDPOSTPROCESSOR HISTO- RYSETSYNC}

This test can be found at ". / raven/tests/framework/PostProcessors/InterfacedPostProce test_historySetSync.xml". This test can be called executing the following command:

./run_tests --re=framework/PostProcessors/InterfacedPostProcesson/Historys

or

./run_framework_tests --re=framework/PostProcessors/InterfacedPo\$tProcesso

- Test Description:

- Tests of the HistorySetSync interfaced post-processor

- Original Author:

- mandd

- Creation date:

- 2016-02-09

- The classes tested in this test are:

- InterfacedPostProcessor

\subsubsection{FRAMEWORK POSTPROCESSORS INTERFACEDPOSTPROCESSOR HS SYNC ALL}

This test can be found at ". / raven/tests / framework/PostProcessors/InterfacedPostProce test_historysetSyncAll.xml". This test can be called executing the following command:

- /run_tests --re=framework/PostProcessors/InterfacedPostProcessor/hs_sync_

or

./run_framework_tests --re=framework/PostProcessors/InterfacedPo terocesso

- Test Description: 
- Tests of the HistorySetSync interfaced post-processor and in particular the all syncMethod

- Original Author:

- talbpaul

- Creation date:

- 2016-04-13

- The classes tested in this test are:

- InterfacedPostProcessor

- Since the creation of this test, the following main revisions have been performed:

1. revision info:

- author : talbpaul

- date : 2019-01-09

- description: moving from ROM-file to ROM-dataobjects

\subsubsection{FRAMEWORK POSTPROCESSORS INTERFACEDPOSTPROCESSOR HS SYNC MIN}

This test can be found at ". / raven/tests / framework/PostProcessors/ InterfacedPostProce test_historysetSyncMin.xml". This test can be called executing the following command:

./run_tests --re=framework/PostProcessors/InterfacedPostProcessor/hs_sync_l

or

./run_framework_tests --re=framework/PostProcessors/InterfacedPoßtProcesso

- Test Description:

- Tests of the HistorySetSync interfaced post-processor and in particular the MIN syncMethod

- Original Author:

- talbpaul

- Creation date:

- 2016-04-14 
- The classes tested in this test are:

- InterfacedPostProcessor

- Since the creation of this test, the following main revisions have been performed:

1. revision info:

- author : talbpaul

- date : 2019-01-09

- description: moving from ROM-file to ROM-dataobjects

\subsubsection{FRAMEWORK POSTPROCESSORS INTERFACEDPOSTPROCESSOR HS SYNC MAX}

This test can be found at ". / raven/tests / framework/PostProcessors/InterfacedPostProce test_historysetSyncMax.xml". This test can be called executing the following command:

. Irun_tests --re=framework/PostProcessors/InterfacedPostProcessor/hs_sync_

or

./run_framework_tests --re=framework/PostProcessors/InterfacedPoßtProcesso

- Test Description:

- Tests of the HistorySetSync interfaced post-processor and in particular the MAX syncMethod

- Original Author:

- talbpaul

- Creation date:

- 2016-04-14

- The classes tested in this test are:

- InterfacedPostProcessor

- Since the creation of this test, the following main revisions have been performed:

1. revision info:

- author: talbpaul

- date : 2019-01-09

- description: moving from ROM-file to ROM-dataobjects 


\subsubsection{FRAMEWORK POSTPROCESSORS INTERFACEDPOSTPROCESSOR HS2PS}

This test can be found at ". /raven/tests/framework/PostProcessors/InterfacedPostProce test_HS2PS.xml". This test can be called executing the following command:

./run_tests --re=framework/PostProcessors/InterfacedPostProcesson/HS2PS

or

./run_framework_tests --re=framework/PostProcessors/InterfacedPoßtProcesso

- Test Description:

- Tests of the HS2PS interfaced post-processor

- Original Author:

- mandd

- Creation date:

- 2016-02-09

- The classes tested in this test are:

- InterfacedPostProcessor

\subsubsection{FRAMEWORK POSTPROCESSORS INTERFACEDPOSTPROCESSOR INTER- FACEDPOSTPROCESSOR POINTSET}

This test can be found at ". / raven/tests/framework/PostProcessors/InterfacedPostProce test_interfacedPP_pointset.xml". This test can be called executing the following command:

./run_tests --re=framework/PostProcessors/InterfacedPostProcessor/interfac

or

./run_framework_tests --re=framework/PostProcessors/InterfacedPoßtProcesso

- Test Description:

- Test of the interfaced post-processor interface to deal with pointSet 
- Original Author:

- mandd

- Creation date:

- 2019-01-16

- The classes tested in this test are:

- InterfacedPostProcessor

\subsubsection{FRAMEWORK POSTPROCESSORS INTERFACEDPOSTPROCESSOR TYPI- CALHISTORYFROMHS}

This test can be found at ". /raven/tests/framework/PostProcessors/InterfacedPostProce test_typicalHistoryFromHS.xml". This test can be called executing the following command:

./run_tests --re=framework/PostProcessors/InterfacedPostProcessol/TypicalH.

or

./run_framework_tests --re=framework/PostProcessors/InterfacedPoßtProcesso

- Test Description:

- Tests of the TypicalHistoryFromHistorySet interfaced post-processor

- Original Author:

- chenj

- Creation date:

- 2016-07-07

- The classes tested in this test are:

- InterfacedPostProcessor

- Since the creation of this test, the following main revisions have been performed:

1. revision info:

- author : talbpaul

- date : 2019-01-30

- description: regolded using central binning system for empirical distributions, also formatted test to raven standard $\mathrm{xml}$ 


\subsubsection{FRAMEWORK POSTPROCESSORS INTERFACEDPOSTPROCESSOR.METADATAUSAGEI}

This test can be found at ". /raven/tests/framework/PostProcessors/InterfacedPostProce test_metadata_usage_in_interfacePP.xml". This test can be called executing the following command:

./run_tests --re=framework/PostProcessors/InterfacedPostProcesson.metadata

Or

./run_framework_tests --re=framework/PostProcessors/InterfacedPoßtProcesso

- Test Description:

- Test of the interfaced post-processor interface internally using metadata coming from RAVEN.

- Original Author:

- mandd

- Creation date:

- 2019-01-16

- The classes tested in this test are:

- Models.PostProcessors.InterfacedPostProcessor

- Since the creation of this test, the following main revisions have been performed:

1. revision info:

- author : alfoa

- date : 2018-05-15

- description: Adding this test description.

\subsubsection{FRAMEWORK POSTPROCESSORS INTERFACEDPOSTPROCESSOR.HSTOPSOPERATOF}

This test can be found at ". /raven/tests/framework/PostProcessors/InterfacedPostProce test_HistorySetToPointSetoperators.xml". This test can be called executing the following command:

. Irun_tests --re=framework/PostProcessors/InterfacedPostProcessor. HStoP Sop 
or

. Irun_framework_tests --re=framework/PostProcessors/InterfacedPo\$tProcesso

- Test Description:

- This test is aimed to show how to use the "HStoPSOperator" InterfacedPostProcessor. The 3 basic functionalities are testes: 1) row-based projection, 2) pivot-value-based projection and 3) operator-based projection (max, min, average)

- Original Author:

- alfoa

- Creation date:

- 2018-02-01

- The classes tested in this test are:

- InterfacedPostProcessor

\subsubsection{FRAMEWORK POSTPROCESSORS INTERFACEDPOSTPROCESSOR.HISTORYSETSAMP AND SYNC}

This test can be found at ". / raven/tests / framework/PostProcessors/InterfacedPostProce test_historySetSampling_and_sync.xml”. This test can be called executing the following command:

. Irun_tests --re=framework/PostProcessors/InterfacedPostProcessor.historys

or

. Irun_framework_tests --re=framework/PostProcessors/InterfacedPoßtProcesso

- Test Description:

- Tests of the HistorySetSampling interfaced post-processor and sub-sequential syncronization of the history set

- Original Author:

- alfoa 
- Creation date:

- 2019-10-08

- The classes tested in this test are:

- InterfacedPostProcessor

- Since the creation of this test, the following main revisions have been performed:

1. revision info:

- author : alfoa

- date : 2019-10-08

- description: Created test to show how to perform the sync

\subsubsection{FRAMEWORK POSTPROCESSORS INTERFACEDPOSTPROCESSOR RISKMEA- SURESDISCRETE}

This test can be found at ". /raven/tests/framework/PostProcessors/RiskMeasuresDiscret test_riskMeasuresDiscrete.xml". This test can be called executing the following command:

./run_tests --re=framework/PostProcessors/InterfacedPostProcesson/RiskMeas

or

./run_framework_tests --re=framework/PostProcessors/InterfacedPoßtProcesso.

- Test Description:

- Tests of the four risk importance measures: Risk Achievement Worth (RAW), Risk Reduction Worth (RRW), Birnbaum (B) and Fussell-Vesely (FV)

- Original Author:

- mandd

- Creation date:

- 2016-10-31

- The classes tested in this test are:

- InterfacedPostProcessor 
- Since the creation of this test, the following main revisions have been performed:

1. revision info:

- author: wangc

- date : 2021-04-22

- description: Move 'RiskMeasuresDiscrete' from InterfacedPostProcessor to PluginType PostProcessor. The subType of this PostProcessor will be changed to 'RiskMeasuresDiscrete', and the 'method' node under this PostProcessor is no longer needed.

\subsubsection{FRAMEWORK POSTPROCESSORS INTERFACEDPOSTPROCESSOR RISKMEA- SURESDISCRETEMULTIPLEIE}

This test can be found at ". / raven/tests/framework/PostProcessors/RiskMeasuresDiscret test_riskMeasuresDiscreteMultipleIE.xml”. This test can be called executing the following command:

. /run_tests --re=framework/PostProcessors/InterfacedPostProcessor/RiskMeas

or

. /run_framework_tests --re=framework/PostProcessors/InterfacedPo\$tProcesso

- Test Description:

- Tests of the four risk importance measures for multiple IEs: Risk Achievement Worth (RAW), Risk Reduction Worth (RRW), Birnbaum (B) and Fussell-Vesely (FV)

- Original Author:

- mandd

- Creation date:

$-2017-02-23$

- The classes tested in this test are:

- InterfacedPostProcessor

- Since the creation of this test, the following main revisions have been performed:

1. revision info:

- author : wangc 
- date : 2021-04-22

- description: Move 'RiskMeasuresDiscrete' from InterfacedPostProcessor to PluginType PostProcessor. The subType of this PostProcessor will be changed to 'RiskMeasuresDiscrete', and the 'method' node under this PostProcessor is no longer needed.

\subsubsection{FRAMEWORK POSTPROCESSORS INTERFACEDPOSTPROCESSOR RISKMEA- SURESTIMEDEP}

This test can be found at ". / raven/tests / framework/PostProcessors/RiskMeasuresDiscret test_riskMonitor.xml". This test can be called executing the following command:

. Irun_tests --re=framework/PostProcessors/InterfacedPostProcesson/RiskMeas

or

. Irun_framework_tests --re=framework/PostProcessors/InterfacedPo\$tProcesso

- Test Description:

- Tests of the four risk importance measures for time dependent data: Risk Achievement Worth (RAW), Risk Reduction Worth (RRW), Birnbaum (B) and Fussell-Vesely (FV)

- Original Author:

- mandd

- Creation date:

- 2017-02-23

- The classes tested in this test are:

- InterfacedPostProcessor

- Since the creation of this test, the following main revisions have been performed:

1. revision info:

- author: wangc

- date : 2021-04-22

- description: Move 'RiskMeasuresDiscrete' from InterfacedPostProcessor to PluginType PostProcessor. The subType of this PostProcessor will be changed to 'RiskMeasuresDiscrete', and the 'method' node under this PostProcessor is no longer needed. 


\subsubsection{FRAMEWORK POSTPROCESSORS HISTORYSETDELAY HISTORYSETDE- LAY}

This test can be found at ". / raven/tests/framework/PostProcessors/HistorySetDelay/ test_historySetDelay.xml". This test can be called executing the following command:

./run_tests --re=framework/PostProcessors/HistorySetDelay/History/SetDelay

or

./run_framework_tests --re=framework/PostProcessors/HistorySetDelay/Histor

- Test Description:

- Tests of the HistorySetDelay interfaced post-processor by loading in a set of sine waves and delaying some of them. The output is the input, but with some parameters lagged and one speed up.

- Original Author:

$-\operatorname{cog} \mathrm{ljj}$

- Creation date:

- 2021-04-19

- The classes tested in this test are:

- HistorySetDelay

\subsubsection{FRAMEWORK POSTPROCESSORS EXTERNALPOSTPROCESSOR.MULTIPLE USAGE}

This test can be found at ". /raven/tests/framework/PostProcessors/ExternalPostProcess multiple_usage.xml". This test can be called executing the following command:

./run_tests --re=framework/PostProcessors/ExternalPostProcessor.nultiple_u.

or

./run_framework_tests --re=framework/PostProcessors/ExternalPost Hrocessor.1

- Test Description: 
- A simple example of writing an external python function and using it inside RAVEN. Here we are testing whether using the same external processor on multiple data objects does not cause naming issues.

- Original Author:

- maljdan

- Creation date:

- 2015-06-28

- The classes tested in this test are:

- PostProcessors.ExternalPostProcessor

- Since the creation of this test, the following main revisions have been performed:

1. revision info:

- author : alfoa

- date : 2019-03-07

- description: Modified syntax in Functions as for issue \#934

\subsubsection{FRAMEWORK POSTPROCESSORS EXTERNALPOSTPROCESSOR.HISTORY SET PARTITIONING}

This test can be found at ". / raven/tests/framework/PostProcessors/ExternalPostProcess test_history_set_partitioning.xml”. This test can be called executing the following command:

. Irun_tests --re=framework/PostProcessors/ExternalPostProcessor.history_se

or

./run_framework_tests --re=framework/PostProcessors/ExternalPosterocessor.

- Test Description:

- An example of using the External Post-Processor to partition a history set by creating a new data object that removes data where the time is less than 0.0001 .

- Original Author:

- alfoa 
- Creation date:

- 2016-09-15

- The classes tested in this test are:

- PostProcessors.ExternalPostProcessor

- Since the creation of this test, the following main revisions have been performed:

1. revision info:

- author: alfoa

- date : 2016-10-04

- description: Adding an additional example that addresses Josh's comment.

2. revision info:

- author : alfoa

- date : 2019-03-07

- description: Modified syntax in Functions as for issue \#934

\subsubsection{FRAMEWORK POSTPROCESSORS EXTERNALPOSTPROCESSOR.HISTORY SET DELETING HISTORIES}

This test can be found at ". / raven/tests/framework/PostProcessors/ExternalPostProcess test_history_set_partitioning_and_remove_some_histories.xml”. This test can be called executing the following command:

./run_tests --re=framework/PostProcessors/ExternalPostProcessor.history_se

or

./run_framework_tests --re=framework/PostProcessors/ExternalPost Hrocessor.

- Test Description:

- An example of using the External Post-Processor to remove some data points from a History Set. This differs from test_history_set_partitioning.xml as that test removed output points only in the time series, whereas this one will remove them from the input space.

- Original Author:

- alfoa 
- Creation date:

- 2016-10-04

- The classes tested in this test are:

- PostProcessors.ExternalPostProcessor

- Since the creation of this test, the following main revisions have been performed:

1. revision info:

- author : maljdan

- date : 2017-01-17

- description: Added an additional example that addresses Josh's comment

2. revision info:

- author : alfoa

- date : 2016-10-04

- description: Adding test descriptions for the tests related to the ExternalPostProcessor

3. revision info:

- author : alfoa

- date : 2019-03-07

- description: Modified syntax in Functions as for issue \#934

\subsubsection{FRAMEWORK PCA GRID IMPORTANCERANKABSCOV}

This test can be found at ". /raven/tests/framework/PostProcessors/pca_postprocessor/ test_postprocessor.xml". This test can be called executing the following command:

./run_tests --re=framework/pca_grid/importanceRankAbsCov

or

./run_framework_tests --re=framework/pca_grid/importanceRankAbsCov

- Test Description:

- Tests the ImportanceRank postprocessor based on MVN distribution with absolute covariance matrix.

- Original Author: 
- wangc

- Creation date:

$-2016-03-15$

- The classes tested in this test are:

- PostProcessors.ImportanceRank

- Since the creation of this test, the following main revisions have been performed:

1. revision info:

- author: wangc

- date : 2017-12-13

- description: convert test to use the new dataobjects

\subsubsection{FRAMEWORK PCA GRID IMPORTANCERANKRELCOV}

This test can be found at ". / raven/tests/framework/PostProcessors/pca_postprocessor/ test_postprocessor_rel.xml". This test can be called executing the following command:

./run_tests --re=framework/pca_grid/importanceRankRelCov

or

./run_framework_tests --re=framework/pca_grid/importanceRankRelchv

- Test Description:

- Tests the ImportanceRank postprocessor based on MVN distribution with relative covariance matrix.

- Original Author:

- wangc

- Creation date:

- 2016-03-15

- The classes tested in this test are:

- PostProcessors.ImportanceRank 
- Since the creation of this test, the following main revisions have been performed:

1. revision info:

- author: wangc

- date : 2017-12-13

- description: convert test to use the new dataobjects

\subsubsection{FRAMEWORK PCA GRID TRANSFORMATIONMATRIX}

This test can be found at ". / raven/tests/framework/PostProcessors/pca_postprocessor/ test_transformation.xml". This test can be called executing the following command:

. /run_tests --re=framework/pca_grid/transformationMatrix

or

. /run_framework_tests --re=framework/pca_grid/transformationMatrix

- Test Description:

- Tests the ImportanceRank postprocessor to output transformation and inverse transformation matrices.

- Original Author:

- wangc

- Creation date:

- 2016-07-15

- The classes tested in this test are:

- PostProcessors.ImportanceRank

- Since the creation of this test, the following main revisions have been performed:

1. revision info:

- author: wangc

- date : 2017-12-13

- description: convert test to use the new dataobjects 


\subsubsection{FRAMEWORK PCA GRID TIMEDEPIMPORTANCERANK}

This test can be found at ". / raven/tests/framework/PostProcessors/pca_postprocessor/ test_time_dependent.xml". This test can be called executing the following command:

. /run_tests --re=framework/pca_grid/timeDepImportanceRank

or

./run_framework_tests --re=framework/pca_grid/timeDepImportanceRank

- Test Description:

- Tests the ImportanceRank postprocessor for time-dependent problems.

- Original Author:

- wangc

- Creation date:

- 2016-03-15

- The classes tested in this test are:

- PostProcessors.ImportanceRank

- Since the creation of this test, the following main revisions have been performed:

1. revision info:

- author: wangc

- date : 2017-12-13

- description: convert test to use the new dataobjects

\subsubsection{FRAMEWORK POSTPROCESSORS DATAMININGPOSTPROCESSOR CLUS- TERING KMEANS}

This test can be found at ". / raven/tests/framework/PostProcessors/DataMiningPostProce clustering/test_dataMiningkMeans.xml”. This test can be called executing the following command:

./run_tests --re=framework/PostProcessors/DataMiningPostProcessof/Clusteri 
or

. Irun_framework_tests --re=framework/PostProcessors/DataMiningPo\$tProcesso

- Test Description:

- Tests clustering with the KMeans model.

- Original Author:

- maljdan

- Creation date:

- 2016-09-09

- The classes tested in this test are:

- DataMining

- Since the creation of this test, the following main revisions have been performed:

1. revision info:

- author: wangc

- date : 2018-09-27

- description: As reported in issue \#805, RAVEN will not allow input DataObject of PostProcessor to be output DataObject, the output data object will be renamed, and the variable name of labels will be explicitly defined.

\subsubsection{FRAMEWORK POSTPROCESSORS DATAMININGPOSTPROCESSOR CLUS- TERING KMEANSPCA}

This test can be found at ". / raven/tests / framework/PostProcessors/DataMiningPostProce Clustering/test_dataMiningKMeansPCA.xml”. This test can be called executing the following command:

. Irun_tests --re=framework/PostProcessors/DataMiningPostProcessor/Clusteri

or

. Irun_framework_tests --re=framework/PostProcessors/DataMiningPoßtProcesso

- Test Description: 
- Tests clustering with KMeans followed by data reduction with PCA.

- Original Author:

- senrs

- Creation date:

- 2015-11-24

- The classes tested in this test are:

- DataMining

- Since the creation of this test, the following main revisions have been performed:

1. revision info:

- author : wangc

- date : 2018-09-27

- description: As reported in issue \#805, RAVEN will not allow input DataObject of PostProcessor to be output DataObject, the output data object will be renamed, and the variable name of labels will be explicitly defined.

\subsubsection{FRAMEWORK POSTPROCESSORS DATAMININGPOSTPROCESSOR CLUS- TERING MINIBATCHKMEANS}

This test can be found at “. / raven/tests / framework/PostProcessors/DataMiningPostProce Clustering/test_dataMiningMiniBatchKMeans.xml". This test can be called executing the following command:

. Irun_tests --re=framework/PostProcessors/DataMiningPostProcesson/Clusteri

or

./run_framework_tests --re=framework/PostProcessors/DataMiningPoßtProcesso

- Test Description:

- Tests clustering with the MiniBatchKMeans model.

- Original Author:

- senrs 
- Creation date:

$-2015-11-24$

- The classes tested in this test are:

- DataMining

- Since the creation of this test, the following main revisions have been performed:

1. revision info:

- author: wangc

- date : 2018-09-27

- description: As reported in issue \#805, RAVEN will not allow input DataObject of PostProcessor to be output DataObject, the output data object will be renamed, and the variable name of labels will be explicitly defined.

\subsubsection{FRAMEWORK POSTPROCESSORS DATAMININGPOSTPROCESSOR CLUS- TERING MEANSHIFT}

This test can be found at ". /raven/tests/framework/PostProcessors/DataMiningPostProce clustering/test_dataMiningMeanShift.xml". This test can be called executing the following command:

./run_tests --re=framework/PostProcessors/DataMiningPostProcesson/Clusteri

or

./run_framework_tests --re=framework/PostProcessors/DataMiningPo\$tProcesso

- Test Description:

- Tests clustering with the MeanShift model.

- Original Author:

- senrs

- Creation date:

- 2015-11-24

- The classes tested in this test are: 
- DataMining

- Since the creation of this test, the following main revisions have been performed:

1. revision info:

- author: wangc

- date : 2018-09-27

- description: As reported in issue \#805, RAVEN will not allow input DataObject of PostProcessor to be output DataObject, the output data object will be renamed, and the variable name of labels will be explicitly defined.

\subsubsection{FRAMEWORK POSTPROCESSORS DATAMININGPOSTPROCESSOR CLUS- TERING AFFINITYPROPOGATION}

This test can be found at ". / raven/tests / framework/PostProcessors/DataMiningPostProce. clustering/test_dataMiningAffinityPropogation.xml”. This test can be called executing the following command:

. Irun_tests --re=framework/PostProcessors/DataMiningPostProcessor/Clusteri

or

. Irun_framework_tests --re=framework/PostProcessors/DataMiningPo\$tProcesso

- Test Description:

- Tests clustering with the AffinityPropogation model.

- Original Author:

- senrs

- Creation date:

- 2015-11-24

- The classes tested in this test are:

- DataMining

- Since the creation of this test, the following main revisions have been performed:

1. revision info:

- author: wangc 
- date : 2018-09-27

- description: As reported in issue \#805, RAVEN will not allow input DataObject of PostProcessor to be output DataObject, the output data object will be renamed, and the variable name of labels will be explicitly defined.

\subsubsection{FRAMEWORK POSTPROCESSORS DATAMININGPOSTPROCESSOR CLUS- TERING DBSCAN}

This test can be found at ". / raven/tests / framework/PostProcessors/DataMiningPostProce Clustering/test_dataMiningDBSCAN.xml". This test can be called executing the following command:

. Irun_tests --re=framework/PostProcessors/DataMiningPostProcesson/Clusteri

or

. Irun_framework_tests --re=framework/PostProcessors/DataMiningPo\$tProcesso

- Test Description:

- Tests clustering with the DBSCAN model.

- Original Author:

- senrs

- Creation date:

- 2015-11-24

- The classes tested in this test are:

- DataMining

- Since the creation of this test, the following main revisions have been performed:

1. revision info:

- author: wangc

- date : 2018-09-27

- description: As reported in issue \#805, RAVEN will not allow input DataObject of PostProcessor to be output DataObject, the output data object will be renamed, and the variable name of labels will be explicitly defined. 


\subsubsection{FRAMEWORK POSTPROCESSORS DATAMININGPOSTPROCESSOR CLUS- TERING SPECTRALCLUSTERING}

This test can be found at ". / raven/tests/framework/PostProcessors/DataMiningPostProce Clustering/test_dataMiningSpectralclustering.xml". This test can be called executing the following command:

. /run_tests --re=framework/PostProcessors/DataMiningPostProcessor/Clusteri

or

./run_framework_tests --re=framework/PostProcessors/DataMiningPo\$tProcesso

- Test Description:

- Tests clustering with SpectralClustering model.

- Original Author:

- senrs

- Creation date:

- 2015-11-24

- The classes tested in this test are:

- DataMining

- Since the creation of this test, the following main revisions have been performed:

1. revision info:

- author: wangc

- date : 2018-09-27

- description: As reported in issue \#805, RAVEN will not allow input DataObject of PostProcessor to be output DataObject, the output data object will be renamed, and the variable name of labels will be explicitly defined.

\subsubsection{FRAMEWORK POSTPROCESSORS DATAMININGPOSTPROCESSOR CLUS- TERING GAUSSIANMIXTURE}

This test can be found at ". / raven/tests/framework/PostProcessors/DataMiningPostProce Clustering/test_dataMiningGaussianMixture.xml”. This test can be called executing the following command: 
./run_tests --re=framework/PostProcessors/DataMiningPostProcesson/Clusteri

or

./run_framework_tests --re=framework/PostProcessors/DataMiningPoßtProcesso

- Test Description:

- Tests mixtures with the GMM model.

- Original Author:

- senrs

- Creation date:

- 2015-11-24

- The classes tested in this test are:

- DataMining

- Since the creation of this test, the following main revisions have been performed:

1. revision info:

- author: wangc

- date : 2018-09-27

- description: As reported in issue \#805, RAVEN will not allow input DataObject of PostProcessor to be output DataObject, the output data object will be renamed, and the variable name of labels will be explicitly defined.

2. revision info:

- author : alfoa

- date : 2019-06-25

- description: init param changed from wc to kmeans because the wc option has been removed in scikitlearn v. 0.21

\subsubsection{FRAMEWORK POSTPROCESSORS DATAMININGPOSTPROCESSOR CLUS- TERING TIME DEP CLUSTERING FILTERED}

This test can be found at ". / raven/tests/framework/PostProcessors/DataMiningPostProce Clustering/test_TD_KMeans_Filtered.xml”. This test can be called executing the following command: 
./run_tests --re=framework/PostProcessors/DataMiningPostProcesson/Clusteri

or

./run_framework_tests --re=framework/PostProcessors/DataMiningPoßtProcesso:

- Test Description:

- Tests clustering with the KMeans model and preprocessing.

- Original Author:

- mandd

- Creation date:

- 2016-03-30

- The classes tested in this test are:

- DataMining

- Since the creation of this test, the following main revisions have been performed:

1. revision info:

- author: wangc

- date : 2018-09-27

- description: As reported in issue \#805, RAVEN will not allow input DataObject of PostProcessor to be output DataObject, the output data object will be renamed, and the variable name of labels will be explicitly defined.

\subsubsection{FRAMEWORK POSTPROCESSORS DATAMININGPOSTPROCESSOR CLUS- TERING VARIATIONALGMM}

This test can be found at ". /raven/tests/framework/PostProcessors/DataMiningPostProce Clustering/test_dataMiningVariationalGMM.xml”. This test can be called executing the following command:

./run_tests --re=framework/PostProcessors/DataMiningPostProcesson/Clusteri

or

./run_framework_tests --re=framework/PostProcessors/DataMiningPoßtProcesso 
- Test Description:

- Tests clustering with VBGMM (Variational Bayesian Gaussian Mixture Model).

- Original Author:

- senrs

- Creation date:

- 2015-11-24

- The classes tested in this test are:

- DataMining

- Since the creation of this test, the following main revisions have been performed:

1. revision info:

- author: wangc

- date : 2018-09-27

- description: As reported in issue \#805, RAVEN will not allow input DataObject of PostProcessor to be output DataObject, the output data object will be renamed, and the variable name of labels will be explicitly defined.

\subsubsection{FRAMEWORK POSTPROCESSORS DATAMININGPOSTPROCESSOR CLUS- TERING AGGLOMERATIVE}

This test can be found at ". / raven/tests / framework/PostProcessors/DataMiningPostProce Clustering/test_dataMiningAgglomerative.xml". This test can be called executing the following command:

./run_tests --re=framework/PostProcessors/DataMiningPostProcesson/Clusteri

or

. Irun_framework_tests --re=framework/PostProcessors/DataMiningPo\$tProcesso

- Test Description:

- Tests clustering using the Agglomerative model.

- Original Author: 
- mandd

- Creation date:

- 2016-07-20

- The classes tested in this test are:

- DataMining

- Since the creation of this test, the following main revisions have been performed:

1. revision info:

- author : maljdan

- date : 2017-08-07

- description: Modifying this test case to exercise putting the data into a new data object different than the input of to the clustering algorithm

\subsubsection{FRAMEWORK POSTPROCESSORS DATAMININGPOSTPROCESSOR CLUS- TERING TIME DEP CLUSTERING FILTERED}

This test can be found at ". / raven/tests / framework/PostProcessors/DataMiningPostProce Clustering/test_TD_MeanShift.xml". This test can be called executing the following command:

. Irun_tests --re=framework/PostProcessors/DataMiningPostProcessor/Clusteri

or

. /run_framework_tests --re=framework/PostProcessors/DataMiningPo\$tProcesso.

- Test Description:

- Tests clustering with the MeanShift model and preprocessing.

- Original Author:

- mandd

- Creation date:

- 2016-07-20

- The classes tested in this test are: 
- DataMining

- Since the creation of this test, the following main revisions have been performed:

1. revision info:

- author: wangc

- date : 2018-09-27

- description: As reported in issue \#805, RAVEN will not allow input DataObject of PostProcessor to be output DataObject, the output data object will be renamed, and the variable name of labels will be explicitly defined.

\subsubsection{FRAMEWORK POSTPROCESSORS DATAMININGPOSTPROCESSOR CLUS- TERING TIME DEP HIERARCHICALSCIPY DTW}

This test can be found at ". / raven/tests/framework/PostProcessors/DataMiningPostProce clustering/test_TD_hierarchical_dtw.xml”. This test can be called executing the following command:

. Irun_tests --re=framework/PostProcessors/DataMiningPostProcessor/Clusteri

or

./run_framework_tests --re=framework/PostProcessors/DataMiningPo\$tProcesso

- Test Description:

- Tests clustering with Hierarchical model and DTW metric.

- Original Author:

- mandd

- Creation date:

- 2016-10-10

- The classes tested in this test are:

- DataMining

- Since the creation of this test, the following main revisions have been performed:

1. revision info:

- author: wangc 
- date : 2018-09-27

- description: As reported in issue \#805, RAVEN will not allow input DataObject of PostProcessor to be output DataObject, the output data object will be renamed, and the variable name of labels will be explicitly defined.

\subsubsection{FRAMEWORK POSTPROCESSORS DATAMININGPOSTPROCESSOR CLUS- TERING TIME DEP HIERARCHICALSCIPY DTW DERIVATIVE}

This test can be found at ". / raven/tests/framework/PostProcessors/DataMiningPostProce Clustering/test_TD_hierarchical_dtw_derivative.xml". This test can be called executing the following command:

. Irun_tests --re=framework/PostProcessors/DataMiningPostProcessor/clusteri

or

. Irun_framework_tests --re=framework/PostProcessors/DataMiningPo\$tProcesso

- Test Description:

- Tests clustering with Hierarchical model and derivative DTW metric.

- Original Author:

- mandd

- Creation date:

- 2016-10-21

- The classes tested in this test are:

- DataMining

- Since the creation of this test, the following main revisions have been performed:

1. revision info:

- author: wangc

- date : 2018-09-27

- description: As reported in issue \#805, RAVEN will not allow input DataObject of PostProcessor to be output DataObject, the output data object will be renamed, and the variable name of labels will be explicitly defined. 


\subsubsection{FRAMEWORK POSTPROCESSORS DATAMININGPOSTPROCESSOR CLUS- TERING HIERARCHICALSCIPY}

This test can be found at ". /raven/tests/framework/PostProcessors/DataMiningPostProce clustering/test_dataMiningHierarchical.xml". This test can be called executing the following command:

./run_tests --re=framework/PostProcessors/DataMiningPostProcesson/Clusteri

or

./run_framework_tests --re=framework/PostProcessors/DataMiningPołtProcesso

- Test Description:

- Tests clustering with the Hierarchical model.

- Original Author:

- mannd

- Creation date:

- 2016-10-10

- The classes tested in this test are:

- DataMining

- Since the creation of this test, the following main revisions have been performed:

1. revision info:

- author: wangc

- date : 2018-09-27

- description: As reported in issue \#805, RAVEN will not allow input DataObject of PostProcessor to be output DataObject, the output data object will be renamed, and the variable name of labels will be explicitly defined.

\subsubsection{FRAMEWORK POSTPROCESSORS DATAMININGPOSTPROCESSOR CLUS- TERING AGGLOMERATIVEDTW}

This test can be found at ". / raven/tests/framework/PostProcessors/DataMiningPostProce Clustering/test_TD_agglomerative_dtw.xml”. This test can be called executing the following command: 
./run_tests --re=framework/PostProcessors/DataMiningPostProcesson/Clusteri

or

./run_framework_tests --re=framework/PostProcessors/DataMiningPoßtProcesso:

- Test Description:

- Tests clustering with Agglomerative model with DTW metric.

- Original Author:

- mandd

- Creation date:

- 2016-10-10

- The classes tested in this test are:

- DataMining

- Since the creation of this test, the following main revisions have been performed:

1. revision info:

- author: wangc

- date : 2018-09-27

- description: As reported in issue \#805, RAVEN will not allow input DataObject of PostProcessor to be output DataObject, the output data object will be renamed, and the variable name of labels will be explicitly defined.

\subsubsection{FRAMEWORK POSTPROCESSORS DATAMININGPOSTPROCESSOR CLUS- TERING AGGLOMERATIVEEUCLIDEAN}

This test can be found at ". /raven/tests/framework/PostProcessors/DataMiningPostProce clustering/test_TD_agglomerative_euclidean.xml”. This test can be called executing the following command:

./run_tests --re=framework/PostProcessors/DataMiningPostProcesson/Clusteri

or

./run_framework_tests --re=framework/PostProcessors/DataMiningPoßtProcesso 
- Test Description:

- Tests clustering with Agglomerative model with Minkowski metric.

- Original Author:

- mandd

- Creation date:

- 2016-10-10

- The classes tested in this test are:

- DataMining

- Since the creation of this test, the following main revisions have been performed:

1. revision info:

- author : maljdan

- date : 2017-08-07

- description: Modifying this test case to exercise putting the data into a new data object different than the input of to the clustering algorithm

2. revision info:

- author : wangc

- date : 2018-09-27

- description: As reported in issue \#805, RAVEN will not allow input DataObject of PostProcessor to be output DataObject, the output data object will be renamed, and the variable name of labels will be explicitly defined.

\subsubsection{FRAMEWORK POSTPROCESSORS DATAMININGPOSTPROCESSOR CLUS- TERING HIERARCHICALSCIPY SKL}

This test can be found at ". / raven/tests / framework/PostProcessors/DataMiningPostProce Clustering/test_dataMiningHierarchical_SKL_Pairwise.xml". This test can be called executing the following command:

./run_tests --re=framework/PostProcessors/DataMiningPostProcessor/Clusteri

or

./run_framework_tests --re=framework/PostProcessors/DataMiningPo\$tProcesso 
- Test Description:

- Tests clustering with the Hierarchical model with the SKL metric.

- Original Author:

- mandd

- Creation date:

- 2016-12-05

- The classes tested in this test are:

- DataMining

- Since the creation of this test, the following main revisions have been performed:

1. revision info:

- author: wangc

- date : 2018-09-27

- description: As reported in issue \#805, RAVEN will not allow input DataObject of PostProcessor to be output DataObject, the output data object will be renamed, and the variable name of labels will be explicitly defined.

\subsubsection{FRAMEWORK POSTPROCESSORS DATAMININGPOSTPROCESSOR CLUS- TERING.INTERACTIVE UI}

This test can be found at ". / raven/tests / framework/PostProcessors/DataMiningPostProce clustering/hierarchical_ui.xml". This test can be called executing the following command:

./run_tests --re=framework/PostProcessors/DataMiningPostProcesson/Clusteri

or

. /run_framework_tests --re=framework/PostProcessors/DataMiningPo\$tProcesso

- Test Description:

- This test exercises the user interface coupled with the Hierarchical Clustering available in RAVEN. This test is very similar to HierarchicalScipy except this adds the ability to test the loading, running, and tear down of the UI associated to that class. 
- Original Author:

- maljdan

- Creation date:

- 2016-05-09

- The classes tested in this test are:

- PostProcessors.DataMining

- Since the creation of this test, the following main revisions have been performed:

1. revision info:

- author: wangc

- date : 2018-09-27

- description: As reported in issue \#805, RAVEN will not allow input DataObject of PostProcessor to be output DataObject, the output data object will be renamed, and the variable name of labels will be explicitly defined.

\subsubsection{FRAMEWORK POSTPROCESSORS DATAMININGPOSTPROCESSOR DIMEN- SIONALITYREDUCTION EXACTPCA MLE}

This test can be found at ". / raven/tests/framework/PostProcessors/DataMiningPostProce DimensionalityReduction/test_dataMiningExactPCA_MLE.xml”. This test can be called executing the following command:

./run_tests --re=framework/PostProcessors/DataMiningPostProcesson/Dimensiol

or

./run_framework_tests --re=framework/PostProcessors/DataMiningPołtProcesso

- Test Description:

- Tests decomposition with PCA and MLE. This test exercises our ability to accept a string for $\mathrm{n} \_$components See Issue \#649

- Original Author:

- maljdan

- Creation date: 
- 2016-08-11

- The classes tested in this test are:

- DataMining

- Since the creation of this test, the following main revisions have been performed:

1. revision info:

- author: wangc

- date : 2018-09-27

- description: As reported in issue \#805, RAVEN will not allow input DataObject of PostProcessor to be output DataObject, the output data object will be renamed, and the variable name of labels will be explicitly defined.

\subsubsection{FRAMEWORK POSTPROCESSORS DATAMININGPOSTPROCESSOR DIMEN- SIONALITYREDUCTION RANDOMIZEDPCA}

This test can be found at ". / raven/tests / framework/PostProcessors/DataMiningPostProce. DimensionalityReduction/test_dataMiningRandomizedPCA.xml”. This test can be called executing the following command:

. Irun_tests --re=framework/PostProcessors/DataMiningPostProcessor/Dimensio

or

. /run_framework_tests --re=framework/PostProcessors/DataMiningPo\$tProcesso

- Test Description:

- Tests decomposition with RandomizedPCA.

- Original Author:

- senrs

- Creation date:

- 2015-11-24

- The classes tested in this test are:

- DataMining 
- Since the creation of this test, the following main revisions have been performed:

1. revision info:

- author: wangc

- date : 2018-09-27

- description: As reported in issue \#805, RAVEN will not allow input DataObject of PostProcessor to be output DataObject, the output data object will be renamed, and the variable name of labels will be explicitly defined.

\subsubsection{FRAMEWORK POSTPROCESSORS DATAMININGPOSTPROCESSOR DIMEN- SIONALITYREDUCTION KERNELPCA}

This test can be found at ". / raven/tests / framework/PostProcessors/DataMiningPostProce DimensionalityReduction/test_dataMiningKernelPCA.xml”. This test can be called executing the following command:

./run_tests --re=framework/PostProcessors/DataMiningPostProcessor/Dimensio

or

. Irun_framework_tests --re=framework/PostProcessors/DataMiningPo\$tProcesso

- Test Description:

- Tests decomposition with KernelPCA

- Original Author:

- senrs

- Creation date:

$-2015-11-24$

- The classes tested in this test are:

- DataMining

- Since the creation of this test, the following main revisions have been performed:

1. revision info:

- author: wangc

- date : 2018-09-27 
- description: As reported in issue \#805, RAVEN will not allow input DataObject of PostProcessor to be output DataObject, the output data object will be renamed, and the variable name of labels will be explicitly defined.

\subsubsection{FRAMEWORK POSTPROCESSORS DATAMININGPOSTPROCESSOR DIMEN- SIONALITYREDUCTION SPARSEPCA}

This test can be found at ". / raven/tests / framework/PostProcessors/DataMiningPostProce. DimensionalityReduction/test_dataMiningSparsePCA.xml”. This test can be called executing the following command:

./run_tests --re=framework/PostProcessors/DataMiningPostProcesson/Dimensio

or

. /run_framework_tests --re=framework/PostProcessors/DataMiningPo\$tProcesso

- Test Description:

- Tests decomposition with SparsePCA

- Original Author:

- senrs

- Creation date:

$-2015-11-24$

- The classes tested in this test are:

- DataMining

- Since the creation of this test, the following main revisions have been performed:

1. revision info:

- author: wangc

- date : 2018-09-27

- description: As reported in issue \#805, RAVEN will not allow input DataObject of PostProcessor to be output DataObject, the output data object will be renamed, and the variable name of labels will be explicitly defined. 


\subsubsection{FRAMEWORK POSTPROCESSORS DATAMININGPOSTPROCESSOR DIMEN- SIONALITYREDUCTION MINIBATCHSPARSEPCA}

This test can be found at ". / raven/tests/framework/PostProcessors/DataMiningPostProce DimensionalityReduction/test_dataMiningMiniBatchSparsePCA.xml". This test can be called executing the following command:

./run_tests --re=framework/PostProcessors/DataMiningPostProcessor/Dimensio or ./run_framework_tests --re=framework/PostProcessors/DataMiningPo\$tProcesso

- Test Description:

- Tests decomposition with MiniBatchSparsePCA

- Original Author:

- senrs

- Creation date:

- 2015-11-24

- The classes tested in this test are:

- DataMining

- Since the creation of this test, the following main revisions have been performed:

1. revision info:

- author: wangc

- date : 2018-09-27

- description: As reported in issue \#805, RAVEN will not allow input DataObject of PostProcessor to be output DataObject, the output data object will be renamed, and the variable name of labels will be explicitly defined.

\subsubsection{FRAMEWORK POSTPROCESSORS DATAMININGPOSTPROCESSOR DIMEN- SIONALITYREDUCTION TRUNCATEDSVD}

This test can be found at ". / raven/tests/framework/PostProcessors/DataMiningPostProce DimensionalityReduction/test_dataMiningTruncatedSVD.xml". This test can be called executing the following command: 
./run_tests --re=framework/PostProcessors/DataMiningPostProcessor/Dimensio

or

./run_framework_tests --re=framework/PostProcessors/DataMiningPoßtProcesso

- Test Description:

- Test decomposition using the TruncatedSVD model.

- Original Author:

- senrs

- Creation date:

- 2015-11-24

- The classes tested in this test are:

- DataMining

- Since the creation of this test, the following main revisions have been performed:

1. revision info:

- author: wangc

- date : 2018-09-27

- description: As reported in issue \#805, RAVEN will not allow input DataObject of PostProcessor to be output DataObject, the output data object will be renamed, and the variable name of labels will be explicitly defined.

\subsubsection{FRAMEWORK POSTPROCESSORS DATAMININGPOSTPROCESSOR DIMEN- SIONALITYREDUCTION FASTICA}

This test can be found at ". /raven/tests/framework/PostProcessors/DataMiningPostProce DimensionalityReduction/test_dataMiningFastICA.xml”. This test can be called executing the following command:

./run_tests --re=framework/PostProcessors/DataMiningPostProcesson/Dimensio

or

./run_framework_tests --re=framework/PostProcessors/DataMiningPoßtProcesso 
- Test Description:

- Tests decomposition using FastICA.

- Original Author:

- senrs

- Creation date:

- 2015-11-24

- The classes tested in this test are:

- DataMining

- Since the creation of this test, the following main revisions have been performed:

1. revision info:

- author: wangc

- date : 2018-09-27

- description: As reported in issue \#805, RAVEN will not allow input DataObject of PostProcessor to be output DataObject, the output data object will be renamed, and the variable name of labels will be explicitly defined.

\subsubsection{FRAMEWORK POSTPROCESSORS DATAMININGPOSTPROCESSOR DIMEN- SIONALITYREDUCTION ISOMAP}

This test can be found at ". / raven/tests / framework/PostProcessors/DataMiningPostProce DimensionalityReduction/test_dataMiningIsomap.xml". This test can be called executing the following command:

./run_tests --re=framework/PostProcessors/DataMiningPostProcessor/Dimensio

or

./run_framework_tests --re=framework/PostProcessors/DataMiningPo\$tProcesso

- Test Description:

- Testing manifold with Isomap.

- Original Author: 
- senrs

- Creation date:

- 2015-11-24

- The classes tested in this test are:

- DataMining

- Since the creation of this test, the following main revisions have been performed:

1. revision info:

- author: wangc

- date : 2018-09-27

- description: As reported in issue \#805, RAVEN will not allow input DataObject of PostProcessor to be output DataObject, the output data object will be renamed, and the variable name of labels will be explicitly defined.

\subsubsection{FRAMEWORK POSTPROCESSORS DATAMININGPOSTPROCESSOR DIMEN- SIONALITYREDUCTION LOCALLYLINEAREMBEDDING}

This test can be found at ". / raven/tests / framework/PostProcessors/DataMiningPostProce. DimensionalityReduction/test_dataMiningLocallyLinearEmbedding.xml”. This test can be called executing the following command:

./run_tests --re=framework/PostProcessors/DataMiningPostProcessor/Dimensio

or

. Irun_framework_tests --re=framework/PostProcessors/DataMiningPo\$tProcesso.

- Test Description:

- Tests manifold with LocallyLinearEmbedding.

- Original Author:

- senrs

- Creation date:

$-2015-11-24$ 
- The classes tested in this test are:

- DataMining

- Since the creation of this test, the following main revisions have been performed:

1. revision info:

- author: wangc

- date : 2018-09-27

- description: As reported in issue \#805, RAVEN will not allow input DataObject of PostProcessor to be output DataObject, the output data object will be renamed, and the variable name of labels will be explicitly defined.

\subsubsection{FRAMEWORK POSTPROCESSORS DATAMININGPOSTPROCESSOR DIMEN- SIONALITYREDUCTION SPECTRALEMBEDDING}

This test can be found at ". / raven/tests/framework/PostProcessors/DataMiningPostProce DimensionalityReduction/test_dataMiningSpectralEmbedding.xml". This test can be called executing the following command:

. Irun_tests --re=framework/PostProcessors/DataMiningPostProcessor/Dimensio

or

. Irun_framework_tests --re=framework/PostProcessors/DataMiningPo\$tProcesso.

- Test Description:

- Tests manifold with SpectralEmbedding.

- Original Author:

- senrs

- Creation date:

- 2015-11-24

- The classes tested in this test are:

- DataMining

- Since the creation of this test, the following main revisions have been performed: 
1. revision info:

- author: wangc

- date : 2018-09-27

- description: As reported in issue \#805, RAVEN will not allow input DataObject of PostProcessor to be output DataObject, the output data object will be renamed, and the variable name of labels will be explicitly defined.

\subsubsection{FRAMEWORK POSTPROCESSORS DATAMININGPOSTPROCESSOR DIMEN- SIONALITYREDUCTION MULTIDIMENSIONALSCALING}

This test can be found at ". / raven/tests/framework/PostProcessors/DataMiningPostProce DimensionalityReduction/test_dataMiningMultiDimensionalScaling.xml”. This test can be called executing the following command:

./run_tests --re=framework/PostProcessors/DataMiningPostProcessor/Dimensio

or

./run_framework_tests --re=framework/PostProcessors/DataMiningPołtProcesso:

- Test Description:

- Tests manifold with MDS

- Original Author:

- senrs

- Creation date:

- 2015-11-24

- The classes tested in this test are:

- DataMining

- Since the creation of this test, the following main revisions have been performed:

1. revision info:

- author: wangc

- date : 2018-09-27

- description: As reported in issue \#805, RAVEN will not allow input DataObject of PostProcessor to be output DataObject, the output data object will be renamed, and the variable name of labels will be explicitly defined. 


\subsubsection{FRAMEWORK POSTPROCESSORS VALIDATION TEST VALIDATION GATE PROBABILISTIC}

This test can be found at ". /raven/tests/framework/PostProcessors/Validation/ test_validation_gate_probabilistic.xml". This test can be called executing the following command:

./run_tests --re=framework/PostProcessors/Validation/test_validation_gate_l

or

./run_framework_tests --re=framework/PostProcessors/Validation/t\&st_valida

- Test Description:

- This test is aimed to show how to use the mechanics of the Validation Post-Processor. For semplicity, this test is using the attenuation model (analytical) and simple Probabilistic Validation metrics. The output name convention is "feature name" "target name" "metric name".

- Original Author:

- alfoa

- Creation date:

- 2021-04-10

- The classes tested in this test are:

- PostProcessors.Validation

- Since the creation of this test, the following main revisions have been performed:

1. revision info:

- author: alfoa

- date : 2021-04-11

- description: Added Modification for new PP API

\subsubsection{FRAMEWORK.TEST TRYCATCH}

This test can be found at ". /raven/tests/framework/TestedScripts/test_trycatch. py". This test can be called executing the following command: 
./run_tests --re=framework.test_trycatch

or

./run_framework_tests --re=framework.test_trycatch

- Test Description:

- This test is aimed to perform Unit test on the MessageHandler It can not be considered as active part of the code but of the regression test system

- Original Author:

- talbpaul

- Creation date:

- 2016-02-26

- The classes tested in this test are:

- MessageHandler

\subsubsection{FRAMEWORK USER GUIDE DATAMINING DATAMININGANALYSIS}

This test can be found at "./raven/tests/framework/user_guide/DataMining/ dataMiningAnalysis.xml". This test can be called executing the following command:

./run_tests --re=framework/user_guide/DataMining/dataMiningAnaly\$is

or

./run_framework_tests --re=framework/user_guide/DataMining/dataMiningAnaly

- Test Description:

- This test is an example for the user guide to draw from. It demonstrates a typical workflow for sampling then making use of the DataMining postprocessor.

- Original Author:

- talbpw 
- Creation date:

- 2017-01-30

- The classes tested in this test are:

- PostProcessor.DataMining

- Since the creation of this test, the following main revisions have been performed:

1. revision info:

- author : burlip

- date : 2019-04-17

- description: modified file to replace the Analyticalbateman example with the projectile example

\subsubsection{FRAMEWORK USER GUIDE RAVENTUTORIAL SINGLERUN}

This test can be found at ". /raven/tests/framework/user_guide/ravenTutorial/ singleRun. xml". This test can be called executing the following command:

./run_tests --re=framework/user_guide/ravenTutorial/singleRun

or

./run_framework_tests --re=framework/user_guide/ravenTutorial/singleRun

- Test Description:

- This test is an example for the user guide to draw from. It demonstrates typical usage of SingleRun.

- Original Author:

- wangc

- Creation date:

- 2017-07-24

- The classes tested in this test are:

- Models.Code 


\subsubsection{FRAMEWORK USER GUIDE SINGLERUNPLOT}

This test can be found at ". / raven/tests/framework/user_guide/ravenTutorial/ singleRunPlotAndPrint. xml". This test can be called executing the following command:

./run_tests --re=framework/user_guide/singleRunPlot

or

./run_framework_tests --re=framework/user_guide/singleRunPlot

- Test Description:

- This test is an example for the user guide to draw from. It demonstrates a streamlined workflow of using the SingleRun step to take a single sample, then plot and print the results.

- Original Author:

- wangc

- Creation date:

- 2017-07-28

- The classes tested in this test are:

- Steps.SingleRun

\subsubsection{FRAMEWORK USER GUIDE SINGLERUNSUBPLOT}

This test can be found at ". / raven/tests/framework/user_guide/ravenTutorial/ singleRunSubPlotsAndSelectivePrint.xml". This test can be called executing the following command:

./run_tests --re=framework/user_guide/singleRunSubPlot

or

./run_framework_tests --re=framework/user_guide/singleRunSubPlot

- Test Description: 
- This test is an example for the user guide to draw from. This advances the simplest SingleRun example by selectively printing and plotting only parts of the data.

- Original Author:

- wangc

- Creation date:

- 2017-07-28

- The classes tested in this test are:

- Steps.SingleRun,OutStreams.Print

\subsubsection{FRAMEWORK USER GUIDE RAVENTUTORIAL MONTECARLO}

This test can be found at ". / raven/tests/framework/user_guide/ravenTutorial/ MonteCarlo.xml". This test can be called executing the following command:

. Irun_tests --re=framework/user_guide/ravenTutorial/MonteCarlo

or

./run_framework_tests --re=framework/user_guide/ravenTutorial/Montecarlo

- Test Description:

- This test is an example for the user guide to draw from. It demonstrates typical usage of the MultiRun with Monte Carlo sampler.

- Original Author:

- wangc

- Creation date:

- 2017-07-24

- The classes tested in this test are:

- Samplers.MonteCarlo 


\subsubsection{FRAMEWORK USER GUIDE RAVENTUTORIAL ROMTRAIN}

This test can be found at ". /raven/tests/framework/user_guide/ravenTutorial/ RomTrain.xml". This test can be called executing the following command:

. /run_tests --re=framework/user_guide/ravenTutorial/RomTrain

or

./run_framework_tests --re=framework/user_guide/ravenTutorial/RomTrain

- Test Description:

- This test is an example for the user guide to draw from. It demonstrates the workflow for obtaining samples, then constructing the ROM and dumping the ROM .

- Original Author:

- wangc

- Creation date:

- 2017-07-26

- The classes tested in this test are:

- Models.ROM

\subsubsection{FRAMEWORK USER GUIDE RAVENTUTORIAL ROMLOAD}

This test can be found at ". /raven/tests/framework/user_guide/ravenTutorial/ RomLoad.xml". This test can be called executing the following command:

./run_tests --re=framework/user_guide/ravenTutorial/RomLoad

or

./run_framework_tests --re=framework/user_guide/ravenTutorial/RontLoad

- Test Description:

- This test is an example for the user guide to draw from. It demonstrates the workflow for loading and sampling ROM. 
- Original Author:

- wangc

- Creation date:

- 2017-07-26

- The classes tested in this test are:

- Models.ROM

\subsubsection{FRAMEWORK USER GUIDE RAVENTUTORIAL POSTPROCESS}

This test can be found at ". / raven/tests/framework/user_guide/ravenTutorial/ PostProcess.xml". This test can be called executing the following command:

. /run_tests --re=framework/user_guide/ravenTutorial/PostProcess

Or

. Irun_framework_tests --re=framework/user_guide/ravenTutorial/Po\$tProcess

- Test Description:

- This test is an example for the user guide to draw from. It performs basic statistics on data taken from the analytic bateman model, and demonstrates a typical workflow for using the PostProcess step.

- Original Author:

- wangc

- Creation date:

- 2017-07-27

- The classes tested in this test are:

- PostProcessors.BasicStatistics

- Since the creation of this test, the following main revisions have been performed:

1. revision info:

- author: wangc 
- date : 2018-12-18

- description: In PR \#882, the standard errors on statistics moments are implemented, these quantities are stored in the pointwise metadata, and will be printed out in the output csv files by default. In order to avoid regolding this test, the optional node 'what' with text values 'input, output' is added. In this case, only the user requested variables as specified in the DataObjects are printed out.

\subsubsection{FRAMEWORK USER GUIDE REDUCEDORDERMODELLING ROMCONSTRUC- TION}

This test can be found at ". /raven/tests/framework/user_guide/ReducedorderModeling/ reducedorderModeling.xml". This test can be called executing the following command:

. Irun_tests --re=framework/user_guide/ReducedorderModelling/ROMC nstructio

or

./run_framework_tests --re=framework/user_guide/ReducedorderModelling/ROMC

- Test Description:

- This test is an example for the user guide to draw from. It demonstrates the workflow for obtaining samples, then constructing both ScitKitLearn and NDinvDistWeight reduced-order models.

- Original Author:

- talbpw

- Creation date:

- 2017-01-27

- The classes tested in this test are:

- Models.ROM

- Since the creation of this test, the following main revisions have been performed:

1. revision info:

- author : burlip

- date : 2019-04-18

- description: modified file to replaced the Analyticalbateman example with the projectile example 


\subsubsection{FRAMEWORK USER GUIDE SIMPLE}

This test can be found at ". / raven/tests/framework/user_guide/EnsembleModel/ basic.xml". This test can be called executing the following command:

./run_tests --re=framework/user_guide/simple

or

./run_framework_tests --re=framework/user_guide/Simple

- Test Description:

- Provides a user case of a simple ensemble model.

- Original Author:

- talbpaul

- Creation date:

- 2018-02-07

- The classes tested in this test are:

- Models.EnsembleModel

\subsubsection{FRAMEWORK USER GUIDE STATISTICALANALYSIS STATISTICALANAL- YSIS}

This test can be found at ". / raven/tests/framework/user_guide/StatisticalAnalysis/ statisticalAnalysis.xml". This test can be called executing the following command:

./run_tests --re=framework/user_guide/StatisticalAnalysis/StatisticalAnaly

or

./run_framework_tests --re=framework/user_guide/StatisticalAnaly $\$$ is/Statis

- Test Description:

- This test is an example for the user guide to draw from. It performs basic statistics on data taken from the analytic bateman model, and demonstrates a typical workflow for using the basic statistics postprocessor. 
- Original Author:

- talbpw

- Creation date:

- 2017-01-27

- The classes tested in this test are:

- PostProcessors.BasicStatistics

- Since the creation of this test, the following main revisions have been performed:

1. revision info:

- author: wangc

- date : 2017-12-20

- description: convert test to use the new DataObjects with the new structure of basic statistic

2. revision info:

- author : burlip

- date : 2019-04-18

- description: modified file to replaced the Analyticalbateman example with the projectile example

\subsubsection{FRAMEWORK USER GUIDE ADAPTIVESAMPLINGSTRATEGIES LIMITSUR- FACESEARCH}

This test can be found at ". / raven/tests / framework/user_guide/AdaptiveSamplingstrateg. adaptiveSamplingLSsearch.xml". This test can be called executing the following command:

./run_tests --re=framework/user_guide/AdaptiveSamplingStrategies/limitsurf

or

./run_framework_tests --re=framework/user_guide/AdaptiveSampling\$trategies

- Test Description:

- This test is an example for the user guide to draw from. It demonstrates a typical workflow for using the LimitSurface capabilities of RAVEN (both the LimitSurfaceSearch sampler and the LimitSurfaceIntegral postprocessor. 
- Original Author:

- talbpw

- Creation date:

- 2017-01-26

- The classes tested in this test are:

- Samplers.LimitSurfaceSearch,PostProcessors.LimitSurfaceIntegral

- Since the creation of this test, the following main revisions have been performed:

1. revision info:

- author : alfoa

- date : 2019-03-07

- description: Modified syntax in Functions as for issue \#934

\subsubsection{FRAMEWORK USER GUIDE SIMPLE}

This test can be found at "./raven/tests/framework/user_guide/optimizing/ simple.xml". This test can be called executing the following command:

. /run_tests --re=framework/user_guide/Simple

or

./run_framework_tests --re=framework/user_guide/Simple

- Test Description:

- Provides a user case of the simplest optimization input.

- Original Author:

- talbpaul

- Creation date:

- 2017-06-30

- The classes tested in this test are:

- Optimizer 
- Since the creation of this test, the following main revisions have been performed:

1. revision info:

- author : talbpaul

- date : 2020-08-21

- description: Optimizer rework compatability

\subsubsection{FRAMEWORK USER GUIDE MULTILEVEL}

This test can be found at "./raven/tests/framework/user_guide/optimizing/ multiple_trajectories.xml”. This test can be called executing the following command:

./run_tests --re=framework/user_guide/Multilevel

or

./run_framework_tests --re=framework/user_guide/Multilevel

- Test Description:

- Provides a user case of multiple-trajectory optimization in action for the user guide.

- Original Author:

- talbpaul

- Creation date:

- 2017-06-30

- The classes tested in this test are:

- Optimizer

- Since the creation of this test, the following main revisions have been performed:

1. revision info:

- author : talbpaul

- date : 2020-08-21

- description: Optimizer rework compatability 


\subsubsection{FRAMEWORK USER GUIDE STEPSIZE}

This test can be found at "./raven/tests/framework/user_guide/optimizing/ step_size.xml". This test can be called executing the following command:

. /run_tests --re=framework/user_guide/Stepsize

or

./run_framework_tests --re=framework/user_guide/Stepsize

- Test Description:

- Provides a user case of providing growth and shrink multiplication factors for gradient search.

- Original Author:

- talbpaul

- Creation date:

- 2017-06-30

- The classes tested in this test are:

- Optimizer

- Since the creation of this test, the following main revisions have been performed:

1. revision info:

- author : talbpaul

- date : 2020-08-21

- description: Optimizer rework compatability

\subsubsection{FRAMEWORK USER GUIDE.CONSTRAIN}

This test can be found at “./raven/tests/framework/user_guide/optimizing/ constrain.xml". This test can be called executing the following command:

. /run_tests --re=framework/user_guide.Constrain

or 
./run_framework_tests --re=framework/user_guide.Constrain

- Test Description:

- Provides a user case of the "constraints" function in action for the user guide.

- Original Author:

- talbpaul

- Creation date:

- 2017-06-30

- The classes tested in this test are:

- Optimizer

- Since the creation of this test, the following main revisions have been performed:

1. revision info:

- author : talbpaul

- date : 2020-08-21

- description: Optimizer rework compatability

2. revision info:

- author : alfoa

- date : 2019-03-07

- description: Modified syntax in Functions as for issue \#934

\subsubsection{FRAMEWORK USER GUIDE FORWARDSAMPLINGSTRATEGIES MONTE- CARLO}

This test can be found at ". / raven/tests/framework/user_guide/ForwardSamplingStrategi forwardSamplingMontecarlo.xml". This test can be called executing the following command:

. Irun_tests --re=framework/user_guide/ForwardSamplingStrategies/MonteCarlo

or

./run_framework_tests --re=framework/user_guide/ForwardSamplingstrategies/l 
- Test Description:

- This test is an example for the user guide to draw from. It demonstrates typical usage of the MonteCarlo sampler to sample a Code model.

- Original Author:

- talbpw

- Creation date:

- 2017-01-26

- The classes tested in this test are:

- Samplers.MonteCarlo

- Since the creation of this test, the following main revisions have been performed:

1. revision info:

- author : burlip

- date : 2019-05-01

- description: modified file to replace the Analyticalbateman example with the projectile example

\subsubsection{FRAMEWORK USER GUIDE FORWARDSAMPLINGSTRATEGIES GRID}

This test can be found at “. / raven/tests/framework/user_guide/ForwardSamplingStrategi forwardSamplingGrid.xml". This test can be called executing the following command:

./run_tests --re=framework/user_guide/ForwardSamplingstrategies/\&rid

or

./run_framework_tests --re=framework/user_guide/ForwardSamplingstrategies/

- Test Description:

- This test is an example for the user guide to draw from. It demonstrates typical usage of the Grid sampler to sample a Code model.

- Original Author:

- talbpw 
- Creation date:

- 2017-01-26

- The classes tested in this test are:

- Samplers.Grid

- Since the creation of this test, the following main revisions have been performed:

1. revision info:

- author : burlip

- date : 2019-05-08

- description: modified file to replace the Analyticalbateman example with the projectile example

\subsubsection{FRAMEWORK USER GUIDE FORWARDSAMPLINGSTRATEGIES STRATI- FIED}

This test can be found at ". /raven/tests/framework/user_guide/ForwardSamplingStrategi forwardSamplingStratified.xml”. This test can be called executing the following command:

./run_tests --re=framework/user_guide/ForwardSamplingstrategies/\$tratified

or

./run_framework_tests --re=framework/user_guide/ForwardSamplingStrategies/

- Test Description:

- This test is an example for the user guide to draw from. It exemplifies using the Stratified sampler on a Code model (the Analytic Bateman in this case).

- Original Author:

- talbpw

- Creation date:

- 2017-01-26

- The classes tested in this test are: 
- Samplers.Stratified

- Since the creation of this test, the following main revisions have been performed:

1. revision info:

- author : burlip

- date : 2019-05-08

- description: modified file to replace the Analyticalbateman example with the projectile example

\subsubsection{FRAMEWORK USER GUIDE FORWARDSAMPLINGSTRATEGIES SPARSEG- RID}

This test can be found at ". / raven/tests / framework/user_guide/ForwardSamplingStrategi forwardSamplingSparseGrid.xml". This test can be called executing the following command:

./run_tests --re=framework/user_guide/ForwardSamplingStrategies/\$parseGrid

or

./run_framework_tests --re=framework/user_guide/ForwardSamplingStrategies/.

- Test Description:

- This test is an example for the user guide to draw from. It typifies a workflow for using the SparseGrid sampler, and constructing a corresponding ROM.

- Original Author:

- talbpw

- Creation date:

- 2017-01-26

- The classes tested in this test are:

- Samplers.SparseGrid

- Since the creation of this test, the following main revisions have been performed:

1. revision info:

- author : burlip 
- date : 2019-05-08

- description: modified file to replace the Analyticalbateman example with the projectile example

\subsubsection{FRAMEWORK.MODELS.EXTERNAL.FMU EXT MODEL}

This test can be found at “./raven/tests/framework/Models/External/fmu_ext_ model_and_use.xml". This test can be called executing the following command:

.Irun_tests --re=framework.Models.External.fmu_ext_model

or

./run_framework_tests --re=framework.Models.External.fmu_ext_model

- Test Description:

- This test is used to exercise the ability or RAVEN to export an FMU of the RAVEN external model class to be used in any FMI FMU compatible framework.

- Original Author:

- alfoa

- Creation date:

- 2021-05-10

- The classes tested in this test are:

- Models.ExternalModel

\subsubsection{FRAMEWORK SINGLERUNS CODEMODEL}

This test can be found at ". /raven/tests/framework/SingleRuns/withCode.xml". This test can be called executing the following command:

./run_tests --re=framework/SingleRuns/codeModel

or

./run_framework_tests --re=framework/SingleRuns/codeModel 
- Test Description:

- This test assures the SingleRun works in conjunction with the Code model.

- Original Author:

- talbpw

- Creation date:

- 2017-02-02

- The classes tested in this test are:

- Steps.SingleRun,Model.Code

\subsubsection{FRAMEWORK SINGLERUNS EXTERNALMODELFAILS}

This test can be found at ". / raven/tests/framework/SingleRuns/withExternalModel. xml". This test can be called executing the following command:

./run_tests --re=framework/SingleRuns/externalModelFails

or

./run_framework_tests --re=framework/SingleRuns/externalModelFails

- Test Description:

- This test assures the SingleRun produces the correct error when paired with the External model.

- Original Author:

- talbpw

- Creation date:

- 2017-01-27

- The classes tested in this test are:

- Steps.SingleRun,Model.ExternalModel 


\subsubsection{FRAMEWORK SINGLERUNS ROMFAILS}

This test can be found at "./raven/tests/framework/SingleRuns/withROM.xml". This test can be called executing the following command:

./run_tests --re=framework/SingleRuns/ROMFails

or

./run_framework_tests --re=framework/SingleRuns/ROMFails

- Test Description:

- This test assures the SingleRun produces the correct error when paired with the ROM model.

- Original Author:

- talbpw

- Creation date:

- 2017-01-27

- The classes tested in this test are:

- Steps.SingleRun,Model.ROM

\subsubsection{FRAMEWORK SINGLERUNS POSTPROCESSORFAILS}

This test can be found at "./raven/tests/framework/SingleRuns/withPP.xml". This test can be called executing the following command:

./run_tests --re=framework/SingleRuns/postProcessorfails

or

./run_framework_tests --re=framework/SingleRuns/postProcessorFails

- Test Description:

- This test assures the SingleRun produces the correct error when paired with the PostProcessor model. 
- Original Author:

- talbpw

- Creation date:

- 2017-01-27

- The classes tested in this test are:

- Steps.SingleRun,Model.PostProcessor

\subsubsection{FRAMEWORK SINGLERUNS DUMMYFAILS}

This test can be found at ". / raven/tests / framework/SingleRuns/withDummy . xml”. This test can be called executing the following command:

. Irun_tests --re=framework/SingleRuns/dummyFails

Or

./run_framework_tests --re=framework/SingleRuns/dummyFails

- Test Description:

- This test assures the SingleRun produces the correct error when paired with the Dummy model.

- Original Author:

- talbpw

- Creation date:

- 2017-01-27

- The classes tested in this test are:

- Steps.SingleRun,Model.Dummy 


\subsubsection{FRAMEWORK OPTIMIZERS SIMULATEDANNEALING.FUNCIONALLYCONSTRAINEDS}

This test can be found at ". / raven/tests/framework/Optimizers/SimulatedAnnealing/ FunctionallyConstrainedSA/test_funcConstrSimulatedAnnealing.xml". This test can be called executing the following command:

./run_tests --re=framework/Optimizers/SimulatedAnnealing.FuncionallyConstr

or

./run_framework_tests --re=framework/Optimizers/SimulatedAnnealing.Funcion

- Test Description:

- This test uses a multidimensional linear function such that the trajectory must pass through a functional constraint to reach the optimal point. The test is designed by @ talbpaul and is applied to simulated annealing.

- Original Author:

- MohammadAbdo

- Creation date:

- 2020-02-05

- The classes tested in this test are:

- Optimizer

\subsubsection{FRAMEWORK OPTIMIZERS GENETICALGORITHMS GA DISCRETEINTWITH- REPLACEMENT}

This test can be found at ". / raven/tests/framework/Optimizers/GeneticAlgorithms/ GA_discreteIntWithReplacement.xml". This test can be called executing the following command:

./run_tests --re=framework/Optimizers/GeneticAlgorithms/GA_discreteIntWith

or

./run_framework_tests --re=framework/Optimizers/GeneticAlgorithm\$/GA_discr 
- Test Description:

- This test is designed to find the optimal solution of a multiple knapsack problem The optimal solution should reach a maximum value of: planValue $=20$

- Original Author:

- mandd

- Creation date:

$-2020-06-24$

- The classes tested in this test are:

- GeneticAlgorithm

\subsubsection{FRAMEWORK OPTIMIZERS GENETICALGORITHMS GA KNAPSACKBASE}

This test can be found at ". / raven/tests / framework/Optimizers/GeneticAlgorithms / GA_knapsackBase.xml". This test can be called executing the following command:

. /run_tests --re=framework/Optimizers/GeneticAlgorithms/GA_knapsadckBase

or

./run_framework_tests --re=framework/Optimizers/GeneticAlgorithm\$/GA_knaps

- Test Description:

- This test is designed to find the optimal solution of a basic knapsack problem

- Original Author:

- mandd

- Creation date:

- 2020-12-02

- The classes tested in this test are:

- GeneticAlgorithm 


\subsubsection{FRAMEWORK OPTIMIZERS GENETICALGORITHMS GA KNAPSACKBASE- UNIFXOVER}

This test can be found at ". /raven/tests/framework/Optimizers/GeneticAlgorithms/ GA_knapsackBaseUnifXover.xml". This test can be called executing the following command:

./run_tests --re=framework/Optimizers/GeneticAlgorithms/GA_knapsackBaseUni

or

./run_framework_tests --re=framework/Optimizers/GeneticAlgorithms/GA_knaps

- Test Description:

- This test is designed to find the optimal solution of a basic knapsack problem

- Original Author:

- mandd

- Creation date:

- 2020-12-02

- The classes tested in this test are:

- GeneticAlgorithm

\subsubsection{FRAMEWORK PCA GRID GRIDPCAVALUE}

This test can be found at “./raven/tests/framework/pca_grid/test_grid_pca_ value.xml". This test can be called executing the following command:

./run_tests --re=framework/pca_grid/gridPCAValue

or

./run_framework_tests --re=framework/pca_grid/gridPCAValue

- Test Description: 
- Tests the value-generated Grid sampler combined with input reductions via PCA method, using multivariate normal distributions.

- Original Author:

- wangc

- Creation date:

- 2015-10-28

- The classes tested in this test are:

- Samplers.Grid

\subsubsection{FRAMEWORK PCA GRID GRIDPCACDF}

This test can be found at ". /raven/tests/framework/pca_grid/test_grid_pca_ cof . xml". This test can be called executing the following command:

./run_tests --re=framework/pca_grid/gridPCACdf

or

. /run_framework_tests --re=framework/pca_grid/gridPCACdf

- Test Description:

- Tests the CDF-generated Grid sampler combined with input reductions via PCA method, using multivariate normal distributions.

- Original Author:

- wangc

- Creation date:

- 2015-10-28

- The classes tested in this test are:

- Samplers.Grid 


\subsubsection{FRAMEWORK PCA GRID MCPCA}

This test can be found at "./raven/tests/framework/pca_grid/test_mc_pca.xml”. This test can be called executing the following command:

./run_tests --re=framework/pca_grid/mcPCA

or

./run_framework_tests --re=framework/pca_grid/mcPCA

- Test Description:

- Tests the MonteCarlo sampler combined with input reductions via PCA method, using multivariate normal distributions.

- Original Author:

- wangc

- Creation date:

$-2015-10-28$

- The classes tested in this test are:

- Samplers.MonteCarlo

\subsubsection{FRAMEWORK PCA ROM INPUTPCAREDUCTION}

This test can be found at ". /raven/tests/framework/pca_rom/test_input_pca . xml". This test can be called executing the following command:

. /run_tests --re=framework/pca_rom/inputPCAReduction

or

./run_framework_tests --re=framework/pca_rom/inputPCAReduction

- Test Description:

- Tests capability of PCA input reduction. 
- Original Author:

- wangc

- Creation date:

- 2015-09-14

- The classes tested in this test are:

- Distributions.MultivariateNormal

\subsubsection{FRAMEWORK PCA ROM MVNABS}

This test can be found at ". / raven/tests / framework/pca_rom/test_MVN_abs.xml”. This test can be called executing the following command:

. /run_tests --re=framework/pca_rom/MVNAbs

or

./run_framework_tests --re=framework/pca_rom/MVNAbs

- Test Description:

- Tests MVN with absolute covariance matrix

- Original Author:

- wangc

- Creation date:

- 2015-09-14

- The classes tested in this test are:

- Distributions.MultivariateNormal 


\subsubsection{FRAMEWORK PCA ROM MVNREL}

This test can be found at ". / raven/tests/framework/pca_rom/test_MVN_rel.xml”. This test can be called executing the following command:

. /run_tests --re=framework/pca_rom/MVNRel

or

./run_framework_tests --re=framework/pca_rom/MVNRel

- Test Description:

- Tests MVN with relative covariance matrix

- Original Author:

- wangc

- Creation date:

- 2015-09-14

- The classes tested in this test are:

- Distributions.MultivariateNormal

\subsubsection{FRAMEWORK PCA ROM.ROM}

This test can be found at ". / raven/tests/framework/pca_rom/test_rom.xml”. This test can be called executing the following command:

./run_tests --re=framework/pca_rom.ROM

or

./run_framework_tests --re=framework/pca_rom.ROM

- Test Description:

- Tests a high-input-dimensionality case to demonstrate input reduction.

- Original Author: 
- wangc

- Creation date:

- 2015-09-14

- The classes tested in this test are:

- Distributions.MultivariateNormal

\subsubsection{FRAMEWORK PCA ROM ONEDIM}

This test can be found at ". / raven/tests / framework/pca_rom/test_one_dim_pca . xml". This test can be called executing the following command:

. /run_tests --re=framework/pca_rom/oneDim

or

./run_framework_tests --re=framework/pca_rom/oneDim

- Test Description:

- Tests fix for Issue \#389 where the code errored when the problem is reduced to 1 dimension.

- Original Author:

- wangc

- Creation date:

- 2015-09-14

- The classes tested in this test are:

- Distributions.MultivariateNormal

\subsubsection{FRAMEWORK OUTSTREAMS.IMAGE GENERATION TEXT}

This test can be found at ". / raven/tests / framework/OutStreams/imageGeneration_ ps. xml". This test can be called executing the following command:

. Irun_tests --re=framework/Outstreams.image_generation_text 
or

./run_framework_tests --re=framework/OutStreams.image_generation_text

- Test Description:

- Test to check the functionalities of OutStreams system in RAVEN of type Plot. This test is aimed to check the capability of RAVEN to create a scatter Plot in PostScript format.

- Original Author:

- maljdan

- Creation date:

- 2016-04-27

- The classes tested in this test are:

- OutStreams.Plot

- Since the creation of this test, the following main revisions have been performed:

1. revision info:

- author : talbpaul

- date : 2016-09-15

- description: Test updates

2. revision info:

- author : alfoa

- date : 2016-10-20

- description: Modified XSD and removed dim attribute from all the tests

3. revision info:

- author : alfoa

- date : 2017-01-21

- description: Adding this test description. 


\subsubsection{FRAMEWORK.IMAGE GENERATION RAW}

This test can be found at ". /raven/tests/framework/Out Streams/test ImageGeneration. py". This test can be called executing the following command:

. /run_tests --re=framework.image_generation_raw

or

-/run_framework_tests --re=framework.image_generation_raw

- Test Description:

- This test the online generation of plots (colorbar plot). It can not be considered part of the active code but of the regression test system.

This test will use ImageMagick's "compare" utility in order to determine if two image files are within some amount of tolerance. This script operates by executing raven on the input file and then using compare to determine if the gold file and the generated file are near identical. If they are near enough to identical this test will report a "pass," otherwise it will return a "fail."

- Original Author:

- maljdan

- Creation date:

- 2016-04-27

- The classes tested in this test are:

- Since the creation of this test, the following main revisions have been performed:

1. revision info:

- author : maljdan

- date : 2016-05-04

- description: Fixing the test for the compare executable to test the gold image against itself, if this returns a non-zero code, then the version of imageMagick cannot be used to get a valid difference. Also, I am removing the difference image and instead doing null: to remove the output file when using compare.

2. revision info: 
- author : alfoa

- date : 2017-01-21

- description: Adding this test description.

\subsubsection{FRAMEWORK OUTSTREAMS.INTERACTIVE PLOTTING}

This test can be found at ". / raven/tests / framework/Out Streams/interactivePlot. xml". This test can be called executing the following command:

. /run_tests --re=framework/OutStreams.interactive_plotting

or

./run_framework_tests --re=framework/Outstreams.interactive_plotting

- Test Description:

- Test to check the functionalities of OutStreams system in RAVEN of type Plot. This test is aimed to check the capability of RAVEN to create a Plot and visualize it on the screen (interactvly)

- Original Author:

- maljdan

- Creation date:

- 2016-06-30

- The classes tested in this test are:

- OutStreams.Plot

- Since the creation of this test, the following main revisions have been performed:

1. revision info:

- author : alfoa

- date : 2016-09-01

- description: Close \#650

2. revision info:

- author : talbpaul

- date : 2016-09-15 
- description: Test updates

3. revision info:

- author : alfoa

- date : 2017-01-21

- description: Adding this test description.

\subsubsection{FRAMEWORK OUTSTREAMS.INVALIDINTERPOLATION}

This test can be found at ". / raven/tests / framework/OutStreams/invalidInterpolation. xml". This test can be called executing the following command:

. /run_tests --re=framework/OutStreams.invalidInterpolation

or

./run_framework_tests --re=framework/OutStreams.invalidInterpolation

- Test Description:

- This test was added to detect a defect in the plotting of something like a contour/surface/pcolor/wireframe where it is not well-defined when a single point is used. See \#832

- Original Author:

- maljdan

- Creation date:

- 2017-03-22

- The classes tested in this test are:

- OutStreams.Plot

- Since the creation of this test, the following main revisions have been performed:

1. revision info:

- author: talbpaul

- date : 2018-01-02

- description: Added input data object to prevent same-input-as-output error 


\subsubsection{FRAMEWORK OUTSTREAMS.TEST SUBPLOT}

This test can be found at "./raven/tests/framework/OutStreams/test_subPlot. xml". This test can be called executing the following command:

. /run_tests --re=framework/Outstreams.test_subPlot

or

. /run_framework_tests --re=framework/Outstreams.test_subPlot

- Test Description:

- Test to check the functionalities of OutStreams system in RAVEN to Plot out the information contained in DataObjects, determining in a sub plotting layout (multiple plots side by side in the same figure)

- Original Author:

- maljdan

- Creation date:

- 2016-04-26

- The classes tested in this test are:

- OutStreams.Plot

- Since the creation of this test, the following main revisions have been performed:

1. revision info:

- author : maljdan

- date : 2016-05-20

- description: Updating the test cases to remove references to Point or History.

2. revision info:

- author : alfoa

- date : 2016-09-01

- description: Close \#650

3. revision info:

- author: alfoa

- date : 2016-10-20 
- description: Modified XSD and removed dim attribute from all the tests

4. revision info:

- author : alfoa

- date : 2017-01-21

- description: Adding this test description.

\subsubsection{FRAMEWORK OUTSTREAMS.IO ROM PICKLE}

This test can be found at ". /raven/tests/framework/OutStreams/test_io_ROM_ pickle.xml". This test can be called executing the following command:

./run_tests --re=framework/OutStreams.io_ROM_pickle

or

./run_framework_tests --re=framework/OutStreams.io_ROM_pickle

- Test Description:

- Test to check the functionalities of OutStreams system in RAVEN to Print out the information contained in DataObjects. In addition, it checks the capability of RAVEN to construct a ROM from a pickle file.

- Original Author:

- maljdan

- Creation date:

- 2016-04-26

- The classes tested in this test are:

- Models.ROM, DataObjects.PointSet, DataObjects.HistorySet, OutStreams.Print

- Since the creation of this test, the following main revisions have been performed:

1. revision info:

- author : alfoa

- date : 2017-01-21

- description: Adding this test description. 


\subsubsection{FRAMEWORK OUTSTREAMS.LEGEND}

This test can be found at "./raven/tests/framework/OutStreams/test_legend. xml". This test can be called executing the following command:

. Irun_tests --re=framework/Outstreams.legend

or

./run_framework_tests --re=framework/OutStreams.legend

- Test Description:

- Test to check the functionalities of OutStreams system in RAVEN of type Plot, when a legend is added.

- Original Author:

- maljdan

- Creation date:

- 2016-04-27

- The classes tested in this test are:

- OutStreams.Plot

- Since the creation of this test, the following main revisions have been performed:

1. revision info:

- author: maljdan

- date : 2016-05-04

- description: Fixing the test for the compare executable to test the gold image against itself, if this returns a non-zero code, then the version of imageMagick cannot be used to get a valid difference. Also, I am removing the difference image and instead doing null: to remove the output file when using compare.

2. revision info:

- author : chenj

- date : 2016-07-07

- description: edit docstring

3. revision info:

- author: alfoa

- date : 2018-05-15

- description: Adding this test description. 


\subsubsection{FRAMEWORK OUTSTREAMS.LINE}

This test can be found at ". /raven/tests / framework / Out Streams / line_plot.xml". This test can be called executing the following command:

. /run_tests --re=framework/Outstreams.line

or

./run_framework_tests --re=framework/Outstreams.line

- Test Description:

- Test to check the functionalities of OutStreams system in RAVEN of line Plot, when HistorySet is used. Fix issue \#722

- Original Author:

- wangc

- Creation date:

- 2018-08-02

- The classes tested in this test are:

- OutStreams.Plot

- Since the creation of this test, the following main revisions have been performed:

1. revision info:

- author : alfoa

- date : 2020-04-07

- description: Modified test to be consistent with newer projectile model

\subsubsection{FRAMEWORK OUTSTREAMS.MULTIPLESCATTEREDDATA}

This test can be found at “. / raven/tests/framework/OutStreams/plot/multipleScatter. xml". This test can be called executing the following command:

. Irun_tests --re=framework/OutStreams.MultiplescatteredData

or 
./run_framework_tests --re=framework/OutStreams.MultiplescatteredData

- Test Description:

- This test assesses the ability of the outstreams to plot two different scatter plots on the same 3D axes

- Original Author:

- MohammadAbdo

- Creation date:

- 2020-04-07

- The classes tested in this test are:

- OutStreams

\subsubsection{FRAMEWORK OUTSTREAMS.SAMPLEPLOT}

This test can be found at "./raven/tests/framework/OutStreams/sample_plot. xml". This test can be called executing the following command:

./run_tests --re=framework/OutStreams. SamplePlot

or

./run_framework_tests --re=framework/OutStreams. SamplePlot

- Test Description:

- Tests use of the SamplePlot plotter. Samples data, then plots the samples using the SamplePlot plotter.

- Original Author:

- talbpaul

- Creation date:

- 2021-04-01

- The classes tested in this test are:

- OutStreams.SamplePlot 


\subsubsection{FRAMEWORK SAMPLERS ROM TIMEDEPROM}

This test can be found at "./raven/tests/framework/ROM/test_t_rom.xml". This test can be called executing the following command:

. /run_tests --re=framework/Samplers/ROM/timeDepRom

or

. /run_framework_tests --re=framework/Samplers/ROM/timeDepRom

- Test Description:

- This tests using a general time-dependent reduced-order model.

- Original Author:

- talbpaul

- Creation date:

- 2015-11-30

- The classes tested in this test are:

- Models.ROM

\subsubsection{FRAMEWORK SAMPLERS ROM TIMEDEPGUASSPOLY}

This test can be found at ". / raven/tests/framework/ROM/test_time_dep_scgpc . xml". This test can be called executing the following command:

. /run_tests --re=framework/Samplers/ROM/timeDepGuassPoly

or

./run_framework_tests --re=framework/Samplers/ROM/timeDepGuassPoly

- Test Description:

- This tests using a time-dependent GaussPolynomialROM.

- Original Author: 
- talbpaul

- Creation date:

- 2016-03-09

- The classes tested in this test are:

- SupervisedLearning.GaussPolynomialROM

- Since the creation of this test, the following main revisions have been performed:

1. revision info:

- author : talbpaul

- date : 2019-01-09

- description: moving from ROM-files to ROM-dataobjects

2. revision info:

- author: wangc

- date : 2018-08-09

- description: Add pivotParameter for time dependent ROM

\subsubsection{FRAMEWORK.ROM.SKL TIME}

This test can be found at ". / raven/tests/framework/ROM/skl_timedep.xml”. This test can be called executing the following command:

. Irun_tests --re=framework.ROM.SKL_time

or

./run_framework_tests --re=framework.ROM.SKL_time

- Test Description:

- Tests the construction and sampling of time-dependent ScitKitLearn ROMs. In particular, tests that if one target (time) has all the same values, but not others, right action is still taken.

- Original Author:

- talbpaul

- Creation date: 
$-2018-01-25$

- The classes tested in this test are:

- SupervisedLearning.SciKitLearn

\subsubsection{FRAMEWORK ROM TIMESERIES POLYEXPONENTIAL.POLYEXPNEAREST}

This test can be found at “. / raven/tests/framework/ROM/TimeSeries/PolyExponential/ test_poly_exponential_nearest.xml”. This test can be called executing the following command:

./run_tests --re=framework/ROM/TimeSeries/PolyExponential.PolyExpNearest

or

./run_framework_tests --re=framework/ROM/TimeSeries/PolyExponentilal.PolyEx

- Test Description:

- This test is aimed to check the mechanics of the PolyExponential ROM using, for the coefficient regression, the nearest algorithm regression

- Original Author:

- alfoa

- Creation date:

$-2018-04-25$

- The classes tested in this test are:

- SupervisedLearning.PolyExponential

- Since the creation of this test, the following main revisions have been performed:

1. revision info:

- author : talbpaul

- date : 2019-01-09

- description: moving from ROM-file to ROM-dataobject

2. revision info:

- author : alfoa

- date : 2018-04-25

- description: Adding this test description. 


\subsubsection{FRAMEWORK ROM TIMESERIES POLYEXPONENTIAL.POLYEXPPOLY}

This test can be found at ". /raven/tests/framework/ROM/TimeSeries/PolyExponential/ test_poly_exponential_poly.xml”. This test can be called executing the following command:

./run_tests --re=framework/ROM/TimeSeries/PolyExponential.PolyExpPoly

or

./run_framework_tests --re=framework/ROM/TimeSeries/PolyExponential.PolyExl

- Test Description:

- This test is aimed to check the mechanics of the PolyExponential ROM using, for the coefficient regression, the polynomial regression (2nd order in this case)

- Original Author:

- alfoa

- Creation date:

$-2018-04-25$

- The classes tested in this test are:

- SupervisedLearning.PolyExponential

- Since the creation of this test, the following main revisions have been performed:

1. revision info:

- author : talbpaul

- date : 2019-01-09

- description: moving from ROM-file to ROM-dataobject

2. revision info:

- author : alfoa

- date : 2018-04-25

- description: Adding this test description. 


\subsubsection{FRAMEWORK ROM TIMESERIES POLYEXPONENTIAL.POLYEXPSPLINE}

This test can be found at ". / raven/tests/framework/ROM/TimeSeries/PolyExponential/ test_poly_exponential_spline.xml”. This test can be called executing the following command:

./run_tests --re=framework/ROM/TimeSeries/PolyExponential.PolyExpspline

or

./run_framework_tests --re=framework/ROM/TimeSeries/PolyExponentilal.PolyEx

- Test Description:

- This test is aimed to check the mechanics of the PolyExponential ROM using, for the coefficient regression, the tensor spline regression

- Original Author:

- alfoa

- Creation date:

$-2018-04-25$

- The classes tested in this test are:

- SupervisedLearning.PolyExponential

- Since the creation of this test, the following main revisions have been performed:

1. revision info:

- author : talbpaul

- date : 2019-01-09

- description: moving from ROM-file to ROM-dataobject

2. revision info:

- author : alfoa

- date : 2018-04-25

- description: Adding this test description. 


\subsubsection{FRAMEWORK ROM TIMESERIES SYNTHETICHISTORY.FOURIER}

This test can be found at ". /raven/tests/framework/ROM/TimeSeries/SyntheticHistory/ fourier.xml". This test can be called executing the following command:

./run_tests --re=framework/ROM/TimeSeries/SyntheticHistory.Fourier

or

./run_framework_tests --re=framework/ROM/TimeSeries/SyntheticHistory.Fouri

- Test Description:

- Tests the SyntheticHistory ROM using only the Fourier TimeSeriesAnalyzer algorithm.

- Original Author:

- talbpaul

- Creation date:

- 2021-01-08

- The classes tested in this test are:

- SupervisedLearning.SyntheticHistory,TSA.Fourier

\subsubsection{FRAMEWORK ROM TIMESERIES SYNTHETICHISTORY.ARMA}

This test can be found at ". / raven/tests/framework/ROM/TimeSeries/SyntheticHistory/ arma.xml". This test can be called executing the following command:

./run_tests --re=framework/ROM/TimeSeries/SyntheticHistory. ARMA

or

./run_framework_tests --re=framework/ROM/TimeSeries/SyntheticHistory.ARMA

- Test Description:

- Tests the SyntheticHistory ROM using only the ARMA TimeSeriesAnalyzer algorithm.

- Original Author: 
- talbpaul

- Creation date:

$-2021-01-27$

- The classes tested in this test are:

- SupervisedLearning.SyntheticHistory,TSA.ARMA

\subsubsection{FRAMEWORK ROM TIMESERIES SYNTHETICHISTORY.FOURIERARMA}

This test can be found at ". / raven/tests/framework/ROM/TimeSeries/SyntheticHistory/ fourier_arma.xml". This test can be called executing the following command:

. /run_tests --re=framework/ROM/TimeSeries/SyntheticHistory.FourierARMA

or

./run_framework_tests --re=framework/ROM/TimeSeries/SyntheticHistory.Fouri

- Test Description:

- Tests the SyntheticHistory ROM using Fourier then ARMA TimeSeriesAnalyzer algorithms.

- Original Author:

- talbpaul

- Creation date:

$-2021-02-11$

- The classes tested in this test are:

- SupervisedLearning.SyntheticHistory,TSA.Fourier,TSA.ARMA

\subsubsection{FRAMEWORK ROM TIMESERIES SYNTHETICHISTORY.WAVELET}

This test can be found at ". / raven/tests / framework/ROM/TimeSeries/SyntheticHistory/ wavelet. $x m l "$. This test can be called executing the following command:

./run_tests --re=framework/ROM/TimeSeries/SyntheticHistory.Wavelet 
or

./run_framework_tests --re=framework/ROM/TimeSeries/SyntheticHistory.Wavel

- Test Description:

- Tests the SyntheticHistory ROM using only the Wavelet TimeSeriesAnalyzer algorithm.

- Original Author:

- dylanjm

- Creation date:

- 2021-03-02

- The classes tested in this test are:

- SupervisedLearning.SyntheticHistory,TSA.Wavelet

\subsubsection{FRAMEWORK ROM TIMESERIES DMD.HIGHORDERDMD}

This test can be found at ". /raven/tests/framework/ROM/TimeSeries/DMD/test_ high_order_dmd.xml". This test can be called executing the following command:

./run_tests --re=framework/ROM/TimeSeries/DMD.HighorderDMD

or

./run_framework_tests --re=framework/ROM/TimeSeries/DMD.HighorderDMD

- Test Description:

- This test is aimed to check the mechanics of the High Order DMD ROM using the standard SVD-based algorithm

- Original Author:

- alfoa

- Creation date:

$-2018-04-25$ 
- The classes tested in this test are:

- SupervisedLearning.DynamicModeDecomposition

- Since the creation of this test, the following main revisions have been performed:

1. revision info:

- author : talbpaul

- date : 2019-01-09

- description: moving from ROM-file to ROM-dataobject

2. revision info:

- author : alfoa

- date : 2018-04-25

- description: Adding this test description.

\subsubsection{FRAMEWORK ROM TIMESERIES DMD.PICKLEDMD}

This test can be found at ". / raven/tests/framework/ROM/TimeSeries/DMD/test_ pickle_dmd.xml”. This test can be called executing the following command:

./run_tests --re=framework/ROM/TimeSeries/DMD.PickleDMD

or

./run_framework_tests --re=framework/ROM/TimeSeries/DMD.PickleDMD

- Test Description:

- This test is aimed to check that the DMD-based surrogate models can be serialized and used in a working calculation flow.

- Original Author:

- alfoa

- Creation date:

- 2018-06-04

- The classes tested in this test are:

- SupervisedLearning.DynamicModeDecomposition 
- Since the creation of this test, the following main revisions have been performed:

1. revision info:

- author : alfoa

- date : 2018-06-04

- description: Adding this test description.

\subsubsection{FRAMEWORK ROM TIMESERIES ARMA.BASIC}

This test can be found at ". / raven/tests/framework/ROM/TimeSeries/ARMA/basic . xml". This test can be called executing the following command:

. /run_tests --re=framework/ROM/TimeSeries/ARMA.Basic

or

./run_framework_tests --re=framework/ROM/TimeSeries/ARMA.Basic

- Test Description:

- This test is aimed to check the possibility to train an ARMA+Fourier model and its capability to generate intrinsically stochastic time series

- Original Author:

- alfoa

- Creation date:

- 2017-02-02

- The classes tested in this test are:

- SupervisedLearning.ARMA

- Since the creation of this test, the following main revisions have been performed:

1. revision info:

- author : alfoa

- date : 2017-02-03

- description: Adding this test description.

2. revision info: 
- author : alfoa

- date : 2017-12-13

- description: adapted for new DataObject structure

3. revision info:

- author : alfoa

- date : 2018-04-26

- description: Modified location of the test (ARMA folder)

4. revision info:

- author : talbpaul

- date : 2018-06-14

- description: Now tests history statistics instead of points

5. revision info:

- author : talbpaul

- date : 2018-12-20

- description: Renamed from ARMA to Basic and added rom printing

6. revision info:

- author: wangc

- date : 2018-12-18

- description: In PR \#882, the standard errors on statistics moments are implemented, these quantities are stored in the pointwise metadata, and will be printed out in the output csv files by default. In order to avoid regolding this test, the optional node 'what' with text values 'input, output' is added. In this case, only the user requested variables as specified in the DataObjects are printed out.

7. revision info:

- author : talbpaul

- date : 2018-09-13

- description: changed Pmax and Pmin to P (same for Q)

8. revision info:

- author : talbpaul

- date : 2019-01-30

- description: reduced test to match what is tested, removed stat printing in favor of rom meta XML

\subsubsection{FRAMEWORK ROM TIMESERIES ARMA.ARMAPARALLEL}

This test can be found at ". / raven/tests/framework/ROM/TimeSeries/ARMA/test_ ARMA_parallel.xml". This test can be called executing the following command: 
./run_tests --re=framework/ROM/TimeSeries/ARMA.ARMAparallel

or

./run_framework_tests --re=framework/ROM/TimeSeries/ARMA.ARMAparallel

- Test Description:

- A copy of framework/ROM/TimeSeries.ARMA where the internal parallel system has been activated in order to test its functionality in conjunction with the ARMA model. This test is aimed to check the possibility to train an ARMA+Fourier model and its capability to generate intrinsically stochastic time series

- Original Author:

- alfoa

- Creation date:

- 2017-02-02

- The classes tested in this test are:

- SupervisedLearning.ARMA

- Since the creation of this test, the following main revisions have been performed:

1. revision info:

- author : alfoa

- date : 2017-02-03

- description: Adding this test description.

2. revision info:

- author : talbpaul

- date : 2017-07-12

- description: Inserted "reseedCopies" option as False, batchSize to 2

3. revision info:

- author : alfoa

- date : 2017-12-13

- description: adapted for new DataObject structure

4. revision info: 
- author : alfoa

- date : 2018-04-26

- description: Modified location of the test (ARMA folder)

5. revision info:

- author : talbpaul

- date : 2018-09-13

- description: changed Pmax and Pmin to P (same for Q)

\subsubsection{FRAMEWORK ROM TIMESERIES ARMA.ARMAPARALLELRESEED}

This test can be found at ". / raven/tests/framework/ROM/TimeSeries/ARMA/arma_ reseed_precursor.xml". This test can be called executing the following command:

./run_tests --re=framework/ROM/TimeSeries/ARMA. ARMAparallelReseed

or

. Irun_framework_tests --re=framework/ROM/TimeSeries/ARMA.ARMAparallelResee

- Test Description:

- Builds a reseeding copy of the ARMA to test multiple evaluations return different values; this run creates the arma and pickles it, also generates gold files for the reseed test. And added sampling for the gold file as well as rommeta.

- Original Author:

- talbpaul

- Creation date:

- 2017-07-12

- The classes tested in this test are:

- SupervisedLearning.ARMA

- Since the creation of this test, the following main revisions have been performed:

1. revision info:

- author : talbpaul

- date : 2017-07-12 
- description: created

2. revision info:

- author : alfoa

- date : 2017-12-13

- description: adapted for new DataObject structure

3. revision info:

- author : alfoa

- date : 2018-04-26

- description: Modified location of the test (ARMA folder)

4. revision info:

- author : talbpaul

- date : 2018-12-20

- description: ROM IO to data object added

5. revision info:

- author : talbpaul

- date : 2018-09-13

- description: changed Pmax and Pmin to P (same for Q)

6. revision info:

- author : zhouj

- date : 2019-05-06

- description: Modified location of the test to arma reseed

\subsubsection{FRAMEWORK ROM TIMESERIES ARMA.ARMARESEEDTEST}

This test can be found at ". / raven/tests/framework/ROM/TimeSeries/ARMA/arma_ reseed_test.xml". This test can be called executing the following command:

. /run_tests --re=framework/ROM/TimeSeries/ARMA. ARMAreseedTest

or

./run_framework_tests --re=framework/ROM/TimeSeries/ARMA.ARMAreseedTest

- Test Description: 
- Runs an unpickled ARMA ROM and assures the histories generated are identical (by setting the batch size to 1 and internalparelle to False in precursor and reseed test). Note that the gold file is created by runing the arma_ressed_precurssor test, signals_0 signals_1 signals_2 signals_3 are gold files. results_0 results_1 results_2 results_3 are the generated unpickled histories by test,then we compare they are the same.

- Original Author:

- talbpaul

- Creation date:

- 2017-07-12

- The classes tested in this test are:

- SupervisedLearning.ARMA

- Since the creation of this test, the following main revisions have been performed:

1. revision info:

- author : talbpaul

- date : 2017-07-12

- description: created

2. revision info:

- author : alfoa

- date : 2017-12-13

- description: adapted for new DataObject structure

3. revision info:

- author : alfoa

- date : 2018-04-26

- description: Modified location of the test (ARMA folder)

4. revision info:

- author : zhouj

- date : 2019-05-06

- description: Modified location of the test to the same with precursor, change batchsize to 1 , delete the internal parallel option, delete distribution block 


\subsubsection{FRAMEWORK ROM TIMESERIES ARMA.SINGLEFOURIER}

This test can be found at ". /raven/tests/framework/ROM/TimeSeries/ARMA/single_ fourier.xml". This test can be called executing the following command:

./run_tests --re=framework/ROM/TimeSeries/ARMA.SingleFourier

or

./run_framework_tests --re=framework/ROM/TimeSeries/ARMA.SingleF qurier

- Test Description:

- Tests that an ARMA can be trained with only a single Fourier base component

- Original Author:

- alfoa

- Creation date:

$-2018-05-21$

- The classes tested in this test are:

- SupervisedLearning.ARMA

- Since the creation of this test, the following main revisions have been performed:

1. revision info:

- author: talbpaul

- date : 2018-09-13

- description: changed Pmax and Pmin to P (same for Q)

\subsubsection{FRAMEWORK ROM TIMESERIES ARMA.MULTIPLEARMA}

This test can be found at ". /raven/tests/framework/ROM/TimeSeries/ARMA/multiple_ arma.xml". This test can be called executing the following command:

./run_tests --re=framework/ROM/TimeSeries/ARMA.MultipleArma

or

./run_framework_tests --re=framework/ROM/TimeSeries/ARMA.MultipleArma 
- Test Description:

- Tests creation of multiple INDEPENDENT target ARMA ROMs from a single training run. While it uses the entries from the correlated ARMA (VARMA) training data, it does not consider the variables correlated.

- Original Author:

- talbpaul

- Creation date:

- 2018-05-09

- The classes tested in this test are:

- SupervisedLearning.ARMA

- Since the creation of this test, the following main revisions have been performed:

1. revision info:

- author: wangc

- date : 2018-12-18

- description: In PR \#882, the standard errors on statistics moments are implemented, these quantities are stored in the pointwise metadata, and will be printed out in the output csv files by default. In order to avoid regolding this test, the optional node 'what' with text values 'input, output' is added. In this case, only the user requested variables as specified in the DataObjects are printed out.

2. revision info:

- author : talbpaul

- date : 2018-09-13

- description: changed Pmax and Pmin to P (same for Q)

\subsubsection{FRAMEWORK ROM TIMESERIES ARMA.MULTIPLEFOURIER}

This test can be found at ". / raven/tests/framework/ROM/TimeSeries/ARMA/multiple_ fourier.xml". This test can be called executing the following command:

./run_tests --re=framework/ROM/TimeSeries/ARMA.MultipleFourier

or

./run_framework_tests --re=framework/ROM/TimeSeries/ARMA.MultipleFourier 


\section{- Test Description:}

- Tests the feature of having independent Fourier modes for each training target. Original data is pure Fourier modes made with dataGenerator. This test is not analytic, but the input training set corralated_0.csv should be very nearly exactly replicated by samples_0.csv.

- Original Author:

- talbpaul

- Creation date:

- 2018-07-26

- The classes tested in this test are:

- SupervisedLearning.ARMA

- Since the creation of this test, the following main revisions have been performed:

1. revision info:

- author : talbpaul

- date : 2018-09-13

- description: changed Pmax and Pmin to P (same for Q)

\subsubsection{FRAMEWORK ROM TIMESERIES ARMA.VARMA}

This test can be found at ". / raven/tests / framework/ROM/TimeSeries/ARMA/varma . xml". This test can be called executing the following command:

./run_tests --re=framework/ROM/TimeSeries/ARMA.VARMA

or

./run_framework_tests --re=framework/ROM/TimeSeries/ARMA.VARMA

- Test Description:

- This test checks the possibility to train a correlated ARMA model and its capability to generate intrinsically stochastic time series with correlated results. The plot output "plot_input.png" shows the original training data, while "plot_VARMA.png" plots the sampled values. B should only be slightly off from A at all points. If this same calculation is done without correlation, both $\mathrm{A}$ and $\mathrm{B}$ independently range over the value 
space, and B can be far from A for each Time value. This provides a quick view norm to see that the correlation is working correctly.

- Original Author:

- talbpaul

- Creation date:

$-2018-05-15$

- The classes tested in this test are:

- SupervisedLearning.ARMA

- Since the creation of this test, the following main revisions have been performed:

1. revision info:

- author : talbpaul

- date : 2019-10-16

- description: split off for smaller mechanics run

2. revision info:

- author: wangc

- date : 2018-12-18

- description: In PR \#882, the standard errors on statistics moments are implemented, these quantities are stored in the pointwise metadata, and will be printed out in the output csv files by default. In order to avoid regolding this test, the optional node 'what' with text values 'input, output' is added. In this case, only the user requested variables as specified in the DataObjects are printed out.

3. revision info:

- author : talbpaul

- date : 2018-09-13

- description: changed Pmax and Pmin to P (same for Q)

\subsubsection{FRAMEWORK ROM TIMESERIES ARMA.VARMAHEAVY}

This test can be found at ". / raven/tests / framework/ROM/TimeSeries/ARMA/varma_ heavy . xml". This test can be called executing the following command:

. / run_tests --re=framework/ROM/TimeSeries/ARMA. VARMAheavy

or 
./run_framework_tests --re=framework/ROM/TimeSeries/ARMA.VARMAheavy

- Test Description:

- This test checks the possibility to train a correlated ARMA model and its capability to generate intrinsically stochastic time series with correlated results. The data is generated using dataGenerator in the working directory. The plot output "plot input.png" shows the original training data, while "plot_VARMA.png" plots the sampled values. $B$ should only be slightly off from $A$ at all points. If this same calculation is done without correlation, both $\mathrm{A}$ and $\mathrm{B}$ independently range over the value space, and $\mathrm{B}$ can be far from A for each Time value. This provides a quick view norm to see that the correlation is working correctly.

- Original Author:

- talbpaul

- Creation date:

$-2018-05-15$

- The classes tested in this test are:

- SupervisedLearning.ARMA

- Since the creation of this test, the following main revisions have been performed:

1. revision info:

- author : talbpaul

- date : 2019-10-16

- description: split off for a heavy test for statistical convergence

2. revision info:

- author: wangc

- date : 2018-12-18

- description: In PR \#882, the standard errors on statistics moments are implemented, these quantities are stored in the pointwise metadata, and will be printed out in the output csv files by default. In order to avoid regolding this test, the optional node 'what' with text values 'input, output' is added. In this case, only the user requested variables as specified in the DataObjects are printed out.

3. revision info:

- author : talbpaul

- date : 2018-09-13

- description: changed Pmax and Pmin to P (same for Q) 


\subsubsection{FRAMEWORK ROM TIMESERIES ARMA.ZEROFILTER}

This test can be found at ". / raven/tests/framework/ROM/TimeSeries/ARMA/zero_ filter.xml". This test can be called executing the following command:

./run_tests --re=framework/ROM/TimeSeries/ARMA.ZeroFilter

or

./run_framework_tests --re=framework/ROM/TimeSeries/ARMA.Zerofilter

- Test Description:

- Tests the ZeroFilter functionality of the ARMA, including as a correlated variable. Note the mean and variance especially of GHI change significantly if the zero-filtering is not working.

- Original Author:

- talbpaul

- Creation date:

- 2018-08-29

- The classes tested in this test are:

- SupervisedLearning.ARMA

- Since the creation of this test, the following main revisions have been performed:

1. revision info:

- author: wangc

- date : 2018-12-18

- description: In PR \#882, the standard errors on statistics moments are implemented, these quantities are stored in the pointwise metadata, and will be printed out in the output csv files by default. In order to avoid regolding this test, the optional node 'what' with text values 'input, output' is added. In this case, only the user requested variables as specified in the DataObjects are printed out.

2. revision info:

- author : talbpaul

- date : 2018-09-13

- description: changed Pmax and Pmin to P (same for Q) 


\subsubsection{FRAMEWORK ROM TIMESERIES ARMA.SEGMENTED}

This test can be found at ". /raven/tests/framework/ROM/TimeSeries/ARMA/segmented. xml". This test can be called executing the following command:

. / run_tests --re=framework/ROM/TimeSeries/ARMA. Segmented

or

./run_framework_tests --re=framework/ROM/TimeSeries/ARMA. Segmented

- Test Description:

- Tests the mechanics of clusterering the "time" subspace of the ARMA ROM.

- Original Author:

- talbpaul

- Creation date:

- 2018-11-20

- The classes tested in this test are:

- SupervisedLearning.ARMA

- Since the creation of this test, the following main revisions have been performed:

1. revision info:

- author: wangc

- date : 2018-12-19

- description: In PR \#882, the standard errors on statistics moments are implemented, these quantities are stored in the pointwise metadata, and will be printed out in the output csv files by default. In order to avoid regolding this test, the optional node 'what' with text values 'input, output' is added. In this case, only the user requested variables as specified in the DataObjects are printed out.

2. revision info:

- author: talbpaul

- date : 2019-01-30

- description: Added a pickling step to check serialization of segmented ARMA.

3. revision info: 
- author : JiaZhouPU

- date : 2019-05-02

- description: Deleted the longest Fourier period for this test, because the phase of this period would have results between PI and negative PI( a result of arctan(infinity) ), and cannnot be detected from the xml check.

\subsubsection{FRAMEWORK ROM TIMESERIES ARMA.CLUSTERED}

This test can be found at". / raven/tests/framework/ROM/TimeSeries/ARMA/clustered. xml". This test can be called executing the following command:

./run_tests --re=framework/ROM/TimeSeries/ARMA. clustered

or

./run_framework_tests --re=framework/ROM/TimeSeries/ARMA.Clustered

- Test Description:

- Tests the mechanics of clusterering the "time" subspace of the ARMA ROM.

- Original Author:

- talbpaul

- Creation date:

- 2018-11-20

- The classes tested in this test are:

- SupervisedLearning.ARMA

- Since the creation of this test, the following main revisions have been performed:

1. revision info:

- author : talbpaul

- date : 2020-01-18

- description: added sampling, clarified to clustered representation 


\subsubsection{FRAMEWORK ROM TIMESERIES ARMA.CLUSTEREDTRUNCATED}

This test can be found at ". /raven/tests/framework/ROM/TimeSeries/ARMA/clustered_ truncated.xml". This test can be called executing the following command:

./run_tests --re=framework/ROM/TimeSeries/ARMA.ClusteredTruncated

or

./run_framework_tests --re=framework/ROM/TimeSeries/ARMA.ClusteredTruncate

- Test Description:

- Tests the mechanics of clusterering the "time" subspace of the ARMA ROM. Resulting histories are represented in the "truncated" format, where each cluster is placed sequentially one after another.

- Original Author:

- talbpaul

- Creation date:

- 2018-11-20

- The classes tested in this test are:

- SupervisedLearning.ARMA

\subsubsection{FRAMEWORK ROM TIMESERIES ARMA.CLUSTEREDRANDCHOICE}

This test can be found at ". / raven/tests/framework/ROM/TimeSeries/ARMA/clustered_ choice_rand.xml". This test can be called executing the following command:

./run_tests --re=framework/ROM/TimeSeries/ARMA.ClusteredRandChoiqe

or

./run_framework_tests --re=framework/ROM/TimeSeries/ARMA.ClusteredRandChoi

- Test Description: 
- Tests the mechanics of clusterering the "time" subspace of the ARMA ROM, with a random choice of the clustered ROMs in the evaluation stage

- Original Author:

- alfoa

- Creation date:

- 2020-04-15

- The classes tested in this test are:

- SupervisedLearning.ARMA

\subsubsection{FRAMEWORK ROM TIMESERIES ARMA.PRESERVECDF}

This test can be found at ". / raven/tests/framework/ROM/TimeSeries/ARMA/preserve_ cof . xml". This test can be called executing the following command:

./run_tests --re=framework/ROM/TimeSeries/ARMA.PreserveCdf

or

./run_framework_tests --re=framework/ROM/TimeSeries/ARMA.PreserveCdf

- Test Description:

- Tests the feature (Models.ARMA.preserveInputCDF) to preserve the CDF of the input training data when producing synthetic histories. The standard is being able to identically match the original data's ValueDuration curve. Currently, there's not a Metric for comparing a single gold standard to several samples; thus we read in a second history set with the ValueDuration curve of the training set repeated 10 times for comparison to the sample ValueDuration curves.

- Original Author:

- talbpaul

- Creation date:

- 2019-01-22

- The classes tested in this test are: 
- SupervisedLearning.ARMA

- Since the creation of this test, the following main revisions have been performed:

1. revision info:

- author : talbpaul

- date : 2019-01-22

- description: created

\subsubsection{FRAMEWORK ROM TIMESERIES ARMA.MULTICYCLE}

This test can be found at ". / raven/tests/framework/ROM/TimeSeries/ARMA/multicycle. xml". This test can be called executing the following command:

. /run_tests --re=framework/ROM/TimeSeries/ARMA.Multicycle

or

./run_framework_tests --re=framework/ROM/TimeSeries/ARMA.Multicyqle

- Test Description:

- Tests sampling the ARMA with multiple cycles in each sample. As a check, assure that for each realization, the ratio between the mean of cycles 1 and 0 should be near 1.5, and the ratio between cycles 2 and 0 should be 2.25. Further, check that when ensembled the pass-through value is usable (mostly a check for ND data).

- Original Author:

- talbpaul

- Creation date:

- 2019-05-14

- The classes tested in this test are:

- SupervisedLearning.ARMA 


\subsubsection{FRAMEWORK ROM TIMESERIES ARMA.MULTICYCLERESEED}

This test can be found at ". / raven/tests/framework/ROM/TimeSeries/ARMA/multicycle_ reseed.xml". This test can be called executing the following command:

./run_tests --re=framework/ROM/TimeSeries/ARMA.MulticycleReseed

or

./run_framework_tests --re=framework/ROM/TimeSeries/ARMA.MulticyqleReseed

- Test Description:

- Tests that the number of sampled cycles can be changed on an ARMA during unserialization. It takes the pickled ARMA from "multicycles" and changes the number of cycles as well as the growth rate. The resulting DataSet CSV should have Cycles 0 and 1 only, and the values for Cycle 1 should be notably higher than Cycle 0 on average. As a check, for each realization, the ratio between cycle 1 and cycle 0 should be near 2.5.

- Original Author:

- talbpaul

- Creation date:

- 2019-05-15

- The classes tested in this test are:

- SupervisedLearning.ARMA

\subsubsection{FRAMEWORK ROM TIMESERIES ARMA.PEAKS}

This test can be found at ". / raven/tests/framework/ROM/TimeSeries/ARMA/peaks . xml". This test can be called executing the following command:

. /run_tests --re=framework/ROM/TimeSeries/ARMA.Peaks

or

./run_framework_tests --re=framework/ROM/TimeSeries/ARMA.Peaks 
- Test Description:

- This test is aimed to check the possibility to train an ARMA+Fourier+Peaks model and its capability to generate intrinsically stochastic time series

- Original Author:

- ZHOUJ

- Creation date:

- 2019-05-29

- The classes tested in this test are:

- SupervisedLearning.ARMA

\subsubsection{FRAMEWORK ROM TIMESERIES ARMA.INTERPOLATED}

This test can be found at ". / raven/tests/framework/ROM/TimeSeries/ARMA/interpolated. xml". This test can be called executing the following command:

. /run_tests --re=framework/ROM/TimeSeries/ARMA. Interpolated

Or

./run_framework_tests --re=framework/ROM/TimeSeries/ARMA.Interpolated

- Test Description:

- Tests the mechanics of providing several set points for ARMAs on different years and interpolating the ARMAs to fill missing years. Note that as implemented, interpolating assumes clustering. The output synthetic.CSV is the truncated output form, with the clusters stacked sequentially. The output resynthetic.csv is the clustered form, with $\_$ROM_Cluster as one of the index dimensions.

- Original Author:

- talbpaul

- Creation date:

- 2019-05-22

- The classes tested in this test are:

- SupervisedLearning.ARMA 


\subsubsection{FRAMEWORK ROM TIMESERIES ARMA.INTERPOLATEDMAXCYCLES}

This test can be found at ". /raven/tests/framework/ROM/TimeSeries/ARMA/interpolated_ maxcycles.xml". This test can be called executing the following command:

./run_tests --re=framework/ROM/TimeSeries/ARMA. InterpolatedMaxCy qles

or

./run_framework_tests --re=framework/ROM/TimeSeries/ARMA.InterpolatedMaxCy

- Test Description:

- Similar to "interpolated" test, but limits the number of cycles (years) sampled. synthet ic . csv should have Year values of $(0,1,2)$ while resynthet ic. csv should have Year values of $(0,1)$. Also tests the nyquistScalar option.

- Original Author:

- talbpaul

- Creation date:

- 2020-01-19

- The classes tested in this test are:

- SupervisedLearning.ROMCollection

\subsubsection{FRAMEWORK ROM MSR.BIWEIGHT}

This test can be found at "./raven/tests/framework/ROM/MSR/test_biweight. xml". This test can be called executing the following command:

./run_tests --re=framework/ROM/MSR.biweight

or

./run_framework_tests --re=framework/ROM/MSR.biweight

- Test Description: 
- An example of using the Morse-Smale regression reduced order model with a biweight kernel function for the kernel density estimator.

Note, all of the tests in MSR operate on a 2D input domain with the goal of fitting a single Gaussian bump. The input dimensions are of largely different scales and one dimension is off-centered from the origin to ensure that normalization is being handled correctly.

- Original Author:

- maljdan

- Creation date:

- 2015-10-06

- The classes tested in this test are:

- SupervisedLearning.MSR

- Since the creation of this test, the following main revisions have been performed:

1. revision info:

- author : maljdan

- date : 2015-10-21

- description: Converting AMSR test files using the provided conversion script.

2. revision info:

- author : talbpaul

- date : 2016-02-08

- description: first update, looking for more failing tests to add

3. revision info:

- author : maljdan

- date : 2016-02-10

- description: Adding functionality to allow a user to specify a filename for an Outstream object that is potentially different than its variable name in the RAVEN input file. Adjusting test cases to exercise this functionality.

4. revision info:

- author : maljdan

- date : 2016-04-06

- description: Updating test cases to reflect the changes to the user input.

5. revision info:

- author : maljdan

- date : 2017-01-19

- description: Adding this test description. 


\subsubsection{FRAMEWORK ROM MSR.COSINE}

This test can be found at "./raven/tests/framework/ROM/MSR/test_cosine.xml”. This test can be called executing the following command:

./run_tests --re=framework/ROM/MSR.cosine

or

./run_framework_tests --re=framework/ROM/MSR.cosine

- Test Description:

- An example of using the Morse-Smale regression reduced order model with a cosine kernel function for the kernel density estimator.

Note, all of the tests in MSR operate on a 2D input domain with the goal of fitting a single Gaussian bump. The input dimensions are of largely different scales and one dimension is off-centered from the origin to ensure that normalization is being handled correctly.

- Original Author:

- maljdan

- Creation date:

$-2015-10-06$

- The classes tested in this test are:

- SupervisedLearning.MSR

- Since the creation of this test, the following main revisions have been performed:

1. revision info:

- author : maljdan

- date : 2015-10-21

- description: Converting AMSR test files using the provided conversion script.

2. revision info:

- author : talbpaul

- date : 2016-02-08

- description: first update, looking for more failing tests to add

3. revision info: 
- author : maljdan

- date : 2016-02-10

- description: Adding functionality to allow a user to specify a filename for an Outstream object that is potentially different than its variable name in the RAVEN input file. Adjusting test cases to exercise this functionality.

4. revision info:

- author : maljdan

- date : 2016-04-06

- description: Updating test cases to reflect the changes to the user input.

5. revision info:

- author : maljdan

- date : 2017-01-19

- description: Adding this test description.

\subsubsection{FRAMEWORK ROM MSR.EPANECHNIKOV}

This test can be found at ". / raven/tests/framework/ROM/MSR/test_Epanechnikov . xml". This test can be called executing the following command:

./run_tests --re=framework/ROM/MSR.Epanechnikov

Or

./run_framework_tests --re=framework/ROM/MSR.Epanechnikov

- Test Description:

- An example of using the Morse-Smale regression reduced order model with an Epanechnikov kernel function for the kernel density estimator.

Note, all of the tests in MSR operate on a 2D input domain with the goal of fitting a single Gaussian bump. The input dimensions are of largely different scales and one dimension is off-centered from the origin to ensure that normalization is being handled correctly.

- Original Author:

- maljdan

- Creation date: 
- 2015-10-06

- The classes tested in this test are:

- SupervisedLearning.MSR

- Since the creation of this test, the following main revisions have been performed:

1. revision info:

- author : maljdan

- date : 2015-10-21

- description: Converting AMSR test files using the provided conversion script.

2. revision info:

- author : talbpaul

- date : 2016-02-08

- description: first update, looking for more failing tests to add

3. revision info:

- author : maljdan

- date : 2016-02-10

- description: Adding functionality to allow a user to specify a filename for an Outstream object that is potentially different than its variable name in the RAVEN input file. Adjusting test cases to exercise this functionality.

4. revision info:

- author : maljdan

- date : 2016-04-06

- description: Updating test cases to reflect the changes to the user input.

5. revision info:

- author : maljdan

- date : 2017-01-19

- description: Adding this test description.

\subsubsection{FRAMEWORK ROM MSR.EXPONENTIAL}

This test can be found at ". /raven/tests/framework/ROM/MSR/test_exponential. xml". This test can be called executing the following command:

./run_tests --re=framework/ROM/MSR.exponential

or 
./run_framework_tests --re=framework/ROM/MSR.exponential

- Test Description:

- An example of using the Morse-Smale regression reduced order model with an exponential kernel function for the kernel density estimator.

Note, all of the tests in MSR operate on a 2D input domain with the goal of fitting a single Gaussian bump. The input dimensions are of largely different scales and one dimension is off-centered from the origin to ensure that normalization is being handled correctly.

- Original Author:

- maljdan

- Creation date:

- 2015-10-06

- The classes tested in this test are:

- SupervisedLearning.MSR

- Since the creation of this test, the following main revisions have been performed:

1. revision info:

- author : maljdan

- date : 2015-10-21

- description: Converting AMSR test files using the provided conversion script.

2. revision info:

- author : talbpaul

- date : 2016-02-08

- description: first update, looking for more failing tests to add

3. revision info:

- author : maljdan

- date : 2016-02-10

- description: Adding functionality to allow a user to specify a filename for an Outstream object that is potentially different than its variable name in the RAVEN input file. Adjusting test cases to exercise this functionality.

4. revision info: 
- author : maljdan

- date : 2016-04-06

- description: Updating test cases to reflect the changes to the user input.

5. revision info:

- author : maljdan

- date : 2017-01-19

- description: Adding this test description.

\subsubsection{FRAMEWORK ROM MSR.GAUSSIAN}

This test can be found at "./raven/tests/framework/ROM/MSR/test_Gaussian. xml". This test can be called executing the following command:

./run_tests --re=framework/ROM/MSR.Gaussian

or

./run_framework_tests --re=framework/ROM/MSR.Gaussian

- Test Description:

- An example of using the Morse-Smale regression reduced order model with a Gaussian kernel function for the kernel density estimator.

Note, all of the tests in MSR operate on a 2D input domain with the goal of fitting a single Gaussian bump. The input dimensions are of largely different scales and one dimension is off-centered from the origin to ensure that normalization is being handled correctly.

- Original Author:

- maljdan

- Creation date:

- 2015-10-06

- The classes tested in this test are:

- SupervisedLearning.MSR

- Since the creation of this test, the following main revisions have been performed:

1. revision info: 
- author : maljdan

- date : 2015-10-21

- description: Converting AMSR test files using the provided conversion script.

2. revision info:

- author : talbpaul

- date : 2016-02-08

- description: first update, looking for more failing tests to add

3. revision info:

- author : maljdan

- date : 2016-02-10

- description: Adding functionality to allow a user to specify a filename for an Outstream object that is potentially different than its variable name in the RAVEN input file. Adjusting test cases to exercise this functionality.

4. revision info:

- author : maljdan

- date : 2016-04-06

- description: Updating test cases to reflect the changes to the user input.

5. revision info:

- author : maljdan

- date : 2017-01-19

- description: Adding this test description.

\subsubsection{FRAMEWORK ROM MSR.LOGISTIC}

This test can be found at "./raven/tests/framework/ROM/MSR/test_logistic. xml". This test can be called executing the following command:

./run_tests --re=framework/ROM/MSR.logistic

or

. Irun_framework_tests --re=framework/ROM/MSR.logistic

- Test Description:

- An example of using the Morse-Smale regression reduced order model with a logistic kernel function for the kernel density estimator. 
Note, all of the tests in MSR operate on a 2D input domain with the goal of fitting a single Gaussian bump. The input dimensions are of largely different scales and one dimension is off-centered from the origin to ensure that normalization is being handled correctly.

- Original Author:

- maljdan

- Creation date:

$-2015-10-06$

- The classes tested in this test are:

- SupervisedLearning.MSR

- Since the creation of this test, the following main revisions have been performed:

1. revision info:

- author : maljdan

- date : 2015-10-21

- description: Converting AMSR test files using the provided conversion script.

2. revision info:

- author : talbpaul

- date : 2016-02-08

- description: first update, looking for more failing tests to add

3. revision info:

- author : maljdan

- date : 2016-02-10

- description: Adding functionality to allow a user to specify a filename for an Outstream object that is potentially different than its variable name in the RAVEN input file. Adjusting test cases to exercise this functionality.

4. revision info:

- author : maljdan

- date : 2016-04-06

- description: Updating test cases to reflect the changes to the user input.

5. revision info:

- author : maljdan

- date : 2017-01-19

- description: Adding this test description. 


\subsubsection{FRAMEWORK ROM MSR.SILVERMAN}

This test can be found at "./raven/tests/framework/ROM/MSR/test_Silverman. xml". This test can be called executing the following command:

. /run_tests --re=framework/ROM/MSR.Silverman

or

./run_framework_tests --re=framework/ROM/MSR.Silverman

- Test Description:

- An example of using the Morse-Smale regression reduced order model with a Silverman kernel function for the kernel density estimator.

Note, all of the tests in MSR operate on a 2D input domain with the goal of fitting a single Gaussian bump. The input dimensions are of largely different scales and one dimension is off-centered from the origin to ensure that normalization is being handled correctly.

- Original Author:

- maljdan

- Creation date:

- 2015-10-06

- The classes tested in this test are:

- SupervisedLearning.MSR

- Since the creation of this test, the following main revisions have been performed:

1. revision info:

- author : maljdan

- date : 2015-10-21

- description: Converting AMSR test files using the provided conversion script.

2. revision info:

- author : talbpaul

- date : 2016-02-08

- description: first update, looking for more failing tests to add

3. revision info: 
- author : maljdan

- date : 2016-02-10

- description: Adding functionality to allow a user to specify a filename for an Outstream object that is potentially different than its variable name in the RAVEN input file. Adjusting test cases to exercise this functionality.

4. revision info:

- author : maljdan

- date : 2016-04-06

- description: Updating test cases to reflect the changes to the user input.

5. revision info:

- author : maljdan

- date : 2017-01-19

- description: Adding this test description.

\subsubsection{FRAMEWORK ROM MSR.TRIANGULAR}

This test can be found at ". / raven/tests/framework/ROM/MSR/test_triangular. xml". This test can be called executing the following command:

. Irun_tests --re=framework/ROM/MSR.triangular

Or

. /run_framework_tests --re=framework/ROM/MSR.triangular

- Test Description:

- An example of using the Morse-Smale regression reduced order model with a triangular kernel function for the kernel density estimator.

Note, all of the tests in MSR operate on a 2D input domain with the goal of fitting a single Gaussian bump. The input dimensions are of largely different scales and one dimension is off-centered from the origin to ensure that normalization is being handled correctly.

- Original Author:

- maljdan

- Creation date: 
- 2015-10-06

- The classes tested in this test are:

- SupervisedLearning.MSR

- Since the creation of this test, the following main revisions have been performed:

1. revision info:

- author : maljdan

- date : 2015-10-21

- description: Converting AMSR test files using the provided conversion script.

2. revision info:

- author : talbpaul

- date : 2016-02-08

- description: first update, looking for more failing tests to add

3. revision info:

- author : maljdan

- date : 2016-02-10

- description: Adding functionality to allow a user to specify a filename for an Outstream object that is potentially different than its variable name in the RAVEN input file. Adjusting test cases to exercise this functionality.

4. revision info:

- author : maljdan

- date : 2016-04-06

- description: Updating test cases to reflect the changes to the user input.

5. revision info:

- author : maljdan

- date : 2017-01-19

- description: Adding this test description.

\subsubsection{FRAMEWORK ROM MSR.TRICUBE}

This test can be found at ". / raven/tests/framework/ROM/MSR/test_tricube.xml”. This test can be called executing the following command:

./run_tests --re=framework/ROM/MSR.tricube

or 
./run_framework_tests --re=framework/ROM/MSR.tricube

- Test Description:

- An example of using the Morse-Smale regression reduced order model with a tricube kernel function for the kernel density estimator.

Note, all of the tests in MSR operate on a 2D input domain with the goal of fitting a single Gaussian bump. The input dimensions are of largely different scales and one dimension is off-centered from the origin to ensure that normalization is being handled correctly.

- Original Author:

- maljdan

- Creation date:

- 2015-10-06

- The classes tested in this test are:

- SupervisedLearning.MSR

- Since the creation of this test, the following main revisions have been performed:

1. revision info:

- author : maljdan

- date : 2015-10-21

- description: Converting AMSR test files using the provided conversion script.

2. revision info:

- author : talbpaul

- date : 2016-02-08

- description: first update, looking for more failing tests to add

3. revision info:

- author : maljdan

- date : 2016-02-10

- description: Adding functionality to allow a user to specify a filename for an Outstream object that is potentially different than its variable name in the RAVEN input file. Adjusting test cases to exercise this functionality.

4. revision info: 
- author : maljdan

- date : 2016-04-06

- description: Updating test cases to reflect the changes to the user input.

5. revision info:

- author : maljdan

- date : 2017-01-19

- description: Adding this test description.

\subsubsection{FRAMEWORK ROM MSR.TRIWEIGHT}

This test can be found at ". /raven/tests/framework/ROM/MSR/test_triweight. xml". This test can be called executing the following command:

. /run_tests --re=framework/ROM/MSR.triweight

or

./run_framework_tests --re=framework/ROM/MSR.triweight

- Test Description:

- An example of using the Morse-Smale regression reduced order model with a triweight kernel function for the kernel density estimator.

Note, all of the tests in MSR operate on a 2D input domain with the goal of fitting a single Gaussian bump. The input dimensions are of largely different scales and one dimension is off-centered from the origin to ensure that normalization is being handled correctly.

- Original Author:

- maljdan

- Creation date:

- 2015-10-06

- The classes tested in this test are:

- SupervisedLearning.MSR

- Since the creation of this test, the following main revisions have been performed:

1. revision info: 
- author : maljdan

- date : 2015-10-21

- description: Converting AMSR test files using the provided conversion script.

2. revision info:

- author : talbpaul

- date : 2016-02-08

- description: first update, looking for more failing tests to add

3. revision info:

- author : maljdan

- date : 2016-02-10

- description: Adding functionality to allow a user to specify a filename for an Outstream object that is potentially different than its variable name in the RAVEN input file. Adjusting test cases to exercise this functionality.

4. revision info:

- author : maljdan

- date : 2016-04-06

- description: Updating test cases to reflect the changes to the user input.

5. revision info:

- author : maljdan

- date : 2017-01-19

- description: Adding this test description.

\subsubsection{FRAMEWORK ROM MSR.UNIFORM}

This test can be found at ". / raven/tests / framework/ROM/MSR/test_uniform.xml”. This test can be called executing the following command:

. /run_tests --re=framework/ROM/MSR.uniform

or

. /run_framework_tests --re=framework/ROM/MSR.uniform

- Test Description:

- An example of using the Morse-Smale regression reduced order model with a uniform kernel function for the kernel density estimator. 
Note, all of the tests in MSR operate on a 2D input domain with the goal of fitting a single Gaussian bump. The input dimensions are of largely different scales and one dimension is off-centered from the origin to ensure that normalization is being handled correctly.

- Original Author:

- maljdan

- Creation date:

$-2015-10-06$

- The classes tested in this test are:

- SupervisedLearning.MSR

- Since the creation of this test, the following main revisions have been performed:

1. revision info:

- author : maljdan

- date : 2015-10-21

- description: Converting AMSR test files using the provided conversion script.

2. revision info:

- author : talbpaul

- date : 2016-02-08

- description: first update, looking for more failing tests to add

3. revision info:

- author : maljdan

- date : 2016-02-10

- description: Adding functionality to allow a user to specify a filename for an Outstream object that is potentially different than its variable name in the RAVEN input file. Adjusting test cases to exercise this functionality.

4. revision info:

- author : maljdan

- date : 2016-04-06

- description: Updating test cases to reflect the changes to the user input.

5. revision info:

- author : maljdan

- date : 2017-01-19

- description: Adding this test description. 


\subsubsection{FRAMEWORK ROM MSR.SVM}

This test can be found at ". / raven/tests/framework/ROM/MSR/test_SVM.xml”. This test can be called executing the following command:

./run_tests --re=framework/ROM/MSR.SVM

or

. /run_framework_tests --re=framework/ROM/MSR.SVM

- Test Description:

- An example of using the Morse-Smale regression reduced order model with a support vector machine.

Note, all of the tests in MSR operate on a 2D input domain with the goal of fitting a single Gaussian bump. The input dimensions are of largely different scales and one dimension is off-centered from the origin to ensure that normalization is being handled correctly.

- Original Author:

- maljdan

- Creation date:

- 2015-10-06

- The classes tested in this test are:

- SupervisedLearning.MSR

- Since the creation of this test, the following main revisions have been performed:

1. revision info:

- author : maljdan

- date : 2015-10-21

- description: Converting AMSR test files using the provided conversion script.

2. revision info:

- author : talbpaul

- date : 2016-02-08

- description: first update, looking for more failing tests to add

3. revision info: 
- author : maljdan

- date : 2016-02-10

- description: Adding functionality to allow a user to specify a filename for an Outstream object that is potentially different than its variable name in the RAVEN input file. Adjusting test cases to exercise this functionality.

4. revision info:

- author : maljdan

- date : 2016-02-10

- description: Using custom sampler blocks for the MSR-SVM tests.

5. revision info:

- author : maljdan

- date : 2016-04-06

- description: Updating test cases to reflect the changes to the user input.

6. revision info:

- author : maljdan

- date : 2017-01-19

- description: Adding this test description.

\subsubsection{FRAMEWORK ROM MSR.SMOOTHSVM}

This test can be found at ". / raven/tests/framework/ROM/MSR/test_smooth_SVM. xml". This test can be called executing the following command:

. Irun_tests --re=framework/ROM/MSR.smoothSVM

or

./run_framework_tests --re=framework/ROM/MSR.smoothSVM

- Test Description:

- An example of using the Morse-Smale regression reduced order model with a support vector machine. This is a smoothed version of MSR where local models are blended together.

Note, all of the tests in MSR operate on a 2D input domain with the goal of fitting a single Gaussian bump. The input dimensions are of largely different scales and one dimension is off-centered from the origin to ensure that normalization is being handled correctly. 
- Original Author:

- maljdan

- Creation date:

- 2015-10-06

- The classes tested in this test are:

- SupervisedLearning.MSR

- Since the creation of this test, the following main revisions have been performed:

1. revision info:

- author : maljdan

- date : 2015-10-21

- description: Converting AMSR test files using the provided conversion script.

2. revision info:

- author : talbpaul

- date : 2016-02-08

- description: first update, looking for more failing tests to add

3. revision info:

- author : maljdan

- date : 2016-02-10

- description: Adding functionality to allow a user to specify a filename for an Outstream object that is potentially different than its variable name in the RAVEN input file. Adjusting test cases to exercise this functionality.

4. revision info:

- author : maljdan

- date : 2016-02-10

- description: Using custom sampler blocks for the MSR-SVM tests.

5. revision info:

- author : maljdan

- date : 2016-04-06

- description: Updating test cases to reflect the changes to the user input.

6. revision info:

- author : maljdan

- date : 2017-01-19

- description: Adding this test description. 


\subsubsection{FRAMEWORK ROM MSR.SMOOTHBIWEIGHT}

This test can be found at "./raven/tests/framework/ROM/MSR/test_smooth_biweight . xml". This test can be called executing the following command:

. Irun_tests --re=framework/ROM/MSR.smoothBiweight

or

./run_framework_tests --re=framework/ROM/MSR.smoothBiweight

- Test Description:

- An example of using the Morse-Smale regression reduced order model with a biweight kernel function for the kernel density estimator. This is a smoothed version of MSR where local models are blended together.

Note, all of the tests in MSR operate on a 2D input domain with the goal of fitting a single Gaussian bump. The input dimensions are of largely different scales and one dimension is off-centered from the origin to ensure that normalization is being handled correctly.

- Original Author:

- maljdan

- Creation date:

- 2015-10-06

- The classes tested in this test are:

- SupervisedLearning.MSR

- Since the creation of this test, the following main revisions have been performed:

1. revision info:

- author : maljdan

- date : 2015-10-21

- description: Converting AMSR test files using the provided conversion script.

2. revision info:

- author : talbpaul

- date : 2016-02-08

- description: first update, looking for more failing tests to add 
3. revision info:

- author : maljdan

- date : 2016-02-10

- description: Adding functionality to allow a user to specify a filename for an Outstream object that is potentially different than its variable name in the RAVEN input file. Adjusting test cases to exercise this functionality.

4. revision info:

- author : maljdan

- date : 2016-04-06

- description: Updating test cases to reflect the changes to the user input.

5. revision info:

- author : maljdan

- date : 2017-01-19

- description: Adding this test description.

\subsubsection{FRAMEWORK ROM MSR.SMOOTHCOSINE}

This test can be found at ". / raven/tests/framework/ROM/MSR/test_smooth_cosine. xml". This test can be called executing the following command:

. /run_tests --re=framework/ROM/MSR.smoothCosine

or

./run_framework_tests --re=framework/ROM/MSR.smoothCosine

- Test Description:

- An example of using the Morse-Smale regression reduced order model with a cosine kernel function for the kernel density estimator. This is a smoothed version of MSR where local models are blended together.

Note, all of the tests in MSR operate on a 2D input domain with the goal of fitting a single Gaussian bump. The input dimensions are of largely different scales and one dimension is off-centered from the origin to ensure that normalization is being handled correctly.

- Original Author:

- maljdan 
- Creation date:

- 2015-10-06

- The classes tested in this test are:

- SupervisedLearning.MSR

- Since the creation of this test, the following main revisions have been performed:

1. revision info:

- author : maljdan

- date : 2015-10-21

- description: Converting AMSR test files using the provided conversion script.

2. revision info:

- author : talbpaul

- date : 2016-02-08

- description: first update, looking for more failing tests to add

3. revision info:

- author : maljdan

- date : 2016-02-10

- description: Adding functionality to allow a user to specify a filename for an Outstream object that is potentially different than its variable name in the RAVEN input file. Adjusting test cases to exercise this functionality.

4. revision info:

- author : maljdan

- date : 2016-04-06

- description: Updating test cases to reflect the changes to the user input.

5. revision info:

- author : maljdan

- date : 2017-01-19

- description: Adding this test description.

\subsubsection{FRAMEWORK ROM MSR.SMOOTHEPANECHNIKOV}

This test can be found at ". /raven/tests/framework/ROM/MSR/test_smooth_Epanechnikov. xml". This test can be called executing the following command:

./run_tests --re=framework/ROM/MSR.smoothEpanechnikov 
or

./run_framework_tests --re=framework/ROM/MSR.smoothEpanechnikov

- Test Description:

- An example of using the Morse-Smale regression reduced order model with an Epanechnikov kernel function for the kernel density estimator. This is a smoothed version of MSR where local models are blended together.

Note, all of the tests in MSR operate on a 2D input domain with the goal of fitting a single Gaussian bump. The input dimensions are of largely different scales and one dimension is off-centered from the origin to ensure that normalization is being handled correctly.

- Original Author:

- maljdan

- Creation date:

- 2015-10-06

- The classes tested in this test are:

- SupervisedLearning.MSR

- Since the creation of this test, the following main revisions have been performed:

1. revision info:

- author : maljdan

- date : 2015-10-21

- description: Converting AMSR test files using the provided conversion script.

2. revision info:

- author : talbpaul

- date : 2016-02-08

- description: first update, looking for more failing tests to add

3. revision info:

- author : maljdan

- date : 2016-02-10

- description: Adding functionality to allow a user to specify a filename for an Outstream object that is potentially different than its variable name in the RAVEN input file. Adjusting test cases to exercise this functionality. 
4. revision info:

- author : maljdan

- date : 2016-04-06

- description: Updating test cases to reflect the changes to the user input.

5. revision info:

- author : maljdan

- date : 2017-01-19

- description: Adding this test description.

\subsubsection{FRAMEWORK ROM MSR.SMOOTHEXPONENTIAL}

This test can be found at ". /raven/tests/framework/ROM/MSR/test_smooth_exponential. xml". This test can be called executing the following command:

./run_tests --re=framework/ROM/MSR.smoothExponential

or

./run_framework_tests --re=framework/ROM/MSR.smoothExponential

- Test Description:

- An example of using the Morse-Smale regression reduced order model with an exponential kernel function for the kernel density estimator. This is a smoothed version of MSR where local models are blended together.

Note, all of the tests in MSR operate on a 2D input domain with the goal of fitting a single Gaussian bump. The input dimensions are of largely different scales and one dimension is off-centered from the origin to ensure that normalization is being handled correctly.

- Original Author:

- maljdan

- Creation date:

- 2015-10-06

- The classes tested in this test are:

- SupervisedLearning.MSR 
- Since the creation of this test, the following main revisions have been performed:

1. revision info:

- author : maljdan

- date : 2015-10-21

- description: Converting AMSR test files using the provided conversion script.

2. revision info:

- author : talbpaul

- date : 2016-02-08

- description: first update, looking for more failing tests to add

3. revision info:

- author : maljdan

- date : 2016-02-10

- description: Adding functionality to allow a user to specify a filename for an Outstream object that is potentially different than its variable name in the RAVEN input file. Adjusting test cases to exercise this functionality.

4. revision info:

- author : maljdan

- date : 2016-04-06

- description: Updating test cases to reflect the changes to the user input.

5. revision info:

- author : maljdan

- date : 2017-01-19

- description: Adding this test description.

\subsubsection{FRAMEWORK ROM MSR.SMOOTHGAUSSIAN}

This test can be found at ". / raven/tests/framework/ROM/MSR/test_smooth_Gaussian. xml". This test can be called executing the following command:

. /run_tests --re=framework/ROM/MSR.smoothGaussian

or

./run_framework_tests --re=framework/ROM/MSR.smoothGaussian

- Test Description: 
- An example of using the Morse-Smale regression reduced order model with a Gaussian kernel function for the kernel density estimator. This is a smoothed version of MSR where local models are blended together.

Note, all of the tests in MSR operate on a 2D input domain with the goal of fitting a single Gaussian bump. The input dimensions are of largely different scales and one dimension is off-centered from the origin to ensure that normalization is being handled correctly.

- Original Author:

- maljdan

- Creation date:

- 2015-10-06

- The classes tested in this test are:

- SupervisedLearning.MSR

- Since the creation of this test, the following main revisions have been performed:

1. revision info:

- author : maljdan

- date : 2015-10-21

- description: Converting AMSR test files using the provided conversion script.

2. revision info:

- author : talbpaul

- date : 2016-02-08

- description: first update, looking for more failing tests to add

3. revision info:

- author : maljdan

- date : 2016-02-10

- description: Adding functionality to allow a user to specify a filename for an Outstream object that is potentially different than its variable name in the RAVEN input file. Adjusting test cases to exercise this functionality.

4. revision info:

- author : maljdan

- date : 2016-04-06

- description: Updating test cases to reflect the changes to the user input.

5. revision info: 
- author : maljdan

- date : 2017-01-19

- description: Adding this test description.

\subsubsection{FRAMEWORK ROM MSR.SMOOTHLOGISTIC}

This test can be found at ". / raven/tests/framework/ROM/MSR/test_smooth_logistic. xml". This test can be called executing the following command:

./run_tests --re=framework/ROM/MSR.smoothLogistic

Or

./run_framework_tests --re=framework/ROM/MSR.smoothLogistic

- Test Description:

- An example of using the Morse-Smale regression reduced order model with a logistic kernel function for the kernel density estimator. This is a smoothed version of MSR where local models are blended together.

Note, all of the tests in MSR operate on a 2D input domain with the goal of fitting a single Gaussian bump. The input dimensions are of largely different scales and one dimension is off-centered from the origin to ensure that normalization is being handled correctly.

- Original Author:

- maljdan

- Creation date:

- 2015-10-06

- The classes tested in this test are:

- SupervisedLearning.MSR

- Since the creation of this test, the following main revisions have been performed:

1. revision info:

- author : maljdan

- date : 2015-10-21

- description: Converting AMSR test files using the provided conversion script. 
2. revision info:

- author : talbpaul

- date : 2016-02-08

- description: first update, looking for more failing tests to add

3. revision info:

- author : maljdan

- date : 2016-02-10

- description: Adding functionality to allow a user to specify a filename for an Outstream object that is potentially different than its variable name in the RAVEN input file. Adjusting test cases to exercise this functionality.

4. revision info:

- author : maljdan

- date : 2016-04-06

- description: Updating test cases to reflect the changes to the user input.

5. revision info:

- author : maljdan

- date : 2017-01-19

- description: Adding this test description.

\subsubsection{FRAMEWORK ROM MSR.SMOOTHSILVERMAN}

This test can be found at “. / raven/tests/framework/ROM/MSR/test_smooth_Silverman. xml". This test can be called executing the following command:

./run_tests --re=framework/ROM/MSR.smoothSilverman

or

./run_framework_tests --re=framework/ROM/MSR.smoothSilverman

- Test Description:

- An example of using the Morse-Smale regression reduced order model with a Silverman kernel function for the kernel density estimator. This is a smoothed version of MSR where local models are blended together.

Note, all of the tests in MSR operate on a 2D input domain with the goal of fitting a single Gaussian bump. The input dimensions are of largely different scales and one dimension is off-centered from the origin to ensure that normalization is being handled correctly. 
- Original Author:

- maljdan

- Creation date:

- 2015-10-06

- The classes tested in this test are:

- SupervisedLearning.MSR

- Since the creation of this test, the following main revisions have been performed:

1. revision info:

- author : maljdan

- date : 2015-10-21

- description: Converting AMSR test files using the provided conversion script.

2. revision info:

- author : talbpaul

- date : 2016-02-08

- description: first update, looking for more failing tests to add

3. revision info:

- author : maljdan

- date : 2016-02-10

- description: Adding functionality to allow a user to specify a filename for an Outstream object that is potentially different than its variable name in the RAVEN input file. Adjusting test cases to exercise this functionality.

4. revision info:

- author : maljdan

- date : 2016-04-06

- description: Updating test cases to reflect the changes to the user input.

5. revision info:

- author : maljdan

- date : 2017-01-19

- description: Adding this test description. 


\subsubsection{FRAMEWORK ROM MSR.SMOOTHTRIANGULAR}

This test can be found at ". / raven/tests/framework/ROM/MSR/test_smooth_triangular. xml". This test can be called executing the following command:

./run_tests --re=framework/ROM/MSR.smoothTriangular

or

. /run_framework_tests --re=framework/ROM/MSR.smoothTriangular

- Test Description:

- An example of using the Morse-Smale regression reduced order model with a triangular kernel function for the kernel density estimator. This is a smoothed version of MSR where local models are blended together.

Note, all of the tests in MSR operate on a 2D input domain with the goal of fitting a single Gaussian bump. The input dimensions are of largely different scales and one dimension is off-centered from the origin to ensure that normalization is being handled correctly.

- Original Author:

- maljdan

- Creation date:

- 2015-10-06

- The classes tested in this test are:

- SupervisedLearning.MSR

- Since the creation of this test, the following main revisions have been performed:

1. revision info:

- author : maljdan

- date : 2015-10-21

- description: Converting AMSR test files using the provided conversion script.

2. revision info:

- author : talbpaul

- date : 2016-02-08

- description: first update, looking for more failing tests to add 
3. revision info:

- author : maljdan

- date : 2016-02-10

- description: Adding functionality to allow a user to specify a filename for an Outstream object that is potentially different than its variable name in the RAVEN input file. Adjusting test cases to exercise this functionality.

4. revision info:

- author : maljdan

- date : 2016-04-06

- description: Updating test cases to reflect the changes to the user input.

5. revision info:

- author : maljdan

- date : 2017-01-19

- description: Adding this test description.

\subsubsection{FRAMEWORK ROM MSR.SMOOTHTRICUBE}

This test can be found at “. / raven/tests/framework/ROM/MSR/test_smooth_tricube. xml". This test can be called executing the following command:

. /run_tests --re=framework/ROM/MSR.smoothTricube

or

./run_framework_tests --re=framework/ROM/MSR.smoothTricube

- Test Description:

- An example of using the Morse-Smale regression reduced order model with a tricube kernel function for the kernel density estimator. This is a smoothed version of MSR where local models are blended together.

Note, all of the tests in MSR operate on a 2D input domain with the goal of fitting a single Gaussian bump. The input dimensions are of largely different scales and one dimension is off-centered from the origin to ensure that normalization is being handled correctly.

- Original Author:

- maljdan 
- Creation date:

- 2015-10-06

- The classes tested in this test are:

- SupervisedLearning.MSR

- Since the creation of this test, the following main revisions have been performed:

1. revision info:

- author : maljdan

- date : 2015-10-21

- description: Converting AMSR test files using the provided conversion script.

2. revision info:

- author : talbpaul

- date : 2016-02-08

- description: first update, looking for more failing tests to add

3. revision info:

- author : maljdan

- date : 2016-02-10

- description: Adding functionality to allow a user to specify a filename for an Outstream object that is potentially different than its variable name in the RAVEN input file. Adjusting test cases to exercise this functionality.

4. revision info:

- author : maljdan

- date : 2016-04-06

- description: Updating test cases to reflect the changes to the user input.

5. revision info:

- author : maljdan

- date : 2017-01-19

- description: Adding this test description.

\subsubsection{FRAMEWORK ROM MSR.SMOOTHTRIWEIGHT}

This test can be found at ". /raven/tests/framework/ROM/MSR/test_smooth_triweight. xml". This test can be called executing the following command:

./run_tests --re=framework/ROM/MSR.smoothTriweight 
or

./run_framework_tests --re=framework/ROM/MSR.smoothTriweight

- Test Description:

- An example of using the Morse-Smale regression reduced order model with a triweight kernel function for the kernel density estimator. This is a smoothed version of MSR where local models are blended together.

Note, all of the tests in MSR operate on a 2D input domain with the goal of fitting a single Gaussian bump. The input dimensions are of largely different scales and one dimension is off-centered from the origin to ensure that normalization is being handled correctly.

- Original Author:

- maljdan

- Creation date:

- 2015-10-06

- The classes tested in this test are:

- SupervisedLearning.MSR

- Since the creation of this test, the following main revisions have been performed:

1. revision info:

- author : maljdan

- date : 2015-10-21

- description: Converting AMSR test files using the provided conversion script.

2. revision info:

- author : talbpaul

- date : 2016-02-08

- description: first update, looking for more failing tests to add

3. revision info:

- author : maljdan

- date : 2016-02-10

- description: Adding functionality to allow a user to specify a filename for an Outstream object that is potentially different than its variable name in the RAVEN input file. Adjusting test cases to exercise this functionality. 
4. revision info:

- author : maljdan

- date : 2016-04-06

- description: Updating test cases to reflect the changes to the user input.

5. revision info:

- author : maljdan

- date : 2017-01-19

- description: Adding this test description.

\subsubsection{FRAMEWORK ROM MSR.SMOOTHUNIFORM}

This test can be found at ". /raven/tests/framework/ROM/MSR/test_smooth_uniform. xml". This test can be called executing the following command:

./run_tests --re=framework/ROM/MSR.smoothUniform

or

./run_framework_tests --re=framework/ROM/MSR.smoothUniform

- Test Description:

- An example of using the Morse-Smale regression reduced order model with a uniform kernel function for the kernel density estimator. This is a smoothed version of MSR where local models are blended together.

Note, all of the tests in MSR operate on a 2D input domain with the goal of fitting a single Gaussian bump. The input dimensions are of largely different scales and one dimension is off-centered from the origin to ensure that normalization is being handled correctly.

- Original Author:

- maljdan

- Creation date:

- 2015-10-06

- The classes tested in this test are:

- SupervisedLearning.MSR 
- Since the creation of this test, the following main revisions have been performed:

1. revision info:

- author : maljdan

- date : 2015-10-21

- description: Converting AMSR test files using the provided conversion script.

2. revision info:

- author : talbpaul

- date : 2016-02-08

- description: first update, looking for more failing tests to add

3. revision info:

- author : maljdan

- date : 2016-02-10

- description: Adding functionality to allow a user to specify a filename for an Outstream object that is potentially different than its variable name in the RAVEN input file. Adjusting test cases to exercise this functionality.

4. revision info:

- author : maljdan

- date : 2016-04-06

- description: Updating test cases to reflect the changes to the user input.

5. revision info:

- author : maljdan

- date : 2017-01-19

- description: Adding this test description.

\subsubsection{FRAMEWORK ROM MSR.PARALLEL}

This test can be found at ". / raven/tests/framework/ROM/MSR/test_local_parallel. xml". This test can be called executing the following command:

./run_tests --re=framework/ROM/MSR.parallel

or

./run_framework_tests --re=framework/ROM/MSR.parallel

- Test Description: 
- A copy of framework/ROM/MSR.uniform where the internal parallel system has been activated in order to test its functionality in conjunction with the MSR.

Note, all of the tests in MSR operate on a 2D input domain with the goal of fitting a single Gaussian bump. The input dimensions are of largely different scales and one dimension is off-centered from the origin to ensure that normalization is being handled correctly.

- Original Author:

- maljdan

- Creation date:

- 2015-10-28

- The classes tested in this test are:

- SupervisedLearning.MSR

- Since the creation of this test, the following main revisions have been performed:

1. revision info:

- author : talbpaul

- date : 2016-02-08

- description: first update, looking for more failing tests to add

2. revision info:

- author : maljdan

- date : 2016-02-10

- description: Adding functionality to allow a user to specify a filename for an Outstream object that is potentially different than its variable name in the RAVEN input file. Adjusting test cases to exercise this functionality.

3. revision info:

- author : maljdan

- date : 2016-04-06

- description: Updating test cases to reflect the changes to the user input.

4. revision info:

- author : maljdan

- date : 2017-01-19

- description: Adding this test description. 


\subsubsection{FRAMEWORK STOCHPOLYPICKLETEST}

This test can be found at ". / raven/tests / framework/ROM/pickleTests/stochpoly_ pickle.xml". This test can be called executing the following command:

. /run_tests --re=framework/stochPolyPickleTest

or

. Irun_framework_tests --re=framework/stochPolyPickleTest

- Test Description:

- This test checks the pickling and unpickling of the GaussPolynomialROM

- Original Author:

- talbpaul

- Creation date:

- 2015-07-07

- The classes tested in this test are:

- SupervisedLearning.GaussPolynomialROM

\subsubsection{FRAMEWORK ROM COLDRESTART}

This test can be found at ". / raven/tests/framework/ROM/pickleTests/restart_ stochpoly_rom.xml". This test can be called executing the following command:

./run_tests --re=framework/ROM/coldRestart

or

./run_framework_tests --re=framework/ROM/coldRestart

- Test Description:

- This tests the ability of a ROM to be unpickled and used with minimal specification, and without the typically-associated Sampler for the GaussPolynomialROM. 
- Original Author:

- talbpaul

- Creation date:

$-2016-03-21$

- The classes tested in this test are:

- SupervisedLearning.GaussPolynomialROM

\subsubsection{FRAMEWORK ROM PICKLETESTS LOADUNTRAINEDERROR}

This test can be found at ". / raven/tests/framework/ROM/pickleTests/untrained_ error.xml". This test can be called executing the following command:

./run_tests --re=framework/ROM/pickleTests/loadUntrainedError

Or

. /run_framework_tests --re=framework/ROM/pickleTests/loadUntrainedError

- Test Description:

- This test checks that a reasonable error is given to the user if an untrained rom is unpickled then sampled.

- Original Author:

- talbpaul

- Creation date:

- 2015-07-07

- The classes tested in this test are:

- Models.ROM 


\subsubsection{FRAMEWORK ROM PICKLETESTS LOADNORROMERROR}

This test can be found at "./raven/tests/framework/ROM/pickleTests/not_a_ rom_error.xml". This test can be called executing the following command:

./run_tests --re=framework/ROM/pickleTests/loadNorROMError

or

./run_framework_tests --re=framework/ROM/pickleTests/loadNorROMEłror

- Test Description:

- This test checks that a reasonable error is given to the user if they attempt to unpickle a pickled object that isn't a ROM.

- Original Author:

- talbpaul

- Creation date:

- 2015-07-07

- The classes tested in this test are:

- Models.ROM

\subsubsection{FRAMEWORK ROM PICKLETESTS LOADNORROMERROR}

This test can be found at "./raven/scripts/externalROMloader.pyload_ROM_externally. xml". This test can be called executing the following command:

./run_tests --re=framework/ROM/pickleTests/loadNorROMError

or

./run_framework_tests --re=framework/ROM/pickleTests/loadNorROMEpror

- Test Description:

- This test checks that a reasonable error is given to the user if they attempt to unpickle a pickled object that isn't a ROM. 
- Original Author:

- talbpaul

- Creation date:

- 2015-07-07

- The classes tested in this test are:

- Models.ROM

\subsubsection{FRAMEWORK SAMPLERS ROM TENSORFLOW KERAS.TF CNN1D}

This test can be found at ". / raven/tests/framework/ROM/tensorflow_keras/cnn1d. xml". This test can be called executing the following command:

./run_tests --re=framework/Samplers/ROM/tensorflow_keras.tf_cnn1d

or

./run_framework_tests --re=framework/Samplers/ROM/tensorflow_keras.tf_cnn1

- Test Description:

- Test the capability of convolutional neural network (CNN) 1D using TensorFlow/Keras Multilayers is used, the CNN-1D will accept (numSamples, timeSteps,Features) as training input, and accept (timeSteps, Features) as prediction input. Ensemble model is used in order to generate time-dependent input for CNN models.

- Original Author:

- wangc

- Creation date:

- 2019-02-07

- The classes tested in this test are:

- Models.ROM 


\subsubsection{FRAMEWORK SAMPLERS ROM TENSORFLOW KERAS.TF MLPC}

This test can be found at ". /raven/tests/framework/ROM/tensorflow_keras/custom_ dataset. xml". This test can be called executing the following command:

./run_tests --re=framework/Samplers/ROM/tensorflow_keras.tf_mlpc

or

.Irun_framework_tests --re=framework/Samplers/ROM/tensorflow_keras.tf_mlpc

- Test Description:

- Test the capability of multilayer perceptron classifier (mlpc) using TensorFlow/Keras The train (row 1-728) and test (row 729-768) data are generated from "Pima Indians Diabetes Database". CustomSampler is used to convert the csv format data into PointSet, and it is also used to test the trained mlpc with the test data. (https://www.kaggle.com/kumargh/pimainc

- Original Author:

- wangc

- Creation date:

- 2019-02-07

- The classes tested in this test are:

- Models.ROM

\subsubsection{FRAMEWORK SAMPLERS ROM TENSORFLOW KERAS.TF LSTM}

This test can be found at “. / raven/tests/framework/ROM/tensorflow_keras/Istm. xml". This test can be called executing the following command:

./run_tests --re=framework/Samplers/ROM/tensorflow_keras.tf_lstm

or

./run_framework_tests --re=framework/Samplers/ROM/tensorflow_keras.tf_lstm

- Test Description: 
- Test the capability of convolutional long short-term memory (lstm) using TensorFlow/Keras Multilayers is used, the lstm will accept (numSamples,timeSteps,Features) as training input, and accept (timeSteps, Features) as prediction input. Ensemble model is used in order to generate time-dependent input for lstm models.

- Original Author:

- wangc

- Creation date:

- 2019-02-07

- The classes tested in this test are:

- Models.ROM

\subsubsection{FRAMEWORK ROM SKLEARN.LINEARSVC}

This test can be found at "./raven/tests/framework/ROM/SKLearn/linearSVC. xml". This test can be called executing the following command:

. /run_tests --re=framework/ROM/SKLearn.linearSVC

or

./run_framework_tests --re=framework/ROM/SKLearn.linearSVC

- Test Description:

- An example exercising supervised sklearn methods, specifically the svm-LinearSVC model is tested here.

Note, all of the tests in SKLearn operate on a 2D input domain with the goal of fitting a paraboloid function. The input dimensions are of largely different scales and one dimension is off-centered from the origin to ensure that normalization is being handled correctly. Classifiers will use this same function to determine if a point is above 0.25 , and multitask methods will additionally fit an additive model $(\mathrm{x}+\mathrm{y})$.

- Original Author:

- maljdan

- Creation date:

- 2016-04-28 
- The classes tested in this test are:

- SupervisedLearning.SciKitLearn

- Since the creation of this test, the following main revisions have been performed:

1. revision info:

- author : maljdan

- date : 2016-05-02

- description: Fixing the random_state of the SVMs.

2. revision info:

- author : maljdan

- date : 2016-05-02

- description: Fixing another SVC case for older scikit-learn versions.

3. revision info:

- author : talbpaul

- date : 2016-09-15

- description: other test updates

4. revision info:

- author : maljdan

- date : 2017-01-19

- description: Adding this test description.

\subsubsection{FRAMEWORK ROM SKLEARN.SVC}

This test can be found at ". / raven/tests/framework/ROM/SKLearn/svc.xml”. This test can be called executing the following command:

. /run_tests --re=framework/ROM/SKLearn.SVC

or

. Irun_framework_tests --re=framework/ROM/SKLearn.SVC

- Test Description:

- An example exercising supervised sklearn methods, specifically the svm-SVC model is tested here. 
Note, all of the tests in SKLearn operate on a 2D input domain with the goal of fitting a paraboloid function. The input dimensions are of largely different scales and one dimension is off-centered from the origin to ensure that normalization is being handled correctly. Classifiers will use this same function to determine if a point is above 0.25 , and multitask methods will additionally fit an additive model $(x+y)$.

- Original Author:

- maljdan

- Creation date:

- 2016-04-28

- The classes tested in this test are:

- SupervisedLearning.SciKitLearn

- Since the creation of this test, the following main revisions have been performed:

1. revision info:

- author : maljdan

- date : 2016-05-02

- description: Fixing the random_state of the SVMs.

2. revision info:

- author : maljdan

- date : 2016-05-02

- description: There is an issue with version compatibility in sklearn, so I am disabling one of the parameters in the input and documenting it.

3. revision info:

- author : talbpaul

- date : 2016-09-15

- description: other test updates

4. revision info:

- author : cogljj

- date : 2016-11-18

- description: Switching floats to integers because they are.

5. revision info:

- author : maljdan

- date : 2017-01-19

- description: Adding this test description. 


\subsubsection{FRAMEWORK ROM SKLEARN.NUSVC}

This test can be found at "./raven/tests/framework/ROM/SKLearn/nuSVC.xml". This test can be called executing the following command:

. Irun_tests --re=framework/ROM/SKLearn.NuSVC

or

./run_framework_tests --re=framework/ROM/SKLearn.NuSVC

- Test Description:

- An example exercising supervised sklearn methods, specifically the svm-NuSVC model is tested here.

Note, all of the tests in SKLearn operate on a 2D input domain with the goal of fitting a paraboloid function. The input dimensions are of largely different scales and one dimension is off-centered from the origin to ensure that normalization is being handled correctly. Classifiers will use this same function to determine if a point is above 0.25 , and multitask methods will additionally fit an additive model $(x+y)$.

- Original Author:

- maljdan

- Creation date:

$-2016-04-28$

- The classes tested in this test are:

- SupervisedLearning.SciKitLearn

- Since the creation of this test, the following main revisions have been performed:

1. revision info:

- author : maljdan

- date : 2016-05-02

- description: Fixing the random_state of the SVMs.

2. revision info:

- author : maljdan

- date : 2016-05-02 
- description: There is an issue with version compatibility in sklearn, so I am disabling one of the parameters in the input and documenting it.

3. revision info:

- author : talbpaul

- date : 2016-09-15

- description: other test updates

4. revision info:

- author : cogljj

- date : 2016-11-18

- description: Switching floats to integers because they are.

5. revision info:

- author : maljdan

- date : 2017-01-19

- description: Adding this test description.

\subsubsection{FRAMEWORK ROM SKLEARN.OCC}

This test can be found at ". / raven/tests/framework/ROM/SKLearn/occ.xml”. This test can be called executing the following command:

. Irun_tests --re=framework/ROM/SKLearn. OCC

or

./run_framework_tests --re=framework/ROM/SKLearn.oCC

- Test Description:

- An example exercising supervised sklearn methods, specifically the linear_model—LinearRegression model is tested here.

Note, all of the tests in SKLearn operate on a 2D input domain with the goal of fitting a paraboloid function. The input dimensions are of largely different scales and one dimension is off-centered from the origin to ensure that normalization is being handled correctly. Classifiers will use this same function to determine if a point is above 0.25 , and multitask methods will additionally fit an additive model $(x+y)$.

- Original Author:

- maljdan 
- Creation date:

- 2016-04-28

- The classes tested in this test are:

- SupervisedLearning.SciKitLearn

- Since the creation of this test, the following main revisions have been performed:

1. revision info:

- author : maljdan

- date : 2016-04-28

- description: Fixing the multiclass SKL types to allow for embedded estimators.

2. revision info:

- author : alfoa

- date : 2016-07-13

- description: removed renormalization

3. revision info:

- author : talbpaul

- date : 2016-09-15

- description: other test updates

4. revision info:

- author : maljdan

- date : 2017-01-19

- description: Adding this test description.

\subsubsection{FRAMEWORK ROM SKLEARN.KNC}

This test can be found at ". /raven/tests/framework/ROM/SKLearn/knc.xml". This test can be called executing the following command:

./run_tests --re=framework/ROM/SKLearn.KNC

or

./run_framework_tests --re=framework/ROM/SKLearn.KNC

- Test Description: 
- An example exercising supervised sklearn methods, specifically the neighbors-KNeighborsClassifier model is tested here.

Note, all of the tests in SKLearn operate on a 2D input domain with the goal of fitting a paraboloid function. The input dimensions are of largely different scales and one dimension is off-centered from the origin to ensure that normalization is being handled correctly. Classifiers will use this same function to determine if a point is above 0.25 , and multitask methods will additionally fit an additive model $(\mathrm{x}+\mathrm{y})$.

- Original Author:

- maljdan

- Creation date:

- 2016-04-28

- The classes tested in this test are:

- SupervisedLearning.SciKitLearn

- Since the creation of this test, the following main revisions have been performed:

1. revision info:

- author : talbpaul

- date : 2016-09-15

- description: other test updates

2. revision info:

- author : maljdan

- date : 2017-01-19

- description: Adding this test description.

\subsubsection{FRAMEWORK ROM SKLEARN.RNC}

This test can be found at ". / raven/tests/framework/ROM/SKLearn/rnc.xml". This test can be called executing the following command:

./run_tests --re=framework/ROM/SKLearn.RNC

or

./run_framework_tests --re=framework/ROM/SKLearn.RNC 


\section{- Test Description:}

- An example exercising supervised sklearn methods, specifically the neighbors-RadiusNeighbors model is tested here.

Note, all of the tests in SKLearn operate on a 2D input domain with the goal of fitting a paraboloid function. The input dimensions are of largely different scales and one dimension is off-centered from the origin to ensure that normalization is being handled correctly. Classifiers will use this same function to determine if a point is above 0.25 , and multitask methods will additionally fit an additive model $(\mathrm{x}+\mathrm{y})$.

- Original Author:

- maljdan

- Creation date:

$-2016-04-28$

- The classes tested in this test are:

- SupervisedLearning.SciKitLearn

- Since the creation of this test, the following main revisions have been performed:

1. revision info:

- author : talbpaul

- date : 2016-09-15

- description: other test updates

2. revision info:

- author : maljdan

- date : 2017-01-19

- description: Adding this test description.

\subsubsection{FRAMEWORK ROM SKLEARN.NCC}

This test can be found at ". / raven/tests/framework/ROM/SKLearn/ncc.xml". This test can be called executing the following command:

. Irun_tests --re=framework/ROM/SKLearn.NCC

or

./run_framework_tests --re=framework/ROM/SKLearn.NCC 


\section{- Test Description:}

- An example exercising supervised sklearn methods, specifically the neighbors-NearestCentroid model is tested here.

Note, all of the tests in SKLearn operate on a 2D input domain with the goal of fitting a paraboloid function. The input dimensions are of largely different scales and one dimension is off-centered from the origin to ensure that normalization is being handled correctly. Classifiers will use this same function to determine if a point is above 0.25 , and multitask methods will additionally fit an additive model $(\mathrm{x}+\mathrm{y})$.

- Original Author:

- maljdan

- Creation date:

$-2016-04-28$

- The classes tested in this test are:

- SupervisedLearning.SciKitLearn

- Since the creation of this test, the following main revisions have been performed:

1. revision info:

- author : talbpaul

- date : 2016-09-15

- description: other test updates

2. revision info:

- author : maljdan

- date : 2017-01-19

- description: Adding this test description.

\subsubsection{FRAMEWORK ROM SKLEARN.OVR}

This test can be found at ". / raven/tests/framework/ROM/SKLearn/ovr.xml". This test can be called executing the following command:

. /run_tests --re=framework/ROM/SKLearn.OVR

or

./run_framework_tests --re=framework/ROM/SKLearn.OVR 


\section{- Test Description:}

- An example exercising supervised sklearn methods, specifically the linear_model—LinearRegression model is tested here.

Note, all of the tests in SKLearn operate on a 2D input domain with the goal of fitting a paraboloid function. The input dimensions are of largely different scales and one dimension is off-centered from the origin to ensure that normalization is being handled correctly. Classifiers will use this same function to determine if a point is above 0.25 , and multitask methods will additionally fit an additive model $(\mathrm{x}+\mathrm{y})$.

- Original Author:

- maljdan

- Creation date:

- 2016-04-28

- The classes tested in this test are:

- SupervisedLearning.SciKitLearn

- Since the creation of this test, the following main revisions have been performed:

1. revision info:

- author : maljdan

- date : 2016-04-28

- description: Fixing the multiclass SKL types to allow for embedded estimators.

2. revision info:

- author : alfoa

- date : 2016-07-13

- description: removed renormalization

3. revision info:

- author : talbpaul

- date : 2016-09-15

- description: other test updates

4. revision info:

- author : maljdan

- date : 2017-01-19

- description: Adding this test description. 


\subsubsection{FRAMEWORK ROM SKLEARN.OVO}

This test can be found at "./raven/tests/framework/ROM/SKLearn/ovo.xml". This test can be called executing the following command:

. /run_tests --re=framework/ROM/SKLearn. OVO

or

. /run_framework_tests --re=framework/ROM/SKLearn. OVO

- Test Description:

- An example exercising supervised sklearn methods, specifically the linear_model—LinearRegression model is tested here.

Note, all of the tests in SKLearn operate on a 2D input domain with the goal of fitting a paraboloid function. The input dimensions are of largely different scales and one dimension is off-centered from the origin to ensure that normalization is being handled correctly. Classifiers will use this same function to determine if a point is above 0.25 , and multitask methods will additionally fit an additive model $(\mathrm{x}+\mathrm{y})$.

- Original Author:

- maljdan

- Creation date:

- 2016-04-28

- The classes tested in this test are:

- SupervisedLearning.SciKitLearn

- Since the creation of this test, the following main revisions have been performed:

1. revision info:

- author : maljdan

- date : 2016-04-28

- description: Fixing the multiclass SKL types to allow for embedded estimators.

2. revision info:

- author : alfoa

- date : 2016-07-13

- description: removed renormalization 
3. revision info:

- author : talbpaul

- date : 2016-09-15

- description: other test updates

4. revision info:

- author : maljdan

- date : 2017-01-19

- description: Adding this test description.

\subsubsection{FRAMEWORK ROM SKLEARN.GAUSSIANNB}

This test can be found at ". /raven/tests/framework/ROM/SKLearn/gaussianNB. xml". This test can be called executing the following command:

. Irun_tests --re=framework/ROM/SKLearn.GaussianNB

or

./run_framework_tests --re=framework/ROM/SKLearn.GaussianNB

- Test Description:

- An example exercising supervised sklearn methods, specifically the naiveBayes_-GaussianNB model is tested here.

Note, all of the tests in SKLearn operate on a 2D input domain with the goal of fitting a paraboloid function. The input dimensions are of largely different scales and one dimension is off-centere from the origin to ensure that normalization is being handled correctly. Classifiers will use this same function to determine if a point is above 0.25 , and multitask methods will additionally fit an additive model $(\mathrm{x}+\mathrm{y})$.

- Original Author:

- maljdan

- Creation date:

- 2016-04-28

- The classes tested in this test are:

- SupervisedLearning.SciKitLearn 
- Since the creation of this test, the following main revisions have been performed:

1. revision info:

- author : talbpaul

- date : 2016-09-15

- description: other test updates

2. revision info:

- author : maljdan

- date : 2017-01-19

- description: Adding this test description.

\subsubsection{FRAMEWORK ROM SKLEARN.BERNOULLINB}

This test can be found at ". / raven/tests/framework/ROM/SKLearn/bernoulliNB. xml". This test can be called executing the following command:

./run_tests --re=framework/ROM/SKLearn.BernoulliNB

or

./run_framework_tests --re=framework/ROM/SKLearn.BernoulliNB

- Test Description:

- An example exercising supervised sklearn methods, specifically the naiveBayes-BernoulliNB model is tested here.

Note, all of the tests in SKLearn operate on a 2D input domain with the goal of fitting a paraboloid function. The input dimensions are of largely different scales and one dimension is off-centered from the origin to ensure that normalization is being handled correctly. Classifiers will use this same function to determine if a point is above 0.25 , and multitask methods will additionally fit an additive model $(\mathrm{x}+\mathrm{y})$.

- Original Author:

- maljdan

- Creation date:

- 2016-04-28

- The classes tested in this test are: 
- SupervisedLearning.SciKitLearn

- Since the creation of this test, the following main revisions have been performed:

1. revision info:

- author : talbpaul

- date : 2016-09-15

- description: other test updates

2. revision info:

- author : maljdan

- date : 2017-01-19

- description: Adding this test description.

\subsubsection{FRAMEWORK ROM SKLEARN.LINEARRIDGEC}

This test can be found at ". / raven/tests/framework/ROM/SKLearn/linearRidgeC. xml". This test can be called executing the following command:

./run_tests --re=framework/ROM/SKLearn.linearRidgeC

or

. Irun_framework_tests --re=framework/ROM/SKLearn.linearRidgeC

- Test Description:

- An example exercising supervised sklearn methods, specifically the linear_model—RidgeClassifier model is tested here.

Note, all of the tests in SKLearn operate on a 2D input domain with the goal of fitting a paraboloid function. The input dimensions are of largely different scales and one dimension is off-centered from the origin to ensure that normalization is being handled correctly. Classifiers will use this same function to determine if a point is above 0.25 , and multitask methods will additionally fit an additive model $(\mathrm{x}+\mathrm{y})$.

- Original Author:

- maljdan

- Creation date:

- 2016-04-28 
- The classes tested in this test are:

- SupervisedLearning.SciKitLearn

- Since the creation of this test, the following main revisions have been performed:

1. revision info:

- author : alfoa

- date : 2016-07-13

- description: removed renormalization

2. revision info:

- author : talbpaul

- date : 2016-09-15

- description: other test updates

3. revision info:

- author : maljdan

- date : 2017-01-19

- description: Adding this test description.

\subsubsection{FRAMEWORK ROM SKLEARN.LINEARRIDGECCV}

This test can be found at “. / raven/tests / framework/ROM/SKLearn/linearRidgeCCV. xml". This test can be called executing the following command:

. /run_tests --re=framework/ROM/SKLearn.linearRidgeCCV

or

. Irun_framework_tests --re=framework/ROM/SKLearn.linearRidgeCCV

- Test Description:

- An example exercising supervised sklearn methods, specifically the linear_model—RidgeClassifierCV model is tested here.

Note, all of the tests in SKLearn operate on a 2D input domain with the goal of fitting a paraboloid function. The input dimensions are of largely different scales and one dimension is off-centered from the origin to ensure that normalization is being handled correctly. Classifiers will use this same function to determine if a point is above 0.25 , and multitask methods will additionally fit an additive model $(\mathrm{x}+\mathrm{y})$. 
- Original Author:

- maljdan

- Creation date:

- 2016-04-28

- The classes tested in this test are:

- SupervisedLearning.SciKitLearn

- Since the creation of this test, the following main revisions have been performed:

1. revision info:

- author : alfoa

- date : 2016-07-13

- description: removed renormalization

2. revision info:

- author : talbpaul

- date : 2016-09-15

- description: other test updates

3. revision info:

- author : maljdan

- date : 2017-01-19

- description: Adding this test description.

\subsubsection{FRAMEWORK ROM SKLEARN.LINEARPAC}

This test can be found at ". /raven/tests/framework/ROM/SKLearn/linearPAC. xml". This test can be called executing the following command:

. Irun_tests --re=framework/ROM/SKLearn.linearPAC

or

. /run_framework_tests --re=framework/ROM/SKLearn.linearPAC

- Test Description: 
- An example exercising supervised sklearn methods, specifically the linear_model—PassiveAggressive model is tested here.

Note, all of the tests in SKLearn operate on a 2D input domain with the goal of fitting a paraboloid function. The input dimensions are of largely different scales and one dimension is off-centered from the origin to ensure that normalization is being handled correctly. Classifiers will use this same function to determine if a point is above 0.25 , and multitask methods will additionally fit an additive model $(\mathrm{x}+\mathrm{y})$.

- Original Author:

- maljdan

- Creation date:

- 2016-04-28

- The classes tested in this test are:

- SupervisedLearning.SciKitLearn

- Since the creation of this test, the following main revisions have been performed:

1. revision info:

- author : maljdan

- date : 2016-05-02

- description: Adding a fixed random_state value to a few of the ROM tests to ensure reproducibility.

2. revision info:

- author : talbpaul

- date : 2016-09-15

- description: other test updates

3. revision info:

- author : maljdan

- date : 2017-01-19

- description: Adding this test description.

\subsubsection{FRAMEWORK ROM SKLEARN.LINEARSGDC}

This test can be found at ". /raven/tests/framework/ROM/SKLearn/linearSGDC . xml". This test can be called executing the following command:

. /run_tests --re=framework/ROM/SKLearn.linearSGDC 
or

./run_framework_tests --re=framework/ROM/SKLearn.linearSGDC

- Test Description:

- An example exercising supervised sklearn methods, specifically the linear_model—SGDClassifier model is tested here.

Note, all of the tests in SKLearn operate on a 2D input domain with the goal of fitting a paraboloid function. The input dimensions are of largely different scales and one dimension is off-centered from the origin to ensure that normalization is being handled correctly. Classifiers will use this same function to determine if a point is above 0.25 , and multitask methods will additionally fit an additive model $(\mathrm{x}+\mathrm{y})$.

- Original Author:

- maljdan

- Creation date:

$-2016-04-28$

- The classes tested in this test are:

- SupervisedLearning.SciKitLearn

- Since the creation of this test, the following main revisions have been performed:

1. revision info:

- author : maljdan

- date : 2016-05-02

- description: Adding a fixed random_state value to a few of the ROM tests to ensure reproducibility.

2. revision info:

- author : talbpaul

- date : 2016-09-15

- description: other test updates

3. revision info:

- author : maljdan

- date : 2017-01-19

- description: Adding this test description. 


\subsubsection{FRAMEWORK ROM SKLEARN.MULTINOMIALNB}

This test can be found at ". / raven/tests/framework/ROM/SKLearn/multinomialNB . xml". This test can be called executing the following command:

./run_tests --re=framework/ROM/SKLearn.MultinomialNB

or

./run_framework_tests --re=framework/ROM/SKLearn.MultinomialNB

- Test Description:

- An example exercising supervised sklearn methods, specifically the naiveBayes—MultinomialNB model is tested here.

Note, all of the tests in SKLearn operate on a 2D input domain with the goal of fitting a paraboloid function. The input dimensions are of largely different scales and one dimension is off-centered from the origin to ensure that normalization is being handled correctly. Classifiers will use this same function to determine if a point is above 0.25 , and multitask methods will additionally fit an additive model $(\mathrm{x}+\mathrm{y})$.

- Original Author:

- maljdan

- Creation date:

- 2016-04-28

- The classes tested in this test are:

- SupervisedLearning.SciKitLearn

- Since the creation of this test, the following main revisions have been performed:

1. revision info:

- author : maljdan

- date : 2016-04-28

- description: Checkpointing. Only a few failing cases left.

2. revision info:

- author : talbpaul

- date : 2016-09-15

- description: other test updates 
3. revision info:

- author : maljdan

- date : 2017-01-19

- description: Adding this test description.

4. revision info:

- author: wangc

- date : 2018-08-08

- description: change the grid values that will within the bounds of distribution

\subsubsection{FRAMEWORK ROM SKLEARN.DTC}

This test can be found at ". / raven/tests/framework/ROM/SKLearn/dtc.xml". This test can be called executing the following command:

./run_tests --re=framework/ROM/SKLearn.DTC

or

./run_framework_tests --re=framework/ROM/SKLearn.DTC

- Test Description:

- An example exercising supervised sklearn methods, specifically the tree-DecisionTreeClassifier model is tested here.

Note, all of the tests in SKLearn operate on a 2D input domain with the goal of fitting a paraboloid function. The input dimensions are of largely different scales and one dimension is off-centered from the origin to ensure that normalization is being handled correctly. Classifiers will use this same function to determine if a point is above 0.25 , and multitask methods will additionally fit an additive model $(\mathrm{x}+\mathrm{y})$.

- Original Author:

- maljdan

- Creation date:

- 2016-04-28

- The classes tested in this test are:

- SupervisedLearning.SciKitLearn 
- Since the creation of this test, the following main revisions have been performed:

1. revision info:

- author : maljdan

- date : 2016-05-02

- description: Adding a fixed random_state value to a few of the ROM tests to ensure reproducibility.

2. revision info:

- author : talbpaul

- date : 2016-09-15

- description: other test updates

3. revision info:

- author : maljdan

- date : 2017-01-19

- description: Adding this test description.

\subsubsection{FRAMEWORK ROM SKLEARN.ETC}

This test can be found at ". / raven/tests/framework/ROM/SKLearn/etc.xml”. This test can be called executing the following command:

. /run_tests --re=framework/ROM/SKLearn.ETC

Or

./run_framework_tests --re=framework/ROM/SKLearn.ETC

- Test Description:

- An example exercising supervised sklearn methods, specifically the tree-ExtraTreeClassifier model is tested here.

Note, all of the tests in SKLearn operate on a 2D input domain with the goal of fitting a paraboloid function. The input dimensions are of largely different scales and one dimension is off-centered from the origin to ensure that normalization is being handled correctly. Classifiers will use this same function to determine if a point is above 0.25 , and multitask methods will additionally fit an additive model $(\mathrm{x}+\mathrm{y})$.

- Original Author:

- maljdan 
- Creation date:

- 2016-04-28

- The classes tested in this test are:

- SupervisedLearning.SciKitLearn

- Since the creation of this test, the following main revisions have been performed:

1. revision info:

- author : maljdan

- date : 2016-05-02

- description: Adding a fixed random_state value to a few of the ROM tests to ensure reproducibility.

2. revision info:

- author : talbpaul

- date : 2016-09-15

- description: other test updates

3. revision info:

- author : maljdan

- date : 2017-01-19

- description: Adding this test description.

\subsubsection{FRAMEWORK ROM SKLEARN.MLPCLASSIFIER}

This test can be found at “. / raven/tests/framework/ROM/SKLearn/mlpClassifier. xml". This test can be called executing the following command:

./run_tests --re=framework/ROM/SKLearn.MLPClassifier

or

./run_framework_tests --re=framework/ROM/SKLearn.MLPClassifier

- Test Description:

- An example exercising supervised sklearn methods, specifically the neural_network.MLPRegressor model is tested here.

Note, all of the tests in SKLearn operate on a 2D input domain with the goal of fitting a paraboloid function. The input dimensions are of largely different scales and one 
dimension is off-centered from the origin to ensure that normalization is being handled correctly. Classifiers will use this same function to determine if a point is above 0.25 , and multitask methods will additionally fit an additive model $(\mathrm{x}+\mathrm{y})$.

- Original Author:

- wangc

- Creation date:

- 2017-10-25

- The classes tested in this test are:

- SupervisedLearning.SciKitLearn

\subsubsection{FRAMEWORK ROM SKLEARN.SVR}

This test can be found at ". / raven/tests/framework/ROM/SKLearn/svr.xml”. This test can be called executing the following command:

./run_tests --re=framework/ROM/SKLearn.SVR

or

./run_framework_tests --re=framework/ROM/SKLearn.SVR

- Test Description:

- An example exercising supervised sklearn methods, specifically the svm-SVR model is tested here.

Note, all of the tests in SKLearn operate on a 2D input domain with the goal of fitting a paraboloid function. The input dimensions are of largely different scales and one dimension is off-centered from the origin to ensure that normalization is being handled correctly. Classifiers will use this same function to determine if a point is above 0.25 , and multitask methods will additionally fit an additive model $(\mathrm{x}+\mathrm{y})$.

- Original Author:

- maljdan

- Creation date:

- 2016-04-28 
- The classes tested in this test are:

- SupervisedLearning.SciKitLearn

- Since the creation of this test, the following main revisions have been performed:

1. revision info:

- author : maljdan

- date : 2016-05-02

- description: There is an issue with version compatibility in sklearn, so I am disabling one of the parameters in the input and documenting it.

2. revision info:

- author : talbpaul

- date : 2016-09-15

- description: other test updates

3. revision info:

- author : cogljj

- date : 2016-11-18

- description: Switching floats to integers because they are.

4. revision info:

- author : maljdan

- date : 2017-01-19

- description: Adding this test description.

\subsubsection{FRAMEWORK ROM SKLEARN.KNR}

This test can be found at ". / raven/tests/framework/ROM/SKLearn/knr.xml". This test can be called executing the following command:

. /run_tests --re=framework/ROM/SKLearn.KNR

or

./run_framework_tests --re=framework/ROM/SKLearn.KNR

- Test Description: 
- An example exercising supervised sklearn methods, specifically the neighbors-KNeighborsRegressor model is tested here.

Note, all of the tests in SKLearn operate on a 2D input domain with the goal of fitting a paraboloid function. The input dimensions are of largely different scales and one dimension is off-centered from the origin to ensure that normalization is being handled correctly. Classifiers will use this same function to determine if a point is above 0.25 , and multitask methods will additionally fit an additive model $(\mathrm{x}+\mathrm{y})$.

- Original Author:

- maljdan

- Creation date:

- 2016-04-28

- The classes tested in this test are:

- SupervisedLearning.SciKitLearn

- Since the creation of this test, the following main revisions have been performed:

1. revision info:

- author : talbpaul

- date : 2016-09-15

- description: other test updates

2. revision info:

- author : maljdan

- date : 2017-01-19

- description: Adding this test description.

\subsubsection{FRAMEWORK ROM SKLEARN.RNR}

This test can be found at ". / raven/tests/framework/ROM/SKLearn/rnr.xml". This test can be called executing the following command:

./run_tests --re=framework/ROM/SKLearn.RNR

or

./run_framework_tests --re=framework/ROM/SKLearn.RNR 


\section{- Test Description:}

- An example exercising supervised sklearn methods, specifically the neighbors-RadiusNeighborsRegr model is tested here.

Note, all of the tests in SKLearn operate on a 2D input domain with the goal of fitting a paraboloid function. The input dimensions are of largely different scales and one dimension is off-centered from the origin to ensure that normalization is being handled correctly. Classifiers will use this same function to determine if a point is above 0.25 , and multitask methods will additionally fit an additive model $(\mathrm{x}+\mathrm{y})$.

- Original Author:

- maljdan

- Creation date:

- 2016-04-28

- The classes tested in this test are:

- SupervisedLearning.SciKitLearn

- Since the creation of this test, the following main revisions have been performed:

1. revision info:

- author : maljdan

- date : 2016-04-28

- description: Fixing RadiusNeigbhorsRegressor test case to use the correct SKLtype in the input file.

2. revision info:

- author : talbpaul

- date : 2016-09-15

- description: other test updates

3. revision info:

- author : maljdan

- date : 2017-01-19

- description: Adding this test description.

\subsubsection{FRAMEWORK ROM SKLEARN.QDA}

This test can be found at ". / raven/tests/framework/ROM/SKLearn/qda.xml”. This test can be called executing the following command: 
. /run_tests --re=framework/ROM/SKLearn.QDA

or

./run_framework_tests --re=framework/ROM/SKLearn.QDA

- Test Description:

- An example exercising supervised sklearn methods, specifically the qda-QDA model is tested here.

Note, all of the tests in SKLearn operate on a 2D input domain with the goal of fitting a paraboloid function. The input dimensions are of largely different scales and one dimension is off-centered from the origin to ensure that normalization is being handled correctly. Classifiers will use this same function to determine if a point is above 0.25 , and multitask methods will additionally fit an additive model $(\mathrm{x}+\mathrm{y})$.

- Original Author:

- maljdan

- Creation date:

- 2016-04-28

- The classes tested in this test are:

- SupervisedLearning.SciKitLearn

- Since the creation of this test, the following main revisions have been performed:

1. revision info:

- author : talbpaul

- date : 2016-09-15

- description: other test updates

2. revision info:

- author : maljdan

- date : 2017-01-19

- description: Adding this test description. 


\subsubsection{FRAMEWORK ROM SKLEARN.GP}

This test can be found at "./raven/tests/framework/ROM/SKLearn/gp.xml”. This test can be called executing the following command:

. /run_tests --re=framework/ROM/SKLearn.GP

or

./run_framework_tests --re=framework/ROM/SKLearn.GP

- Test Description:

- An example exercising supervised sklearn methods, specifically the GaussianProcess-GaussianProce model is tested here.

Note, all of the tests in SKLearn operate on a 2D input domain with the goal of fitting a paraboloid function. The input dimensions are of largely different scales and one dimension is off-centered from the origin to ensure that normalization is being handled correctly. Classifiers will use this same function to determine if a point is above 0.25 , and multitask methods will additionally fit an additive model $(\mathrm{x}+\mathrm{y})$.

- Original Author:

- maljdan

- Creation date:

- 2016-04-28

- The classes tested in this test are:

- SupervisedLearning.SciKitLearn

- Since the creation of this test, the following main revisions have been performed:

1. revision info:

- author : talbpaul

- date : 2016-09-15

- description: other test updates

2. revision info:

- author : maljdan

- date : 2017-01-19

- description: Adding this test description. 


\subsubsection{FRAMEWORK ROM SKLEARN.LINEARARD}

This test can be found at "./raven/tests/framework/ROM/SKLearn/linearARD . xml". This test can be called executing the following command:

. /run_tests --re=framework/ROM/SKLearn.linearARD

or

. /run_framework_tests --re=framework/ROM/SKLearn.linearARD

- Test Description:

- An example exercising supervised sklearn methods, specifically the linear_model—ARDRegression model is tested here.

Note, all of the tests in SKLearn operate on a 2D input domain with the goal of fitting a paraboloid function. The input dimensions are of largely different scales and one dimension is off-centered from the origin to ensure that normalization is being handled correctly. Classifiers will use this same function to determine if a point is above 0.25 , and multitask methods will additionally fit an additive model $(\mathrm{x}+\mathrm{y})$.

- Original Author:

- maljdan

- Creation date:

- 2016-04-28

- The classes tested in this test are:

- SupervisedLearning.SciKitLearn

- Since the creation of this test, the following main revisions have been performed:

1. revision info:

- author : alfoa

- date : 2016-07-13

- description: removed renormalization

2. revision info:

- author : talbpaul

- date : 2016-09-15

- description: other test updates 
3. revision info:

- author : maljdan

- date : 2017-01-19

- description: Adding this test description.

\subsubsection{FRAMEWORK ROM SKLEARN.LINEARBAYESIANRIDGE}

This test can be found at ". / raven/tests/framework/ROM/SKLearn/linearBayesianRidge. xml". This test can be called executing the following command:

./run_tests --re=framework/ROM/SKLearn. linearBayesianRidge

or

./run_framework_tests --re=framework/ROM/SKLearn.linearBayesianRi dge

- Test Description:

- An example exercising supervised sklearn methods, specifically the linear_model—BayesianRidge model is tested here.

Note, all of the tests in SKLearn operate on a 2D input domain with the goal of fitting a paraboloid function. The input dimensions are of largely different scales and one dimension is off-centered from the origin to ensure that normalization is being handled correctly. Classifiers will use this same function to determine if a point is above 0.25 , and multitask methods will additionally fit an additive model $(\mathrm{x}+\mathrm{y})$.

- Original Author:

- maljdan

- Creation date:

- 2016-04-28

- The classes tested in this test are:

- SupervisedLearning.SciKitLearn

- Since the creation of this test, the following main revisions have been performed:

1. revision info:

- author : talbpaul

- date : 2016-09-15 
- description: other test updates

2. revision info:

- author : maljdan

- date : 2017-01-19

- description: Adding this test description.

\subsubsection{FRAMEWORK ROM SKLEARN.LINEARELASTICNET}

This test can be found at ". / raven/tests/framework/ROM/SKLearn/linearElasticNet. xml". This test can be called executing the following command:

./run_tests --re=framework/ROM/SKLearn.linearElasticNet

or

./run_framework_tests --re=framework/ROM/SKLearn.linearElasticNet

- Test Description:

- An example exercising supervised sklearn methods, specifically the linear_model—ElasticNet model is tested here.

Note, all of the tests in SKLearn operate on a 2D input domain with the goal of fitting a paraboloid function. The input dimensions are of largely different scales and one dimension is off-centered from the origin to ensure that normalization is being handled correctly. Classifiers will use this same function to determine if a point is above 0.25 , and multitask methods will additionally fit an additive model $(\mathrm{x}+\mathrm{y})$.

- Original Author:

- maljdan

- Creation date:

- 2016-04-28

- The classes tested in this test are:

- SupervisedLearning.SciKitLearn

- Since the creation of this test, the following main revisions have been performed:

1. revision info: 
- author : alfoa

- date : 2016-07-13

- description: removed renormalization

2. revision info:

- author : talbpaul

- date : 2016-09-15

- description: other test updates

3. revision info:

- author : maljdan

- date : 2017-01-19

- description: Adding this test description.

\subsubsection{FRAMEWORK ROM SKLEARN.LINEARELASTICNETCV}

This test can be found at ". / raven/tests / framework/ROM/SKLearn/linearElasticNetCV. xml". This test can be called executing the following command:

./run_tests --re=framework/ROM/SKLearn.linearElasticNetCV

or

./run_framework_tests --re=framework/ROM/SKLearn.linearElasticNetCV

- Test Description:

- An example exercising supervised sklearn methods, specifically the linear_model—ElasticNetCV model is tested here.

Note, all of the tests in SKLearn operate on a 2D input domain with the goal of fitting a paraboloid function. The input dimensions are of largely different scales and one dimension is off-centered from the origin to ensure that normalization is being handled correctly. Classifiers will use this same function to determine if a point is above 0.25 , and multitask methods will additionally fit an additive model $(\mathrm{x}+\mathrm{y})$.

- Original Author:

- maljdan

- Creation date:

- 2016-04-28 
- The classes tested in this test are:

- SupervisedLearning.SciKitLearn

- Since the creation of this test, the following main revisions have been performed:

1. revision info:

- author : talbpaul

- date : 2016-09-15

- description: other test updates

2. revision info:

- author : maljdan

- date : 2017-01-19

- description: Adding this test description.

\subsubsection{FRAMEWORK ROM SKLEARN.LINEARLARS}

This test can be found at ". / raven/tests/framework/ROM/SKLearn/linearLARS . xml". This test can be called executing the following command:

. /run_tests --re=framework/ROM/SKLearn.linearLARS

or

./run_framework_tests --re=framework/ROM/SKLearn.linearLARS

- Test Description:

- An example exercising supervised sklearn methods, specifically the linear_model_Lars model is tested here.

Note, all of the tests in SKLearn operate on a 2D input domain with the goal of fitting a paraboloid function. The input dimensions are of largely different scales and one dimension is off-centered from the origin to ensure that normalization is being handled correctly. Classifiers will use this same function to determine if a point is above 0.25 , and multitask methods will additionally fit an additive model $(x+y)$.

- Original Author:

- maljdan

- Creation date: 
- 2016-04-28

- The classes tested in this test are:

- SupervisedLearning.SciKitLearn

- Since the creation of this test, the following main revisions have been performed:

1. revision info:

- author : talbpaul

- date : 2016-09-15

- description: other test updates

2. revision info:

- author : maljdan

- date : 2017-01-19

- description: Adding this test description.

\subsubsection{FRAMEWORK ROM SKLEARN.LINEARLARSCV}

This test can be found at ". / raven/tests/framework/ROM/SKLearn/linearLARSCV. xml". This test can be called executing the following command:

. /run_tests --re=framework/ROM/SKLearn.linearLARSCV

or

./run_framework_tests --re=framework/ROM/SKLearn.linearLARSCV

- Test Description:

- An example exercising supervised sklearn methods, specifically the linear_model—LarsCV model is tested here.

Note, all of the tests in SKLearn operate on a 2D input domain with the goal of fitting a paraboloid function. The input dimensions are of largely different scales and one dimension is off-centered from the origin to ensure that normalization is being handled correctly. Classifiers will use this same function to determine if a point is above 0.25 , and multitask methods will additionally fit an additive model $(\mathrm{x}+\mathrm{y})$.

- Original Author:

- maljdan 
- Creation date:

- 2016-04-28

- The classes tested in this test are:

- SupervisedLearning.SciKitLearn

- Since the creation of this test, the following main revisions have been performed:

1. revision info:

- author : talbpaul

- date : 2016-09-15

- description: other test updates

2. revision info:

- author : maljdan

- date : 2017-01-19

- description: Adding this test description.

\subsubsection{FRAMEWORK ROM SKLEARN.LINEARLASSO}

This test can be found at ". /raven/tests/framework/ROM/SKLearn/linearLasso. xml". This test can be called executing the following command:

./run_tests $--r e=f r a m e w o r k / R O M / S K L e a r n . l i n e a r L a s s o$

or

./run_framework_tests --re=framework/ROM/SKLearn.linearLasso

- Test Description:

- An example exercising supervised sklearn methods, specifically the linear_model—Lasso model is tested here.

Note, all of the tests in SKLearn operate on a 2D input domain with the goal of fitting a paraboloid function. The input dimensions are of largely different scales and one dimension is off-centered from the origin to ensure that normalization is being handled correctly. Classifiers will use this same function to determine if a point is above 0.25 , and multitask methods will additionally fit an additive model $(\mathrm{x}+\mathrm{y})$.

- Original Author: 
- maljdan

- Creation date:

- 2016-04-28

- The classes tested in this test are:

- SupervisedLearning.SciKitLearn

- Since the creation of this test, the following main revisions have been performed:

1. revision info:

- author : alfoa

- date : 2016-07-13

- description: removed renormalization

2. revision info:

- author : talbpaul

- date : 2016-09-15

- description: other test updates

3. revision info:

- author : maljdan

- date : 2017-01-19

- description: Adding this test description.

\subsubsection{FRAMEWORK ROM SKLEARN.LINEARLASSOCV}

This test can be found at ". / raven/tests/framework/ROM/SKLearn/linearLassoCV. xml". This test can be called executing the following command:

. /run_tests --re=framework/ROM/SKLearn.linearLassoCV

or

. /run_framework_tests --re=framework/ROM/SKLearn.linearLassoCV

- Test Description: 
- An example exercising supervised sklearn methods, specifically the linear_model_LassoCV model is tested here.

Note, all of the tests in SKLearn operate on a 2D input domain with the goal of fitting a paraboloid function. The input dimensions are of largely different scales and one dimension is off-centered from the origin to ensure that normalization is being handled correctly. Classifiers will use this same function to determine if a point is above 0.25 , and multitask methods will additionally fit an additive model $(\mathrm{x}+\mathrm{y})$.

- Original Author:

- maljdan

- Creation date:

- 2016-04-28

- The classes tested in this test are:

- SupervisedLearning.SciKitLearn

- Since the creation of this test, the following main revisions have been performed:

1. revision info:

- author : talbpaul

- date : 2016-09-15

- description: other test updates

2. revision info:

- author : maljdan

- date : 2017-01-19

- description: Adding this test description.

\subsubsection{FRAMEWORK ROM SKLEARN.LINEARLASSOLARS}

This test can be found at ". / raven/tests/framework/ROM/SKLearn/ I inearLassoLARS . xml". This test can be called executing the following command:

./run_tests --re=framework/ROM/SKLearn.linearLassoLARS

or

. /run_framework_tests --re=framework/ROM/SKLearn.linearLassoLARS 
- Test Description:

- An example exercising supervised sklearn methods, specifically the linear_model_LLassoLars model is tested here.

Note, all of the tests in SKLearn operate on a 2D input domain with the goal of fitting a paraboloid function. The input dimensions are of largely different scales and one dimension is off-centered from the origin to ensure that normalization is being handled correctly. Classifiers will use this same function to determine if a point is above 0.25 , and multitask methods will additionally fit an additive model $(x+y)$.

- Original Author:

- maljdan

- Creation date:

- 2016-04-28

- The classes tested in this test are:

- SupervisedLearning.SciKitLearn

- Since the creation of this test, the following main revisions have been performed:

1. revision info:

- author : talbpaul

- date : 2016-09-15

- description: other test updates

2. revision info:

- author : maljdan

- date : 2017-01-19

- description: Adding this test description.

\subsubsection{FRAMEWORK ROM SKLEARN.LINEARLASSOLARSCV}

This test can be found at ". / raven/tests/framework/ROM/SKLearn/ I inearLassoLARSCV. xml". This test can be called executing the following command:

./run_tests --re=framework/ROM/SKLearn.linearLassoLARSCV

or

. /run_framework_tests --re=framework/ROM/SKLearn.linearLassoLARSqV 
- Test Description:

- An example exercising supervised sklearn methods, specifically the linear_model—LassoLarsCV model is tested here.

Note, all of the tests in SKLearn operate on a 2D input domain with the goal of fitting a paraboloid function. The input dimensions are of largely different scales and one dimension is off-centered from the origin to ensure that normalization is being handled correctly. Classifiers will use this same function to determine if a point is above 0.25 , and multitask methods will additionally fit an additive model $(x+y)$.

- Original Author:

- maljdan

- Creation date:

$-2016-04-28$

- The classes tested in this test are:

- SupervisedLearning.SciKitLearn

- Since the creation of this test, the following main revisions have been performed:

1. revision info:

- author: talbpaul

- date : 2016-09-15

- description: other test updates

2. revision info:

- author : maljdan

- date : 2017-01-19

- description: Adding this test description.

\subsubsection{FRAMEWORK ROM SKLEARN.LINEARLASSOLARSIC}

This test can be found at ". / raven/tests/framework/ROM/SKLearn/I inearLassoLARS IC. xml". This test can be called executing the following command:

./run_tests --re=framework/ROM/SKLearn.linearLassoLARSIC

or

. /run_framework_tests --re=framework/ROM/SKLearn.linearLassoLARSIC 
- Test Description:

- An example exercising supervised sklearn methods, specifically the linear_model_LassoLarsIC model is tested here.

Note, all of the tests in SKLearn operate on a 2D input domain with the goal of fitting a paraboloid function. The input dimensions are of largely different scales and one dimension is off-centered from the origin to ensure that normalization is being handled correctly. Classifiers will use this same function to determine if a point is above 0.25 , and multitask methods will additionally fit an additive model $(\mathrm{x}+\mathrm{y})$.

- Original Author:

- maljdan

- Creation date:

$-2016-04-28$

- The classes tested in this test are:

- SupervisedLearning.SciKitLearn

- Since the creation of this test, the following main revisions have been performed:

1. revision info:

- author : talbpaul

- date : 2016-09-15

- description: other test updates

2. revision info:

- author : maljdan

- date : 2017-01-19

- description: Adding this test description.

\subsubsection{FRAMEWORK ROM SKLEARN.LINEARREGRESSION}

This test can be found at ". / raven/tests/framework/ROM/SKLearn/linearRegression. xml". This test can be called executing the following command:

./run_tests --re=framework/ROM/SKLearn.linearRegression

or

./run_framework_tests --re=framework/ROM/SKLearn.linearRegression 
- Test Description:

- An example exercising supervised sklearn methods, specifically the linear_model—LinearRegression model is tested here.

Note, all of the tests in SKLearn operate on a 2D input domain with the goal of fitting a paraboloid function. The input dimensions are of largely different scales and one dimension is off-centered from the origin to ensure that normalization is being handled correctly. Classifiers will use this same function to determine if a point is above 0.25 , and multitask methods will additionally fit an additive model $(\mathrm{x}+\mathrm{y})$.

- Original Author:

- maljdan

- Creation date:

- 2016-04-28

- The classes tested in this test are:

- SupervisedLearning.SciKitLearn

- Since the creation of this test, the following main revisions have been performed:

1. revision info:

- author : alfoa

- date : 2016-07-13

- description: removed renormalization

2. revision info:

- author : talbpaul

- date : 2016-09-15

- description: other test updates

3. revision info:

- author : maljdan

- date : 2017-01-19

- description: Adding this test description.

\subsubsection{FRAMEWORK ROM SKLEARN.LINEARLOGISTICREGRESSION}

This test can be found at “. / raven/tests/framework/ROM/SKLearn/linearLogisticRegressic xml". This test can be called executing the following command: 
./run_tests --re=framework/ROM/SKLearn.linearLogisticRegression

or

./run_framework_tests --re=framework/ROM/SKLearn.linearLogisticRegression

- Test Description:

- An example exercising supervised sklearn methods, specifically the linear_model—LogisticRegression model is tested here.

Note, all of the tests in SKLearn operate on a 2D input domain with the goal of fitting a paraboloid function. The input dimensions are of largely different scales and one dimension is off-centere from the origin to ensure that normalization is being handled correctly. Classifiers will use this same function to determine if a point is above 0.25 , and multitask methods will additionally fit an additive model $(\mathrm{x}+\mathrm{y})$.

- Original Author:

- maljdan

- Creation date:

- 2016-04-28

- The classes tested in this test are:

- SupervisedLearning.SciKitLearn

- Since the creation of this test, the following main revisions have been performed:

1. revision info:

- author : talbpaul

- date : 2016-09-15

- description: other test updates

2. revision info:

- author : maljdan

- date : 2017-01-19

- description: Adding this test description. 


\subsubsection{FRAMEWORK ROM SKLEARN.LINEARMULTITASKLASSO}

This test can be found at ". /raven/tests/framework/ROM/SKLearn/linearMultiTaskLasso. xml". This test can be called executing the following command:

./run_tests --re=framework/ROM/SKLearn.linearMultiTaskLasso

or

. /run_framework_tests --re=framework/ROM/SKLearn.linearMultiTasktasso

- Test Description:

- An example exercising supervised sklearn methods, specifically the linear_model—MultiTaskLasso model is tested here.

Note, all of the tests in SKLearn operate on a 2D input domain with the goal of fitting a paraboloid function. The input dimensions are of largely different scales and one dimension is off-centered from the origin to ensure that normalization is being handled correctly. Classifiers will use this same function to determine if a point is above 0.25 , and multitask methods will additionally fit an additive model $(\mathrm{x}+\mathrm{y})$.

- Original Author:

- maljdan

- Creation date:

- 2016-04-28

- The classes tested in this test are:

- SupervisedLearning.SciKitLearn

- Since the creation of this test, the following main revisions have been performed:

1. revision info:

- author : alfoa

- date : 2016-07-13

- description: removed renormalization

2. revision info:

- author : talbpaul

- date : 2016-09-15

- description: other test updates 
3. revision info:

- author : maljdan

- date : 2017-01-19

- description: Adding this test description.

\subsubsection{FRAMEWORK ROM SKLEARN.LINEARMULTITASKELASTICNET}

This test can be found at ". / raven/tests/framework/ROM/SKLearn/linearMultiTaskElasticl xml". This test can be called executing the following command:

. Irun_tests --re=framework/ROM/SKLearn.linearMultiTaskElasticNet

or

./run_framework_tests --re=framework/ROM/SKLearn.linearMultiTaskElasticNet

- Test Description:

- An example exercising supervised sklearn methods, specifically the linear_model—MultiTaskElasticN model is tested here.

Note, all of the tests in SKLearn operate on a 2D input domain with the goal of fitting a paraboloid function. The input dimensions are of largely different scales and one dimension is off-centered from the origin to ensure that normalization is being handled correctly. Classifiers will use this same function to determine if a point is above 0.25 , and multitask methods will additionally fit an additive model $(\mathrm{x}+\mathrm{y})$.

- Original Author:

- maljdan

- Creation date:

- 2016-04-28

- The classes tested in this test are:

- SupervisedLearning.SciKitLearn

- Since the creation of this test, the following main revisions have been performed:

1. revision info:

- author : maljdan

- date : 2016-04-28 
- description: Adding gold files and fixing MultiTaskElasticNet

2. revision info:

- author : alfoa

- date : 2016-07-13

- description: removed renormalization

3. revision info:

- author : talbpaul

- date : 2016-09-15

- description: other test updates

4. revision info:

- author : maljdan

- date : 2017-01-19

- description: Adding this test description.

\subsubsection{FRAMEWORK ROM SKLEARN.LINEAROMP}

This test can be found at "./raven/tests/framework/ROM/SKLearn/linearOMP . xml". This test can be called executing the following command:

. /run_tests --re=framework/ROM/SKLearn.linearOMP

or

. /run_framework_tests --re=framework/ROM/SKLearn.linearOMP

- Test Description:

- An example exercising supervised sklearn methods, specifically the linear_model—OrthogonalMatchir model is tested here.

Note, all of the tests in SKLearn operate on a 2D input domain with the goal of fitting a paraboloid function. The input dimensions are of largely different scales and one dimension is off-centered from the origin to ensure that normalization is being handled correctly. Classifiers will use this same function to determine if a point is above 0.25 , and multitask methods will additionally fit an additive model $(\mathrm{x}+\mathrm{y})$.

- Original Author:

- maljdan 
- Creation date:

- 2016-04-28

- The classes tested in this test are:

- SupervisedLearning.SciKitLearn

- Since the creation of this test, the following main revisions have been performed:

1. revision info:

- author : talbpaul

- date : 2016-09-15

- description: other test updates

2. revision info:

- author : maljdan

- date : 2017-01-19

- description: Adding this test description.

\subsubsection{FRAMEWORK ROM SKLEARN.LINEAROMPCV}

This test can be found at "./raven/tests/framework/ROM/SKLearn/linearOMPCV. xml". This test can be called executing the following command:

./run_tests --re=framework/ROM/SKLearn.linearOMPCV

or

./run_framework_tests --re=framework/ROM/SKLearn.linearOMPCV

- Test Description:

- An example exercising supervised sklearn methods, specifically the linear_model—OrthogonalMatchir model is tested here.

Note, all of the tests in SKLearn operate on a 2D input domain with the goal of fitting a paraboloid function. The input dimensions are of largely different scales and one dimension is off-centered from the origin to ensure that normalization is being handled correctly. Classifiers will use this same function to determine if a point is above 0.25 , and multitask methods will additionally fit an additive model $(\mathrm{x}+\mathrm{y})$.

- Original Author: 
- maljdan

- Creation date:

- 2016-04-28

- The classes tested in this test are:

- SupervisedLearning.SciKitLearn

- Since the creation of this test, the following main revisions have been performed:

1. revision info:

- author : talbpaul

- date : 2016-09-15

- description: other test updates

2. revision info:

- author : maljdan

- date : 2017-01-19

- description: Adding this test description.

\subsubsection{FRAMEWORK ROM SKLEARN.LINEARPERCEPTRON}

This test can be found at ". / raven/tests / framework/ROM/SKLearn/linearPerceptron. xml". This test can be called executing the following command:

./run_tests --re=framework/ROM/SKLearn.linearPerceptron

Or

. /run_framework_tests --re=framework/ROM/SKLearn.linearPerceptron

- Test Description:

- An example exercising supervised sklearn methods, specifically the linear_model-Perceptron model is tested here.

Note, all of the tests in SKLearn operate on a 2D input domain with the goal of fitting a paraboloid function. The input dimensions are of largely different scales and one dimension is off-centered from the origin to ensure that normalization is being handled correctly. Classifiers will use this same function to determine if a point is above 0.25 , and multitask methods will additionally fit an additive model $(\mathrm{x}+\mathrm{y})$. 
- Original Author:

- maljdan

- Creation date:

- 2016-04-28

- The classes tested in this test are:

- SupervisedLearning.SciKitLearn

- Since the creation of this test, the following main revisions have been performed:

1. revision info:

- author : talbpaul

- date : 2016-09-15

- description: other test updates

2. revision info:

- author : maljdan

- date : 2017-01-19

- description: Adding this test description.

\subsubsection{FRAMEWORK ROM SKLEARN.LINEARRIDGER}

This test can be found at ". / raven/tests/framework/ROM/SKLearn/linearRidgeR. xml". This test can be called executing the following command:

./run_tests --re=framework/ROM/SKLearn.linearRidgeR

or

./run_framework_tests --re=framework/ROM/SKLearn.linearRidgeR

- Test Description:

- An example exercising supervised sklearn methods, specifically the linear_model—Ridge model is tested here.

Note, all of the tests in SKLearn operate on a 2D input domain with the goal of fitting a paraboloid function. The input dimensions are of largely different scales and one dimension is off-centered from the origin to ensure that normalization is being handled correctly. Classifiers will use this same function to determine if a point is above 0.25 , and multitask methods will additionally fit an additive model $(\mathrm{x}+\mathrm{y})$. 
- Original Author:

- maljdan

- Creation date:

- 2016-04-28

- The classes tested in this test are:

- SupervisedLearning.SciKitLearn

- Since the creation of this test, the following main revisions have been performed:

1. revision info:

- author : talbpaul

- date : 2016-09-15

- description: other test updates

2. revision info:

- author : maljdan

- date : 2017-01-19

- description: Adding this test description.

\subsubsection{FRAMEWORK ROM SKLEARN.LINEARRIDGERCV}

This test can be found at ". / raven/tests / framework/ROM/SKLearn/ linearRidgeRCV. xml". This test can be called executing the following command:

./run_tests --re=framework/ROM/SKLearn.linearRidgeRCV

or

./run_framework_tests --re=framework/ROM/SKLearn.linearRidgeRCV

- Test Description:

- An example exercising supervised sklearn methods, specifically the linear_model—RidgeCV model is tested here.

Note, all of the tests in SKLearn operate on a 2D input domain with the goal of fitting a paraboloid function. The input dimensions are of largely different scales and one dimension is off-centered from the origin to ensure that normalization is being handled correctly. Classifiers will use this same function to determine if a point is above 0.25 , and multitask methods will additionally fit an additive model $(\mathrm{x}+\mathrm{y})$. 
- Original Author:

- maljdan

- Creation date:

- 2016-04-28

- The classes tested in this test are:

- SupervisedLearning.SciKitLearn

- Since the creation of this test, the following main revisions have been performed:

1. revision info:

- author : alfoa

- date : 2016-07-13

- description: removed renormalization

2. revision info:

- author : talbpaul

- date : 2016-09-15

- description: other test updates

3. revision info:

- author : maljdan

- date : 2017-01-19

- description: Adding this test description.

\subsubsection{FRAMEWORK ROM SKLEARN.LINEARPAR}

This test can be found at "./raven/tests/framework/ROM/SKLearn/linearPAR. xml". This test can be called executing the following command:

./run_tests --re=framework/ROM/SKLearn.linearPAR

or

. /run_framework_tests --re=framework/ROM/SKLearn.linearPAR

- Test Description: 
- An example exercising supervised sklearn methods, specifically the linear_model—PassiveAggressivel model is tested here.

Note, all of the tests in SKLearn operate on a 2D input domain with the goal of fitting a paraboloid function. The input dimensions are of largely different scales and one dimension is off-centered from the origin to ensure that normalization is being handled correctly. Classifiers will use this same function to determine if a point is above 0.25 , and multitask methods will additionally fit an additive model $(\mathrm{x}+\mathrm{y})$.

- Original Author:

- maljdan

- Creation date:

- 2016-04-28

- The classes tested in this test are:

- SupervisedLearning.SciKitLearn

- Since the creation of this test, the following main revisions have been performed:

1. revision info:

- author : maljdan

- date : 2016-05-02

- description: Adding a fixed random_state value to a few of the ROM tests to ensure reproducibility.

2. revision info:

- author : talbpaul

- date : 2016-09-15

- description: other test updates

3. revision info:

- author : maljdan

- date : 2017-01-19

- description: Adding this test description.

\subsubsection{FRAMEWORK ROM SKLEARN.LINEARSGDR}

This test can be found at "./raven/tests/framework/ROM/SKLearn/linearSGDR. xml". This test can be called executing the following command:

./run_tests --re=framework/ROM/SKLearn.linearSGDR 
or

./run_framework_tests --re=framework/ROM/SKLearn.linearSGDR

- Test Description:

- An example exercising supervised sklearn methods, specifically the linear_model—SGDRegressor model is tested here.

Note, all of the tests in SKLearn operate on a 2D input domain with the goal of fitting a paraboloid function. The input dimensions are of largely different scales and one dimension is off-centered from the origin to ensure that normalization is being handled correctly. Classifiers will use this same function to determine if a point is above 0.25 , and multitask methods will additionally fit an additive model $(\mathrm{x}+\mathrm{y})$.

- Original Author:

- maljdan

- Creation date:

- 2016-04-28

- The classes tested in this test are:

- SupervisedLearning.SciKitLearn

- Since the creation of this test, the following main revisions have been performed:

1. revision info:

- author : maljdan

- date : 2016-05-02

- description: Adding a fixed random_state value to a few of the ROM tests to ensure reproducibility.

2. revision info:

- author : talbpaul

- date : 2016-09-15

- description: other test updates

3. revision info:

- author : maljdan

- date : 2017-01-19

- description: Adding this test description. 


\subsubsection{FRAMEWORK ROM SKLEARN.NEURAL NETWORK}

This test can be found at ". / raven/tests/framework/ROM/SKLearn/mlpRegressor. xml". This test can be called executing the following command:

. Irun_tests --re=framework/ROM/SKLearn.neural_network

or

./run_framework_tests --re=framework/ROM/SKLearn.neural_network

- Test Description:

- An example exercising supervised sklearn methods, specifically the neural_network-MLPRegressor model is tested here.

Note, all of the tests in SKLearn operate on a 2D input domain with the goal of fitting a paraboloid function. The input dimensions are of largely different scales and one dimension is off-centered from the origin to ensure that normalization is being handled correctly. Classifiers will use this same function to determine if a point is above 0.25 , and multitask methods will additionally fit an additive model $(\mathrm{x}+\mathrm{y})$.

- Original Author:

- wangc

- Creation date:

$-2017-10-25$

- The classes tested in this test are:

- SupervisedLearning.SciKitLearn

\subsubsection{FRAMEWORK ROM TENSORSPLINE.GRIDSPLINE}

This test can be found at ". /raven/tests/framework/ROM/TensorSpline/tensor_ spline_grid.xml". This test can be called executing the following command:

./run_tests --re=framework/ROM/TensorSpline.GridSpline

or

./run_framework_tests --re=framework/ROM/TensorSpline.GridSpline 
- Test Description:

- This case is aimed to test the functionalities of the Tensor Spline Surrogate Model. It trains the Tensor Spline on a grid generated sampling the "projectile" model and inquires it on a refined Grid.

- Original Author:

- alfoa

- Creation date:

- 2018-04-12

- The classes tested in this test are:

- SupervisedLearning.NDSpline

- Since the creation of this test, the following main revisions have been performed:

1. revision info:

- author : alfoa

- date : 2018-04-12

- description: Created test and added description

\subsubsection{FRAMEWORK ROM TENSORSPLINE.NOGRIDSPLINE}

This test can be found at ". / raven/tests/framework/ROM/TensorSpline/tensor_ spline_no_grid_mc.xml”. This test can be called executing the following command:

./run_tests --re=framework/ROM/TensorSpline.NoGridSpline

or

./run_framework_tests --re=framework/ROM/TensorSpline.NoGridSpline

- Test Description:

- This case is aimed to test the functionalities of the Tensor Spline Surrogate Model. It trains the Tensor Spline on a MC generated sampling the "projectile" model and inquires it on a Grid. This tests the capability to accept not cartesian grids as training sets. It is important to notice that this tests demonstrates that the mechanics work but does not suggest to train the NDSpline ROM on not a cartesian grid. 
- Original Author:

- alfoa

- Creation date:

- 2018-04-12

- The classes tested in this test are:

- SupervisedLearning.NDSpline

- Since the creation of this test, the following main revisions have been performed:

1. revision info:

- author : alfoa

- date : 2018-04-12

- description: Created test and added description

\subsubsection{FRAMEWORK SAMPLERS ROM SOBOL STATICSOBOLROMSMOLYAK}

This test can be found at". / raven/tests/framework/ROM/Sobol/test_sobol_rom . xml". This test can be called executing the following command:

. Irun_tests --re=framework/Samplers/ROM/Sobol/staticSobolRomSmolyak

or

./run_framework_tests --re=framework/Samplers/ROM/Sobol/staticSoldolRomSmol

- Test Description:

- This test checks the operation of the sampler and ROM together.

- Original Author:

- talbpaul

- Creation date:

- 2016-03-03

- The classes tested in this test are:

- Samplers.Sobol,SupervisedLearning.HDMRRom 
- Since the creation of this test, the following main revisions have been performed:

1. revision info:

- author : talbpaul

- date : 2018-12-20

- description: moved ROM metadata writing from direct-to-file to direct-to-dataobject

\subsubsection{FRAMEWORK SAMPLERS ROM SOBOL STATICSOBOLROMSMOLYAK}

This test can be found at ". / raven/tests / framework/ROM/Sobol/test_sobol_tensor. xml". This test can be called executing the following command:

. Irun_tests --re=framework/Samplers/ROM/Sobol/staticSobolRomSmolyak

or

. Irun_framework_tests --re=framework/Samplers/ROM/Sobol/staticSoldolRomSmol

- Test Description:

- This test checks the operation of the sampler and model, using the tensor sparse grid.

- Original Author:

- talbpaul

- Creation date:

- 2015-07-07

- The classes tested in this test are:

- Samplers.Sobol,SupervisedLearning.HDMRRom

- Since the creation of this test, the following main revisions have been performed:

1. revision info:

- author: talbpaul

- date : 2018-12-20

- description: moved ROM metadata writing from direct-to-file to direct-to-dataobject 


\subsubsection{FRAMEWORK SAMPLERS ROM SOBOL ADAPTIVESOBOLMAXRUNS}

This test can be found at ". /raven/tests/framework/ROM/Sobol/test_adapt_sobol_ maxruns.xml". This test can be called executing the following command:

./run_tests --re=framework/Samplers/ROM/Sobol/AdaptiveSobolMaxRuns

or

./run_framework_tests --re=framework/Samplers/ROM/Sobol/Adaptive\$obolMaxRu

- Test Description:

- This tests using the AdaptiveSobol sampler to construct HDMRRom ROMs, but restricted by a specified number of samples (maxRuns in the sampler).

- Original Author:

- talbpaul

- Creation date:

- 2016-02-08

- The classes tested in this test are:

- Samplers.AdaptiveSobol,SupervisedLearning.HDMRRom

- Since the creation of this test, the following main revisions have been performed:

1. revision info:

- author : talbpaul

- date : 2018-12-20

- description: moved ROM metadata writing from direct-to-file to direct-to-dataobject

\subsubsection{FRAMEWORK SAMPLERS ROM SOBOL ADAPTIVESOBOLPARALLEL}

This test can be found at “./raven/tests/framework/ROM/Sobol/test_adapt_sobol_ parallel.xml". This test can be called executing the following command:

./run_tests --re=framework/Samplers/ROM/Sobol/AdaptiveSobolparallel

or 
./run_framework_tests --re=framework/Samplers/ROM/Sobol/Adaptive\$obolparal.

- Test Description:

- This tests using the AdaptiveSobol sampler to construct HDMRRom ROMs, in parallel.

- Original Author:

- talbpaul

- Creation date:

- 2016-02-08

- The classes tested in this test are:

- Samplers.AdaptiveSobol,SupervisedLearning.HDMRRom

- Since the creation of this test, the following main revisions have been performed:

1. revision info:

- author : talbpaul

- date : 2018-12-20

- description: moved ROM metadata writing from direct-to-file to direct-to-dataobject

\subsubsection{FRAMEWORK SAMPLERS ROM SOBOL TIMEDEPENDENT}

This test can be found at "./raven/tests/framework/ROM/Sobol/test_time_dep_ sobol.xml". This test can be called executing the following command:

./run_tests --re=framework/Samplers/ROM/Sobol/TimeDependent

or

./run_framework_tests --re=framework/Samplers/ROM/Sobol/TimeDependent

- Test Description:

- This test checks the construction of a time-dependent HDMRRom.

- Original Author: 
- talbpaul

- Creation date:

- 2016-03-09

- The classes tested in this test are:

- SupervisedLearning.HDMRRom

- Since the creation of this test, the following main revisions have been performed:

1. revision info:

- author: wangc

- date : 2018-08-09

- description: Add pivotParameter for time dependent ROM

2. revision info:

- author : talbpaul

- date : 2018-12-20

- description: moved ROM metadata writing from direct-to-file to direct-to-dataobject

\subsubsection{FRAMEWORK HYBRIDMODEL.HYBRIDMODEL}

This test can be found at ". / raven/tests / framework/hybridModel/test_hybrid_ model.xml". This test can be called executing the following command:

./run_tests --re=framework/hybridModel.hybridModel

Or

./run_framework_tests --re=framework/hybridModel.hybridModel

- Test Description:

- Example of usage of the Hybrid Model capability in RAVEN This test is intended to check the functionality of Hybrid Model.

- Original Author:

- wangc

- Creation date: 
- 2017-09-13

- The classes tested in this test are:

- Models.HybridModel, Models.ExternalModel, Models.ROM

- Since the creation of this test, the following main revisions have been performed:

1. revision info:

- author: wangc

- date : 2019-07-09

- description: Move cross validation assemble from HybridModel to ROM

\subsubsection{FRAMEWORK HYBRIDMODEL.ROMTRAIN}

This test can be found at ". /raven/tests/framework/hybridModel/test_train_ rom. xml". This test can be called executing the following command:

./run_tests --re=framework/hybridModel.romTrain

or

. /run_framework_tests --re=framework/hybridModel.romTrain

- Test Description:

- This test is intended to check the rom train capability of the Hybrid Model.

- Original Author:

- wangc

- Creation date:

- 2017-09-20

- The classes tested in this test are:

- Models.HybridModel, Models.ExternalModel, Models.ROM

- Since the creation of this test, the following main revisions have been performed:

1. revision info:

- author: wangc

- date : 2019-07-09

- description: Move cross validation asssemble from HybridModel to ROM 


\subsubsection{FRAMEWORK HYBRIDMODEL.RUNCODE}

This test can be found at "./raven/tests/framework/hybridModel/test_code. xml". This test can be called executing the following command:

. /run_tests --re=framework/hybridModel.runcode

or

. Irun_framework_tests --re=framework/hybridModel.runCode

- Test Description:

- This test is aimed to test the HybridModel system with the generic code system.

- Original Author:

- wangc

- Creation date:

- 2017-09-21

- The classes tested in this test are:

- Models.HybridModel, Models.Code.GenericCode

- Since the creation of this test, the following main revisions have been performed:

1. revision info:

- author: wangc

- date : 2019-07-09

- description: Move cross validation asssemble from HybridModel to ROM

\subsubsection{FRAMEWORK HYBRIDMODEL.ROM ROM}

This test can be found at ". / raven/tests / framework/hybridModel/test_rom_rom . xml". This test can be called executing the following command:

./run_tests --re=framework/hybridModel.rom_rom

or

./run_framework_tests --re=framework/hybridModel.rom_rom 
- Test Description:

- Example of usage of the Hybrid Model capability in RAVEN This test is intended to check the functionality of Hybrid Model. Use ROM to train ROM

- Original Author:

- wangc

- Creation date:

- 2017-09-13

- The classes tested in this test are:

- Models.HybridModel, Models.ROM

- Since the creation of this test, the following main revisions have been performed:

1. revision info:

- author : alfoa

- date : 2017-12-20

- description: replaced median absolute error with average absolute error score

2. revision info:

- author: wangc

- date : 2019-07-09

- description: Move cross validation asssemble from HybridModel to ROM

\subsubsection{FRAMEWORK HYBRIDMODEL.LOGICALMODEL}

This test can be found at “. / raven/tests / framework/hybridModel/test_logical_ model .xml". This test can be called executing the following command:

./run_tests --re=framework/hybridModel.logicalModel

or

./run_framework_tests --re=framework/hybridModel.logicalModel

- Test Description: 
- Example of usage of the Logical Model capability in RAVEN This test is intended to check the functionality of Logical Model with three external models "sum", "minus" and "multiply". A control function "control" is required to select the external model to run based on the values of input variables $\mathrm{x}$ and $\mathrm{y}$. If $\mathrm{x} i \vec{i}$ and $\mathrm{y} i, 1$, the model "sum" will be selected. if $x i, 0$ and $y$ is less than or equal 1 , the model "multiply" will be selected. In other conditions, the model "minus" will be selected.

- Original Author:

- wangc

- Creation date:

- 2020-03-31

- The classes tested in this test are:

- Models.LogicalModel

\subsubsection{FRAMEWORK HYBRIDMODEL.LOGICALCODE}

This test can be found at ". / raven/tests / framework/hybridModel/test_logical_ code.xml". This test can be called executing the following command:

./run_tests --re=framework/hybridModel.logicalcode

Or

./run_framework_tests --re=framework/hybridModel.logicalcode

- Test Description:

- Example of usage of the Logical Model capability in RAVEN This test is intended to check the functionality of Logical Model with the generic code system. Two simple python codes are used in this test. One is an exponential function of input variables $\mathrm{x}$ and $\mathrm{y}$, the other one is a polynomial function of input variables $\mathrm{x}$ and $\mathrm{y}$. A control function "control" utilizes the input values of $\mathrm{x}$ and $\mathrm{y}$ to determine which code to run. If $x i, 0.5$ and $y i 1.5$, the polynomial model 'poly' will be executed, otherwise the exponential model 'exp' will be executed.

- Original Author:

- wangc 
- Creation date:

- 2020-05-07

- The classes tested in this test are:

- Models.LogicalModel

\subsubsection{FRAMEWORK HYBRIDMODEL.LOGICALROM}

This test can be found at ". / raven/tests/framework/hybridModel/test_logical_ rom.xml". This test can be called executing the following command:

./run_tests --re=framework/hybridModel.logicalRom

or

./run_framework_tests --re=framework/hybridModel.logicalRom

- Test Description:

- Example of usage of the Logical Model capability in RAVEN This test is intended to check the functionality of Logical Model with ROMs. A control function "control" is required to select the right model to run based on the values of input variables leftTemperature and rightTemperatureDist. If leftTemperature in $(860,1340)$ and rightTemperatureDist in $(740,1460)$, the ROM "svr" will be selected, else the model "thermalConductivityComputation" will be selected.

- Original Author:

- wangc

- Creation date:

$-2020-05-11$

- The classes tested in this test are:

- Models.LogicalModel 


\subsubsection{FRAMEWORK PCASAMPLER PCAINDEX}

This test can be found at “./raven/tests/framework/pcaSampler/test_pca_index. xml". This test can be called executing the following command:

. /run_tests --re=framework/pcaSampler/pcaIndex

or

. /run_framework_tests --re=framework/pcaSampler/pcaIndex

- Test Description:

- Tests use of the PCA method to sample in surrogate input space.

- Original Author:

- wangc

- Creation date:

- 2016-02-16

- The classes tested in this test are:

- Distributions.MultivariateNormal,Samplers

\subsubsection{FRAMEWORK PCASAMPLER MULTIPCA}

This test can be found at "./raven/tests/framework/pcaSampler/test_multi_ pca.xml". This test can be called executing the following command:

./run_tests --re=framework/pcasampler/multiPCA

or

./run_framework_tests --re=framework/pcasampler/multiPCA

- Test Description:

- Tests use of multiple PCA surrogates in one sampling scheme.

- Original Author: 
- wangc

- Creation date:

- 2016-02-16

- The classes tested in this test are:

- Distributions.MultivariateNormal,Samplers.MonteCarlo

\subsubsection{FRAMEWORK PCASAMPLER GRIDPCA}

This test can be found at “. / raven/tests / framework/pcaSampler/test_pca_index_ grid.xml". This test can be called executing the following command:

. /run_tests --re=framework/pcaSampler/gridPCA

or

./run_framework_tests --re=framework/pcaSampler/gridPCA

- Test Description:

- Tests use of the PCA method to sample in gridded surrogate input space.

- Original Author:

- wangc

- Creation date:

- 2016-02-16

- The classes tested in this test are:

- Distributions.MultivariateNormal,Samplers.Grid

\subsubsection{FRAMEWORK SAMPLERS.DATAOBJECTCONSTANTS}

This test can be found at ". / raven/tests / framework/Samplers/dataobject_constants. xml". This test can be called executing the following command:

. /run_tests --re=framework/Samplers.DataObjectConstants 
Or

./run_framework_tests --re=framework/Samplers.DataObjectConstant

- Test Description:

- Tests using a DataObject external to a Step as a source of constants for a Sampler. The models are very simple, so to regold, assure the correct values pass through for the constants and final result is obtained according to the models.

- Original Author:

- talbpaul

- Creation date:

- 2018-11-14

- The classes tested in this test are:

- Sampler

\subsubsection{FRAMEWORK SAMPLERS ENSEMBLEDSAMPLER.ENSEMBLESAMPLERDUMMY}

This test can be found at ". / raven/tests / framework/Samplers /EnsembledSampler/ test_EnsembleSamplerDummy . xml". This test can be called executing the following command:

./run_tests --re=framework/Samplers/EnsembledSampler.EnsembleSamplerDummy

or

./run_framework_tests --re=framework/Samplers/EnsembledSampler.EnsembleSam

- Test Description:

- An example of using the EnsembleForward sampler aimed to combine multiple forward sampling tecniques (MonteCarlo, Grid, Stratified, etc.). This test uses a Dummy Model in order to check that the sampled values are correcly passed to the Model entity.

- Original Author:

- alfoa 
- Creation date:

- 2016-04-20

- The classes tested in this test are:

- Samplers.EnsembleForward, Models.Dummy

- Since the creation of this test, the following main revisions have been performed:

1. revision info:

- author : alfoa

- date : 2016-04-20

- description: Finalized EnsembleSampler

2. revision info:

- author: alfoa

- date : 2016-05-25

- description: Finalized merge request. Closes \#559

3. revision info:

- author : talbpaul

- date : 2016-09-15

- description: Test updates

4. revision info:

- author : alfoa

- date : 2017-01-21

- description: Adding this test description.

5. revision info:

- author : talbpaul

- date : 2017-09-27

- description: Added seed to samplerInit for testing

6. revision info:

- author : talbpaul

- date : 2018-01-02

- description: added input data object to prevent same-input-as-output error 


\subsubsection{FRAMEWORK SAMPLERS ENSEMBLEDSAMPLER.ENSEMBLESAMPLEREXTMODEL}

This test can be found at ". / raven/tests / framework/Samplers/EnsembledSampler/ test_EnsembleSamplerExtModel.xml". This test can be called executing the following command:

./run_tests --re=framework/Samplers/EnsembledSampler.EnsembleSamplerExtMod

or

./run_framework_tests --re=framework/Samplers/EnsembledSampler.EnsembleSam

- Test Description:

- An example of using the EnsembleForward sampler aimed to combine multiple forward sampling tecniques (MonteCarlo, Grid, Stratified, etc.). This test uses an ExternalModel in order to testify that this sampling strategy can work with RAVEN internal developed Models.

- Original Author:

- alfoa

- Creation date:

- 2016-04-20

- The classes tested in this test are:

- Samplers.EnsembleForward, Models.ExternalModel

- Since the creation of this test, the following main revisions have been performed:

1. revision info:

- author : alfoa

- date : 2016-04-20

- description: Finalized EnsembleForward Sampler

2. revision info:

- author : talbpaul

- date : 2016-09-15

- description: Test updates

3. revision info:

- author : alfoa

- date : 2017-01-21

- description: Adding this test description. 


\subsubsection{FRAMEWORK SAMPLERS ENSEMBLEDSAMPLER.ENSEMBLESAMPLERDUMMY}

This test can be found at ". / raven/tests / framework/Samplers/EnsembledSampler/ test_EnsembleSamplerFunctions.xml”. This test can be called executing the following command:

./run_tests --re=framework/Samplers/EnsembledSampler.EnsembleSamplerDummy

or

./run_framework_tests --re=framework/Samplers/EnsembledSampler.EnsembleSam

- Test Description:

- This test uses a Dummy Model in order to check that the values generated by functions in the ensemble sampler are correctly passed to the Model entity.

- Original Author:

- mandd

- Creation date:

$-2017-05-25$

- The classes tested in this test are:

- Samplers.EnsembleForward, Models.Dummy

- Since the creation of this test, the following main revisions have been performed:

1. revision info:

- author : talbpaul

- date : 2018-01-02

- description: added input data object to prevent same-input-as-output error

2. revision info:

- author : alfoa

- date : 2019-03-07

- description: Modified syntax in Functions as for issue \#934 


\subsubsection{FRAMEWORK SAMPLERS ENSEMBLEDSAMPLER.ENSEMBLESAMPLERCONSTANTS}

This test can be found at ". / raven/tests / framework/Samplers/EnsembledSampler/ test_EnsembleSamplerConstants.xml". This test can be called executing the following command:

./run_tests --re=framework/Samplers/EnsembledSampler.EnsembleSamplerConsta

or

./run_framework_tests --re=framework/Samplers/EnsembledSampler.EnsembleSam

- Test Description:

- Tests the use of constants in the EnsembleForward sampler.

- Original Author:

- talbpaul

- Creation date:

- 2017-08-30

- The classes tested in this test are:

- Samplers.EnsembleForward

- Since the creation of this test, the following main revisions have been performed:

1. revision info:

- author : talbpaul

- date : 2018-01-02

- description: added input data object to prevent same-input-as-output error

\subsubsection{FRAMEWORK SAMPLERS VECTORSAMPLES MC}

This test can be found at "./raven/tests/framework/Samplers/VectorSamples/ mc.xml". This test can be called executing the following command:

./run_tests --re=framework/Samplers/VectorSamples/MC

or 
./run_framework_tests --re=framework/Samplers/VectorSamples/MC

- Test Description:

- Tests sampling a vector variable.

- Original Author:

- talbpaul

- Creation date:

- 2018-03-21

- The classes tested in this test are:

- Samplers.MonteCarlo

\subsubsection{FRAMEWORK SAMPLERS ADAPTIVEBATCH.GREEDYDISTANCE}

This test can be found at "./raven/tests/framework/Samplers/AdaptiveBatch/ test_greedy_d.xml". This test can be called executing the following command:

. /run_tests --re=framework/Samplers/AdaptiveBatch.GreedyDistance

or

./run_framework_tests --re=framework/Samplers/AdaptiveBatch.GreedyDistance

- Test Description:

- An example of using the limit surface post-processor using the greedy selection algorithm with the distance scoring function. That is, the point to be selected will be furthest from all existing points that have been or are being evaluated. Note, all of the tests in AdaptiveBatch operate on a 2D input domain with the goal of extracting a circular limit surface with radius 0.5 and centered at the origin, $(0,0)$.

- Original Author:

- maljdan

- Creation date: 
$-2015-11-03$

- The classes tested in this test are:

- Samplers.LimitSurfaceSearch

- Since the creation of this test, the following main revisions have been performed:

1. revision info:

- author : maljdan

- date : 2015-11-20

- description: Collapsing the batch sampling into the limit surface class, updating documentation, XSD, and the test cases to reflect this change.

2. revision info:

- author : talbpaul

- date : 2016-02-08

- description: First update, looking for more failing tests to add.

3. revision info:

- author : maljdan

- date : 2016-04-06

- description: Updating test cases to reflect the changes to the user input.

4. revision info:

- author : maljdan

- date : 2017-01-18

- description: Adding test description.

5. revision info:

- author : alfoa

- date : 2019-03-07

- description: Modified syntax in Functions as for issue \#934

\subsubsection{FRAMEWORK SAMPLERS ADAPTIVEBATCH.GREEDYDISTANCEPERSISTENCE}

This test can be found at ". /raven/tests/framework/Samplers/AdaptiveBatch/ test_greedy_dp.xml”. This test can be called executing the following command:

. Irun_tests --re=framework/Samplers/AdaptiveBatch.GreedyDistancepersistenc

or 
./run_framework_tests --re=framework/Samplers/AdaptiveBatch.GreedyDistance

- Test Description:

- An example of using the limit surface post-processor using the greedy selection algorithm with the distance+persistence scoring function. That is, the point to be selected will be a combination of distance from other points and it will have low persistence, that is to say that its label has changed frequently indicating it is in an uncertain area. Note, all of the tests in AdaptiveBatch operate on a 2D input domain with the goal of extracting a circular limit surface with radius 0.5 and centered at the origin, $(0,0)$.

- Original Author:

- maljdan

- Creation date:

- 2015-11-03

- The classes tested in this test are:

- Samplers.LimitSurfaceSearch

- Since the creation of this test, the following main revisions have been performed:

1. revision info:

- author : maljdan

- date : 2015-11-20

- description: Collapsing the batch sampling into the limit surface class, updating documentation, XSD, and the test cases to reflect this change.

2. revision info:

- author : talbpaul

- date : 2016-02-08

- description: First update, looking for more failing tests to add.

3. revision info:

- author : maljdan

- date : 2016-04-06

- description: Updating test cases to reflect the changes to the user input.

4. revision info:

- author : maljdan 
- date : 2017-01-18

- description: Adding test description.

5. revision info:

- author : alfoa

- date : 2019-03-07

- description: Modified syntax in Functions as for issue \#934

\subsubsection{FRAMEWORK SAMPLERS ADAPTIVEBATCH.MAXPDISTANCE}

This test can be found at "./raven/tests/framework/Samplers/AdaptiveBatch/ test_maxp_d.xml". This test can be called executing the following command:

./run_tests --re=framework/Samplers/AdaptiveBatch.MaxPDistance

or

./run_framework_tests --re=framework/Samplers/AdaptiveBatch.MaxpDistance

- Test Description:

- An example of using the limit surface post-processor with a maximum persistence batch selection algorithm with the distance scoring function. That is, the points to be selected will be toplogically distinct maxima in terms of distance from already sampled points. This differs from the maximum value maxima as this looks at points with the highest topological persistence. Note, all of the tests in AdaptiveBatch operate on a 2D input domain with the goal of extracting a circular limit surface with radius 0.5 and centered at the origin, $(0,0)$.

- Original Author:

- maljdan

- Creation date:

- 2015-11-03

- The classes tested in this test are:

- Samplers.LimitSurfaceSearch

- Since the creation of this test, the following main revisions have been performed:

1. revision info: 
- author : maljdan

- date : 2015-11-20

- description: Collapsing the batch sampling into the limit surface class, updating documentation, XSD, and the test cases to reflect this change.

2. revision info:

- author : talbpaul

- date : 2016-02-08

- description: First update, looking for more failing tests to add.

3. revision info:

- author : maljdan

- date : 2016-04-06

- description: Updating test cases to reflect the changes to the user input.

4. revision info:

- author : maljdan

- date : 2017-01-18

- description: Adding test description.

5. revision info:

- author : alfoa

- date : 2019-03-07

- description: Modified syntax in Functions as for issue \#934

\subsubsection{FRAMEWORK SAMPLERS ADAPTIVEBATCH.MAXPDISTANCEPERSISTENCE}

This test can be found at "./raven/tests/framework/Samplers/AdaptiveBatch/ test_maxp_dp.xml”. This test can be called executing the following command:

. /run_tests --re=framework/Samplers/AdaptiveBatch.MaxPDistancePersistence

or

./run_framework_tests --re=framework/Samplers/AdaptiveBatch.MaxpDistancePe

- Test Description:

- An example of using the limit surface post-processor with a maximum persistence batch selection algorithm with the distance+persistence scoring function. That is, the points to be selected will be toplogically distinct maxima in terms of a combination of 
distance from already sampled points and also the temporal inconsistency of the label for this point (points with more volatility are preferred). This differs from the maximum value maxima as this looks at points with the highest topological persistence. Note, all of the tests in AdaptiveBatch operate on a 2D input domain with the goal of extracting a circular limit surface with radius 0.5 and centered at the origin, $(0,0)$.

- Original Author:

- maljdan

- Creation date:

$-2015-11-03$

- The classes tested in this test are:

- Samplers.LimitSurfaceSearch

- Since the creation of this test, the following main revisions have been performed:

1. revision info:

- author : maljdan

- date : 2015-11-20

- description: Collapsing the batch sampling into the limit surface class, updating documentation, XSD, and the test cases to reflect this change.

2. revision info:

- author : talbpaul

- date : 2016-02-08

- description: First update, looking for more failing tests to add.

3. revision info:

- author : maljdan

- date : 2016-04-06

- description: Updating test cases to reflect the changes to the user input.

4. revision info:

- author : maljdan

- date : 2017-01-18

- description: Adding test description.

5. revision info:

- author : alfoa

- date : 2019-03-07

- description: Modified syntax in Functions as for issue \#934 


\subsubsection{FRAMEWORK SAMPLERS ADAPTIVEBATCH.MAXVDISTANCE}

This test can be found at "./raven/tests/framework/Samplers/AdaptiveBatch/ test_maxv_d.xml". This test can be called executing the following command:

./run_tests --re=framework/Samplers/AdaptiveBatch.MaxVDistance

or

./run_framework_tests --re=framework/Samplers/AdaptiveBatch.MaxVDistance

- Test Description:

- An example of using the limit surface post-processor with a maximum value batch selection algorithm with the distance scoring function. That is, the points to be selected will be toplogically distinct maxima in terms of distance from already sampled points. This differs from the maximum persistence maxima as this looks at distinct local maxima with the highest value. Note, all of the tests in AdaptiveBatch operate on a 2D input domain with the goal of extracting a circular limit surface with radius 0.5 and centered at the origin, $(0,0)$.

- Original Author:

- maljdan

- Creation date:

$-2015-11-03$

- The classes tested in this test are:

- Samplers.LimitSurfaceSearch

- Since the creation of this test, the following main revisions have been performed:

1. revision info:

- author : maljdan

- date : 2015-11-20

- description: Collapsing the batch sampling into the limit surface class, updating documentation, XSD, and the test cases to reflect this change.

2. revision info:

- author : talbpaul

- date : 2016-02-08 
- description: First update, looking for more failing tests to add.

3. revision info:

- author : maljdan

- date : 2016-04-06

- description: Updating test cases to reflect the changes to the user input.

4. revision info:

- author : maljdan

- date : 2017-01-18

- description: Adding test description.

5. revision info:

- author : alfoa

- date : 2019-03-07

- description: Modified syntax in Functions as for issue \#934

\subsubsection{FRAMEWORK SAMPLERS ADAPTIVEBATCH.MAXVDISTANCE}

This test can be found at ". / raven/tests/framework/Samplers/AdaptiveBatch/ test_maxv_dp.xml". This test can be called executing the following command:

./run_tests --re=framework/Samplers/AdaptiveBatch.MaxVDistance

Or

./run_framework_tests --re=framework/Samplers/AdaptiveBatch.MaxVDistance

- Test Description:

- An example of using the limit surface post-processor with a maximum value batch selection algorithm with the distance+persistence scoring function. That is, the points to be selected will be toplogically distinct maxima in terms of a combination of distance from already sampled points and also the temporal inconsistency of the label for this point (points with more volatility are preferred). This differs from the maximum persistence maxima as this looks at distinct local maxima with the highest value. Note, all of the tests in AdaptiveBatch operate on a 2D input domain with the goal of extracting a circular limit surface with radius 0.5 and centered at the origin, $(0,0)$.

- Original Author:

- maljdan 
- Creation date:

- 2015-11-03

- The classes tested in this test are:

- Samplers.LimitSurfaceSearch

- Since the creation of this test, the following main revisions have been performed:

1. revision info:

- author : maljdan

- date : 2015-11-20

- description: Collapsing the batch sampling into the limit surface class, updating documentation, XSD, and the test cases to reflect this change.

2. revision info:

- author : talbpaul

- date : 2016-02-08

- description: First update, looking for more failing tests to add.

3. revision info:

- author : maljdan

- date : 2016-04-06

- description: Updating test cases to reflect the changes to the user input.

4. revision info:

- author : maljdan

- date : 2017-01-18

- description: Adding test description.

5. revision info:

- author : alfoa

- date : 2019-03-07

- description: Modified syntax in Functions as for issue \#934

\subsubsection{FRAMEWORK SAMPLERS ADAPTIVEBATCH.PRERANKINGSIMPLIFICATION}

This test can be found at "./raven/tests/framework/Samplers/AdaptiveBatch/ test_pre.xml". This test can be called executing the following command:

./run_tests --re=framework/Samplers/AdaptiveBatch.PreRankingSimplification 
or

./run_framework_tests --re=framework/Samplers/AdaptiveBatch.PreRankingSimp

- Test Description:

- A copy of framework/Samplers/AdaptiveBatch.MaxPDistance (test_maxp_d.xml) except it has been modified to provide a simplification value which is used to set a level for persistence simplification before extracting local maximum from either the maxP or $\max V$ batch strategies. In this example, the value is 0.5 , so any persistence value less than 0.5 of the scoring function's range of values is removed and thus we may end up with very few local maxima candidates. Note, all of the tests in AdaptiveBatch operate on a $2 \mathrm{D}$ input domain with the goal of extracting a circular limit surface with radius 0.5 and centered at the origin, $(0,0)$.

- Original Author:

- maljdan

- Creation date:

- 2015-11-03

- The classes tested in this test are:

- Samplers.LimitSurfaceSearch

- Since the creation of this test, the following main revisions have been performed:

1. revision info:

- author : maljdan

- date : 2015-11-20

- description: Collapsing the batch sampling into the limit surface class, updating documentation, XSD, and the test cases to reflect this change.

2. revision info:

- author : talbpaul

- date : 2016-02-08

- description: First update, looking for more failing tests to add.

3. revision info:

- author : maljdan

- date : 2016-04-06

- description: Updating test cases to reflect the changes to the user input. 
4. revision info:

- author : maljdan

- date : 2017-01-18

- description: Adding test description.

5. revision info:

- author : alfoa

- date : 2019-03-07

- description: Modified syntax in Functions as for issue \#934

\subsubsection{FRAMEWORK SAMPLERS ADAPTIVEBATCH.POSTRANKINGTHRESHOLDING}

This test can be found at "./raven/tests/framework/Samplers/AdaptiveBatch/ test_post.xml". This test can be called executing the following command:

./run_tests --re=framework/Samplers/AdaptiveBatch.PostRankingThresholding

or

./run_framework_tests --re=framework/Samplers/AdaptiveBatch.PostRankingThr

- Test Description:

- A copy of framework/Samplers/AdaptiveBatch.MaxPDistance (test_maxp_d.xml) except it has been modified to provide a threshold value which is used to reject samples whose score is less than a given percentage of the scoring range. Thus, if the threshold is .75 and the scores range from $0-10$, then any score less than 7.5 will be rejected. Note, all of the tests in AdaptiveBatch operate on a 2D input domain with the goal of extracting a circular limit surface with radius 0.5 and centered at the origin, $(0,0)$.

- Original Author:

- maljdan

- Creation date:

- 2015-11-03

- The classes tested in this test are:

- Samplers.LimitSurfaceSearch

- Since the creation of this test, the following main revisions have been performed: 
1. revision info:

- author : maljdan

- date : 2015-11-20

- description: Collapsing the batch sampling into the limit surface class, updating documentation, XSD, and the test cases to reflect this change.

2. revision info:

- author : talbpaul

- date : 2016-02-08

- description: First update, looking for more failing tests to add.

3. revision info:

- author : maljdan

- date : 2016-04-06

- description: Updating test cases to reflect the changes to the user input.

4. revision info:

- author : maljdan

- date : 2017-01-18

- description: Adding test description.

5. revision info:

- author : alfoa

- date : 2019-03-07

- description: Modified syntax in Functions as for issue \#934

\subsubsection{FRAMEWORK SAMPLERS ADAPTIVEBATCH.THICKENEDBAND}

This test can be found at "./raven/tests/framework/Samplers/AdaptiveBatch/ test_thick.xml". This test can be called executing the following command:

./run_tests --re=framework/Samplers/AdaptiveBatch.ThickenedBand

or

./run_framework_tests --re=framework/Samplers/AdaptiveBatch. ThickenedBand

- Test Description: 
- A copy of framework/Samplers/AdaptiveBatch.MaxPDistance (test_maxp_d.xml) except it has been modified to provide a thickened band of candidates for scoring rather than only the points that lie on the limit surface of the current reduced order model representing the data. Note, all of the tests in AdaptiveBatch operate on a 2D input domain with the goal of extracting a circular limit surface with radius 0.5 and centered at the origin, $(0,0)$.

- Original Author:

- maljdan

- Creation date:

$-2015-11-03$

- The classes tested in this test are:

- Samplers.LimitSurfaceSearch

- Since the creation of this test, the following main revisions have been performed:

1. revision info:

- author : maljdan

- date : 2015-11-20

- description: Collapsing the batch sampling into the limit surface class, updating documentation, XSD, and the test cases to reflect this change.

2. revision info:

- author : talbpaul

- date : 2016-02-08

- description: First update, looking for more failing tests to add.

3. revision info:

- author : maljdan

- date : 2016-04-06

- description: Updating test cases to reflect the changes to the user input.

4. revision info:

- author : maljdan

- date : 2017-01-18

- description: Adding test description.

5. revision info:

- author : alfoa

- date : 2019-03-07

- description: Modified syntax in Functions as for issue \#934 


\subsubsection{FRAMEWORK SAMPLERS ADAPTIVEBATCH.INVALIDPRERANKINGSIMPLIFICATION}

This test can be found at "./raven/tests/framework/Samplers/AdaptiveBatch/ test_invalid_pre.xml". This test can be called executing the following command:

./run_tests --re=framework/Samplers/AdaptiveBatch. InvalidPreRankingsimplif.

or

./run_framework_tests --re=framework/Samplers/AdaptiveBatch.InvalidPreRank

- Test Description:

- An example of using the limit surface post-processor with an invalid simplification value. This should degrade gracefully by defaulting to a simplification of 0 and warning the user of the change made to the execution. Note, all of the tests in AdaptiveBatch operate on a 2D input domain with the goal of extracting a circular limit surface with radius 0.5 and centered at the origin, $(0,0)$.

- Original Author:

- maljdan

- Creation date:

- 2015-11-03

- The classes tested in this test are:

- Samplers.LimitSurfaceSearch

- Since the creation of this test, the following main revisions have been performed:

1. revision info:

- author: maljdan

- date : 2015-11-20

- description: Collapsing the batch sampling into the limit surface class, updating documentation, XSD, and the test cases to reflect this change.

2. revision info:

- author: talbpaul

- date : 2016-02-08

- description: First update, looking for more failing tests to add.

3. revision info: 
- author : maljdan

- date : 2016-04-06

- description: Updating test cases to reflect the changes to the user input.

4. revision info:

- author : maljdan

- date : 2017-01-18

- description: Adding test description.

5. revision info:

- author : alfoa

- date : 2019-03-07

- description: Modified syntax in Functions as for issue \#934

\subsubsection{FRAMEWORK SAMPLERS ADAPTIVEBATCH.INVALIDPOSTRANKINGSIMPLIFICATIO}

This test can be found at ". /raven/tests/framework/Samplers/AdaptiveBatch/ test_invalid_post.xml". This test can be called executing the following command:

./run_tests --re=framework/Samplers/AdaptiveBatch. InvalidPostRankingsimpli or

. Irun_framework_tests --re=framework/Samplers/AdaptiveBatch.InvalidPostRan

- Test Description:

- An example of using the limit surface post-processor with an invalid threshold value. This should degrade gracefully by defaulting to a threshold of 0 and warning the user of the change made to the execution. Note, all of the tests in AdaptiveBatch operate on a $2 \mathrm{D}$ input domain with the goal of extracting a circular limit surface with radius 0.5 and centered at the origin, $(0,0)$.

- Original Author:

- maljdan

- Creation date:

$-2015-11-03$

- The classes tested in this test are: 
- Samplers.LimitSurfaceSearch

- Since the creation of this test, the following main revisions have been performed:

1. revision info:

- author : maljdan

- date : 2015-11-20

- description: Collapsing the batch sampling into the limit surface class, updating documentation, XSD, and the test cases to reflect this change.

2. revision info:

- author : talbpaul

- date : 2016-02-08

- description: First update, looking for more failing tests to add.

3. revision info:

- author : maljdan

- date : 2016-04-06

- description: Updating test cases to reflect the changes to the user input.

4. revision info:

- author : maljdan

- date : 2017-01-18

- description: Adding test description.

5. revision info:

- author : alfoa

- date : 2019-03-07

- description: Modified syntax in Functions as for issue \#934

\subsubsection{FRAMEWORK SAMPLERS ADAPTIVEBATCH.INVALIDMAXBATCHSIZE}

This test can be found at ". / raven/tests/framework/Samplers/AdaptiveBatch/ test_invalid_mbs.xml”. This test can be called executing the following command:

./run_tests --re=framework/Samplers/AdaptiveBatch. InvalidMaxBatchsize

or

.Irun_framework_tests --re=framework/Samplers/AdaptiveBatch.InvalidMaxBatc

- Test Description: 
- An example of using the limit surface post-processor with an invalid maximum batch size. This should degrade gracefully by defaulting to a maximum batch size of 1 and warning the user of the change made to the execution. Note, all of the tests in AdaptiveBatch operate on a 2D input domain with the goal of extracting a circular limit surface with radius 0.5 and centered at the origin, $(0,0)$.

- Original Author:

- maljdan

- Creation date:

- 2015-11-03

- The classes tested in this test are:

- Samplers.LimitSurfaceSearch

- Since the creation of this test, the following main revisions have been performed:

1. revision info:

- author : maljdan

- date : 2015-11-20

- description: Collapsing the batch sampling into the limit surface class, updating documentation, XSD, and the test cases to reflect this change.

2. revision info:

- author : talbpaul

- date : 2016-02-08

- description: First update, looking for more failing tests to add.

3. revision info:

- author : maljdan

- date : 2016-04-06

- description: Updating test cases to reflect the changes to the user input.

4. revision info:

- author : maljdan

- date : 2017-01-18

- description: Adding test description.

5. revision info:

- author : alfoa

- date : 2019-03-07

- description: Modified syntax in Functions as for issue \#934 


\subsubsection{FRAMEWORK SAMPLERS ADAPTIVEBATCH.INVALIDSCORINGSTRATEGY}

This test can be found at "./raven/tests/framework/Samplers/AdaptiveBatch/ test_invalid_score.xml". This test can be called executing the following command:

. /run_tests --re=framework/Samplers/AdaptiveBatch. InvalidScoring\$trategy

or

./run_framework_tests --re=framework/Samplers/AdaptiveBatch.InvalidScoring

- Test Description:

- An example of using the limit surface post-processor with an invalid scoring strategy. This should print a message and exit. Note, all of the tests in AdaptiveBatch operate on a $2 \mathrm{D}$ input domain with the goal of extracting a circular limit surface with radius 0.5 and centered at the origin, $(0,0)$.

- Original Author:

- maljdan

- Creation date:

$-2015-11-03$

- The classes tested in this test are:

- Samplers.LimitSurfaceSearch

- Since the creation of this test, the following main revisions have been performed:

1. revision info:

- author : maljdan

- date : 2015-11-20

- description: Collapsing the batch sampling into the limit surface class, updating documentation, XSD, and the test cases to reflect this change.

2. revision info:

- author : talbpaul

- date : 2016-02-08

- description: First update, looking for more failing tests to add.

3. revision info: 
- author : maljdan

- date : 2016-04-06

- description: Updating test cases to reflect the changes to the user input.

4. revision info:

- author : maljdan

- date : 2017-01-18

- description: Adding test description.

5. revision info:

- author : alfoa

- date : 2019-03-07

- description: Modified syntax in Functions as for issue \#934

\subsubsection{FRAMEWORK SAMPLERS ADAPTIVEBATCH.INVALIDBATCHSTRATEGY}

This test can be found at ". /raven/tests/framework/Samplers/AdaptiveBatch/ test_invalid_batch.xml”. This test can be called executing the following command:

. /run_tests --re=framework/Samplers/AdaptiveBatch. InvalidBatchstrategy

or

./run_framework_tests --re=framework/Samplers/AdaptiveBatch.InvalidBatchSt

- Test Description:

- An example of using the limit surface post-processor with an invalid batch strategy which will fail with an error message. Note, all of the tests in AdaptiveBatch operate on a $2 \mathrm{D}$ input domain with the goal of extracting a circular limit surface with radius 0.5 and centered at the origin, $(0,0)$.

- Original Author:

- maljdan

- Creation date:

- 2015-11-03

- The classes tested in this test are:

- Samplers.LimitSurfaceSearch 
- Since the creation of this test, the following main revisions have been performed:

1. revision info:

- author : maljdan

- date : 2015-11-20

- description: Collapsing the batch sampling into the limit surface class, updating documentation, XSD, and the test cases to reflect this change.

2. revision info:

- author : talbpaul

- date : 2016-02-08

- description: First update, looking for more failing tests to add.

3. revision info:

- author : maljdan

- date : 2017-01-18

- description: Adding test description.

4. revision info:

- author : alfoa

- date : 2019-03-07

- description: Modified syntax in Functions as for issue \#934

\subsubsection{FRAMEWORK SAMPLERS ADAPTIVEBATCH.INVALIDPOSTRANKINGSIMPLIFICATIO}

This test can be found at ". /raven/tests/framework/Samplers/AdaptiveBatch/ test_invalid_thick.xml". This test can be called executing the following command:

. Irun_tests --re=framework/Samplers/AdaptiveBatch. InvalidPostRankingSimpli

Or

. Irun_framework_tests --re=framework/Samplers/AdaptiveBatch.InvalidPostRan

\section{- Test Description:}

- An example of using the limit surface post-processor with an invalid threshold value. This should degrade gracefully by defaulting to a threshold of 0 and warning the user of the change made to the execution. Note, all of the tests in AdaptiveBatch operate on a $2 \mathrm{D}$ input domain with the goal of extracting a circular limit surface with radius 0.5 and centered at the origin, $(0,0)$. 
- Original Author:

- maljdan

- Creation date:

- 2015-11-03

- The classes tested in this test are:

- Samplers.LimitSurfaceSearch

- Since the creation of this test, the following main revisions have been performed:

1. revision info:

- author: maljdan

- date : 2015-11-20

- description: Collapsing the batch sampling into the limit surface class, updating documentation, XSD, and the test cases to reflect this change.

2. revision info:

- author : maljdan

- date : 2015-11-23

- description: Adjusting the invalid thickness example to replicate the default distancePersistence scoring function rather than the distance scoring function (which fails on the HPC for some yet undiscovered reason).

3. revision info:

- author : talbpaul

- date : 2016-02-08

- description: First update, looking for more failing tests to add.

4. revision info:

- author : maljdan

- date : 2016-04-06

- description: Updating test cases to reflect the changes to the user input.

5. revision info:

- author : maljdan

- date : 2017-01-18

- description: Adding test description.

6. revision info:

- author : alfoa

- date : 2019-03-07

- description: Modified syntax in Functions as for issue \#934 


\subsubsection{FRAMEWORK SAMPLERS NOVARS.MC}

This test can be found at "./raven/tests/framework/Samplers/NoVars/mc.xml". This test can be called executing the following command:

./run_tests --re=framework/Samplers/NoVars.MC

or

. /run_framework_tests --re=framework/Samplers/NoVars.MC

- Test Description:

- Tests using no variables as inputs to the MonteCarlo sampler. While not precisely analytic, the samples pointset should contain two realizations, each with values $1,2,3$ for $\mathrm{x} 1, \mathrm{x} 2$, and $\mathrm{x} 3$.

- Original Author:

- talbpaul

- Creation date:

- 2019-11-05

- The classes tested in this test are:

- Samplers.MonteCarlo

\subsubsection{FRAMEWORK SAMPLERS NOVARS.GRID}

This test can be found at ". / raven/tests / framework/Samplers /NoVars/grid.xml". This test can be called executing the following command:

. Irun_tests --re=framework/Samplers/NoVars.Grid

or

./run_framework_tests --re=framework/Samplers/NoVars.Grid

- Test Description: 
- Tests using no variables as inputs to the Grid sampler. While not precisely analytic, the samples pointset should contain one realization, with values 1, 2, 3 for $\mathrm{x} 1, \mathrm{x} 2$, and $\mathrm{x} 3$.

- Original Author:

- talbpaul

- Creation date:

- 2019-11-05

- The classes tested in this test are:

- Samplers.Grid

\subsubsection{FRAMEWORK SAMPLERS NOVARS.STRATIFIED}

This test can be found at ". / raven/tests / framework/Samplers/NoVars/stratified. xml". This test can be called executing the following command:

. Irun_tests --re=framework/Samplers/NoVars.Stratified

or

./run_framework_tests --re=framework/Samplers/NoVars.Stratified

- Test Description:

- Tests using no variables as inputs to the Stratified sampler. While not precisely analytic, the samples pointset should contain one realization, with values 1, 2, 3 for $\mathrm{x} 1$, $\mathrm{x} 2$, and $\mathrm{x} 3$.

- Original Author:

- talbpaul

- Creation date:

- 2019-11-05

- The classes tested in this test are:

- Samplers.Stratified 


\subsubsection{FRAMEWORK SAMPLERS RESTART.AMC}

This test can be found at “. / raven/tests/framework/Samplers/Restart/adaptive_ mc.xml". This test can be called executing the following command:

. /run_tests --re=framework/Samplers/Restart.AMC

or

./run_framework_tests --re=framework/Samplers/Restart.AMC

- Test Description:

- Tests restarting an Adaptive Monte Carlo sampling from restart. makeCoarse samples initial data, then makeRestart makes additional samples, restarting from the first set of samples. makeFine does all the samples without restart for comparison. The model for coarse always returns a value of 1 , while the model for restart returns a value of 2 , so you can tell which samples came from which sampling strategy. Further, the solution export for the restart should contain all the sample points, not merely the newly-sampled points.

- Original Author:

- talbpaul

- Creation date:

- 2020-07-16

- The classes tested in this test are:

- Samplers.AdaptiveMonteCarlo

\subsubsection{FRAMEWORK SAMPLERS RESTART.STOCHPOLY}

This test can be found at "./raven/tests/framework/Samplers/Restart/test_ restart_stochpoly.xml". This test can be called executing the following command:

./run_tests --re=framework/Samplers/Restart.StochPoly

or

./run_framework_tests --re=framework/Samplers/Restart.StochPoly 
- Test Description:

- The essence of this test is to demonstrate the input space when generated from restart or from scratch are identical. The external models are organized so that outputs from the restart data have an output value of 1.0, and from the higher-fidelity sampler have an output value of 2.0. Obviously using different models with restarts is a terrible idea in general, but makes this test work. In the end, "fine.csv" and "restart.csv" should have identical input space, but different output space; all the output of "fine.csv" should be 2.0, while the restarted points in "restart.csv" should be 1.0

- Original Author:

- talbpaul

- Creation date:

- 2015-07-07

- The classes tested in this test are:

- Samplers.SparseGridCollocation

\subsubsection{FRAMEWORK SAMPLERS RESTART.SOBOL}

This test can be found at "./raven/tests/framework/Samplers/Restart/test_ restart_sobol.xml". This test can be called executing the following command:

./run_tests --re=framework/Samplers/Restart. Sobol

or

./run_framework_tests --re=framework/Samplers/Restart.sobol

- Test Description:

- The essence of this test is to demonstrate the input space when generated from restart or from scratch are identical. The external models are organized so that outputs from the restart data have an output value of 1.0, and from the higher-fidelity sampler have an output value of 2.0. Obviously using different models with restarts is a terrible idea in general, but makes this test work. In the end, "fine.csv" and "restart.csv" should have identical input space, but different output space; all the output of "fine.csv" should be 2.0, while the restarted points in "restart.csv" should be 1.0

- Original Author: 
- talbpaul

- Creation date:

- 2015-07-07

- The classes tested in this test are:

- Samplers.Sobol

\subsubsection{FRAMEWORK SAMPLERS RESTART.GRID}

This test can be found at "./raven/tests/framework/Samplers/Restart/test_ restart_Grid.xml". This test can be called executing the following command:

./run_tests --re=framework/Samplers/Restart.Grid

Or

./run_framework_tests --re=framework/Samplers/Restart.Grid

- Test Description:

- The essence of this test is to demonstrate the input space when generated from restart or from scratch are identical. The external models are organized so that outputs from the restart data have an output value of 1.0, and from the higher-fidelity sampler have an output value of 2.0. Obviously using different models with restarts is a terrible idea in general, but makes this test work. In the end, "fine.csv" and "restart.csv" should have identical input space, but different output space; all the output of "fine.csv" should be 2.0, while the restarted points in "restart.csv" should be 1.0

- Original Author:

- talbpaul

- Creation date:

- 2015-07-07

- The classes tested in this test are:

- Samplers.Grid 


\subsubsection{FRAMEWORK SAMPLERS RESTART.LOADFROMLARGECSV}

This test can be found at ". /raven/tests/framework/Samplers/Restart/large_ load_from_csv.xml”. This test can be called executing the following command:

. /run_tests --re=framework/Samplers/Restart.LoadFromLargeCSV

or

. Irun_framework_tests --re=framework/Samplers/Restart.LoadFromLargeCSV

- Test Description:

- At one point there was performance issues restarting from a large amount of data that is loaded from a CSV. This test exists to ensure loading from a large CSV behaves consistently.

- Original Author:

- talbpaul

- Creation date:

- 2016-06-30

- The classes tested in this test are:

- Samplers.Grid

\subsubsection{FRAMEWORK SAMPLERS CATEGORICAL.STRINGS}

This test can be found at ". / raven/tests/framework/Samplers/Categorical/string_ vars. xml". This test can be called executing the following command:

./run_tests --re=framework/Samplers/Categorical.Strings

or

./run_framework_tests --re=framework/Samplers/Categorical.Strings

- Test Description: 
- Tests the ability to use the Categorical distribution along with Functions to essentially sample strings for variables. The model run is a simple projectile motion problem that can be run in either "stepper"; or "analytic" mode. The output of the model is "height"; as a time-dependent variable. The analytic mode is exact, but the stepper mode is intentionally chosen to be a poor approximation (too few steps taken).

- Original Author:

- talbpaul

- Creation date:

- 2018-01-31

- The classes tested in this test are:

- Samplers.Categorical,Function

- Since the creation of this test, the following main revisions have been performed:

1. revision info:

- author : alfoa

- date : 2019-03-07

- description: Modified syntax in Functions as for issue \#934

\subsubsection{FRAMEWORK SAMPLERS CATEGORICAL.RESTART}

This test can be found at ". / raven/tests / framework/Samplers/Categorical/string_ vars_restart.xml”. This test can be called executing the following command:

./run_tests --re=framework/Samplers/Categorical.Restart

or

./run_framework_tests --re=framework/Samplers/Categorical.Restart

- Test Description:

- Tests restarting from input spaces with string variables (from categorical+function) as well as restarting a HistorySet. The difference between input and output spaces is in the output variable "restartID". In the data being restarted from, the value is 1.0, while in the new samples taken, the value is 2.0. The restart history CSVs that should have a "1.0" restart ID are $0,2,6,8,9,11,15,17,18,20,24,26$ The restart history CSVs that should have a "2.0" restart ID are 1,3,4,5,7,10,12,13,14,16,19,21,22,23,25 
- Original Author:

- talbpaul

- Creation date:

$-2018-01-31$

- The classes tested in this test are:

- Samplers.Categorical,Function

- Since the creation of this test, the following main revisions have been performed:

1. revision info:

- author: alfoa

- date : 2019-03-07

- description: Modified syntax in Functions as for issue \#934

\subsubsection{FRAMEWORK SAMPLERS CATEGORICAL.RESTARTMISSINGVARS}

This test can be found at ". / raven/tests/framework/Samplers/Categorical/missing_ variables.xml". This test can be called executing the following command:

./run_tests --re=framework/Samplers/Categorical.RestartMissingVans

or

./run_framework_tests --re=framework/Samplers/Categorical.RestartMissingVa

- Test Description:

- Tests restarting from input spaces with string variables (from categorical+function) but with variables missing from the restart (should fail). The difference between input and output spaces is in the output variable "restartID". In the data being restarted from, the value is 1.0, while in the new samples taken, the value is 2.0. The restart history CSVs that should have a "1.0"; restart ID are 0,2,6,8,9,11,15,17,18,20,24,26 The restart history CSVs that should have a " 2.0 "; restart ID are 1,3,4,5,7,10,12,13,14,16,19,21,22,23,25

- Original Author:

- talbpaul

- Creation date: 
- 2018-03-14

- The classes tested in this test are:

- Samplers.Categorical,Function

- Since the creation of this test, the following main revisions have been performed:

1. revision info:

- author : alfoa

- date : 2019-03-07

- description: Modified syntax in Functions as for issue \#934

\subsubsection{FRAMEWORK SAMPLERS CUSTOMSAMPLER.CUSTOMSAMPLERFROMFILE}

This test can be found at ". /raven/tests/framework/Samplers/CustomSampler/ test_Custom_Sampler_File.xml". This test can be called executing the following command:

./run_tests --re=framework/Samplers/CustomSampler.customSamplerFromFile

or

. Irun_framework_tests --re=framework/Samplers/CustomSampler.customSamplerF

- Test Description:

- An example of using the Custom Sampler object. In this test the realization coordinates are collected from a Files object of type CSV.

- Original Author:

- alfoa

- Creation date:

- 2016-05-24

- The classes tested in this test are:

- Samplers.CustomSampler, Files.Input

- Since the creation of this test, the following main revisions have been performed:

1. revision info: 
- author : alfoa

- date : 2016-05-25

- description: Fixed absolute path

2. revision info:

- author : talbpaul

- date : 2016-09-15

- description: Test updates

3. revision info:

- author : alfoa

- date : 2017-01-21

- description: Adding this test description.

4. revision info:

- author : talbpaul

- date : 2018-05-02

- description: Added nameInSource test to get "x" from "xalias"

\subsubsection{FRAMEWORK SAMPLERS CUSTOMSAMPLER.CUSTOMSAMPLERFROMDATAOBJECT}

This test can be found at ". /raven/tests/framework/Samplers/CustomSampler/ test_Custom_Sampler_DataObject.xml". This test can be called executing the following command:

. Irun_tests --re=framework/Samplers/CustomSampler.customSamplerF homDataObj

or

. /run_framework_tests --re=framework/Samplers/CustomSampler.customSamplerF

- Test Description:

- An example of using the Custom Sampler object. In this test the realization coordinates are directly taken from a DataObjec of type PointSet

- Original Author:

- alfoa

- Creation date:

$-2016-05-25$ 
- The classes tested in this test are:

- Samplers.CustomSampler, DataObjects.PointSet

- Since the creation of this test, the following main revisions have been performed:

1. revision info:

- author : alfoa

- date : 2016-05-25

- description: Fixed absolute path

2. revision info:

- author : talbpaul

- date : 2016-09-15

- description: Test updates

3. revision info:

- author : alfoa

- date : 2017-01-21

- description: Adding this test description.

4. revision info:

- author : talbpaul

- date : 2018-05-02

- description: Added nameInSource test renaming "x" to "xnew"

\subsubsection{FRAMEWORK SAMPLERS CUSTOMSAMPLER.CUSTOMSAMPLERFROMDATAOBJECT}

This test can be found at ". /raven/tests/framework/Samplers/CustomSampler/ from_dataset.xml". This test can be called executing the following command:

. Irun_tests --re=framework/Samplers/CustomSampler. customSamplerF romDataObj

or

./run_framework_tests --re=framework/Samplers/CustomSampler.customSamplerF

- Test Description:

- An example of using the Custom Sampler object. In this test the realization coordinates are directly taken from a DataObjec of type PointSet 
- Original Author:

- alfoa

- Creation date:

- 2016-05-25

- The classes tested in this test are:

- Samplers.CustomSampler, DataObjects.PointSet

- Since the creation of this test, the following main revisions have been performed:

1. revision info:

- author : alfoa

- date : 2016-05-25

- description: Fixed absolute path

2. revision info:

- author : talbpaul

- date : 2016-09-15

- description: Test updates

3. revision info:

- author : alfoa

- date : 2017-01-21

- description: Adding this test description.

4. revision info:

- author : talbpaul

- date : 2020-01-07

- description: Adding a constant

\subsubsection{FRAMEWORK SAMPLERS CUSTOMSAMPLER.BYINDEX}

This test can be found at ". /raven/tests/framework/Samplers/CustomSampler/ by_index.xml". This test can be called executing the following command:

./run_tests --re=framework/Samplers/CustomSampler.ByIndex

or

./run_framework_tests --re=framework/Samplers/CustomSampler.ByIndex 
- Test Description:

- An example of using the Custom Sampler object. In this test we take a single realization by index from a data object.

- Original Author:

- talbpaul

- Creation date:

- 2018-11-08

- The classes tested in this test are:

- Samplers.CustomSampler, DataObjects.PointSet

\subsubsection{FRAMEWORK SAMPLERS SPARSEGRID SMOLYAKGRIDTEST}

This test can be found at ". / raven/tests / framework/Samplers/SparseGrid/test_ sparse_grid.xml". This test can be called executing the following command:

. /run_tests --re=framework/Samplers/SparseGrid/SmolyakGridTest

or

./run_framework_tests --re=framework/Samplers/SparseGrid/SmolyakqridTest

- Test Description:

- This tests using Smolyak-style SparseGridCollocation.

- Original Author:

- talbpaul

- Creation date:

- 2015-07-07

- The classes tested in this test are:

- Samplers.SparseGridCollocation 


\subsubsection{FRAMEWORK SAMPLERS SPARSEGRID TENSORGRIDTEST}

This test can be found at ". / raven/tests/framework/Samplers/SparseGrid/test_ tensor_grid.xml”. This test can be called executing the following command:

. /run_tests --re=framework/Samplers/SparseGrid/TensorGridTest

or

./run_framework_tests --re=framework/Samplers/SparseGrid/TensorGridTest

- Test Description:

- This tests using non-sparse tensor collocation.

- Original Author:

- talbpaul

- Creation date:

- 2015-07-07

- The classes tested in this test are:

- Samplers.SparseGridCollocation

\subsubsection{FRAMEWORK SAMPLERS SPARSEGRID TENSORPRDUCTGRID}

This test can be found at ". / raven/tests/framework/Samplers/SparseGrid/test_ index_TP.Xml". This test can be called executing the following command:

./run_tests --re=framework/Samplers/SparseGrid/tensorPrductGrid

or

./run_framework_tests --re=framework/Samplers/SparseGrid/tensorPrductGrid

- Test Description:

- This tests the creation of a TensorProduct construction sparse grid.

- Original Author: 
- talbpaul

- Creation date:

- 2015-09-11

- The classes tested in this test are:

- IndexSets.TensorProduct

\subsubsection{FRAMEWORK SAMPLERS SPARSEGRID TOTALDEGREEGRID}

This test can be found at ". / raven/tests / framework/Samplers / SparseGrid/test_ index_TD.xml". This test can be called executing the following command:

./run_tests --re=framework/Samplers/SparseGrid/totalDegreeGrid

Or

./run_framework_tests --re=framework/Samplers/SparseGrid/totalDeqreeGrid

- Test Description:

- This tests the creation of a TotalDegree construction sparse grid.

- Original Author:

- talbpaul

- Creation date:

- 2015-09-11

- The classes tested in this test are:

- IndexSets.TotalDegree

\subsubsection{FRAMEWORK SAMPLERS SPARSEGRID HYPERBOLICCROSSGRID}

This test can be found at ". / raven/tests/framework/Samplers/SparseGrid/test_ index_HC.xml". This test can be called executing the following command:

. /run_tests --re=framework/Samplers/SparseGrid/hyperbolicCrossGrid 
or

. Irun_framework_tests --re=framework/Samplers/SparseGrid/hyperbolicCrossGr

- Test Description:

- This tests the creation of a HyperbolicCross construction sparse grid.

- Original Author:

- talbpaul

- Creation date:

- 2015-09-11

- The classes tested in this test are:

- IndexSets.HyperbolicCross

\subsubsection{FRAMEWORK SAMPLERS SPARSEGRID CUSTOMGRID}

This test can be found at ". / raven/tests/framework/Samplers/SparseGrid/test_ index_custom.xml". This test can be called executing the following command:

./run_tests --re=framework/Samplers/SparseGrid/customGrid

or

./run_framework_tests --re=framework/Samplers/SparseGrid/customGrid

- Test Description:

- This tests the creation of a Custom sparse grid.

- Original Author:

- talbpaul

- Creation date:

- 2015-09-11

- The classes tested in this test are:

- IndexSets.Custom 


\subsubsection{FRAMEWORK SAMPLERS SPARSEGRID ANISOTROPICGRID}

This test can be found at ". / raven/tests/framework/Samplers/SparseGrid/test_ index_anisotropic.xml". This test can be called executing the following command:

./run_tests --re=framework/Samplers/SparseGrid/anisotropicGrid

or

./run_framework_tests --re=framework/Samplers/SparseGrid/anisotrqpicGrid

- Test Description:

- This tests the creation of an anisotropic sparse grid (using the interpolation weights in the ROM).

- Original Author:

- talbpaul

- Creation date:

- 2015-09-11

- The classes tested in this test are:

- IndexSets

\subsubsection{FRAMEWORK SAMPLERS SPARSEGRID UNIFORM}

This test can be found at ". / raven/tests / framework/Samplers / SparseGrid/test_ scgpc_uniform.xml". This test can be called executing the following command:

./run_tests --re=framework/Samplers/SparseGrid/uniform

or

./run_framework_tests --re=framework/Samplers/SparseGrid/uniform

- Test Description:

- This tests using SparseGridCollocation with uniformly-distributed inputs. 
- Original Author:

- talbpaul

- Creation date:

- 2015-09-11

- The classes tested in this test are:

- Samplers.SparseGridCollocation

\subsubsection{FRAMEWORK SAMPLERS SPARSEGRID UNIFORM CC}

This test can be found at ". / raven/tests/framework/Samplers/SparseGrid/test_ scgpc_uniform_cc.xml". This test can be called executing the following command:

./run_tests --re=framework/Samplers/SparseGrid/uniform_cc

Or

./run_framework_tests --re=framework/Samplers/SparseGrid/uniform_cC

- Test Description:

- This tests using SparseGridCollocation with Clenshaw Curtis points and weights (on uniformly-distributed variables).

- Original Author:

- talbpaul

- Creation date:

- 2015-09-11

- The classes tested in this test are:

- Samplers.SparseGridCollocation 


\subsubsection{FRAMEWORK SAMPLERS SPARSEGRID NORMAL}

This test can be found at ". / raven/tests / framework/Samplers / SparseGrid/test_ scgpc_normal.xml". This test can be called executing the following command:

. /run_tests --re=framework/Samplers/SparseGrid/normal

or

./run_framework_tests --re=framework/Samplers/SparseGrid/normal

- Test Description:

- This tests using SparseGridCollocation with normally-distributed inputs.

- Original Author:

- talbpaul

- Creation date:

- 2015-09-11

- The classes tested in this test are:

- Samplers.SparseGridCollocation

\subsubsection{FRAMEWORK SAMPLERS SPARSEGRID GAMMA}

This test can be found at ". / raven/tests / framework/Samplers / SparseGrid/test_ scgpc_gamma.xml". This test can be called executing the following command:

./run_tests --re=framework/Samplers/SparseGrid/gamma

or

./run_framework_tests --re=framework/Samplers/SparseGrid/gamma

- Test Description:

- This tests using SparseGridCollocation with gamma-distributed inputs.

- Original Author: 
- talbpaul

- Creation date:

- 2015-09-11

- The classes tested in this test are:

- Samplers.SparseGridCollocation

\subsubsection{FRAMEWORK SAMPLERS SPARSEGRID BETA}

This test can be found at ". / raven/tests / framework/Samplers / SparseGrid/test_ scgpc_beta.xml". This test can be called executing the following command:

./run_tests --re=framework/Samplers/SparseGrid/beta

Or

./run_framework_tests --re=framework/Samplers/SparseGrid/beta

- Test Description:

- This tests using SparseGridCollocation with beta-distributed inputs.

- Original Author:

- talbpaul

- Creation date:

- 2015-09-11

- The classes tested in this test are:

- Samplers.SparseGridCollocation

\subsubsection{FRAMEWORK SAMPLERS SPARSEGRID BETANORM}

This test can be found at ". / raven/tests/framework/Samplers/SparseGrid/test_ scgpc_betanorm.xml”. This test can be called executing the following command:

./run_tests --re=framework/Samplers/SparseGrid/betanorm 
or

./run_framework_tests --re=framework/Samplers/SparseGrid/betanorm

- Test Description:

- This tests using SparseGridCollocation with truncated-normal-beta distributed inputs.

- Original Author:

- talbpaul

- Creation date:

- 2015-09-11

- The classes tested in this test are:

- Samplers.SparseGridCollocation

\subsubsection{FRAMEWORK SAMPLERS SPARSEGRID TRIANG}

This test can be found at ". / raven/tests / framework/Samplers/SparseGrid/test_ scgpc_triang.xml". This test can be called executing the following command:

./run_tests --re=framework/Samplers/SparseGrid/triang

or

./run_framework_tests --re=framework/Samplers/SparseGrid/triang

- Test Description:

- This tests using SparseGridCollocation with triangular-distributed inputs.

- Original Author:

- talbpaul

- Creation date:

- 2015-09-11

- The classes tested in this test are:

- Samplers.SparseGridCollocation 


\subsubsection{FRAMEWORK SAMPLERS SPARSEGRID EXPONENTIAL}

This test can be found at ". / raven/tests/framework/Samplers / SparseGrid/test_ scgpc_expon.xml". This test can be called executing the following command:

./run_tests --re=framework/Samplers/SparseGrid/exponential

or

./run_framework_tests --re=framework/Samplers/SparseGrid/exponential

- Test Description:

- This tests using SparseGridCollocation with exponential-distributed inputs.

- Original Author:

- talbpaul

- Creation date:

$-2015-09-11$

- The classes tested in this test are:

- Samplers.SparseGridCollocation

\subsubsection{FRAMEWORK SAMPLERS SPARSEGRID ADAPTIVEONVARIANCE}

This test can be found at ". / raven/tests/framework/Samplers/SparseGrid/test_ adaptive_stochpoly_var.xml". This test can be called executing the following command:

./run_tests --re=framework/Samplers/SparseGrid/AdaptiveonVariance

or

./run_framework_tests --re=framework/Samplers/SparseGrid/AdaptiveonVarianc

- Test Description:

- This tests the adaptive sparse grid with adaptive samples chosen and converged according to variance. 
- Original Author:

- talbpaul

- Creation date:

- 2015-07-07

- The classes tested in this test are:

- Samplers.AdaptiveSparseGrid

\subsubsection{FRAMEWORK SAMPLERS ADAPTIVELIMITSURFACESEARCH.TESTLIMITSURFACEM}

This test can be found at ". / raven/tests/framework/Samplers/AdaptiveLimitSurfaceSearcl test_limit_surface_multi_grid_acc.xml”. This test can be called executing the following command:

./run_tests --re=framework/Samplers/AdaptiveLimitSurfaceSearch.t\&stLimitSu

or

./run_framework_tests --re=framework/Samplers/AdaptiveLimitSurfaceSearch.t

- Test Description:

- This test is aimed to check the capability of RAVEN to perform an adaptive sampling strategy of type LimitSurfaceSearch when using the convergence acceleration scheme of type Multi-Grid.

- Original Author:

- alfoa

- Creation date:

- 2015-09-10

- The classes tested in this test are:

- Samplers.LimitSurfaceSearch, Functions.External

- Since the creation of this test, the following main revisions have been performed:

1. revision info: 
- author : cogljj

- date : 2015-09-29

- description: Modifing external modules to use relative to working directory.

2. revision info:

- author: alfoa

- date : 2015-10-05

- description: Modified multigrid test to make it faster

3. revision info:

- author : maljdan

- date : 2016-05-20

- description: Updating the test cases to remove references to Point or History.

4. revision info:

- author : maljdan

- date : 2016-11-08

- description: Serializing the adaptive tests and regolding the multigrid case since I can validate the results look good. I am still trying to understand what the correct results for the 3D case are.

5. revision info:

- author : alfoa

- date : 2017-01-21

- description: Adding this test description.

6. revision info:

- author : alfoa

- date : 2017-12-12

- description: Adapted for new DataObject structure

7. revision info:

- author : alfoa

- date : 2019-03-07

- description: Modified syntax in Functions as for issue \#934

\subsubsection{FRAMEWORK SAMPLERS ADAPTIVELIMITSURFACESEARCH.ADAPTIVE SAMPLER EXT MODEL}

This test can be found at ". / raven/tests / framework/Samplers / AdaptiveLimitSurfaceSearcl test_adaptive_sampler.xml”. This test can be called executing the following command: 
./run_tests --re=framework/Samplers/AdaptiveLimitSurfaceSearch.adlaptive_sar

or

./run_framework_tests --re=framework/Samplers/AdaptiveLimitSurfaceSearch.a

- Test Description:

- This test is aimed to test the capability of RAVEN to employ a goal oriented sampling. It tests the LimitSurfaceSearch algorithm using an external model as "system code"

- Original Author:

- alfoa

- Creation date:

- 2015-04-09

- The classes tested in this test are:

- Samplers.LimitSurfaceSearch, Models.ExternalModel, Models.ROM

- Since the creation of this test, the following main revisions have been performed:

1. revision info:

- author : alfoa

- date : 2017-12-12

- description: Adapted for new DataObject structure

2. revision info:

- author : alfoa

- date : 2019-03-07

- description: Modified syntax in Functions as for issue \#934

\subsubsection{FRAMEWORK SAMPLERS ADAPTIVELIMITSURFACESEARCH.ADAPTIVE SAMPLER NO CROSSING}

This test can be found at ". / raven/tests / framework/Samplers/AdaptiveLimitSurfaceSearch test_limit_surface_no_crossing_transition.xml”. This test can be called executing the following command: 
./run_tests --re=framework/Samplers/AdaptiveLimitSurfaceSearch.adlaptive_sar

or

./run_framework_tests --re=framework/Samplers/AdaptiveLimitSurfaceSearch.a

- Test Description:

- This test is aimed to test that the LimitSurfaceSearch Sampler is able to successfully detect a failure of the adaptive search (No limit surface crossed). This test is connected to the defect mitigation reported in the issue \#704

- Original Author:

- alfoa

- Creation date:

- 2018-08-25

- The classes tested in this test are:

- Samplers.LimitSurfaceSearch, Models.ExternalModel, Models.ROM

- Since the creation of this test, the following main revisions have been performed:

1. revision info:

- author : alfoa

- date : 2018-08-25

- description: Added the test for testing the mitigation for the issue \#704

2. revision info:

- author : alfoa

- date : 2019-03-07

- description: Modified syntax in Functions as for issue \#934

\subsubsection{FRAMEWORK SAMPLERS ADAPTIVELIMITSURFACESEARCH.LIMIT SUR- FACE 1 DIMENSIONAL}

This test can be found at ". / raven/tests / framework/Samplers/AdaptiveLimitSurfaceSearcl test_adaptive_sampler_1_d_limit_surface.xml”. This test can be called executing the following command: 
./run_tests --re=framework/Samplers/AdaptiveLimitSurfaceSearch.lilmit_surfa

or

./run_framework_tests --re=framework/Samplers/AdaptiveLimitSurfadeSearch.1

- Test Description:

- This test is aimed to test the capability of RAVEN to employ a goal oriented sampling. It tests the LimitSurfaceSearch algorithm using an external model as "system code". In addition it ensures that a 1Dimensional Limit surface can be generated (1 uncertainty).

- Original Author:

- alfoa

- Creation date:

- 2018-11-29

- The classes tested in this test are:

- Samplers.LimitSurfaceSearch, Models.ExternalModel, Models.ROM

- Since the creation of this test, the following main revisions have been performed:

1. revision info:

- author : alfoa

- date : 2018-11-29

- description: New test for addressing issue \#864

2. revision info:

- author : alfoa

- date : 2019-03-07

- description: Modified syntax in Functions as for issue \#934

\subsubsection{FRAMEWORK SAMPLERS DYNAMICEVENTTREES.TESTCLASSICDETSHORTRAVEN}

This test can be found at ". / raven/tests / framework/Samplers / DynamicEvent Trees/ testDETshort. xml". This test can be called executing the following command:

./run_tests --re=framework/Samplers/DynamicEventTrees.testClassigDETshortR 
or

./run_framework_tests --re=framework/Samplers/DynamicEventTrees.testclassi

- Test Description:

- An example of using the Dynamic Event Tree Methodology with an external Code (RELAP7-like in this case). This test is a fast executing one that is aimed to check the mechanics of the DET methodology.

- Original Author:

- alfoa

- Creation date:

- 2015-10-05

- The classes tested in this test are:

- Samplers.DynamicEventTree, Models.Code

- Since the creation of this test, the following main revisions have been performed:

1. revision info:

- author : maljdan

- date : 2016-04-06

- description: Updating test cases to reflect the changes to the user input

2. revision info:

- author : talbpaul

- date : 2016-04-26

- description: Added check for pre-existing backup files when validating

3. revision info:

- author : alfoa

- date : 2016-04-27

- description: New subdirectories structure

4. revision info:

- author : maljdan

- date : 2016-08-03

- description: Removing PrintCSV PostProcessor from the DET test cases.

5. revision info: 
- author : maljdan

- date : 2016-08-08

- description: Fixing DET test case.

6. revision info:

- author : alfoa

- date : 2016-10-20

- description: Modified XSD and removed dim attribute from all the tests

7. revision info:

- author : alfoa

- date : 2017-01-21

- description: Adding this test description.

8. revision info:

- author : alfoa

- date : 2018-01-24

- description: Modification for new DataObject and new option to specify printing and plotting subdirectories (attribute "dir")

9. revision info:

- author : talbpaul

- date : 2018-01-26

- description: Added maxqueuesize 1 to be consistent with historical behavior

\subsubsection{FRAMEWORK SAMPLERS DYNAMICEVENTTREES.TESTHYBRIDDETSHORTRAVEN}

This test can be found at ". / raven/tests / framework/Samplers/DynamicEventTrees / testHybridDET.xml". This test can be called executing the following command:

. Irun_tests --re=framework/Samplers/DynamicEventTrees.testHybridDETshortRA

or

. Irun_framework_tests --re=framework/Samplers/DynamicEventTrees. testHybridl

- Test Description:

- An example of using the Dynamic Event Tree Methodology (Hybrid Dynamic Event Tree) with an external Code (RELAP7-like in this case). This test is a fast executing one that is aimed to check the mechanics of the DET methodology, when epistemic uncertanties need to be modeled. 
- Original Author:

- alfoa

- Creation date:

- 2015-10-05

- The classes tested in this test are:

- Samplers.DynamicEventTree, Models.Code

- Since the creation of this test, the following main revisions have been performed:

1. revision info:

- author : cogljj

- date : 2016-03-21

- description: Fixing dynamic event tree RELAP-7 inputs.

2. revision info:

- author : maljdan

- date : 2016-04-06

- description: Updating test cases to reflect the changes to the user input.

3. revision info:

- author : talbpaul

- date : 2016-04-26

- description: Added check for pre-existing backup files when validating

4. revision info:

- author: alfoa

- date : 2016-04-27

- description: New subdirectories structure

5. revision info:

- author : maljdan

- date : 2016-08-03

- description: Removing PrintCSV PostProcessor from the DET test cases.

6. revision info:

- author : alfoa

- date : 2016-10-20

- description: Modified XSD and removed dim attribute from all the tests

7. revision info: 
- author : alfoa

- date : 2017-01-21

- description: Adding this test description.

\subsubsection{FRAMEWORK TESTADAPTIVEDYNAMICEVENTTREERAVEN}

This test can be found at ". / raven/tests / framework/Samplers / DynamicEventTrees / test_adaptive_det_simple.xml". This test can be called executing the following command:

. /run_tests --re=framework/testAdaptiveDynamicEventTreeRAVEN

or

./run_framework_tests --re=framework/testAdaptiveDynamicEventTreeRAVEN

- Test Description:

- This test is aimed to test the capability of RAVEN to employ the Adaptive Dynamic Event Tree Sampling strategy (using RELAP7 as system code)

- Original Author:

-@alfoa

- Creation date:

$-2015-01-26$

- The classes tested in this test are:

- Samplers.AdaptiveDET

- Since the creation of this test, the following main revisions have been performed:

1. revision info:

_ author : @alfoa

- date : 2016-04-27

- description: Updating the test in order to accept the new subdirectories' structure

2. revision info:

- author : alfoa

- date : 2019-03-07

- description: Modified syntax in Functions as for issue \#934 


\subsubsection{FRAMEWORK TESTADAPTIVEHYBRIDDYNAMICEVENTTREERAVEN}

This test can be found at ". / raven/tests / framework/Samplers / DynamicEventTrees / test_adaptive_hybrid_det.xml". This test can be called executing the following command:

. /run_tests --re=framework/testAdaptiveHybridDynamicEventTreeRAVEN

or

./run_framework_tests --re=framework/testAdaptiveHybridDynamicEvent TreeRAV

- Test Description:

- This test is aimed to test the capability of RAVEN to employ the Adaptive Hybrid Dynamic Event Tree Sampling strategy (using RELAP7 as system code)

- Original Author:

-@alfoa

- Creation date:

$-2015-01-26$

- The classes tested in this test are:

- Samplers.AdaptiveDET

- Since the creation of this test, the following main revisions have been performed:

1. revision info:

- author: @alfoa

- date : 2016-04-27

- description: Updating the test in order to accept the new subdirectories' structure

2. revision info:

- author : alfoa

- date : 2019-03-07

- description: Modified syntax in Functions as for issue \#934 


\subsubsection{FRAMEWORK SAMPLERS SOBOL SOBOL}

This test can be found at “. / raven/tests / framework/Samplers/Sobol/test_sobol_ sampler.xml". This test can be called executing the following command:

. /run_tests --re=framework/Samplers/Sobol/Sobol

or

./run_framework_tests --re=framework/Samplers/Sobol/Sobol

- Test Description:

- This tests using the Sobol static sampler with the basic syntax.

- Original Author:

- talbpaul

- Creation date:

- 2015-09-14

- The classes tested in this test are:

- Samplers.Sobol

\subsubsection{FRAMEWORK SAMPLERS.ADAPTIVEMONTECARLO}

This test can be found at ". / raven/tests / framework/Samplers / AdaptiveMonteCarlo/ test_adaptive_MC_sampler.xml”. This test can be called executing the following command:

./run_tests --re=framework/Samplers.AdaptiveMonteCarlo

or

./run_framework_tests --re=framework/Samplers.AdaptiveMonteCarlo

- Test Description:

- This tests the capability of RAVEN to use the AdaptiveMonteCarlo sampler. Converged on expectedValue of y1 and y2, as well as the sigma of y1 and y2. 
- Original Author:

- ZHOUJ2

- Creation date:

- 2020-02-06

- The classes tested in this test are:

- Samplers.AdaptiveMonteCarlo, Models.ExternalModel

\subsubsection{FRAMEWORK MCMC.METROPOLISOBSERVED}

This test can be found at ". / raven/tests/framework/MCMC/test_mh_with_observations . xml". This test can be called executing the following command:

./run_tests --re=framework/MCMC.Metropolisobserved

or

./run_framework_tests --re=framework/MCMC.Metropolisobserved

- Test Description:

- Test the Markov Chain Monte Carlo algorithm: Metropolis Sampling assume xi, yi-rho $\operatorname{Normal}(\mathrm{mu}, \mathrm{cov})$, where $\mathrm{mu}=[0,0]$, and $\operatorname{cov}=[[1.0$, rho], [rho, 1.0]] 1000 samples is generated using np.random.multivariate normal as the observed data. A non-informative prior for covariance matrices is the Jeffreys prior (see Gelman et al., 1995), which is of the form $1 / \operatorname{det}(\mathrm{Cov})^{* *}(3 / 2)$. A uniform distribution $[-0.07,0.07]$ is used as the proposal distribution.

- Original Author:

- wangc

- Creation date:

- 2020-07-27

- The classes tested in this test are:

- MCMC.Metropolis 


\subsubsection{FRAMEWORK MCMC.METROPOLISONEDIM}

This test can be found at ". / raven/tests/framework/MCMC/test_1D.xml". This test can be called executing the following command:

. /run_tests --re=framework/MCMC.MetropolisoneDim

or

./run_framework_tests --re=framework/MCMC.MetropolisoneDim

- Test Description:

- Test the Markov Chain Monte Carlo algorithm: Metropolis Sampling Test to see if there is any degradation in Metropolis Sampling A large number (80000) of samples is used to test the efficiency of Metropolis Sampler. It takes about 550 second in Mac machine. If the time to run this test exceed 700s, some investigation should be conducted to resolve the issue. Heavy test: 550s on Mac, set MaxTime to 700s

- Original Author:

- wangc

- Creation date:

- 2020-12-20

- The classes tested in this test are:

- MCMC.Metropolis

\subsubsection{FRAMEWORK MCMC.METROPOLISONEDIMTUNE}

This test can be found at "./raven/tests/framework/MCMC/test_1D_tune.xml". This test can be called executing the following command:

. Irun_tests --re=framework/MCMC.MetropolisoneDimTune

or

./run_framework_tests --re=framework/MCMC.MetropolisoneDimTune

- Test Description: 
- Test the Markov Chain Monte Carlo algorithm: Metropolis Sampling specifically testing the auto tuning of scaling parameter with provided tune interval.

- Original Author:

- wangc

- Creation date:

- 2020-12-20

- The classes tested in this test are:

- MCMC.Metropolis

\subsubsection{FRAMEWORK MCMC.METROPOLISTENDIM}

This test can be found at ". / raven/tests/framework/MCMC/test_10D.xml". This test can be called executing the following command:

. /run_tests --re=framework/MCMC.MetropolisTenDim

Or

./run_framework_tests --re=framework/MCMC.MetropolisTenDim

- Test Description:

- Test the Markov Chain Monte Carlo algorithm: Metropolis Sampling for high-dimensional input space (10-dim): likelihood function: N-Dimensional Gaussian distribution with mu $\operatorname{Normal}(0,10)$, analytical mean values: [-3.23840979, 4.32107943, 0.18209698, 5.10287563, - 2.01392171, 10.67490632, -0.26232032, 5.92550919, 7.70044106, 6.86019041] var LogNormal(0, 1.5), analytical std values: [ 1.58121149, 1.30760725, 0.25210521, 4.22805349, 2.75537484, 15.26527159, 0.16109538, 1.0258734, 0.0772615, 4.86960071] Prior on mean is $\mathrm{U}(-500,500)$

- Original Author:

- wangc

- Creation date:

$-2020-12-20$

- The classes tested in this test are:

- MCMC.Metropolis 


\subsubsection{FRAMEWORK MCMC.METROPOLISTENDIMMVN}

This test can be found at "./raven/tests/framework/MCMC/test_10D_mvn.xml". This test can be called executing the following command:

. Irun_tests --re=framework/MCMC.MetropolisTenDimMVN

or

./run_framework_tests --re=framework/MCMC.Metropolis TenDimMVN

- Test Description:

- Test the Markov Chain Monte Carlo algorithm: Adaptive Metropolis Sampling likelihood function: N-Dimensional Gaussian distribution with input space (10-dim): mu $\operatorname{Normal}(0,10)$, analytical mean values: [-3.23840979, 4.32107943, 0.18209698, $5.10287563,-2.01392171,10.67490632,-0.26232032,5.92550919,7.70044106,6.86019041]$ var $\operatorname{LogNormal}(0,1.5)$, analytical std values: [ $1.58121149,1.30760725,0.25210521$, 4.22805349, 2.75537484, 15.26527159, 0.16109538, 1.0258734, 0.0772615, 4.86960071] Prior on mean is $\mathrm{U}(-500,500)$

- Original Author:

- wangc

- Creation date:

- 2020-12-20

- The classes tested in this test are:

- MCMC.Metropolis

\subsubsection{FRAMEWORK MCMC.METROPOLISTENDIMPROPMVN}

This test can be found at "./raven/tests/framework/MCMC/test_10D_proposal_ mvn. xml". This test can be called executing the following command:

. /run_tests --re=framework/MCMC.MetropolisTenDimPropMVN

or

. /run_framework_tests --re=framework/MCMC.MetropolisTenDimPropMVN 
- Test Description:

- Test the Markov Chain Monte Carlo algorithm: Adaptive Metropolis Sampling likelihood function: N-Dimensional Gaussian distribution with input space (10-dim): mu Normal(0, 10), analytical mean values: [-3.23840979, 4.32107943, 0.18209698, 5.10287563, - 2.01392171, 10.67490632, -0.26232032, 5.92550919, 7.70044106, 6.86019041] var $\operatorname{LogNormal}(0,1.5)$, analytical std values: [ 1.58121149, 1.30760725, 0.25210521, 4.22805349, 2.75537484, 15.26527159, 0.16109538, 1.0258734, 0.0772615, 4.86960071] Prior on mean is $\mathrm{U}(-500,500)$

- Original Author:

- wangc

- Creation date:

- 2020-12-20

- The classes tested in this test are:

- MCMC.Metropolis

\subsubsection{FRAMEWORK MCMC.ADAPTIVEMETROPOLIS}

This test can be found at ". / raven/tests/framework/MCMC/test_amh.xml". This test can be called executing the following command:

./run_tests --re=framework/MCMC.AdaptiveMetropolis

or

./run_framework_tests --re=framework/MCMC.AdaptiveMetropolis

- Test Description:

- Test the Markov Chain Monte Carlo algorithm: Adaptive Metropolis Sampling A 2D multivariate normal distribution is used as the likelihood function. mean: [5, 5], $\operatorname{cov}=[[1,0.9],[0.9,1]]$ Both input parameters have the standard normal distribution as their prior distribution. The proposal distributions for the input variables are also standard normal distribution. "TargetEvaluation" is used to collect the inputs and outputs from the likelihood model. "SolutionExport" is used to store the posterior values for the input variables, i.e. "xin" and "yin"

- Original Author: 
- wangc

- Creation date:

- 2020-12-20

- The classes tested in this test are:

- MCMC.Metropolis

\subsubsection{FRAMEWORK MCMC.ADAPTIVEMETROPOLISMVN}

This test can be found at "./raven/tests/framework/MCMC/test_amh_mvn.xml". This test can be called executing the following command:

. Irun_tests --re=framework/MCMC.AdaptiveMetropolisMVN

Or

./run_framework_tests --re=framework/MCMC.AdaptiveMetropolisMVN

- Test Description:

- Test the Markov Chain Monte Carlo algorithm: Adaptive Metropolis Sampling A 2D multivariate normal distribution is used as the likelihood function. mean: $[5,5]$, $\operatorname{cov}=[[1,0.9],[0.9,1]]$ Input parameters have the multivariate normal distribution as their prior distribution. The proposal distributions for the input variables are also standard normal distribution. "TargetEvaluation" is used to collect the inputs and outputs from the likelihood model. "SolutionExport" is used to store the posterior values for the input variables, i.e. "xin" and "yin"

- Original Author:

- wangc

- Creation date:

- 2020-12-20

- The classes tested in this test are:

- MCMC.Metropolis 


\subsubsection{FRAMEWORK MCMC.ADAPTIVEMETROPOLISPROPMVN}

This test can be found at “./raven/tests/framework/MCMC/test_amh_prop_mvn. xml". This test can be called executing the following command:

. Irun_tests --re=framework/MCMC.AdaptiveMetropolisPropMVN

or

./run_framework_tests --re=framework/MCMC.AdaptiveMetropolisPropMVN

- Test Description:

- Test the Markov Chain Monte Carlo algorithm: Adaptive Metropolis Sampling A 2D multivariate normal distribution is used as the likelihood function. mean: [5, 5], $\operatorname{cov}=[[1,0.9],[0.9,1]]$ Both input parameters have the standard normal distribution as their prior distribution. The proposal distributions for the input variables multivariate normal distribution. "TargetEvaluation" is used to collect the inputs and outputs from the likelihood model. "SolutionExport" is used to store the posterior values for the input variables, i.e. "xin" and "yin"

- Original Author:

- wangc

- Creation date:

- 2020-12-20

- The classes tested in this test are:

- MCMC.Metropolis

\subsubsection{FRAMEWORK PCA LHS LHSVALUE}

This test can be found at “. / raven/tests / framework/pca_LHS/test_LHS_pca_value. xml". This test can be called executing the following command:

. Irun_tests --re=framework/pca_LHS/LHSValue

or

./run_framework_tests --re=framework/pca_LHS/LHSValue 
- Test Description:

- Tests use of the PCA method to sample in LHS surrogate input space, with grid constructed by value.

- Original Author:

- wangc

- Creation date:

- 2015-11-18

- The classes tested in this test are:

- Distributions.MultivariateNormal,Samplers.Stratified,PostProcessors.BasicStatistics

- Since the creation of this test, the following main revisions have been performed:

1. revision info:

- author: wangc

- date : 2017-12-20

- description: convert test to use the new DataObjects with the new structure of basic statistic

\subsubsection{FRAMEWORK PCA LHS LHSCDF}

This test can be found at ". / raven/tests / framework/pca_LHS/test_LHS_pca_cdf . xml". This test can be called executing the following command:

./run_tests --re=framework/pca_LHS/LHSCdf

or

./run_framework_tests --re=framework/pca_LHS/LHSCdf

- Test Description:

- Tests use of the PCA method to sample in LHS surrogate input space, with grid constructed via $\mathrm{CDF}$.

- Original Author:

- wangc 
- Creation date:

- 2015-11-18

- The classes tested in this test are:

- Distributions.MultivariateNormal,Samplers.Stratified,PostProcessors.BasicStatistics

- Since the creation of this test, the following main revisions have been performed:

1. revision info:

- author: wangc

- date : 2017-12-20

- description: convert test to use the new DataObjects with the new structure of basic statistic

\subsubsection{FRAMEWORK PCA LHS STRATIFIEDVALUE}

This test can be found at "./raven/tests/framework/pca_LHS/test_stratified_ pca_value.xml". This test can be called executing the following command:

./run_tests --re=framework/pca_LHS/stratifiedValue

or

./run_framework_tests --re=framework/pca_LHS/stratifiedValue

- Test Description:

- Tests use of the PCA method to sample in LHS surrogate input space with grid by value.

- Original Author:

- wangc

- Creation date:

- 2015-11-18

- The classes tested in this test are:

- Distributions.MultivariateNormal,Samplers.Stratified 


\subsubsection{FRAMEWORK PCA LHS STRATIFIEDCDF}

This test can be found at ". / raven/tests/framework/pca_LHS/test_stratified_ pca_cdf.xml". This test can be called executing the following command:

./run_tests --re=framework/pca_LHS/stratifiedCdf

or

./run_framework_tests --re=framework/pca_LHS/stratifiedCdf

- Test Description:

- Tests use of the PCA method to sample in LHS surrogate input space with grid by CDF.

- Original Author:

- wangc

- Creation date:

- 2015-11-18

- The classes tested in this test are:

- Distributions.MultivariateNormal,Samplers.Stratified

\subsubsection{FRAMEWORK PCA LHS LHS}

This test can be found at ". / raven/tests/framework/pca_LHS/test_LHS.xml". This test can be called executing the following command:

. /run_tests --re=framework/pca_LHS/LHS

or

./run_framework_tests --re=framework/pca_LHS/LHS

- Test Description:

- Tests use of the PCA method to sample in LHS surrogate input space.

- Original Author: 
- wangc

- Creation date:

- 2015-11-18

- The classes tested in this test are:

- Distributions.MultivariateNormal,Samplers.Stratified,PostProcessors.BasicStatistics

- Since the creation of this test, the following main revisions have been performed:

1. revision info:

- author: wangc

- date : 2017-12-20

- description: convert test to use the new DataObjects with the new structure of basic statistic

\subsubsection{FRAMEWORK PCA LHS LHSCORRELATED}

This test can be found at ". / raven/tests / framework/pca_LHS/test_LHS_correlated_ cdf . xml". This test can be called executing the following command:

. /run_tests --re=framework/pca_LHS/LHSCorrelated

or

./run_framework_tests --re=framework/pca_LHS/LHSCorrelated

- Test Description:

- Tests use of the PCA method to sample in LHS surrogate input space. The distributions are a multivariate normal.

- Original Author:

- wangc

- Creation date:

- 2015-11-18

- The classes tested in this test are:

- Distributions.MultivariateNormal,Samplers.Stratified,PostProcessors.BasicStatistics 
- Since the creation of this test, the following main revisions have been performed:

1. revision info:

- author: wangc

- date : 2017-12-20

- description: convert test to use the new DataObjects with the new structure of basic statistic

\subsubsection{FRAMEWORK PCA LHS LHSUNCORRELATED}

This test can be found at ". / raven/tests / framework/pca_LHS/test_LHS_uncorrelated_ cdf . xml". This test can be called executing the following command:

./run_tests --re=framework/pca_LHS/LHSuncorrelated

or

./run_framework_tests --re=framework/pca_LHS/LHSuncorrelated

- Test Description:

- Benchmark for test of the PCA method to sample in LHS surrogate input space.

- Original Author:

- wangc

- Creation date:

$-2015-11-18$

- The classes tested in this test are:

- Distributions.MultivariateNormal,Samplers.Stratified,PostProcessors.BasicStatistics

- Since the creation of this test, the following main revisions have been performed:

1. revision info:

- author: wangc

- date : 2017-12-20

- description: convert test to use the new DataObjects with the new structure of basic statistic 


\subsubsection{FRAMEWORK DATAOBJECTS.TEST OUTPUT POINT ATTRIBUTES}

This test can be found at ". / raven/tests/framework/DataObjects/pointSetAttributes . xml". This test can be called executing the following command:

./run_tests --re=framework/DataObjects.test_output_point_attributes

or

./run_framework_tests --re=framework/DataObjects.test_output_point_attribu

- Test Description:

- Test to check the functionalities of DataObjects preprocess the data applying simple operator (e.g. max value, average, min value, etc.), in the options XML node. In this case it tests the attributes for PointSet.

- Original Author:

- alfoa

- Creation date:

- 2018-02-01

- The classes tested in this test are:

- DataObjects.PointSet, Models.ExternalModel

\subsubsection{FRAMEWORK DATAOBJECTS.TEST OUTPUT ATTRIBUTES}

This test can be found at ". / raven/tests/framework/DataOb jects/historySetAttributes. xml". This test can be called executing the following command:

./run_tests --re=framework/DataObjects.test_output_attributes

or

./run_framework_tests --re=framework/Dataobjects.test_output_atthibutes

- Test Description: 
- Test to check the functionalities of DataObjects preprocess the data applying simple operator (e.g. max value, average, min value, etc.), in the options XML node. In this case it tests the attributes for HistorySet.

- Original Author:

- alfoa

- Creation date:

- 2018-02-01

- The classes tested in this test are:

- DataObjects.HistorySet, Models.ExternalModel

\subsubsection{FRAMEWORK DATAOBJECTS.TEST OUTPUT FROM DB POINT ATTRIBUTES}

This test can be found at ". / raven/tests / framework/DataObjects/pointSetFromDBAttribut xml". This test can be called executing the following command:

.Irun_tests --re=framework/Dataobjects.test_output_from_db_point_attribute

or

.Irun_framework_tests --re=framework/Dataobjects.test_output_from_db_point

- Test Description:

- Test to check the functionalities of DataObjects preprocess the data applying simple operator (e.g. max value, average, min value, etc.), in the options XML node. In this case it tests the attributes for PointSet coming from a Database.

- Original Author:

- alfoa

- Creation date:

- 2018-02-01

- The classes tested in this test are:

- DataObjects.PointSet, Models.ExternalModel 


\subsubsection{FRAMEWORK DATAOBJECTS.TEST OUTPUT FROM DB HISTORY ATTRIBUTES}

This test can be found at ". / raven/tests/framework/DataObjects/historySetFromDBAttribu xml". This test can be called executing the following command:

./run_tests --re=framework/DataObjects.test_output_from_db_history_attribu

or

./run_framework_tests --re=framework/DataObjects.test_output_fron_db_histo

- Test Description:

- Test to check the functionalities of DataObjects preprocess the data applying simple operator (e.g. max value, average, min value, etc.), in the options XML node. In this case it tests the attributes for HistorySet coming from a DataBase.

- Original Author:

- alfoa

- Creation date:

- 2018-02-01

- The classes tested in this test are:

- DataObjects.HistorySet, Models.ExternalModel

\subsubsection{FRAMEWORK DATAOBJECTS.DATAOBJECT METADATA}

This test can be found at “. /raven/tests/framework/DataObjects/test_DataObject_ metadata.xml". This test can be called executing the following command:

./run_tests --re=framework/DataObjects.dataObject_metadata

or

./run_framework_tests --re=framework/DataObjects.dataObject_metadlata

- Test Description: 
- Test to check the functionalities of DataObjects.PointSet, when metadata need to be dumped out. In this test, the PointProbability generated during the employment of a sampling strategy is dumped in the CSV files, even if it is part of the metadata container.

- Original Author:

- maljdan

- Creation date:

- 2016-06-23

- The classes tested in this test are:

- DataObjects.PointSet

- Since the creation of this test, the following main revisions have been performed:

1. revision info:

- author : maljdan

- date : 2016-06-23

- description: Moving test case into a specific DataObjects folder

2. revision info:

- author : alfoa

- date : 2017-01-21

- description: Adding this test description.

\subsubsection{FRAMEWORK DATAOBJECTS.LOAD CSV}

This test can be found at "./raven/tests/framework/DataObjects/test_load_ CSV.xml". This test can be called executing the following command:

./run_tests --re=framework/Dataobjects.load_csv

or

./run_framework_tests --re=framework/DataObjects.load_csv

- Test Description:

- Test to check the functionalities of DataObjects.PointSet to be constructed by an extern input file (csv) 
- Original Author:

- maljdan

- Creation date:

- 2016-06-23

- The classes tested in this test are:

- DataObjects.PointSet

- Since the creation of this test, the following main revisions have been performed:

1. revision info:

- author : alfoa

- date : 2017-01-21

- description: Adding this test description.

\subsubsection{FRAMEWORK DATAOBJECTS.LOAD CSV HISTORY}

This test can be found at "./raven/tests/framework/DataObjects/test_load_ CSV_history.xml". This test can be called executing the following command:

. /run_tests --re=framework/Dataobjects.load_csv_history

or

./run_framework_tests --re=framework/Dataobjects.load_csv_history

- Test Description:

- Test to check the functionalities of DataObjects.HistorySet to be constructed by an extern input file (csv)

- Original Author:

- maljdan

- Creation date:

- 2016-06-23

- The classes tested in this test are: 
- DataObjects.HistorySet

- Since the creation of this test, the following main revisions have been performed:

1. revision info:

- author : alfoa

- date : 2017-01-21

- description: Adding this test description.

\subsubsection{FRAMEWORK DATAOBJECTS.LOAD CSV HISTORY}

This test can be found at ". / raven/tests / framework/DataObjects/load_csv_dataset. xml". This test can be called executing the following command:

./run_tests --re=framework/DataObjects.load_csv_history

or

./run_framework_tests --re=framework/Dataobjects.load_csv_history

- Test Description:

- Test to check the functionalities of DataObjects.DataSet to be constructed by an extern input file (csv)

- Original Author:

- maljdan

- Creation date:

- 2018-04-26

- The classes tested in this test are:

- DataObjects.DataSet

\subsubsection{FRAMEWORK DATAOBJECTS.LOAD TWO CSVS}

This test can be found at "./raven/tests/framework/DataObjects/test_load_ two_cSVs.xml". This test can be called executing the following command:

./run_tests --re=framework/DataObjects.load_two_csvs 
or

./run_framework_tests --re=framework/DataObjects.load_two_csvs

- Test Description:

- This test verifies that multiple files can be loaded into a single point set without destroying the information from each other.

- Original Author:

- maljdan

- Creation date:

- 2017-06-26

- The classes tested in this test are:

- DataObjects.PointSet

\subsubsection{FRAMEWORK DATAOBJECTS.LOAD TWO CSV HISTORIES}

This test can be found at "./raven/tests/framework/DataObjects/test_load_ two_csv_histories.xml". This test can be called executing the following command:

./run_tests --re=framework/DataObjects.load_two_csv_histories

or

./run_framework_tests --re=framework/DataObjects.load_two_csv_hi gtories

- Test Description:

- This test verifies that multiple csv files can be loaded into a single history set without destroying the information from each other.

- Original Author:

- maljdan

- Creation date:

- 2017-06-26

- The classes tested in this test are:

- DataObjects.HistorySet 


\subsubsection{FRAMEWORK DATAOBJECTS.LOAD TWO CSV HISTORIES FAIL}

This test can be found at “. / raven/tests/framework/DataObjects/load_two_csv_ histories_fail.xml". This test can be called executing the following command:

./run_tests --re=framework/Dataobjects.load_two_csv_histories_fail

or

./run_framework_tests --re=framework/DataObjects.load_two_csv_hi łtories_fa

- Test Description:

- The input variable "b" is missing in the second history set, replaced by "e", so the result dataobject fails to construct. This test checks the error received.

- Original Author:

- talbpaul

- Creation date:

$-2018-01-23$

- The classes tested in this test are:

- DataObjects.HistorySet

\subsubsection{FRAMEWORK DATAOBJECTS.LOAD PRINT LARGE NUM VARS}

This test can be found at "./raven/tests/framework/Dataobjects/test_large_ num_vars.xml". This test can be called executing the following command:

./run_tests --re=framework/Dataobjects.load_print_large_num_vars

or

./run_framework_tests --re=framework/DataObjects.load_print_large_num_vars

- Test Description:

- Test to check the performance of load and print of DataSet 
- Original Author:

- wangc

- Creation date:

- 2018-11-11

- The classes tested in this test are:

- DataObjects.DataSet

\subsubsection{FRAMEWORK DATAOBJECTS.LOAD PRINT LARGE NUM VARS HEAVY}

This test can be found at ". /raven/tests/framework/DataObjects/test_large_ num_vars_heavy.xml". This test can be called executing the following command:

./run_tests --re=framework/Dataobjects.load_print_large_num_vars_heavy

or

.Irun_framework_tests --re=framework/DataObjects.load_print_large_num_vars

- Test Description:

- Test to check the performance of load and print of DataSet

- Original Author:

- wangc

- Creation date:

- 2018-11-11

- The classes tested in this test are:

- DataObjects.DataSet 


\subsubsection{FRAMEWORK DATAOBJECTS.STRINGIO}

This test can be found at "./raven/tests/framework/DataObjects/string_io. xml". This test can be called executing the following command:

. /run_tests --re=framework/DataObjects.StringIO

or

. /run_framework_tests --re=framework/DataObjects.StringIO

- Test Description:

- Tests that strings can be part of the output space.

- Original Author:

- talbpaul

- Creation date:

- 2020-07-07

- The classes tested in this test are:

- DataObjects.DataSet

\subsubsection{FRAMEWORK VARIABLEGROUPS SETOPERATIONS}

This test can be found at ". / raven/tests/framework/VariableGroups/sets.xml". This test can be called executing the following command:

./run_tests --re=framework/VariableGroups/Setoperations

or

./run_framework_tests --re=framework/VariableGroups/Setoperation

- Test Description: 
- Tests complex VariableGroup construction using plus, minus, intersect, and symmetric difference operations. Uses these constructs in the DataObjects.

To test correct operation, check the headers of the output data objects for the right variable lists, both the names themeselves and the order of the columns.

Additional unit tests for VariableGroups are in the raven/tests/framework/unit_tests/utils/testXmlUtils.p file.

- Original Author:

- talbpaul

- Creation date:

- 2016-02-08

- The classes tested in this test are:

- VariableGroups

- Since the creation of this test, the following main revisions have been performed:

1. revision info:

- author : talbpaul

- date : 2018-09-25

- description: implicit nested group usage instead of explicit

2. revision info:

- author : talbpaul

- date : 2020-10-15

- description: empty vg with comment

\subsubsection{FRAMEWORK VARIABLEGROUPS ROM}

This test can be found at ". /raven/tests/framework/VariableGroups/rom.xml". This test can be called executing the following command:

./run_tests --re=framework/VariableGroups/ROM

or

./run_framework_tests --re=framework/VariableGroups/ROM

- Test Description: 
- tests variable groups when used as part of a ROM or external model

- Original Author:

- talbpaul

- Creation date:

- 2016-02-08

- The classes tested in this test are:

- VariableGroups

\subsubsection{FRAMEWORK VARIABLEGROUPS EXTERNALNODES}

This test can be found at ". / raven/tests/framework/VariableGroups/extnodes . xml". This test can be called executing the following command:

. /run_tests --re=framework/VariableGroups/ExternalNodes

Or

. /run_framework_tests --re=framework/VariableGroups/ExternalNodes

- Test Description:

- tests variable groups used in external XML

- Original Author:

- talbpaul

- Creation date:

- 2016-02-08

- The classes tested in this test are:

- VariableGroups 


\subsubsection{FRAMEWORK VARIABLEGROUPS ORDEREDVARIABLES}

This test can be found at "./raven/tests/framework/VariableGroups/ordered. xml". This test can be called executing the following command:

. /run_tests --re=framework/VariableGroups/OrderedVariables

or

. Irun_framework_tests --re=framework/VariableGroups/OrderedVariables

- Test Description:

- tests order preservation of variables in variable groups

- Original Author:

- talbpaul

- Creation date:

- 2016-02-08

- The classes tested in this test are:

- VariableGroups

\subsubsection{FRAMEWORK.TEST XSD INPUT DATA}

This test can be found at ". / raven/tests / framework/TestXSD/TestDataRead.py". This test can be called executing the following command:

./run_tests --re=framework.test_xsd_input_data

or

./run_framework_tests --re=framework.test_xsd_input_data

- Test Description:

- This test is aimed to check the functionality of the XSD python validator

- Original Author: 
$-\operatorname{cog} \mathrm{ljj}$

- Creation date:

- 2016-04-11

- The classes tested in this test are:

- None

- Since the creation of this test, the following main revisions have been performed:

1. revision info:

- author : cogljj

- date : 2016-04-12

- description: Adding a findFirst function to the xml reader.

2. revision info:

- author : cogljj

- date : 2016-04-12

- description: Renaming text to value in ParameterInput

3. revision info:

- author : cogljj

- date : 2016-07-05

- description: Add ability to run without lxml. The first checkes for lxml and skips the test if missing. The second checkes for lxml before running part of the test.

4. revision info:

- author : alfoa

- date : 2017-01-21

- description: Adding this test description.

5. revision info:

- author: alfoa

- date : 2018-01-31

- description: Modified for new dataobject and correct check of boolean types

\subsubsection{CROW.TEST NORMAL}

This test can be found at ". / raven/tests/framework/TestXSD/TestStrictCheck. py". This test can be called executing the following command: 
. Irun_tests --re=crow.test_normal

or

./run_framework_tests --re=crow.test_normal

- Test Description:

- This test is a Unit Test for the RAVEN input checker. This test is aimed to check that the input checker (Strict mode) is able to detect input errors.

- Original Author:

$-\operatorname{cogljj}$

- Creation date:

- 2017-08-30

- The classes tested in this test are:

- Since the creation of this test, the following main revisions have been performed:

1. revision info:

- author : alfoa

- date : 2018-05-15

- description: Adding this test description.

\subsubsection{FRAMEWORK.TEST XSD INPUT FAILS}

This test can be found at ". / raven/tests/framework/TestXSD/TestFails.py". This test can be called executing the following command:

./run_tests --re=framework.test_xsd_input_fails

or

./run_framework_tests --re=framework.test_xsd_input_fails

- Test Description: 
- This test is aimed to check the functionality of the XSD python validator (failure)

- Original Author:

$-\operatorname{cog} \mathrm{jj}$

- Creation date:

- 2016-04-11

- The classes tested in this test are:

- Since the creation of this test, the following main revisions have been performed:

1. revision info:

- author : alfoa

- date : 2017-01-21

- description: Adding this test description.

\subsubsection{FRAMEWORK PCA ADAPTIVE SGC TEST ADAPTIVE SGC PCA ANALYTIC}

This test can be found at "./raven/tests/framework/pca_adaptive_sgc/test_ adaptive_sgc_pca_analytic.xml”. This test can be called executing the following command:

.Irun_tests --re=framework/pca_adaptive_sgc/test_adaptive_sgc_pco__analytic

or

./run_framework_tests --re=framework/pca_adaptive_sgc/test_adaptîve_sgc_pc

- Test Description:

- Tests use of the PCA method for adaptive sparse grids on polynomial models.

- Original Author:

- wangc

- Creation date:

- 2015-11-30 
- The classes tested in this test are:

- Distributions.MultivariateNormal,Samplers.AdaptiveSparseGrid,SupervisedLearning.GaussPolynomi

- Since the creation of this test, the following main revisions have been performed:

1. revision info:

- author : talbpaul

- date : 2019-01-08

- description: moved from ROM-File to ROM-DataObject

\subsubsection{FRAMEWORK PCA ADAPTIVE SGC TEST ADAPTIVE SGC ATTENU COR- RELATION}

This test can be found at “./raven/tests/framework/pca_adaptive_sgc/test_ adaptive_sgc_attenu_correlation.xml". This test can be called executing the following command:

.Irun_tests --re=framework/pca_adaptive_sgc/test_adaptive_sgc_attenu_corre

or

.Irun_framework_tests --re=framework/pca_adaptive_sgc/test_adaptifve_sgc_at

- Test Description:

- Tests use of the PCA method for adaptive sparse grids on exponential decay models with nonzero covariance.

- Original Author:

- wangc

- Creation date:

- 2015-11-30

- The classes tested in this test are:

- Distributions.MultivariateNormal,Samplers.AdaptiveSparseGrid,SupervisedLearning.GaussPolynomi

- Since the creation of this test, the following main revisions have been performed:

1. revision info: 
- author : talbpaul

- date : 2019-01-08

- description: moving from ROM-file to ROM-dataobject

\subsubsection{FRAMEWORK ALIASSYSTEMTESTS.TESTROMALIASED}

This test can be found at "./raven/tests/framework/aliasSystemTests/test_ rom_aliased.xml". This test can be called executing the following command:

. Irun_tests --re=framework/aliasSystemTests.testROMAliased

Or

./run_framework_tests --re=framework/aliasSystemTests.testROMAliased

- Test Description:

- An example of using the the alias system in RAVEN with a ROM Model. This capability allows the user to alias Models variables within the RAVEN framework

- Original Author:

- alfoa

- Creation date:

- 2016-11-18

- The classes tested in this test are:

- Models.ROM, Models.AliasSystem

- Since the creation of this test, the following main revisions have been performed:

1. revision info:

- author : alfoa

- date : 2016-11-22

- description: Closes \#754

2. revision info:

- author : alfoa

- date : 2017-01-21

- description: Adding this test description. 


\subsubsection{FRAMEWORK ALIASSYSTEMTESTS.TESTEXTERNALMODELALIASED}

This test can be found at "./raven/tests/framework/aliasSystemTests/test_ external_model_aliased.xml". This test can be called executing the following command:

./run_tests --re=framework/aliasSystemTests.testExternalModelAliassed

or

./run_framework_tests --re=framework/aliasSystemTests.testExternalModelAli

- Test Description:

- An example of using the the alias system in RAVEN with an External Model. This capability allows the user to alias Models variables within the RAVEN framework

- Original Author:

- alfoa

- Creation date:

- 2016-11-18

- The classes tested in this test are:

- Models.ExternalModel, Models.AliasSystem

- Since the creation of this test, the following main revisions have been performed:

1. revision info:

- author : alfoa

- date : 2016-11-22

- description: Closes \#754

2. revision info:

- author: alfoa

- date : 2016-11-28

- description: Added XSD schema and Closes \#756

3. revision info:

- author : alfoa

- date : 2017-01-21 
- description: Adding this test description.

4. revision info:

- author : talbpaul

- date : 2018-04-04

- description: added awful.variable+name for hard-testing input alias

\subsubsection{FRAMEWORK ALIASSYSTEMTESTS.TESTENSEMBLEMODELALIASED}

This test can be found at "./raven/tests/framework/aliasSystemTests/test_ ensemble_model_aliased.xml". This test can be called executing the following command:

./run_tests --re=framework/aliasSystemTests.testEnsembleModelAliased

or

. /run_framework_tests --re=framework/aliasSystemTests.testEnsembleModelAli

- Test Description:

- An example of using the the alias system in RAVEN with an Ensemble Model. This capability allows the user to alias Models variables within the RAVEN framework

- Original Author:

- alfoa

- Creation date:

- 2016-11-30

- The classes tested in this test are:

- Models.EnsembleModel, Models.AliasSystem

- Since the creation of this test, the following main revisions have been performed:

1. revision info:

- author : alfoa

- date : 2017-01-21

- description: Adding this test description. 


\subsubsection{FRAMEWORK.DISTRIBUTIONS.CATEGORICAL}

This test can be found at ". /raven/tests/framework/Distributions/test_distributionsCat xml". This test can be called executing the following command:

. /run_tests --re=framework.Distributions.categorical

or

./run_framework_tests --re=framework.Distributions.categorical

- Test Description:

- This test is aimed to test the capability of RAVEN to use 1D Categorical distributions.

- Original Author:

- mandd

- Creation date:

- 2016-11-02

- The classes tested in this test are:

- Distributions.Categorical

- Since the creation of this test, the following main revisions have been performed:

1. revision info:

- author : alfoa

- date : 2017-01-21

- description: Adding this test description.

2. revision info:

- author: wangc

- date : 2017-12-20

- description: convert test to use the new DataObjects with the new structure of basic statistic

3. revision info:

- author: wangc

- date : 2018-12-18 
- description: In PR \#882, the standard errors on statistics moments are implemented, these quantities are stored in the pointwise metadata, and will be printed out in the output csv files by default. In order to avoid regolding this test, the optional node 'what' with text values 'input, output' is added. In this case, only the user requested variables as specified in the DataObjects are printed out.

\subsubsection{FRAMEWORK.DISTRIBUTIONS.LAPLACE}

This test can be found at ". / raven/tests / framework/Distributions/test_distributionsLar xml". This test can be called executing the following command:

. Irun_tests --re=framework. Distributions.laplace

or

./run_framework_tests --re=framework. Distributions.laplace

- Test Description:

- This test is aimed to test the capability of RAVEN to use 1D Laplace distributions.

- Original Author:

$-\operatorname{cog} l \mathrm{jj}$

- Creation date:

- 2017-04-26

- The classes tested in this test are:

- Distributions.Laplace

\subsubsection{FRAMEWORK.DISTRIBUTIONS.GEOMETRIC}

This test can be found at ". / raven/tests/framework/Distributions/test_distributionsGe xml". This test can be called executing the following command:

. Irun_tests --re=framework.Distributions.geometric

or

./run_framework_tests --re=framework. Distributions.geometric 
- Test Description:

- This test is aimed to test the capability of RAVEN to use 1D Geometric distribution.

- Original Author:

$-\operatorname{cog} \mathrm{jjj}$

- Creation date:

- 2017-04-26

- The classes tested in this test are:

- Distributions.Geometric

\subsubsection{FRAMEWORK.DISTRIBUTIONS.CATEGORICAL}

This test can be found at ". / raven/tests/framework/Distributions/test_distributionsMa xml". This test can be called executing the following command:

. /run_tests --re=framework.Distributions.categorical

or

./run_framework_tests --re=framework.Distributions.categorical

- Test Description:

- This test is aimed to test the capability of RAVEN to use 1D Markov Categorical distributions.

- Original Author:

- wangc

- Creation date:

- 2018-01-24

- The classes tested in this test are:

- Distributions.Markov 


\subsubsection{FRAMEWORK.DISTRIBUTIONS.LOGUNIFORM}

This test can be found at ". /raven/tests/framework/Distributions/test_distributionsLo xml". This test can be called executing the following command:

./run_tests --re=framework.Distributions.logUniform

or

./run_framework_tests --re=framework.Distributions.logUniform

- Test Description:

- This test checks the capability of RAVEN to use 1D LogUniform distributions (natural).

- Original Author:

- mandd

- Creation date:

- 2018-05-07

- The classes tested in this test are:

- Distributions.LogUniform

\subsubsection{FRAMEWORK.DISTRIBUTIONS.LOG10UNIFORM}

This test can be found at ". / raven/tests / framework/Distributions/test_distributionsLos xml". This test can be called executing the following command:

./run_tests --re=framework.Distributions.log10uniform

or

./run_framework_tests --re=framework.Distributions.log10Uniform

- Test Description:

- This test checks the capability of RAVEN to use 1D LogUniform distributions (decimal base). 
- Original Author:

- mandd

- Creation date:

- 2018-05-07

- The classes tested in this test are:

- Distributions.LogUniform

\subsubsection{FRAMEWORK.DISTRIBUTIONS.UNIFORMDISCRETE}

This test can be found at ". / raven/tests / framework/Distributions/test_distributionsUn xml". This test can be called executing the following command:

. Irun_tests --re=framework.Distributions.uniformDiscrete

Or

. /run_framework_tests --re=framework.Distributions.uniformDiscrete

- Test Description:

- This test checks the capability of RAVEN to use UniformDiscrete distribution (strategy=withReplacement).

- Original Author:

- mandd

- Creation date:

- 2020-06-05

- The classes tested in this test are:

- Distributions.UniformDiscrete 


\subsubsection{FRAMEWORK.DISTRIBUTIONS.UNIFORMDISCRETEWITHOUTREPLACEMENT}

This test can be found at "./raven/tests/framework/Distributions/test_distributionsun xml". This test can be called executing the following command:

./run_tests --re=framework. Distributions . uniformDiscreteWithoutReplacement

or

./run_framework_tests --re=framework.Distributions.uniformDiscreteWithoutR

- Test Description:

- This test checks the capability of RAVEN to use UniformDiscrete distribution (strategy=withoutReplacement)..

- Original Author:

- mandd

- Creation date:

- 2020-06-05

- The classes tested in this test are:

- Distributions.UniformDiscrete

\subsubsection{FRAMEWORK.DISTRIBUTIONS.UNIFORMDISCRETEWITHOUTREPLACEMENTSMAL}

This test can be found at "./raven/tests/framework/Distributions/test_distributionsUn xml". This test can be called executing the following command:

./run_tests --re=framework. Distributions . uniformDiscreteWithoutReplacement.

or

./run_framework_tests --re=framework.Distributions.uniformDiscreteWithoutR

- Test Description:

- This test checks the capability of RAVEN to use UniformDiscrete distribution (case where the number of variables linked to the UniformDiscrete distribution support cardinality). 
- Original Author:

- mandd

- Creation date:

- 2020-06-05

- The classes tested in this test are:

- Distributions.UniformDiscrete

\subsubsection{2ＦRAMEWORK.DISTRIBUTIONS.UNIFORMDISCRETEWITHOUTREPLACEMENTFLOAT}

This test can be found at ". / raven/tests/framework/Distributions/test_distributionsUn xml". This test can be called executing the following command:

. Irun_tests --re=framework.Distributions.uniformDiscreteWithoutReplacement

or

./run_framework_tests --re=framework.Distributions.uniformDiscreteWithoutR

- Test Description:

- This test checks the capability of RAVEN to use UniformDiscrete distribution when the support is float and not integer.

- Original Author:

- mandd

- Creation date:

$-2020-06-05$

- The classes tested in this test are:

- Distributions.UniformDiscrete 


\subsubsection{CROW.TEST IMPORT}

This test can be found at "./raven/tests/crow/test_import.py". This test can be called executing the following command:

. Irun_tests --re=crow.test_import

or

. Irun_framework_tests --re=crow.test_import

- Test Description:

- This test is a Unit Test for the crow swig classes. It tests the import of the crow library

- Original Author:

$-\operatorname{cog} \mathrm{ljj}$

- Creation date:

- 2017-03-24

- The classes tested in this test are:

- crow

- Since the creation of this test, the following main revisions have been performed:

1. revision info:

- author: alfoa

- date : 2018-05-15

- description: Adding this test description.

\subsubsection{CROW.TEST UTILS}

This test can be found at ". /raven/tests/crow/test_utils.py". This test can be called executing the following command:

. Irun_tests $--r e=c r o w . t e s t \_u t i l s$

or

./run_framework_tests --re=crow.test_utils 
- Test Description:

- This test is a Unit Test for the crow swig classes. It tests that the classes that are swigged in CROW are accessable by Python

- Original Author:

$-\operatorname{cogljj}$

- Creation date:

- 2017-03-24

- The classes tested in this test are:

- crow

- Since the creation of this test, the following main revisions have been performed:

1. revision info:

- author : alfoa

- date : 2018-05-15

- description: Adding this test description.

\subsubsection{CROW.TEST NORMAL}

This test can be found at "./raven/tests/crow/test_normal.py". This test can be called executing the following command:

. /run_tests --re=crow.test_normal

or

. /run_framework_tests --re=crow.test_normal

- Test Description:

- This test is a Unit Test for the crow swig classes. It tests that the normal distribution is accessable by Python

- Original Author:

- cogljj

- Creation date: 
- 2017-03-24

- The classes tested in this test are:

- crow

- Since the creation of this test, the following main revisions have been performed:

1. revision info:

- author : alfoa

- date : 2018-05-15

- description: Adding this test description.

\subsubsection{CROW.TEST LAPLACE}

This test can be found at ". /raven/tests/crow/test_laplace.py". This test can be called executing the following command:

. Irun_tests --re=crow.test_laplace

or

./run_framework_tests --re=crow.test_laplace

- Test Description:

- This test is a Unit Test for the crow swig classes. It tests that the laplace distribution is accessable by Python

- Original Author:

$-\operatorname{cog} \mathrm{ljj}$

- Creation date:

- 2017-03-24

- The classes tested in this test are:

- crow

- Since the creation of this test, the following main revisions have been performed:

1. revision info:

- author : alfoa

- date : 2018-05-15

- description: Adding this test description. 


\subsubsection{CROW.TEST GEOMETRIC}

This test can be found at ". / raven/tests/crow/test_geometric.py". This test can be called executing the following command:

. /run_tests --re=crow.test_geometric

or

. /run_framework_tests --re=crow.test_geometric

- Test Description:

- This test is a Unit Test for the crow swig classes. It tests that the geometric distribution is accessable by Python

- Original Author:

$-\operatorname{cog} \mathrm{ljj}$

- Creation date:

- 2017-03-24

- The classes tested in this test are:

- crow

- Since the creation of this test, the following main revisions have been performed:

1. revision info:

- author : alfoa

- date : 2018-05-15

- description: Adding this test description.

\subsubsection{CROW.TEST SVD}

This test can be found at ". / raven/tests/crow/test_eigen_svd.py". This test can be called executing the following command:

. /run_tests --re=crow.test_svd

or 
. Irun_framework_tests --re=crow.test_svd

- Test Description:

- This test is a Unit Test for the crow swig classes. It tests that the MultiVariate Normal distribution is accessable by Python and that the SVD on the covariance is performable in Python

- Original Author:

$-\operatorname{cog} l \mathrm{jj}$

- Creation date:

- 2017-03-24

- The classes tested in this test are:

- crow

- Since the creation of this test, the following main revisions have been performed:

1. revision info:

- author : alfoa

- date : 2018-05-15

- description: Adding this test description.

\subsubsection{CROW.TEST REDUCTION}

This test can be found at ". / raven/tests/crow/test_reduction.py". This test can be called executing the following command:

. Irun_tests --re=crow.test_reduction

or

./run_framework_tests --re=crow.test_reduction

- Test Description:

- This test is a Unit Test for the crow swig classes. It tests that the MultiVariate Normal distribution is accessable by Python and that the dimensionality reduction based on the covariance is correctly performed. 
- Original Author:

$-\operatorname{cog} \mathrm{ljj}$

- Creation date:

- 2017-03-24

- The classes tested in this test are:

- crow

- Since the creation of this test, the following main revisions have been performed:

1. revision info:

- author : alfoa

- date : 2018-05-15

- description: Adding this test description.

\subsubsection{CROW.TEST PCA INDEX}

This test can be found at “./raven/tests/crow/test_pca_index.py". This test can be called executing the following command:

./run_tests --re=crow.test_pca_index

or

./run_framework_tests --re=crow.test_pca_index

- Test Description:

- This test is a Unit Test for the crow swig classes. It tests that the MultiVariate Normal distribution is accessable by Python and that PCA back transformation is correctly implemented

- Original Author:

$-\operatorname{cog} \mathrm{ljj}$

- Creation date:

- 2017-03-24

- The classes tested in this test are: 
- crow

- Since the creation of this test, the following main revisions have been performed:

1. revision info:

- author : alfoa

- date : 2018-05-15

- description: Adding this test description.

\subsubsection{CROW.TEST MVN PCA}

This test can be found at "./raven/tests/crow/test_mvn_pca.py". This test can be called executing the following command:

. Irun_tests --re=crow.test_mvn_pca

or

./run_framework_tests --re=crow.test_mvn_pca

- Test Description:

- This test is a Unit Test for the crow swig classes. It tests that the MultiVariate Normal distribution is accessable by Python and that PCA is correctly performed and that the back transformation is correctly implemented

- Original Author:

$-\operatorname{cog} l \mathrm{lj}$

- Creation date:

$-2017-03-24$

- The classes tested in this test are:

- crow

- Since the creation of this test, the following main revisions have been performed:

1. revision info:

- author : alfoa

- date : 2018-05-15

- description: Adding this test description. 


\subsubsection{CROW.TEST INVERSEMARGINALFORPCA}

This test can be found at "./raven/tests/crow/test_inverseMarginalForPCA. py". This test can be called executing the following command:

. Irun_tests --re=crow.test_inverseMarginalforPCA

or

./run_framework_tests --re=crow.test_inverseMarginalforPCA

- Test Description:

- This test is a Unit Test for the crow swig classes. It tests that the MultiVariate Normal distribution is accessable by Python and that PCA is correctly performed and that the inverse marginal distribution is computable in the transformed space

- Original Author:

$-\operatorname{cog} \mathrm{ljj}$

- Creation date:

$-2017-03-24$

- The classes tested in this test are:

- crow

- Since the creation of this test, the following main revisions have been performed:

1. revision info:

- author : alfoa

- date : 2018-05-15

- description: Adding this test description.

\subsubsection{CROW.TEST CELLPROBABILITYWEIGHT}

This test can be found at "./raven/tests/crow/test_cellProbabilityWeight. py". This test can be called executing the following command:

./run_tests --re=crow.test_cellProbabilityWeight 
or

./run_framework_tests --re=crow.test_cellProbabilityWeight

- Test Description:

- This test is a Unit Test for the crow swig classes. It tests that the MultiVariate Normal distribution is accessable by Python and that the probability weights (cell probability) is correctly computed.

- Original Author:

$-\operatorname{cog} \mathrm{ljj}$

- Creation date:

- 2017-03-24

- The classes tested in this test are:

- crow

- Since the creation of this test, the following main revisions have been performed:

1. revision info:

- author : alfoa

- date : 2018-05-15

- description: Adding this test description.

\subsubsection{CROW.TEST MARGINALCDFFORPCA}

This test can be found at ". / raven/tests/crow/test_marginalCdfForPCA.py". This test can be called executing the following command:

. /run_tests --re=crow.test_marginalCdfForPCA

or

./run_framework_tests --re=crow.test_marginalCdfForPCA

- Test Description: 
- This test is a Unit Test for the crow swig classes. It tests that the MultiVariate Normal distribution is accessable by Python and that PCA is correctly performed and that the CDF marginal distribution is computable in the transformed space

- Original Author:

$-\operatorname{cog} l \mathrm{jj}$

- Creation date:

- 2017-03-24

- The classes tested in this test are:

- crow

- Since the creation of this test, the following main revisions have been performed:

1. revision info:

- author : alfoa

- date : 2018-05-15

- description: Adding this test description.

\subsubsection{CROW.TEST TRANSFORMATIONMATRIX}

This test can be found at ". / raven/tests / crow/test_transformationMatrix.py". This test can be called executing the following command:

. Irun_tests --re=crow.test_transformationMatrix

or

./run_framework_tests --re=crow.test_transformationMatrix

- Test Description:

- This test is a Unit Test for the crow swig classes. It tests that the MultiVariate Normal distribution is accessable by Python and that transformation matrix is accessable and correctly computed.

- Original Author:

$-\operatorname{cogljj}$ 
- Creation date:

$-2017-03-24$

- The classes tested in this test are:

- crow

- Since the creation of this test, the following main revisions have been performed:

1. revision info:

- author : alfoa

- date : 2018-05-15

- description: Adding this test description.

\subsubsection{CROW.TEST SVD INDEX}

This test can be found at “./raven/tests/crow/test_svd_index.py". This test can be called executing the following command:

./run_tests --re=crow.test_svd_index

or

./run_framework_tests --re=crow.test_svd_index

- Test Description:

- This test is a Unit Test for the crow swig classes. It tests that the MultiVariate Normal distribution is accessable by Python and that the SVD indexes are accessable

- Original Author:

$-\operatorname{cog} \mathrm{ljj}$

- Creation date:

$-2017-03-24$

- The classes tested in this test are:

- crow

- Since the creation of this test, the following main revisions have been performed: 
1. revision info:

- author : alfoa

- date : 2018-05-15

- description: Adding this test description.

\subsubsection{CROW.TEST TRANSFORMATIONMATRIX INDEX}

This test can be found at "./raven/tests/crow/test_transformationMatrix_index. py". This test can be called executing the following command:

./run_tests --re=crow.test_transformationMatrix_index

or

./run_framework_tests --re=crow.test_transformationMatrix_index

- Test Description:

- This test is a Unit Test for the crow swig classes. It tests that the MultiVariate Normal distribution is accessable by Python and that transformation matrix indexes are accessable and correctly reported.

- Original Author:

$-\operatorname{cog} l j \mathrm{j}$

- Creation date:

- 2017-03-24

- The classes tested in this test are:

- crow

- Since the creation of this test, the following main revisions have been performed:

1. revision info:

- author : alfoa

- date : 2018-05-15

- description: Adding this test description. 


\subsubsection{CROW.TEST INVERSE TRANSFORMATIONMATRIX}

This test can be found at "./raven/tests/crow/test_inverse_transformation_ matrix.py". This test can be called executing the following command:

. Irun_tests --re=crow.test_inverse_transformationMatrix

or

./run_framework_tests --re=crow.test_inverse_transformationMatrix

- Test Description:

- This test is a Unit Test for the crow swig classes. It tests that the MultiVariate Normal distribution is accessable by Python and that the inverse transformation matrix is correctly computed.

- Original Author:

$-\operatorname{cog} \mathrm{ljj}$

- Creation date:

- 2017-03-24

- The classes tested in this test are:

- crow

- Since the creation of this test, the following main revisions have been performed:

1. revision info:

- author : alfoa

- date : 2018-05-15

- description: Adding this test description.

\subsubsection{CROW.TEST INVERSE TRANSFORMATIONMATRIX}

This test can be found at "./raven/tests/crow/test_symmetric_psd.py". This test can be called executing the following command:

./run_tests --re=crow.test_inverse_transformationMatrix 
Or

./run_framework_tests --re=crow.test_inverse_transformationMatrix

- Test Description:

- This test is a Unit Test for the crow swig classes. It tests that the MultiVariate Normal distribution is accessable by Python and that the lef singular vectors are correctly reported

- Original Author:

$-\operatorname{cog} \mathrm{ljj}$

- Creation date:

$-2017-03-24$

- The classes tested in this test are:

- crow

- Since the creation of this test, the following main revisions have been performed:

1. revision info:

- author : alfoa

- date : 2018-05-15

- description: Adding this test description.

\subsubsection{DOC WORKSHOP OPTIMIZER INPUTS.OPTIMIZER}

This test can be found at "./raven/doc/workshop/optimizer/Inputs/1_optimize. xml". This test can be called executing the following command:

./run_tests --re=doc/workshop/optimizer/Inputs .optimizer

or

./run_framework_tests --re=doc/workshop/optimizer/Inputs.optimizer

- Test Description:

- Workshop test: test Optimizer capability 
- Original Author:

- wangc

- Creation date:

- 2018-09-21

- The classes tested in this test are:

- Samplers.Optimizer

\subsubsection{DOC WORKSHOP STATICDATAANALYSIS INPUTS.DMGM1}

This test can be found at "./raven/doc/workshop/staticDataAnalysis/inputs/ t.xml". This test can be called executing the following command:

./run_tests --re=doc/workshop/staticDataAnalysis/inputs.dmGM1

or

./run_framework_tests --re=doc/workshop/staticDataAnalysis/input\$.dmGM1

- Test Description:

- Workshop test: DataMining with Gaussian Process

- Original Author:

- wangc

- Creation date:

- 2018-09-21

- The classes tested in this test are:

- PostProcessors.DataMining

- Since the creation of this test, the following main revisions have been performed:

1. revision info:

- author: wangc

- date : 2018-09-27

- description: As reported in issue \#805, RAVEN will not allow input DataObject of PostProcessor to be output DataObject, the output data object will be renamed, and the variable name of labels will be explicitly defined. 


\subsubsection{DOC WORKSHOP STATICDATAANALYSIS INPUTS.DMICA}

This test can be found at "./raven/doc/workshop/staticDataAnalysis/inputs/ test_dataMiningExactICA.xml". This test can be called executing the following command:

./run_tests --re=doc/workshop/staticDataAnalysis/inputs.dmICA

or

./run_framework_tests --re=doc/workshop/staticDataAnalysis/input\$.dmICA

- Test Description:

- Workshop test: DataMining with ICA method

- Original Author:

- wangc

- Creation date:

- 2018-09-21

- The classes tested in this test are:

- PostProcessors.DataMining

- Since the creation of this test, the following main revisions have been performed:

1. revision info:

- author: wangc

- date : 2018-09-27

- description: As reported in issue \#805, RAVEN will not allow input DataObject of PostProcessor to be output DataObject, the output data object will be renamed, and the variable name of labels will be explicitly defined.

\subsubsection{DOC WORKSHOP STATICDATAANALYSIS INPUTS.DMICA}

This test can be found at "./raven/doc/workshop/staticDataAnalysis/inputs/ test_dataMiningExactPCA.xml". This test can be called executing the following command: 
./run_tests --re=doc/workshop/staticDataAnalysis/inputs.dmICA

or

./run_framework_tests --re=doc/workshop/staticDataAnalysis/input\$.dmICA

- Test Description:

- Workshop test: DataMining with ICA method

- Original Author:

- wangc

- Creation date:

- 2018-09-21

- The classes tested in this test are:

- PostProcessors.DataMining

- Since the creation of this test, the following main revisions have been performed:

1. revision info:

- author: wangc

- date : 2018-09-27

- description: As reported in issue \#805, RAVEN will not allow input DataObject of PostProcessor to be output DataObject, the output data object will be renamed, and the variable name of labels will be explicitly defined.

\subsubsection{DOC WORKSHOP STATICDATAANALYSIS INPUTS.DMGM2}

This test can be found at ". / raven/doc/workshop/staticDataAnalysis/inputs / test_dataMiningGaussianMixture.xml". This test can be called executing the following command:

. /run_tests --re=doc/workshop/staticDataAnalysis/inputs.dmGM2

or

./run_framework_tests --re=doc/workshop/staticDataAnalysis/input\$.dmGM2 
- Test Description:

- Workshop test: DataMining with Gaussian Mixture method

- Original Author:

- wangc

- Creation date:

- 2018-09-21

- The classes tested in this test are:

- PostProcessors.DataMining

- Since the creation of this test, the following main revisions have been performed:

1. revision info:

- author: wangc

- date : 2018-09-27

- description: As reported in issue \#805, RAVEN will not allow input DataObject of PostProcessor to be output DataObject, the output data object will be renamed, and the variable name of labels will be explicitly defined.

\subsubsection{DOC WORKSHOP STATICDATAANALYSIS INPUTS.KMEANS}

This test can be found at "./raven/doc/workshop/staticDataAnalysis/inputs/ test_dataMiningKMeans.xml". This test can be called executing the following command:

./run_tests --re=doc/workshop/staticDataAnalysis/inputs.KMeans

or

./run_framework_tests --re=doc/workshop/staticDataAnalysis/input\$.KMeans

- Test Description:

- Workshop test: DataMining with KMeans method

- Original Author:

- wangc

- Creation date: 
- 2018-09-21

- The classes tested in this test are:

- PostProcessors.DataMining

- Since the creation of this test, the following main revisions have been performed:

1. revision info:

- author: wangc

- date : 2018-09-27

- description: As reported in issue \#805, RAVEN will not allow input DataObject of PostProcessor to be output DataObject, the output data object will be renamed, and the variable name of labels will be explicitly defined.

\subsubsection{DOC WORKSHOP STATICDATAANALYSIS INPUTS.MEANSHIFT}

This test can be found at "./raven/doc/workshop/staticDataAnalysis/inputs/ test_dataMiningMeanShift_complete.xml". This test can be called executing the following command:

./run_tests --re=doc/workshop/staticDataAnalysis/inputs.MeanShift

or

. /run_framework_tests --re=doc/workshop/staticDataAnalysis/input\$.MeanShif

- Test Description:

- Workshop test: DataMining with MeanShift method

- Original Author:

- wangc

- Creation date:

- 2018-09-21

- The classes tested in this test are:

- PostProcessors.DataMining

- Since the creation of this test, the following main revisions have been performed: 
1. revision info:

- author: wangc

- date : 2018-09-27

- description: As reported in issue \#805, RAVEN will not allow input DataObject of PostProcessor to be output DataObject, the output data object will be renamed, and the variable name of labels will be explicitly defined.

\subsubsection{DOC WORKSHOP ADVANCEDRELIABILITY EXERCISES INPUTS.ADAPT}

This test can be found at ". /raven/doc/workshop/advancedReliability/exercises/ Inputs/1_adaptive_sampling.xml". This test can be called executing the following command:

./run_tests --re=doc/workshop/advancedReliability/exercises/Inputs. Adapt

or

./run_framework_tests --re=doc/workshop/advancedReliability/exerfises/Inpu

- Test Description:

- Workshop test: advanced reliability test using Limit Surface Search

- Original Author:

- wangc

- Creation date:

- 2018-09-21

- The classes tested in this test are:

- Sampler.LimitSurfaceSearch

\subsubsection{DOC WORKSHOP ADVANCEDRELIABILITY EXERCISES INPUTS.LS}

This test can be found at ". / raven/doc/workshop/advancedReliability/exercises/ Inputs/2_ls_probability_computation.xml". This test can be called executing the following command:

./run_tests --re=doc/workshop/advancedReliability/exercises/Inputs. LS 
or

./run_framework_tests --re=doc/workshop/advancedReliability/exercises/Inpu

- Test Description:

- Workshop test: advanced reliability test using Limit Surface Integral PostProcessor

- Original Author:

- wangc

- Creation date:

- 2018-09-21

- The classes tested in this test are:

- PostProcessor.LimitSurfaceIntegral

\subsubsection{DOC WORKSHOP ADVANCEDRELIABILITY INPUTS.DPRA}

This test can be found at ". / raven/doc/workshop/advancedReliability/inputs / dynamicPRA.xml". This test can be called executing the following command:

./run_tests --re=doc/workshop/advancedReliability/inputs.dPRA

or

./run_framework_tests --re=doc/workshop/advancedReliability/inputs.dPRA

- Test Description:

- Workshop test: advanced reliability test using dynamic PRA

- Original Author:

- wangc

- Creation date:

- 2018-09-21

- The classes tested in this test are: 
- None

- Since the creation of this test, the following main revisions have been performed:

1. revision info:

- author: wangc

- date : 2018-12-18

- description: In PR \#882, the standard errors on statistics moments are implemented, these quantities are stored in the pointwised metadata, and will be printed out in the output csv files by default. In order to avoid regolding this test, the optional node 'what' with text values 'input, output' is added. In this case, only the user requested variables as specified in the DataObjects are printed out.

\subsubsection{DOC WORKSHOP ADVANCEDRELIABILITY INPUTS.RUNLS}

This test can be found at ". / raven/doc/workshop/advancedReliability/inputs / runLS.xml". This test can be called executing the following command:

./run_tests --re=doc/workshop/advancedReliability/inputs.runLs

or

./run_framework_tests --re=doc/workshop/advancedReliability/inputs.runLS

- Test Description:

- Workshop test: advanced reliability test with Limit surface search

- Original Author:

- wangc

- Creation date:

- 2018-09-21

- The classes tested in this test are:

- None 


\subsubsection{DOC WORKSHOP ADVANCEDRELIABILITY INPUTS.RUNLS}

This test can be found at “. /raven/doc/workshop/advancedReliability/inputs/ pbFromLS . xml". This test can be called executing the following command:

./run_tests --re=doc/workshop/advancedReliability/inputs.runLS

or

./run_framework_tests --re=doc/workshop/advancedReliability/inputs.runLS

- Test Description:

- Workshop test: advanced reliability test with Limit surface integral postprocessor

- Original Author:

- wangc

- Creation date:

- 2018-09-21

- The classes tested in this test are:

- None

\subsubsection{DOC WORKSHOP ENSEMBLEMODELS INPUTS ENSEMBLEEXAMPLE.ENSEMBLE}

This test can be found at ". / raven/doc/workshop/ensembleModels/inputs/ensembleExample, 1_ensemble_model.xml". This test can be called executing the following command:

. /run_tests --re=doc/workshop/ensembleModels/inputs/ensembleExample. ensemb.

or

./run_framework_tests --re=doc/workshop/ensembleModels/inputs/en fembleExam

- Test Description:

- Workshop test: test EnsembleModel Capability

- Original Author: 
- wangc

- Creation date:

- 2018-09-21

- The classes tested in this test are:

- Models.EnsembleModel

\subsubsection{DOC WORKSHOP ENSEMBLEMODELS INPUTS HYBRIDEXAMPLE.HYBRID}

This test can be found at ". / raven/doc/workshop/ensembleModels / inputs / hybridExample/ test_hybrid_model.xml". This test can be called executing the following command:

./run_tests --re=doc/workshop/ensembleModels/inputs/hybridExample.hybrid

or

./run_framework_tests --re=doc/workshop/ensembleModels/inputs/hybridExampl

- Test Description:

- Workshop test: test HybridModel Capability

- Original Author:

- wangc

- Creation date:

- 2018-09-21

- The classes tested in this test are:

- Models.HybridModel

\subsubsection{DOC WORKSHOP STOCHASTICCOLLOCATION INPUTS.SCTRAIN1}

This test can be found at ". / raven/doc/workshop/stochasticCollocation/inputs / scgpc_trainer_1.xml”. This test can be called executing the following command:

./run_tests --re=doc/workshop/stochasticCollocation/inputs.scTrain1 
Or

./run_framework_tests --re=doc/workshop/stochasticCollocation/inquts.scTra.

- Test Description:

- Workshop test: using sthochastic collocation sampler to train and evaluate a Gaussian Polynomial ROM

- Original Author:

- wangc

- Creation date:

- 2018-09-21

- The classes tested in this test are:

- Models.ROM

- Since the creation of this test, the following main revisions have been performed:

1. revision info:

- author : talbpaul

- date : 2019-01-08

- description: moved from ROM- $i$ XML to ROM- $¿$ DataObject

\subsubsection{DOC WORKSHOP STOCHASTICCOLLOCATION INPUTS.SCTRAIN2}

This test can be found at ". /raven/doc/workshop/stochasticCollocation/inputs/ scgpc_trainer_2.xml". This test can be called executing the following command:

./run_tests --re=doc/workshop/stochasticCollocation/inputs.scTrain2

or

./run_framework_tests --re=doc/workshop/stochasticCollocation/inputs.scTra

- Test Description:

- Workshop test: using sthochastic collocation sampler to train and evaluate a Gaussian Polynomial ROM 
- Original Author:

- wangc

- Creation date:

- 2018-09-21

- The classes tested in this test are:

- Models.ROM

- Since the creation of this test, the following main revisions have been performed:

1. revision info:

- author : talbpaul

- date : 2019-01-08

- description: moved from ROM- $\_$XML to ROM- $¿$ DataObject

\subsubsection{DOC WORKSHOP STOCHASTICCOLLOCATION INPUTS.SCTRAIN3}

This test can be found at ". / raven/doc/workshop/stochasticCollocation/inputs / scgpc_trainer_3.xml”. This test can be called executing the following command:

./run_tests --re=doc/workshop/stochasticCollocation/inputs.scTrain3

Or

./run_framework_tests --re=doc/workshop/stochasticCollocation/inputs.scTra.

- Test Description:

- Workshop test: using sthochastic collocation sampler to train and evaluate a Gaussian Polynomial ROM

- Original Author:

- wangc

- Creation date:

- 2018-09-21

- The classes tested in this test are: 
- Models.ROM

- Since the creation of this test, the following main revisions have been performed:

1. revision info:

- author : talbpaul

- date : 2019-01-08

- description: moved from ROM- $i X M L$ to ROM- $¿$ DataObject

\subsubsection{DOC WORKSHOP TIMEDEPDATAMINING INPUTS.BSTATT}

This test can be found at "./raven/doc/workshop/timeDepDataMining/inputs/ time_dep.xml". This test can be called executing the following command:

./run_tests --re=doc/workshop/timeDepDataMining/inputs.bstat T

or

./run_framework_tests --re=doc/workshop/timeDepDataMining/inputs.bstat $\mathrm{b}$

- Test Description:

- Workshop test: Time dependent basic statistics analysis

- Original Author:

- wangc

- Creation date:

- 2018-09-21

- The classes tested in this test are:

- PostProcessors.BasicStatistics

- Since the creation of this test, the following main revisions have been performed:

1. revision info:

- author: wangc

- date : 2018-12-18

- description: In PR \#882, the standard errors on statistics moments are implemented, these quantities are stored in the pointwised metadata, and will be printed out in the output csv files by default. In order to avoid regolding this test, the optional node 'what' with text values 'input, output' is added. In this case, only the user requested variables as specified in the DataObjects are printed out. 


\subsubsection{DOC WORKSHOP TIMEDEPDATAMINING INPUTS.KMEANST}

This test can be found at "./raven/doc/workshop/timeDepDataMining/inputs/ timeSliceKMeans. xml". This test can be called executing the following command:

./run_tests --re=doc/workshop/timeDepDataMining/inputs.KMeansT

or

./run_framework_tests --re=doc/workshop/timeDepDataMining/inputs.KMeanst

- Test Description:

- Workshop test: Time dependent data mining with KMeans analysis

- Original Author:

- wangc

- Creation date:

- 2018-09-21

- The classes tested in this test are:

- PostProcessors.DataMining

- Since the creation of this test, the following main revisions have been performed:

1. revision info:

- author: wangc

- date : 2018-09-27

- description: As reported in issue \#805, RAVEN will not allow input DataObject of PostProcessor to be output DataObject, the output data object will be renamed, and the variable name of labels will be explicitly defined.

\subsubsection{DOC WORKSHOP TIMEDEPDATAMINING INPUTS.DTW}

This test can be found at "./raven/doc/workshop/timeDepDataMining/inputs/ TD_hierarchical_dtw.xml". This test can be called executing the following command:

./run_tests --re=doc/workshop/timeDepDataMining/inputs.DTW 
or

./run_framework_tests --re=doc/workshop/timeDepDataMining/inputs.DTW

- Test Description:

- Workshop test: Time dependent data mining with DTW metric analysis

- Original Author:

- wangc

- Creation date:

- 2018-09-21

- The classes tested in this test are:

- PostProcessors.DataMining, Metrics.DTW

- Since the creation of this test, the following main revisions have been performed:

1. revision info:

- author : wangc

- date : 2018-09-27

- description: As reported in issue \#805, RAVEN will not allow input DataObject of PostProcessor to be output DataObject, the output data object will be renamed, and the variable name of labels will be explicitly defined.

\subsubsection{DOC WORKSHOP REDUCEDORDERMODELING INPUTS EXAMPLES.SAMPLE}

This test can be found at ". / raven/doc/workshop/reducedOrderModeling/inputs / examples/1_sample_Function.xml". This test can be called executing the following command:

./run_tests --re=doc/workshop/reducedOrderModeling/inputs/examples.sample or

./run_framework_tests --re=doc/workshop/reducedorderModeling/inpyts/exampl

- Test Description: 
- Workshop test: simple sampler test

- Original Author:

- wangc

- Creation date:

- 2018-09-21

- The classes tested in this test are:

- None

\subsubsection{DOC WORKSHOP REDUCEDORDERMODELING INPUTS EXAMPLES.TRAIN}

This test can be found at ". / raven/doc/workshop/reducedOrderModeling/inputs / examples/2_train_rom.xml". This test can be called executing the following command:

./run_tests --re=doc/workshop/reducedOrderModeling/inputs/examples.train

or

./run_framework_tests --re=doc/workshop/reducedorderModeling/inpyts/exampl

- Test Description:

- Workshop test: train a ROM

- Original Author:

- wangc

- Creation date:

- 2018-09-21

- The classes tested in this test are:

- Models.ROM 


\subsubsection{DOC WORKSHOP REDUCEDORDERMODELING INPUTS EXAMPLES.PICKLE}

This test can be found at ". / raven/doc/workshop/reducedorderModeling/inputs / examples/3_pickle_rom.xml". This test can be called executing the following command:

./run_tests --re=doc/workshop/reducedorderModeling/inputs/examples.pickle

or

./run_framework_tests --re=doc/workshop/reducedorderModeling/inpyts/exampl

- Test Description:

- Workshop test: pickle a ROM

- Original Author:

- wangc

- Creation date:

- 2018-09-21

- The classes tested in this test are:

- Models.ROM

\subsubsection{DOC WORKSHOP REDUCEDORDERMODELING INPUTS EXAMPLES.LOAD}

This test can be found at ". / raven/doc/workshop/reducedorderModeling/inputs / examples/4_load_rom.xml". This test can be called executing the following command:

./run_tests --re=doc/workshop/reducedorderModeling/inputs/examples. load

or

./run_framework_tests --re=doc/workshop/reducedorderModeling/inpyts/exampl

- Test Description:

- Workshop test: load a pickled ROM

- Original Author: 
- wangc

- Creation date:

- 2018-09-21

- The classes tested in this test are:

- Models.ROM

\subsubsection{DOC WORKSHOP REDUCEDORDERMODELING INPUTS EXAMPLES.SROM}

This test can be found at ". / raven/doc/workshop/reducedorderModeling/inputs / examples/5_sample_rom.xml". This test can be called executing the following command:

. /run_tests --re=doc/workshop/reducedOrderModeling/inputs/examples.srom

or

./run_framework_tests --re=doc/workshop/reducedorderModeling/inpyts/exampl

- Test Description:

- Workshop test: sample a trained rom

- Original Author:

- wangc

- Creation date:

- 2018-09-21

- The classes tested in this test are:

- Models.ROM

\subsubsection{DOC WORKSHOP REDUCEDORDERMODELING INPUTS EXAMPLES.SPROM}

This test can be found at ". / raven/doc/workshop/reducedOrderModeling/inputs / examples/6_sample_prom.xml". This test can be called executing the following command:

./run_tests --re=doc/workshop/reducedOrderModeling/inputs/examples.sprom 
or

./run_framework_tests --re=doc/workshop/reducedorderModeling/inputs/exampl

- Test Description:

- Workshop test: sample a pickled rom

- Original Author:

- wangc

- Creation date:

- 2018-09-21

- The classes tested in this test are:

- Models.ROM

\subsubsection{DOC WORKSHOP REDUCEDORDERMODELING INPUTS EXAMPLES.EVAL}

This test can be found at ". / raven/doc/workshop/reducedorderModeling/inputs / examples/7_evaluate_rom.xml". This test can be called executing the following command:

./run_tests --re=doc/workshop/reducedorderModeling/inputs/examples s.eval

or

./run_framework_tests --re=doc/workshop/reducedorderModeling/inpyts/exampl

- Test Description:

- Workshop test: use rom for prediction

- Original Author:

- wangc

- Creation date:

- 2018-09-21

- The classes tested in this test are:

- Models.ROM 


\subsubsection{DOC WORKSHOP REDUCEDORDERMODELING INPUTS EXAMPLES.SC}

This test can be found at ". / raven/doc/workshop/reducedorderModeling/inputs / examples/9_sc_gpc.xml". This test can be called executing the following command:

./run_tests --re=doc/workshop/reducedorderModeling/inputs/examples.sc

or

./run_framework_tests --re=doc/workshop/reducedorderModeling/inputs/exampl

- Test Description:

- Workshop test: Using Stochastic Collocation to train a Gaussian Polynomial ROM

- Original Author:

- wangc

- Creation date:

- 2018-09-21

- The classes tested in this test are:

- Models.ROM

\subsubsection{DOC WORKSHOP CODECOUPLING EXERCISES.CODEINTERFACE}

This test can be found at ". /raven/doc/workshop/codeCoupling/exercises/sample_ new_code_interface.xml". This test can be called executing the following command:

./run_tests --re=doc/workshop/codeCoupling/exercises.codeInterfage

or

./run_framework_tests --re=doc/workshop/codeCoupling/exercises.codeInterfa

- Test Description:

- Workshop test: test code coupling with projectile model

- Original Author: 
- wangc

- Creation date:

- 2018-09-21

- The classes tested in this test are:

- Models.Code

\subsubsection{DOC WORKSHOP CODECOUPLING INPUT.GENERICINTERFACE}

This test can be found at ". / raven/doc/workshop/codeCoupling/inputs/example_ using_generic_interface.xml". This test can be called executing the following command:

./run_tests --re=doc/workshop/codeCoupling/input.genericInterface

or

./run_framework_tests --re=doc/workshop/codeCoupling/input.generilcInterfac

- Test Description:

- Workshop test: test code coupling with Projectile model using generic code interface

- Original Author:

- wangc

- Creation date:

- 2018-09-21

- The classes tested in this test are:

- Models.Code

\subsubsection{DOC WORKSHOP CODECOUPLING INPUT.BATEMANINTERFACE}

This test can be found at ". / raven/doc/workshop/codeCoupling/inputs / example_ using_new_interface.xml". This test can be called executing the following command:

./run_tests --re=doc/workshop/codeCoupling/input.batemanInterface 
or

./run_framework_tests --re=doc/workshop/codeCoupling/input.batemanInterfac

- Test Description:

- Workshop test: test code coupling with Projectile model using bateman code interface

- Original Author:

- wangc

- Creation date:

- 2018-09-21

- The classes tested in this test are:

- Models.Code

\subsubsection{FRAMEWORK CODEINTERFACETESTS ACCELERATEDCFD.PERTURBACFD1}

This test can be found at ". / raven/AcceleratedCFD/test_perturb_acdf.xml". This test can be called executing the following command:

./run_tests --re=framework/CodeInterfaceTests/AcceleratedCFD.perturbACFD1

or

./run_framework_tests --re=framework/CodeInterfaceTests/AcceleratedCFD.per

- Test Description:

- An example of using the AcceleratedCFD code interface. This test is aimed to test the mechanics of the interface (no executable).

- Original Author:

- alfoa

- Creation date:

- 2020-11-03

- The classes tested in this test are:

- Models.Code.AcceleratedCFD 


\section{Undocumented tests}

Currently, There are 22 undocumented tests:

1. Folder: /Users/alfoa/projects/raven_github/raven/plugins/SR2ML/tests/ maintenanceModels. Tests:

- ./raven/plugins/SR2ML/tests/maintenanceModels/test_PMmodel_ operating.xml

- . /raven/plugins/SR2ML/tests/maintenanceModels/test_PMmodel_ standby $\cdot \mathrm{xml}$

2. Folder: /Users/alfoa/projects/raven_github/raven/plugins/LoGos/tests/ MilestoneTestsSeptFY19/use_case_II. Tests:

- . /raven/plugins/LOGOS/tests/MilestoneTestsSeptFY19/use_case_ II/raven_capital_budgeting_II.xml

3. Folder: /Users/alfoa/projects/raven_github/raven/plugins/Logos/tests/ MilestoneTestsSeptFY19/use_case_III. Tests:

- . /raven/plugins/LOGOS/tests/MilestoneTestsSeptFY19/use_case_ III/raven_capital_budgeting_III.xml

4. Folder: /Users/alfoa/projects/raven_github/raven/plugins/HERON/tests/ unit_tests. Tests:

- . /raven/plugins/HERON/tests/unit_tests/testComponent.py

5. Folder: /Users/alfoa/projects/raven_github/raven/plugins/TEAL/tests. Tests:

- ./raven/plugins/TEAL/tests/HourlyObjectOrientedTest.py

6. Folder: /Users/alfoa/projects/raven_github/raven/tests/framework/ unit_tests/utils. Tests:

- ./raven/tests/framework/unit_tests/utils/testFrontUtils.py

7. Folder: /Users/alfoa/projects/raven_github/raven/tests/framework/ unit_tests/Optimizers. Tests:

- . /raven/tests/framework/unit_tests/Optimizers/testFinitedifference. py 
8. Folder: /Users/alfoa/projects/raven_github/raven/tests/framework/ Models/External. Tests:

-./raven/tests/framework/Models/External/load_and_run_fmu.py

9. Folder: /Users/alfoa/projects/raven_github/raven/tests/framework/ Input Templates. Tests:

- ./raven/tests/framework/InputTemplates/uq_maker.py

10. Folder: /Users/alfoa/projects/raven_github/raven/tests/framework/ Input Templates/UQTemplate. Tests:

-./raven/tests/framework/InputTemplates/UQTemplate/new_uq.xml

11. Folder: /Users/alfoa/projects/raven_github/raven/tests/reg_self_ tests. Tests:

- ./raven/tests/reg_self_tests/test_ordered_csv.py

12. Folder: /Users/alfoa/projects/raven_github/raven/doc/workshop/forwardSampl inputs. Tests:

- ./raven/doc/workshop/forwardSampling/inputs/AssembleTests . py

- ./raven/doc/workshop/forwardSampling/inputs/test_1_sample_ and_plot.xml

- ./raven/doc/workshop/forwardSampling/inputs/test_2_normal_ distribution.xml

- ./raven/doc/workshop/forwardSampling/inputs/test_3_initial_ height.xml

- ./raven/doc/workshop/forwardSampling/inputs/test_4_grid_sampler. xml

- ./raven/doc/workshop/forwardSampling/inputs/test_5_basic_stats . xml

- ./raven/doc/workshop/forwardSampling/inputs/test_6_point_sets . xml

- ./raven/doc/workshop/forwardSampling/inputs/test_7_history_ sets. $\mathrm{xml}$

- ./raven/doc/workshop/forwardSampling/inputs/test_8_history_ sync.xml

- ./raven/doc/workshop/forwardSampling/inputs/test_9_time_stats . $\mathrm{xml}$ 


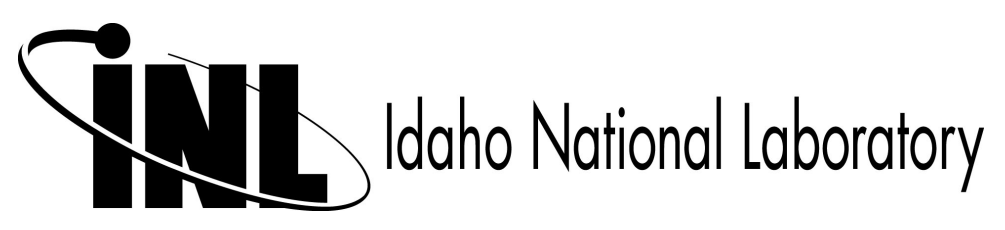

